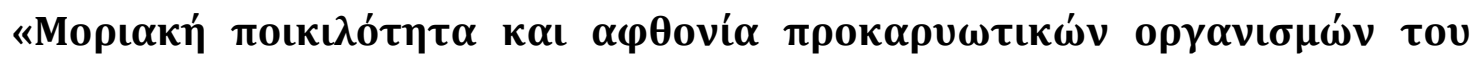

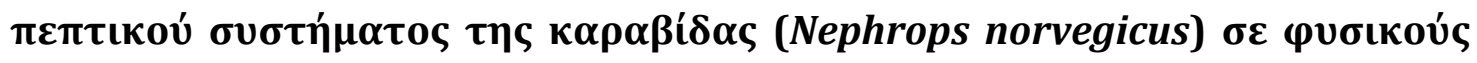

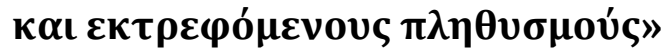




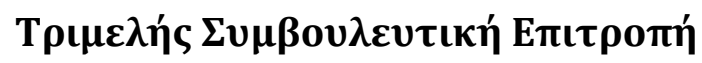

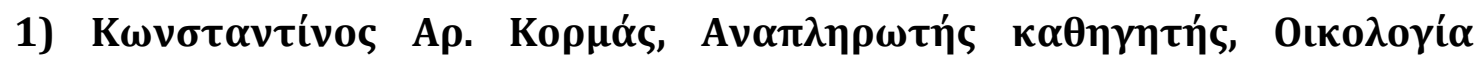

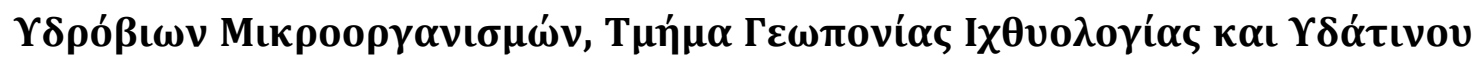

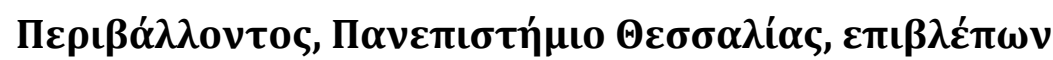

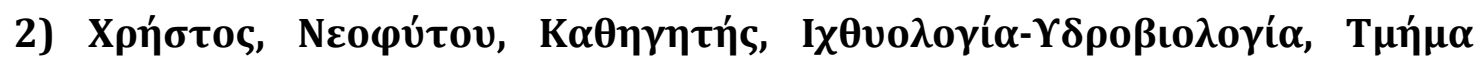

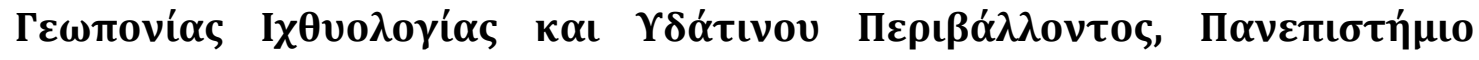
$\Theta \varepsilon \sigma \sigma \alpha \lambda i ́ \alpha \varsigma, \mu \varepsilon ́ \lambda o \varsigma$

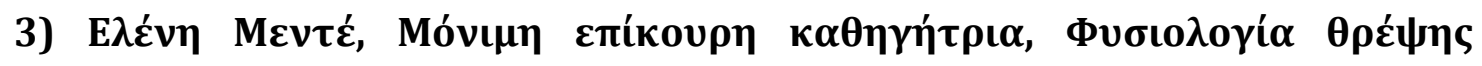

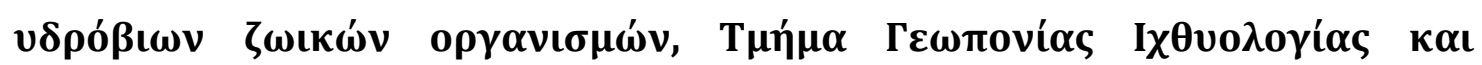

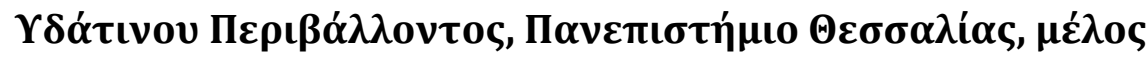

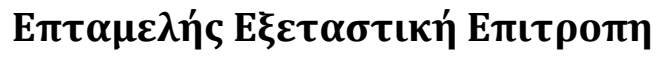

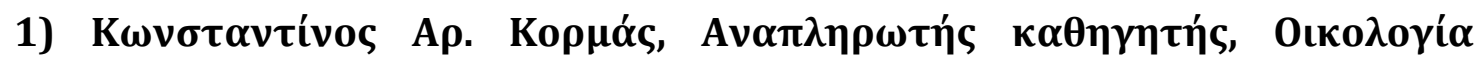

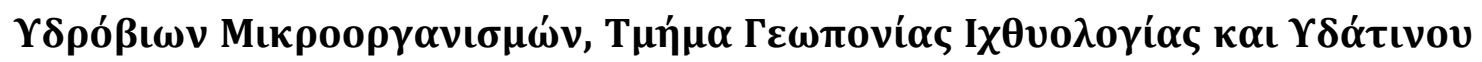

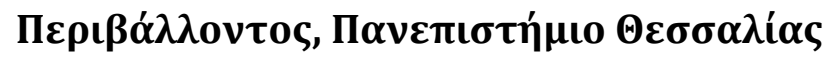

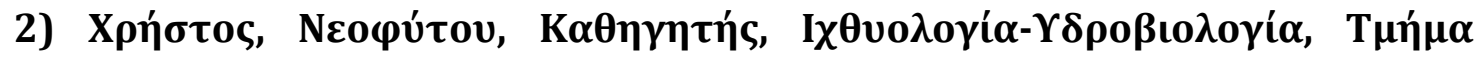

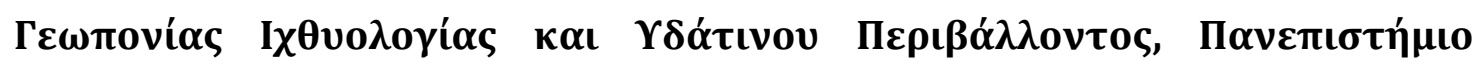
$\Theta \varepsilon \sigma \sigma \alpha \lambda i ́ \alpha \varsigma$

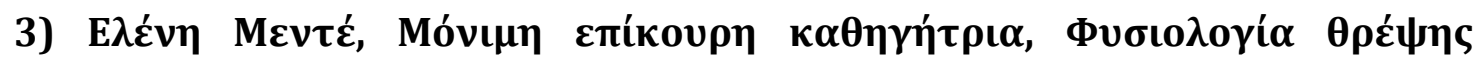

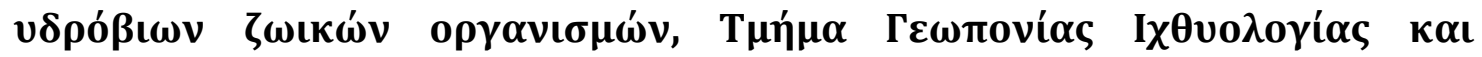

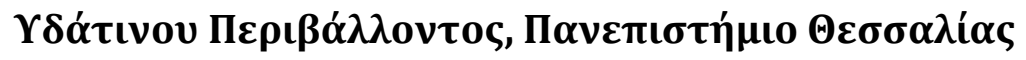

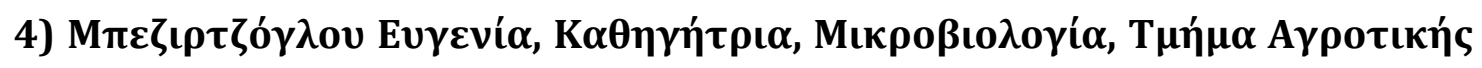

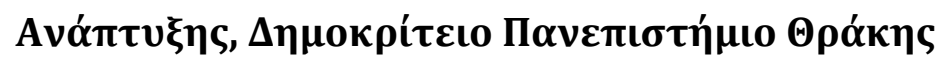

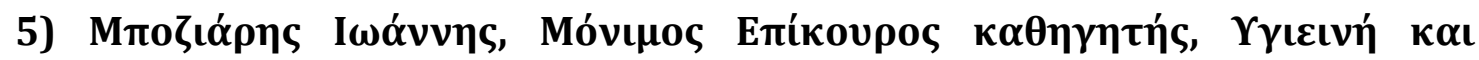

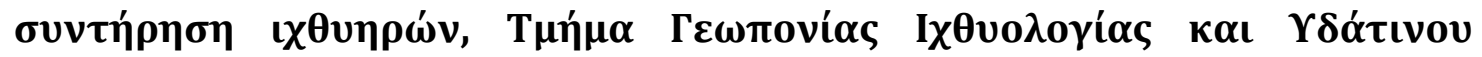

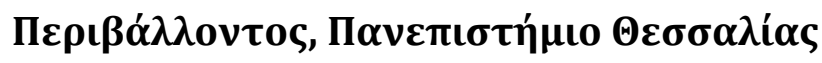

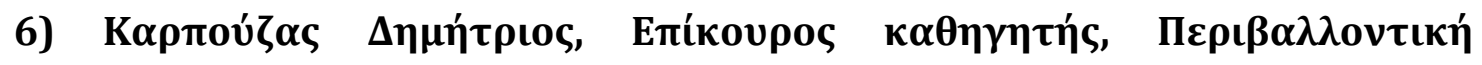

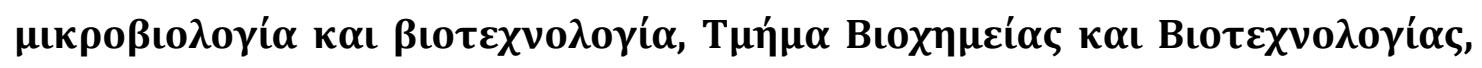

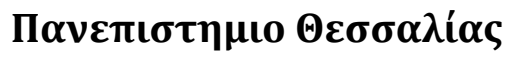

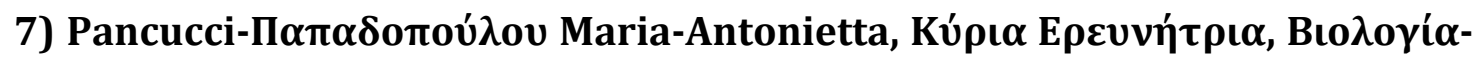

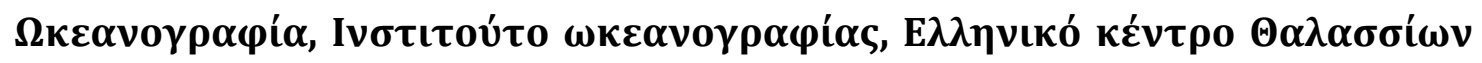

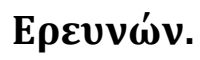




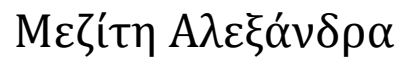

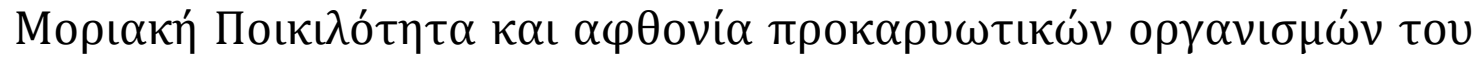

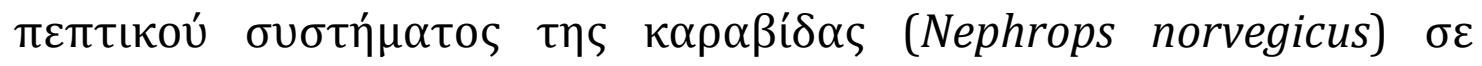

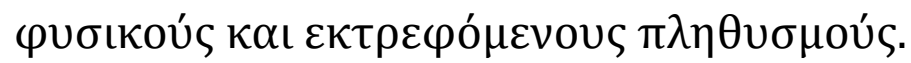




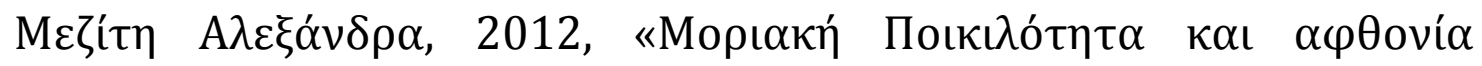

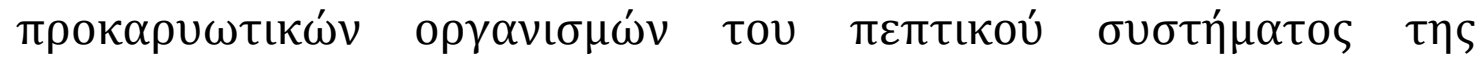

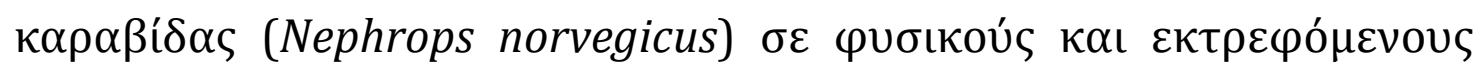
$\pi \lambda \eta \theta v \sigma \mu o u ́ \varsigma »$

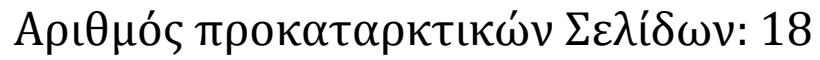

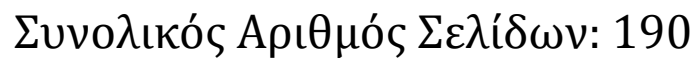

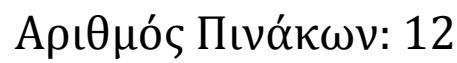

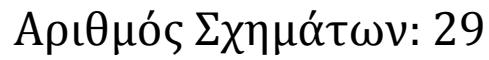

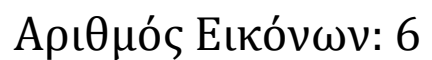

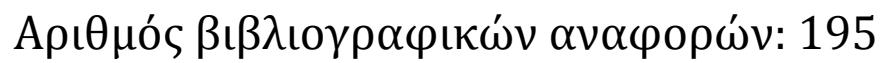




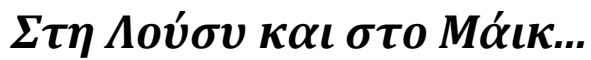

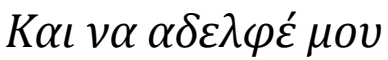

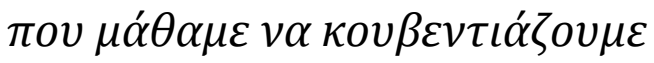

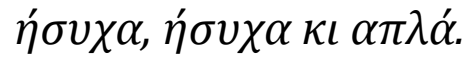

$K \alpha \tau \alpha \lambda \alpha \beta \alpha \iota v o ́ \mu \alpha \sigma \tau \varepsilon \tau \omega ́ \rho \alpha$ $\delta \varepsilon \chi \rho \varepsilon \iota \alpha ́ \zeta$

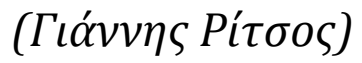




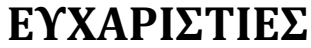

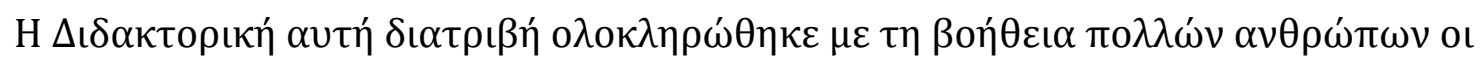

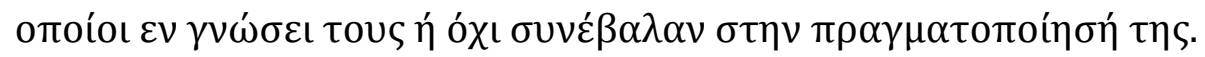

$\Theta \alpha \eta^{\prime} \theta \varepsilon \lambda \alpha \lambda o \iota \pi o ́ v v \alpha \varepsilon v \chi \alpha \rho\left\llcorner\sigma \tau \sigma^{\prime} \sigma \omega:\right.$

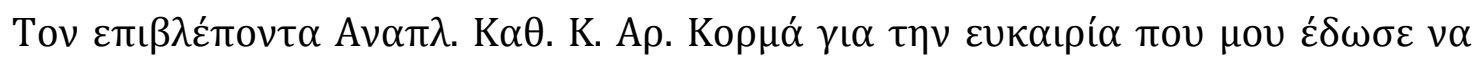

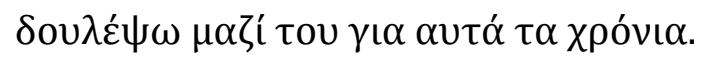

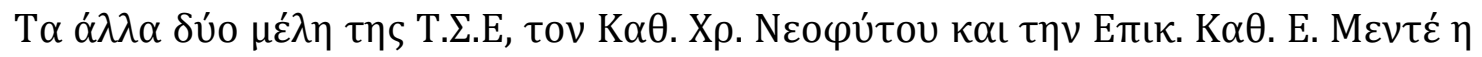

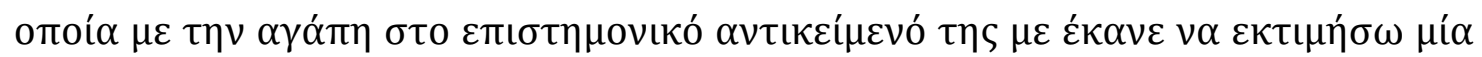

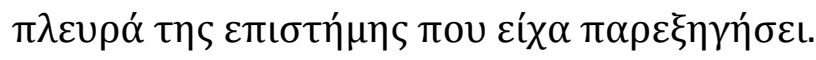

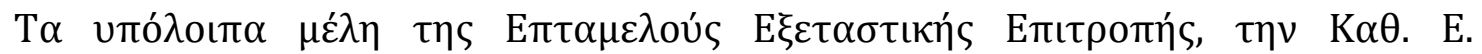

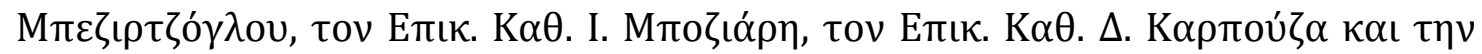

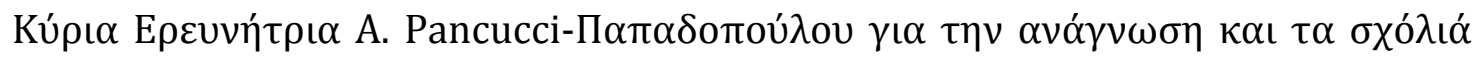

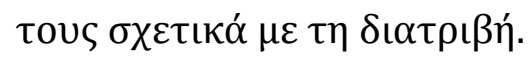

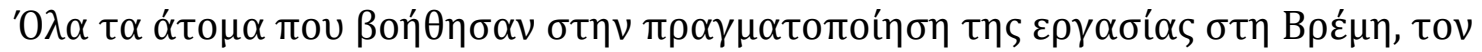

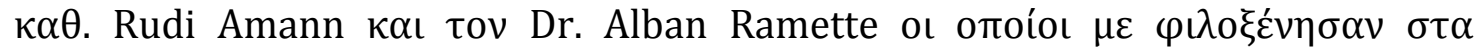

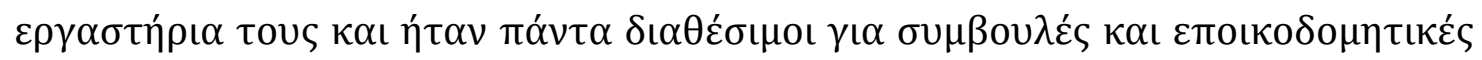

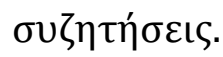

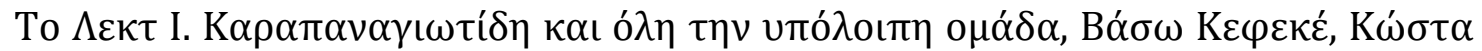

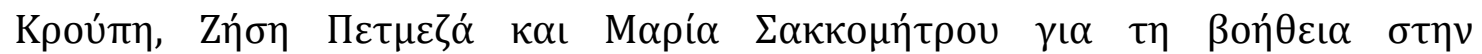

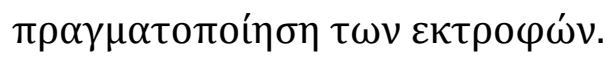

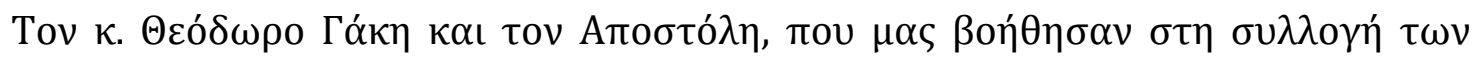

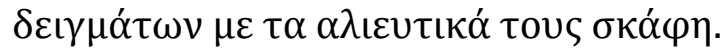

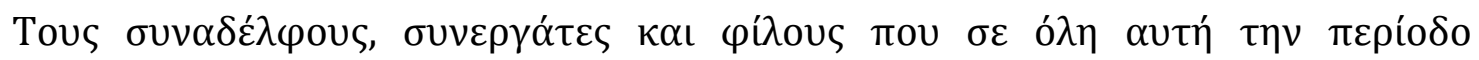

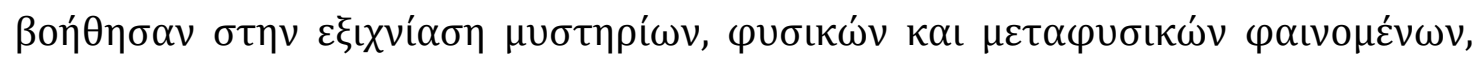

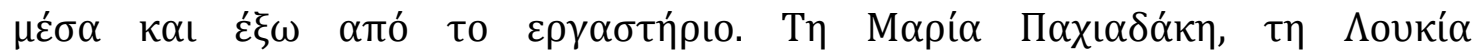




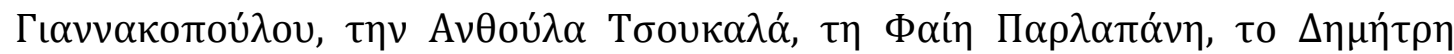

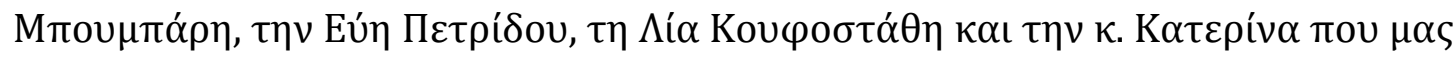
$\pi \rho o ́ \sigma \varepsilon \chi \varepsilon \sigma \alpha \nu \pi \alpha \iota \delta$ เó $\rceil\rceil$. .

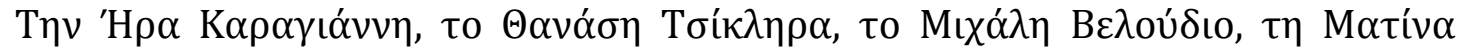

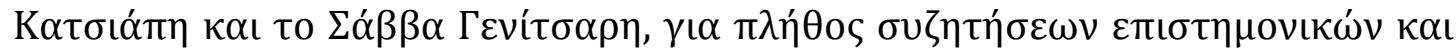

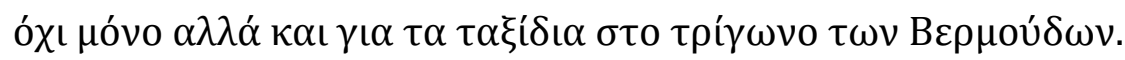

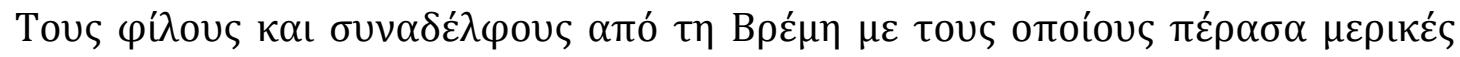

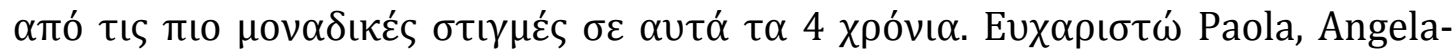

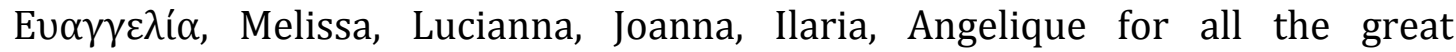
moments!!!

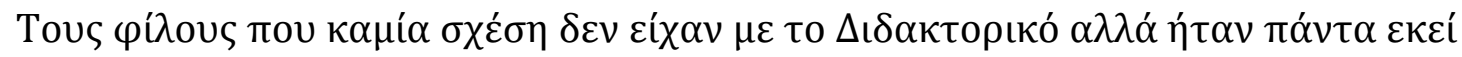

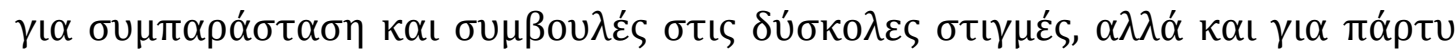

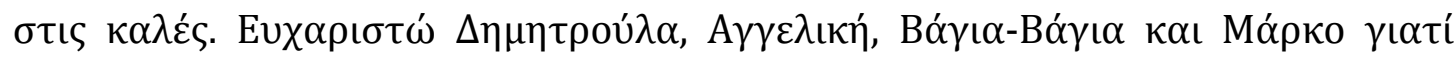

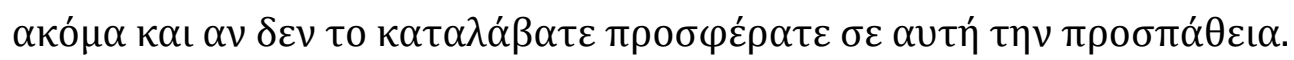

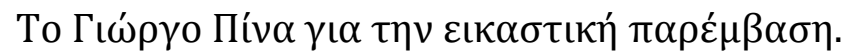

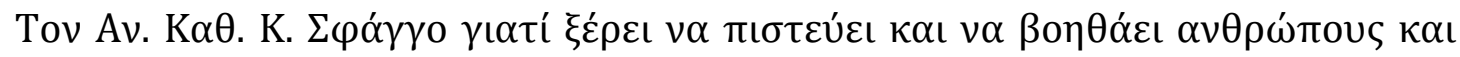

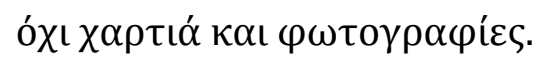

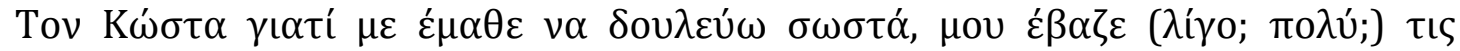

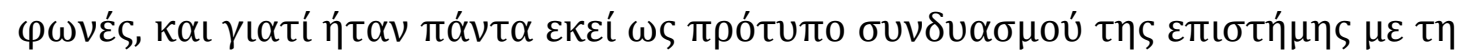
$\zeta \omega \eta \dot{~}$

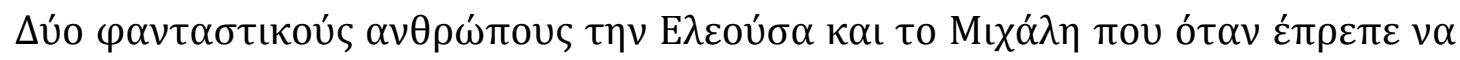

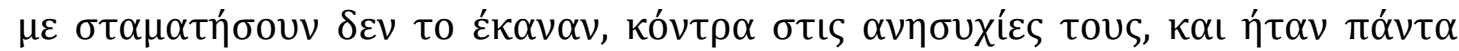

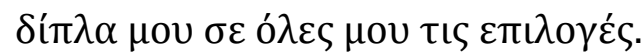

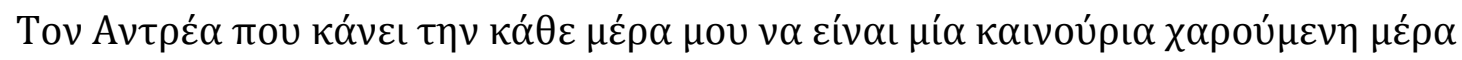
$(\pi . \pi) ! ! !$ 


\section{ПЕРІ $\Lambda$ НН}

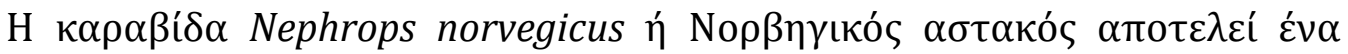

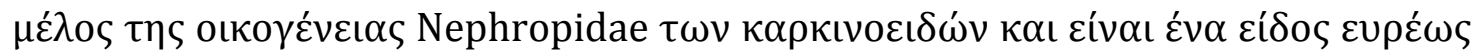

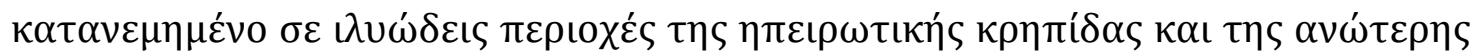

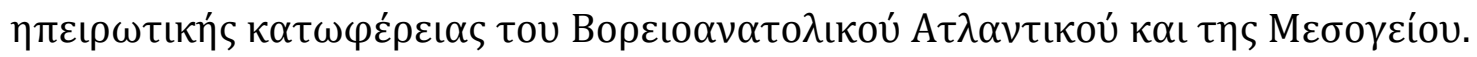

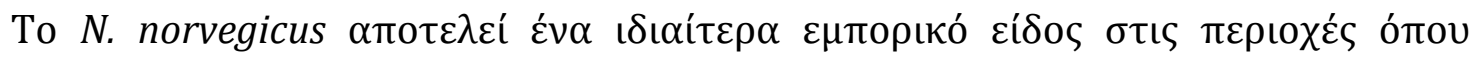

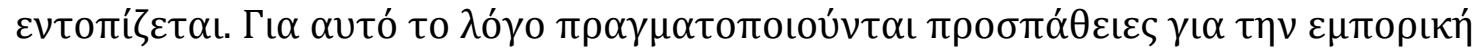

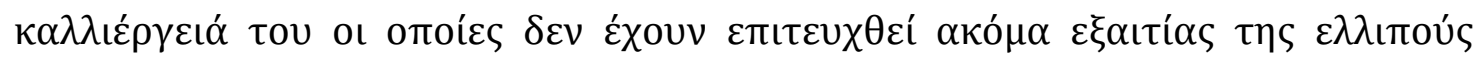

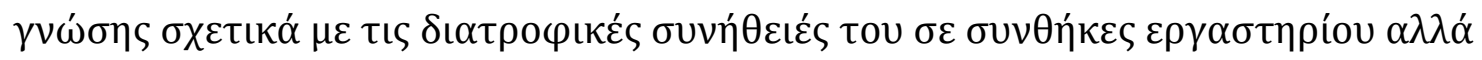

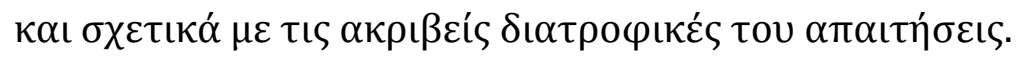

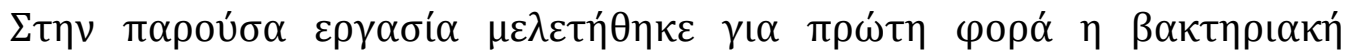

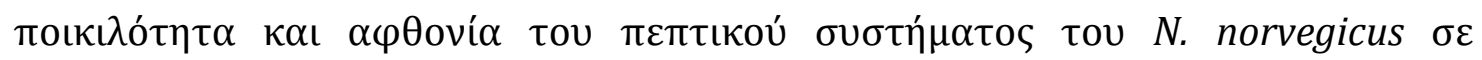

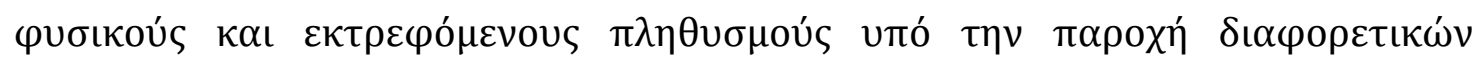

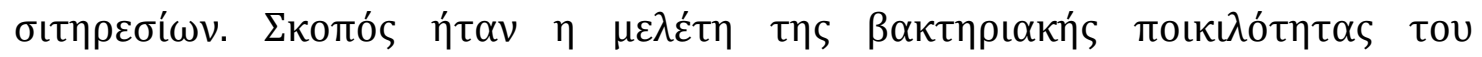

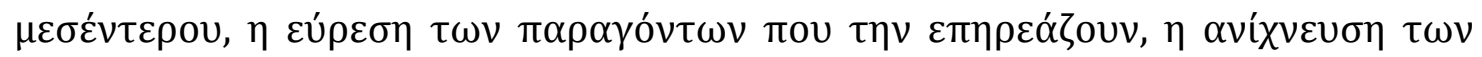

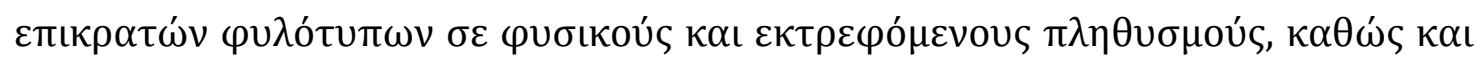

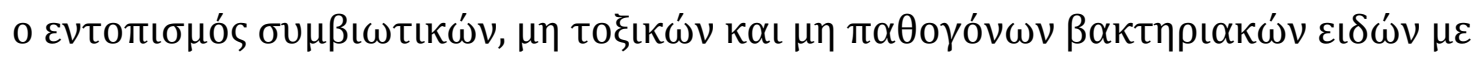

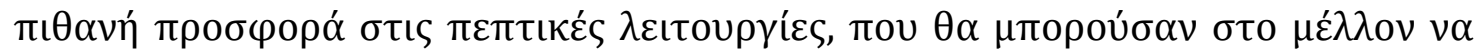

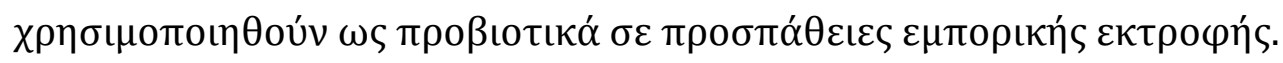

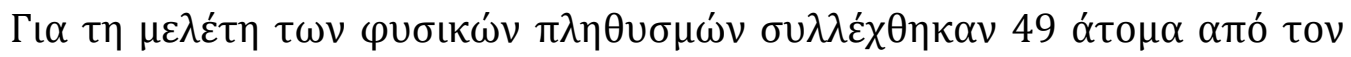

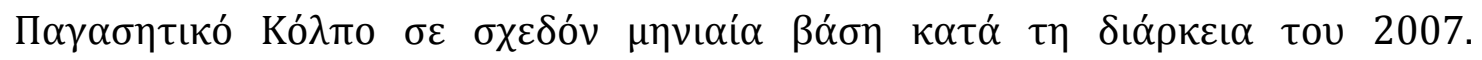

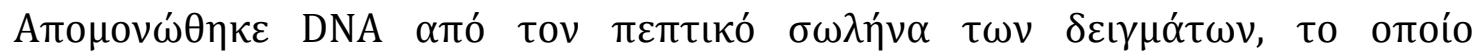

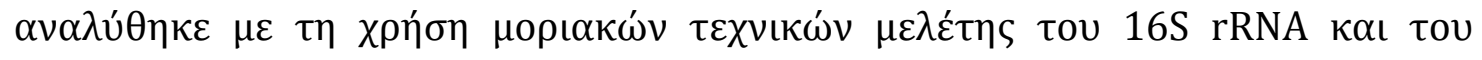




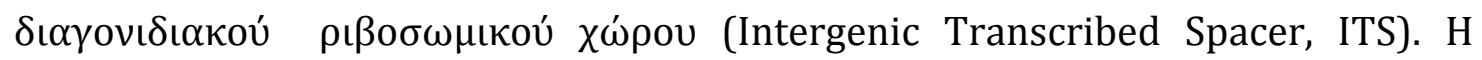

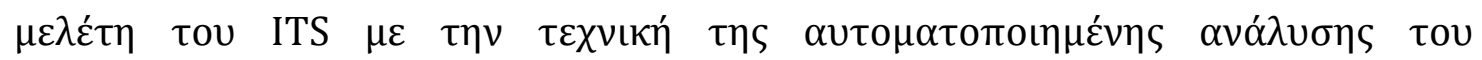

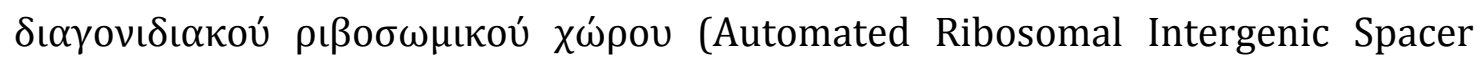

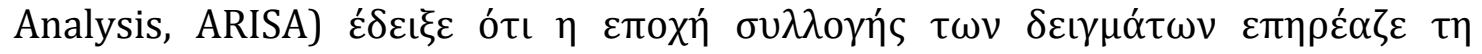

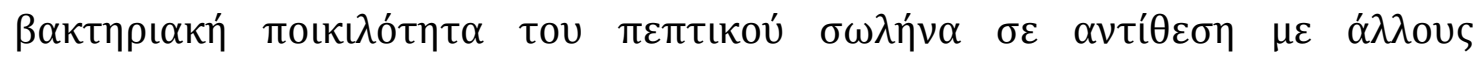

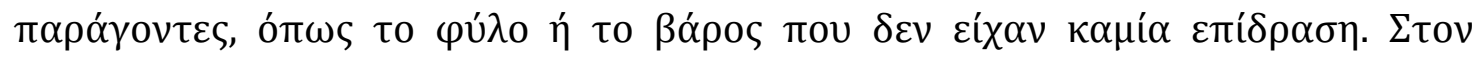

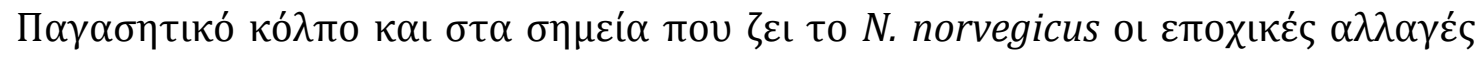

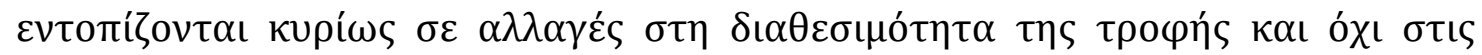

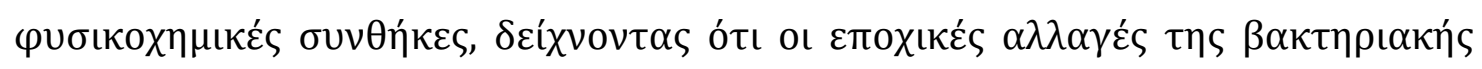

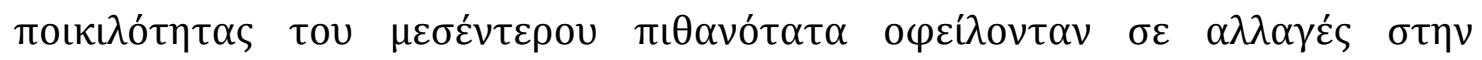

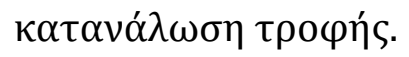

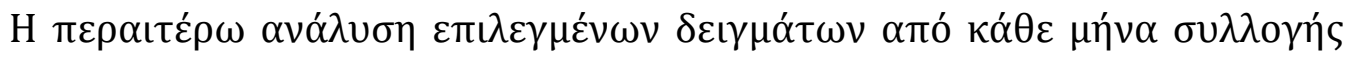

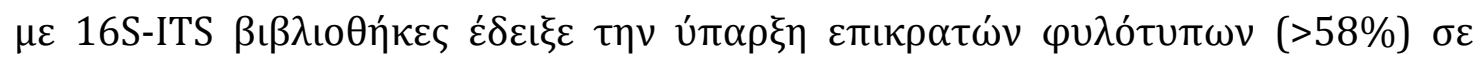

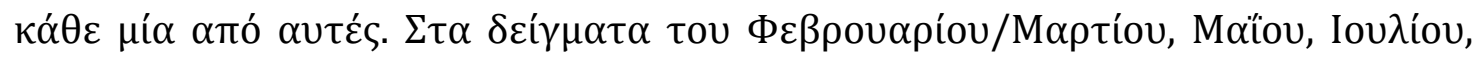

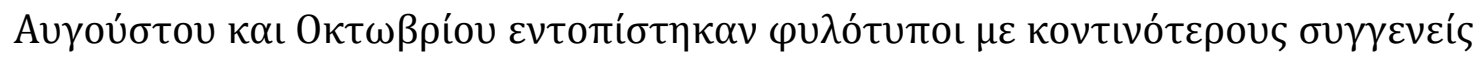

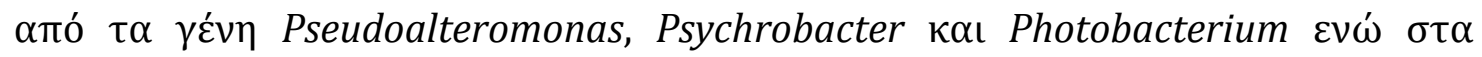

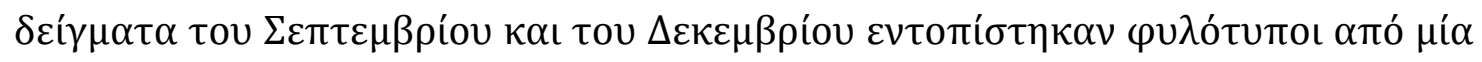

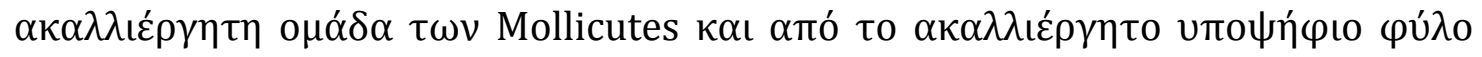
CK-1C4-19, $\alpha v \tau i ́ \sigma \tau o \iota \chi \alpha . ~ H ~ \pi \alpha \rho o v \sigma i ́ \alpha \tau \omega \nu \alpha \kappa \alpha \lambda \lambda \iota \varepsilon ́ \rho \gamma \eta \tau \omega \nu$ Mollicutes $\sigma \tau \alpha \delta \varepsilon \dot{\gamma} \gamma \mu \alpha \tau \alpha$

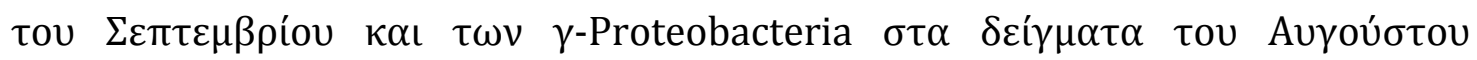

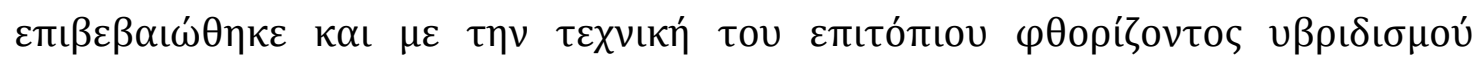

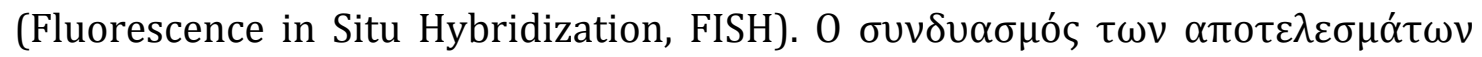

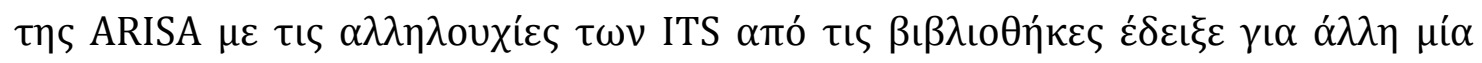

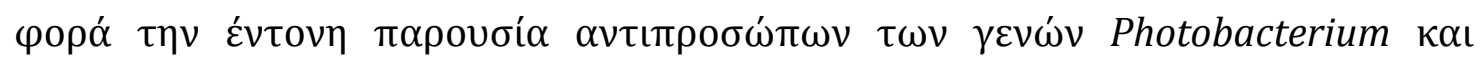

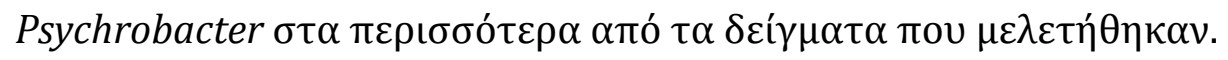


Гı

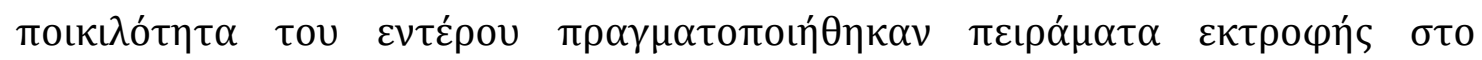

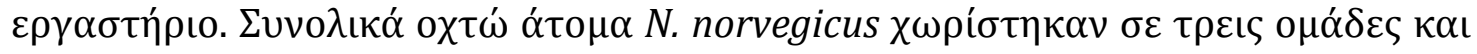

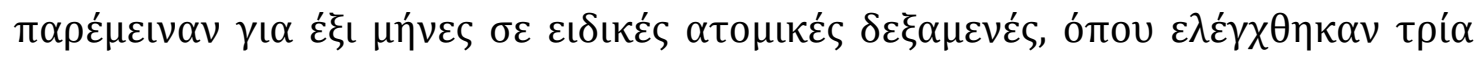

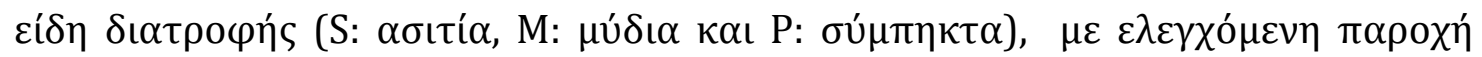

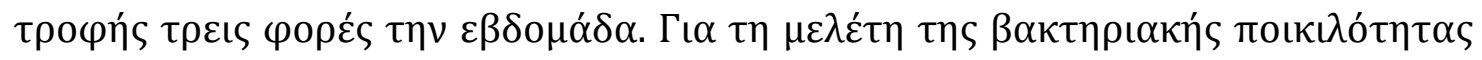

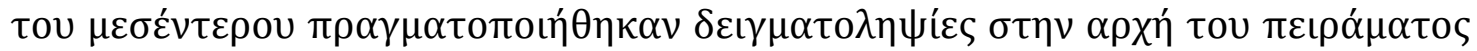

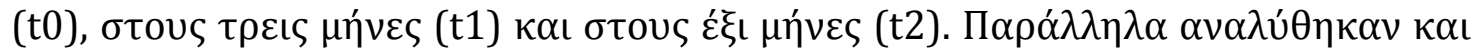

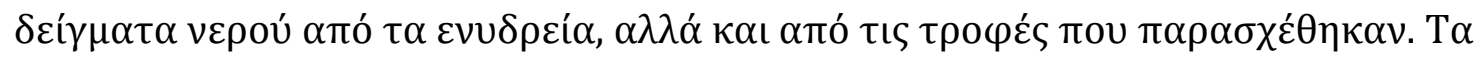

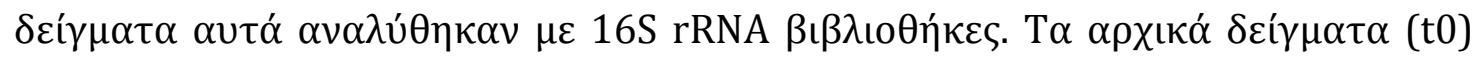

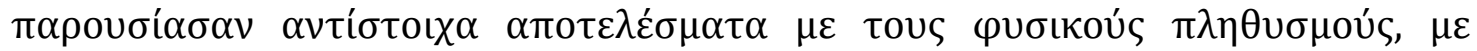

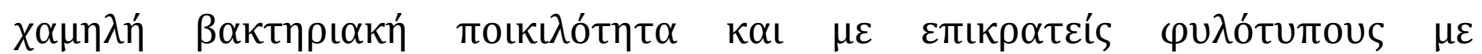

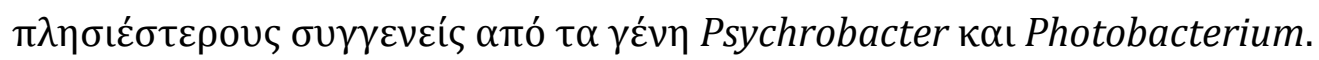

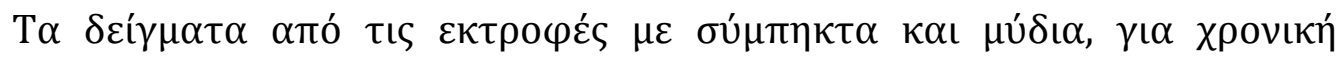

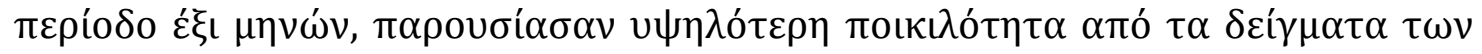

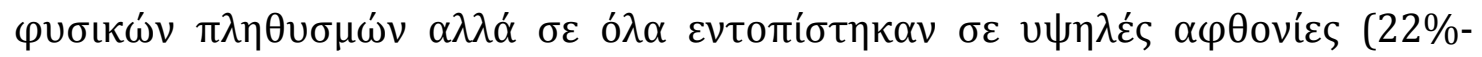

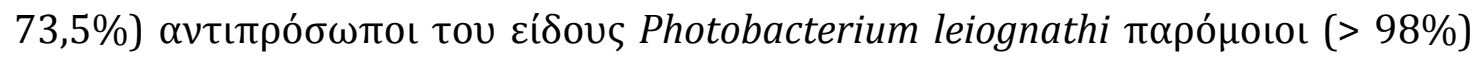

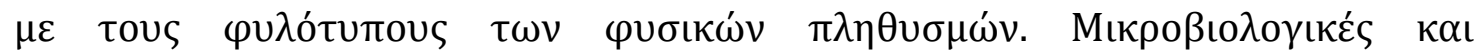

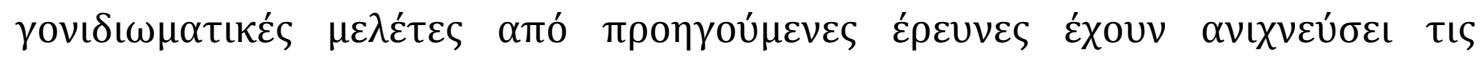

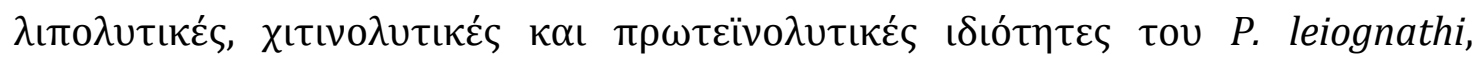

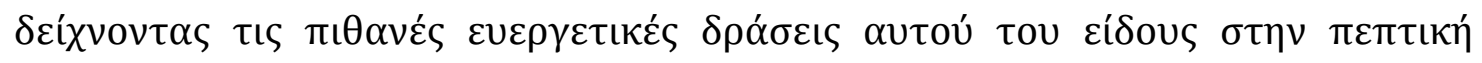
$\lambda \varepsilon\llcorner\tau o u \rho \gamma i ́ \alpha$ tou N. norvegicus.

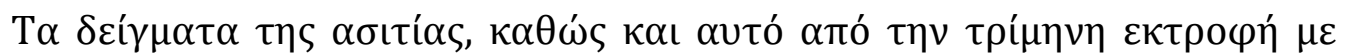

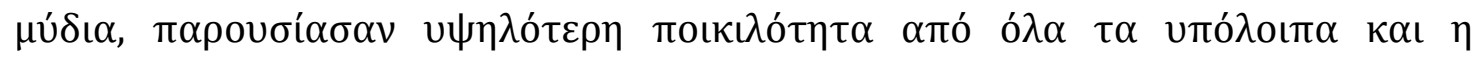

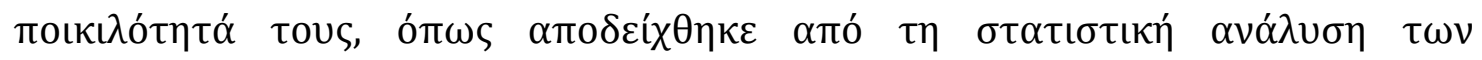




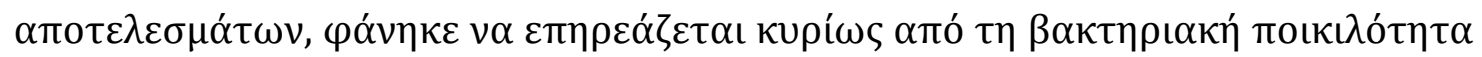

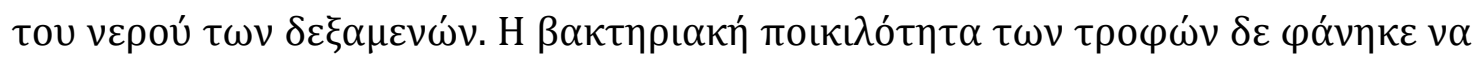

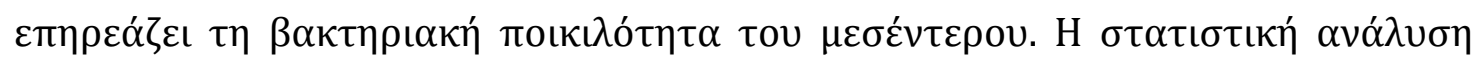
$\tau \omega \nu$ a

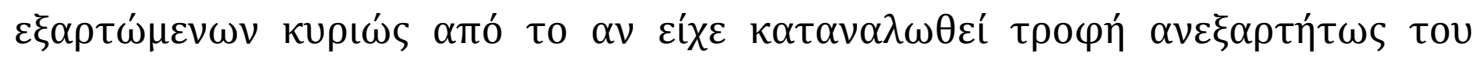

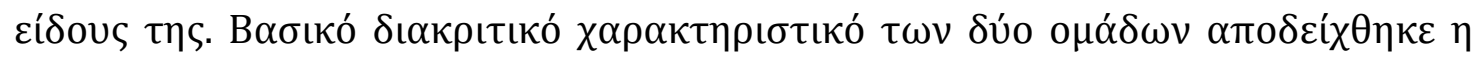

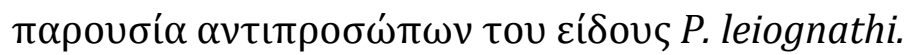

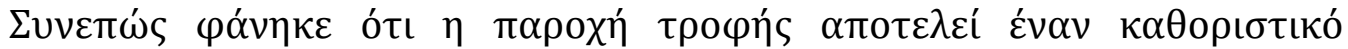

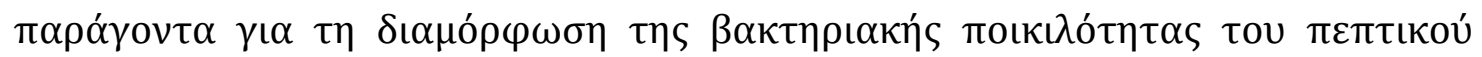

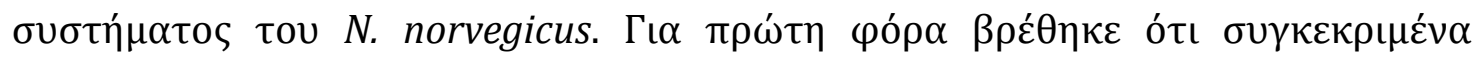

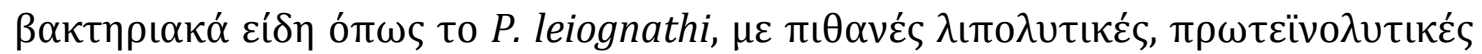

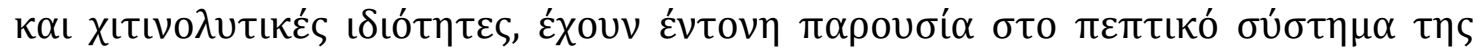

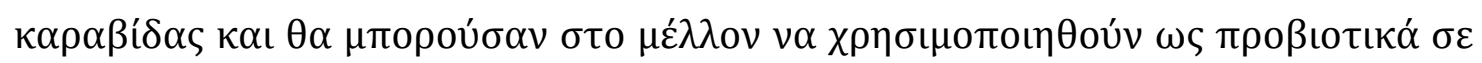

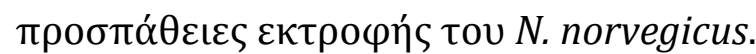

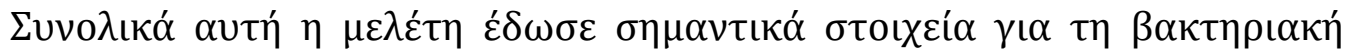

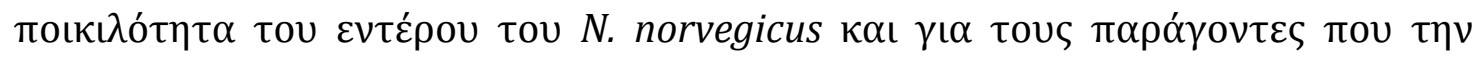

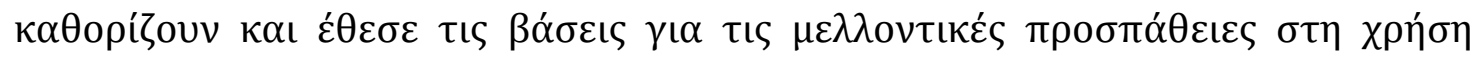

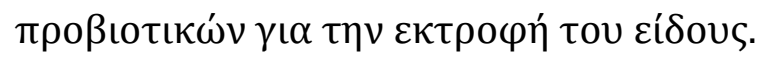

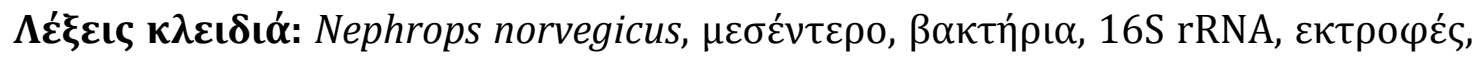

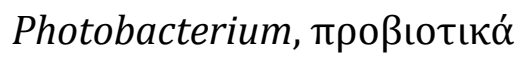




\section{ПEPIEXOMENA}

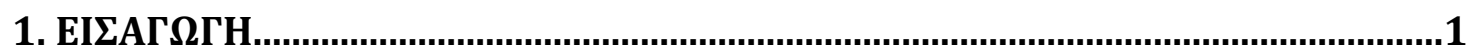

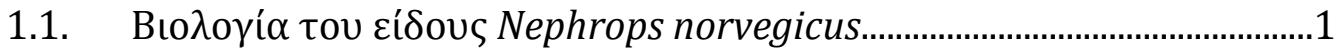

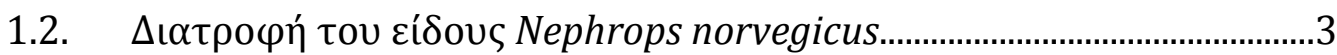

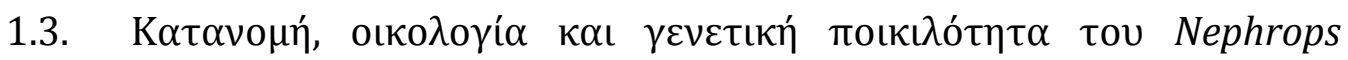

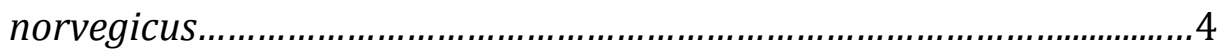

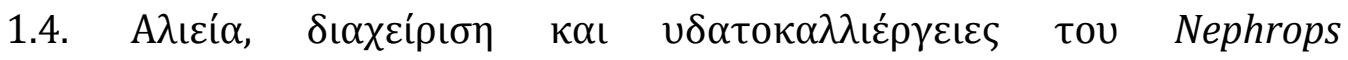
norvegicus.

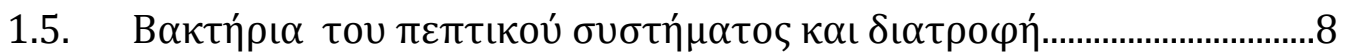

1.6. В Вкєฑ์́

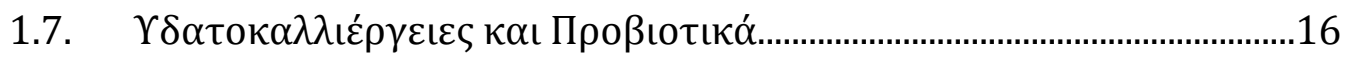

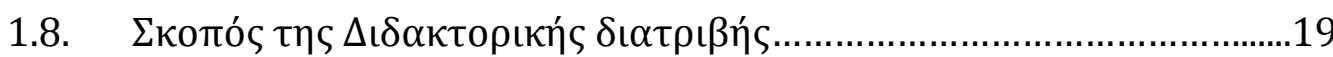

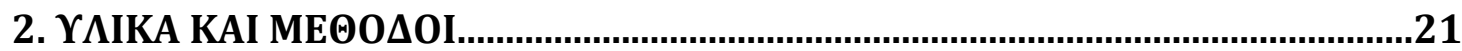

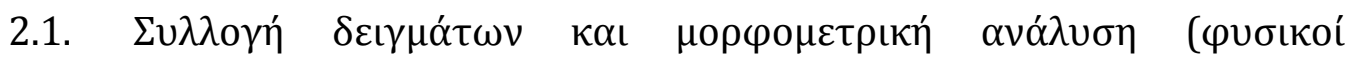

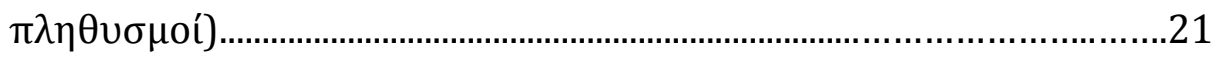

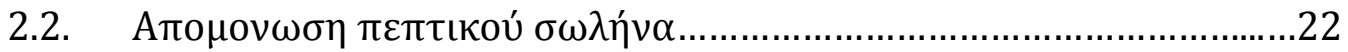

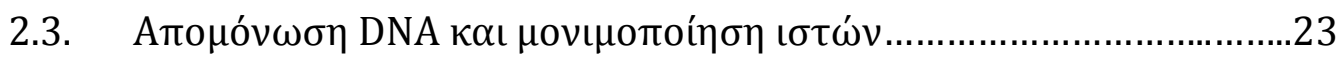

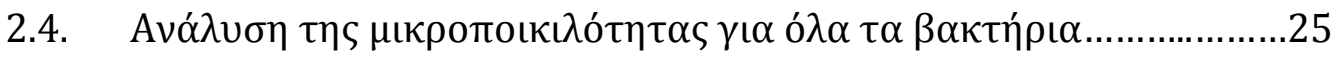

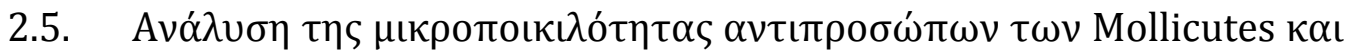

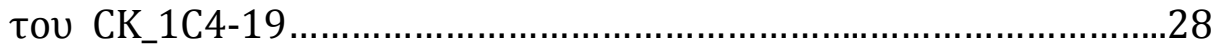

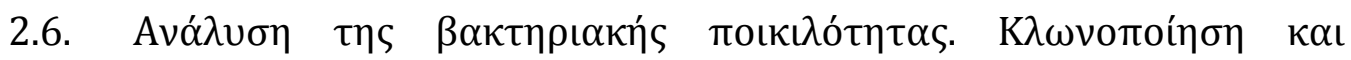

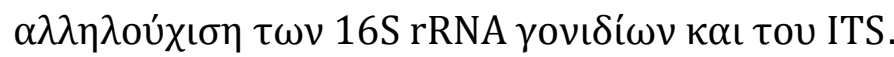

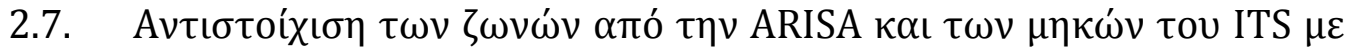

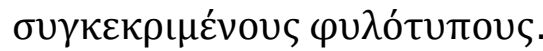
.33

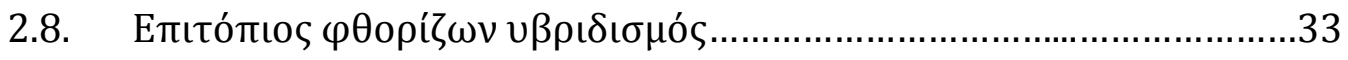

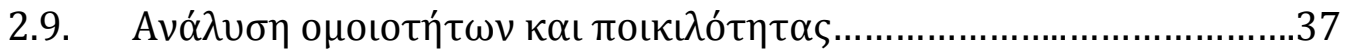




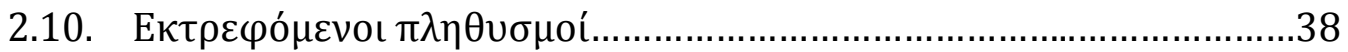

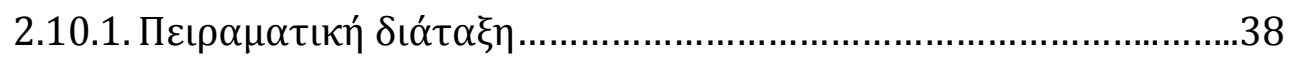

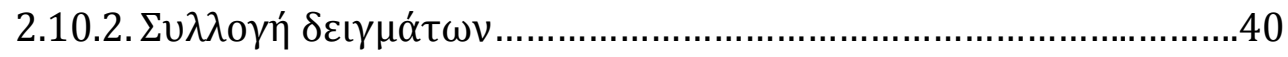

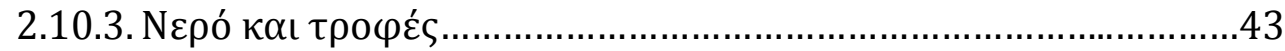

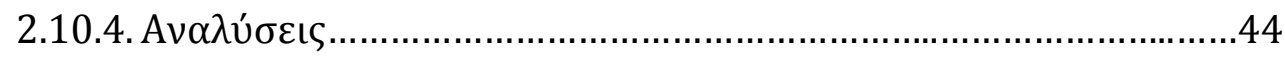

3. АПОТЕ ЕЕМАТА.................................................................................48

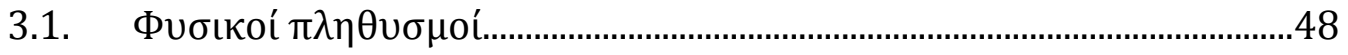

3.1.1. Avó่

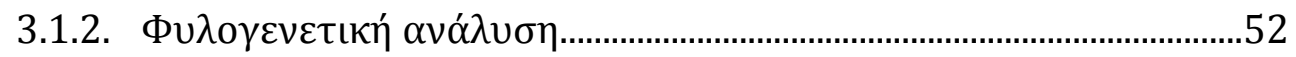

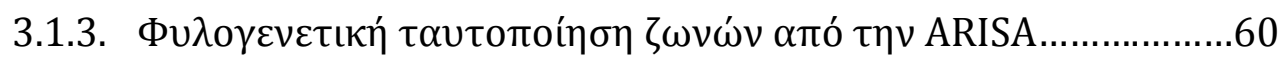

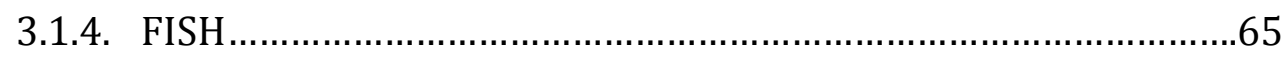

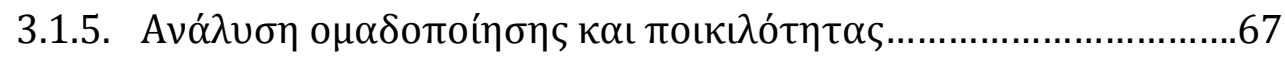

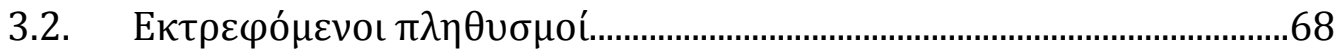

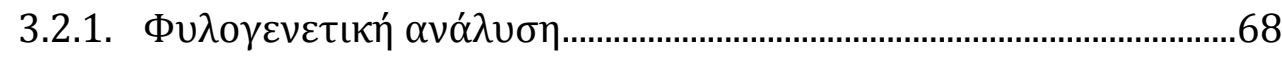

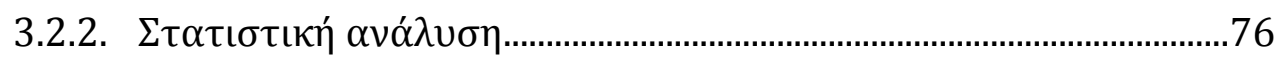

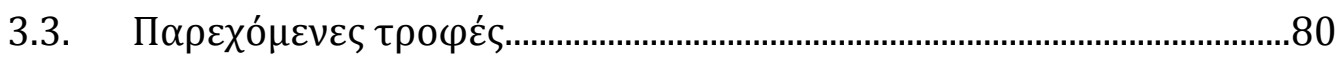

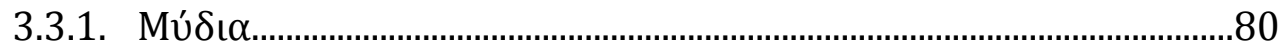

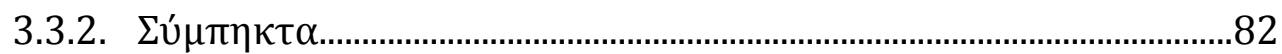

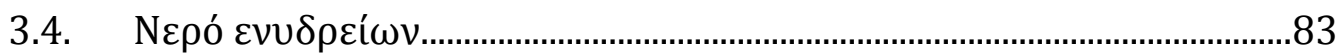

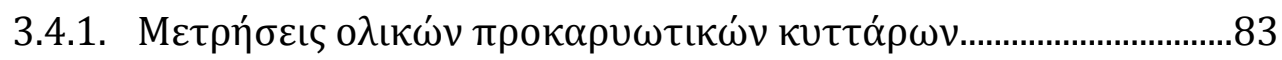

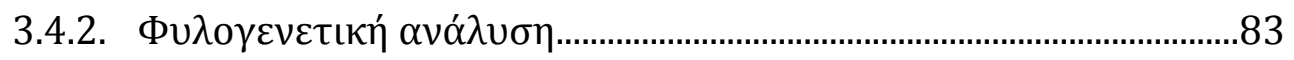

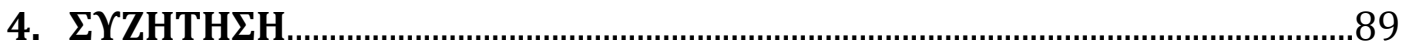

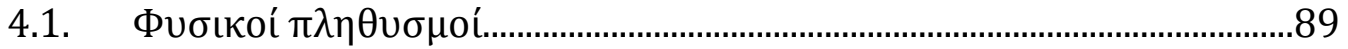

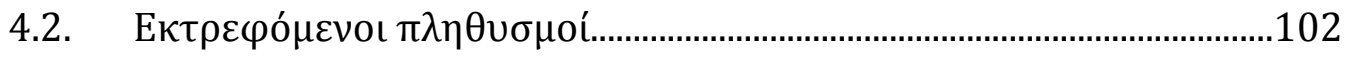

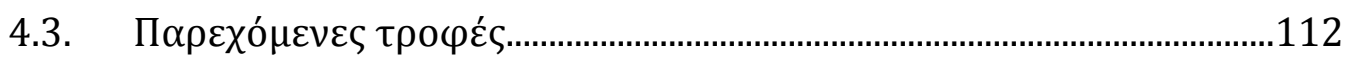

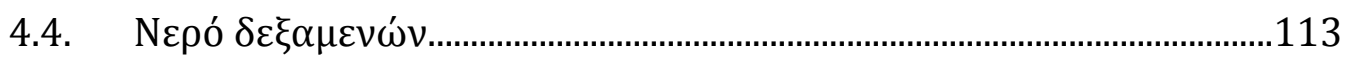

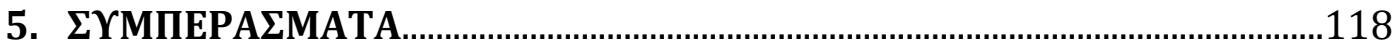

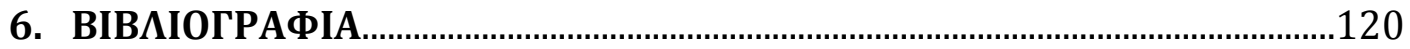

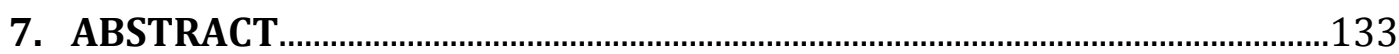

\section{ПАРАРТНМА}




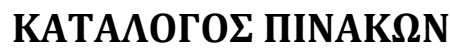

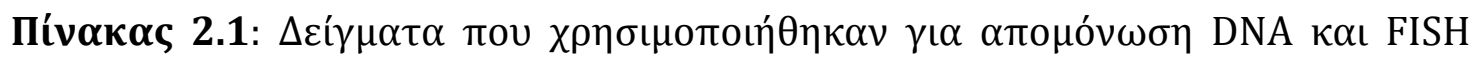

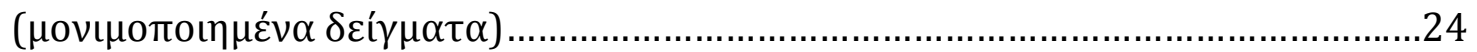

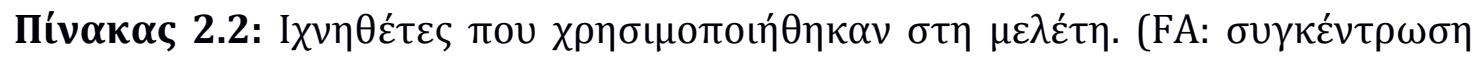
$\varphi о \rho \mu \alpha \mu$ เ́́ov, $\mathrm{W}=\mathrm{A} / \mathrm{T})$

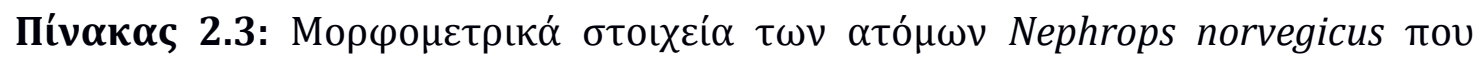

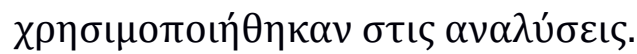
. .41

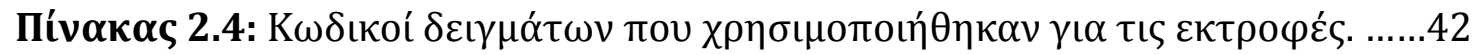

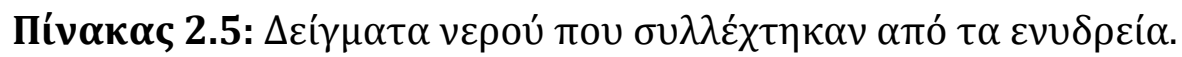

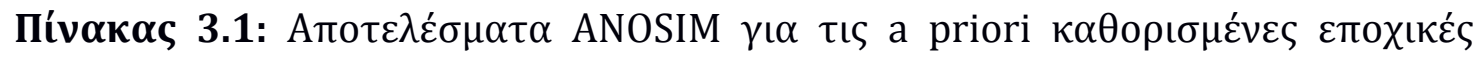

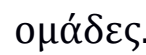

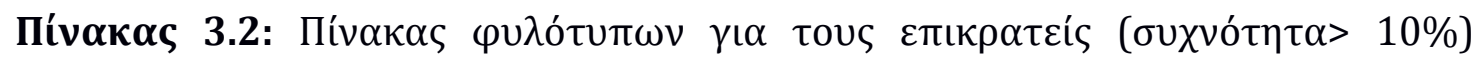

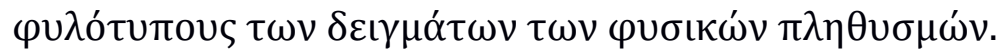

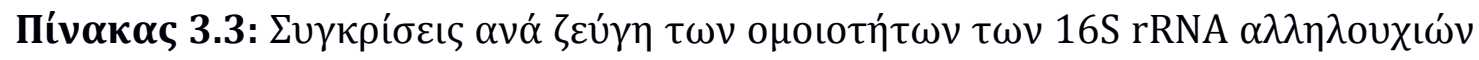

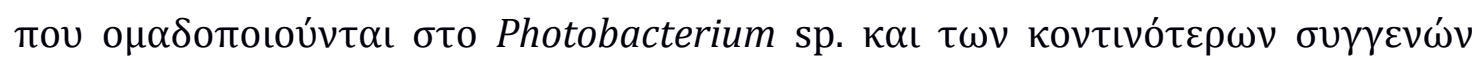
tous .57

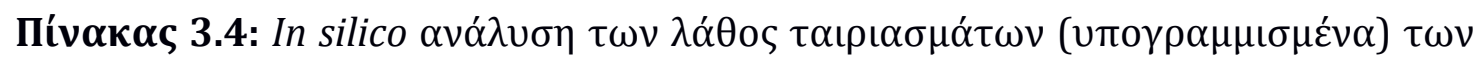

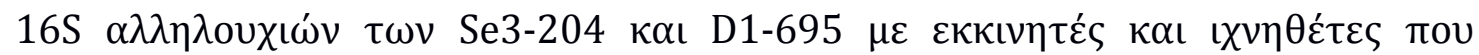

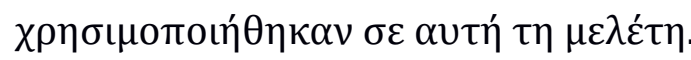
.60

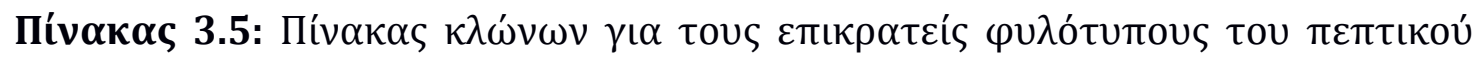

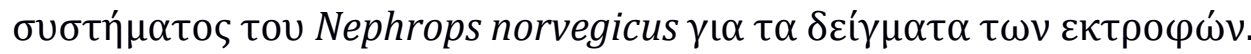
.70

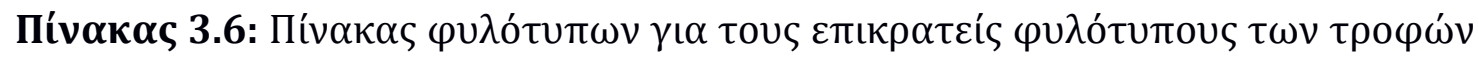

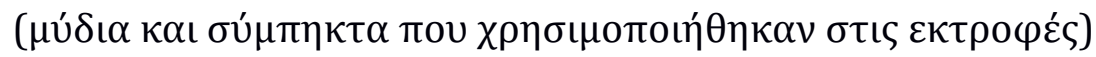
.82

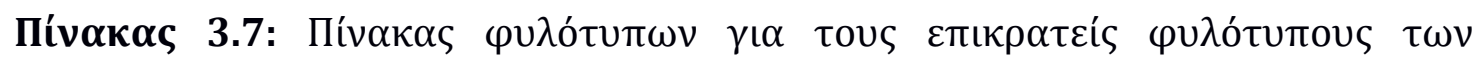

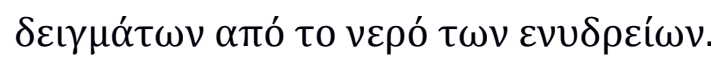
.86

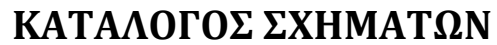

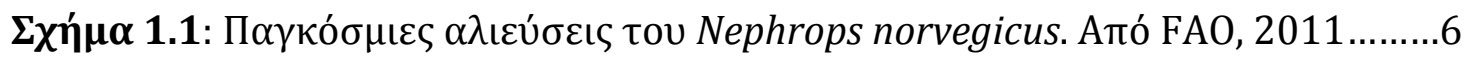




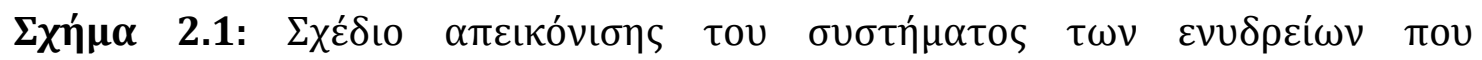

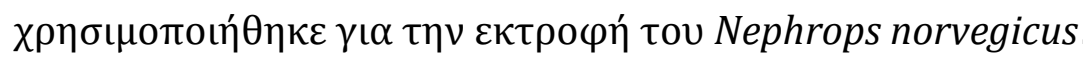

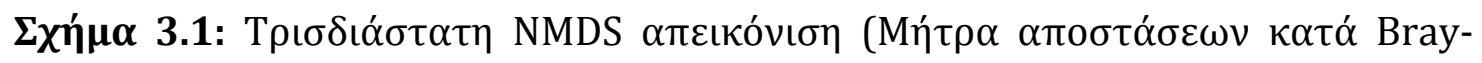

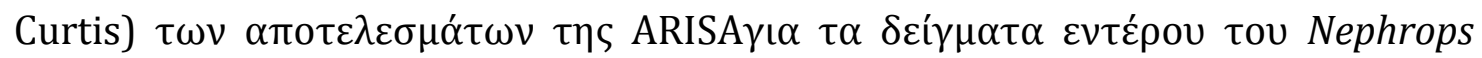
norvegicus (stress $=0,17$ ).

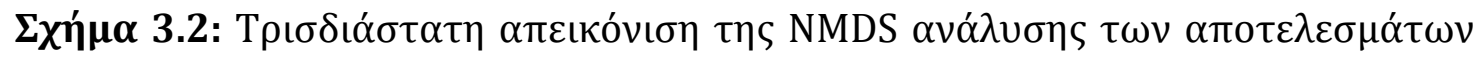

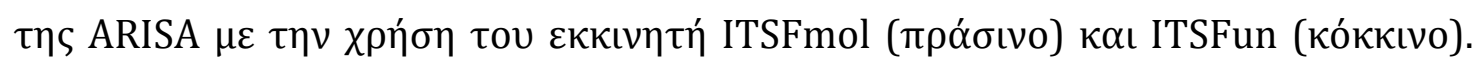

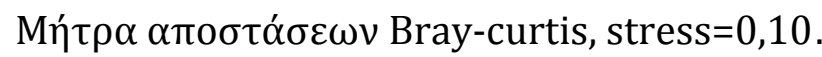
50

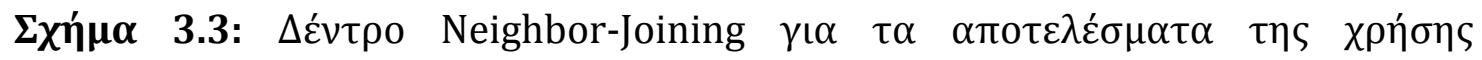

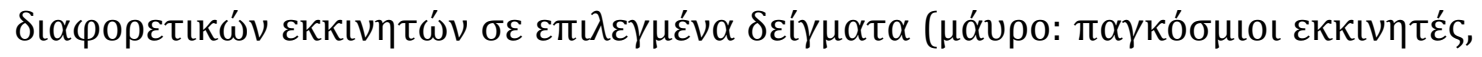

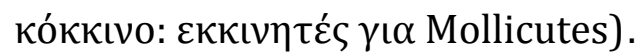

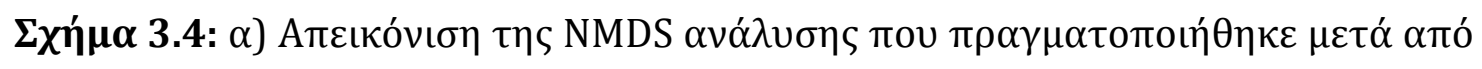

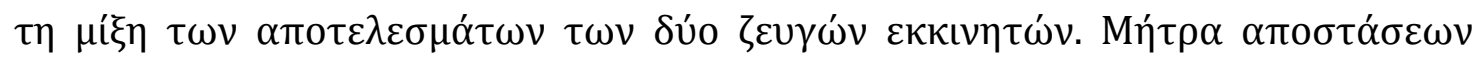

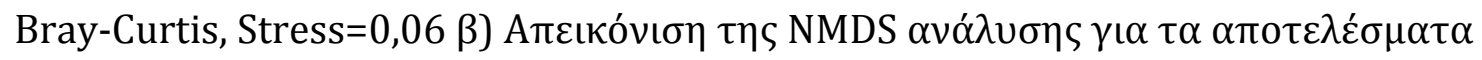

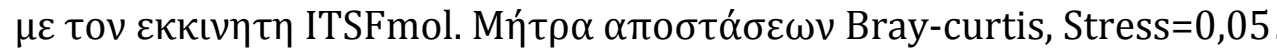
.52

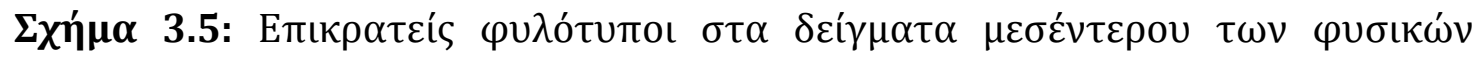

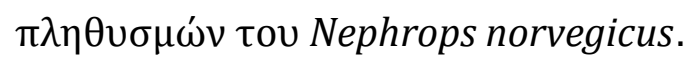
55

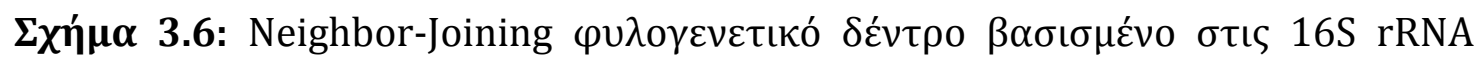

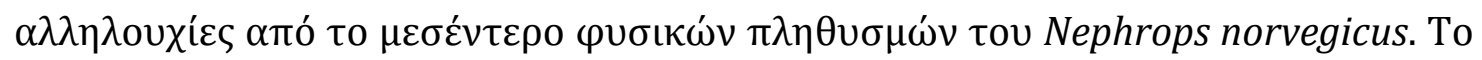

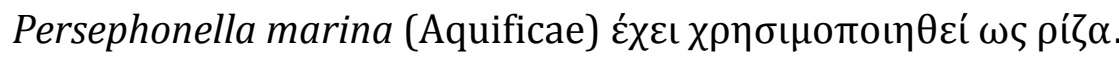
.56

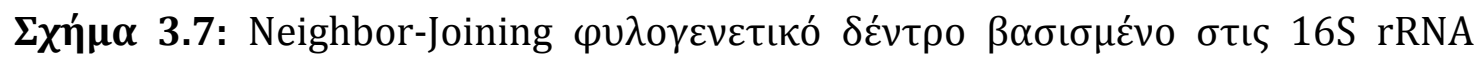

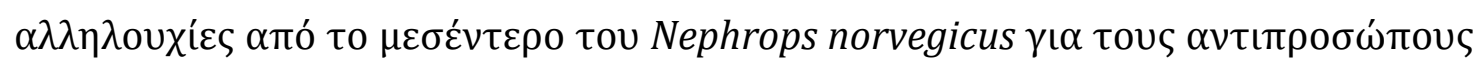

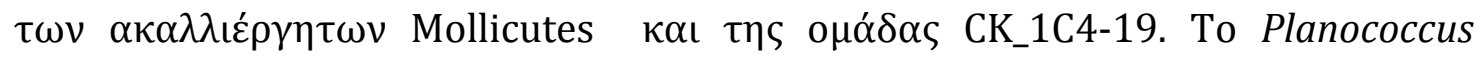

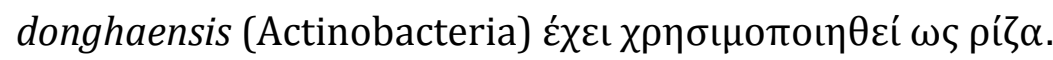
.58

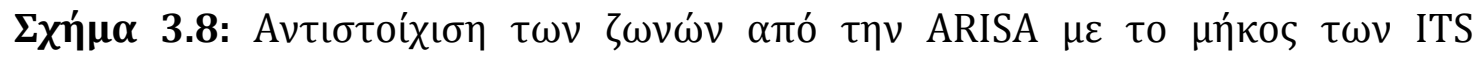

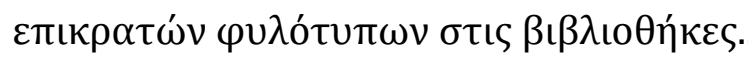

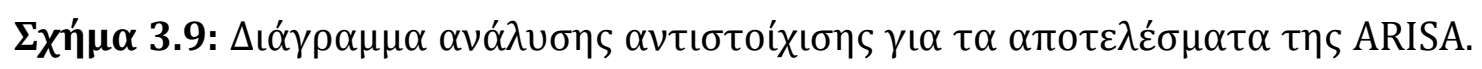

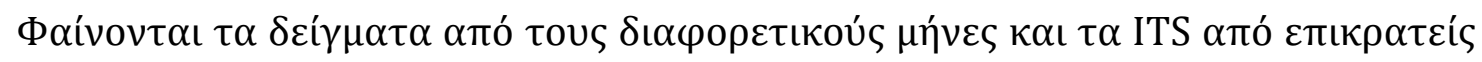

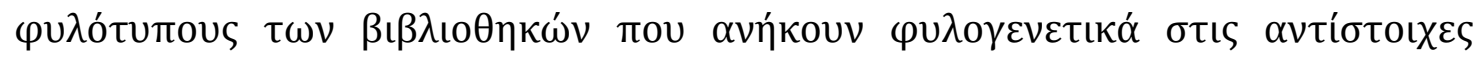
o $\mu \alpha ́ \delta \varepsilon \varsigma$

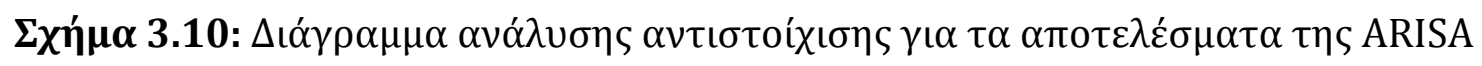

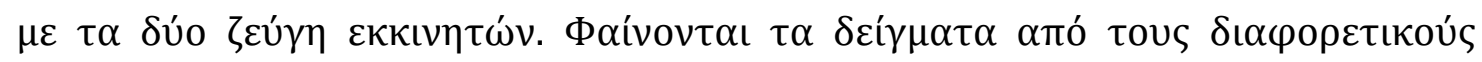

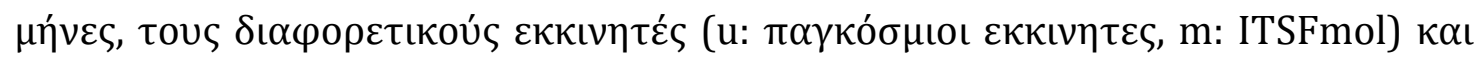




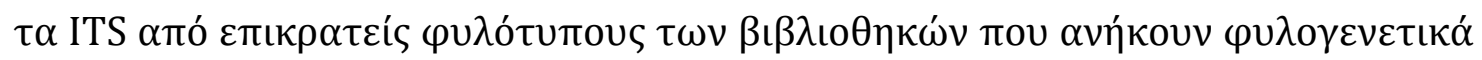

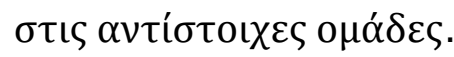

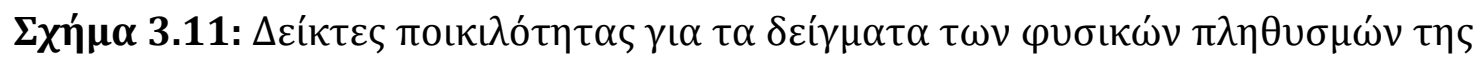
к $\alpha \rho \beta \hat{i} \delta \alpha \varsigma$ Nephrops norvegicus.

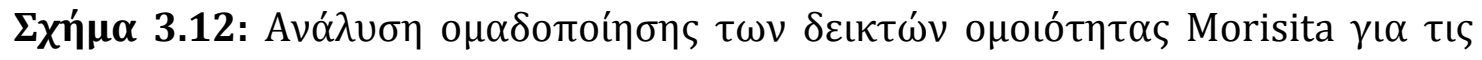

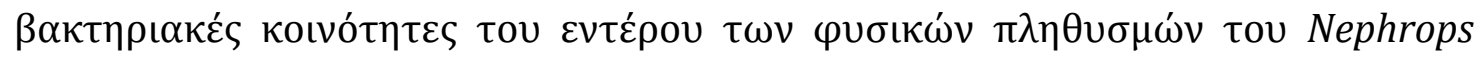
norvegicus.

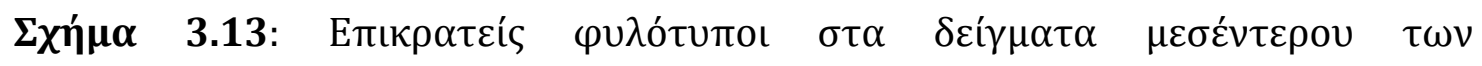

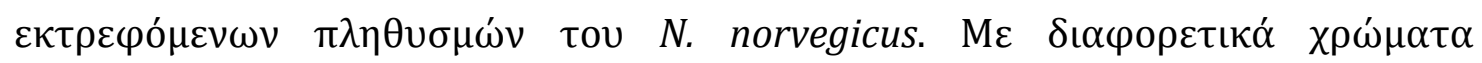

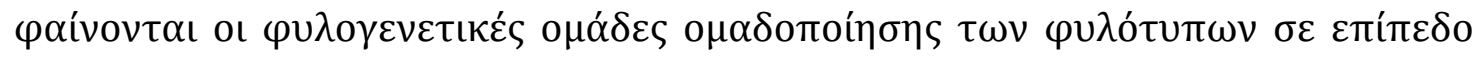

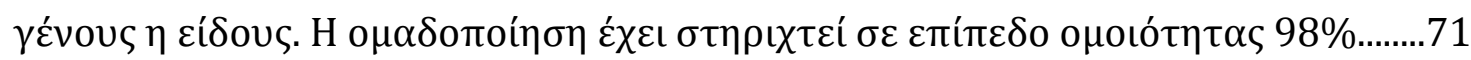

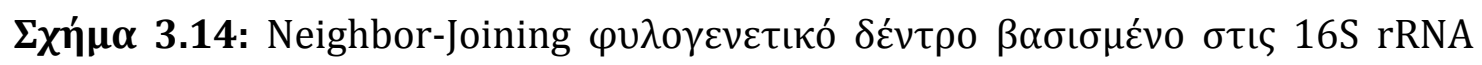

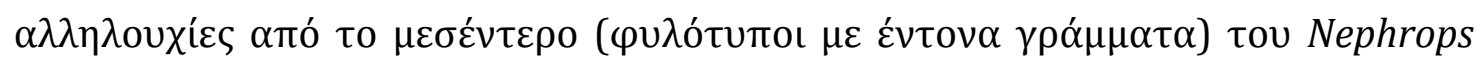

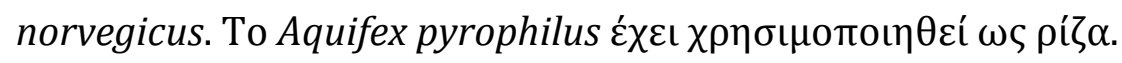
.72

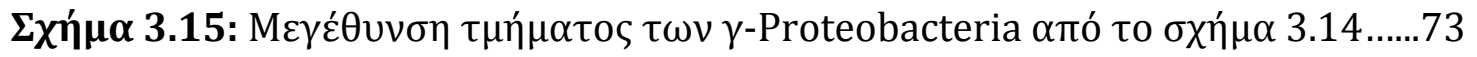

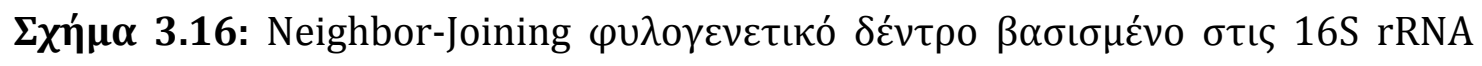

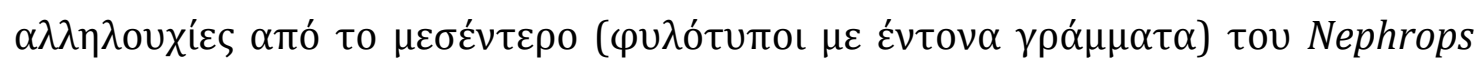

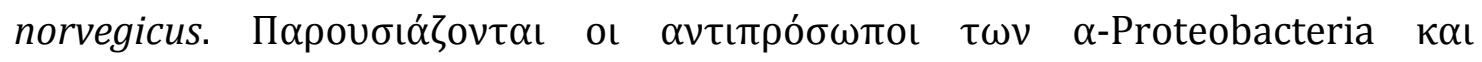

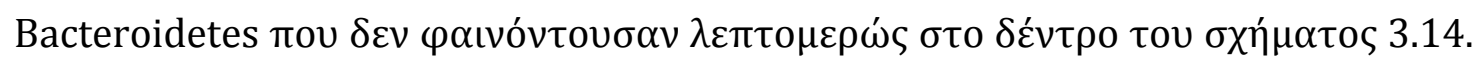

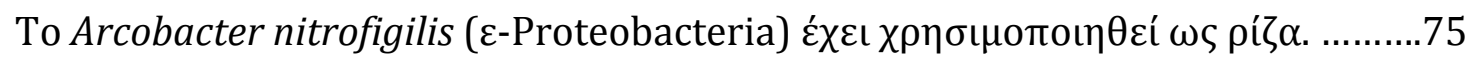

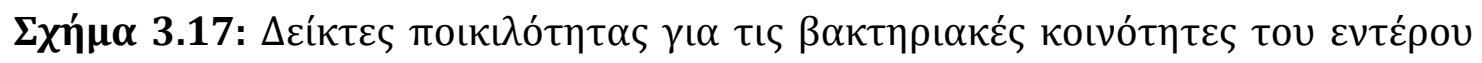

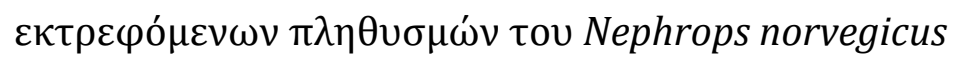
76

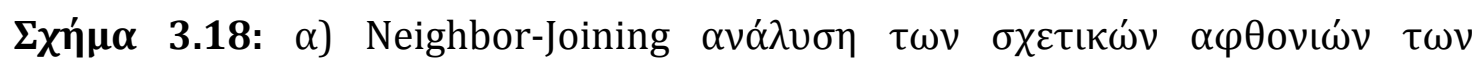

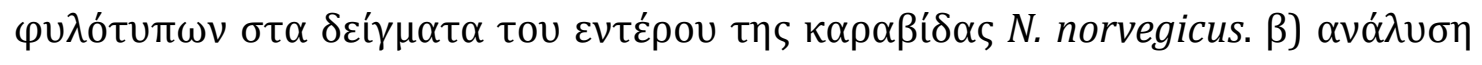

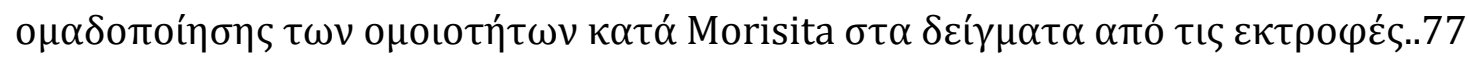

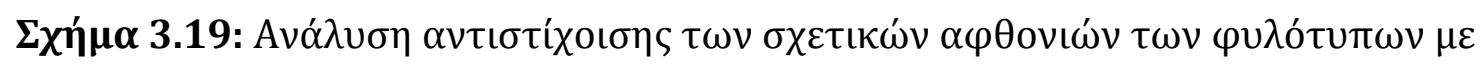

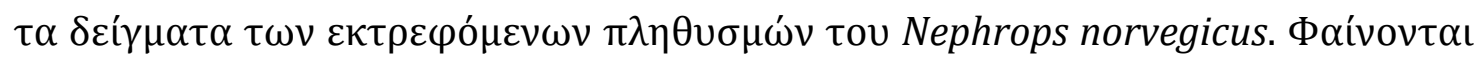

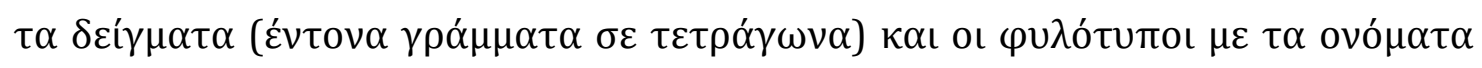

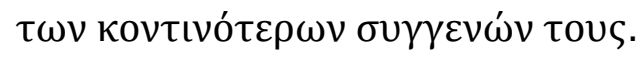
.78

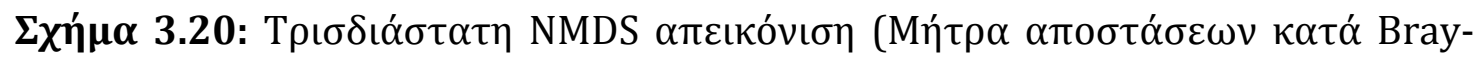

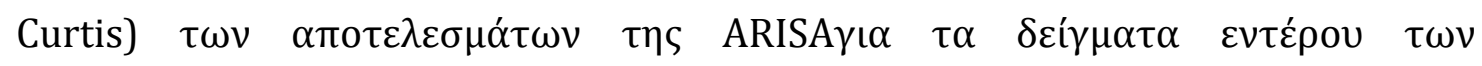

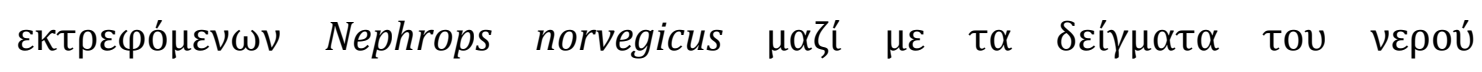
(stress $=0,17$ ). 


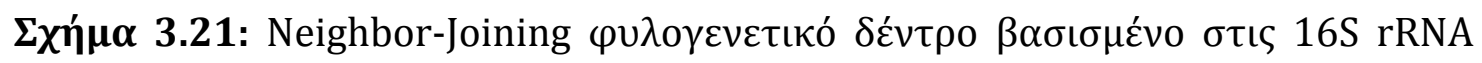

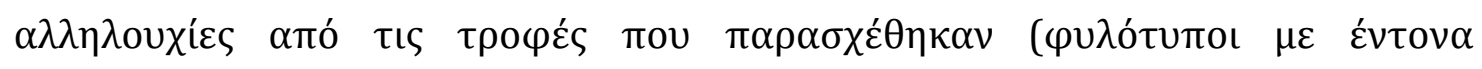

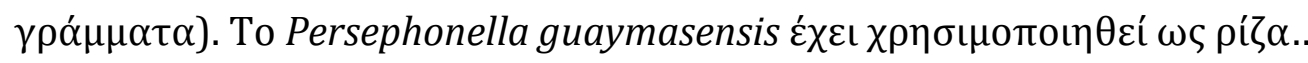
. .81

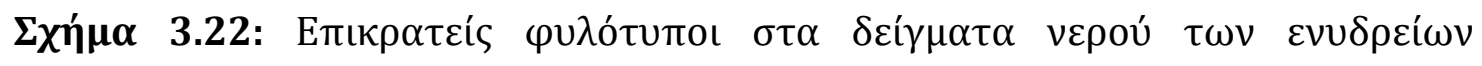

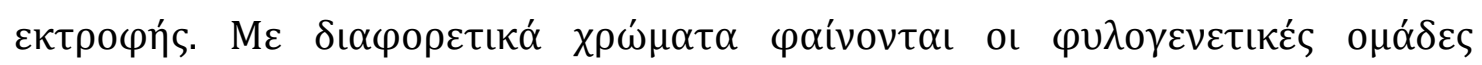

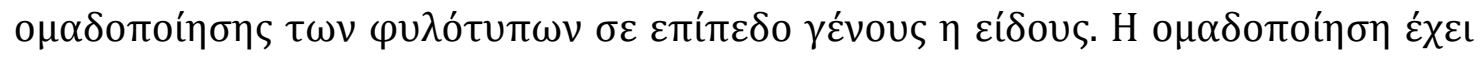

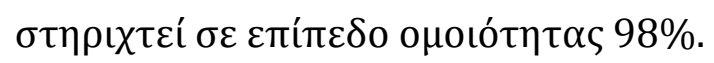
.84

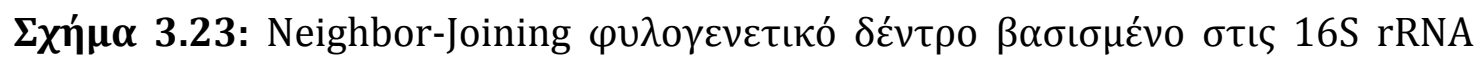

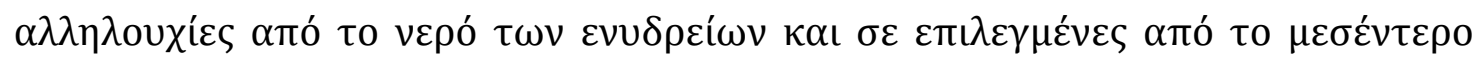

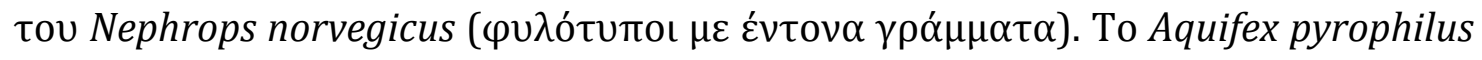

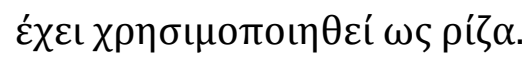

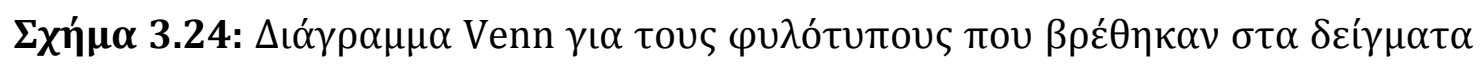

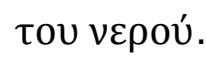
.87

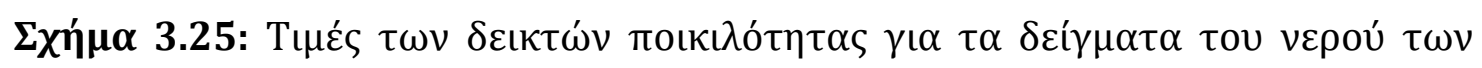
$\varepsilon v \cup \delta \rho \varepsilon i ́ \omega v$ 88

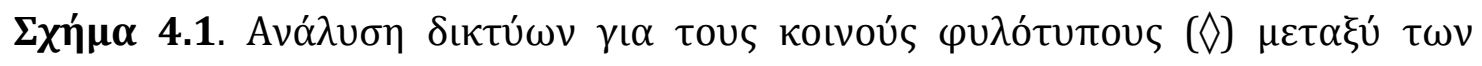

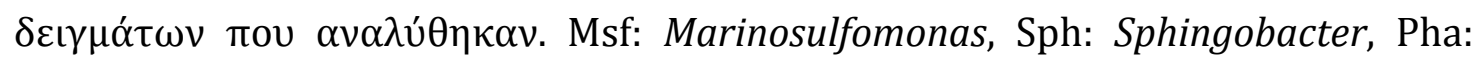
Phaeobacter, Mrv: Marivita, Psrp: Psychroserpens, Prop: Propionibacterium, She: Shewanella, Vib: Vibrio, Ple: Photobacterium leiognathi, CK: CK_1C4_19, Hep:

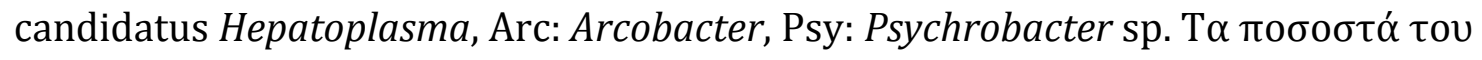

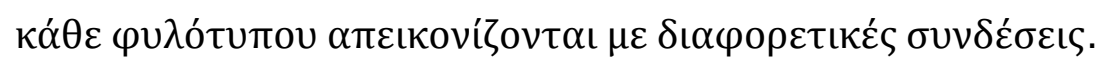
90

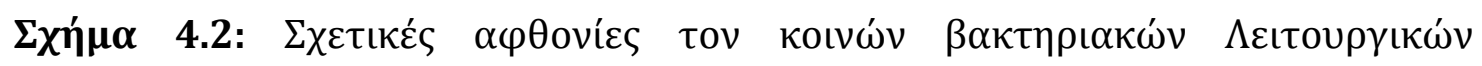

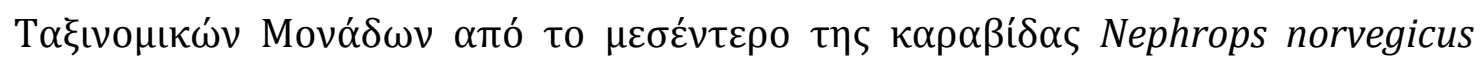

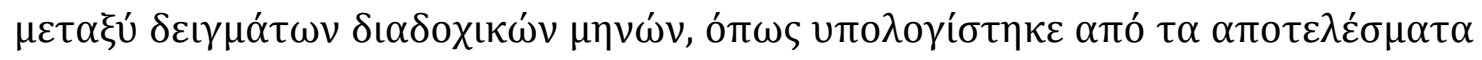
tᄁS ARISA. .93

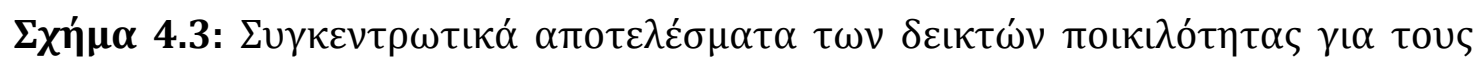

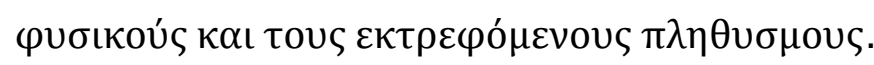
104

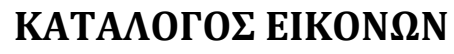

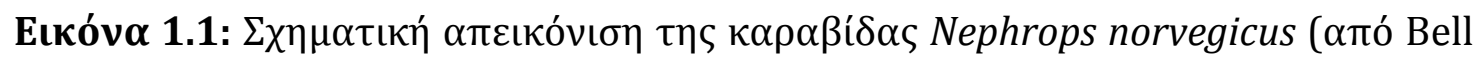
et al. 2006). 1 


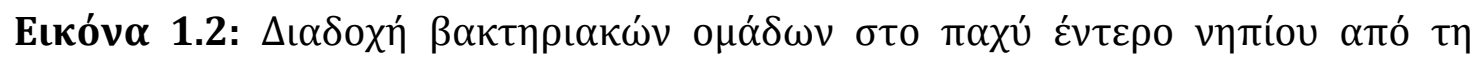

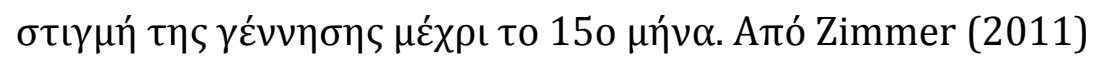

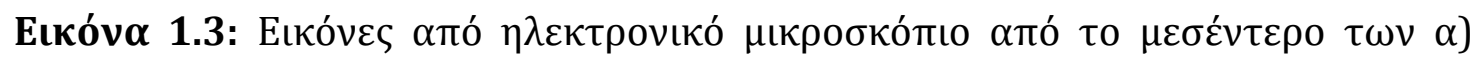
Calocaris mesandreae, $\beta$ ) Calianassa subterranean $\kappa \alpha \mathrm{\iota} \gamma$ ) Upogebia stellata. $\mathrm{H}$

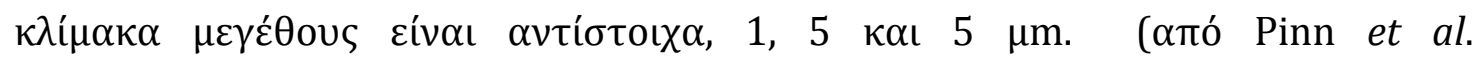
1999) 13

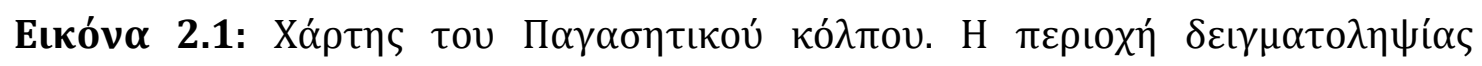

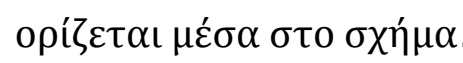

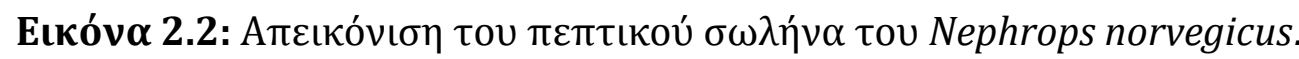
.23

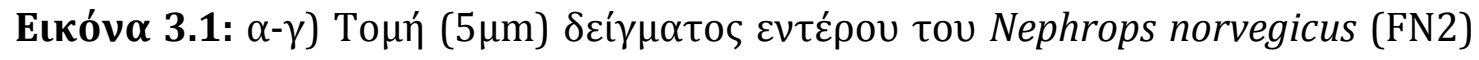

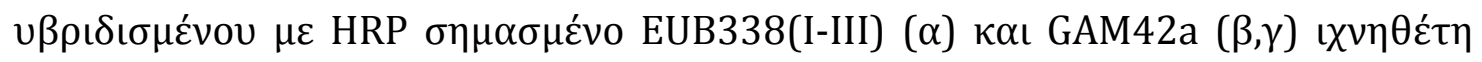

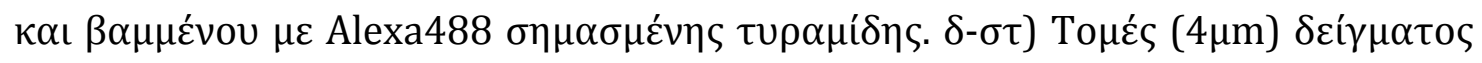

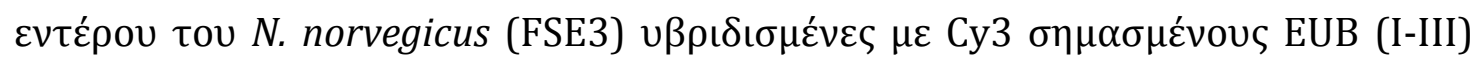

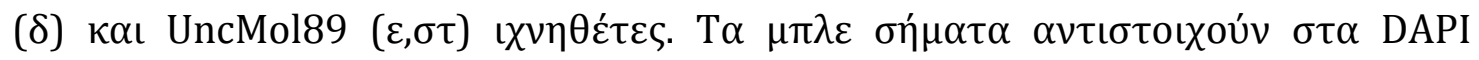

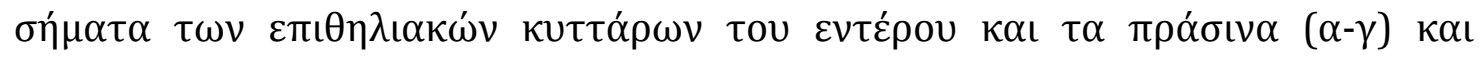

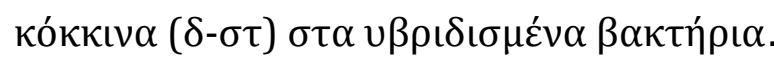
.66

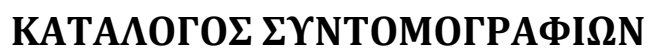

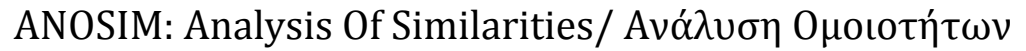

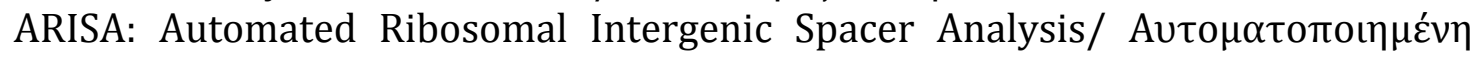

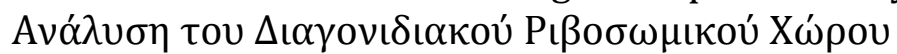

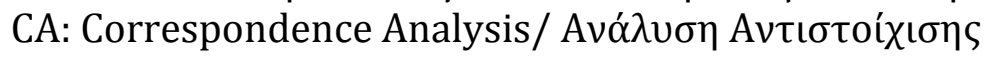

CARD-FISH: Catalyzed reporter deposition-FISH/ FISH $\mu \varepsilon \alpha \pi o ́ \theta \varepsilon \sigma \eta ~ \kappa \alpha \tau \alpha \lambda u ́ \tau \eta$

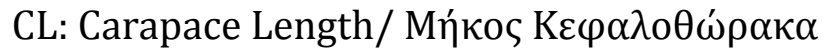

DAPI: Diaminidophenylindole/ $\Delta \iota \alpha \mu \iota v o \varphi \varepsilon v v \lambda \iota v \delta o ́ \lambda \eta$

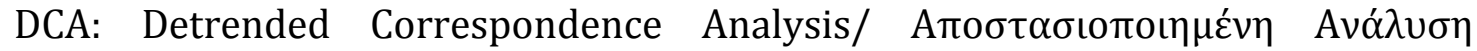

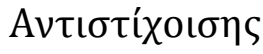

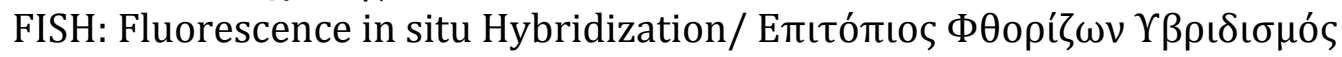

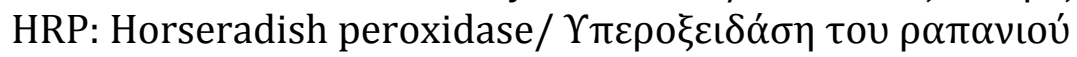

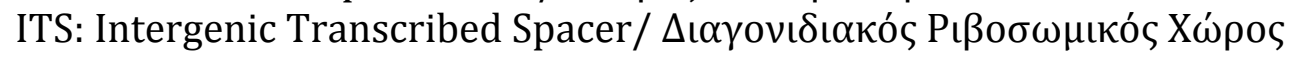

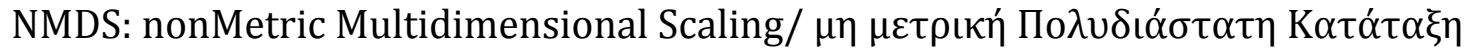

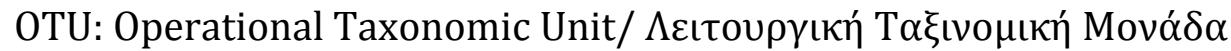

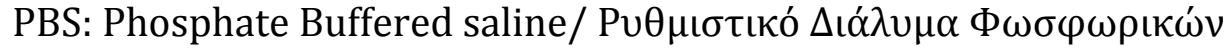

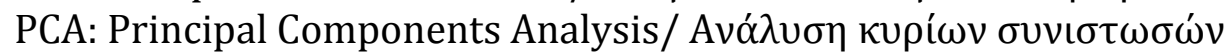

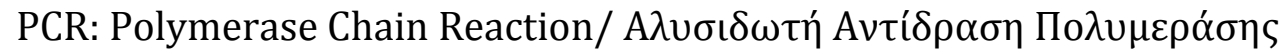

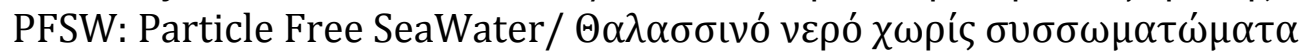

RDA: Redundancy Analysis/ Avó $\lambda u \sigma \eta \pi \lambda \varepsilon o v \alpha \sigma \mu o u ́$

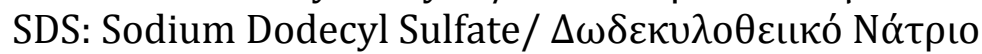




\section{1. $\mathrm{EI \Sigma A \Gamma \Omega \Gamma H}$}

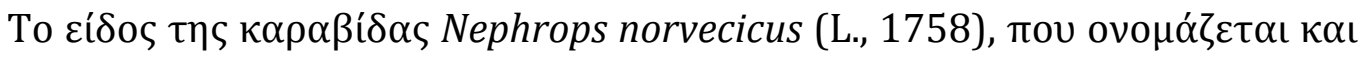

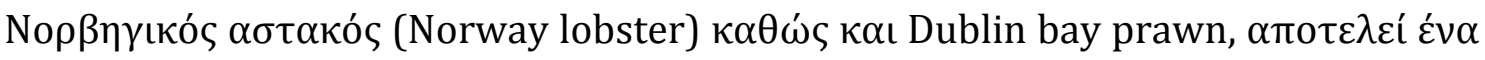

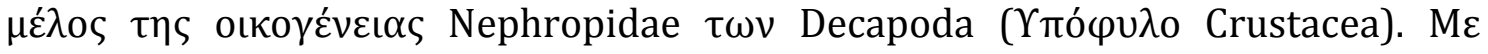

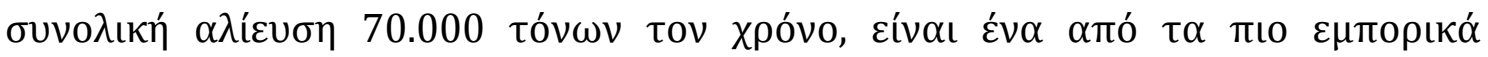

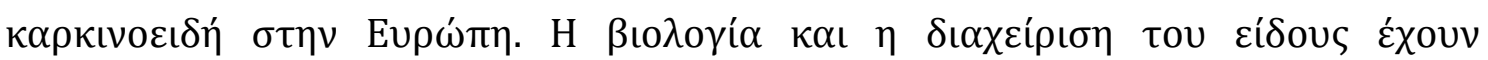

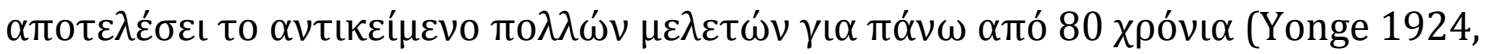
Farmer 1974, Farmer 1975, Chapman 1980, Sarda 1995, Graham \& Ferro 2004).

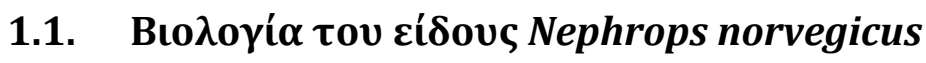

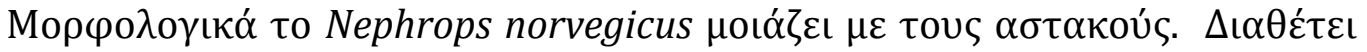

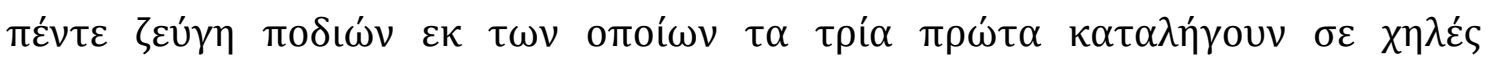

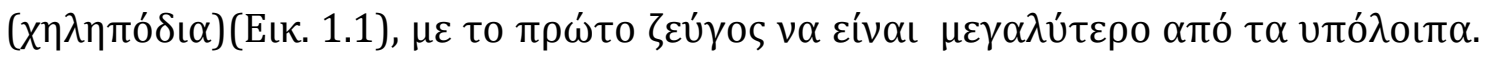

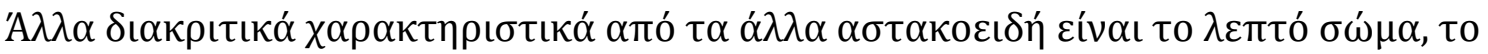

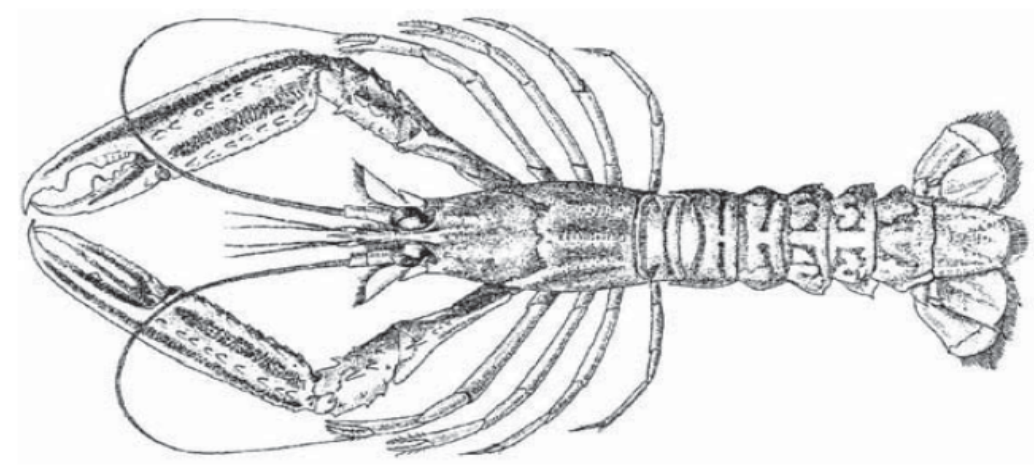

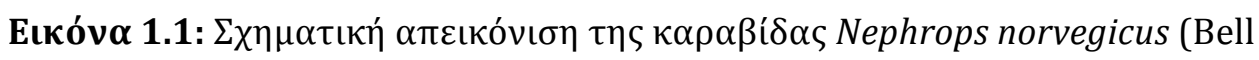
et al. 2006) 


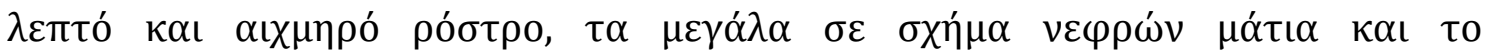

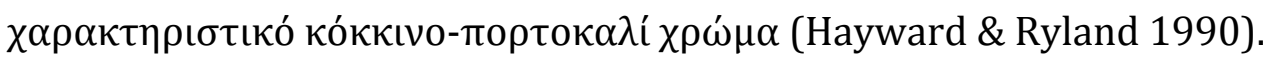

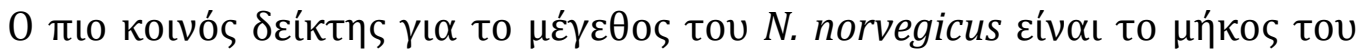

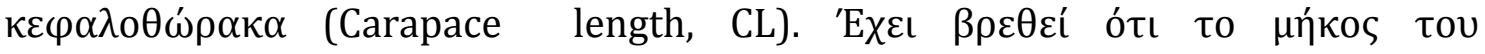

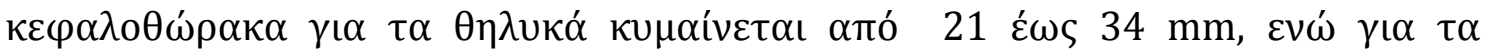

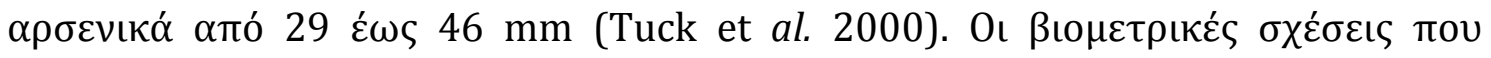

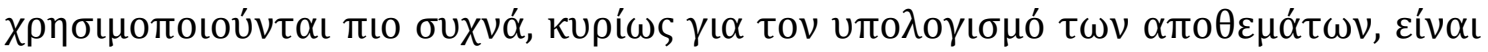

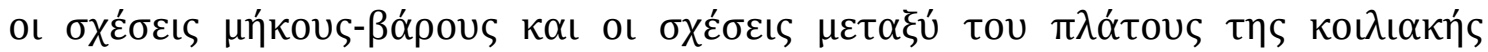

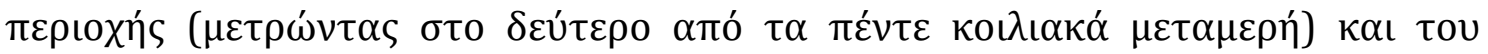

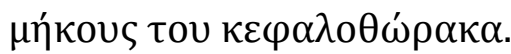

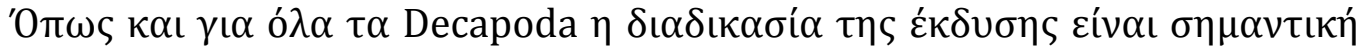

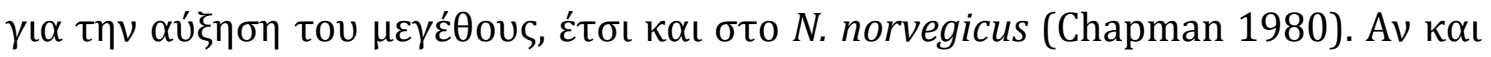

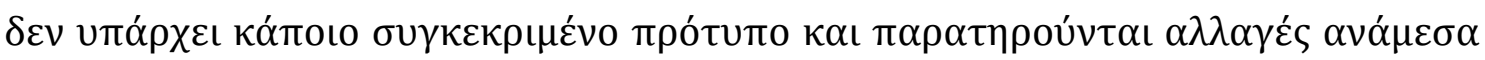

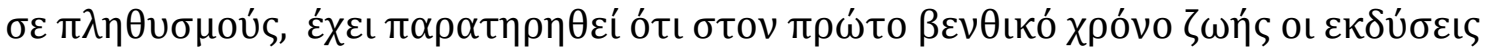

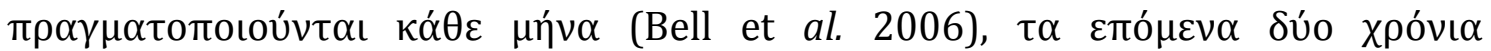

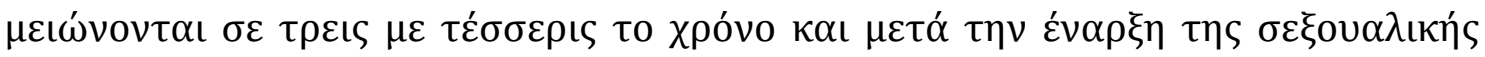

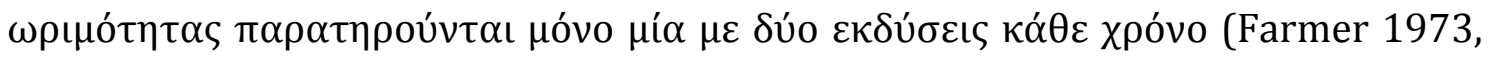

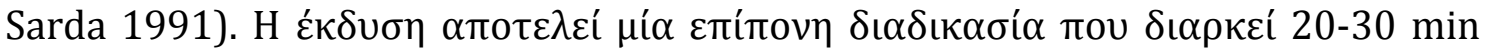

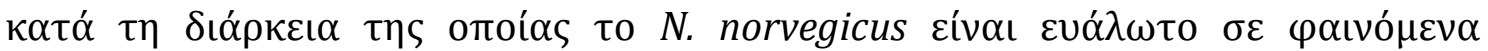

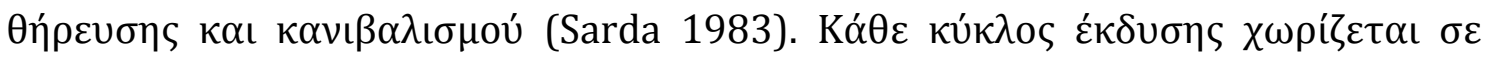

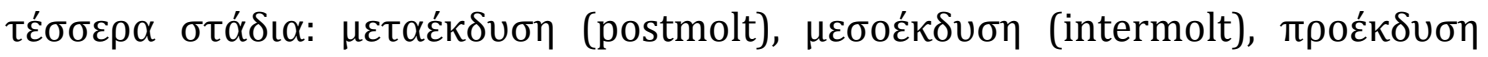

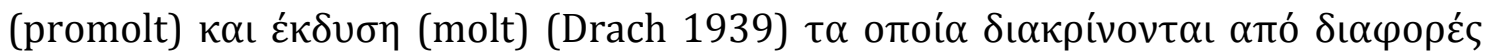

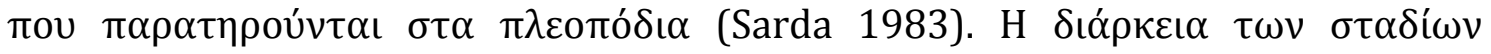

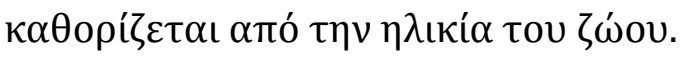




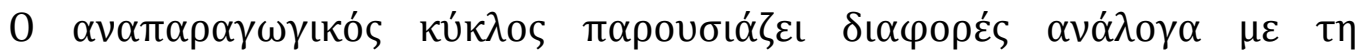

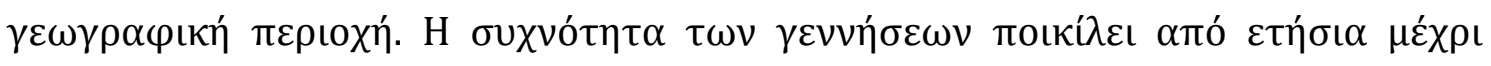

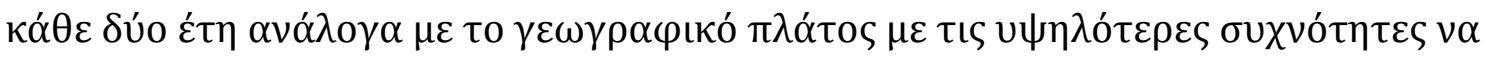

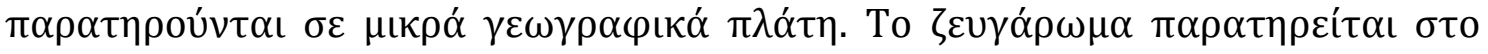

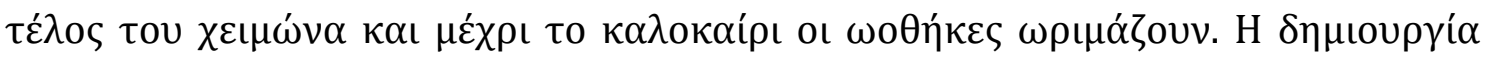

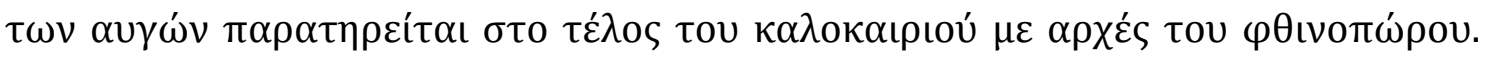

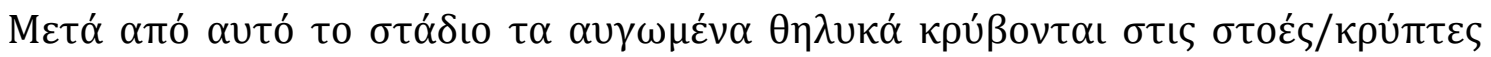

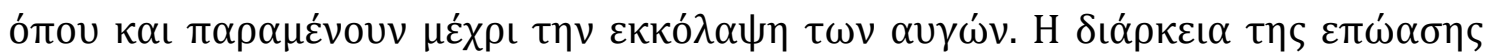

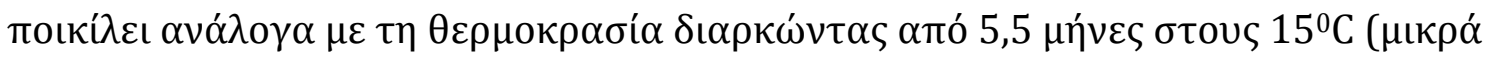

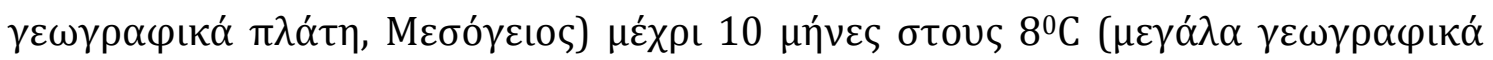

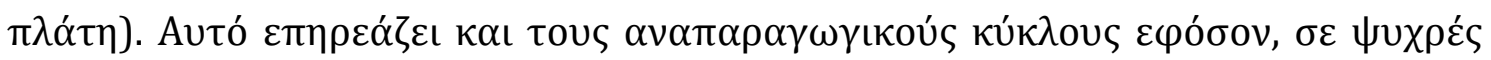

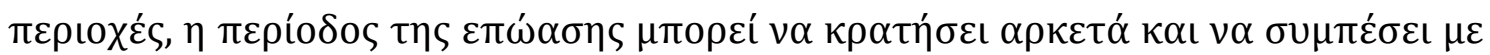

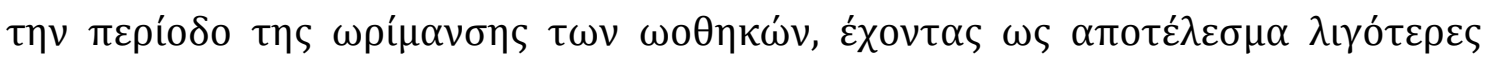

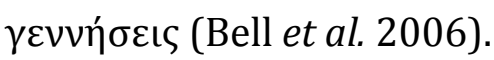

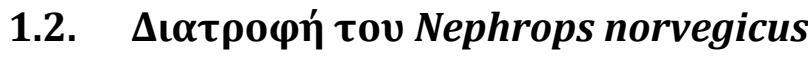

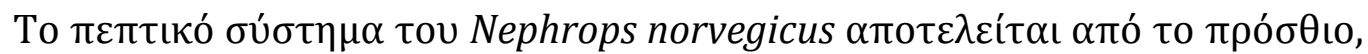

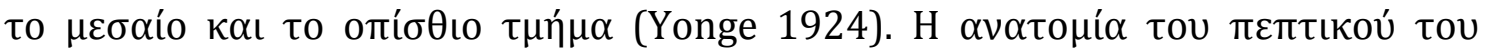

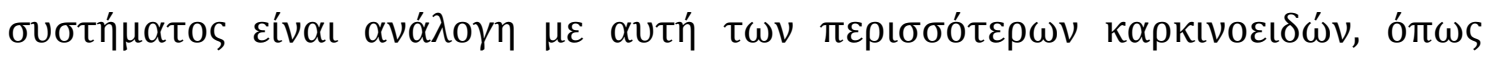

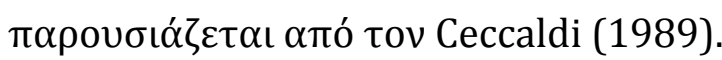

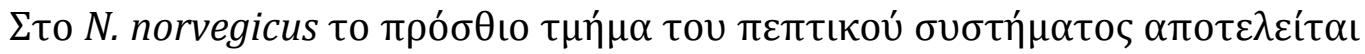

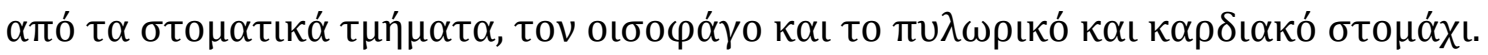

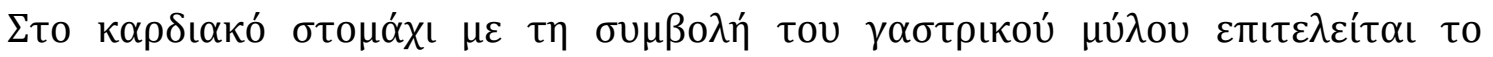




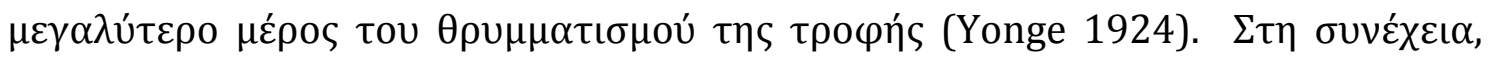

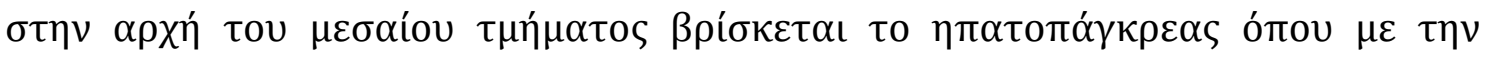

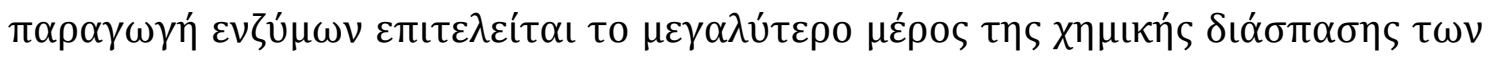

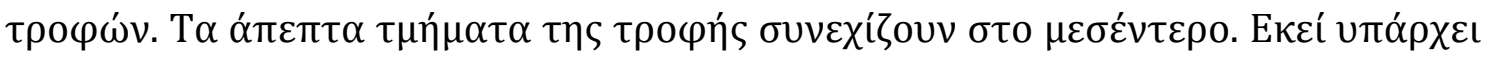

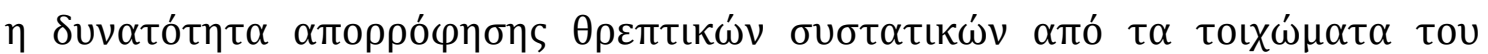

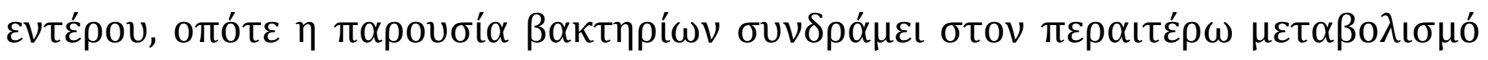

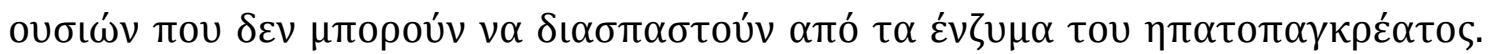

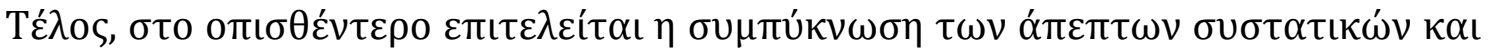

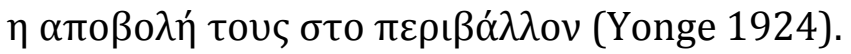

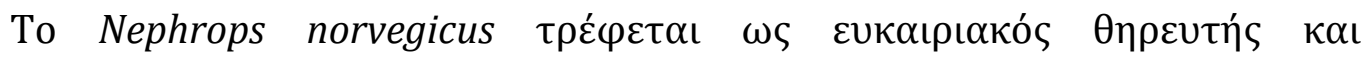

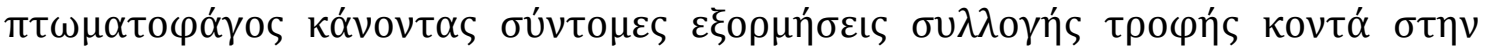

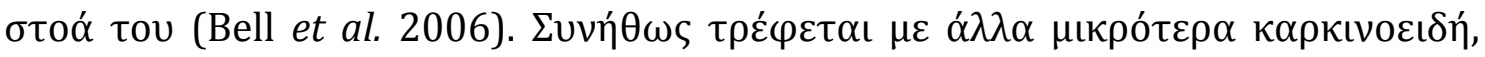

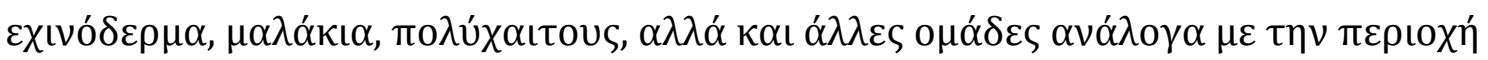

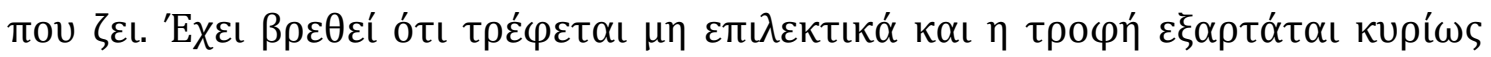

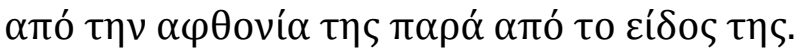

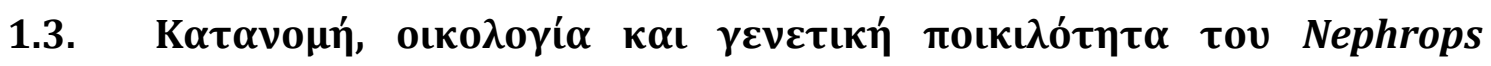 norvegicus}

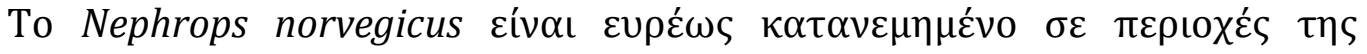

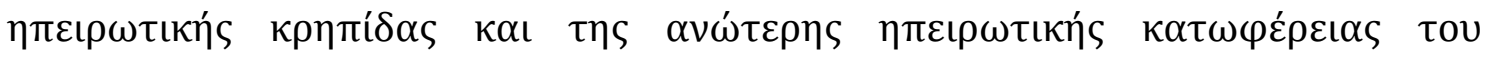

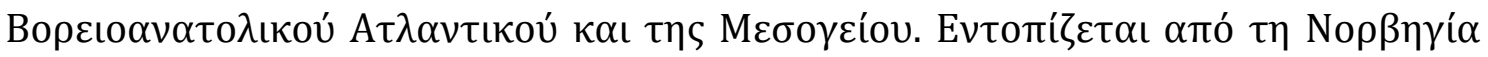

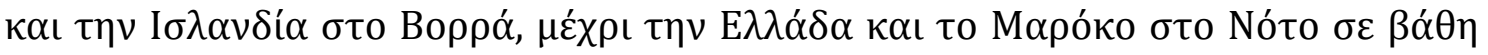

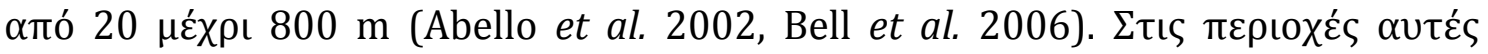

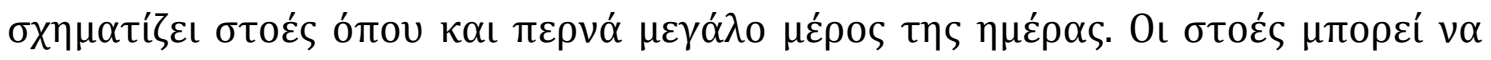




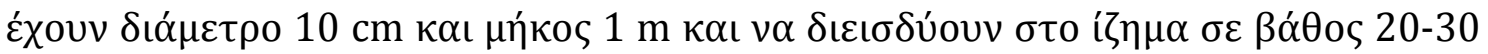

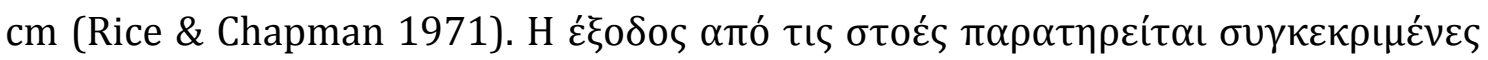

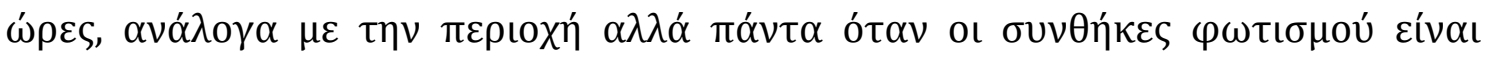

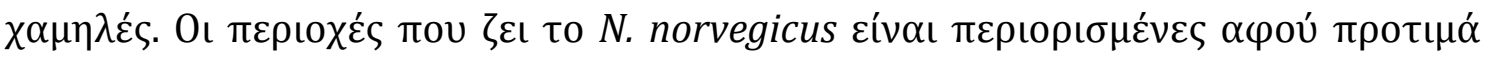

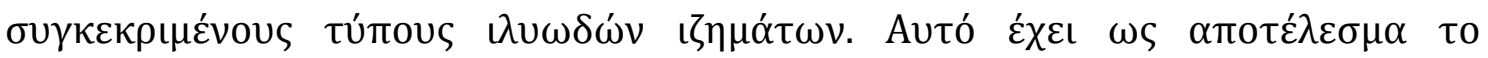

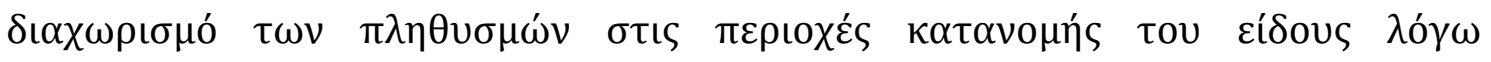

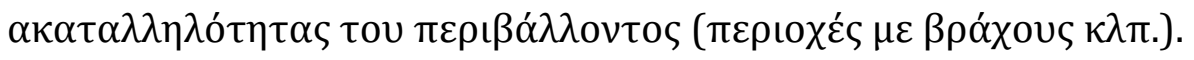

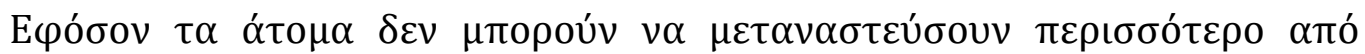

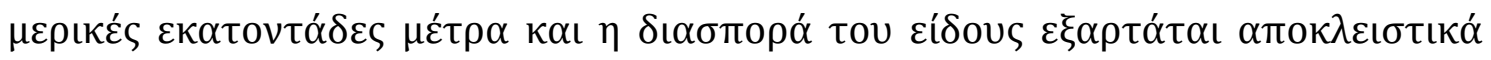

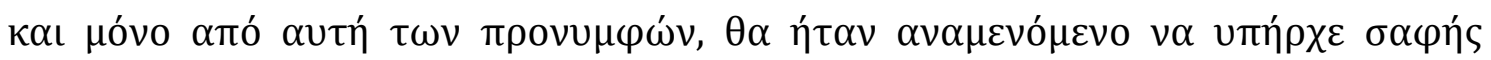

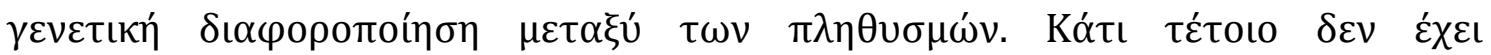

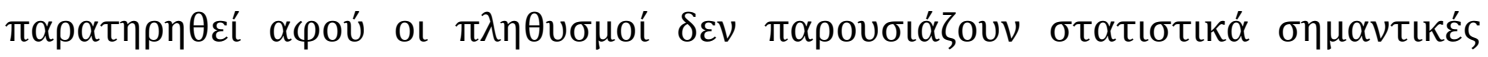

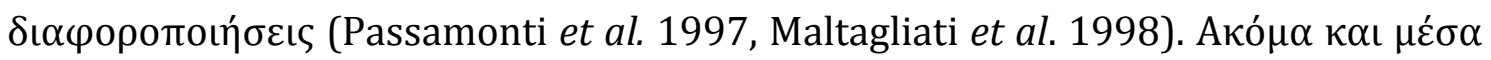

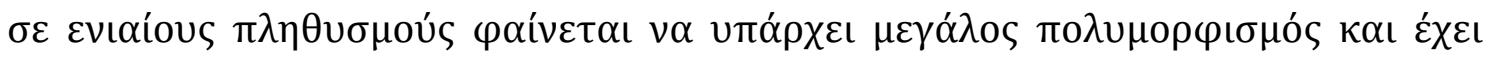

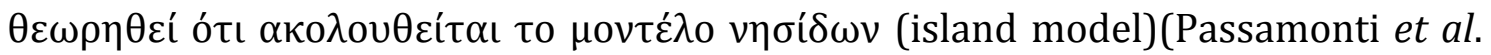

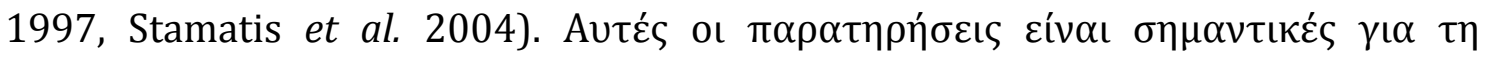

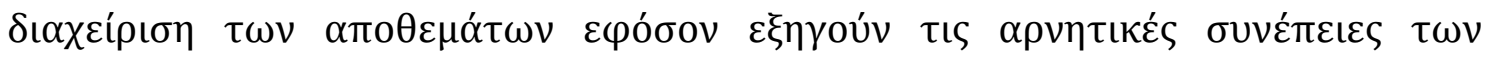

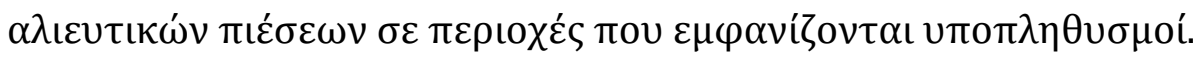

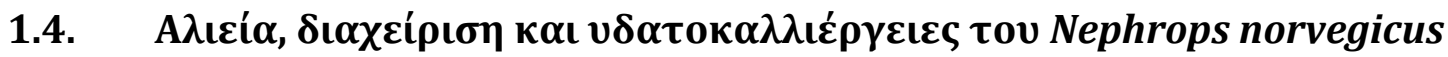

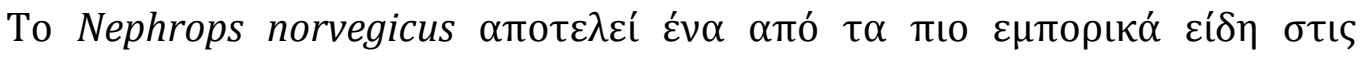

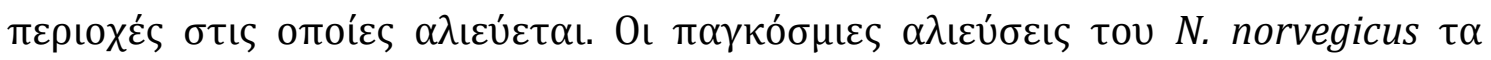

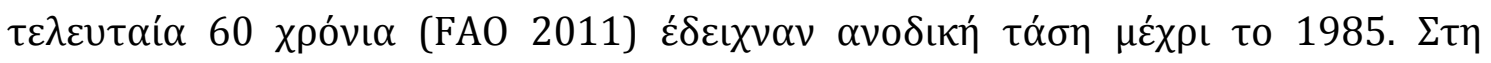




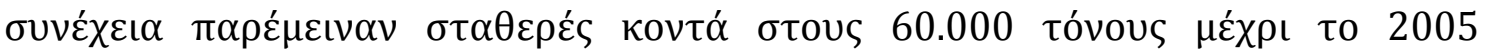

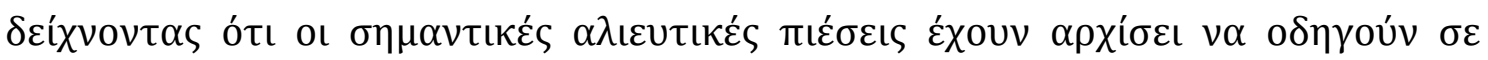

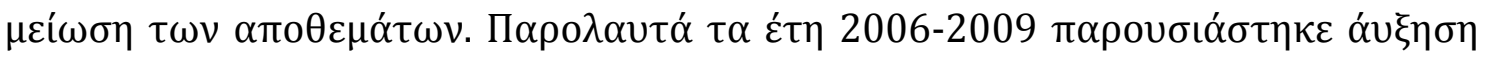

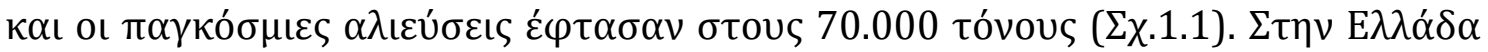

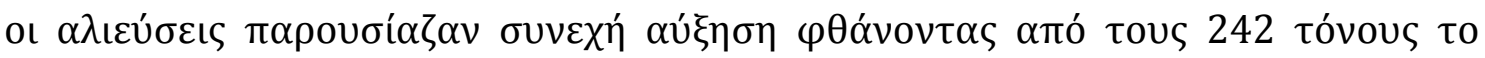

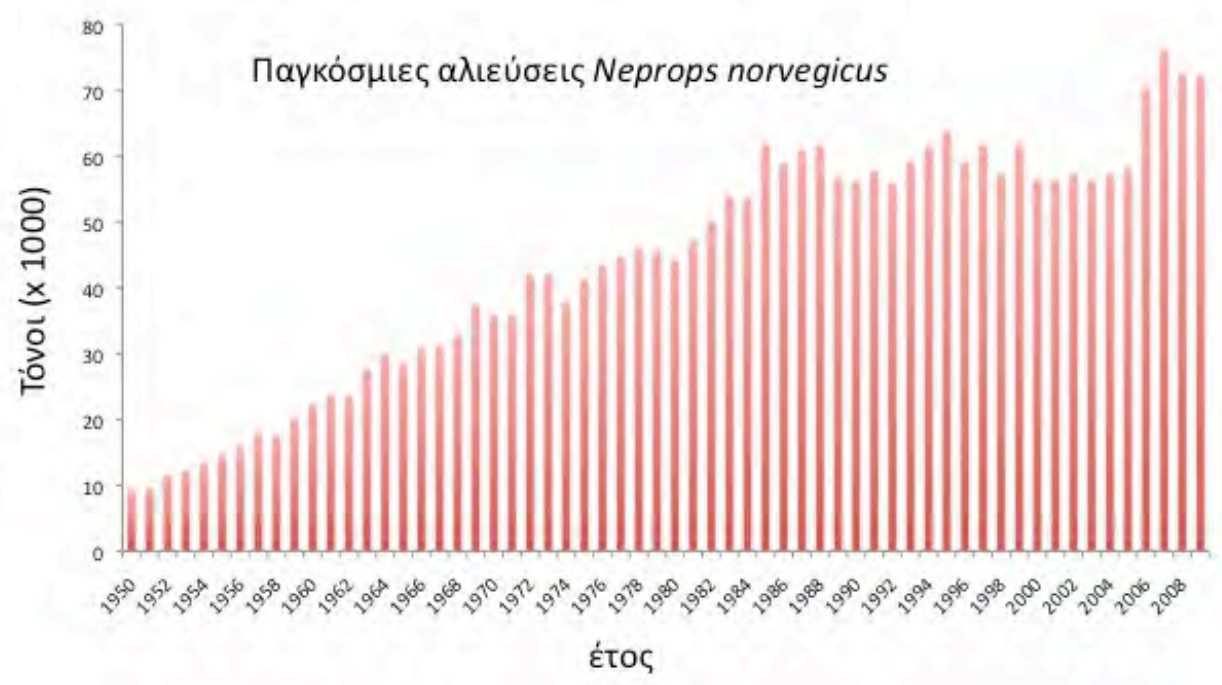

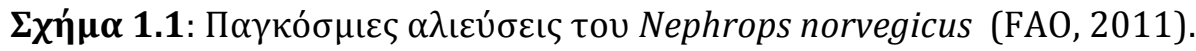

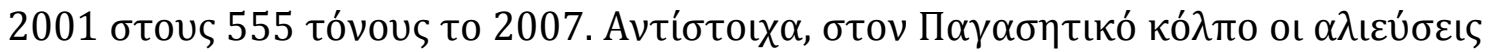

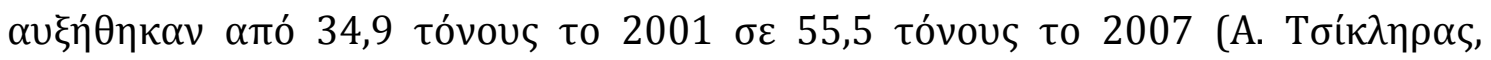

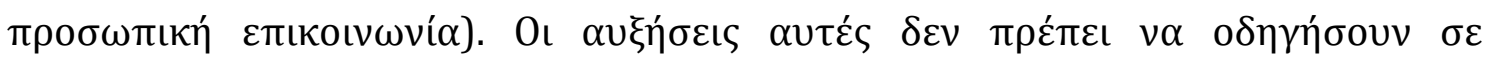

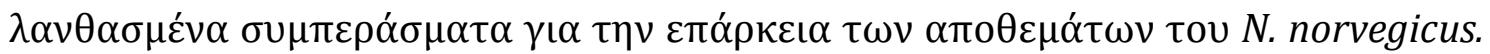

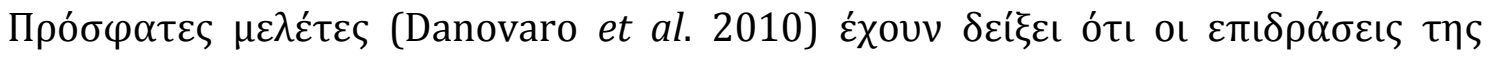

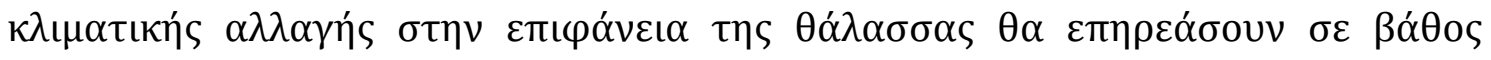

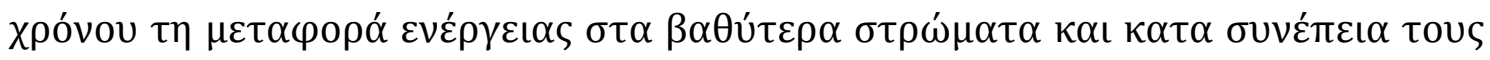




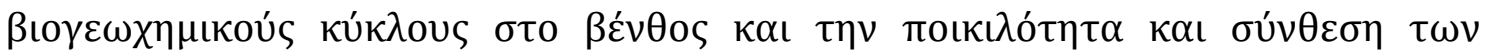

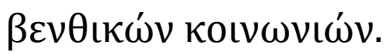

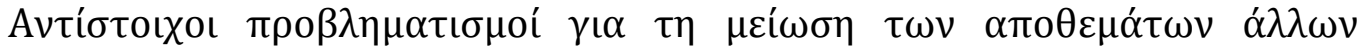

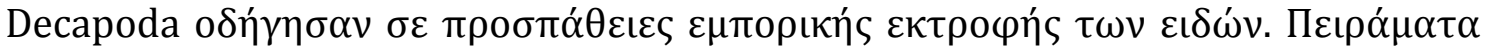

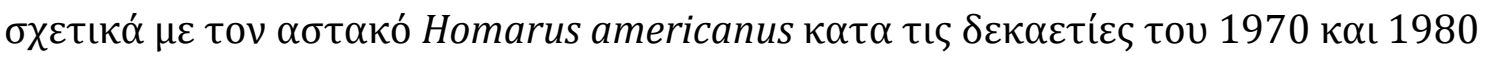

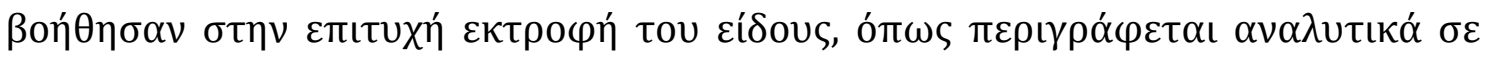

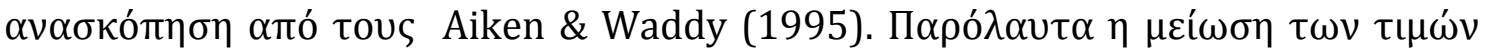

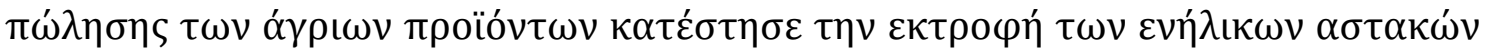

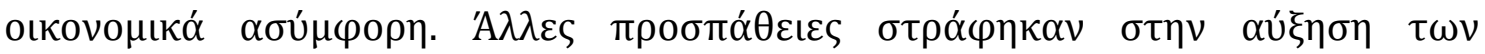

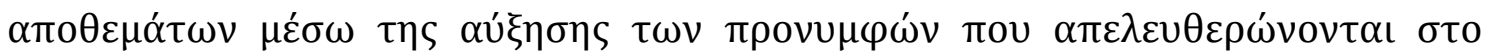

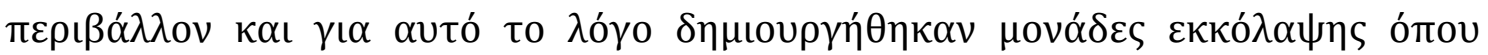

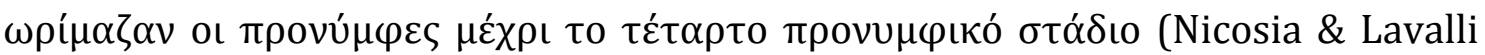

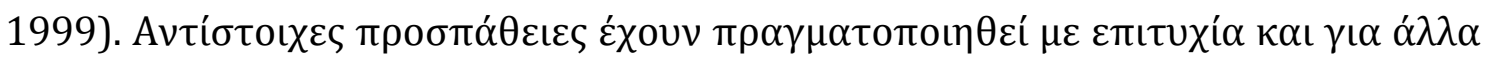

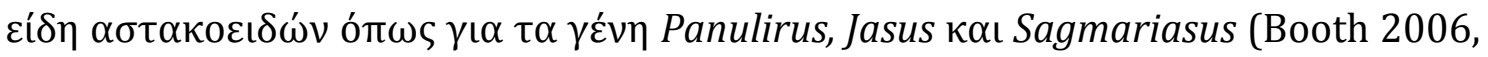
Phillips \& Melville-Smith 2006).

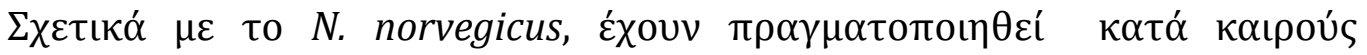

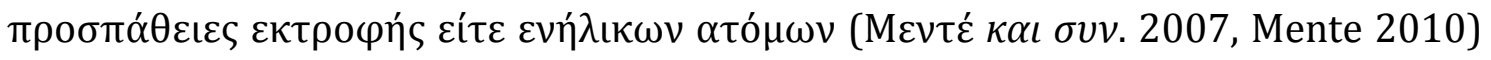

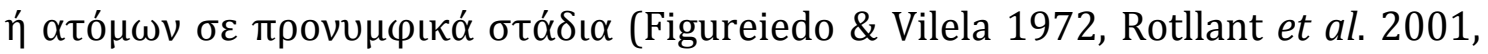

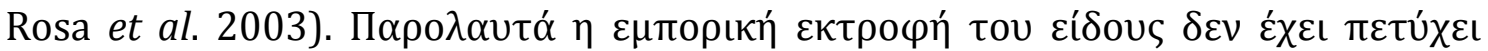

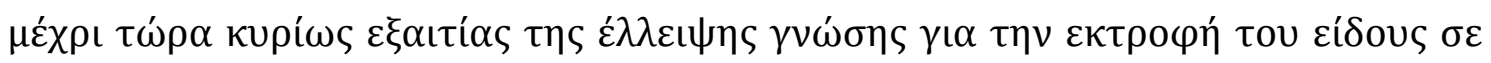

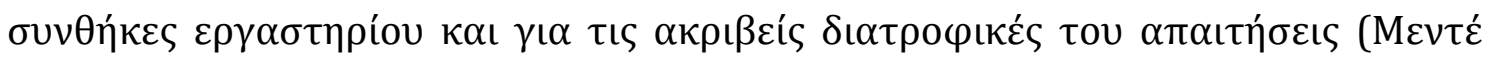

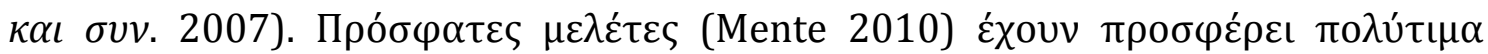

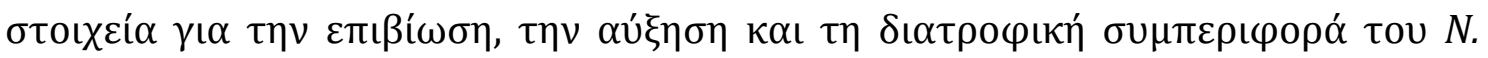




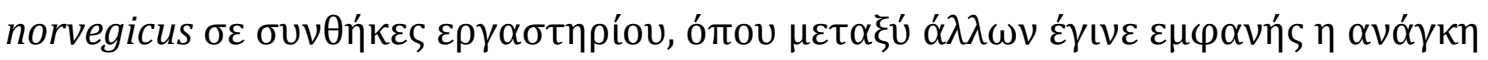

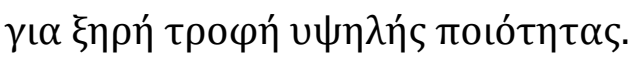

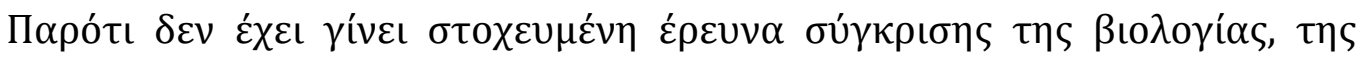

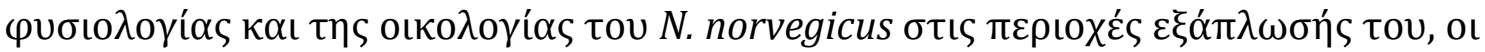

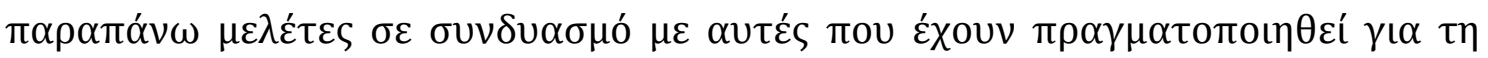

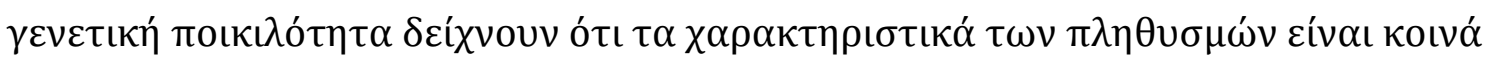

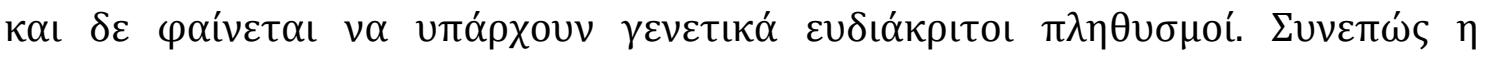

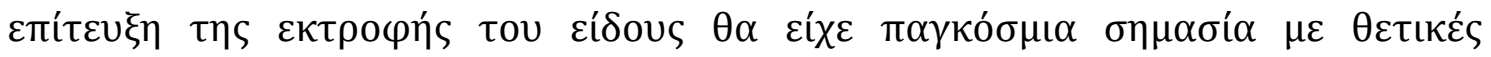

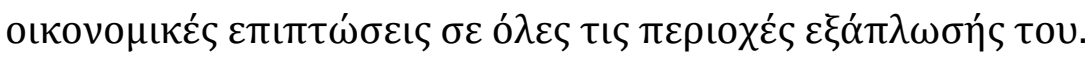

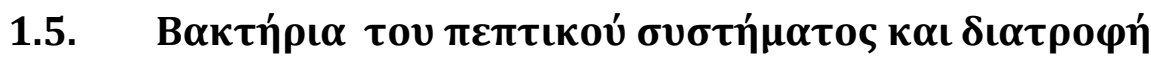

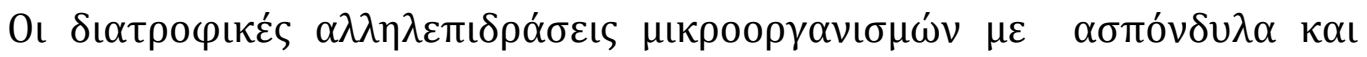

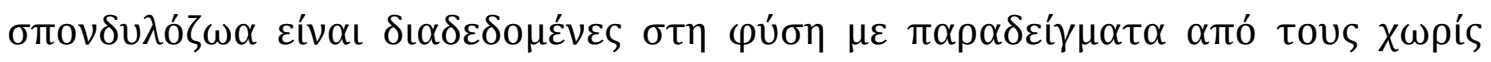

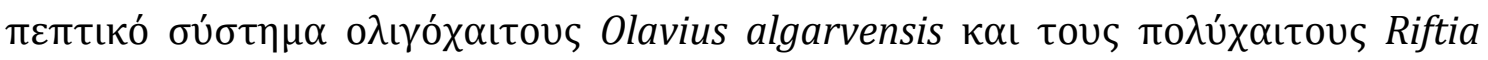

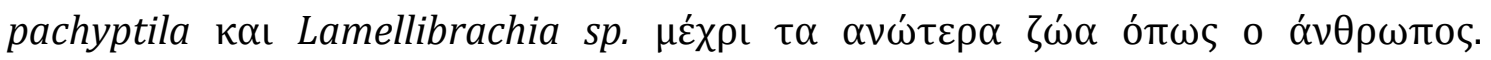

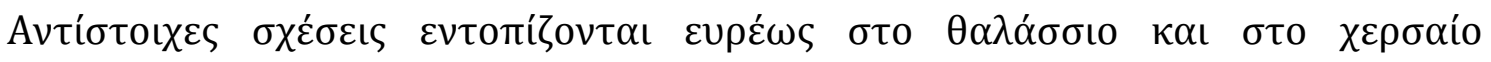

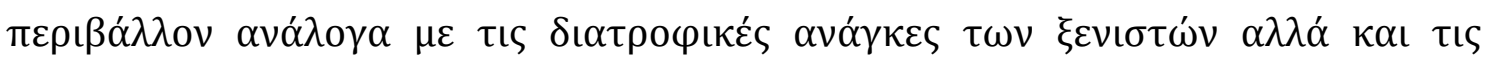

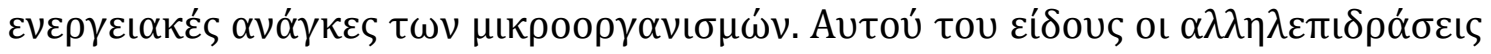

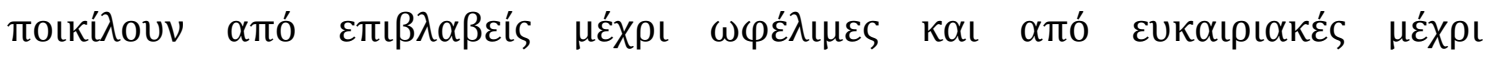

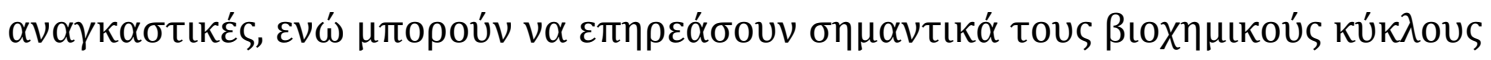

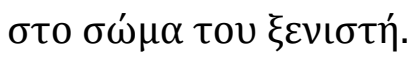

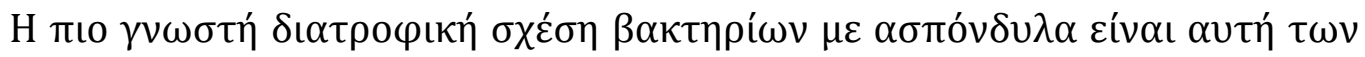

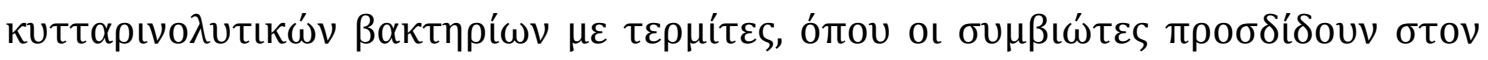




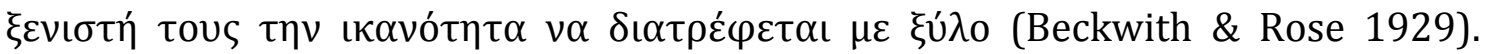

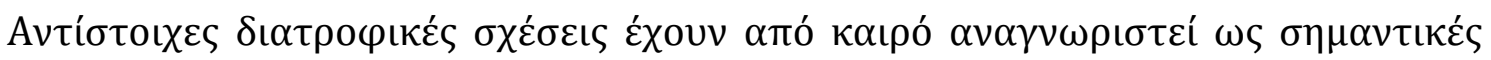

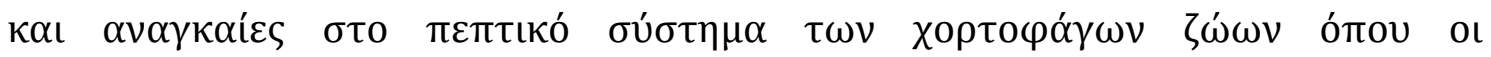

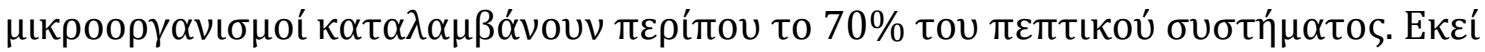

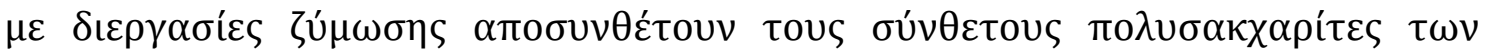

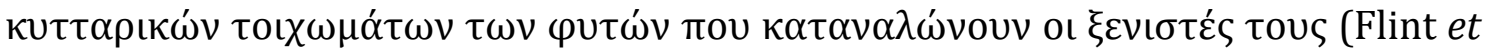
al. 2008).

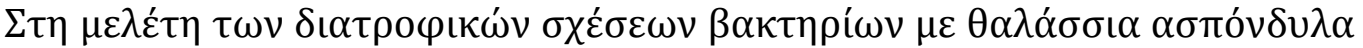

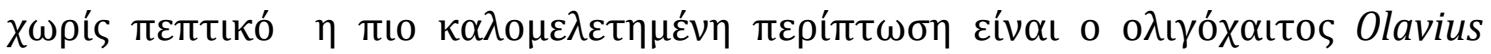

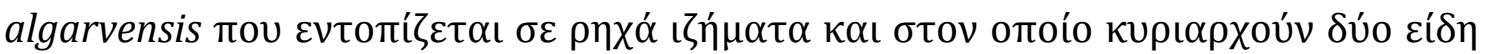

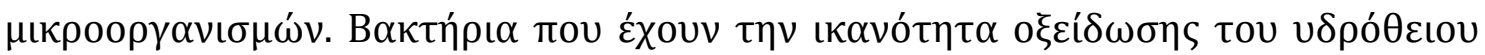

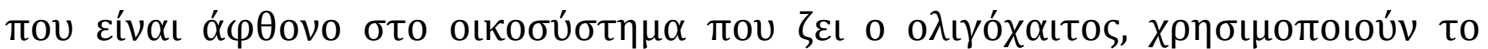

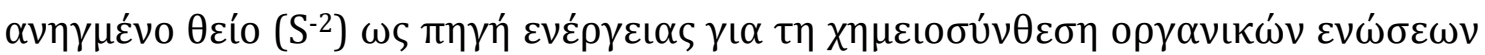

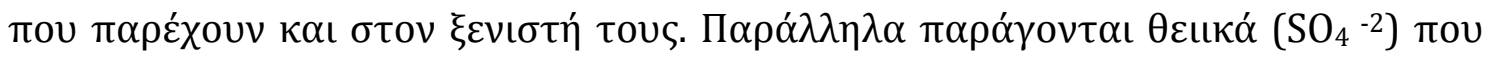

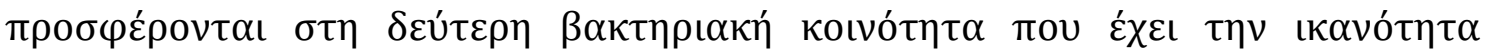

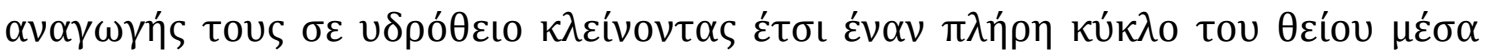

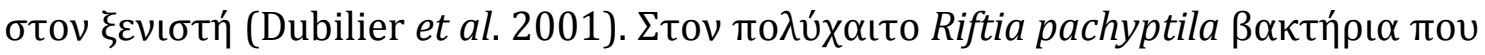

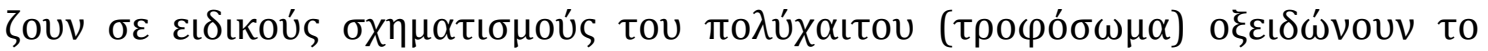

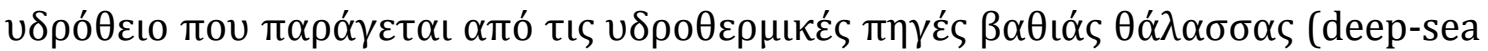

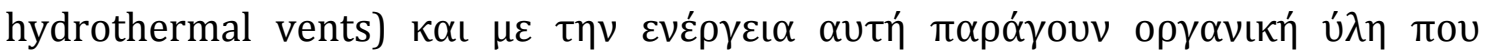

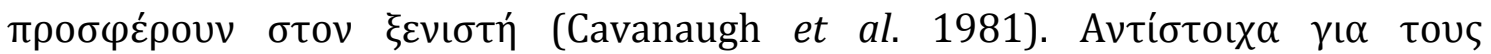

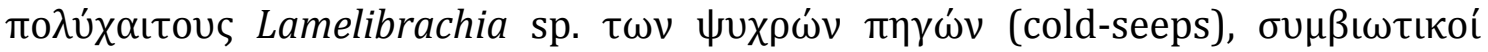

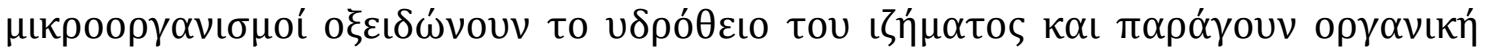

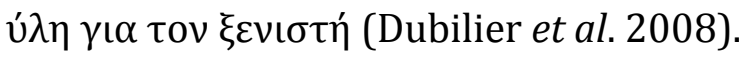




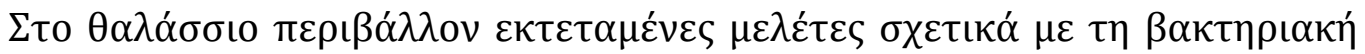

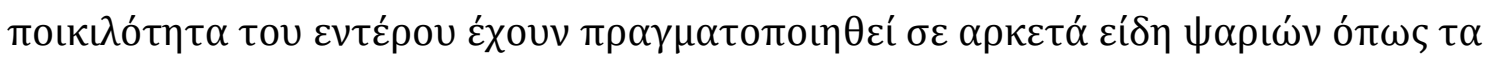

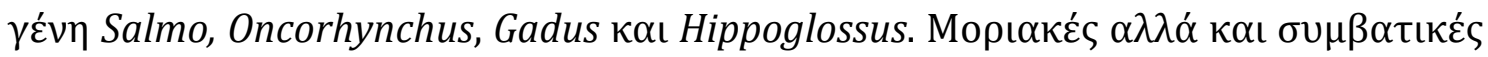

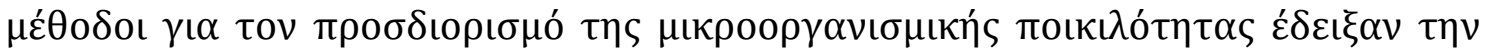

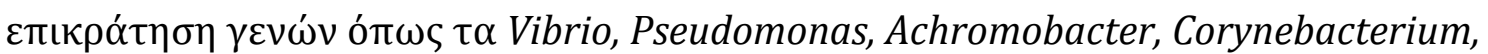
Alteromonas, Photobacterium, Psychrobacter, Mycoplasma, Stenotrophomonas к $\alpha$

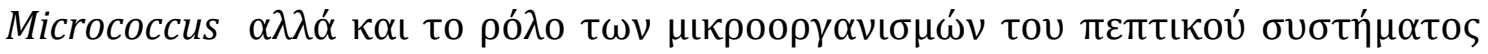

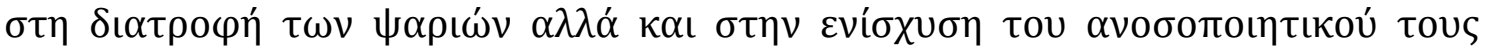

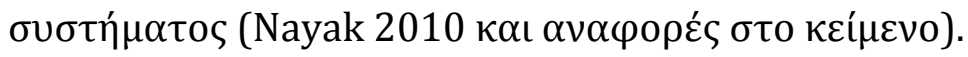

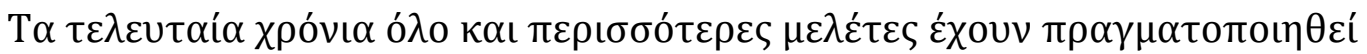

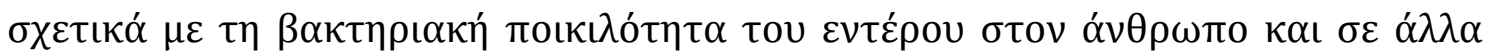
$\theta \eta \lambda \alpha \sigma \tau \iota \kappa \alpha ́$ (Turnbaugh et al. 2006, Ley et al. 2008). Oı Ley et al. (2008) $\varepsilon \delta \varepsilon เ \xi \alpha \nu$

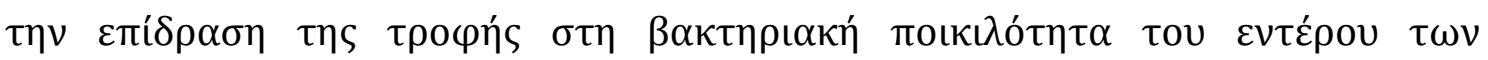

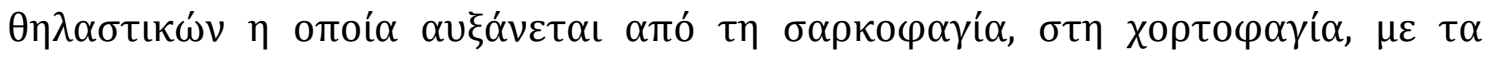

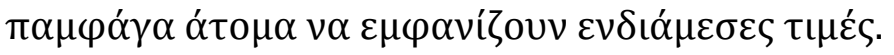

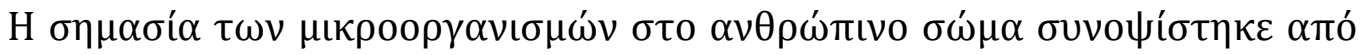

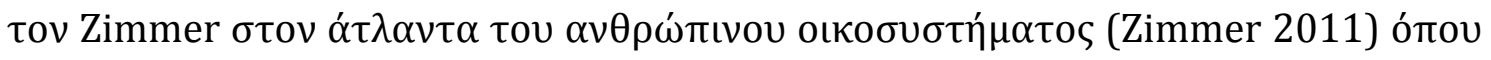

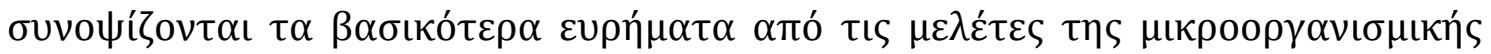

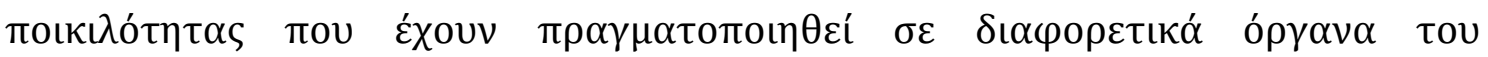

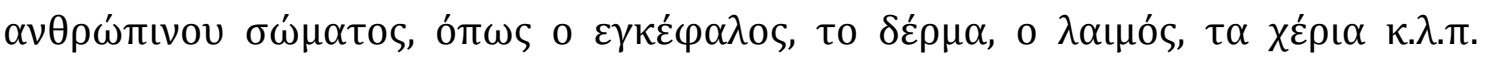

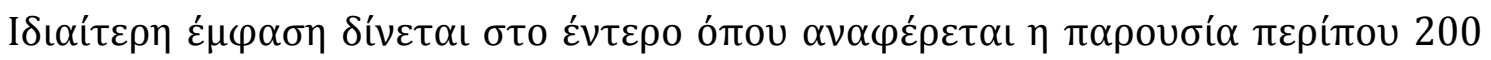

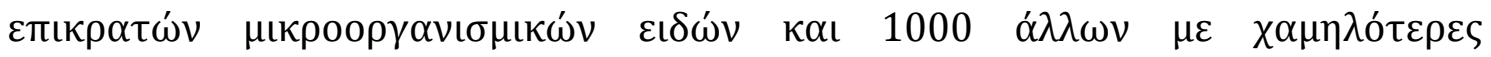

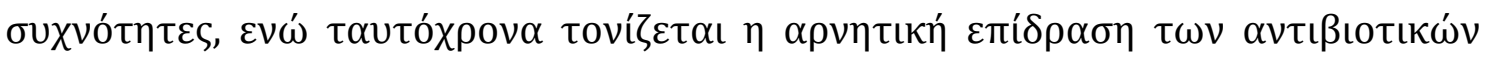

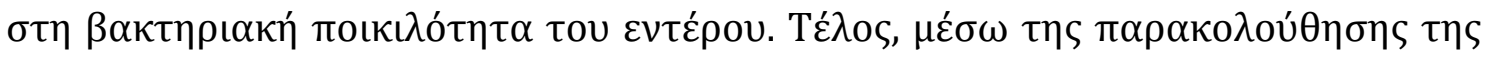




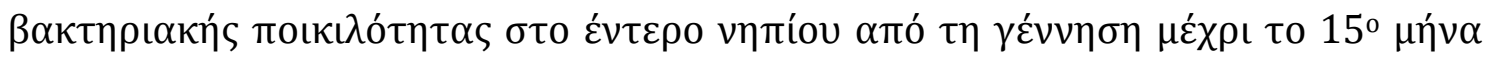

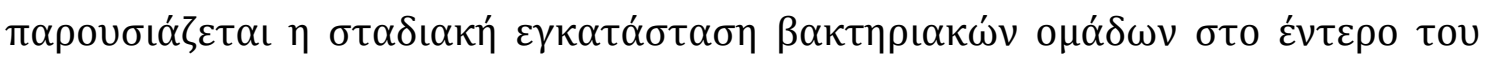

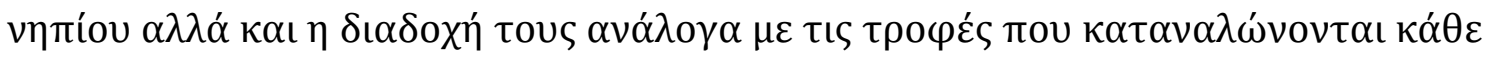
фора́ (Еıк. 1.2).

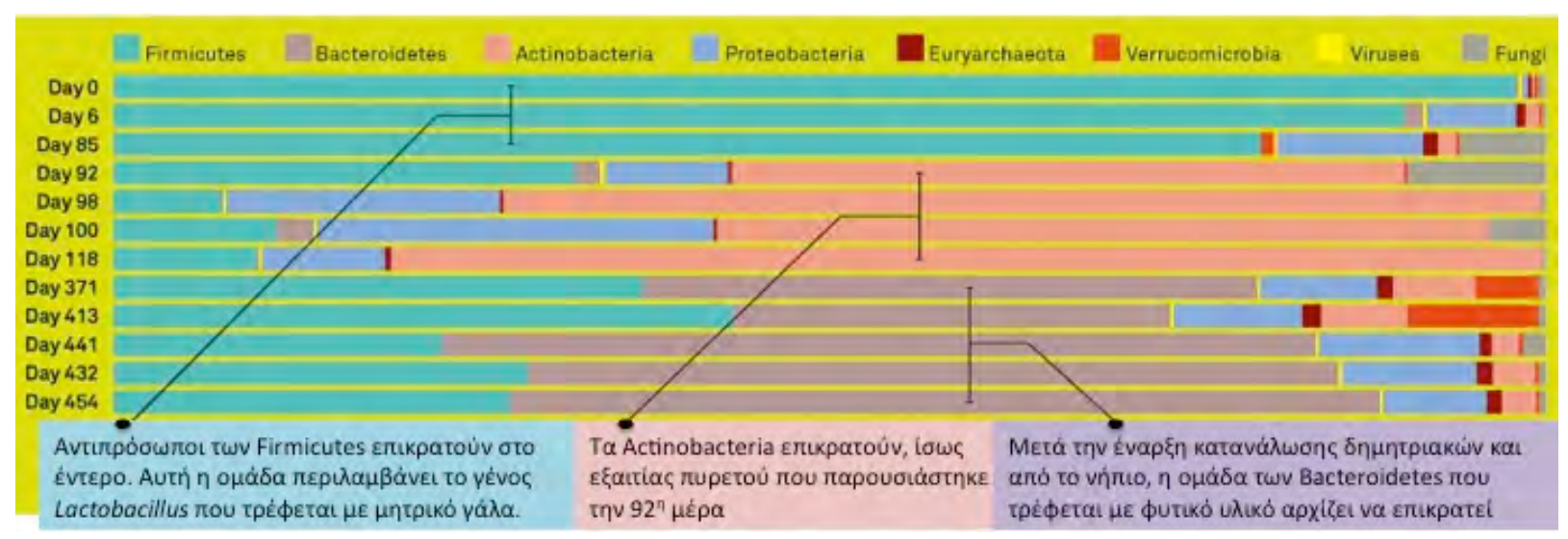

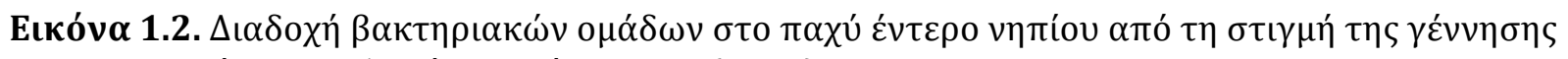

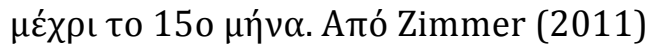

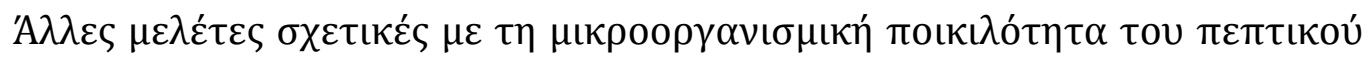

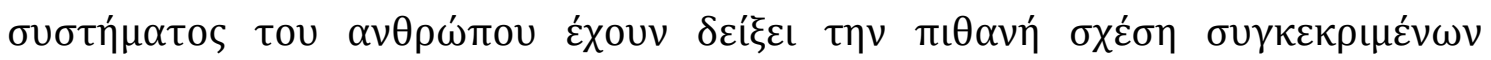

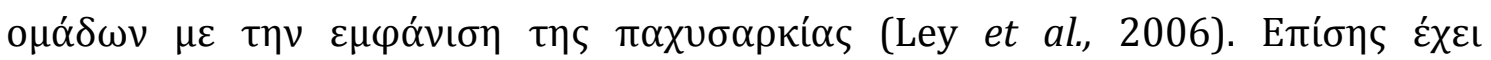

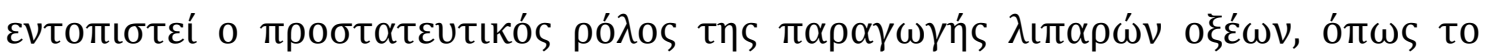

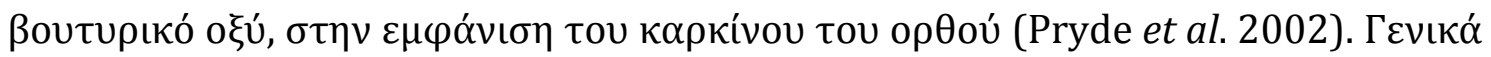

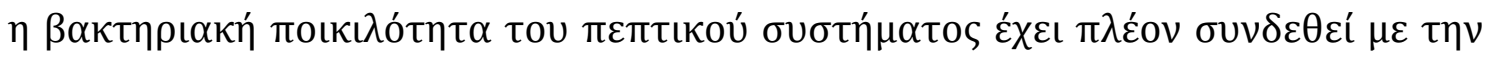

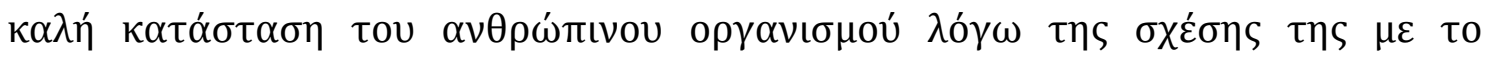

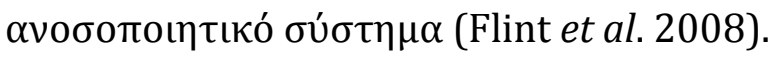

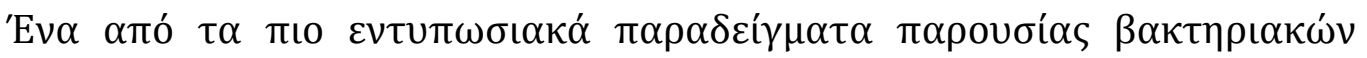

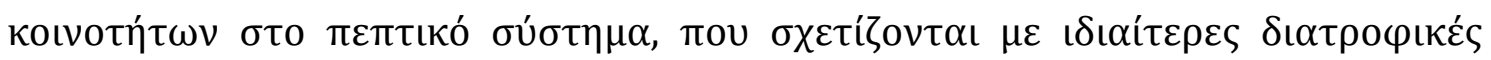




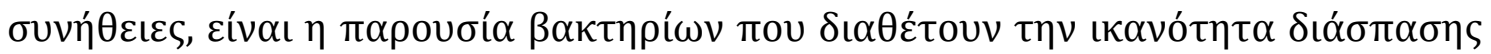

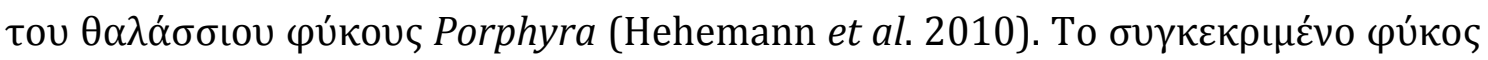

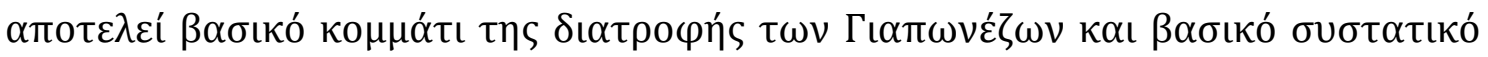

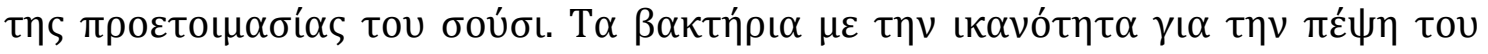

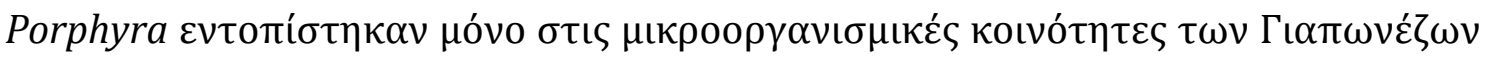

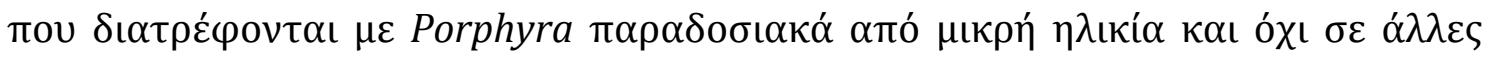

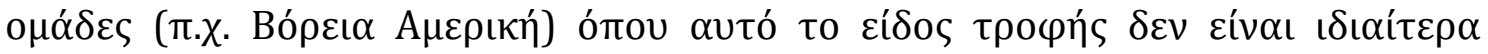
$\delta\llcorner\alpha \delta \varepsilon \delta \circ \mu \varepsilon ́ v o$.

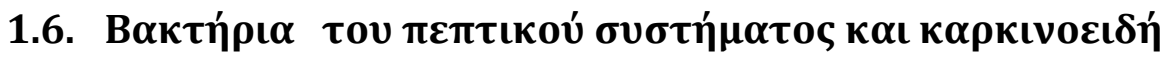

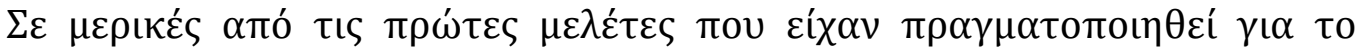

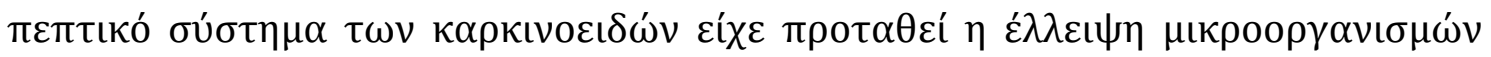

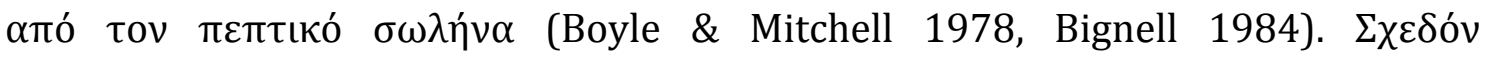

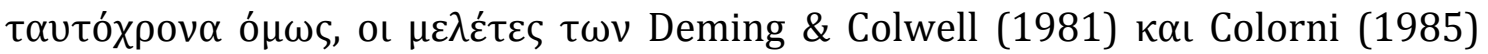

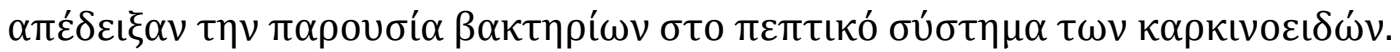

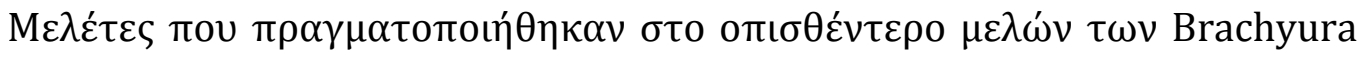

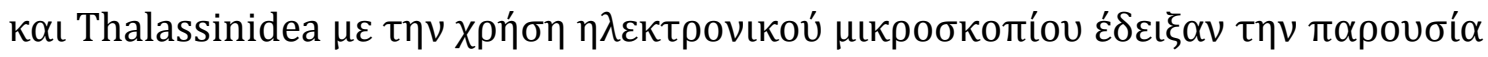

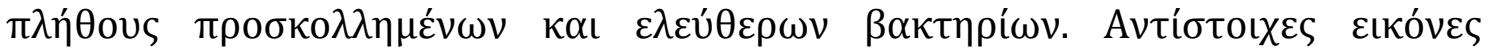

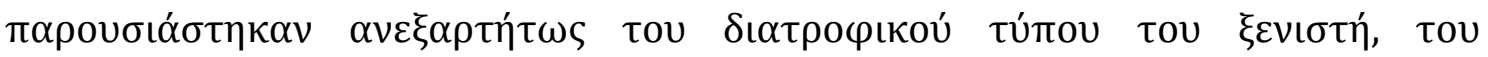

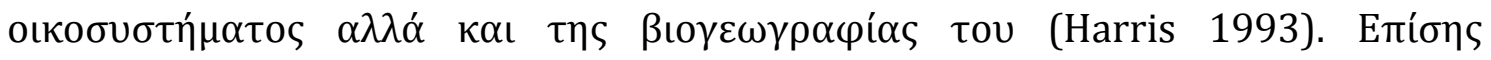

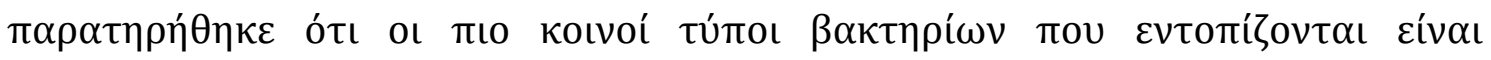

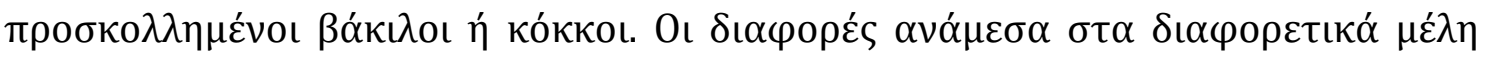

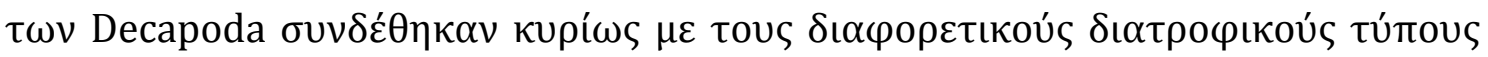




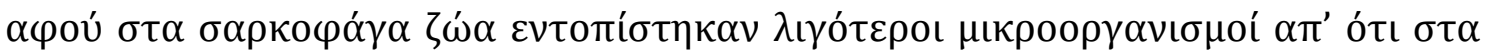
$\theta \rho v \mu \mu \alpha \tau о \varphi \alpha ́ \gamma \alpha$.

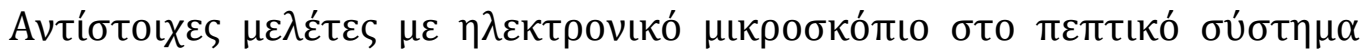

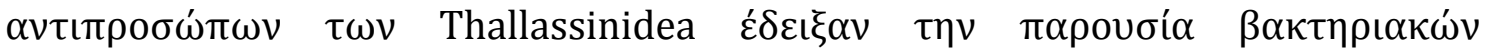

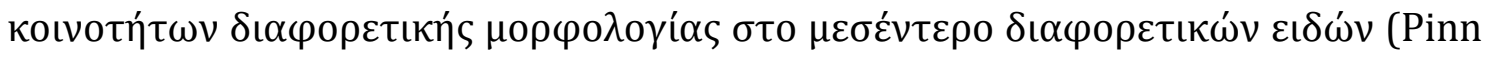

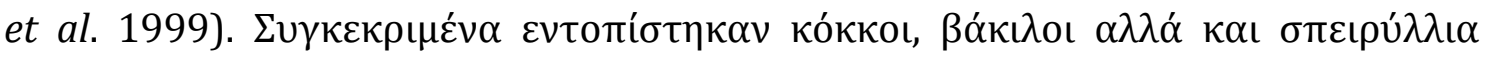
(Еเк. 1.3).
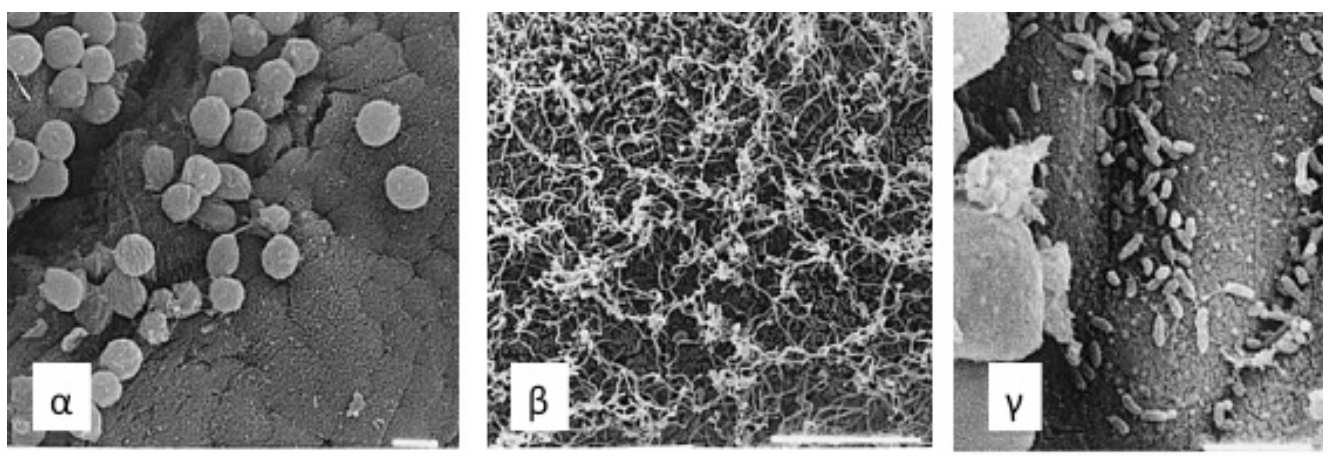

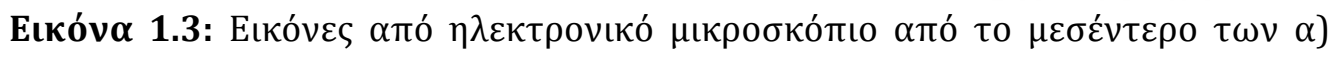
Calocaris mesandreae, $\beta$ ) Calianassa subterranean $\kappa \alpha$ เ) Upogebia

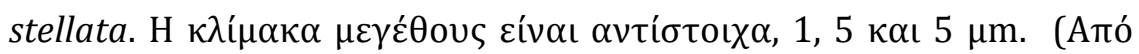
Pinn et al. 1999)

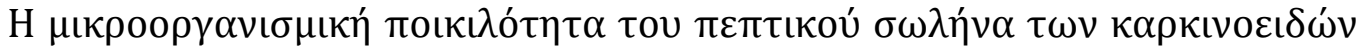

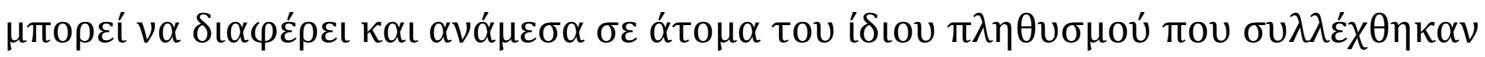

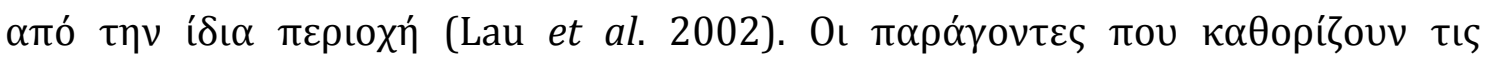

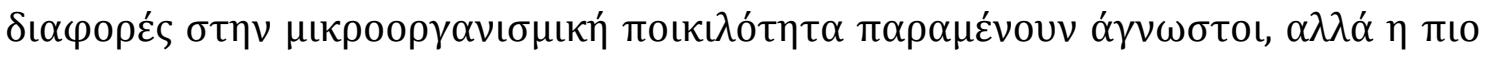

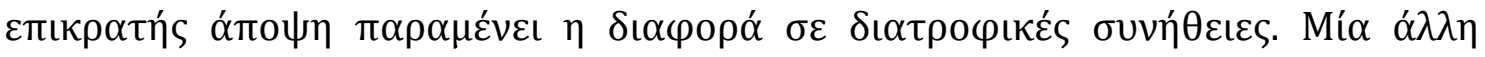

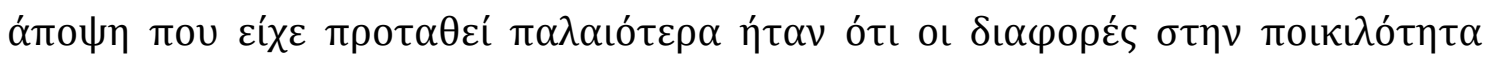

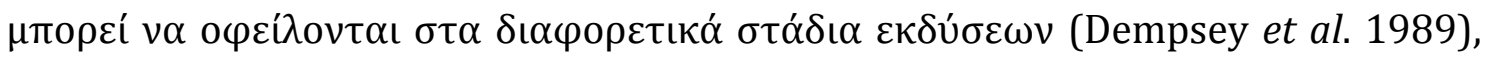




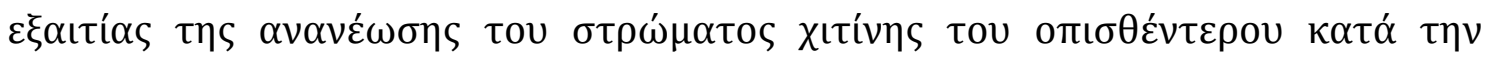

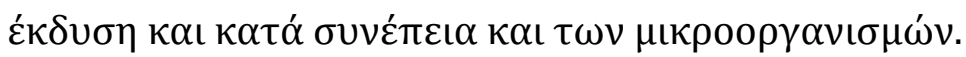

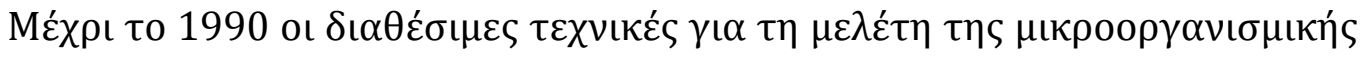

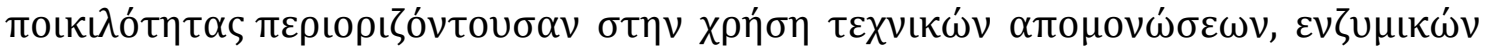

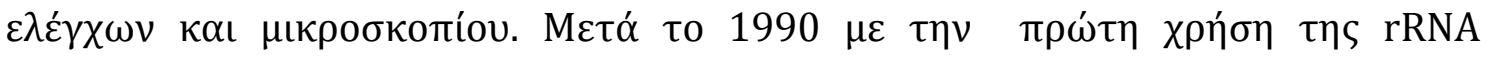

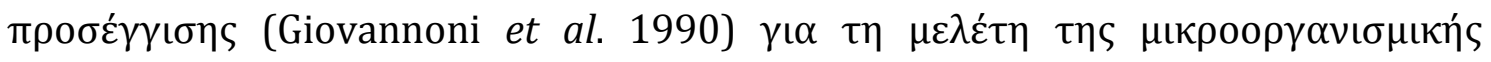

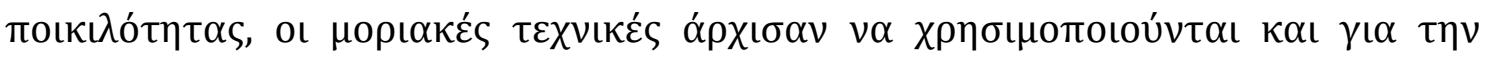

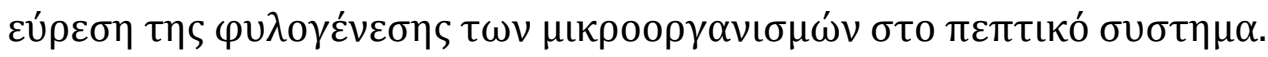

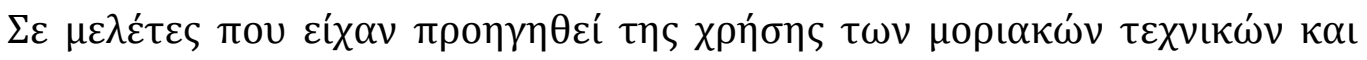

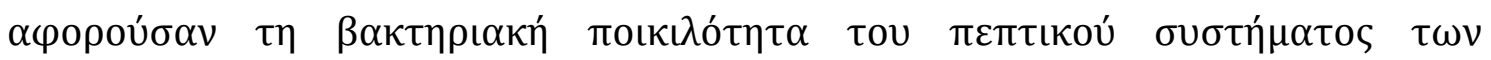

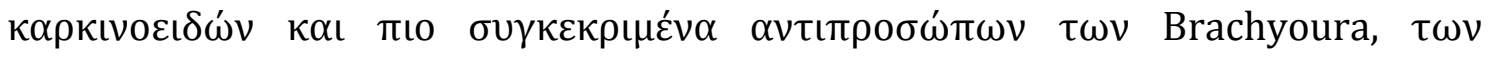

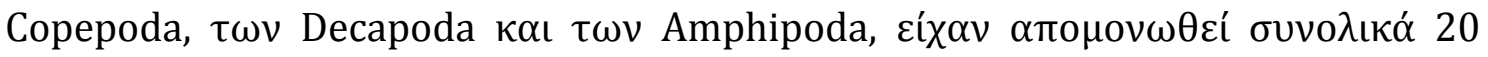

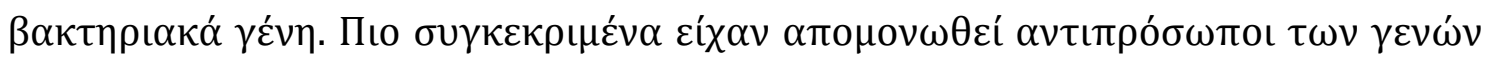
Vibrio, Pseudomonas, Photobacterium, Aeromonas, Citrobacter, Moraxella, Enterobacterium, Acinetobacter, Oceanospirillium, Alteromonas, Xanthomonas ( $\gamma$ Proteobacteria) Flavobacterium, Cytophaga, (Bacteroidetes), Micrococcus, Corynobacterium (Actinobacteria), Staphylococcus, Bacillus, (Firmicutes), Alcaligenes, Chromobacterium, Achromobacter ( $\beta$-Proteobacteria) (Harris 1993).

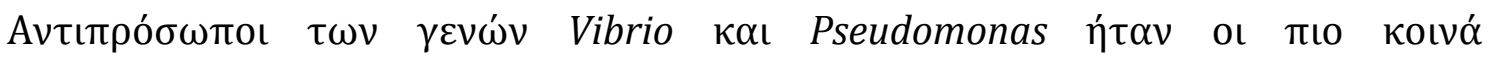

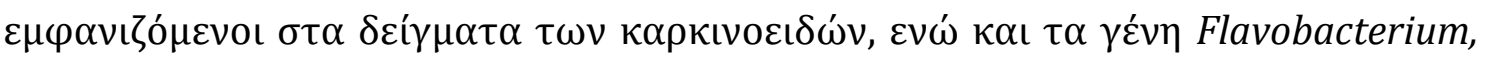

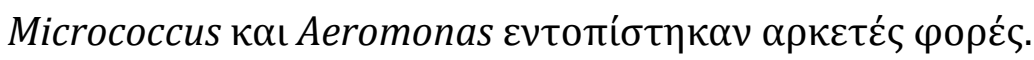

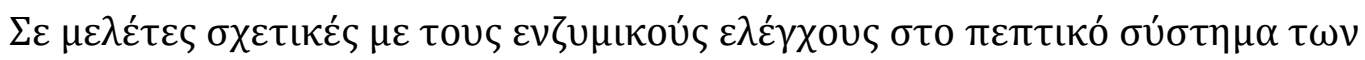

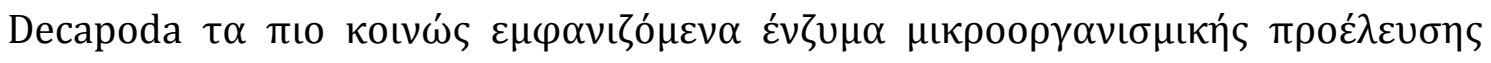

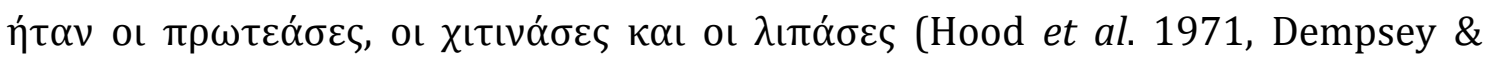




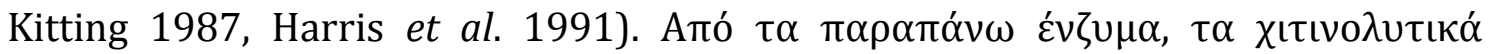

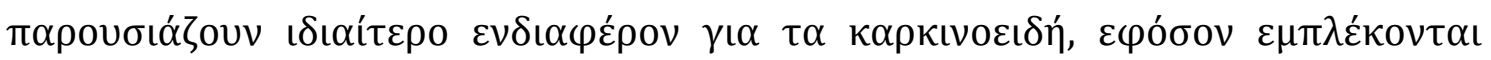

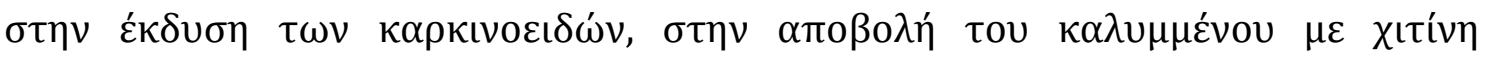

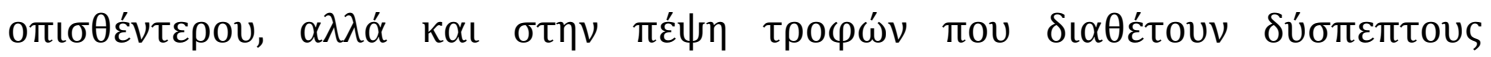
$\varepsilon \xi \omega \sigma \kappa \varepsilon \lambda \varepsilon \tau o u ́ s$.

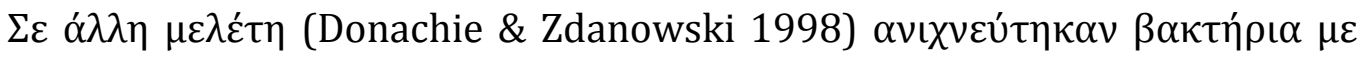

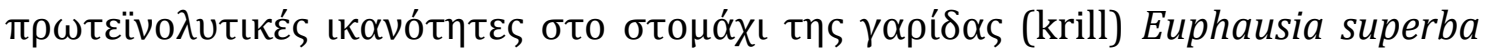

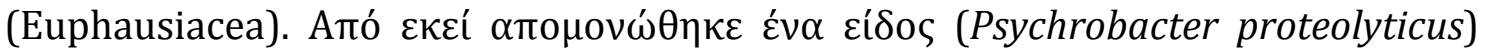

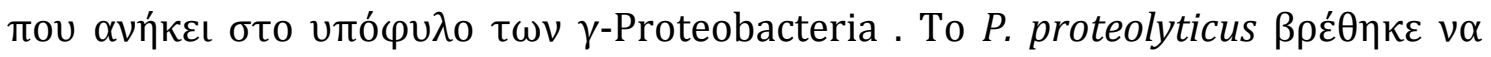

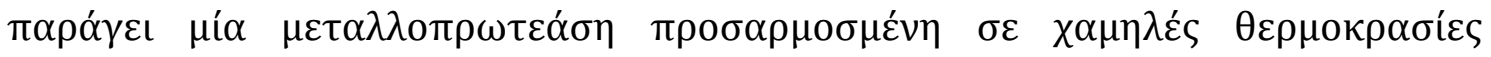

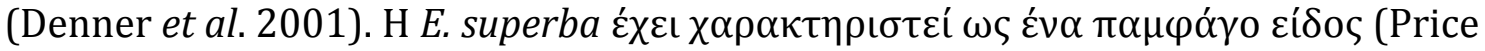

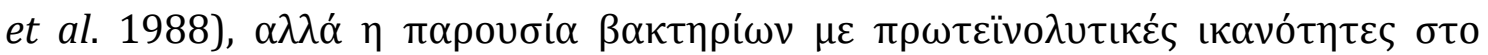

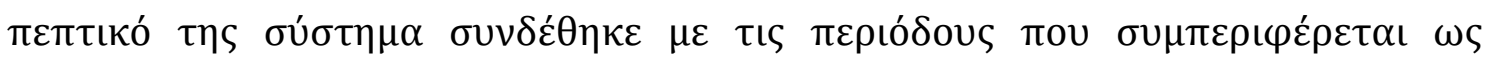

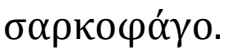

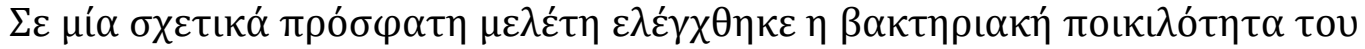

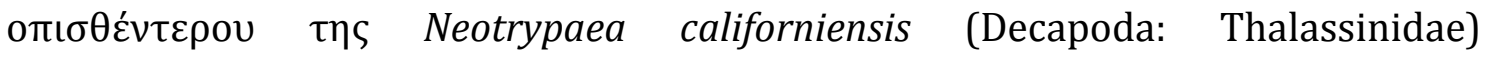

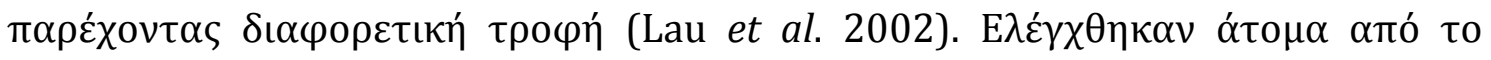

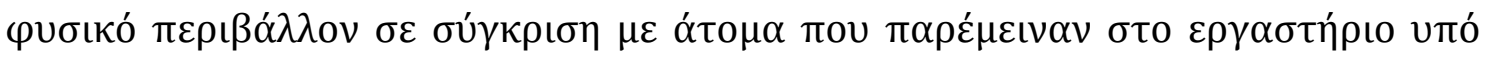

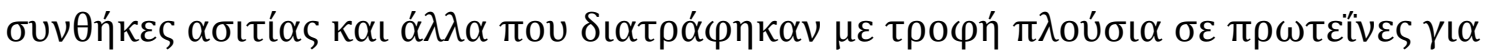

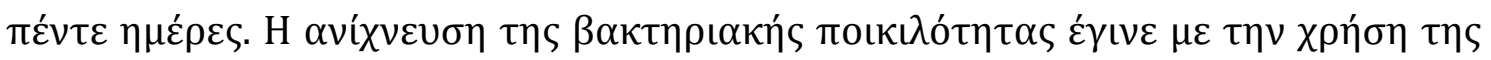

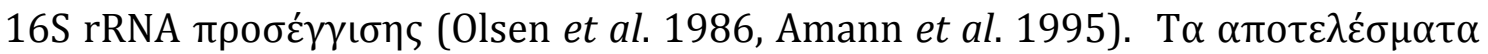

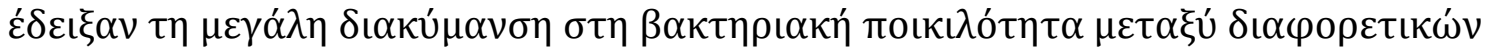

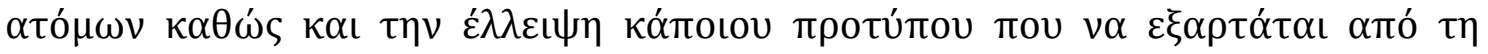

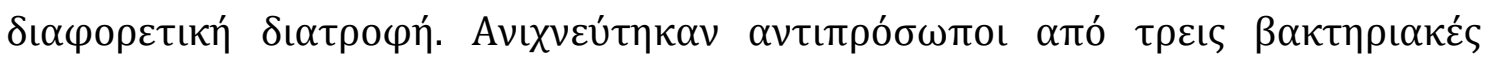


o $\alpha_{\alpha} \delta \varepsilon \varsigma, \quad$ Proteobacteria ( $\left.\alpha-, \quad \gamma^{-} \quad \kappa \alpha \iota \quad \varepsilon-\right), \quad$ CFB (Cytophaga-Flavobacteria-

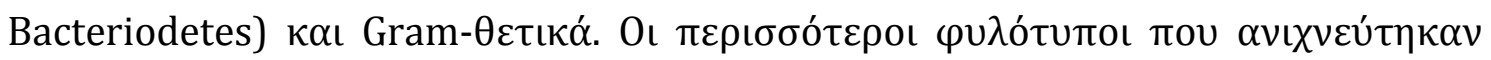

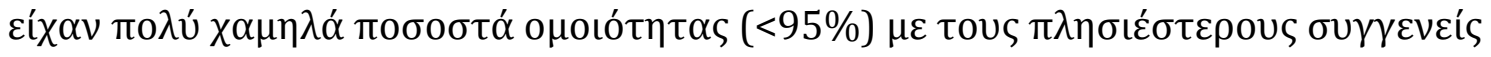

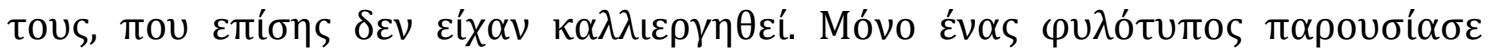

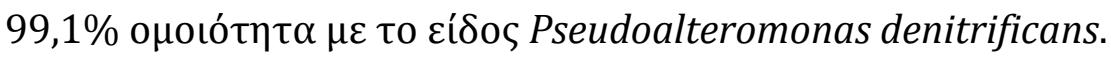

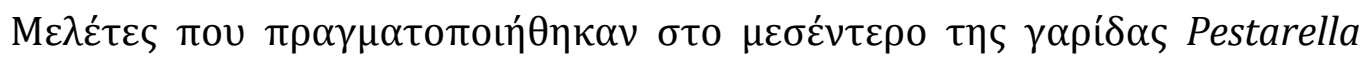

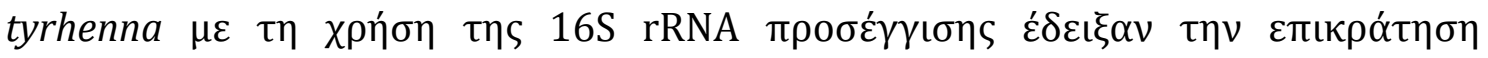
$\varphi$

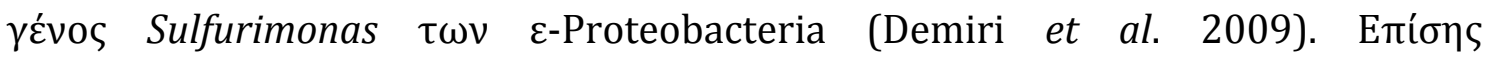

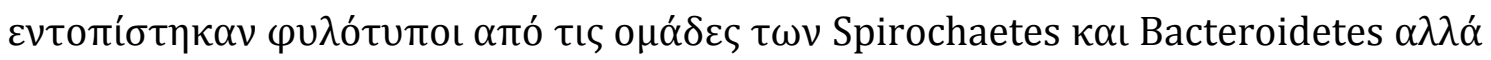

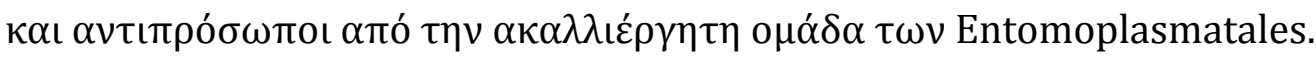

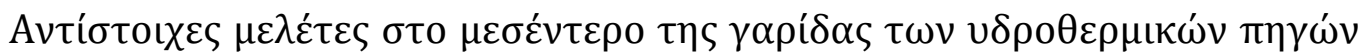

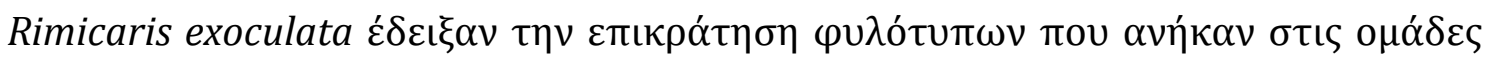
$\tau \omega \nu \varepsilon$-Proteobacteria, Defferibacteres $\kappa \alpha \iota$ Entomoplasmatales (Zbinden \&

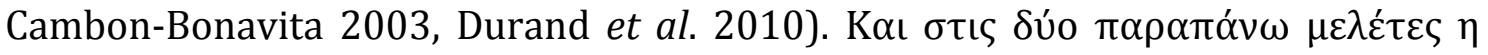

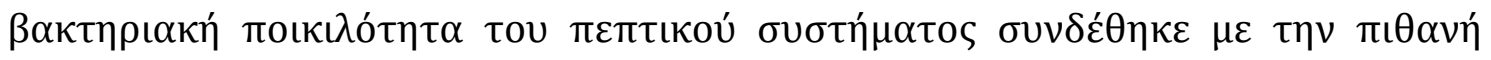

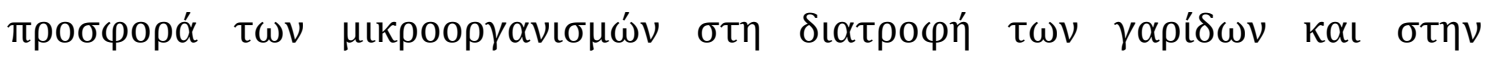

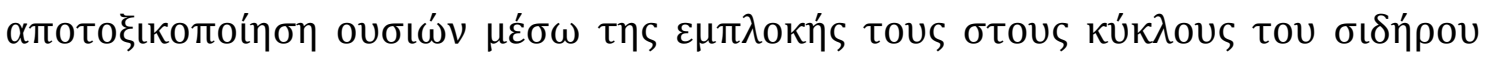
(Fe) каı тou $\theta$ cíou (S).

\section{7. Ү Ү}

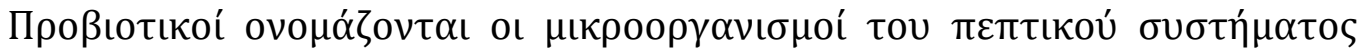

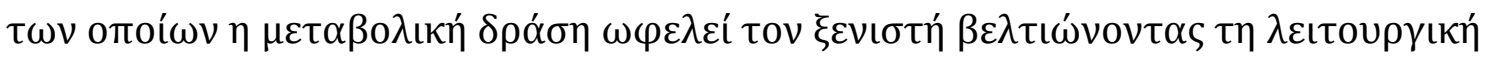




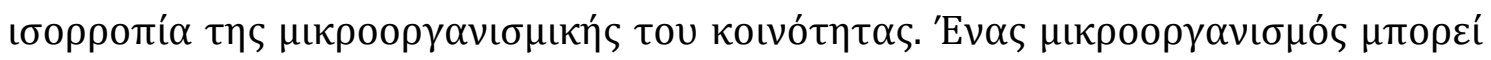

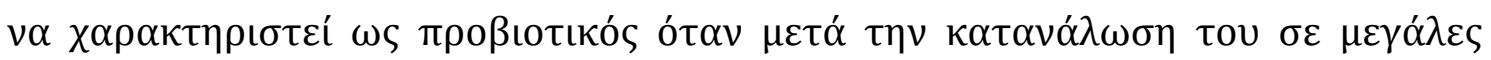

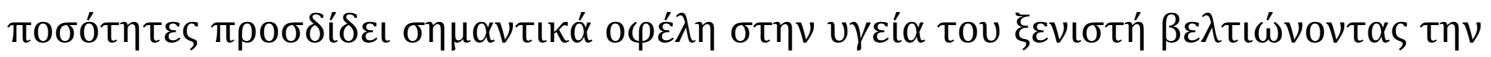

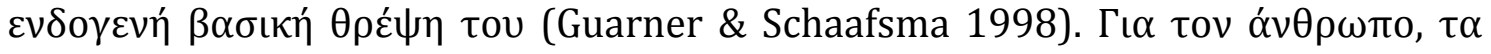

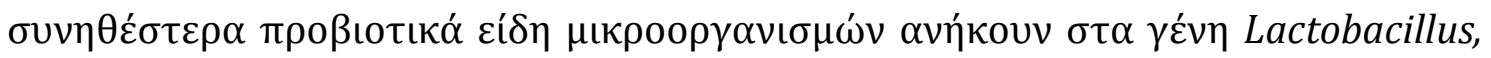
Bifidobacterium каı Enterococcus (Fuller 1991).

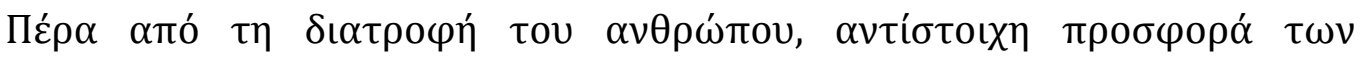

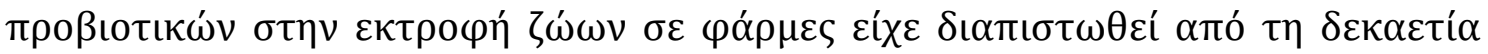

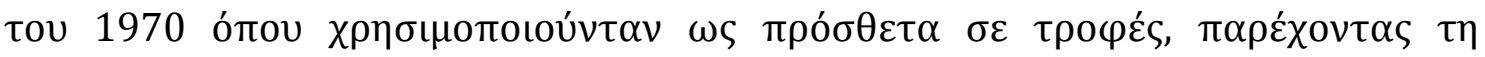

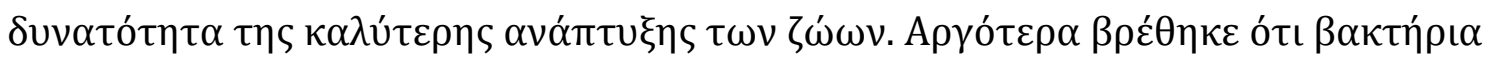

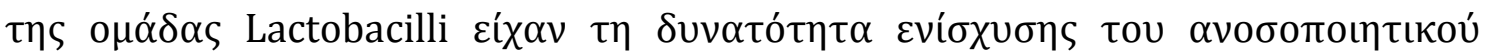

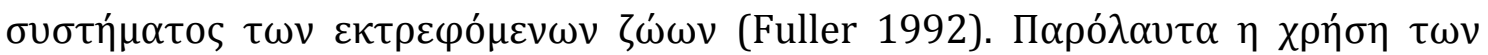
$\pi \rho \circ \beta \iota \tau \tau$ เ

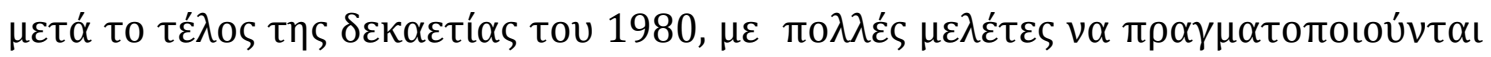

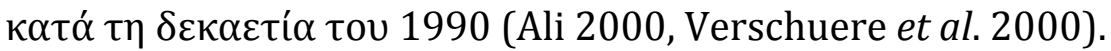

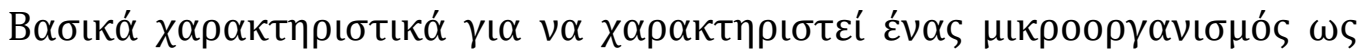

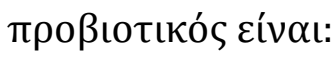

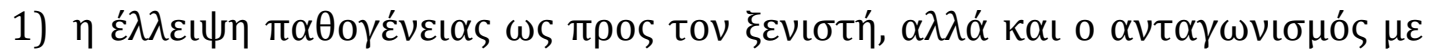

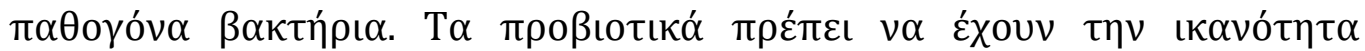

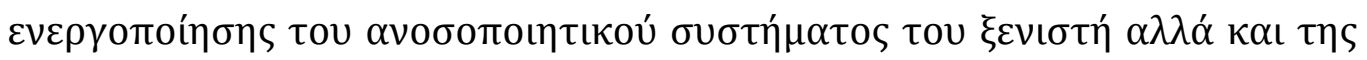

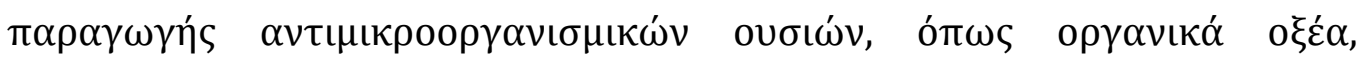

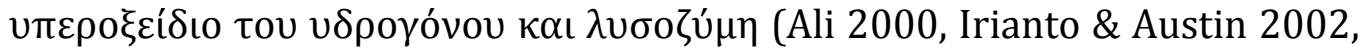
Irianto \& Austin 2003) 


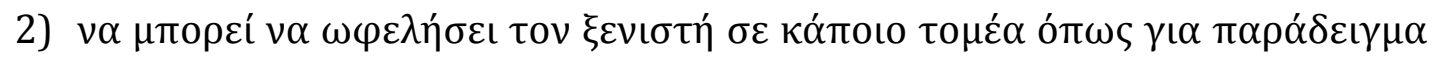

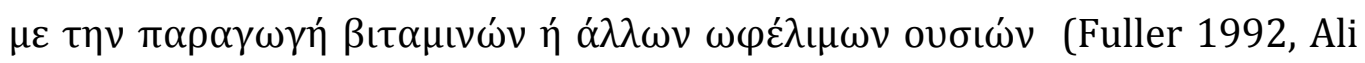
2000, Irianto \& Austin 2002)

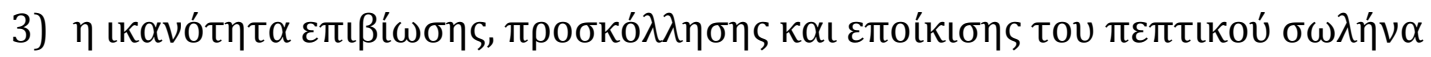

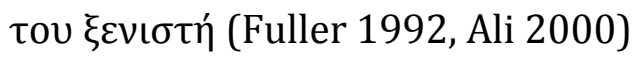

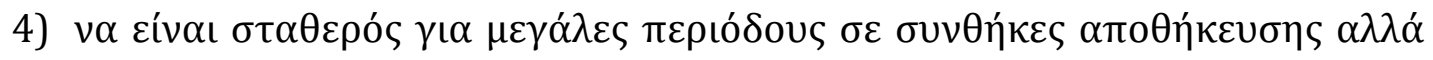

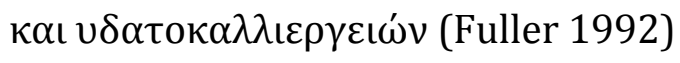

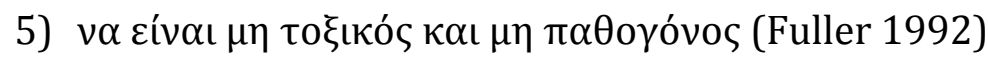

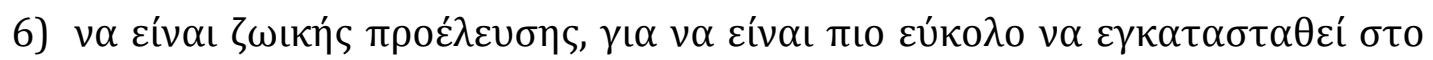

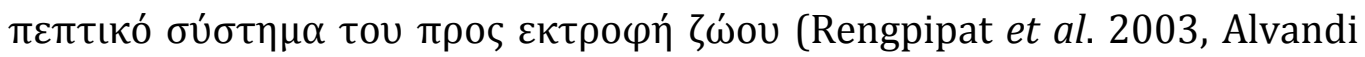
et al. 2004)

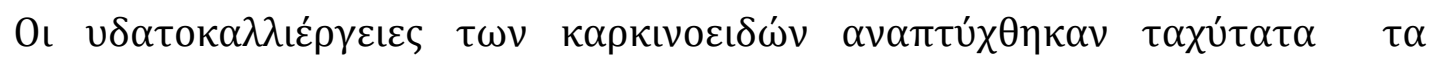

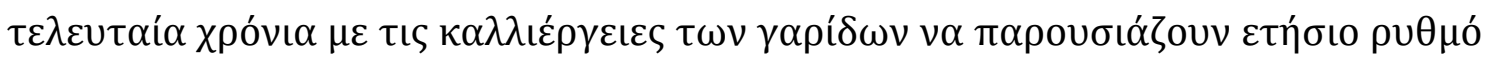

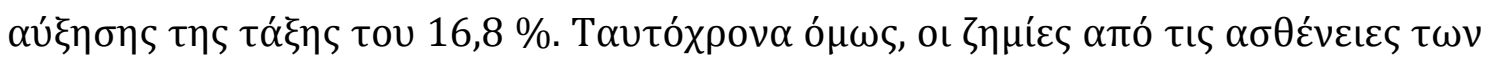

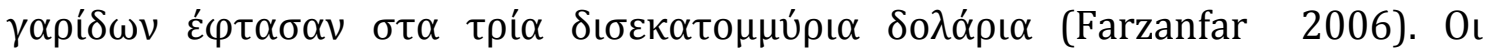

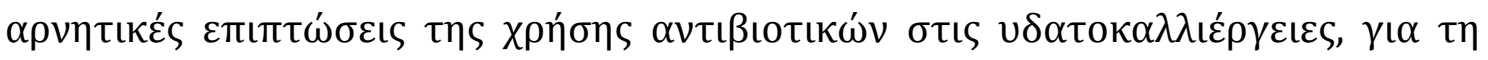

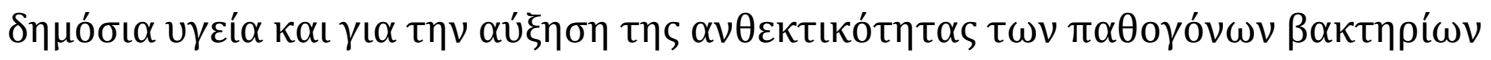

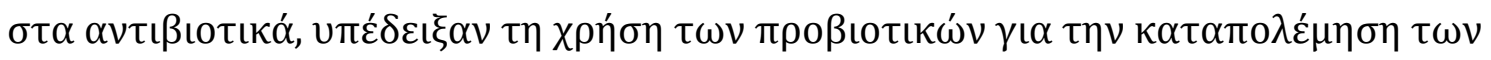
$\alpha \sigma \theta \varepsilon v \varepsilon ı \omega ́ v ~ \sigma \tau \iota \varsigma ~ v \delta \alpha \tau о \kappa \alpha \lambda \lambda \iota \varepsilon ́ p \gamma \varepsilon เ \varepsilon \varsigma$ (Vaseeharan \& Ramasamy 2003).

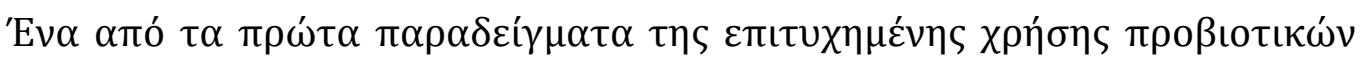

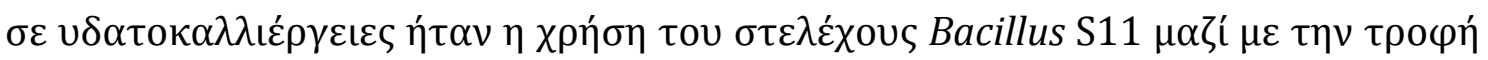

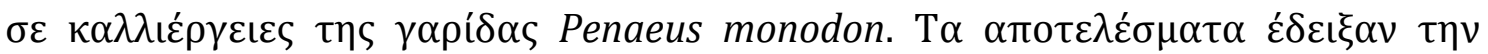

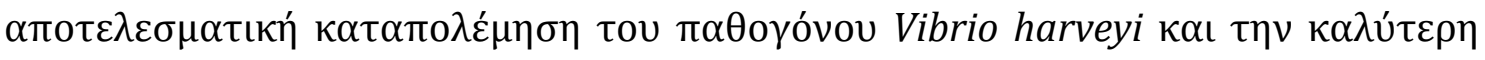

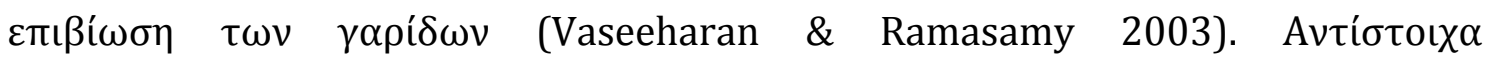




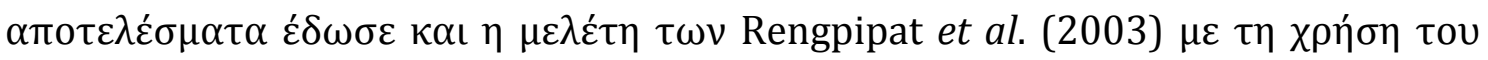

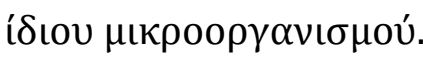

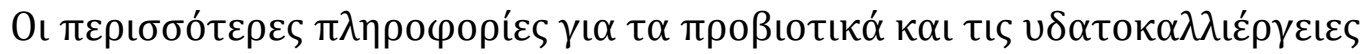

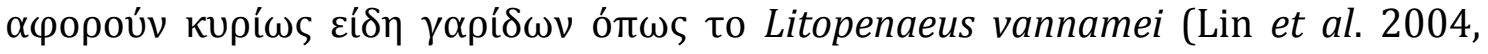
Balcázar et al. 2007, Chiu et al. 2007) Kaı to Penaeus monodon (Dalmin et al. 2001,

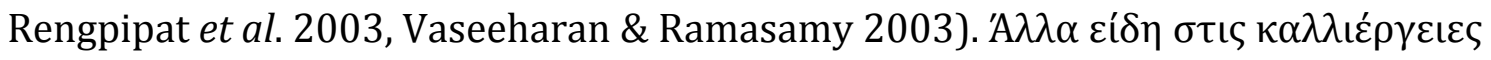

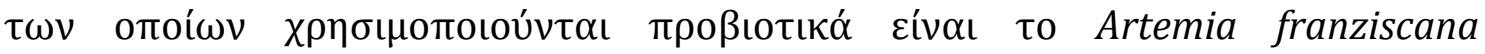

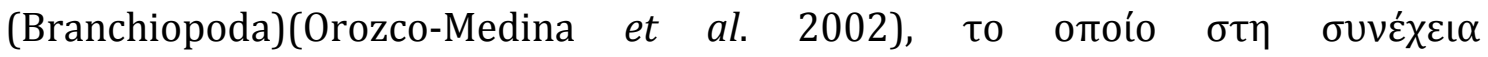

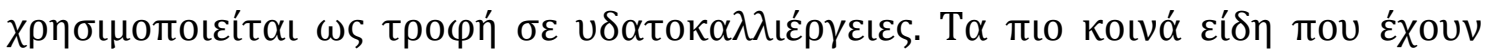

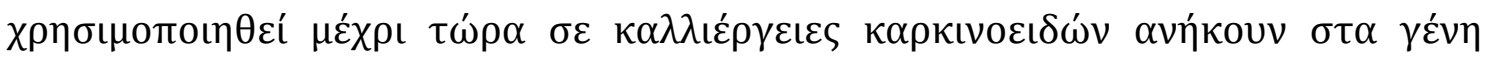

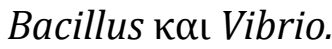

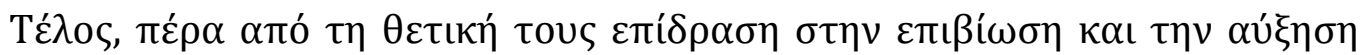

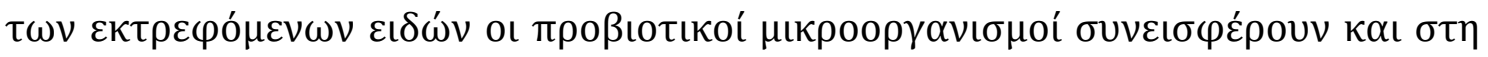

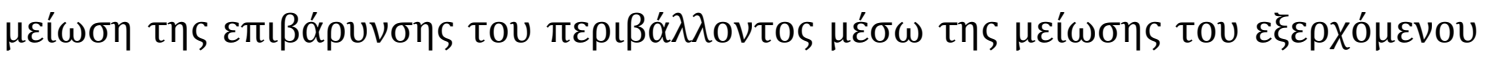

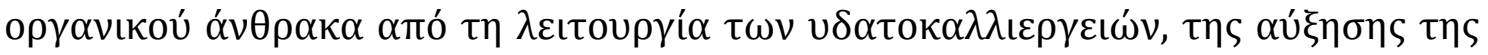

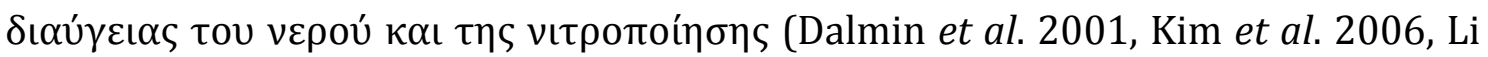
et al. 2006).

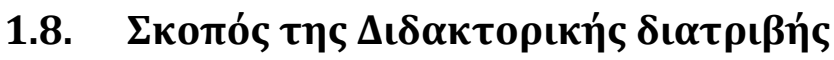

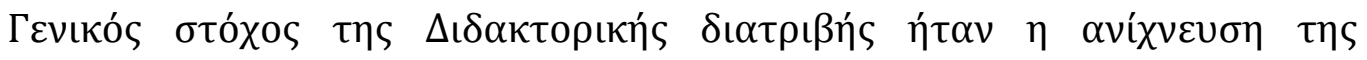

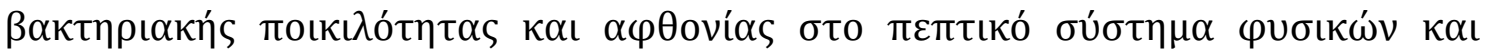

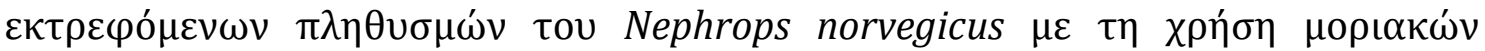
$\tau \varepsilon \chi \nu เ \kappa \omega ́ v$. 


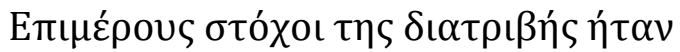

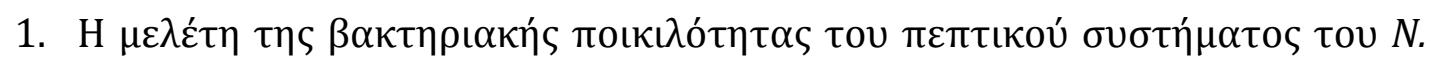

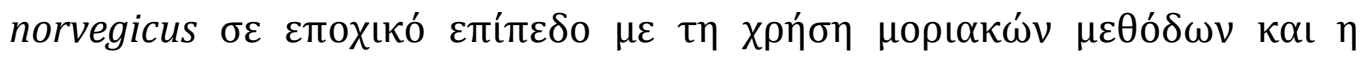

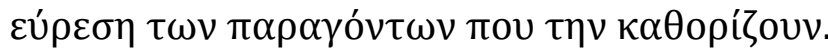

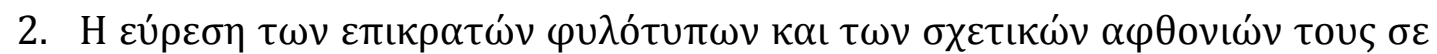

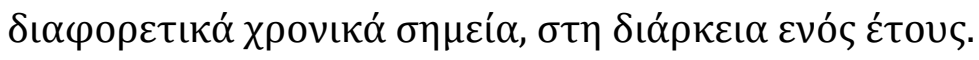

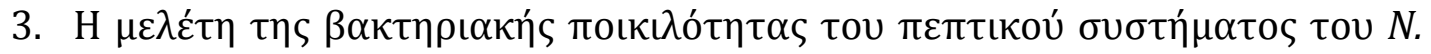

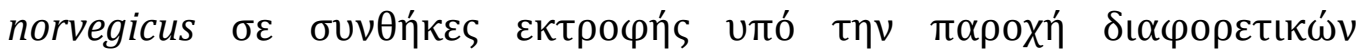

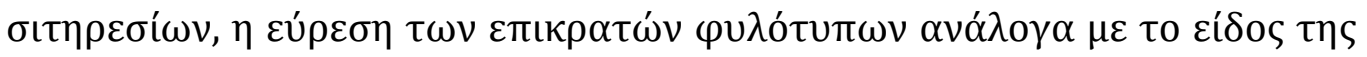

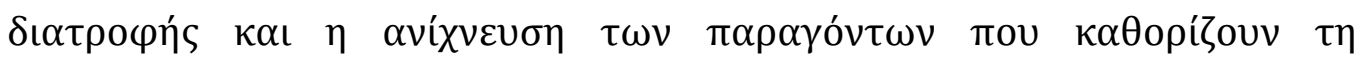

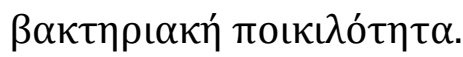

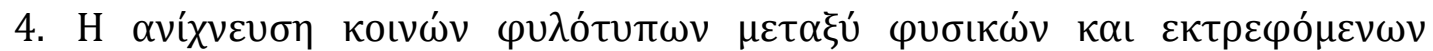
$\pi \lambda \eta \theta v \sigma \mu \omega ́ v$.

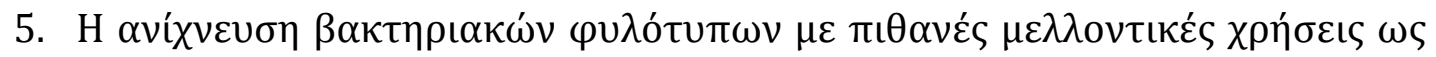

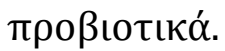




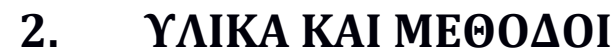

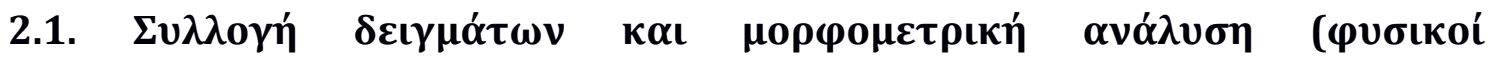 $\pi \lambda \eta \theta v \sigma \mu o i ́)$}

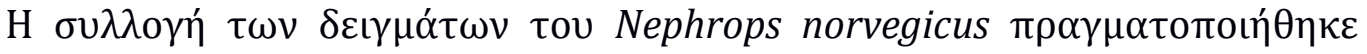

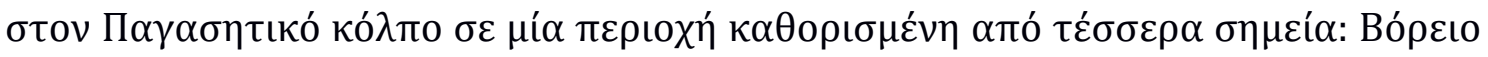

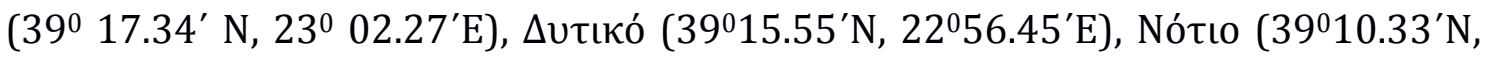

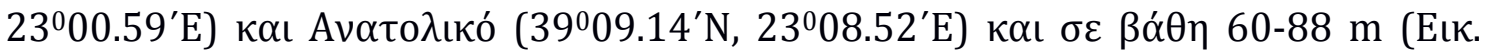

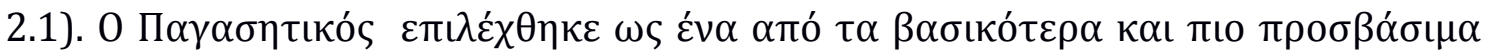

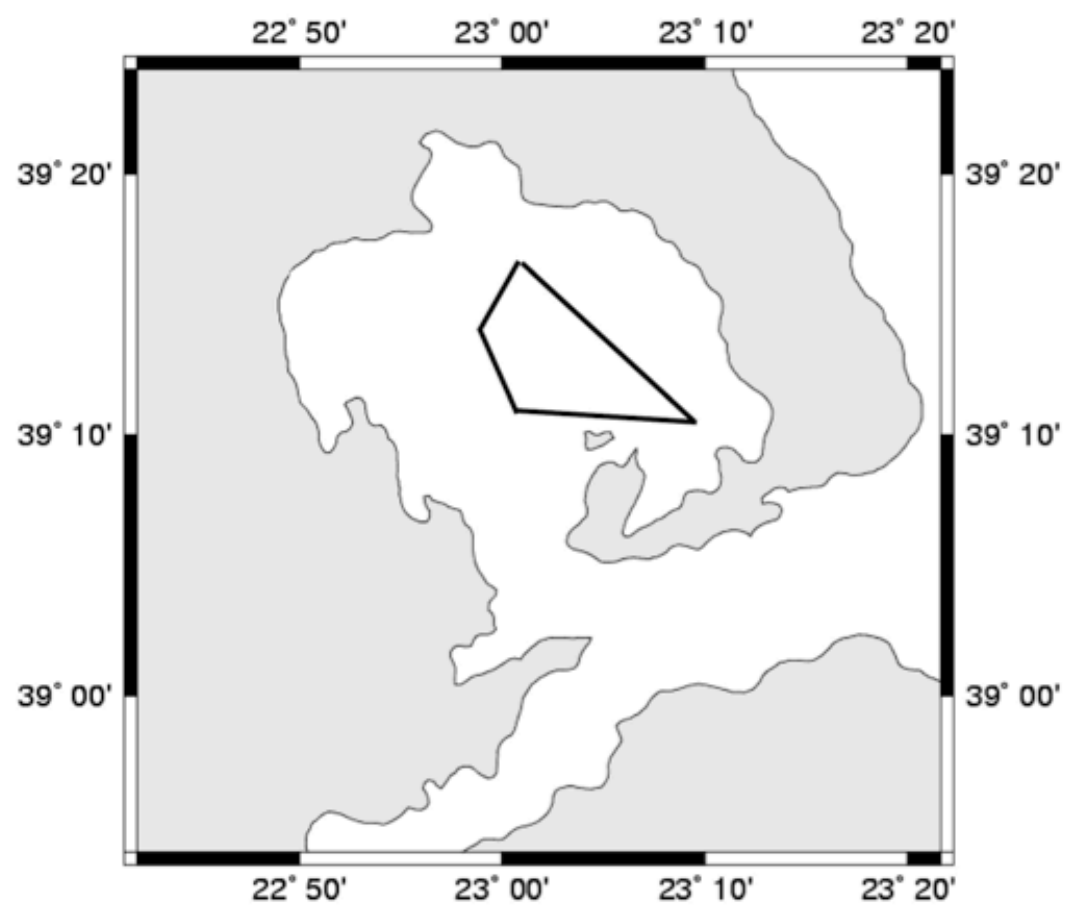

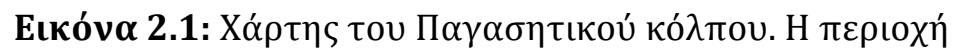

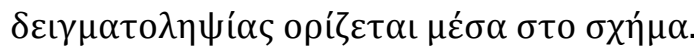




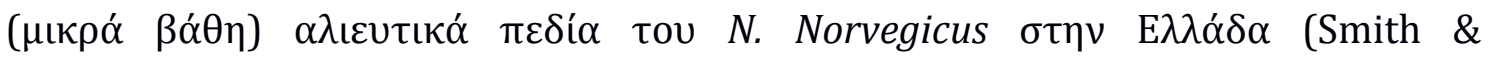
Papadopoulou 2003).

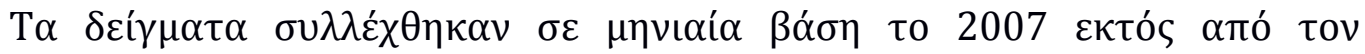

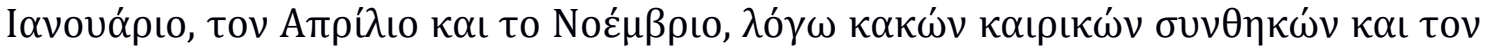

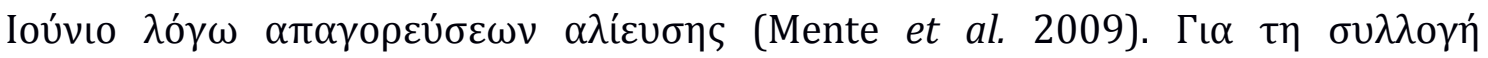

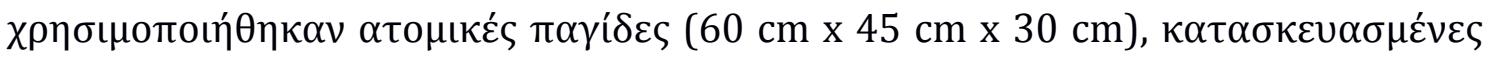

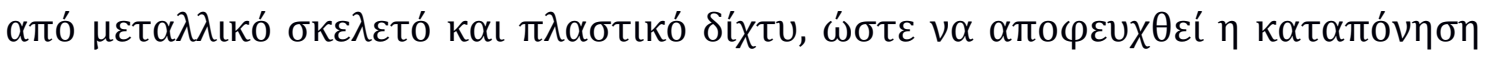

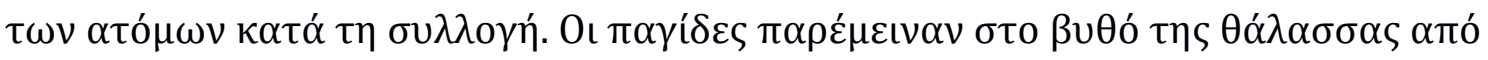

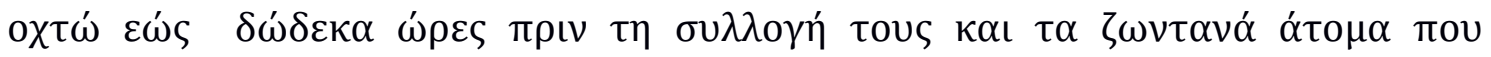

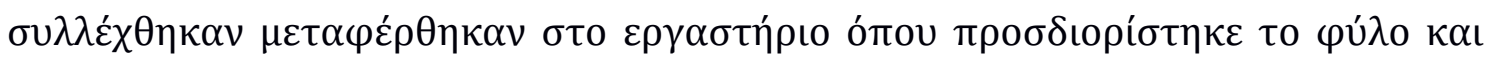

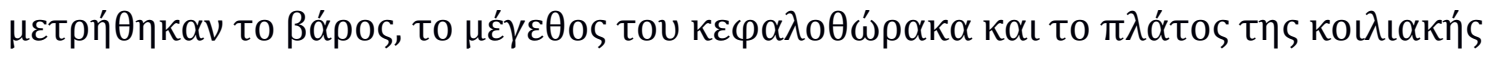

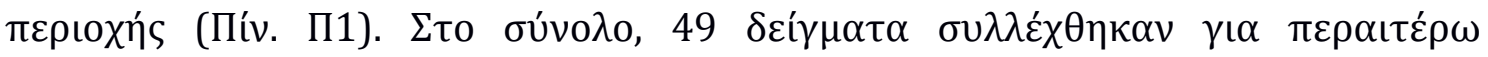
$\alpha \nu \alpha ́ \lambda v \sigma \eta$.

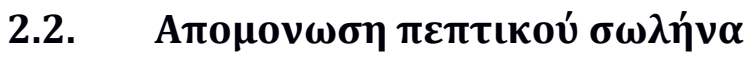

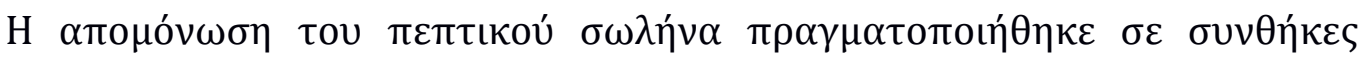

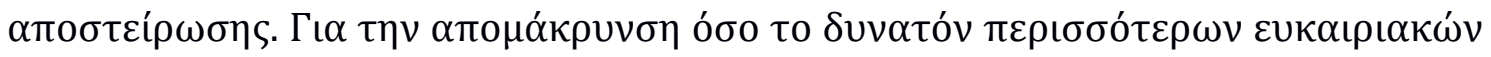

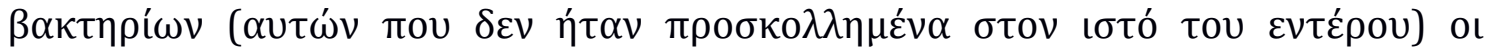

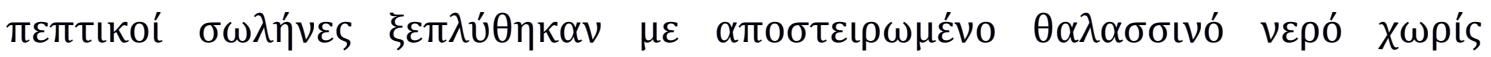

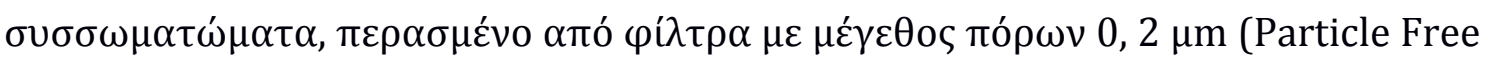

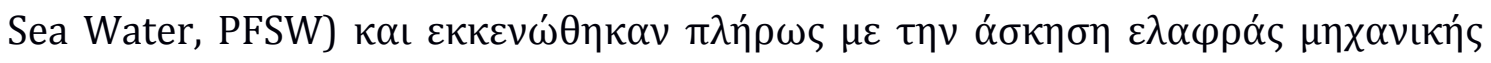

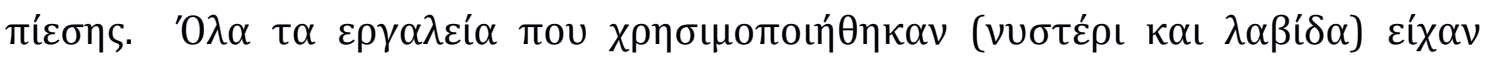

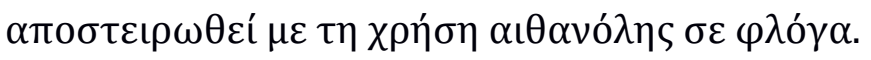




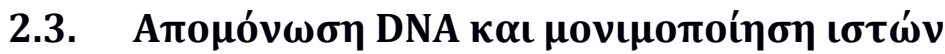

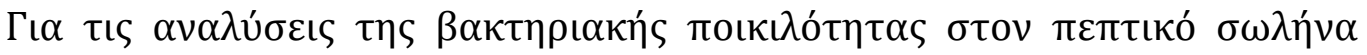

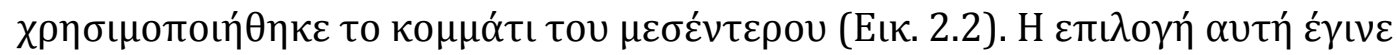

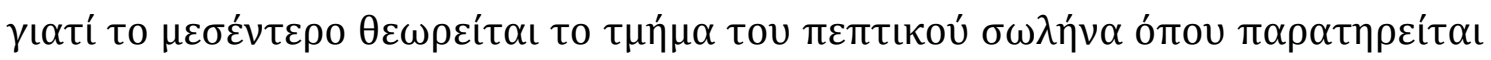

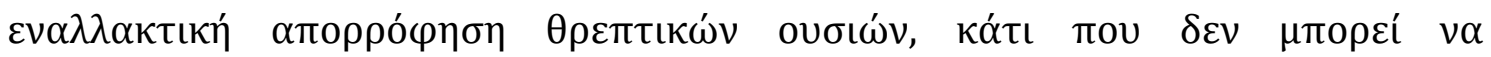

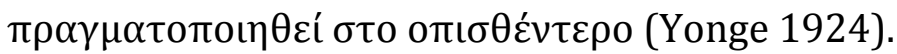

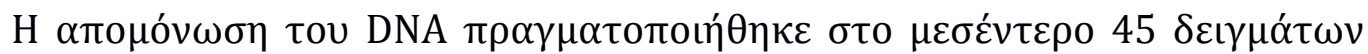

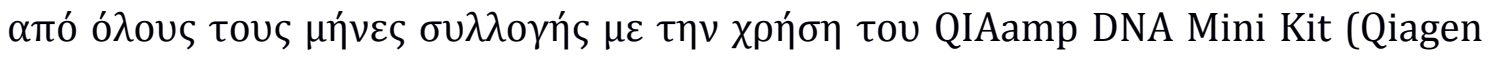

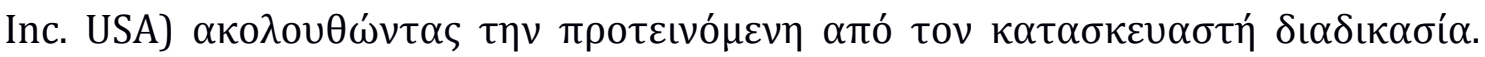

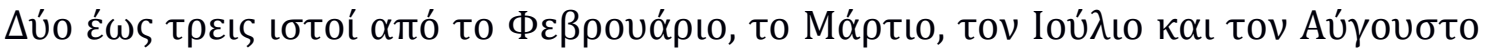

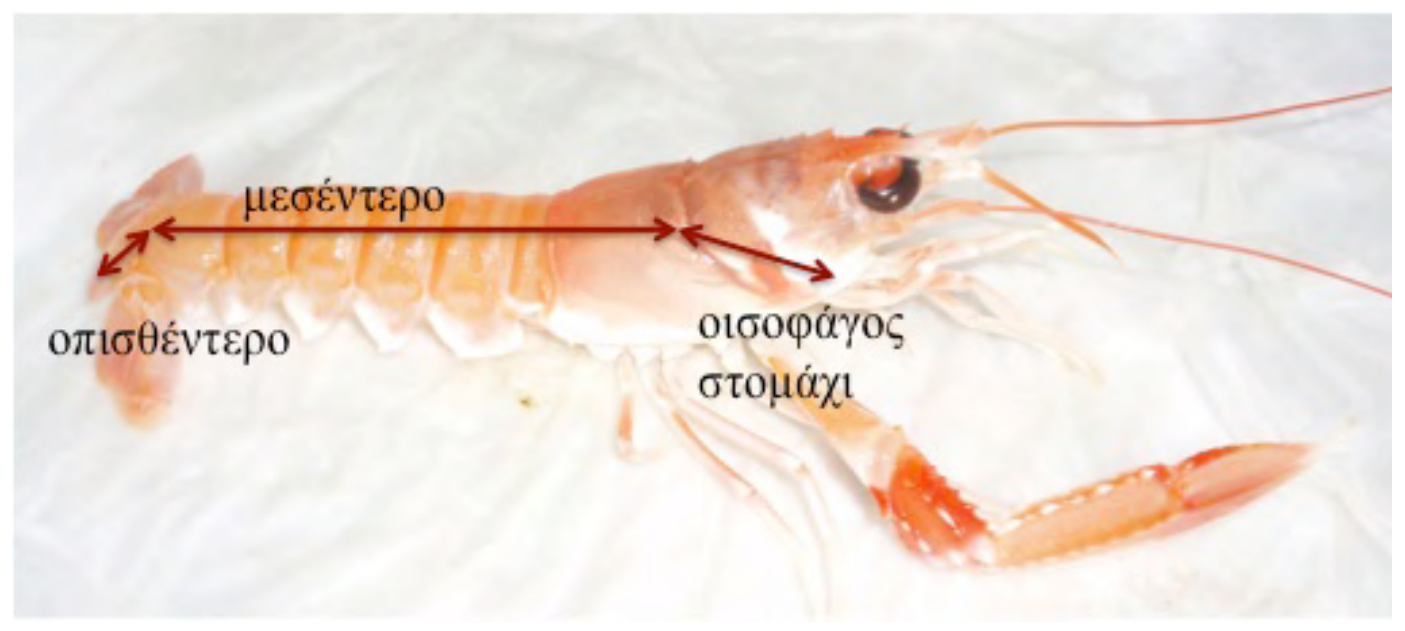

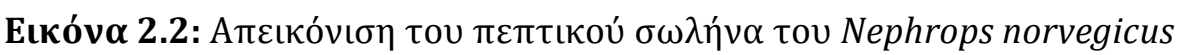

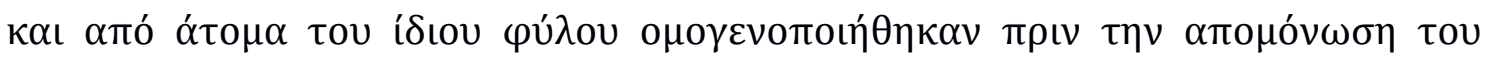




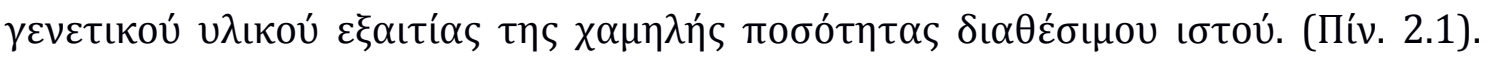

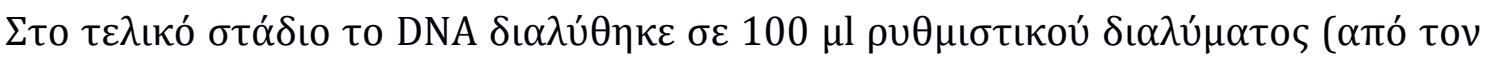

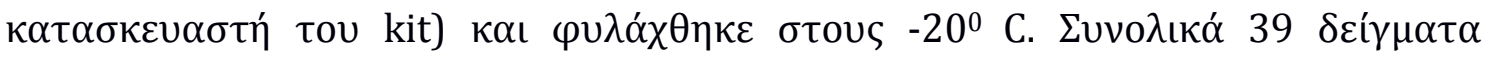

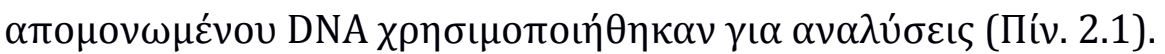

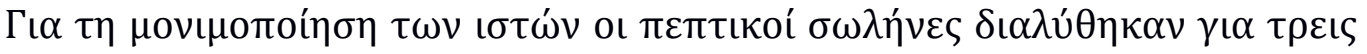

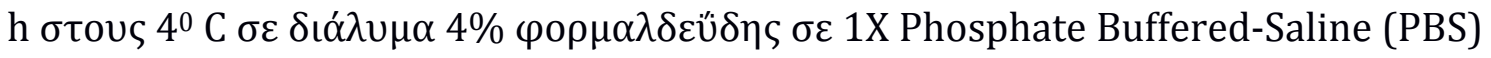

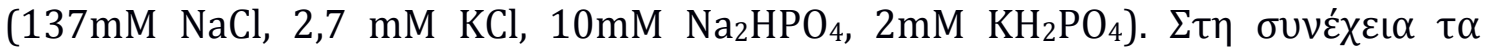

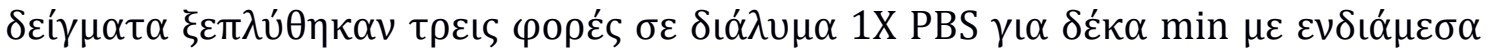

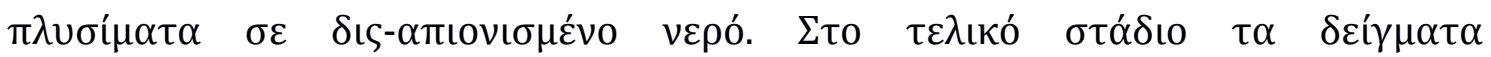

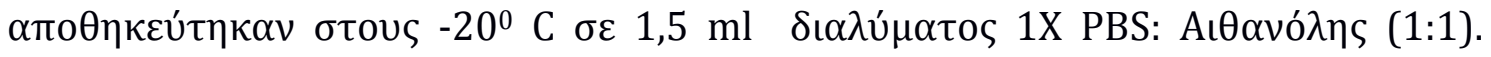

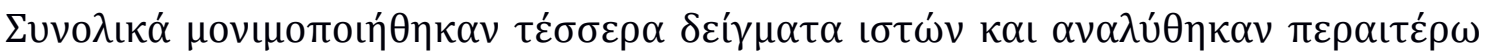

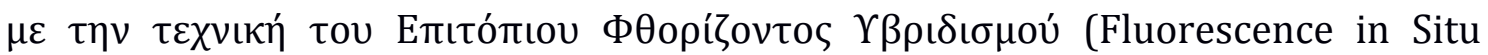
Hybridization, FISH) (Пív. 2.1.).

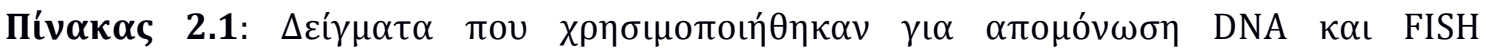

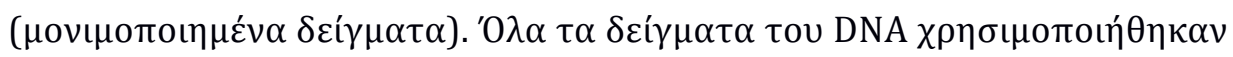

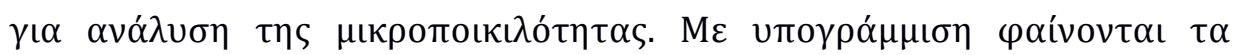

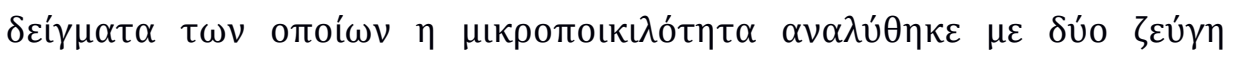

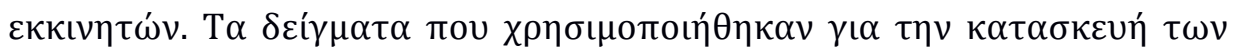

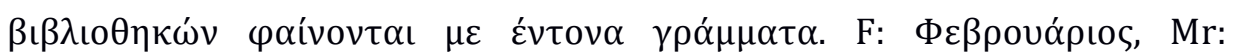

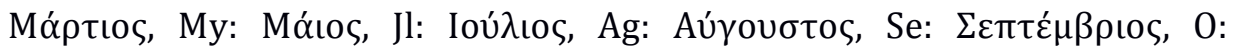

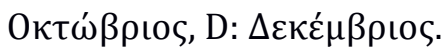

\begin{tabular}{|c|c|c|c|}
\hline Mńvas & 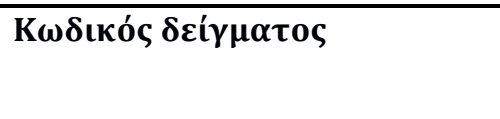 & 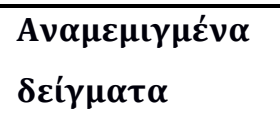 & 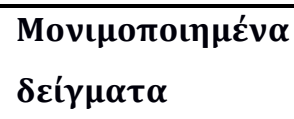 \\
\hline 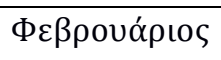 & - & 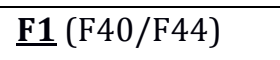 & \\
\hline Móptios & Mr26 & Mr1 (Mr4/Mr10) & \\
\hline \multirow[t]{2}{*}{ Móıos } & My27/My44/My45/My46/My4 & & \\
\hline & 7 & & \\
\hline
\end{tabular}




\begin{tabular}{|c|c|c|c|}
\hline Mńvas & 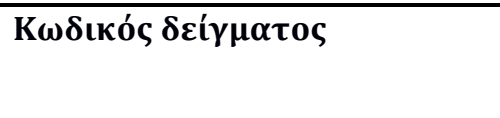 & 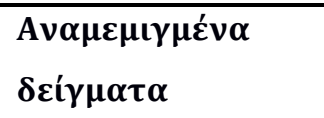 & 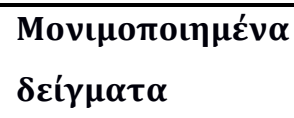 \\
\hline Ioú̉ıs & $\mathrm{Jl1} / \mathrm{Jl} 3$ & Jl4 (Jl14/Jl17/Jl19) & \\
\hline 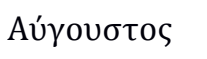 & 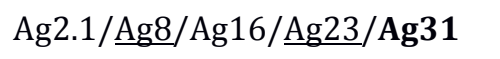 & Ag1 (Ag4/Ag6/Ag9) & FN2/FN3 \\
\hline$\Sigma \varepsilon \pi \tau \varepsilon \dot{\mu} \mu \beta \rho\llcorner$ & $\begin{array}{l}\mathrm{Se} 1 / \mathrm{Se} 2 / \underline{\mathrm{Se}} 3 / \mathrm{Se} 4 / \mathrm{Se} 5 / \mathrm{Se} 6 / \mathrm{Se} 7 \\
/ \mathrm{Se} 9 / \mathrm{Se} 11 / \underline{\mathrm{Se} 12}\end{array}$ & & FSe3/FSe1 \\
\hline 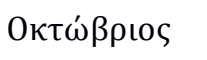 & $\underline{01 / 02} / 03 / 04 / 05 / 06$ & & \\
\hline$\Delta \varepsilon \kappa \varepsilon ́ \mu \beta \rho ı \varsigma$ & $\underline{\text { D1/D2/D3/D4/D6/D7 }}$ & & \\
\hline
\end{tabular}

\subsection{Avód}

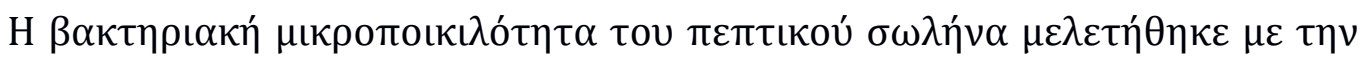

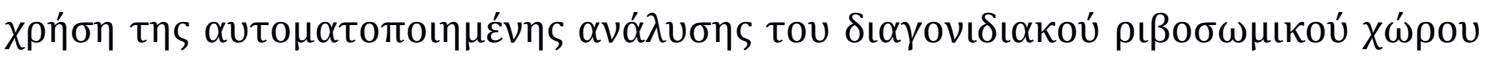
(Automated Ribosomal Intergenic Spacer Analysis, ARISA) (Fischer \& Triplett

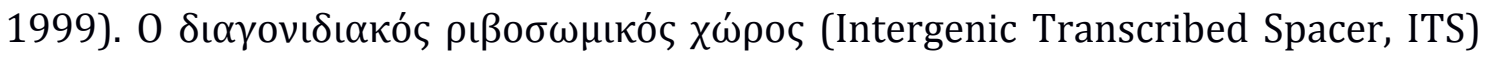

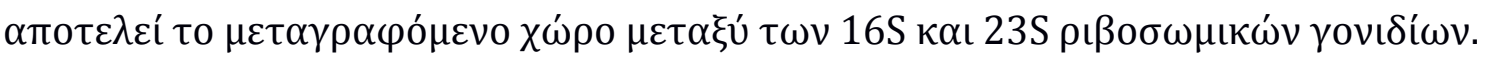

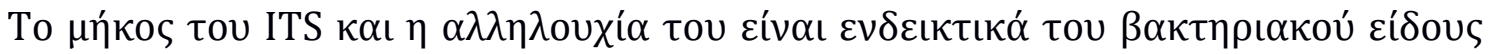

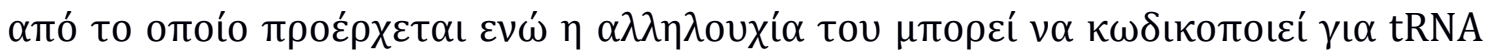

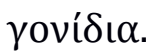

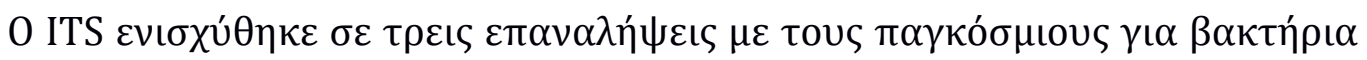

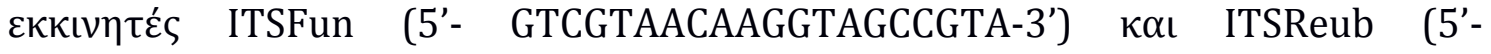

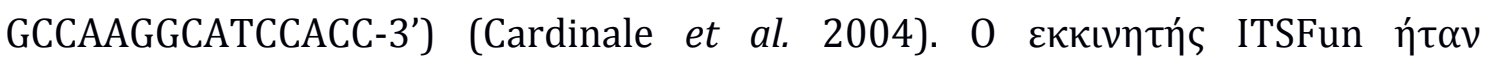

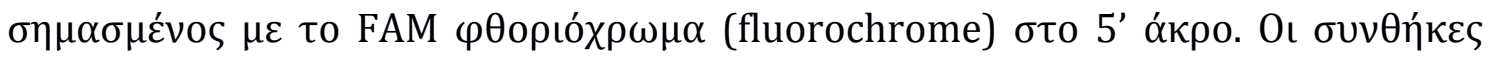

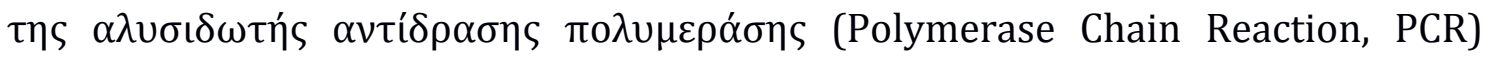

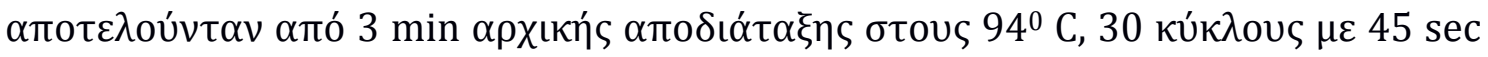

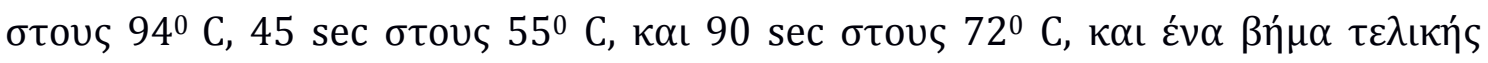




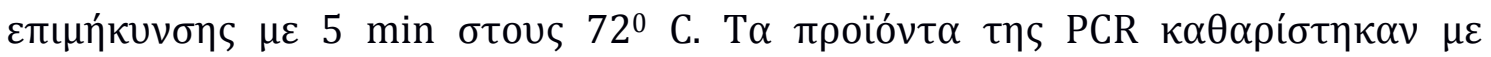

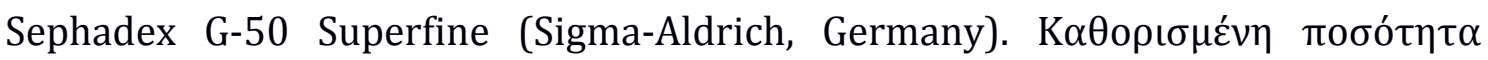

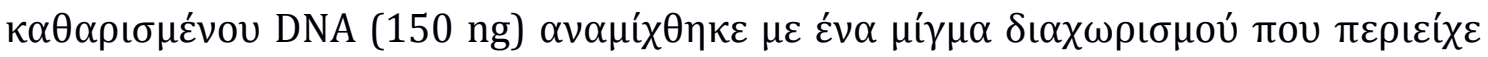

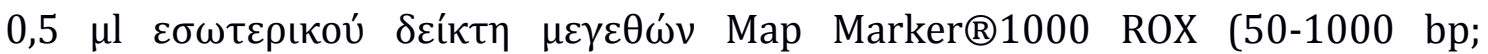
BioVentures Inc., Washington, DC, USA), 0,5 $\mu$ l tracking dye (BioVentures) к $\alpha$ ı $14 \mu \mathrm{l}$ $\alpha \pi ı v ı \sigma \mu \varepsilon ́ v o v$ Hi-Di $\varphi о \rho \mu \alpha \mu$ เ́íov (Applied Biosystems, Foster City, CA, USA). 0

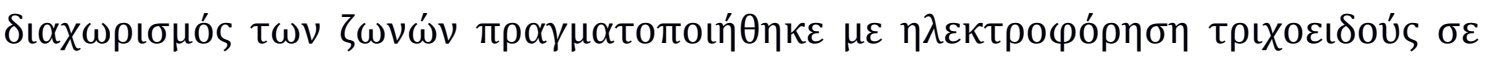

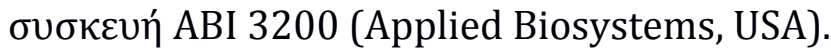

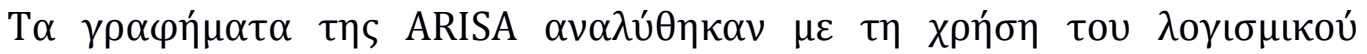

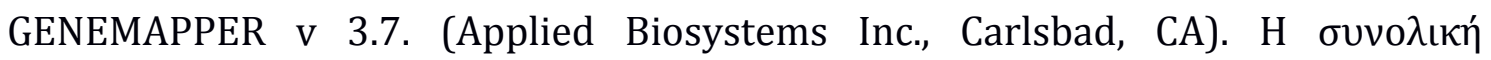

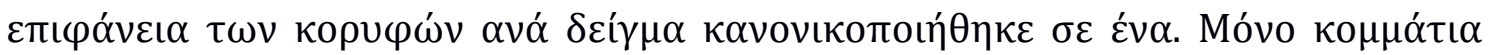

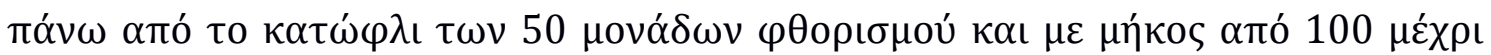

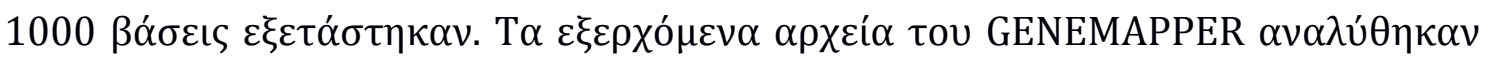

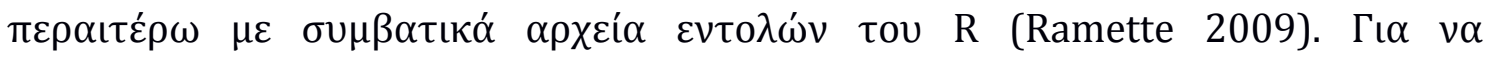

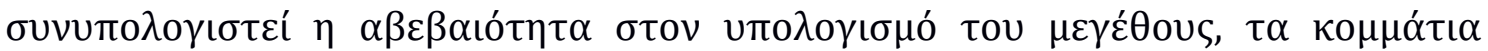

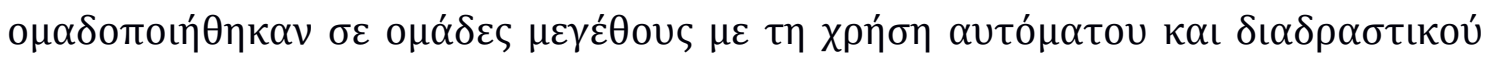

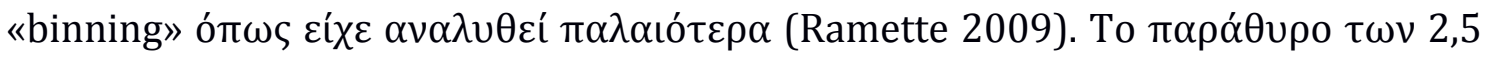

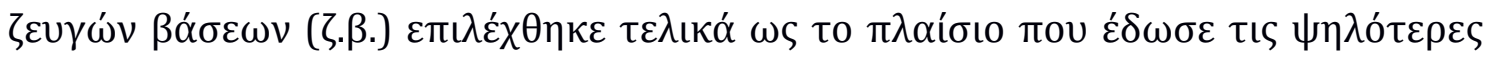

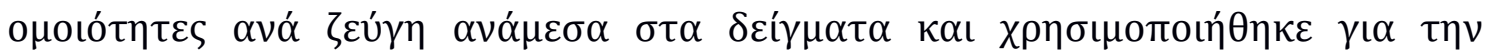

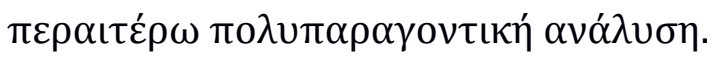

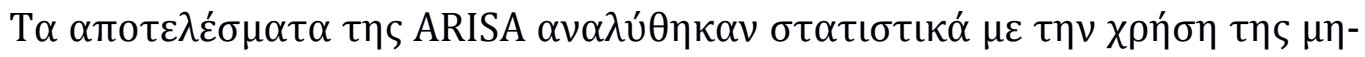

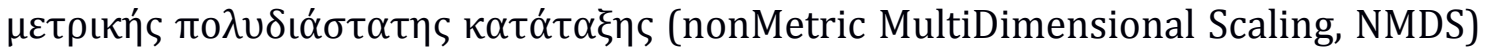

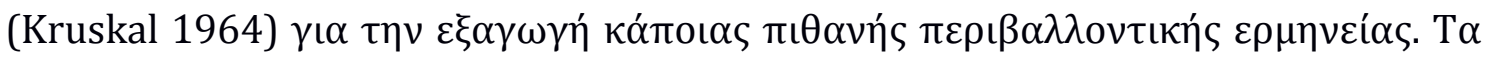

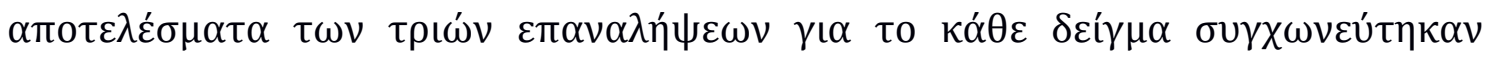




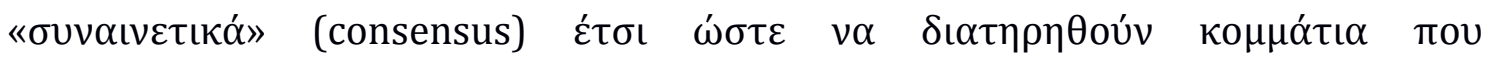

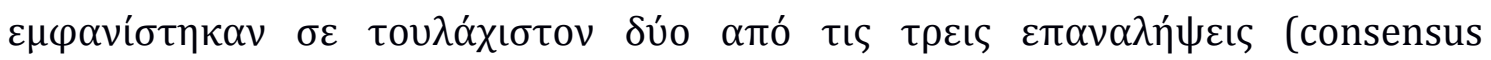

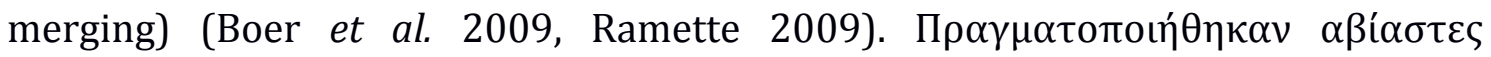

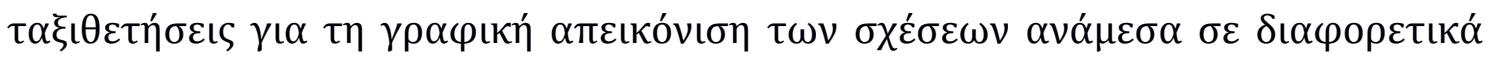

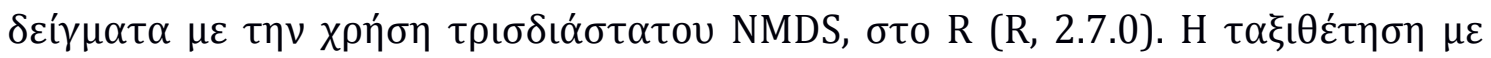

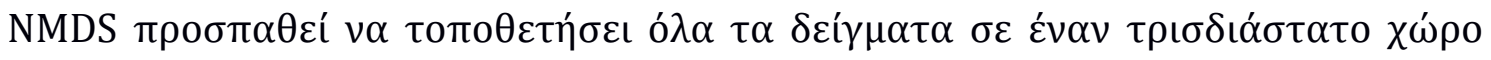

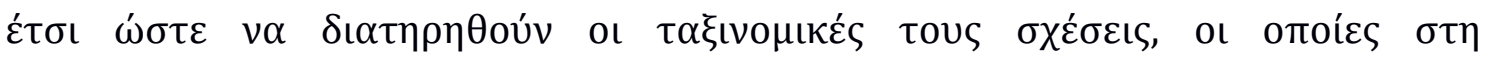

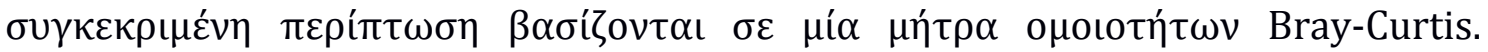

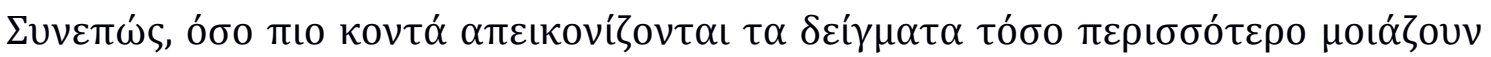

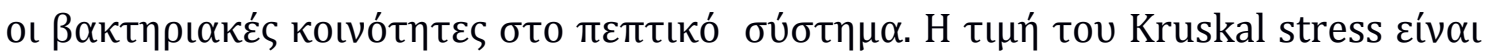

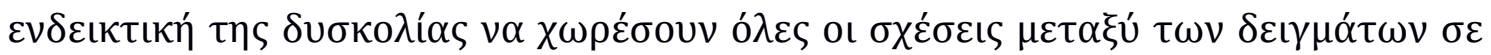

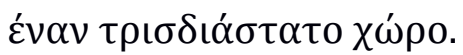

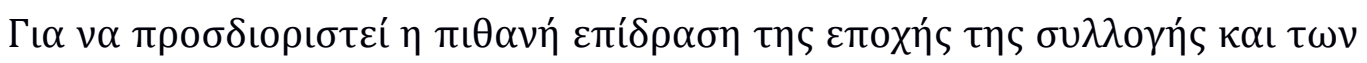

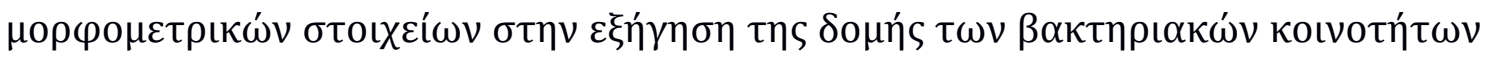

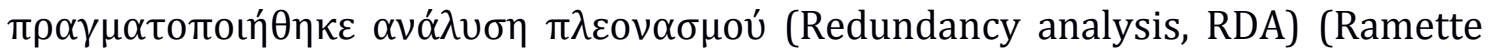

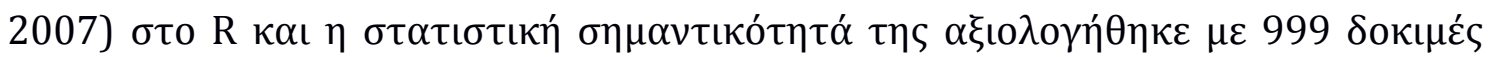
$\mu \varepsilon \tau \alpha \theta \varepsilon \dot{\sigma \varepsilon \varepsilon \omega \nu \text {. H RDA } \theta \varepsilon \omega \rho \varepsilon i ́ \tau \alpha \iota ~ \varepsilon \pi \varepsilon ́ \kappa \tau \alpha \sigma \eta ~ \tau \eta \varsigma ~ \alpha v \alpha ́ \lambda v \sigma \eta \varsigma ~ \kappa u \rho i ́ \omega v ~ \sigma u v เ \sigma \tau \omega \sigma \omega ́ v ~}$

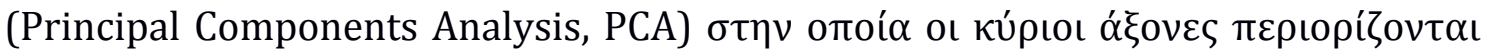

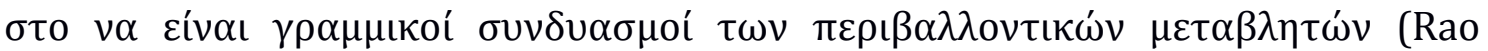

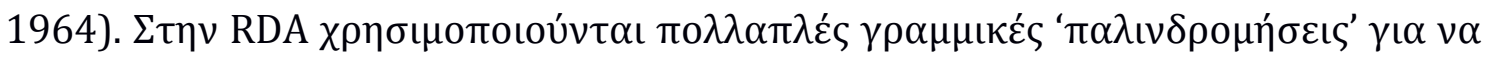

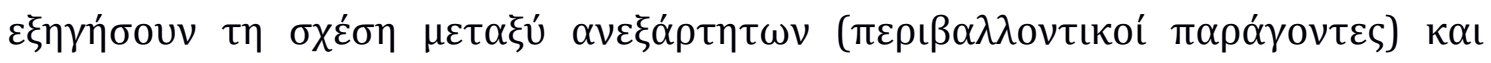

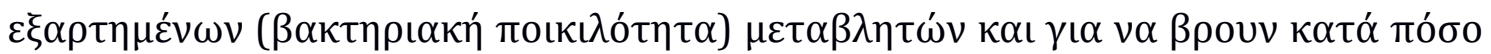

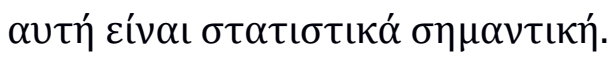




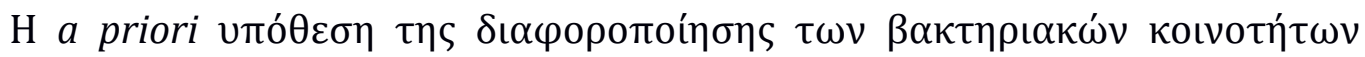

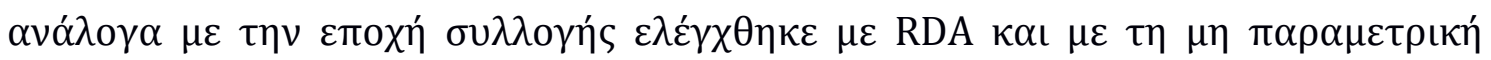

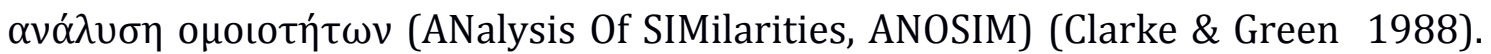

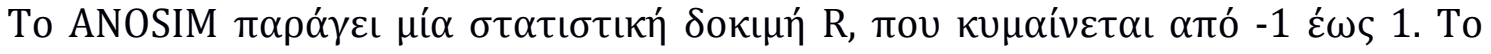

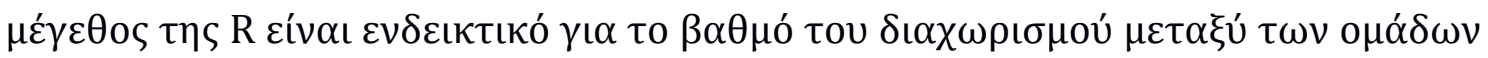

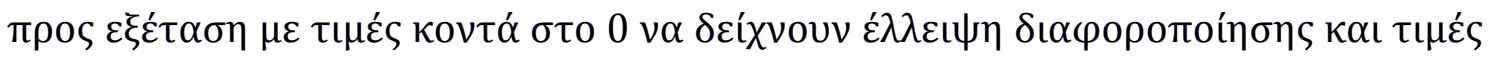

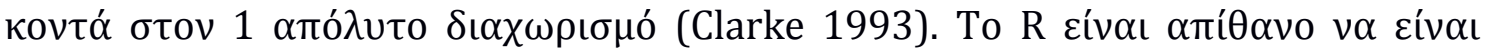

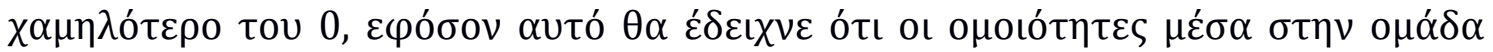

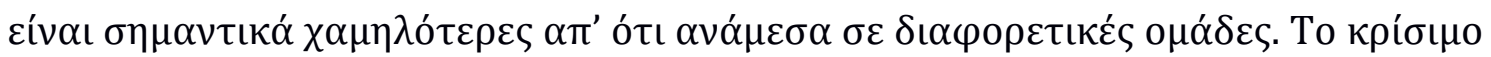

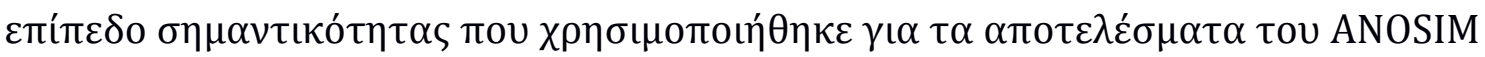

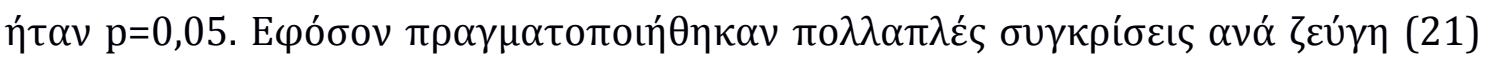

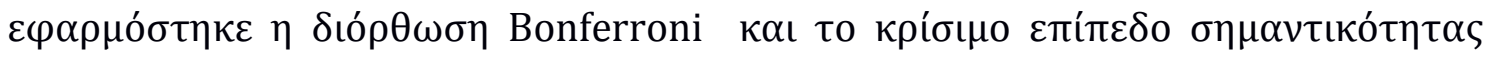

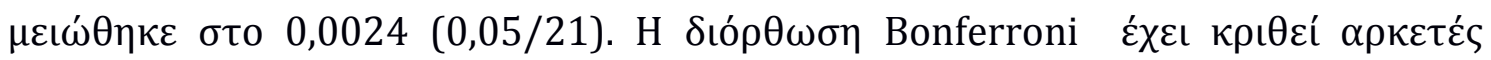

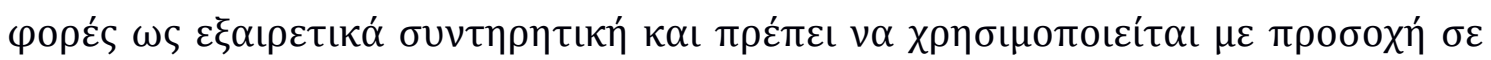

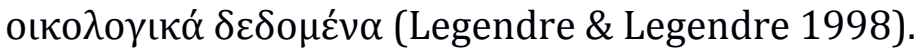

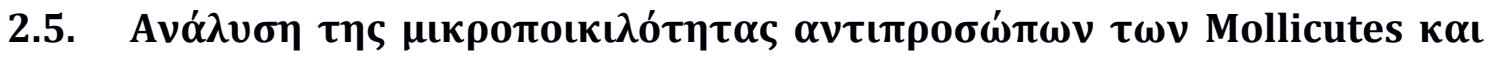 Tov CK_1C4-19}

H $\varphi$

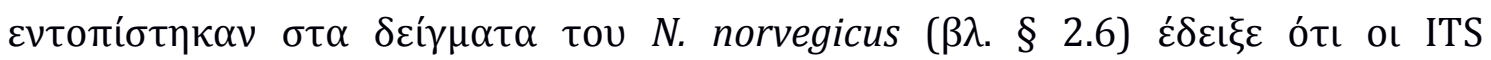

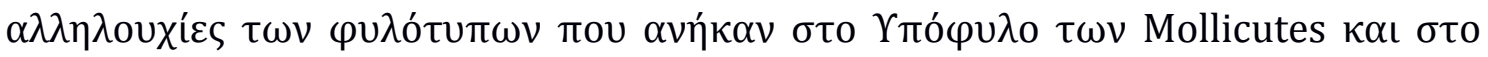

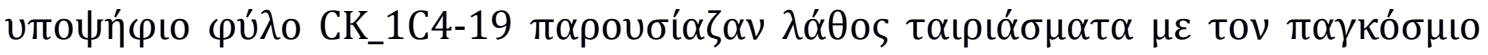

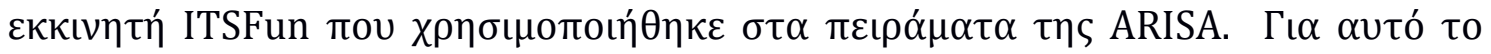

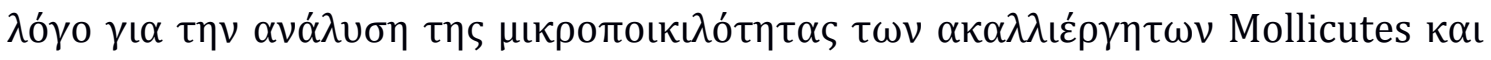




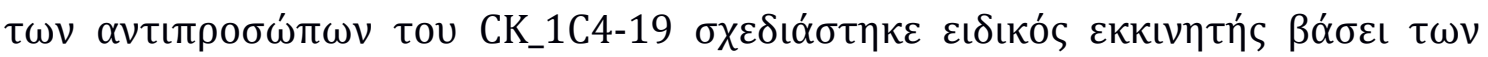

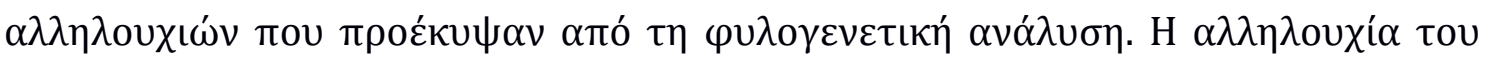

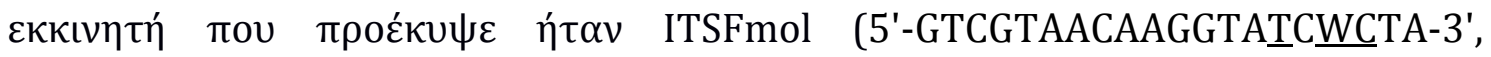

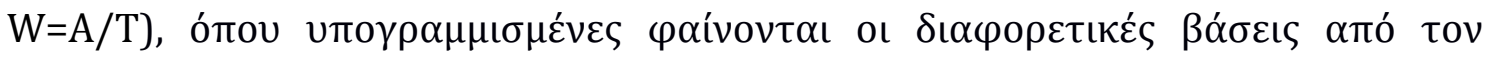

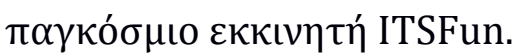

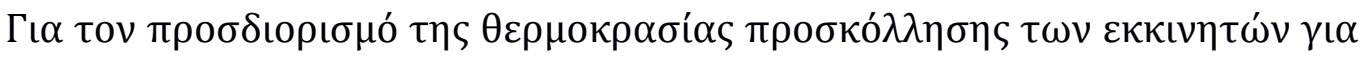

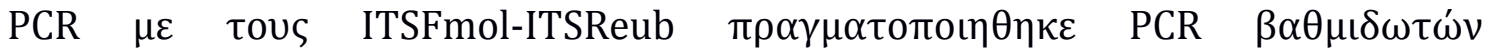

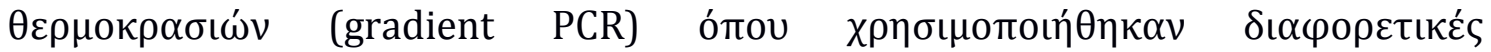

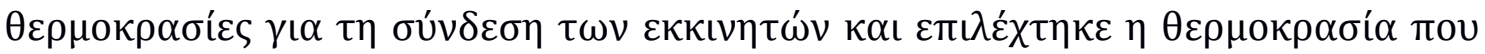

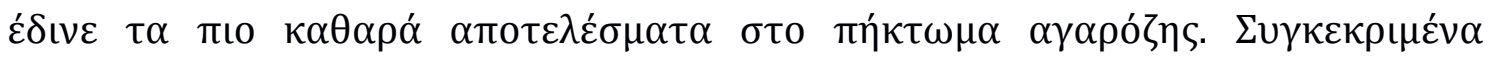

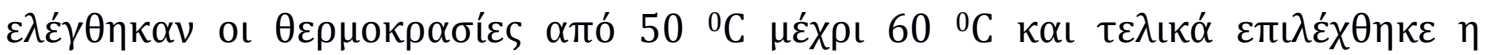
$\theta \varepsilon \rho \mu о \kappa \rho \alpha \sigma i ́ \alpha \tau \omega \nu 56{ }^{\circ} \mathrm{C}$.

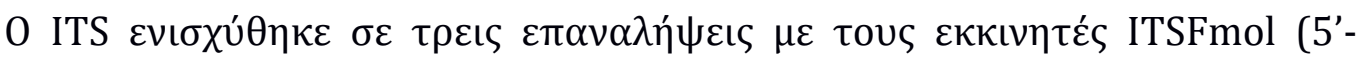

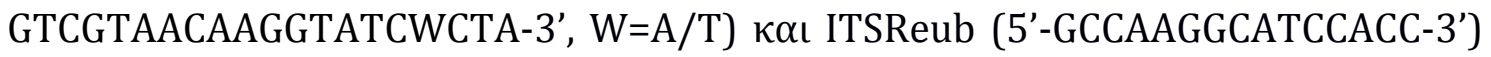

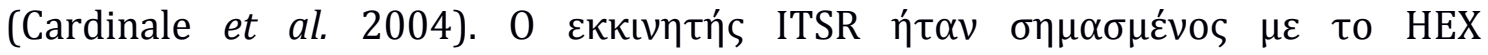

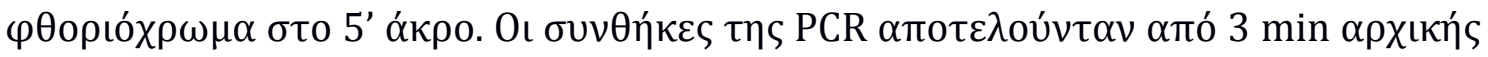

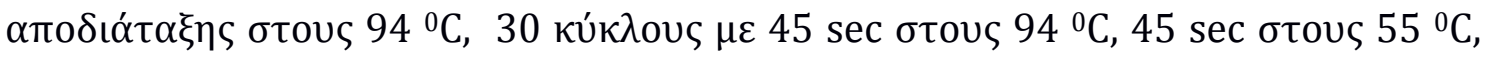

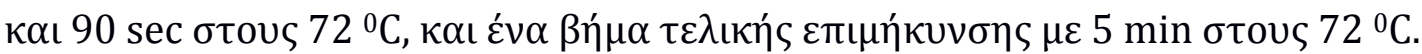

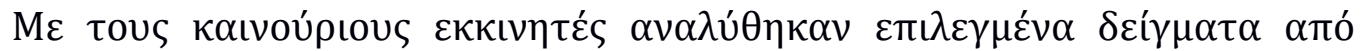

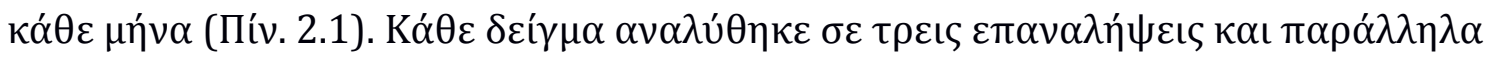

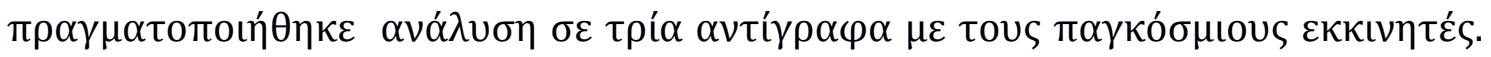

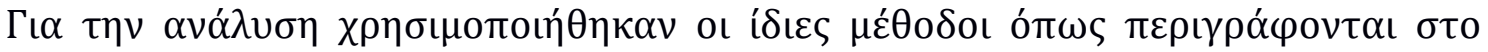

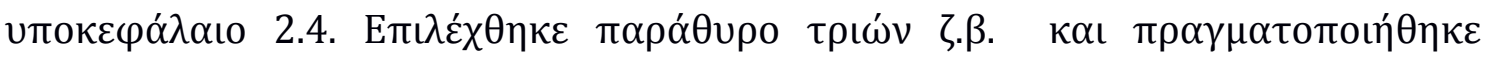

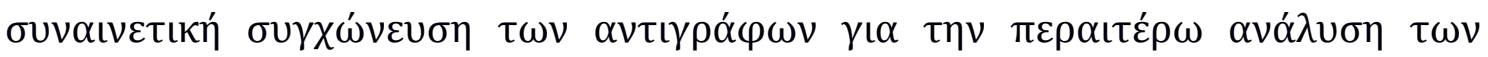




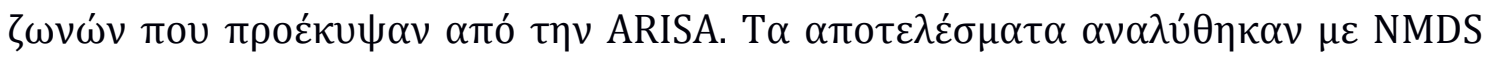

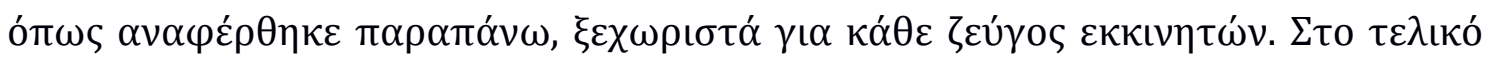

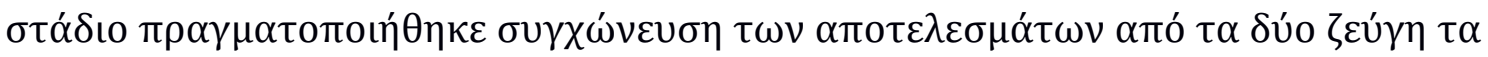

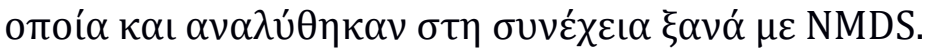

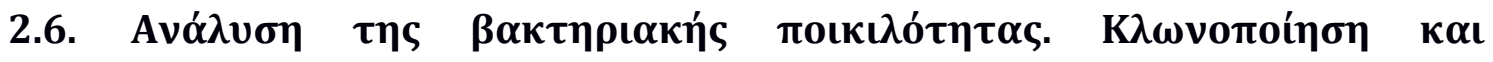

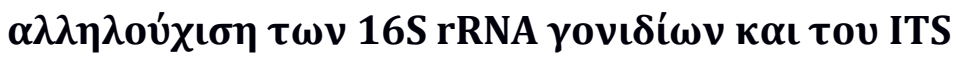

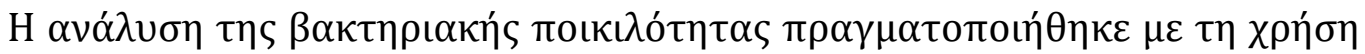

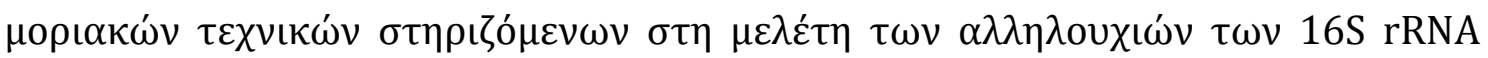

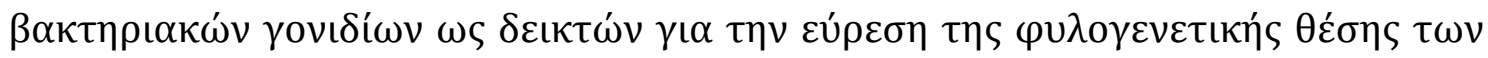

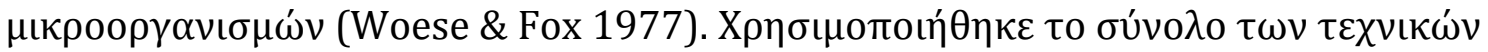

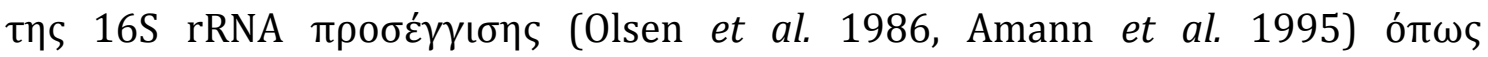

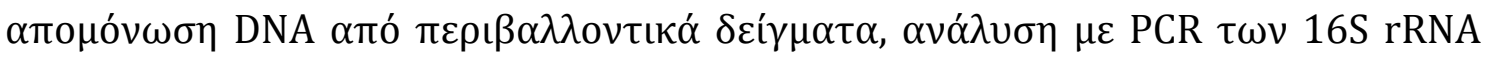

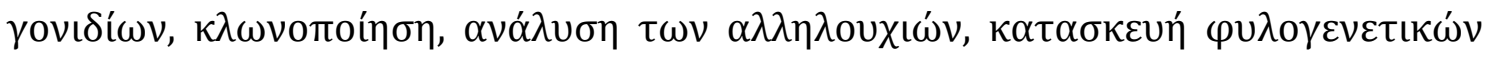

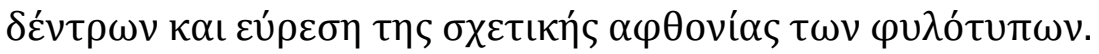

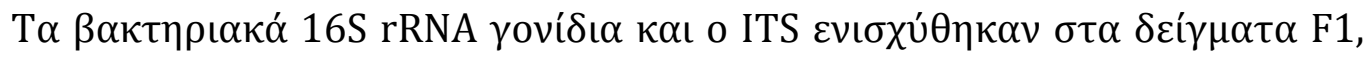

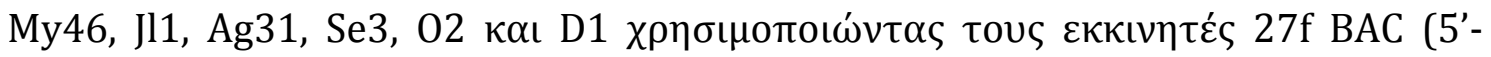
AGAGTTTGATCMTGGCTCAG-3'， M=A/C) (Lane 1991) каı ITSReub. To

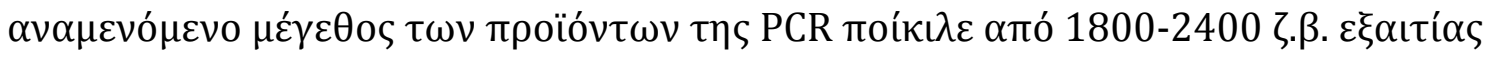

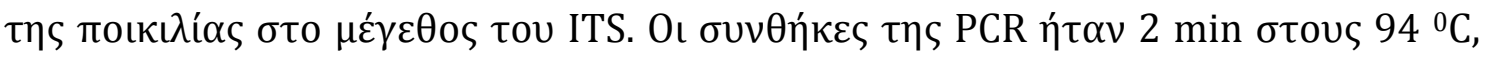

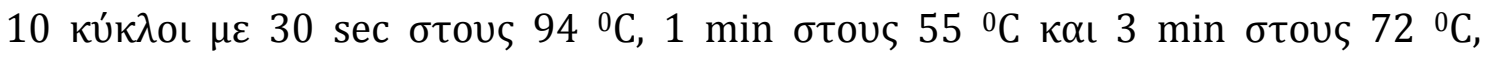

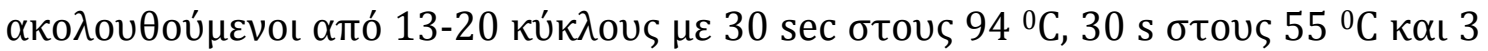

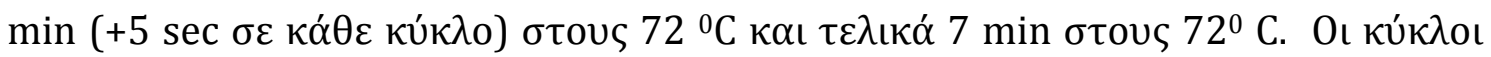

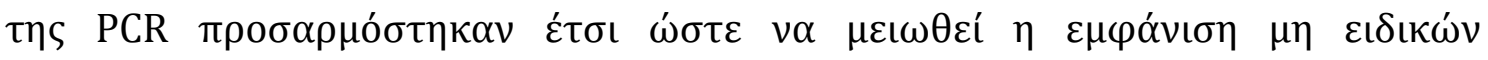




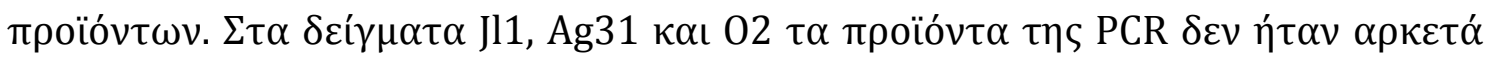

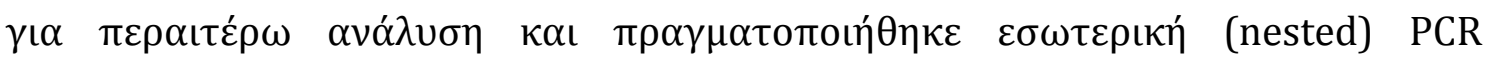

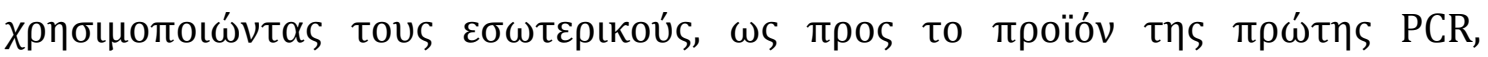

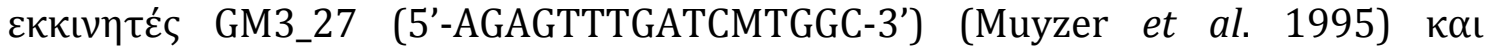
GM4_1492 (5-TACCTTGTTACGACTT-3') (Kane et al. 1993). To $\alpha v \alpha \mu \varepsilon v o ́ p \varepsilon v o$

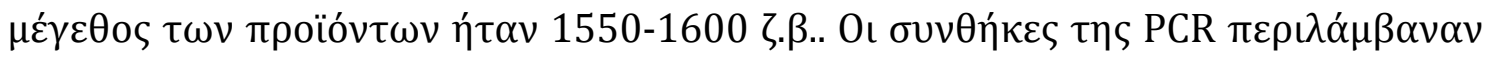

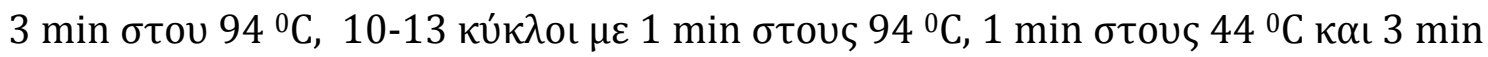

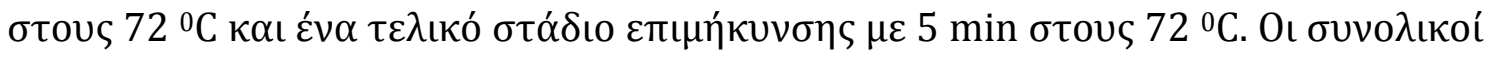

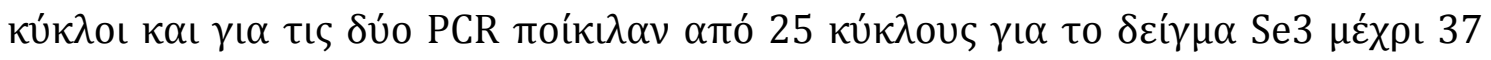

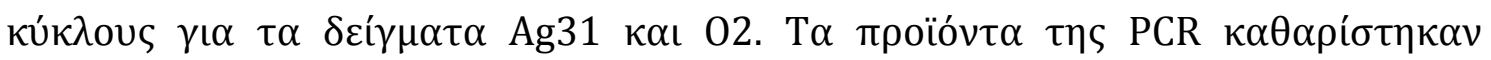

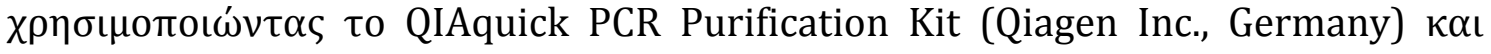

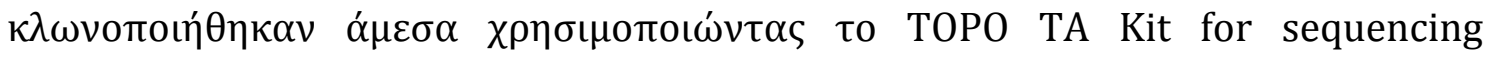

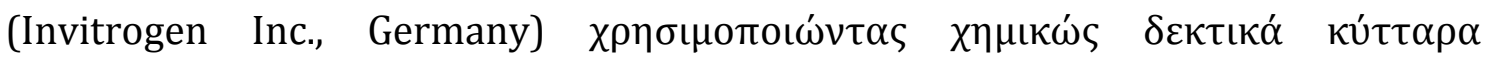

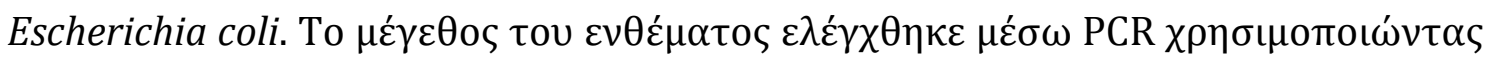

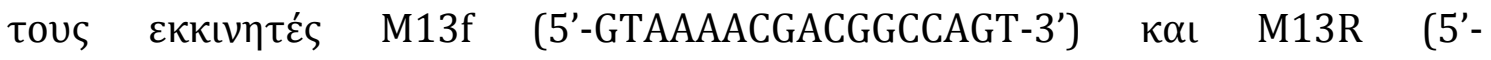

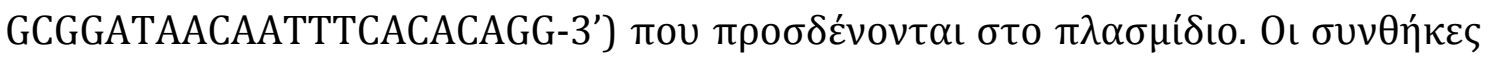

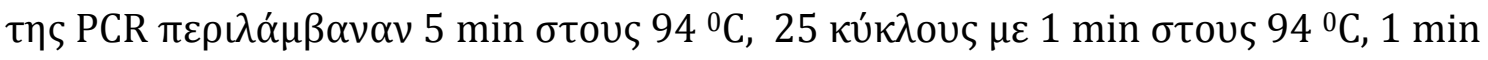

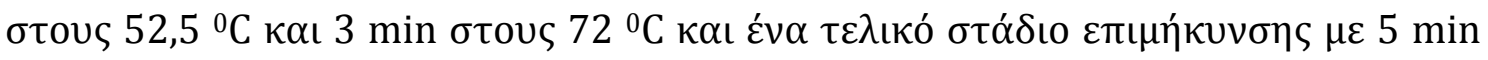

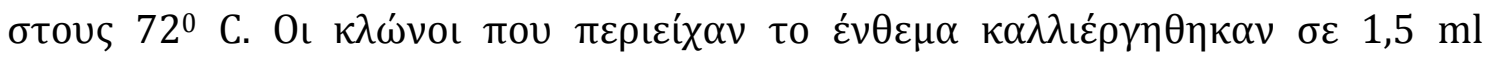

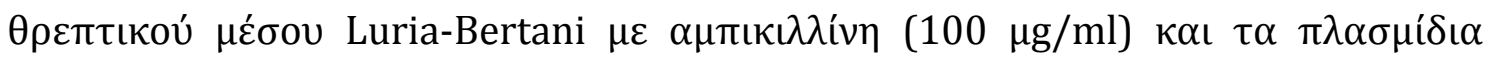

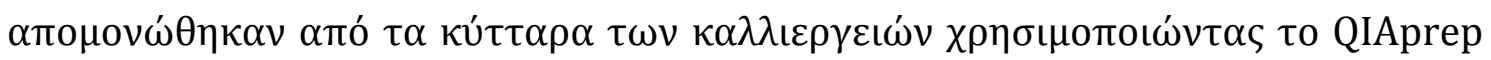

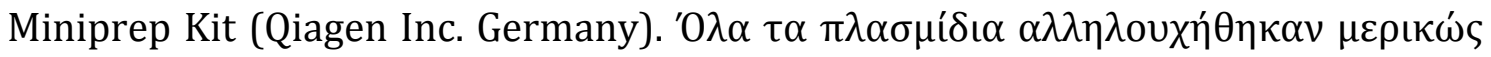

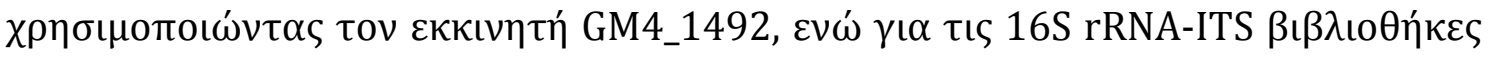

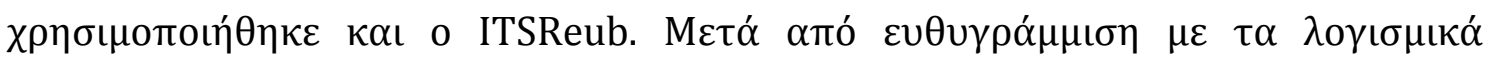


SEQUENCHER 4.6 (Gene Codes Corporation, USA) кaı CLUSTALW (Larkin et al.

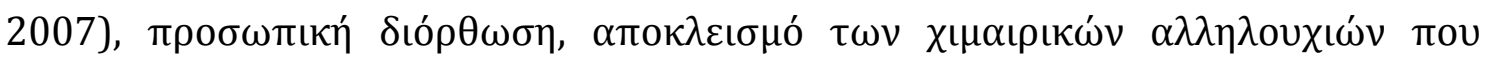

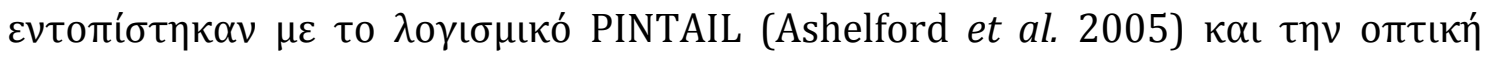

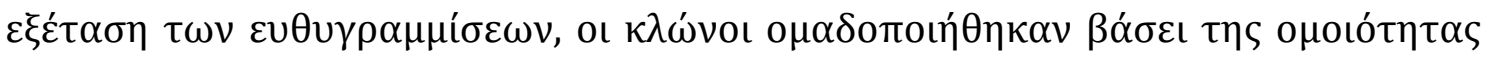

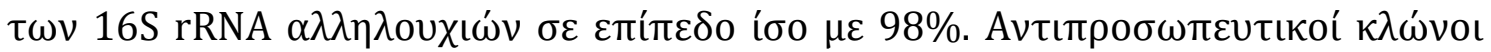

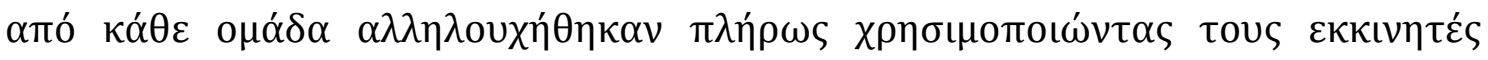
G5_341f (5'-CCTACGGGAGGCAGCAG-3'), GM1R (5'-ATTACCGCGGCTGCTGG-3') к $\alpha$ 907R (5'-CCGTCAATTCCTTTRAGTTT-3')(Muyzer et al. 1995).

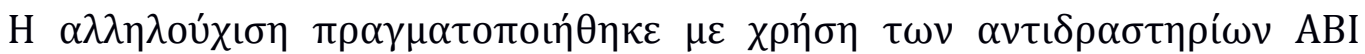

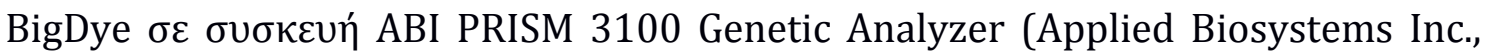

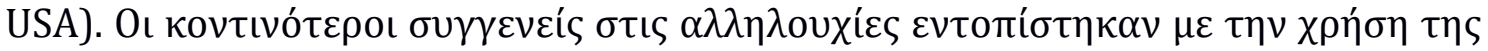

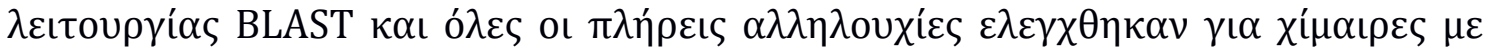

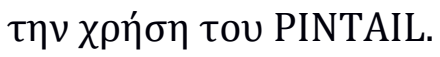

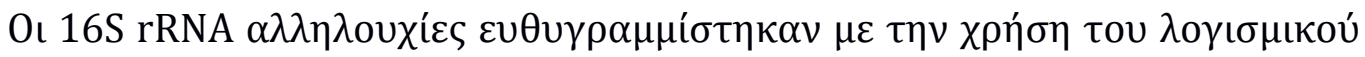

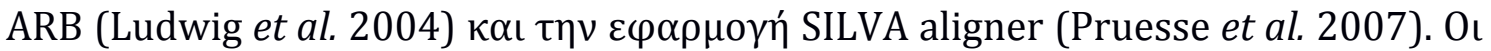

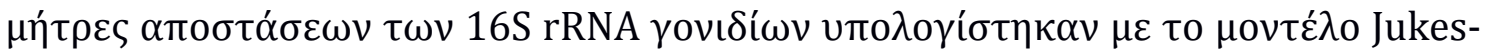

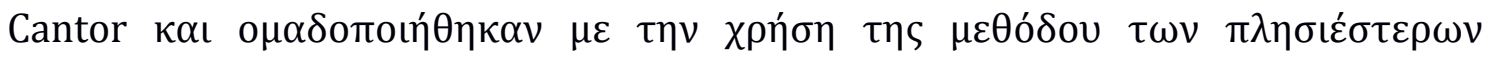

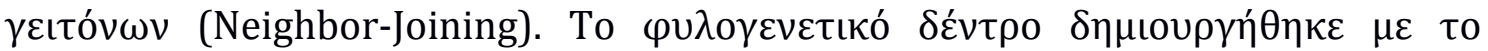

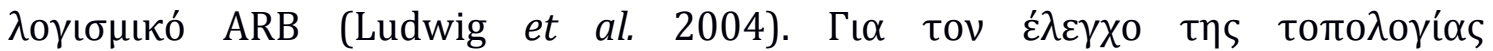

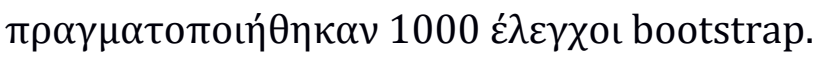

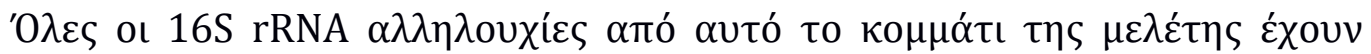

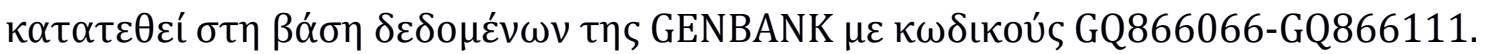




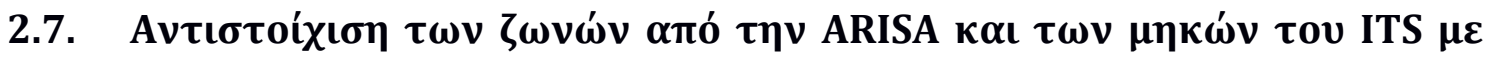

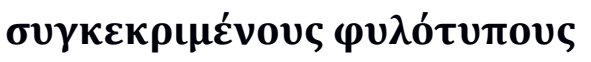

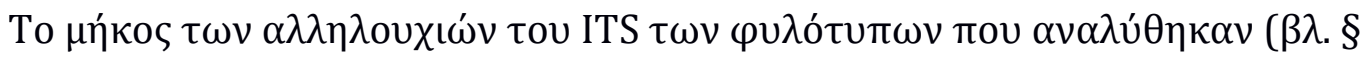

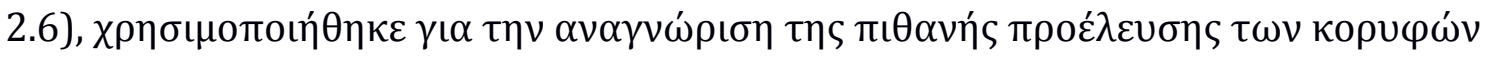

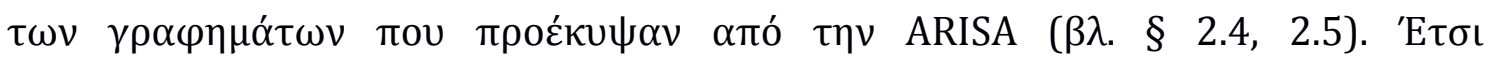

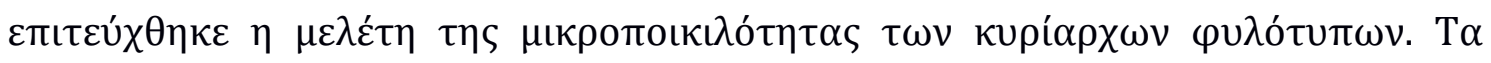

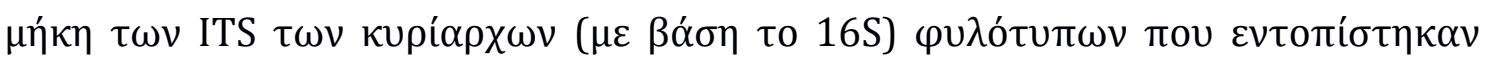

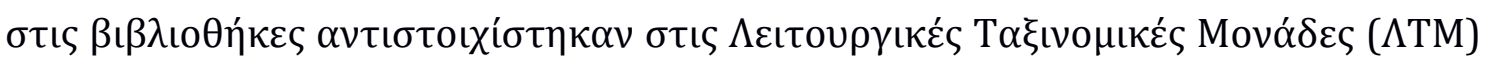

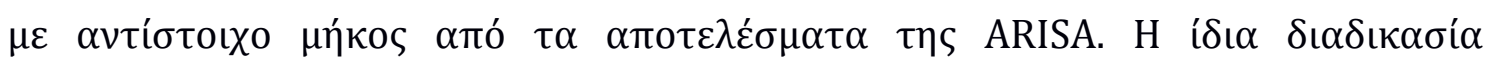

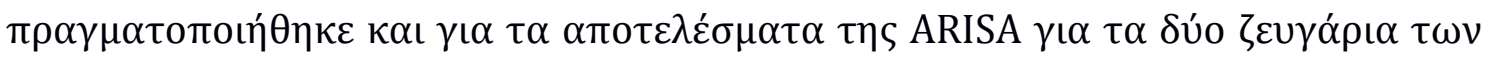

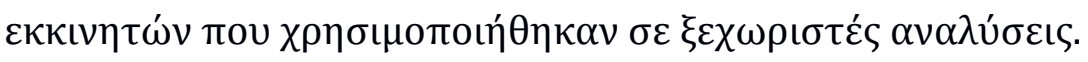

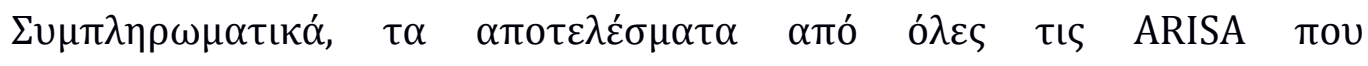

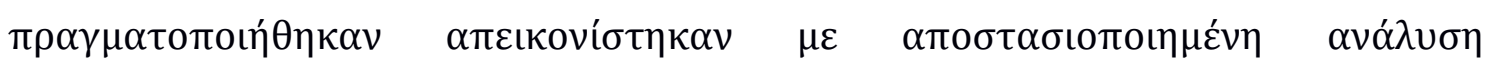

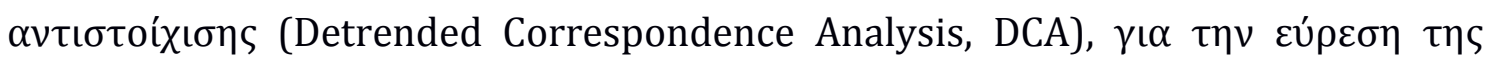

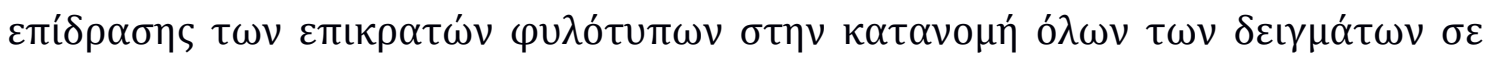

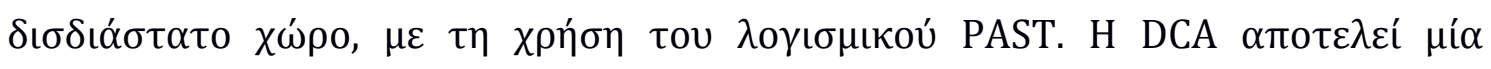

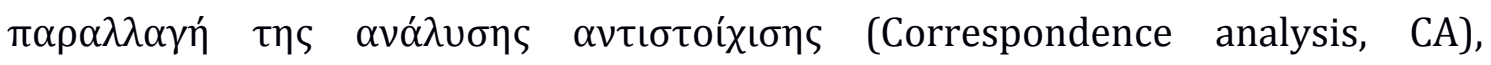

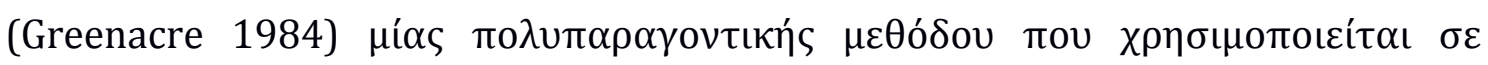

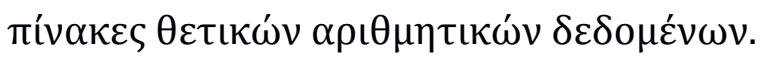

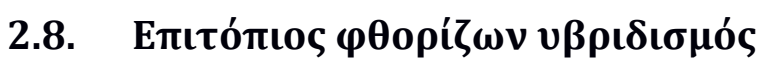

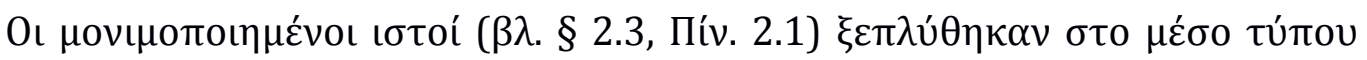

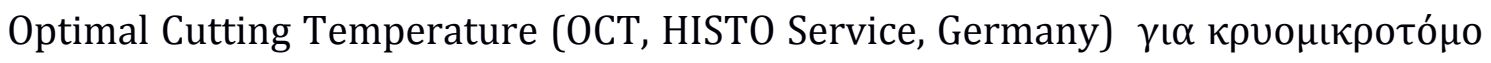




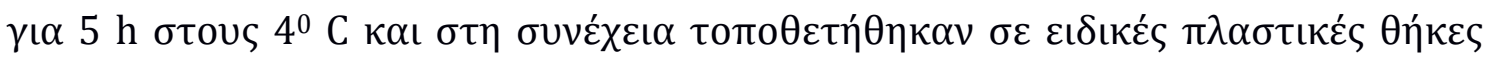

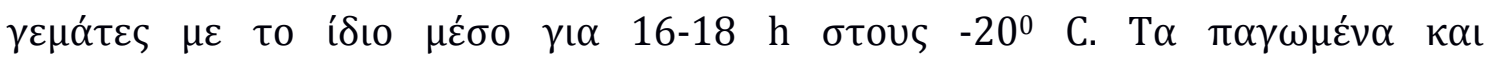

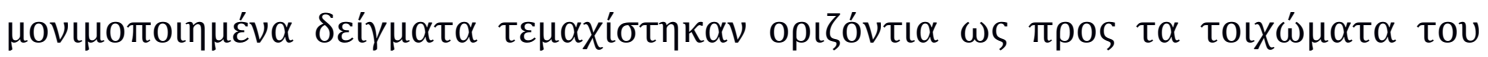

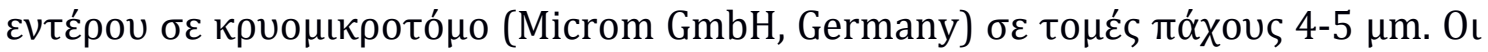

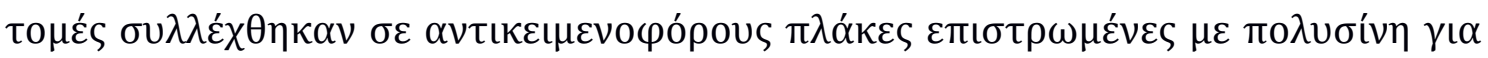

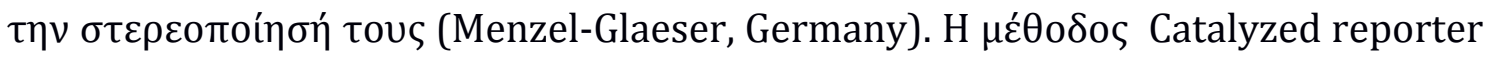

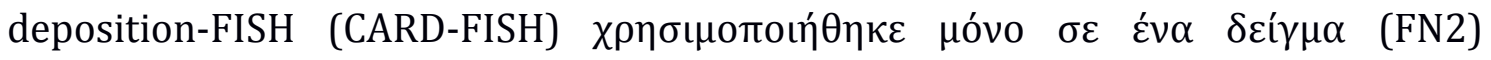

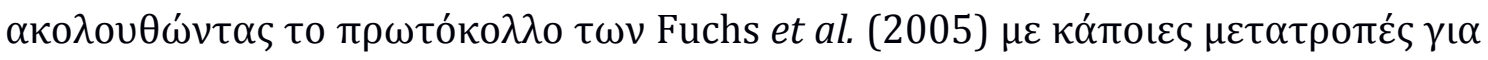

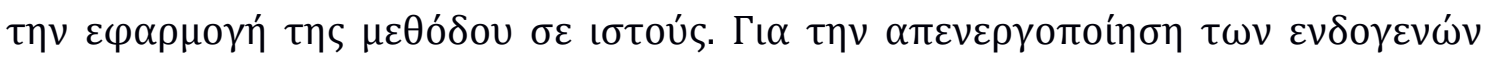

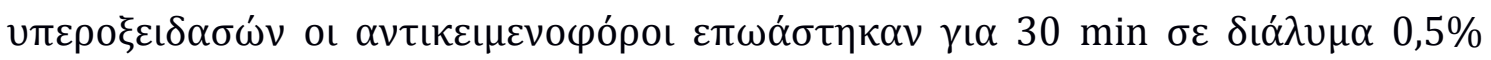

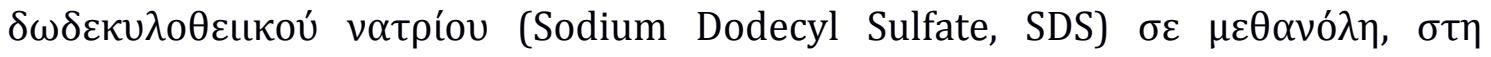

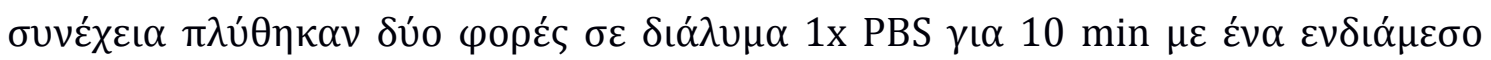

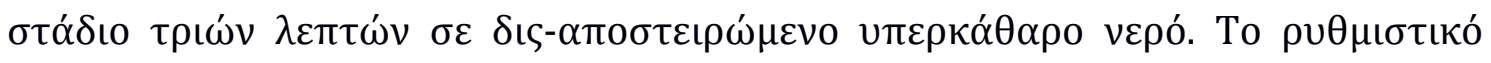

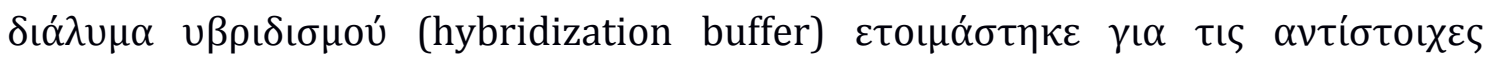

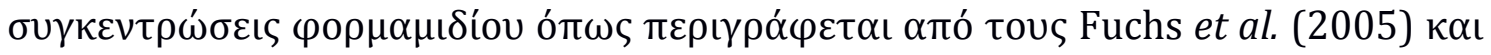

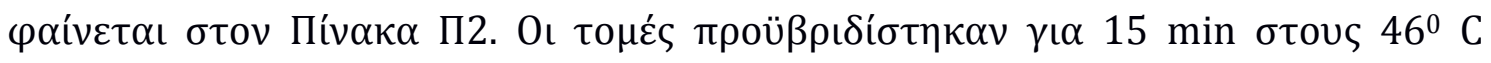

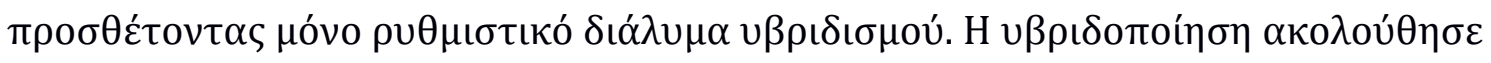

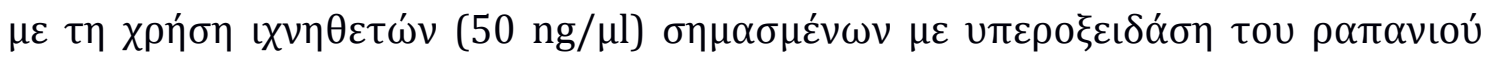

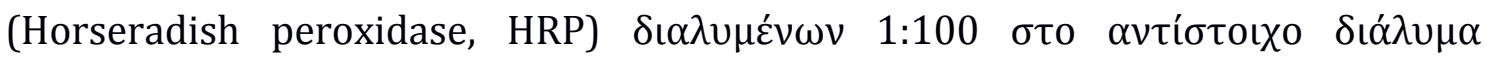

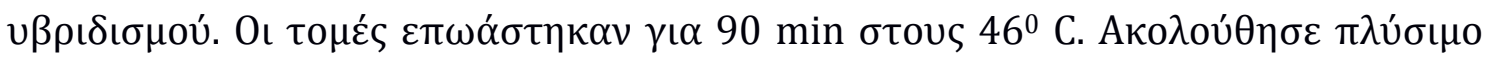

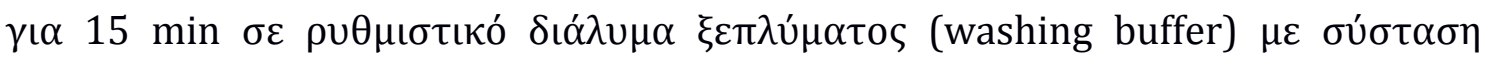

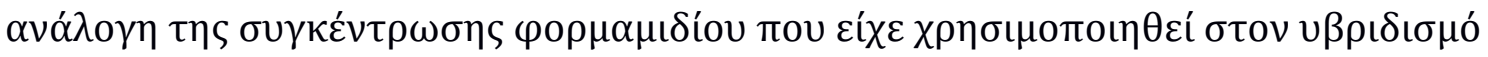

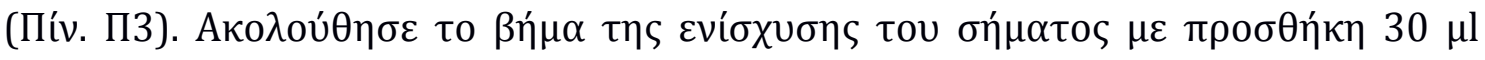

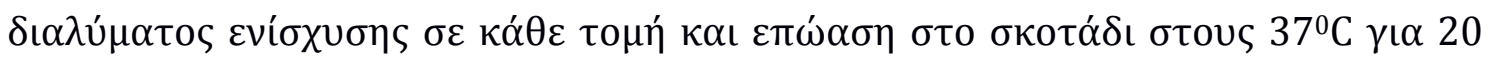




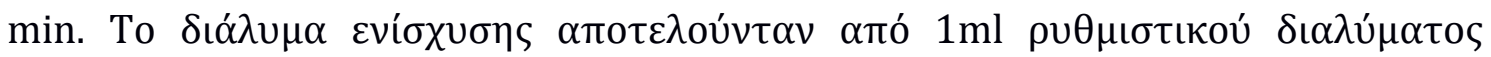

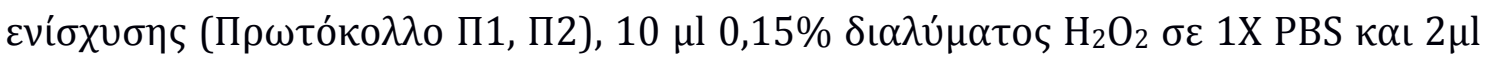

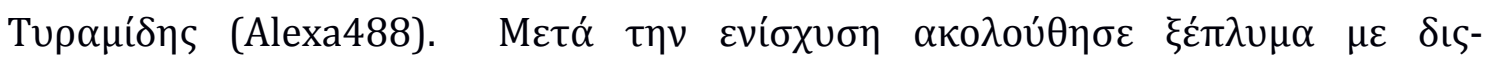

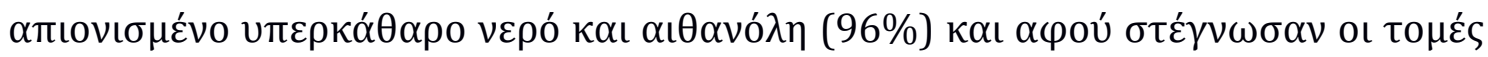

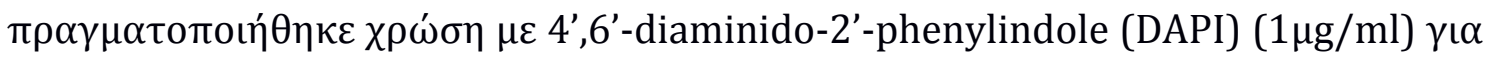
10 min.

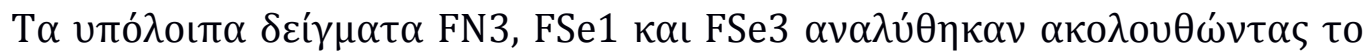

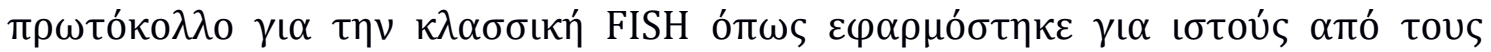

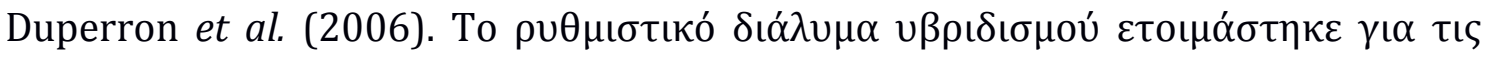

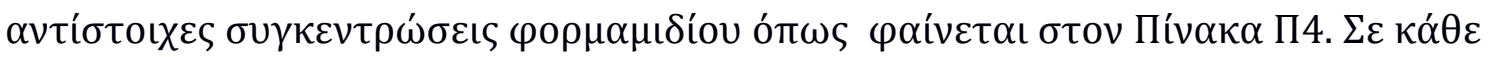

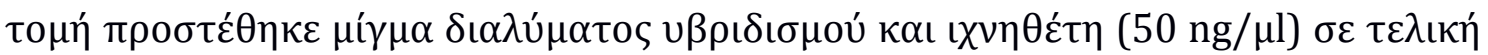

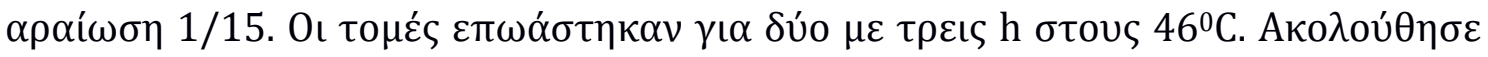

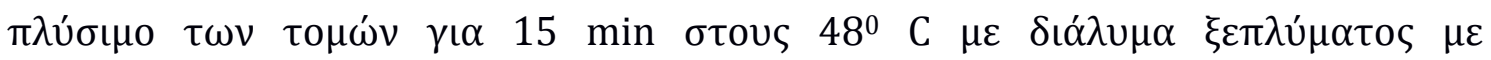

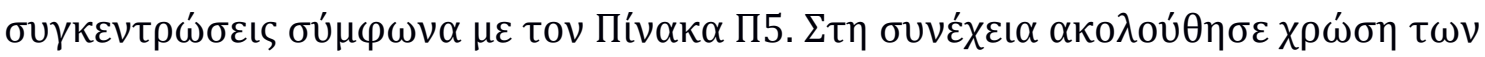

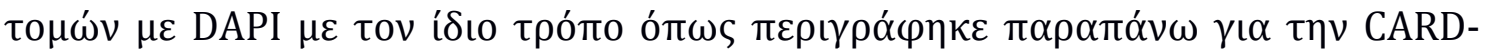

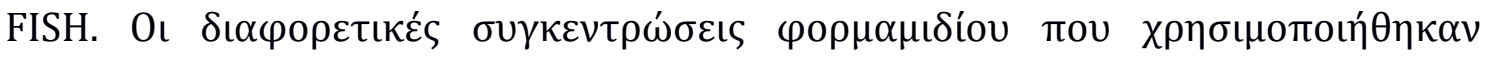

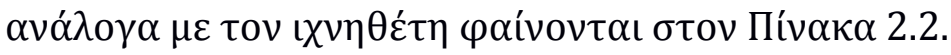

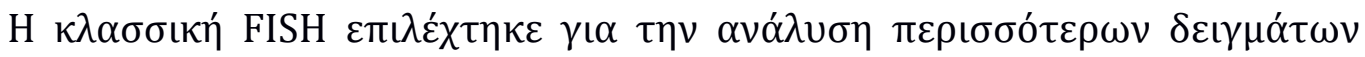

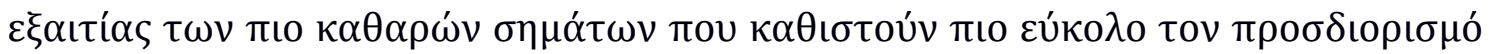

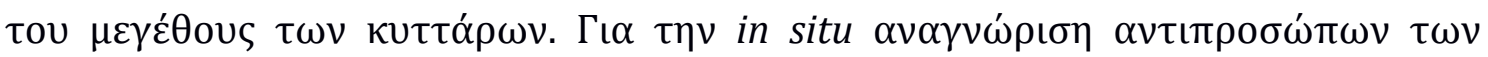

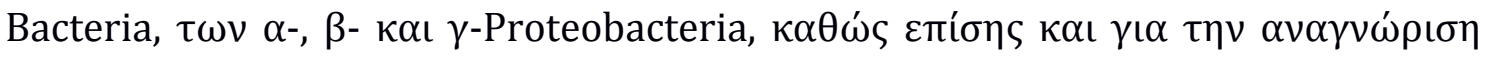

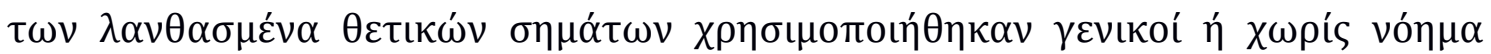

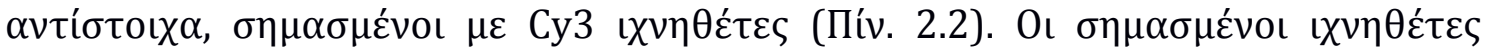

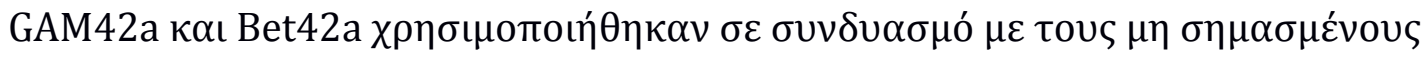




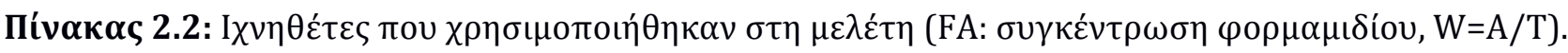

\begin{tabular}{|c|c|c|c|c|c|}
\hline I $\chi v \eta \theta \varepsilon ́ \tau \eta S$ & 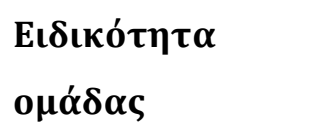 & $A \lambda \lambda \eta \lambda o v \chi \chi^{\prime} \alpha\left(5^{\prime}-3^{\prime}\right)$ & 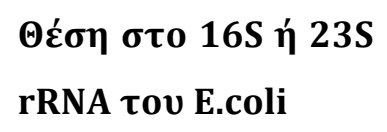 & FA\% & 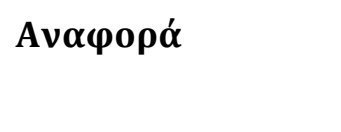 \\
\hline $\begin{array}{l}\text { EUB338 (I- } \\
\text { III) }\end{array}$ & $\begin{array}{l}\sigma \chi \varepsilon \delta o ́ v \quad \text { ó } \lambda \alpha \quad \tau \alpha \\
\beta \alpha \kappa \tau \eta ́ \rho ı \alpha\end{array}$ & GCWGCCWCCCGTAGGWGT & $338-355$ & 35 & Daims et al. 1999 \\
\hline NON338 & 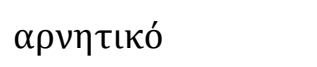 & ACTCCTACGGGAGGCAGC & - & 35 & Amann et al. 1990 \\
\hline GAM42a & $\gamma$-Proteobacteria & GCCTTCCCACATCGTTT & 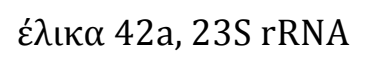 & 35 & Manz et al. 1992 \\
\hline BET42a & $\beta$-Proteobacteria & GCCTTCCCACTTCGTTT & 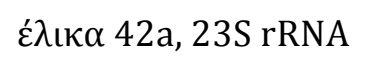 & 35 & Manz et al. 1992 \\
\hline ALF968 (*) & $\alpha$-Proteobacteria & GGTAAGGTTCTGCGCGTT & 968 & 35 & Glöckner et al. 1999 \\
\hline UncMol89 & $\begin{array}{l}\text { Фиһótuтоs Se3- } \\
204\end{array}$ & $\begin{array}{l}\text { CGTTCGCCACTAACACCAAA } \\
\text { ATC }\end{array}$ & 89 & 35 & 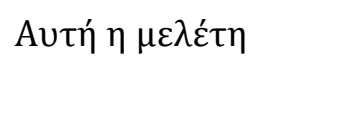 \\
\hline
\end{tabular}

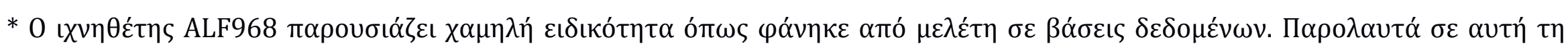

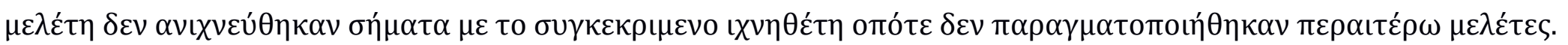




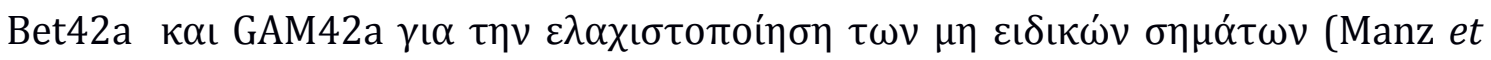
al., 1992).

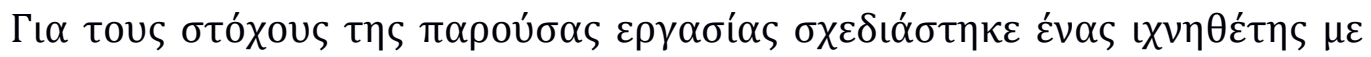

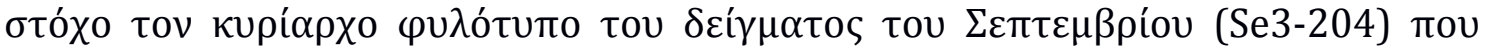

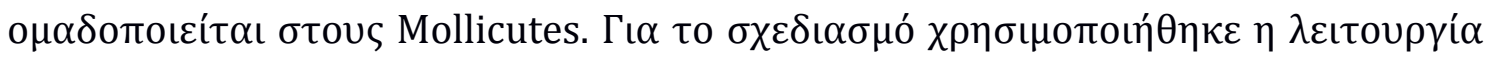

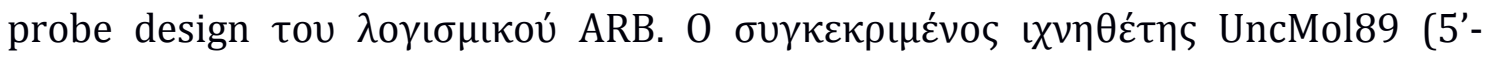

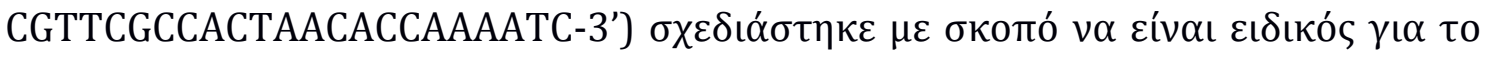

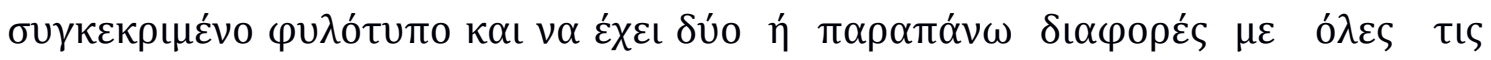

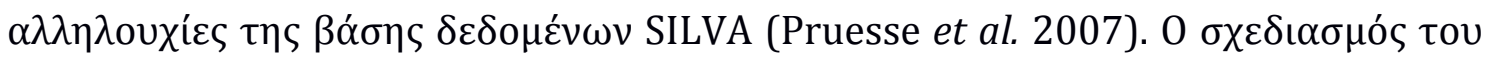

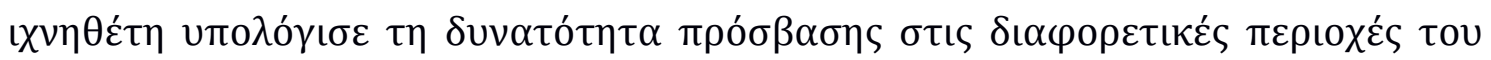

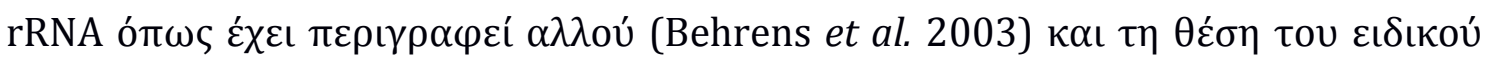

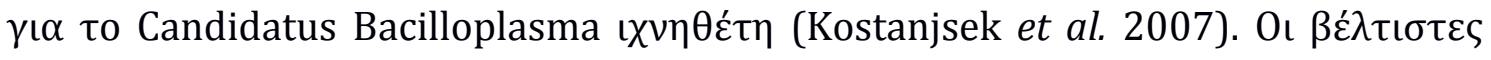

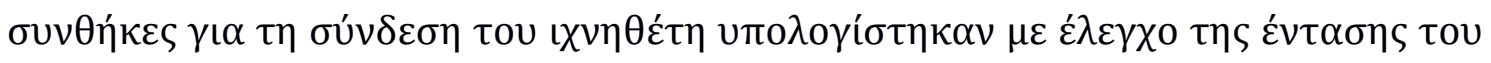

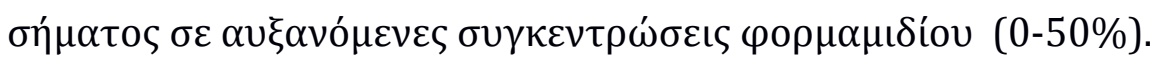

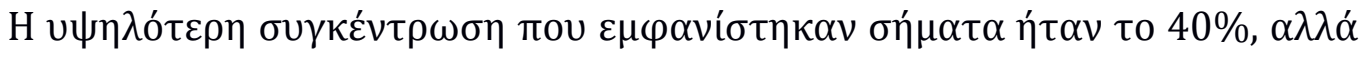

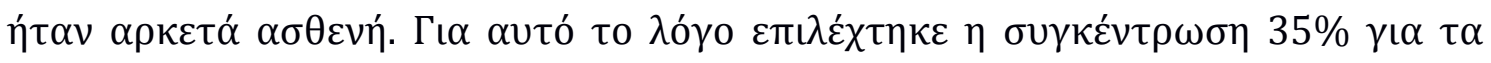

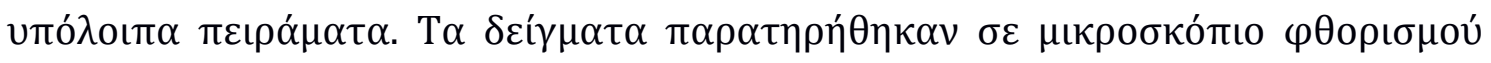

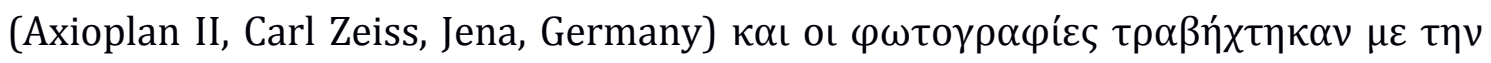

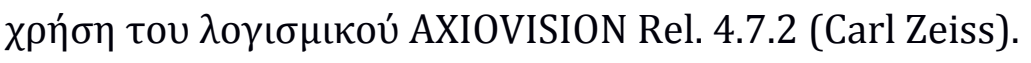

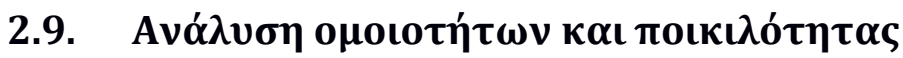

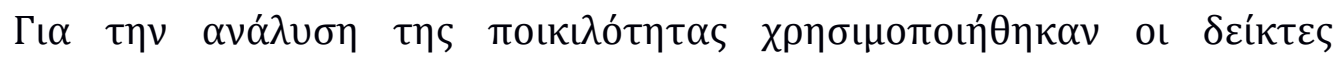

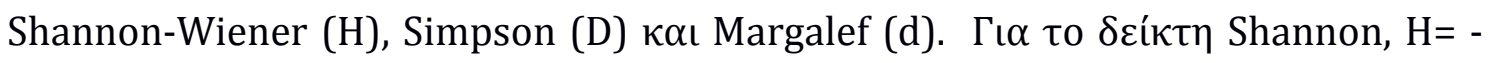




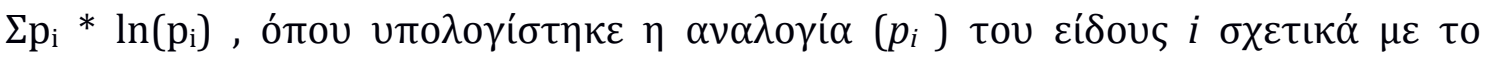

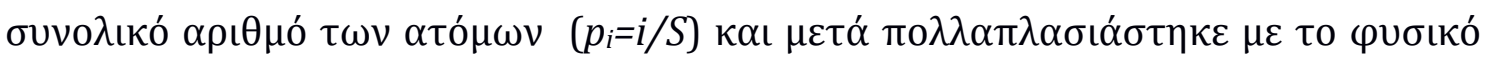

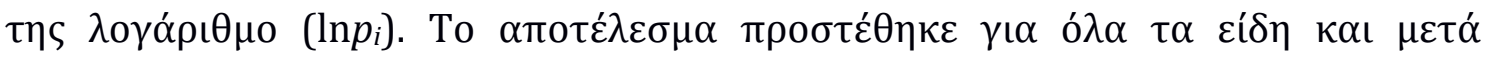

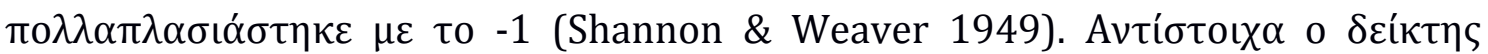

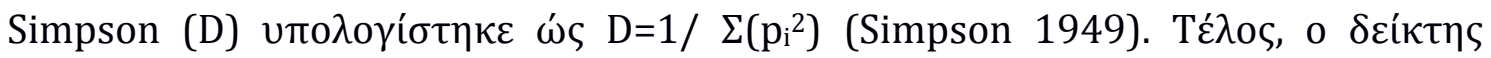

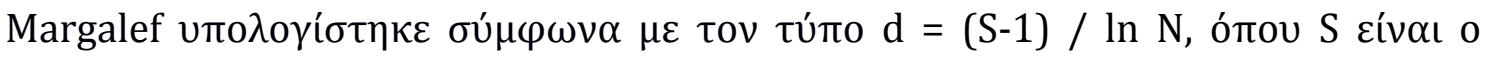

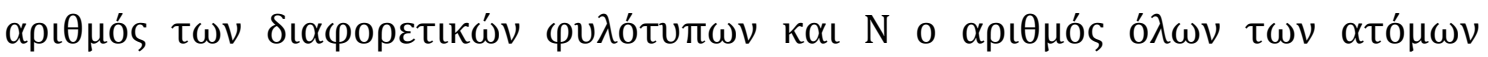
(Margalef 1958).

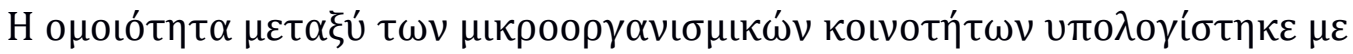

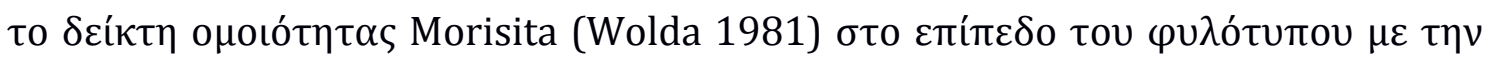

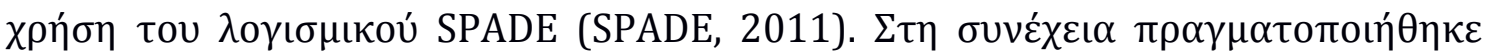

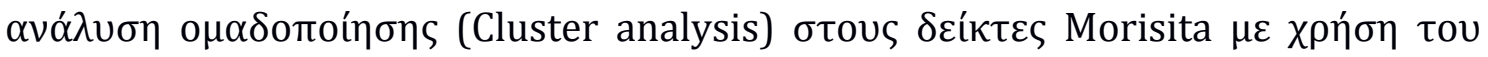

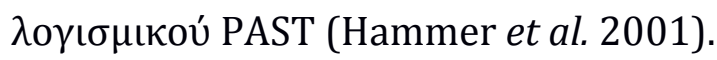

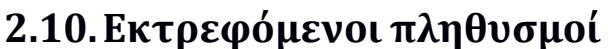

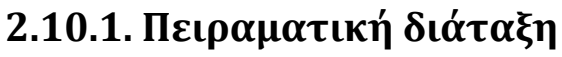

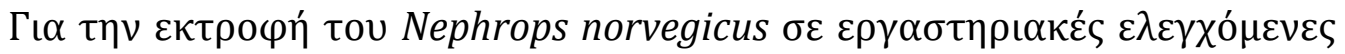

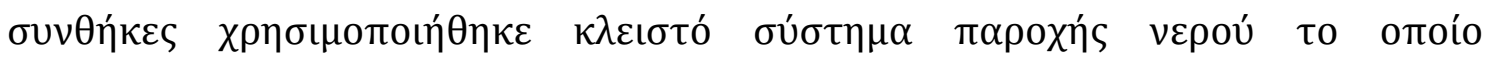

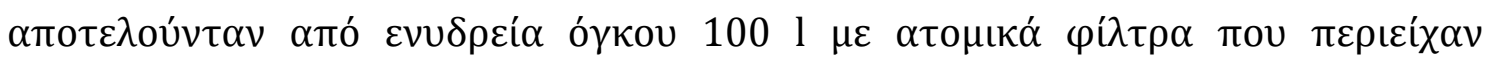

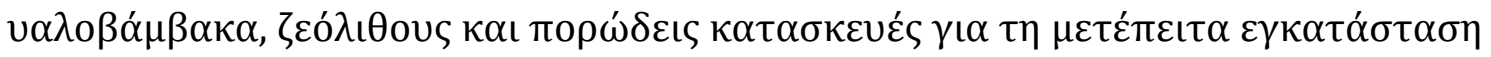

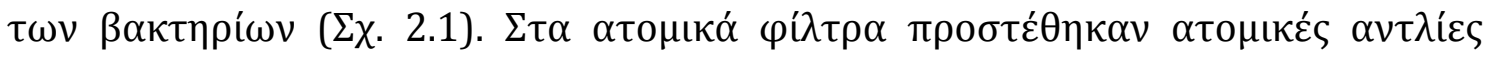

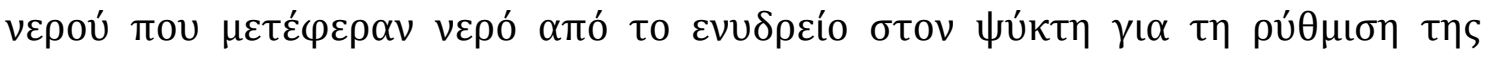




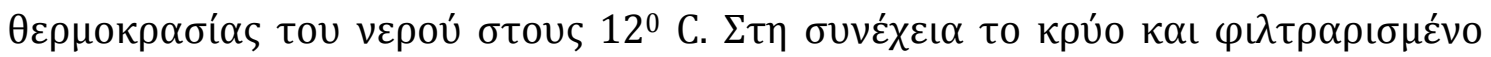

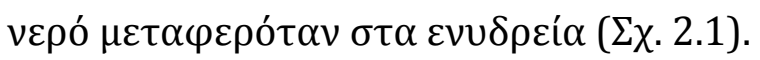

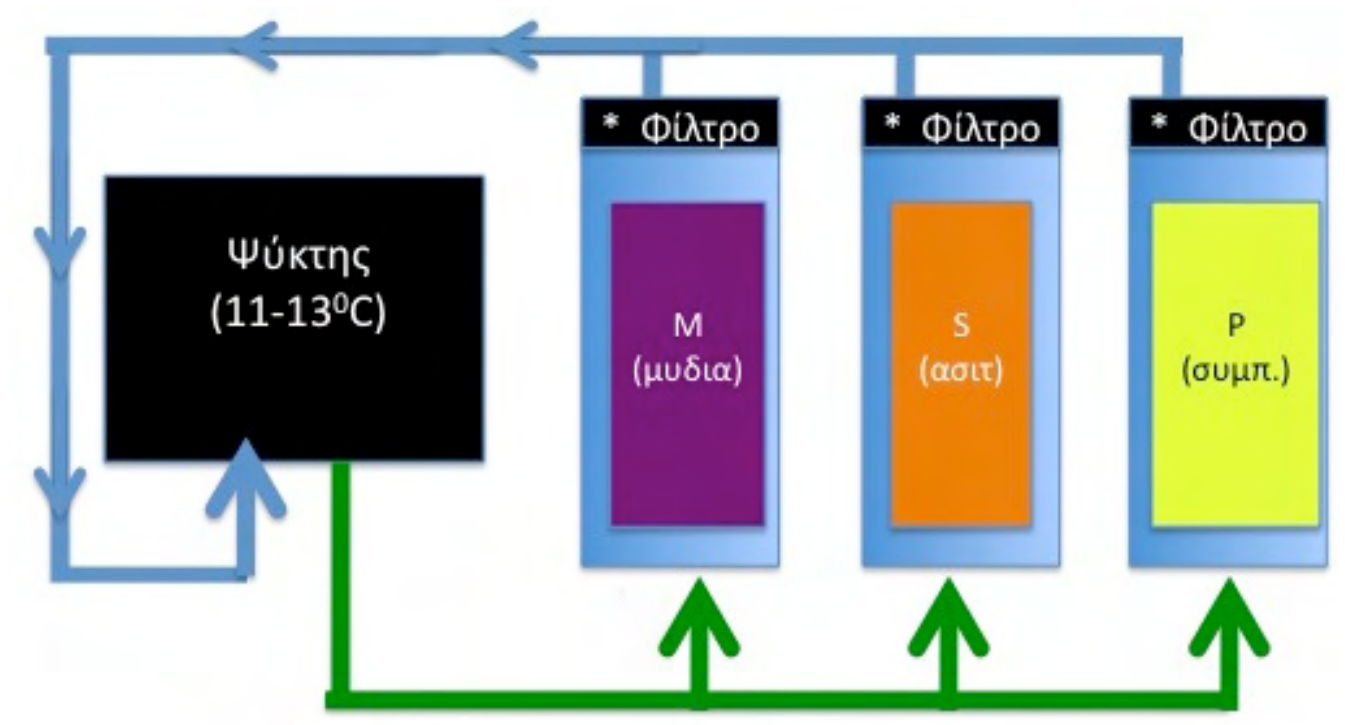

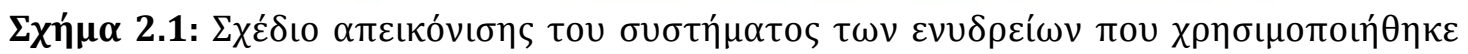

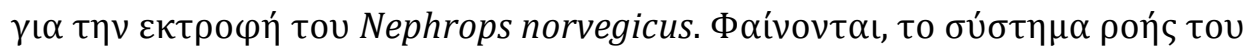

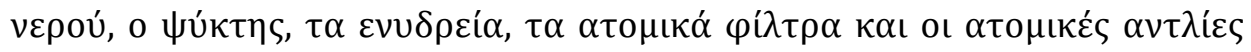

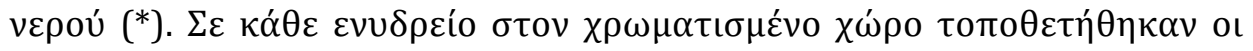

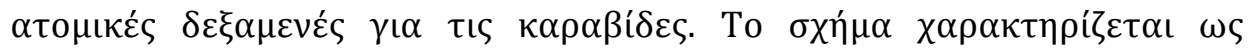

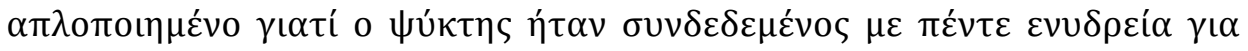

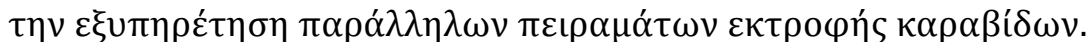

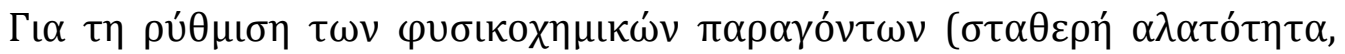

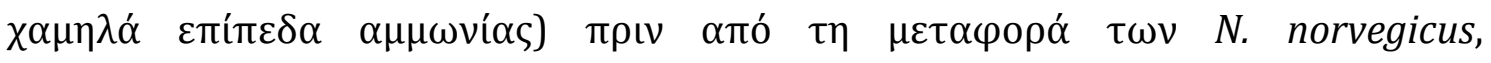

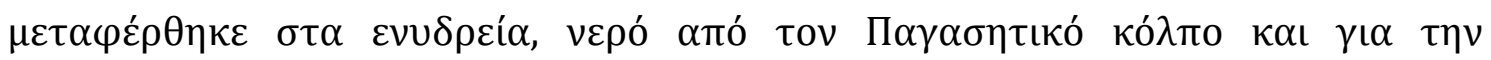

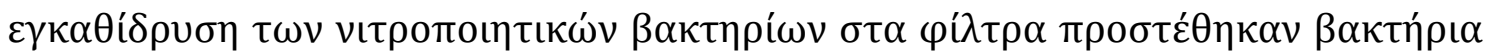

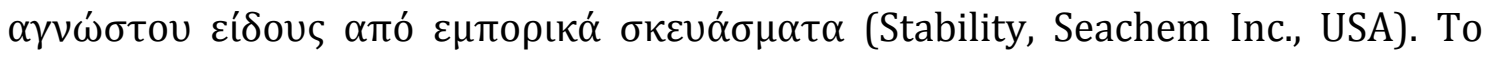

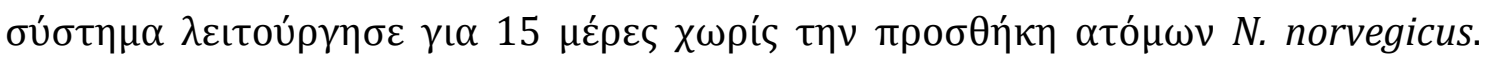

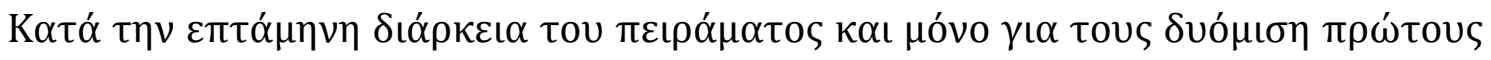




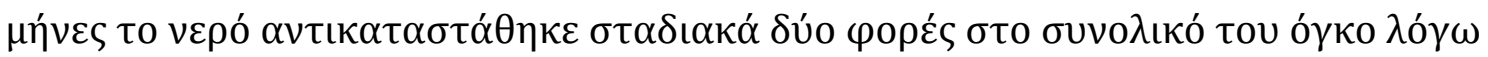

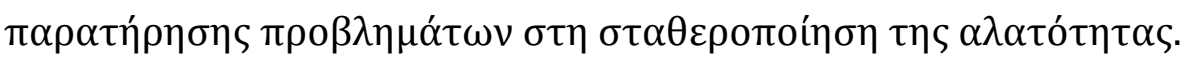

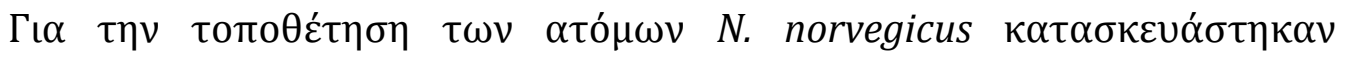

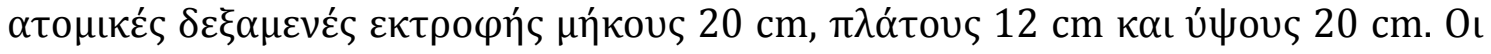

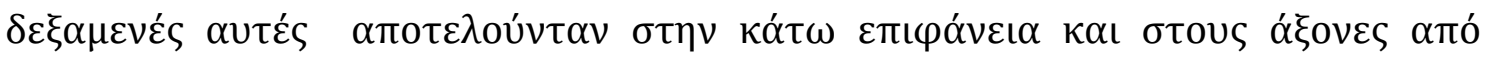

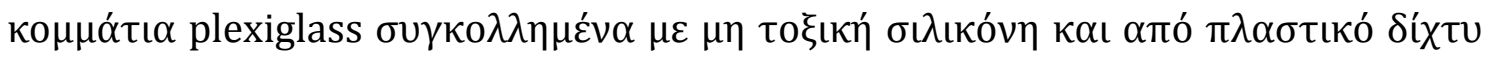

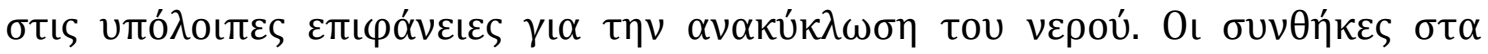

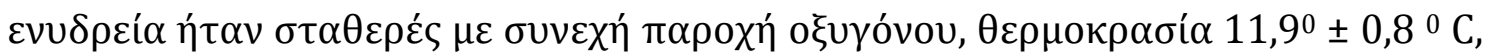

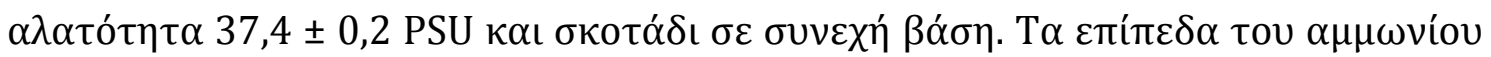

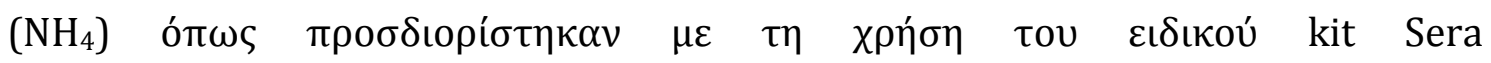

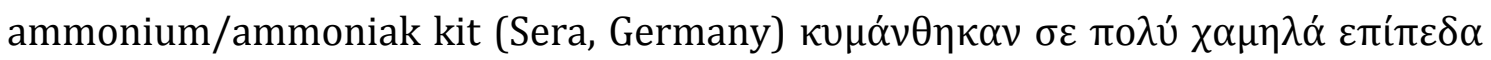

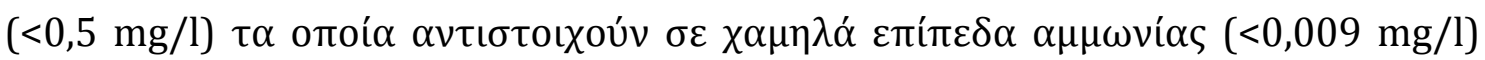

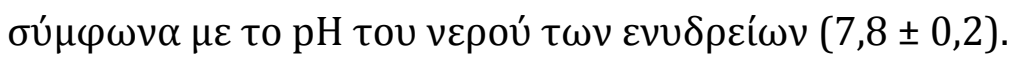

\subsection{2. $\Sigma v \lambda \lambda o \gamma \eta ́$ $\delta \varepsilon เ \gamma \mu \alpha ̛ ́ \tau \omega v$}

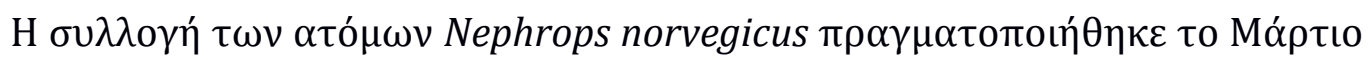

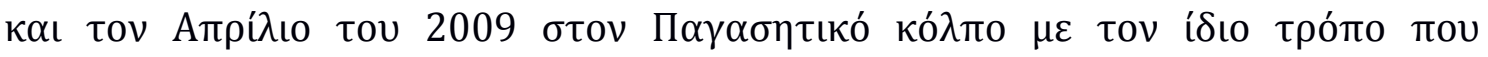

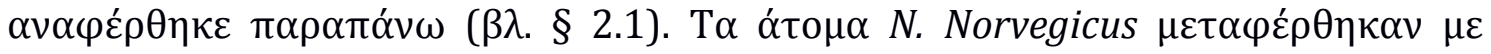

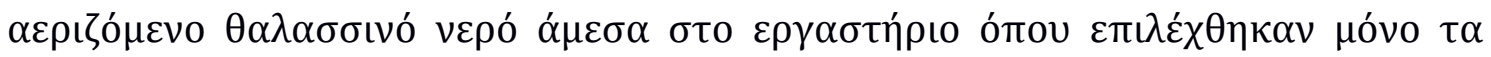

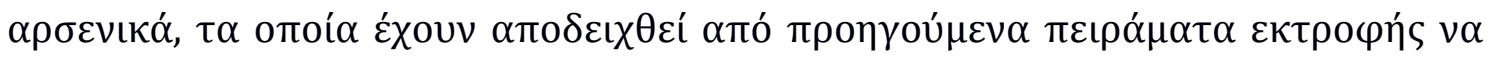

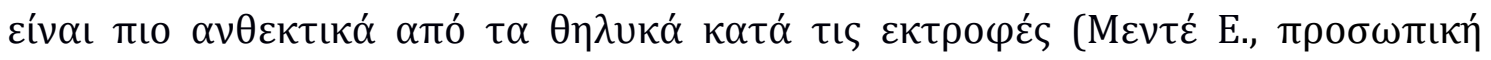

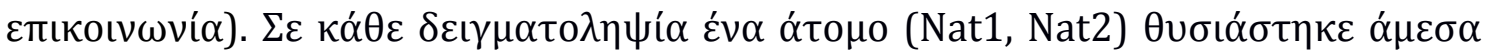




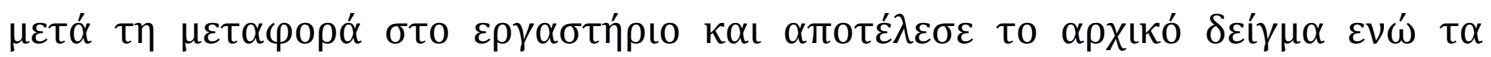

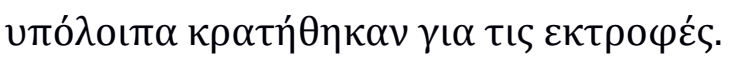

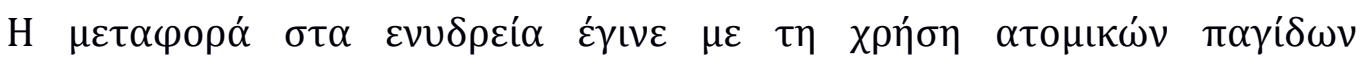

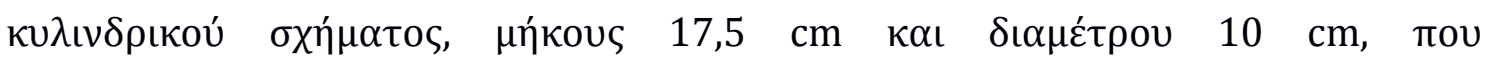

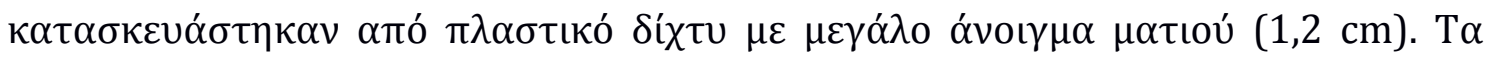

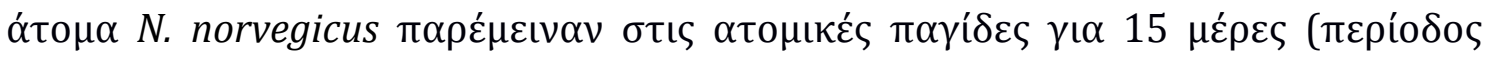

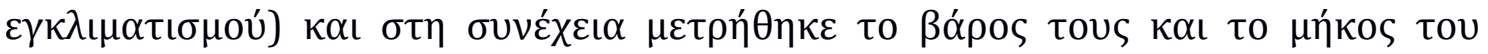

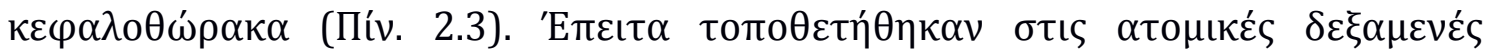

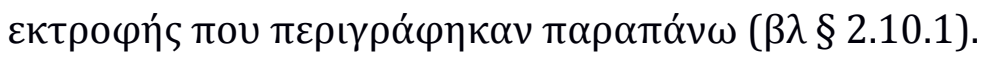

\begin{tabular}{|c|c|c|}
\hline & & \\
\hline S3m1 & 72,17 & 48,9 \\
\hline S6m1 & 58,47 & 44 \\
\hline S6m2 & 47,06 & 43,7 \\
\hline M3m1 & 29,68 & 34,3 \\
\hline M6m1 & 49,35 & 41,6 \\
\hline M6m2 & 51,34 & 41,6 \\
\hline P6m1 & 43,93 & 36,4 \\
\hline P6m2 & 41,91 & 37,5 \\
\hline
\end{tabular}

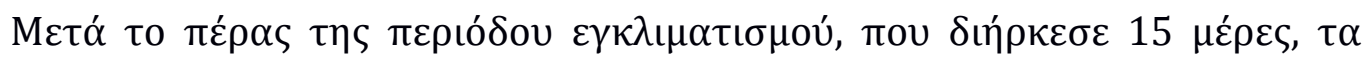

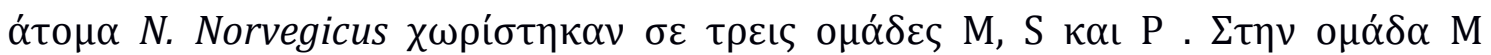




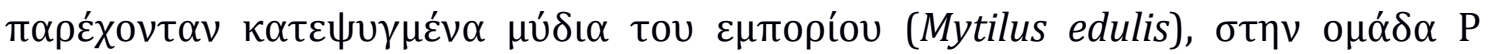

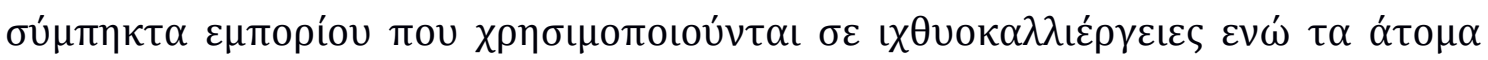

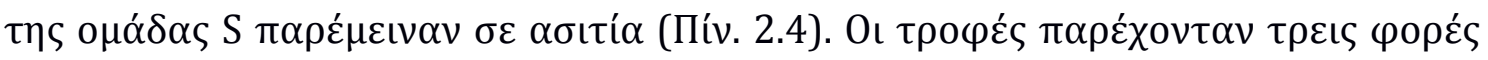

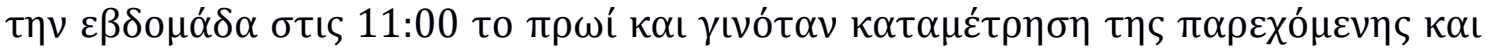

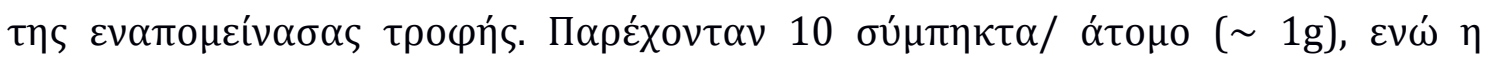

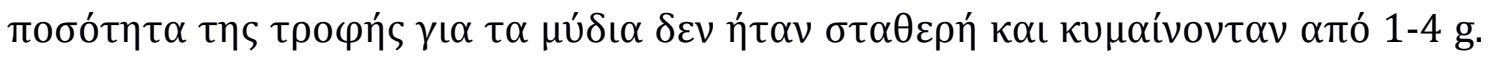

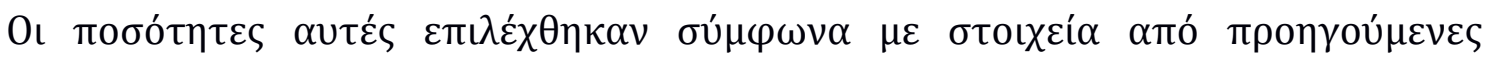

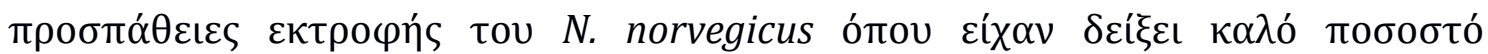

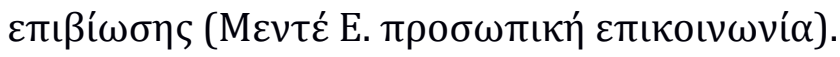

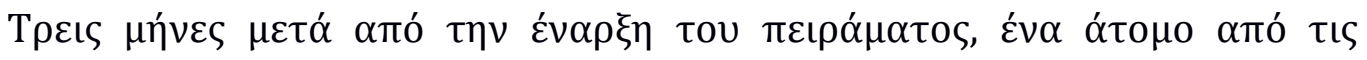

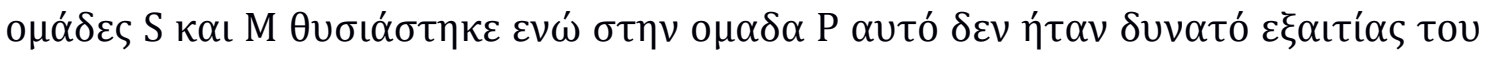

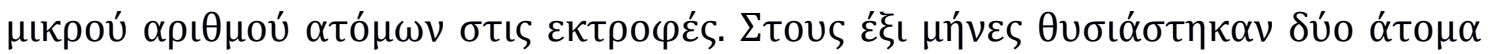

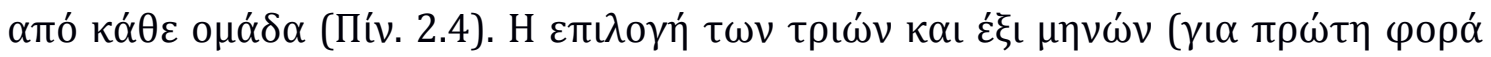

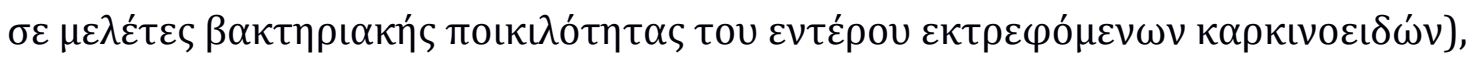

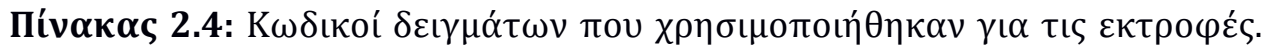

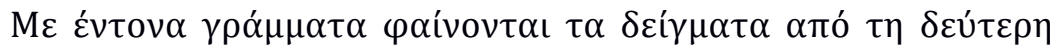
$\delta \varepsilon เ \gamma \mu \alpha \tau o \lambda \eta \psi i ́ \alpha(A \pi \rho i ́ \lambda ı$ เos 2009).

\begin{tabular}{|c|c|c|c|}
\hline$\Delta \varepsilon i ́ \gamma \mu \alpha$ & $t_{0}(\alpha \rho \chi \eta ́)$ & $t_{1}(3 \mu \eta ́ v \varepsilon \varsigma)$ & $t_{2}(6 \mu \eta ́ v \varepsilon \varsigma)$ \\
\hline 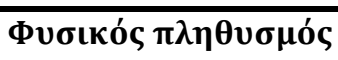 & Nat1/Nat2 & & \\
\hline Aбıтía & & $\mathrm{S} 3 \mathrm{~m} 1$ & $\mathrm{~S} 6 \mathrm{~m} 1 / \mathrm{S} 6 \mathrm{~m} 2$ \\
\hline 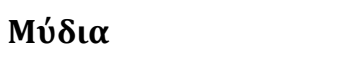 & & M3m1 & $\mathrm{M} 6 \mathrm{~m} 1 / \mathrm{M} 6 \mathrm{~m} 2$ \\
\hline$\Sigma u ́ \mu \pi \eta \kappa \tau \alpha$ & & & P6m1/P6m2 \\
\hline
\end{tabular}




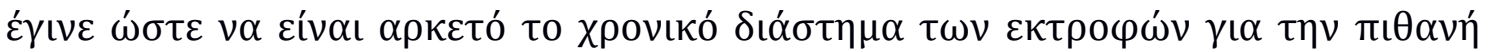

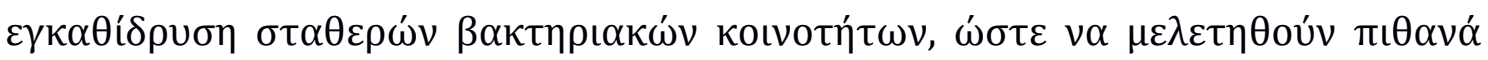

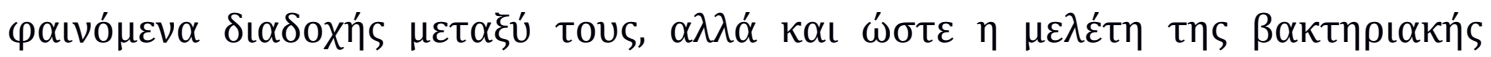

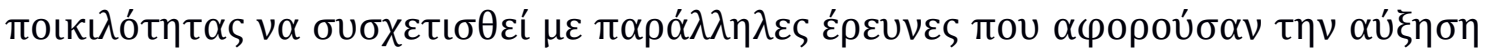

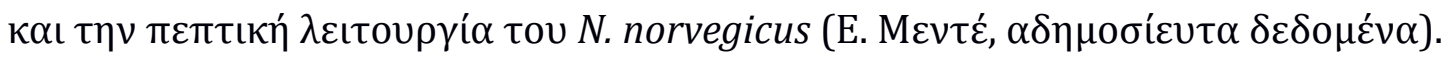

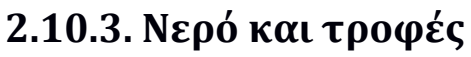

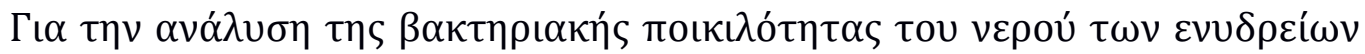

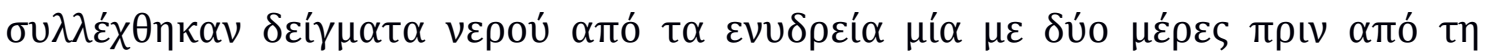

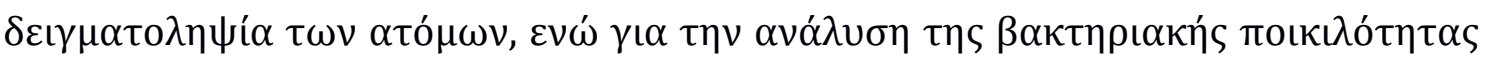

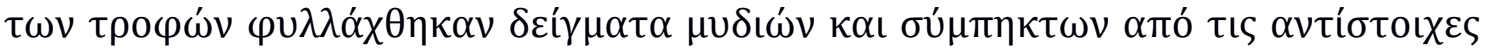

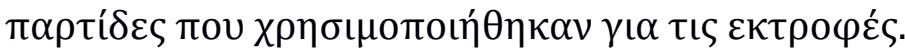

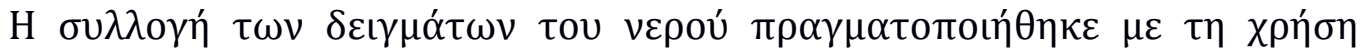

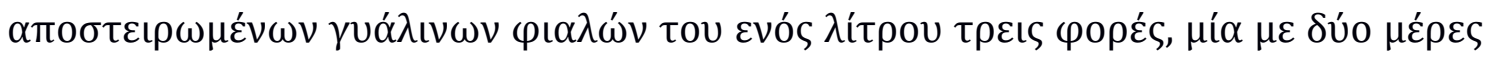

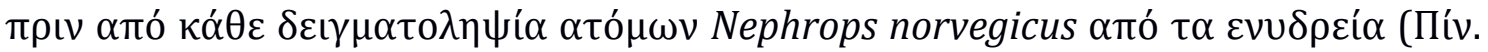

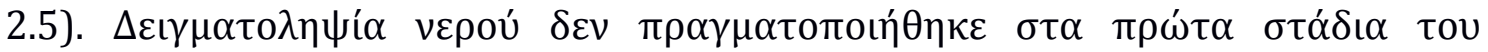

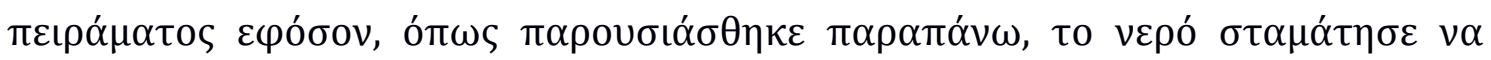

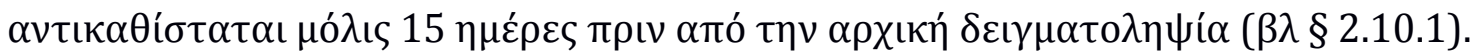

Гı

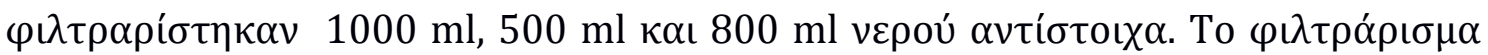

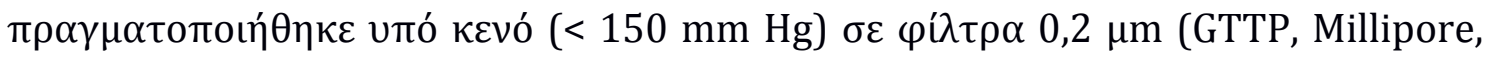

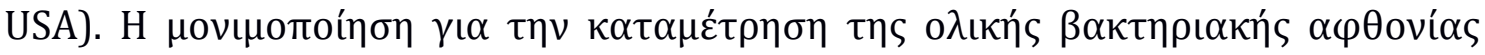




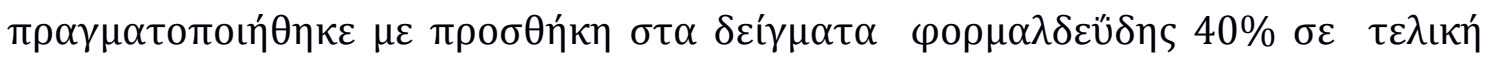

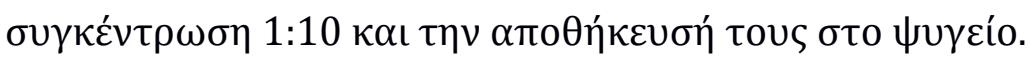

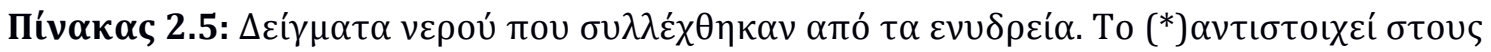

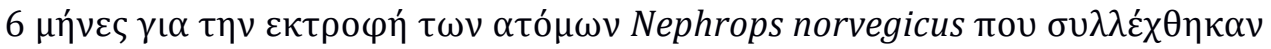
$\sigma \tau \eta \delta \varepsilon v ́ \tau \varepsilon \rho \eta \delta \varepsilon เ \gamma \mu \alpha \tau o \lambda \eta \psi i ́ \alpha$.

\begin{tabular}{|c|c|c|c|}
\hline$\Delta \varepsilon^{\prime} \gamma \mu \alpha$ & $\Delta \varepsilon \iota \gamma \mu \alpha \tau 0 \lambda \eta \psi i ́ \alpha$ & 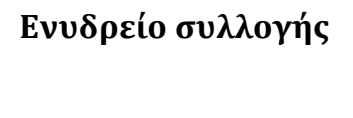 & 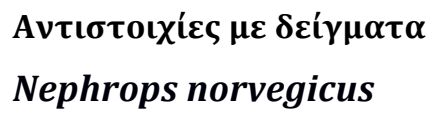 \\
\hline Wt2 & 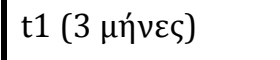 & Aбıтí $\alpha$ & M3m1, S3m1 \\
\hline Wt3 & 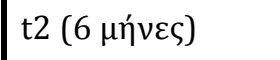 & Aбıтí $\alpha$ & M6m1, M6m2, S6m1, S6m2 \\
\hline Wt4 & 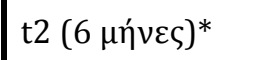 & $\Sigma u^{\prime} \mu \pi \eta \kappa \tau \alpha$ & P6m1, P6m2 \\
\hline
\end{tabular}

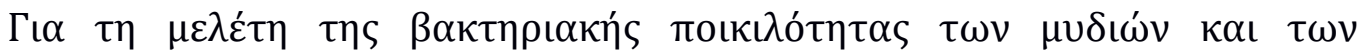

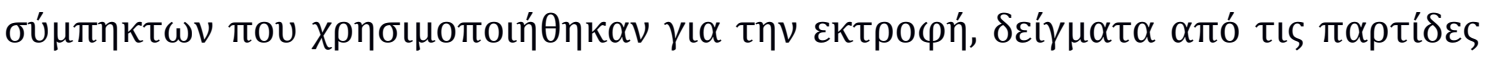

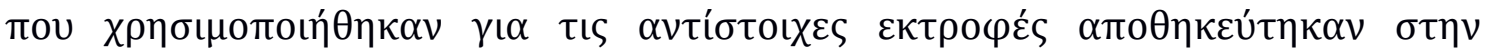

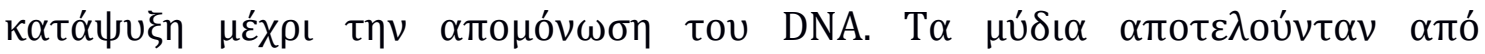

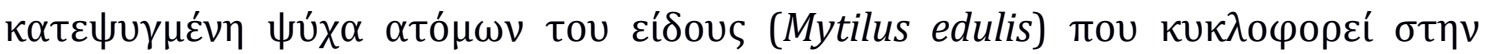

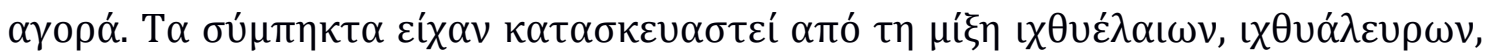

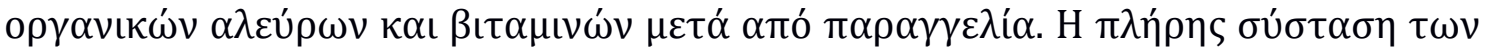

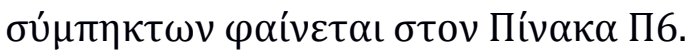

\subsubsection{Av $\alpha \lambda u ́ \sigma \varepsilon เ \varsigma$}

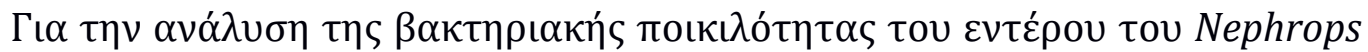

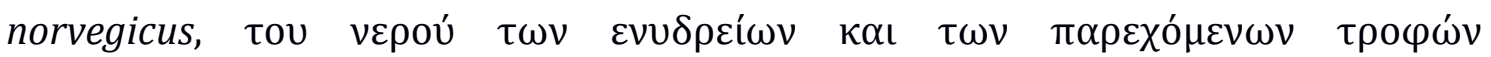

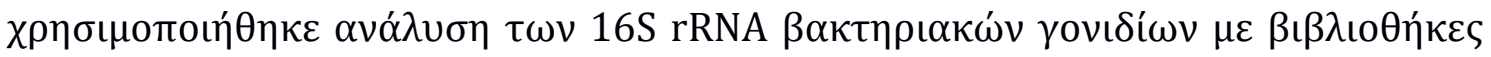




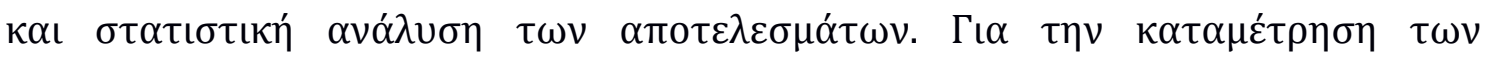

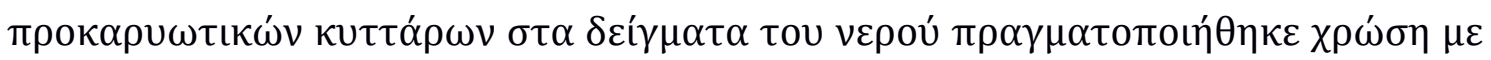
DAPI.

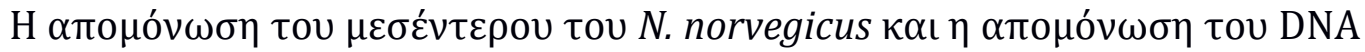

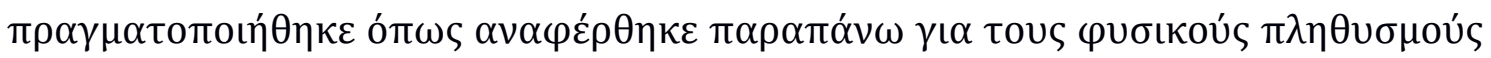

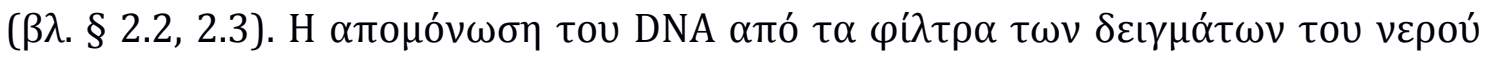

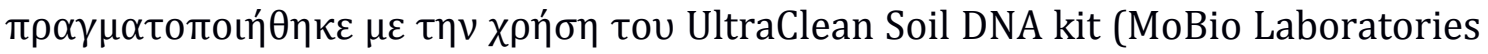

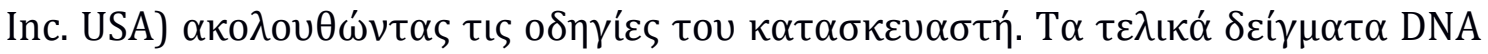

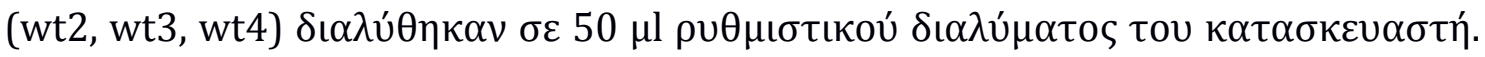

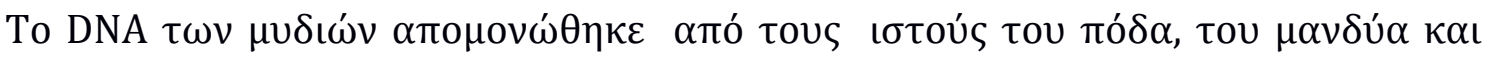

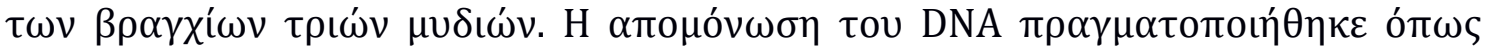

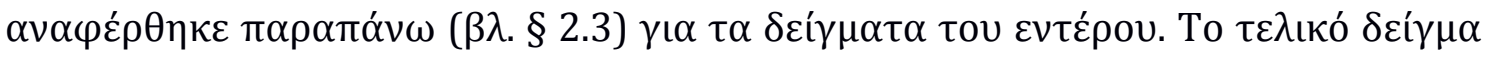

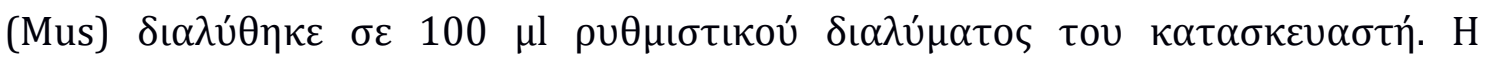

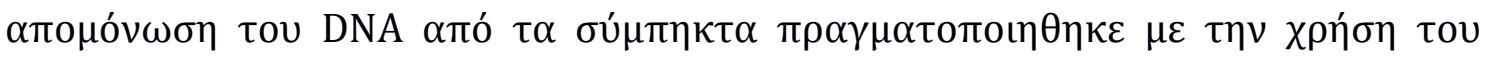

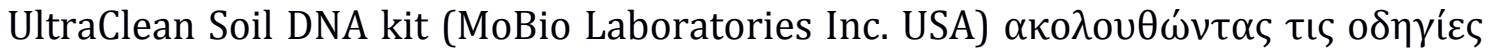

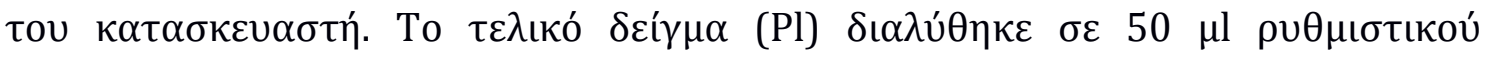

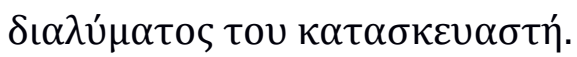

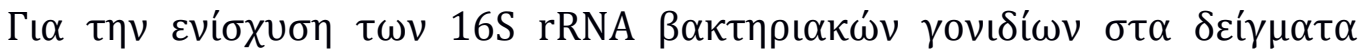

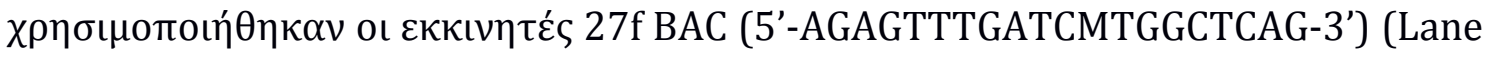
1991) кol 907r (5'-CCCGTCAATTCCTTTRAGTTT-3') (Muyzer et al. 1995). To

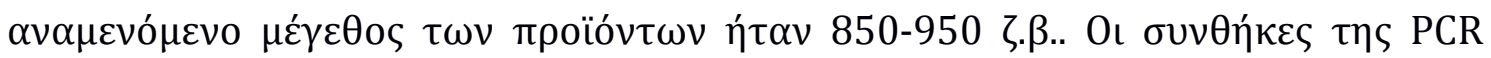

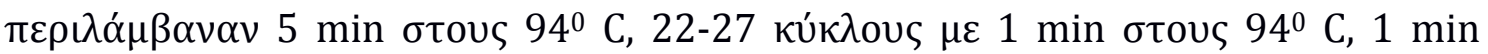

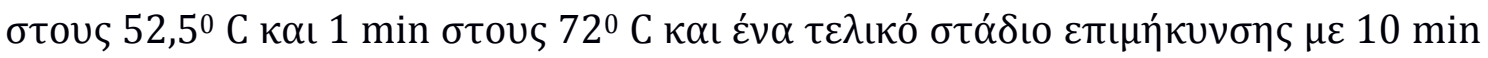

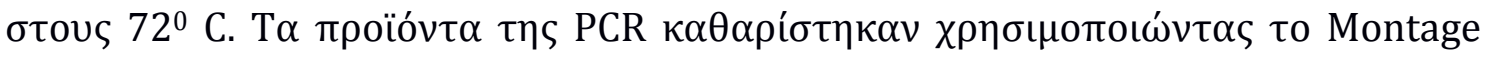




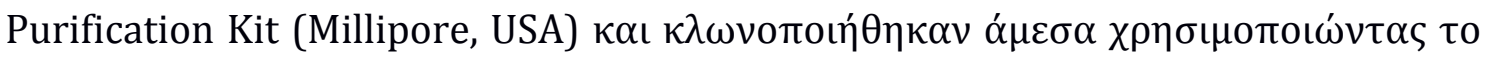

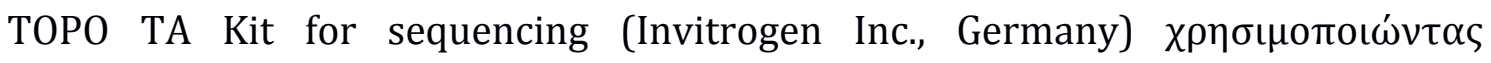

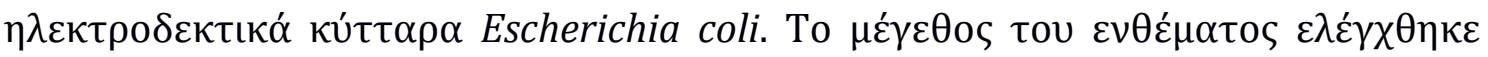

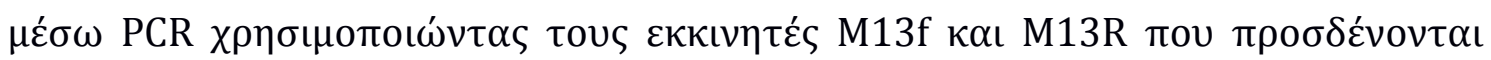

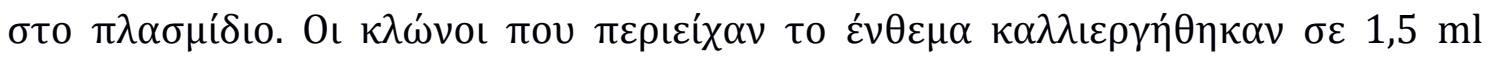

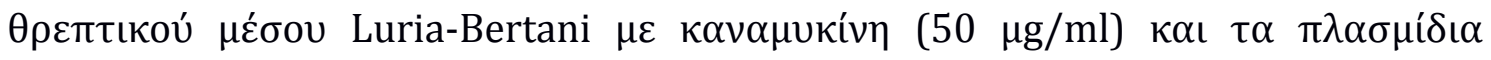

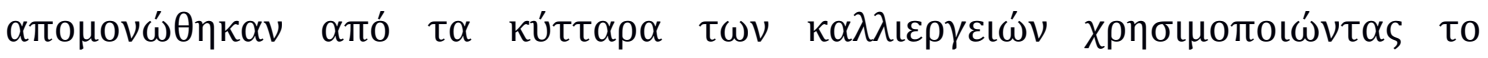
Nucleospin Plasmid QuickPure kit (Macherey-Nagel GmbH and Co, KG, Germany).

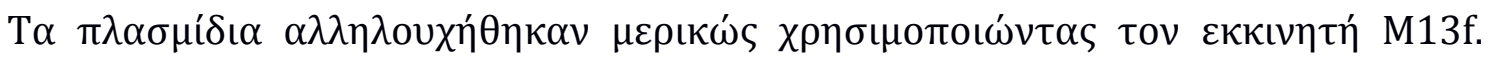

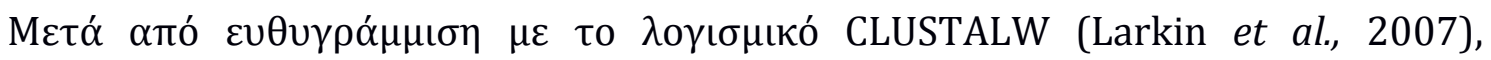

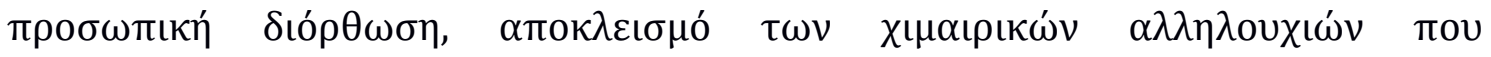

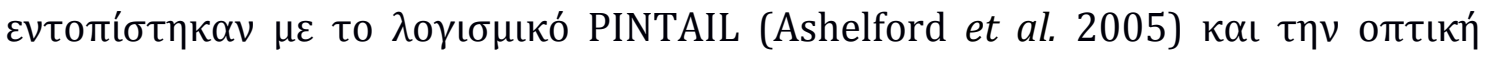

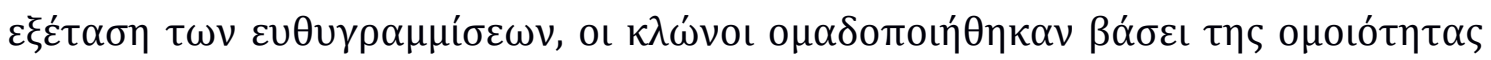

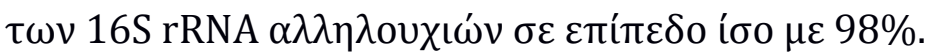

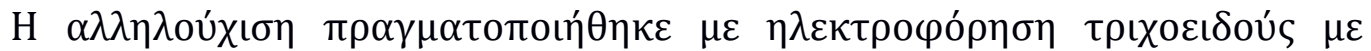
$\chi \rho \eta ́ \sigma \eta \tau \omega v \alpha v \tau \iota \delta \rho \alpha \sigma \tau \eta \rho i ́ \omega v$ BigDye Terminator kit (Applied Biosystems Inc., USA)

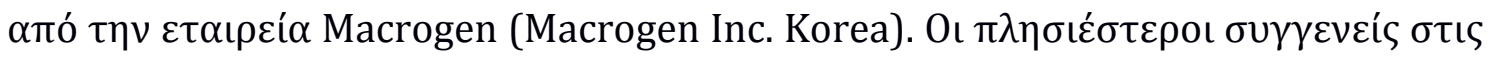

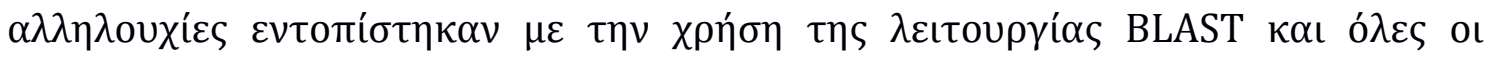

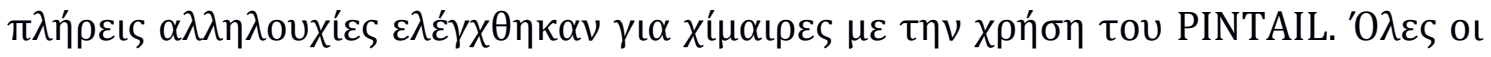

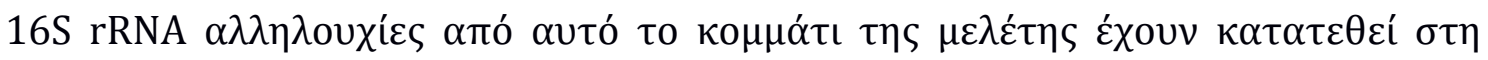

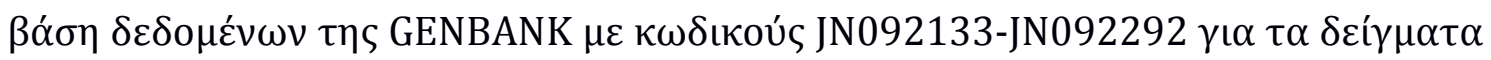

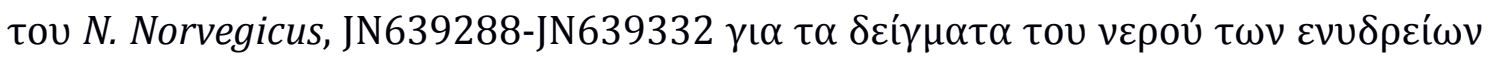

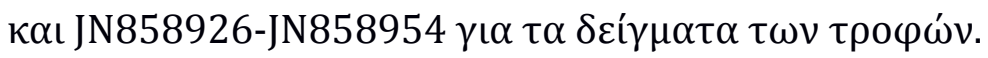




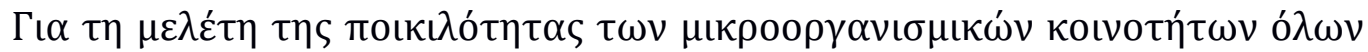

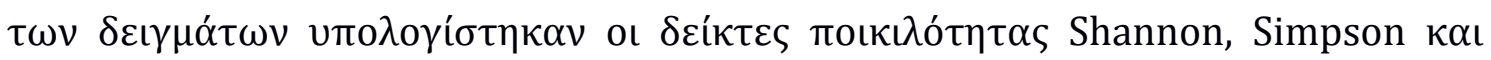

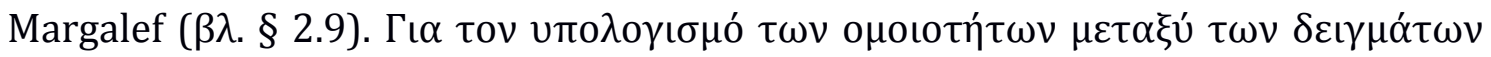

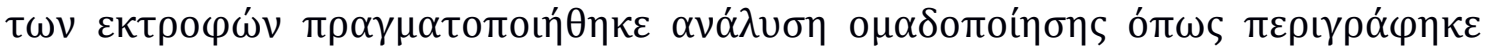

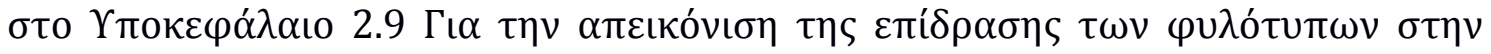

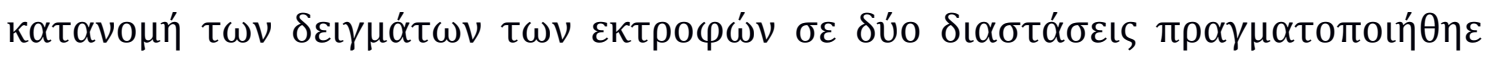

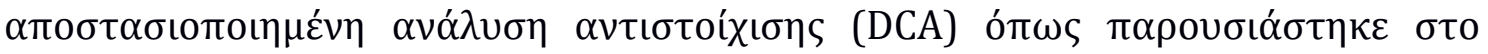

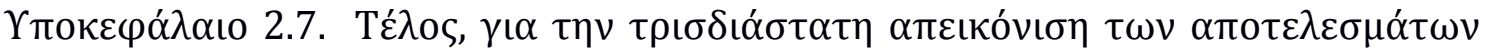

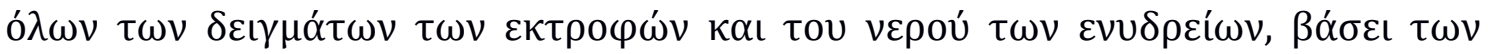

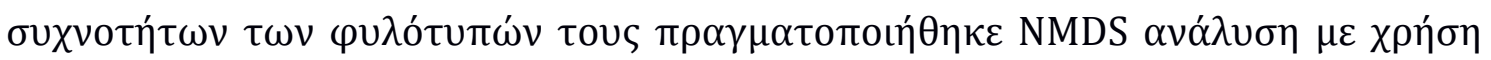

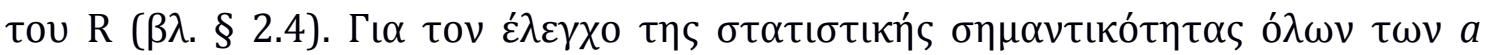

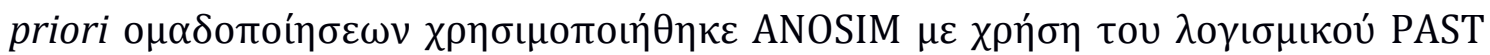
$(\beta \lambda . \S 2.4)$.

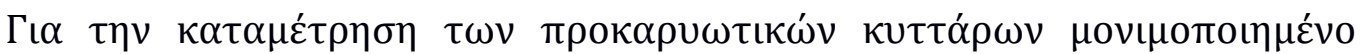

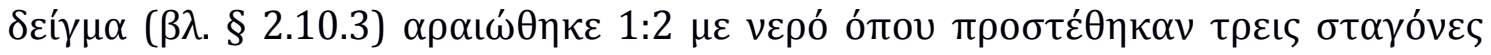

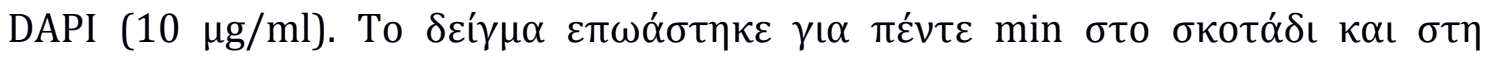

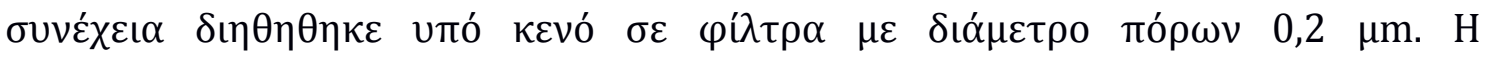

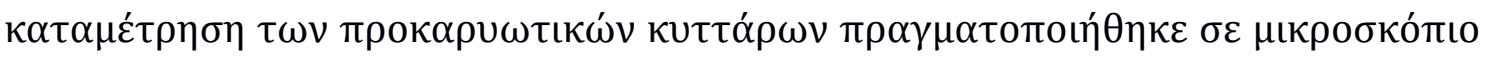

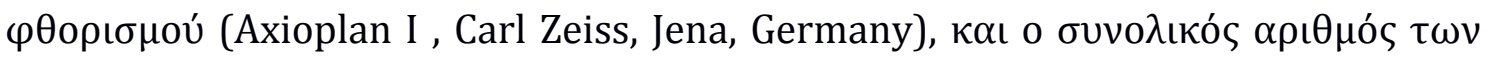

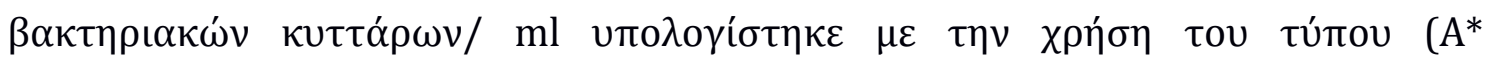

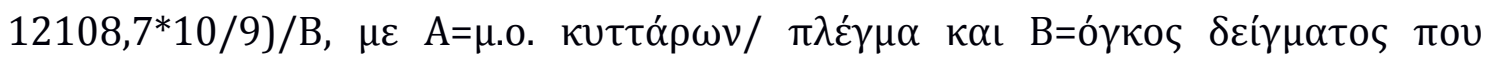
$\varphi і \lambda \tau \rho \alpha \rho i ́ \sigma \tau \eta \kappa \varepsilon$. 


\section{3. АПОТЕИЕЕMATA}

\section{1. Фvбเкоí $\pi \lambda \eta \theta v \sigma \mu o i ́$}

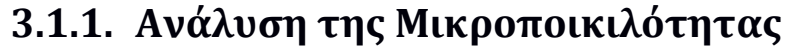

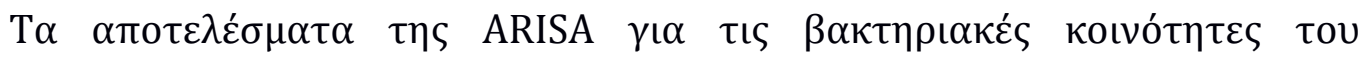

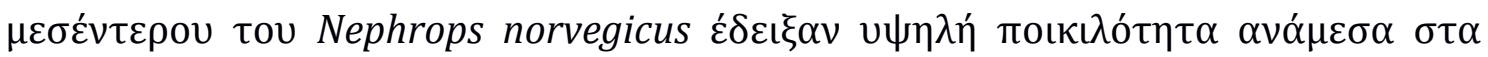

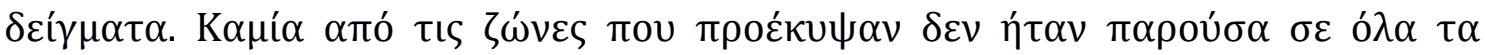

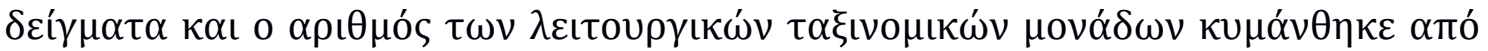

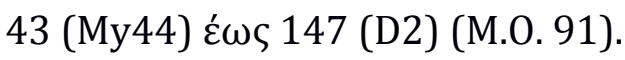

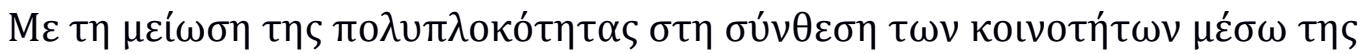

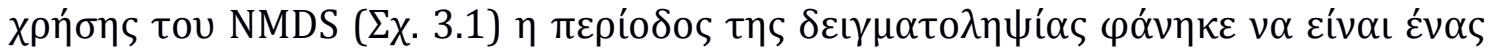

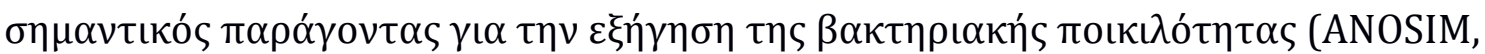

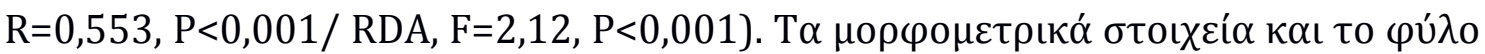

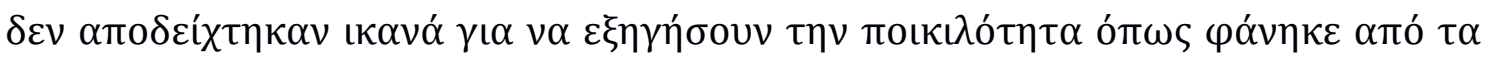

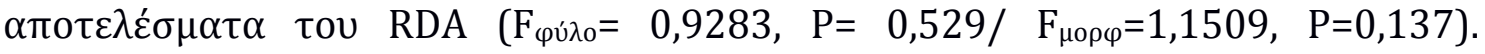

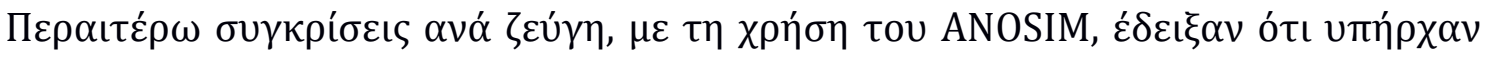

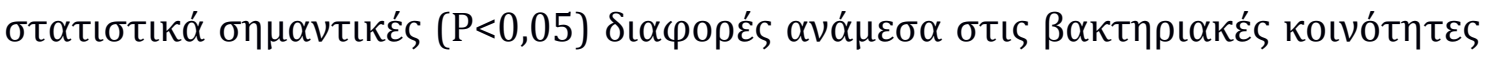
$\tau \omega \nu \pi \varepsilon \rho\llcorner\sigma \sigma o ́ \tau \varepsilon \rho \omega \nu ~ \mu \eta v \omega ́ v ~(\Pi i ́ v .3 .1)$.

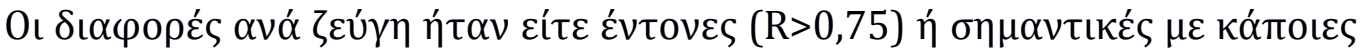

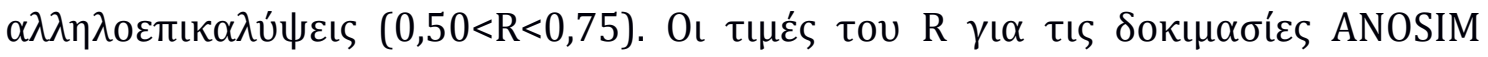

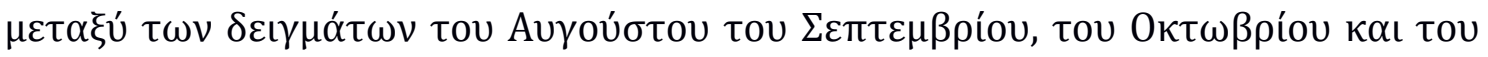

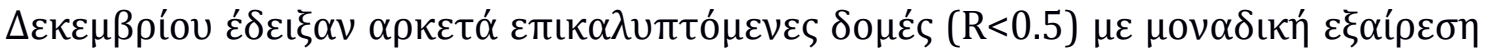

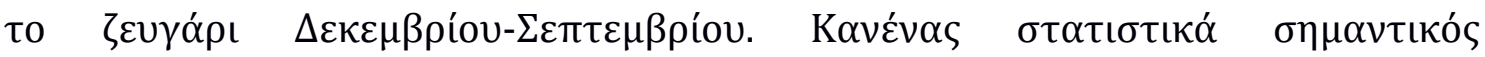




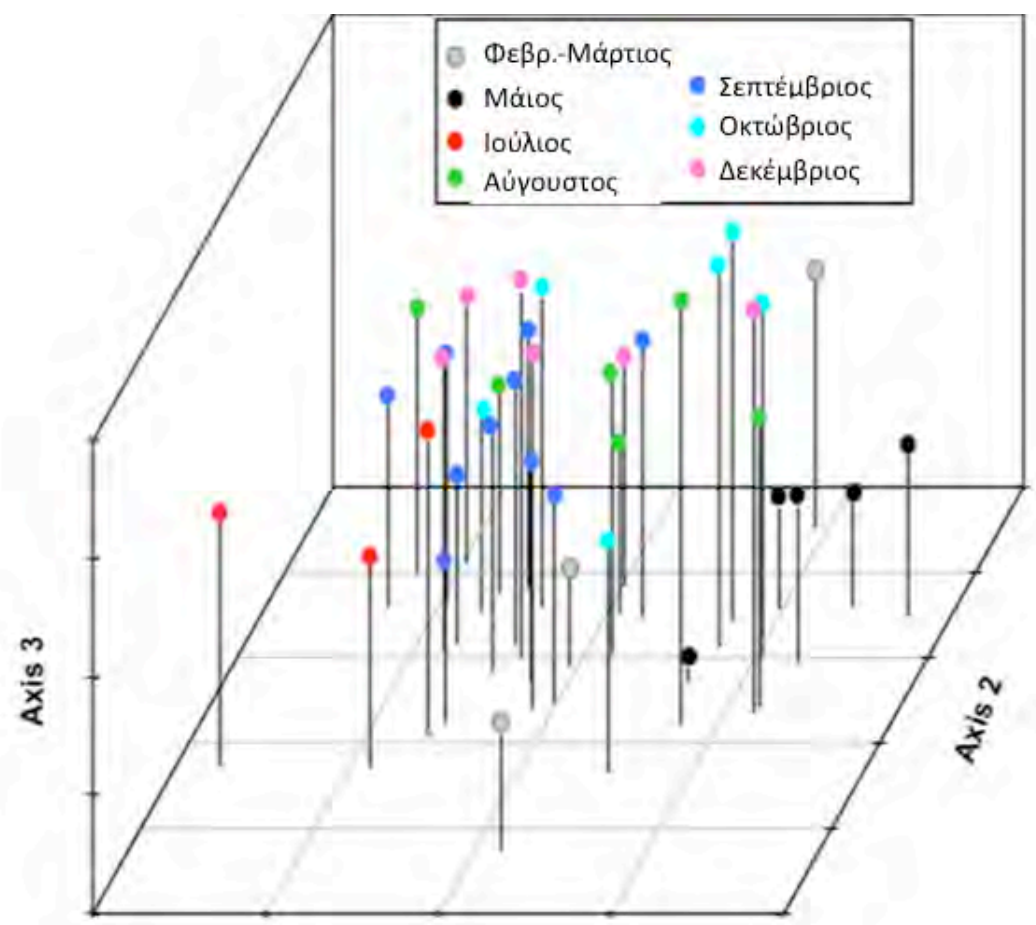

Axis 1

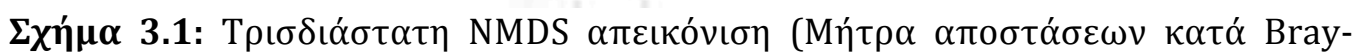

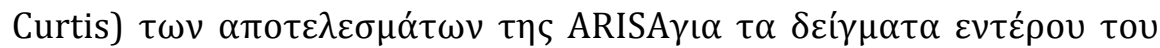

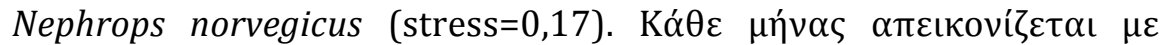

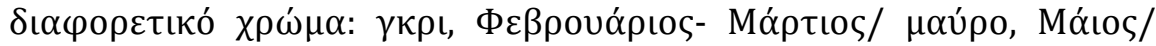

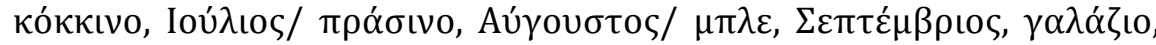

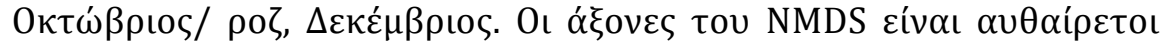

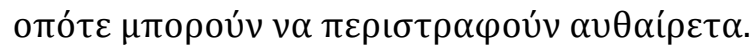

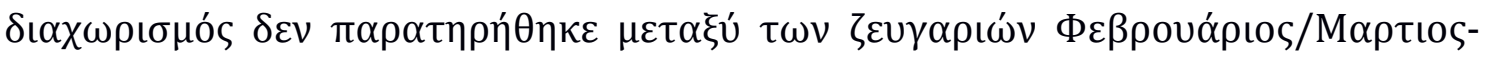

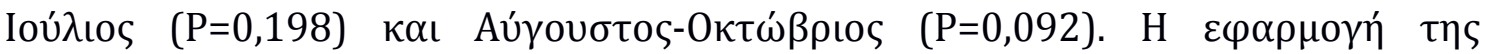

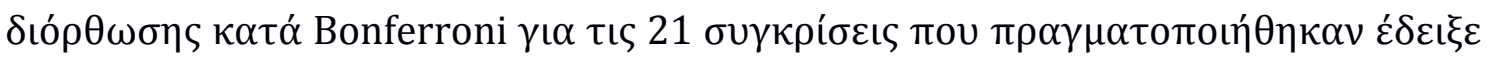

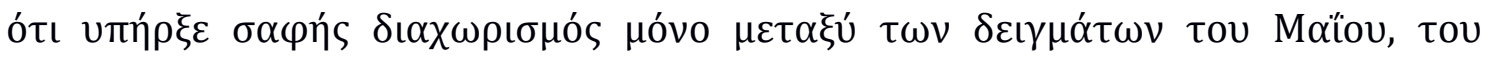

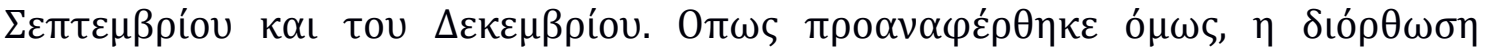

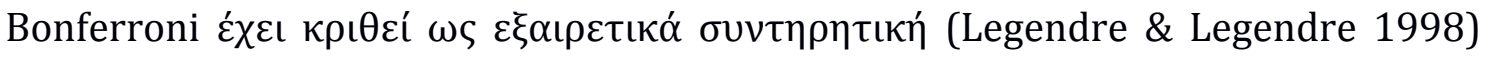

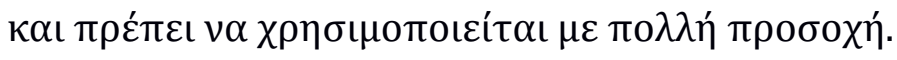




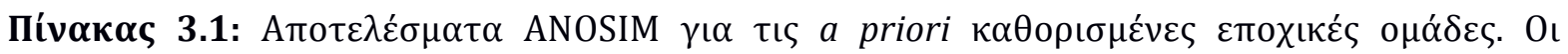

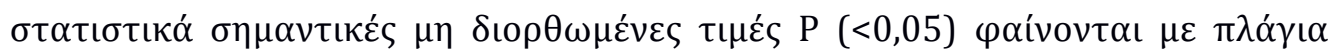

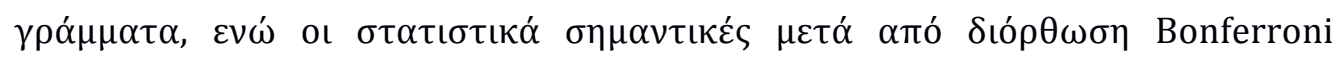

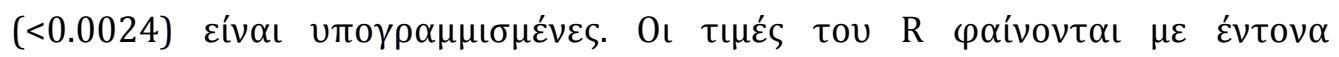
$\gamma \rho \alpha \dot{\mu} \mu \mu \alpha \tau \alpha$.

\begin{tabular}{|c|c|c|c|c|c|c|c|}
\hline & 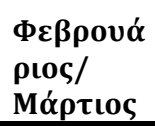 & Mó́tos & Iov́גıos & 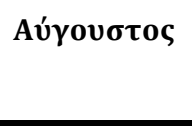 & $\begin{array}{l}\Sigma \varepsilon \pi \tau \varepsilon \dot{\mu} \mu \beta \rho t \\
\text { o૬ }\end{array}$ & 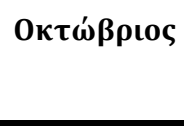 & 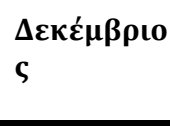 \\
\hline 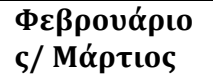 & & 0.0166 & 0.1983 & 0.0484 & 0.0083 & 0.0231 & 0.0105 \\
\hline Mó́tos & 0.590 & & 0.0183 & 0.0044 & $\underline{0.0002}$ & 0.0026 & $\underline{0.0023}$ \\
\hline Iovגıs & 0.444 & 0.969 & & 0.0113 & $\overline{0.0027}$ & 0.013 & $\overline{0.0133}$ \\
\hline 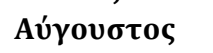 & 0.376 & 0.605 & 0.654 & & 0.0138 & 0.0916 & 0.0154 \\
\hline$\Sigma \varepsilon \pi \tau \varepsilon \dot{\varepsilon} \mu \beta \rho\llcorner о \zeta$ & 0.721 & 0.939 & 0.840 & 0.293 & & 0.0043 & 0.0003 \\
\hline 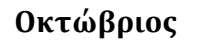 & 0.500 & 0.872 & 0.697 & 0.161 & 0.403 & & 0.0302 \\
\hline 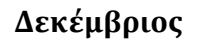 & 0.611 & 0.923 & 0.765 & 0.387 & 0.521 & 0.280 & \\
\hline
\end{tabular}

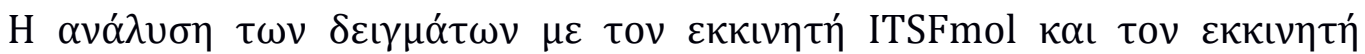

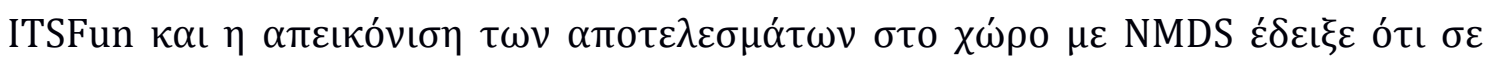

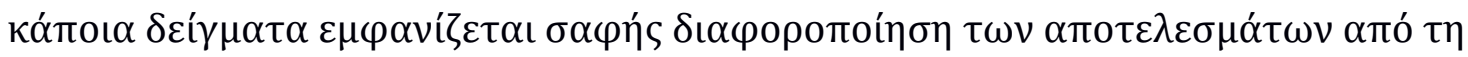

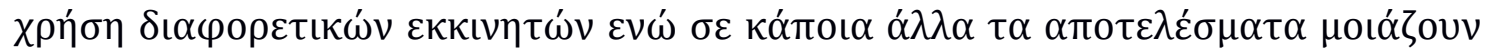

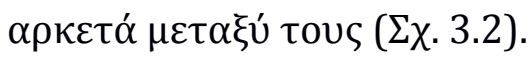

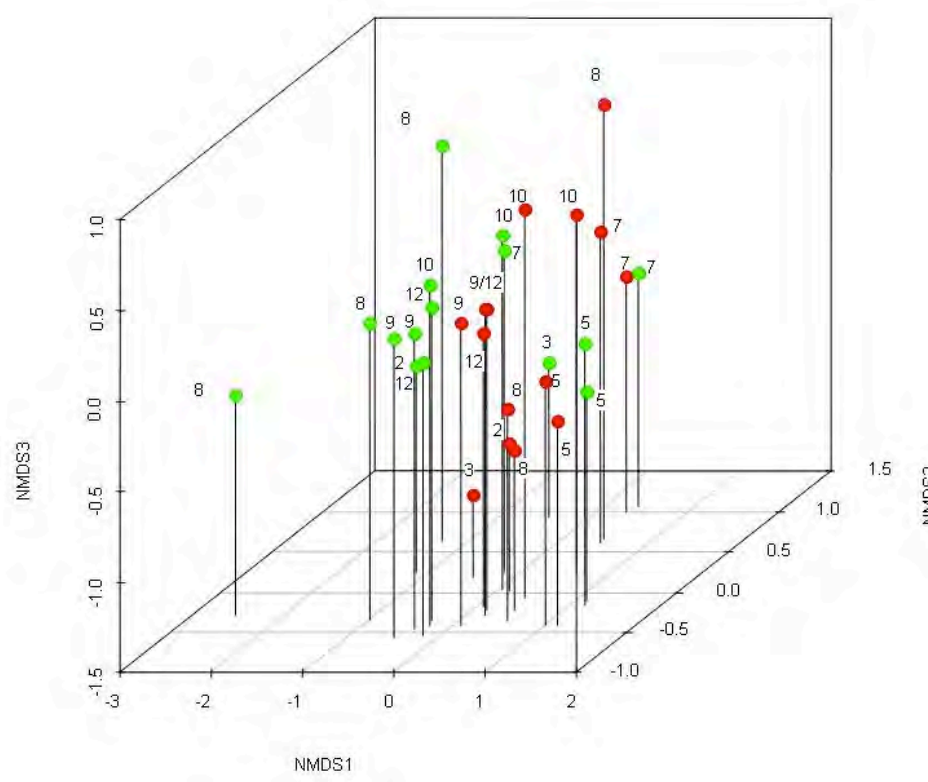

$\Sigma \chi \eta \dot{\mu \alpha} \quad 3.2:$ :

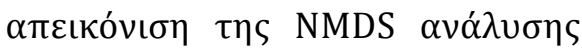

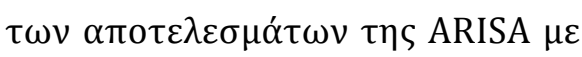

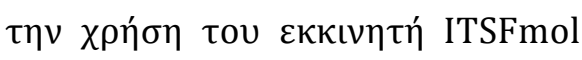

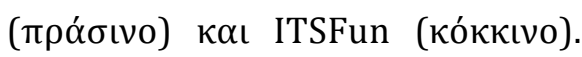

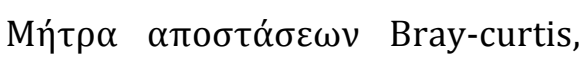
stress $=0,10 . \quad$ Oı $\quad \alpha \rho \iota \theta \mu o i ́$

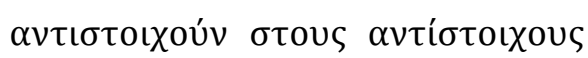

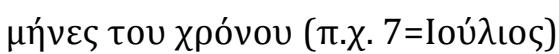




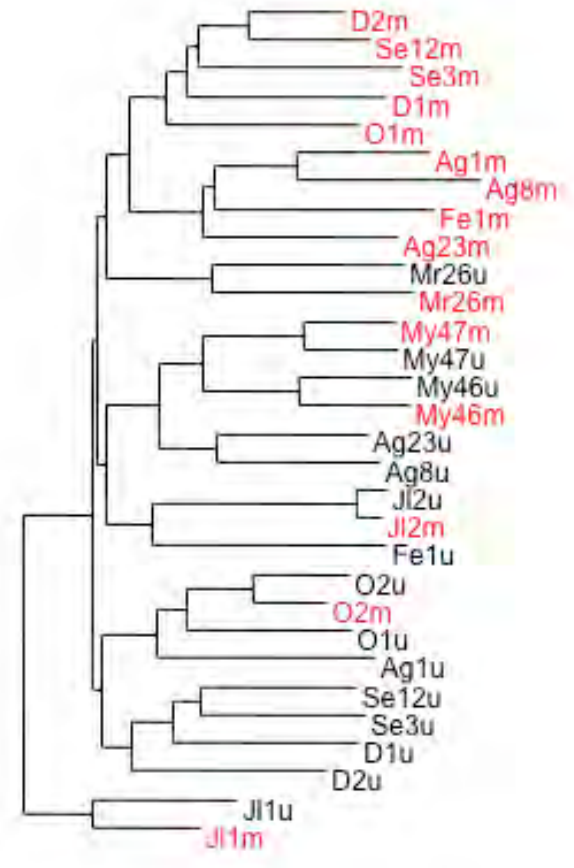

$\Sigma \chi \eta^{\prime} \mu \alpha$ 3.3: $\Delta \varepsilon \dot{v} \tau \tau \rho$ Neighbor-Joining $\gamma \iota \alpha$ $\tau \alpha \alpha \pi 0 \tau \varepsilon \lambda \varepsilon \dot{\varepsilon} \sigma \mu \alpha \tau \alpha \tau \eta \varsigma$

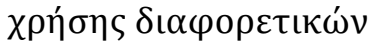

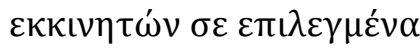
$\delta \varepsilon \dot{\gamma} \gamma \mu \alpha \tau \alpha$ ( $\mu \alpha \dot{v} \nu \rho:$ $\pi \alpha \gamma \kappa o ́ \sigma \mu t о เ ~ \varepsilon \kappa \kappa เ v \eta \tau \varepsilon ́ \varsigma$,

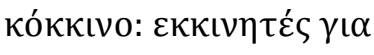
Mollicutes)

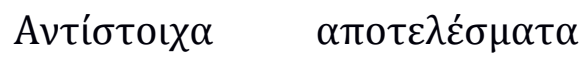

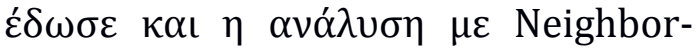
Joining $(\Sigma \chi .3 .3)$. Пıо $\sigma u \gamma \kappa \varepsilon \kappa \rho ц \varepsilon ́ v \alpha$

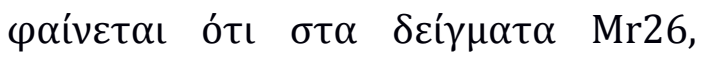

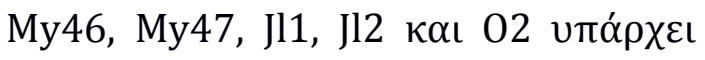

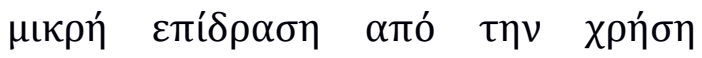

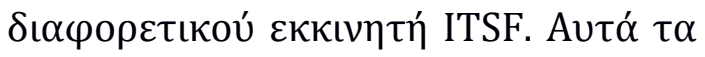

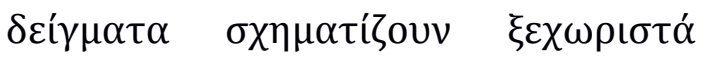

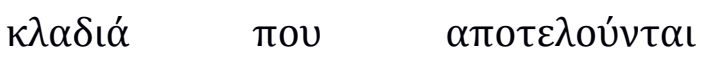

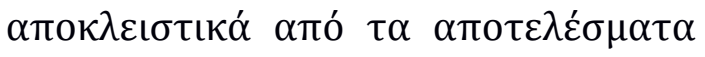

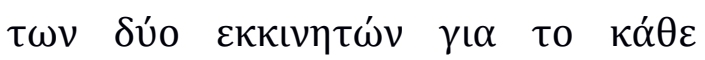
$\delta \varepsilon i ́ \gamma \mu \alpha$.

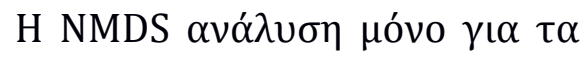

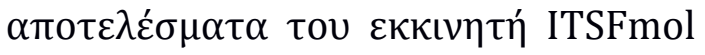

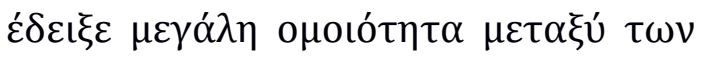

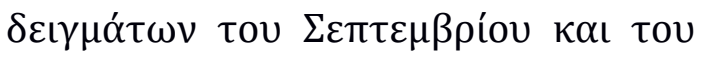

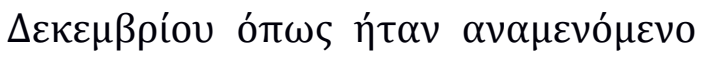

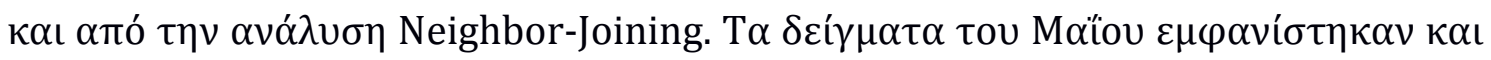

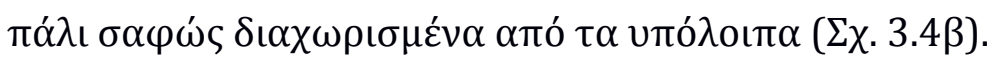

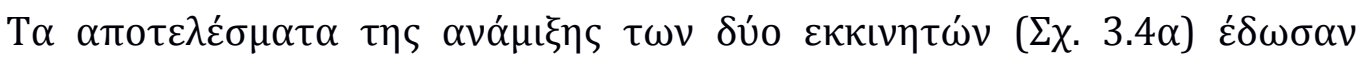

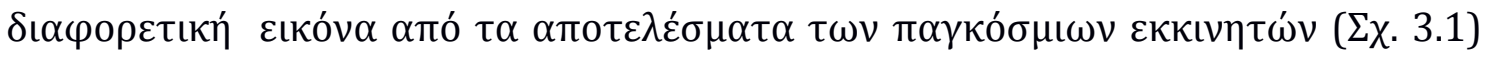

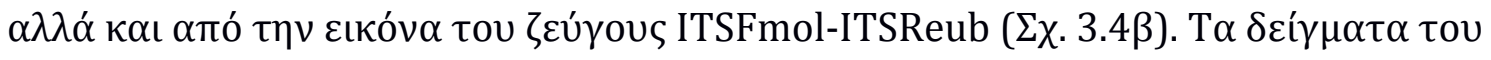

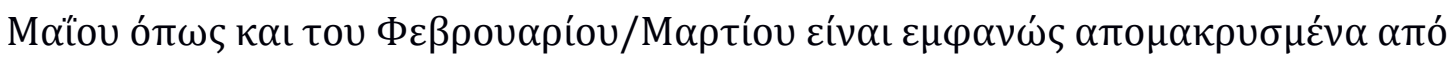




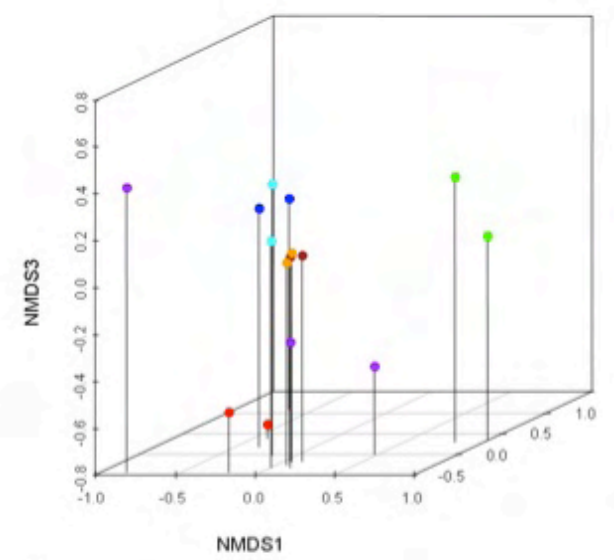

$\alpha$

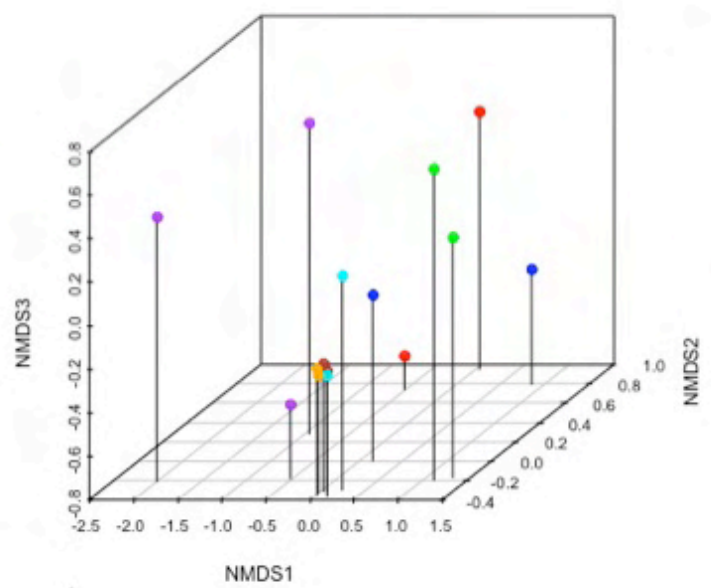

$\beta$

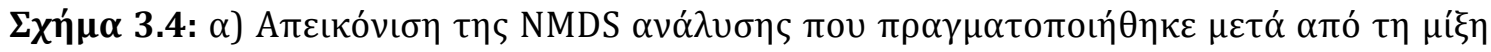

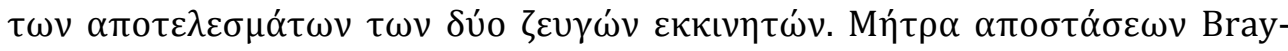

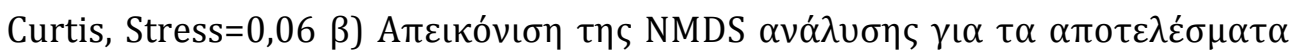

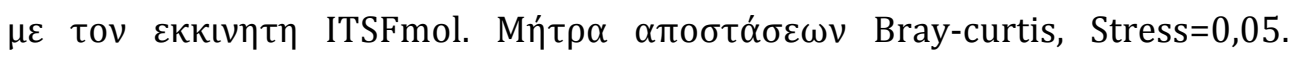

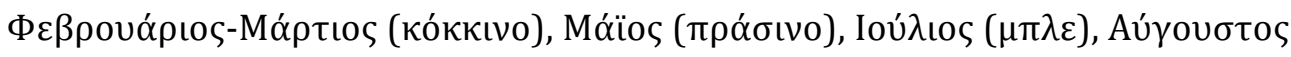

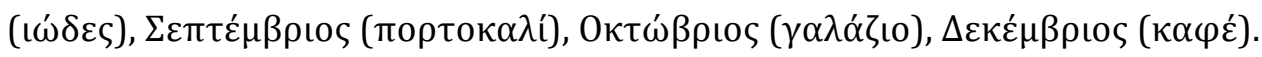

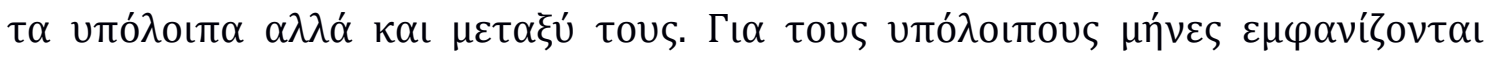

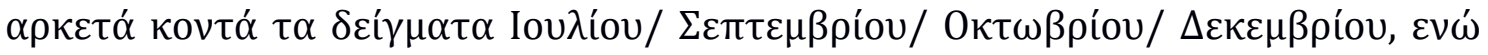

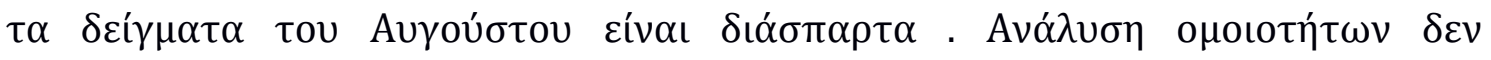

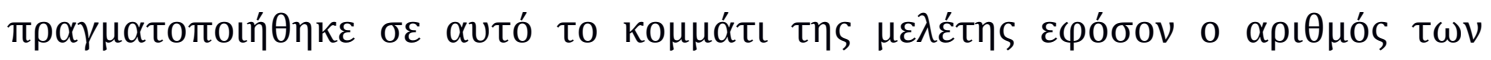

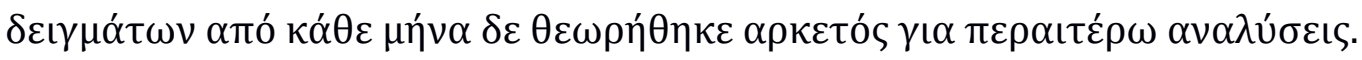

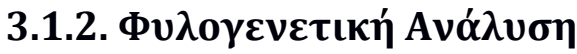

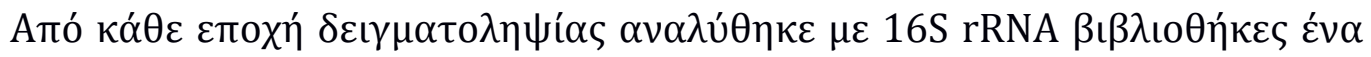

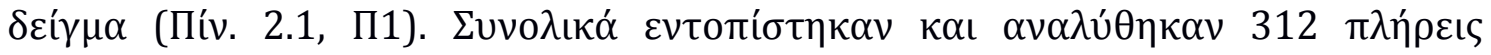




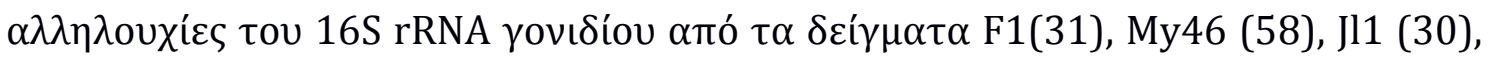
Ag31 (22), Se3(53), 02 (25) каı D1 (93).

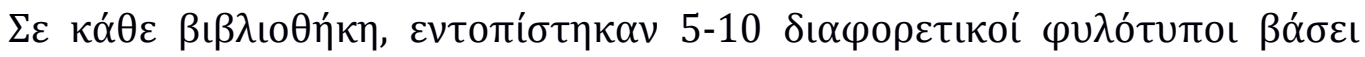

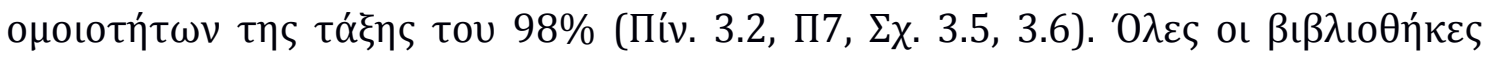

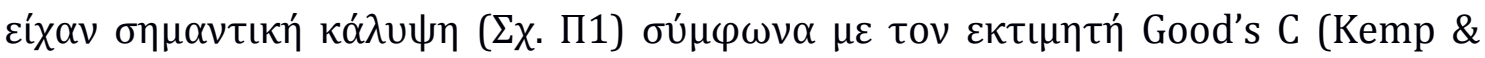

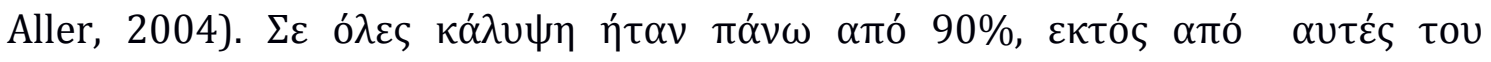

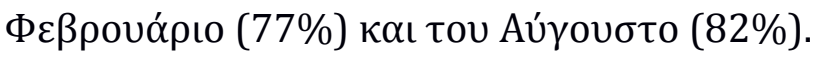

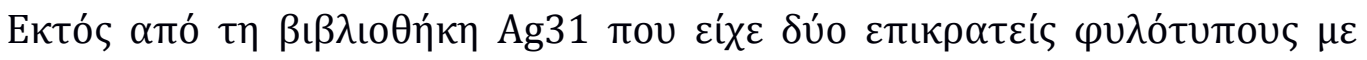

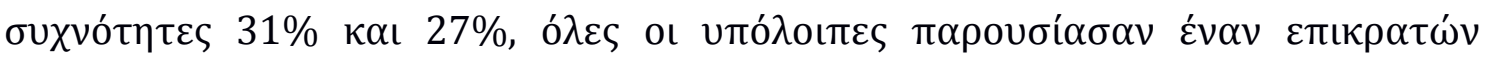

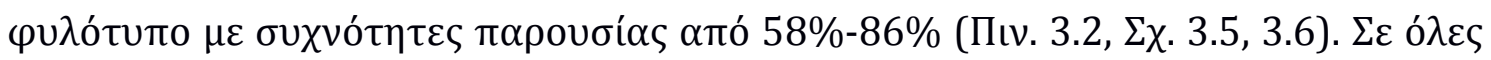

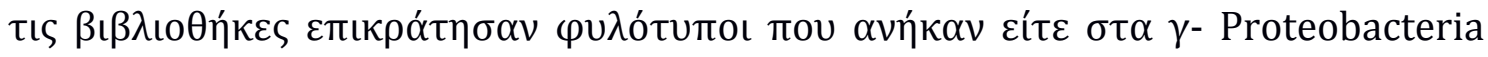

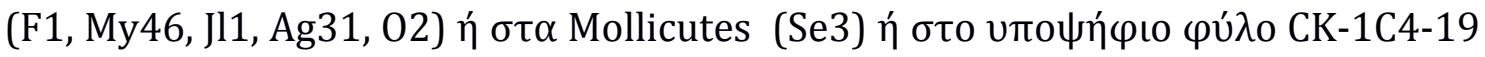

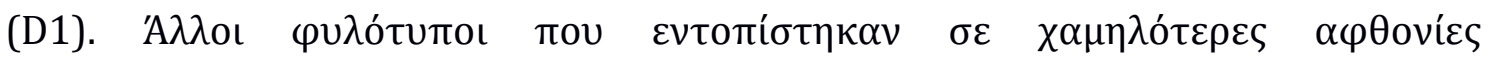

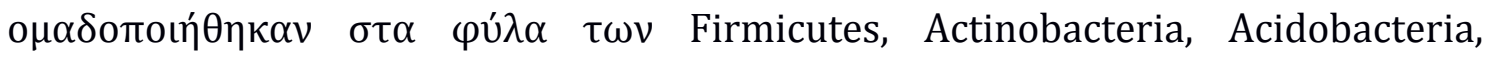

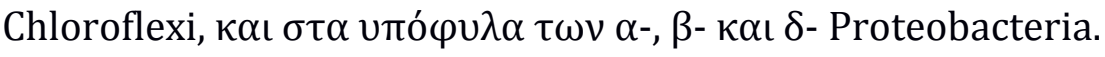

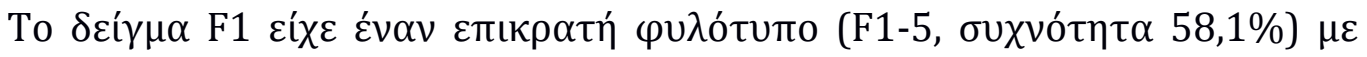

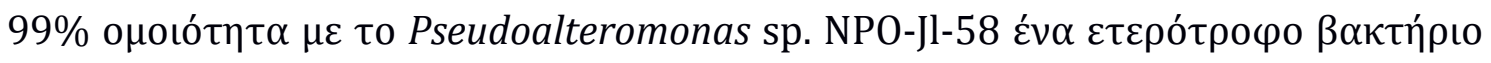

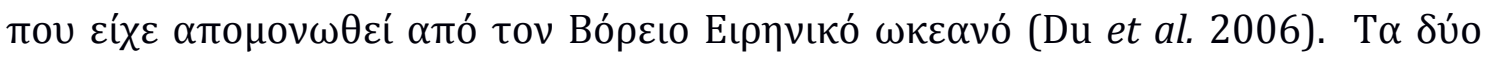

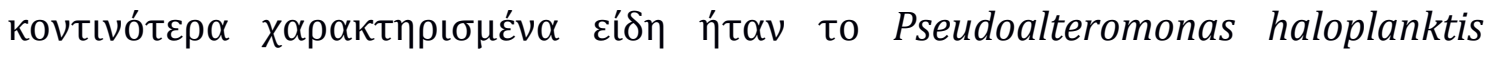
TAC125 (Medigue et al. 2005) каı то Pseudoalteromonas elyakovii (Sawabe et al.

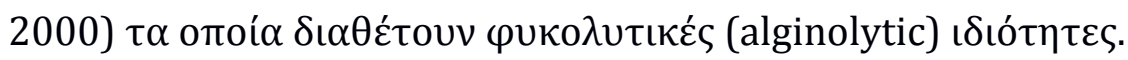

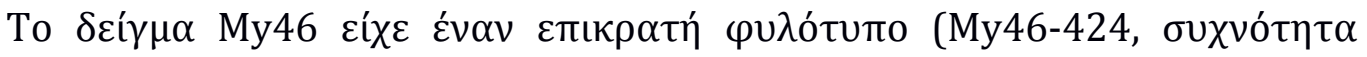

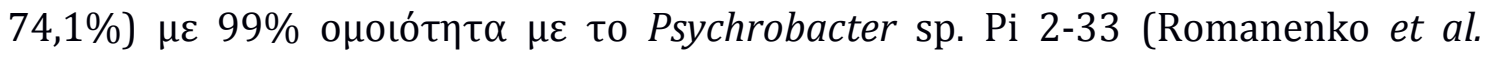

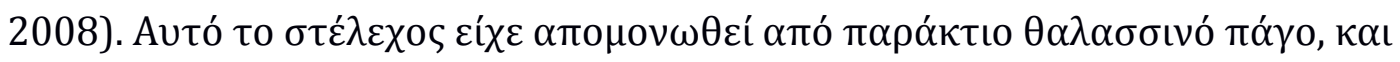




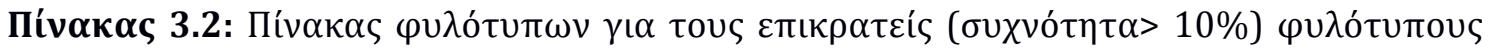

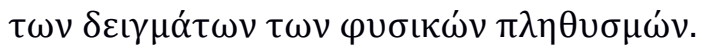

\begin{tabular}{|c|c|c|c|c|}
\hline 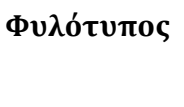 & $\Sigma v \chi v o ́ \tau \eta \tau \alpha$ & 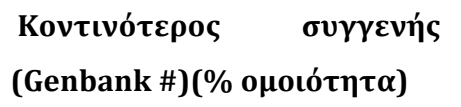 & 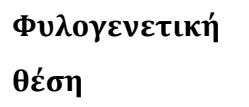 & 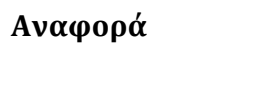 \\
\hline F1-5 & $58,1 \%$ & $\begin{array}{l}\text { Pseudoalteromonas sp. NPO-Jl- } \\
58(\text { AY745828)(99) }\end{array}$ & $\gamma$-Proteobacteria & Du et al. 2006 \\
\hline F1-4 & $12,9 \%$ & $\begin{array}{l}\text { Psychrobacter aquimaris, KOPRI } \\
24929 \text { (EF101547) (99) }\end{array}$ & $\gamma$-Proteobacteria & Lee et al. 2006 \\
\hline My46-424 & $74,1 \%$ & $\begin{array}{l}\text { Psychrobacter sp. Pi 2-33 } \\
(\text { AB365059)(99) }\end{array}$ & $\gamma$-Proteobacteria & $\begin{array}{l}\text { Romanenko et al. } \\
2008\end{array}$ \\
\hline My46-492 & $19,0 \%$ & Vibrio lentus (AY292936)(99) & $\gamma$-Proteobacteria & $\begin{array}{l}\text { Nishiguchi \& Nair } \\
2003\end{array}$ \\
\hline Jl1-36 & $73,3 \%$ & $\begin{array}{l}\text { Photobacterium } \\
\text { (AY292917)(99) }\end{array}$ & $\gamma$-Proteobacteria & $\begin{array}{l}\text { Nishiguchi \& Nair } \\
2003\end{array}$ \\
\hline Jl1-4 & $13,3 \%$ & $\begin{array}{l}\text { Photobacterium sp. JT-ISH-224 } \\
\text { (AB293986) (99) }\end{array}$ & $\gamma$-Proteobacteria & $\begin{array}{l}\text { Tsukamoto et al. } \\
2008\end{array}$ \\
\hline Ag31-3 & $31,9 \%$ & $\begin{array}{l}\text { Photobacterium leiognathi } \\
\text { strain RM1 (AY292947)(99) }\end{array}$ & $\gamma$-Proteobacteria & $\begin{array}{l}\text { Nishiguchi \& Nair } \\
2003\end{array}$ \\
\hline Ag31-6 & $27,3 \%$ & $\begin{array}{l}\text { Photobacterium } \\
\text { (AY292917)(98) }\end{array}$ & $\gamma$-Proteobacteria & $\begin{array}{l}\text { Nishiguchi \& Nair } \\
2003\end{array}$ \\
\hline Ag31-15 & $13,6 \%$ & Vibrio lentus (AY292936) (98) & $\gamma$-Proteobacteria & $\begin{array}{l}\text { Nishiguchi \& Nair } \\
2003\end{array}$ \\
\hline Se3-204 & $83,0 \%$ & $\begin{array}{l}\text { Uncultured } \\
\text { (DQ340200) (90) }\end{array}$ & Mollicutes & Bano et al. 2007 \\
\hline $02-1$ & $76,0 \%$ & $\begin{array}{l}\text { Photobacterium leiognathi } \\
\text { strain RM1(AY292947)(99) }\end{array}$ & $\gamma$-Proteobacteria & $\begin{array}{l}\text { Nishiguchi \& Nair } \\
2003\end{array}$ \\
\hline $02-8$ & $16,0 \%$ & $\begin{array}{l}\text { Vibrio splendidus isolate PB1- } \\
\text { 10rrnH (EU091332)(99) }\end{array}$ & $\gamma$-Proteobacteria & Jensen et al. 2009 \\
\hline D1-695 & $86,0 \%$ & $\begin{array}{l}\text { Uncultured bacterium clone } \\
\text { CK_1_C4_1(EU488044)(87) }\end{array}$ & CK-1C4-19 & $\begin{array}{l}\text { Green-Garcia } \\
2008\end{array}$ \\
\hline
\end{tabular}


F1

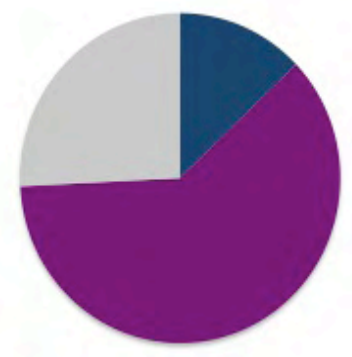

D1

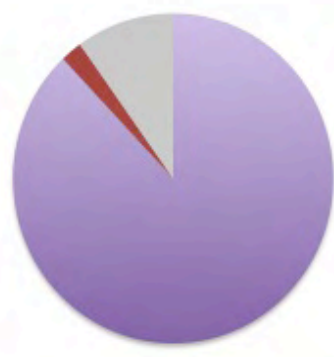

02

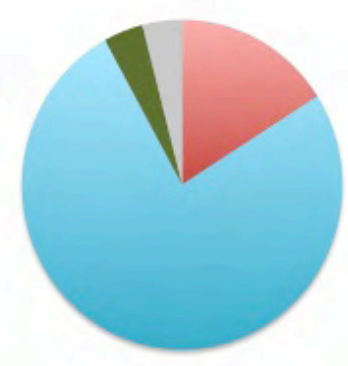

- Psychrobacter aquimaris

- Vibrio splendidus

= Psychrobacter sp.

= CK-1C4-19

= P. leiognathi subsp. (1)

$=$ P. leiognathi subsp.(2)

- V.salmonicida

- Dechloromonas sp.

- Pseudoalteromonas sp.

- Se3-204

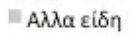

\section{Se3}

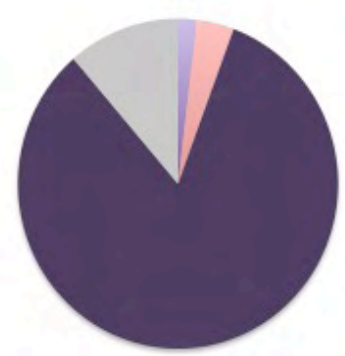

My46

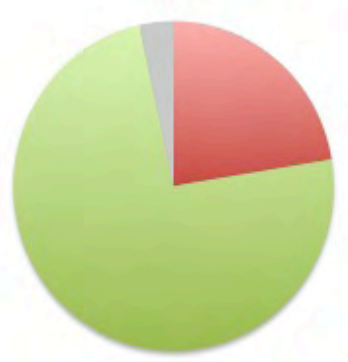

J1

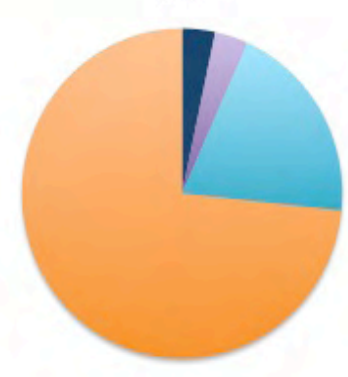

Ag31

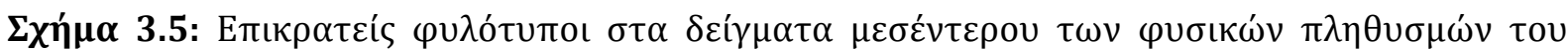

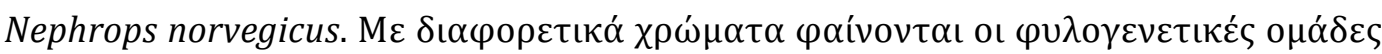

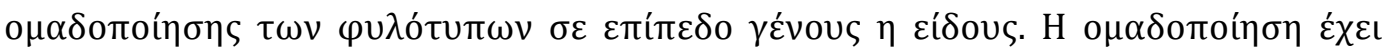

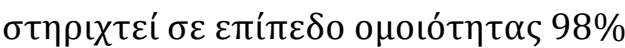




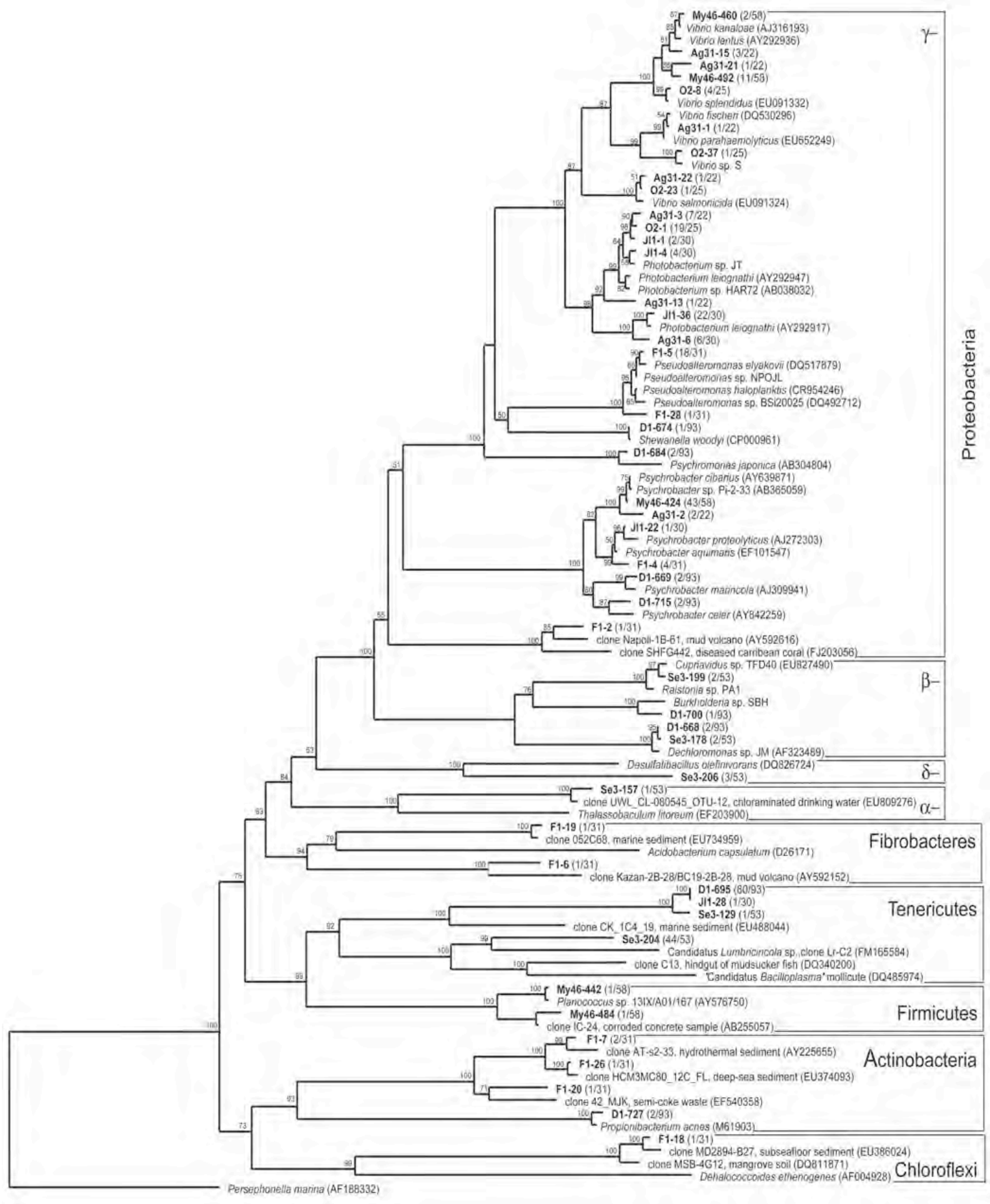

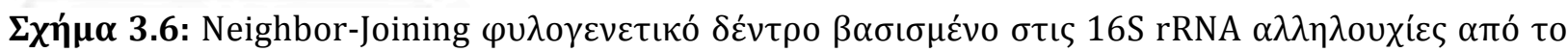

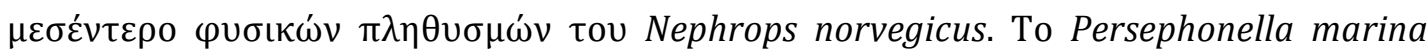

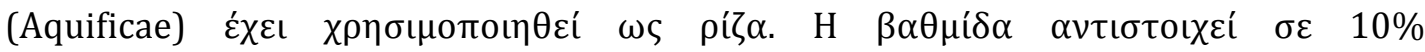

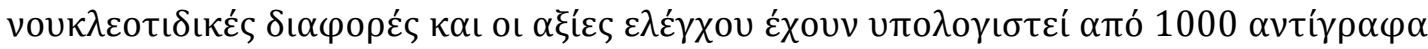

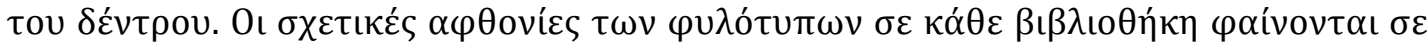

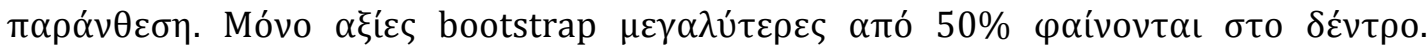




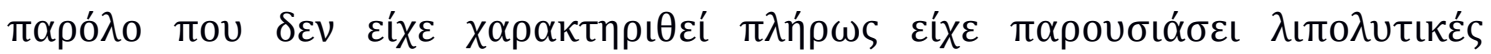

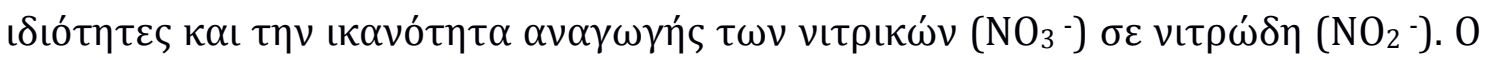

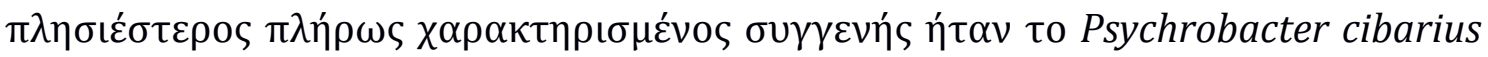

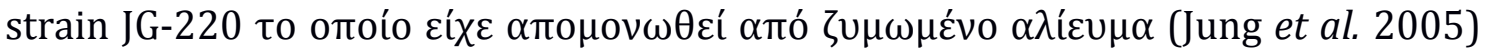

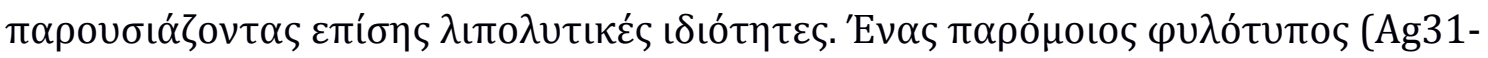

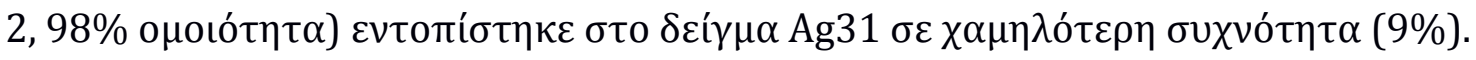

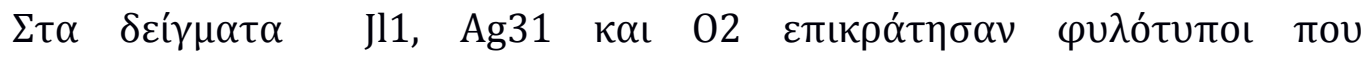

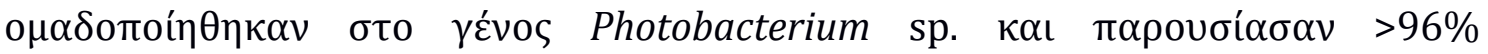

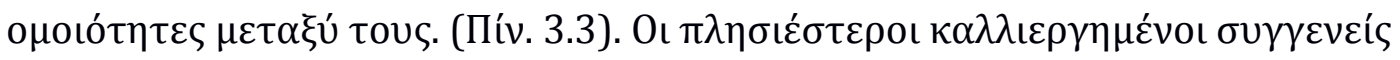

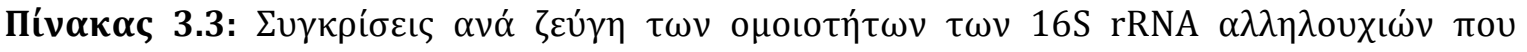

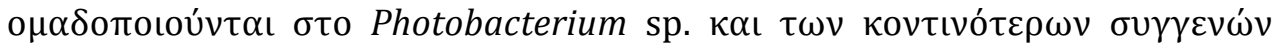

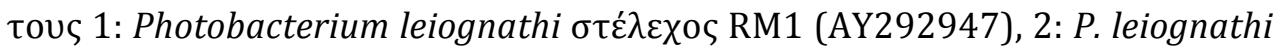
(AY292917), 3: Photobacterium sp. HAR23 (AB038031), 4: Photobacterium

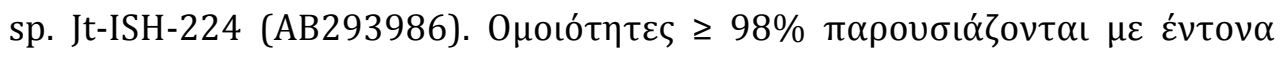
$\gamma \rho \alpha \dot{\mu} \mu \mu \alpha \tau \alpha$.

\begin{tabular}{|c|c|c|c|c|c|c|c|c|c|c|c|}
\hline 1 & 2 & 3 & 4 & Jl1-36 & J11-4 & JI1-1 & Ag31-3 & Ag31-6 & Ag31-13 & $02-1$ & 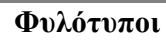 \\
\hline \multirow[t]{10}{*}{-} & 96 & 99 & 98 & 96 & 99 & 99 & 99 & 96 & 97 & 99 & 1 \\
\hline & - & 96 & 96 & 99 & 96 & 96 & 96 & 98 & 96 & 96 & 2 \\
\hline & & - & 98 & 96 & 99 & 99 & 98 & 97 & 98 & 99 & 3 \\
\hline & & & - & 96 & 99 & 99 & 98 & 96 & 97 & 99 & 4 \\
\hline & & & & - & 96 & 96 & 96 & 98 & 96 & 96 & Jl1-36 \\
\hline & & & & & - & 98 & 98 & 96 & 97 & 98 & J11-4 \\
\hline & & & & & & - & 99 & 96 & 97 & 99 & JI1-1 \\
\hline & & & & & & & - & 96 & 97 & 99 & Ag31-3 \\
\hline & & & & & & & & - & 96 & 96 & Ag31-6 \\
\hline & & & & & & & & & - & 97 & Ag31-13 \\
\hline
\end{tabular}




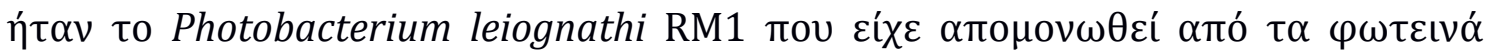

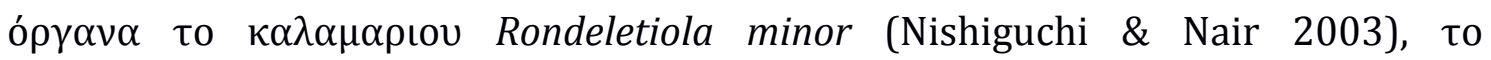

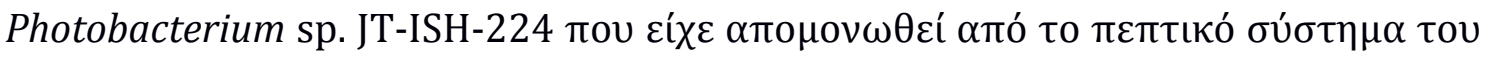

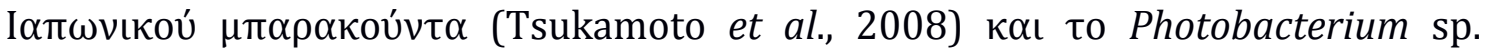

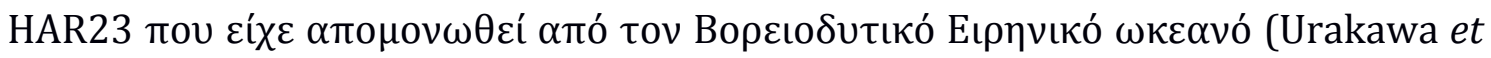
al. 1999).

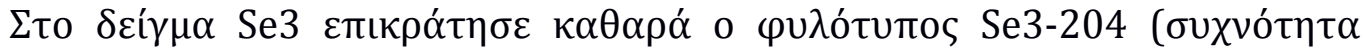

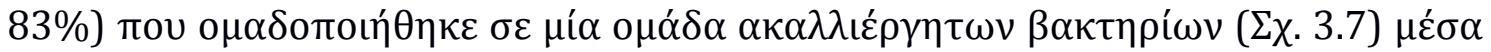

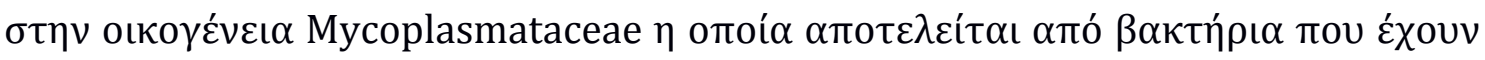

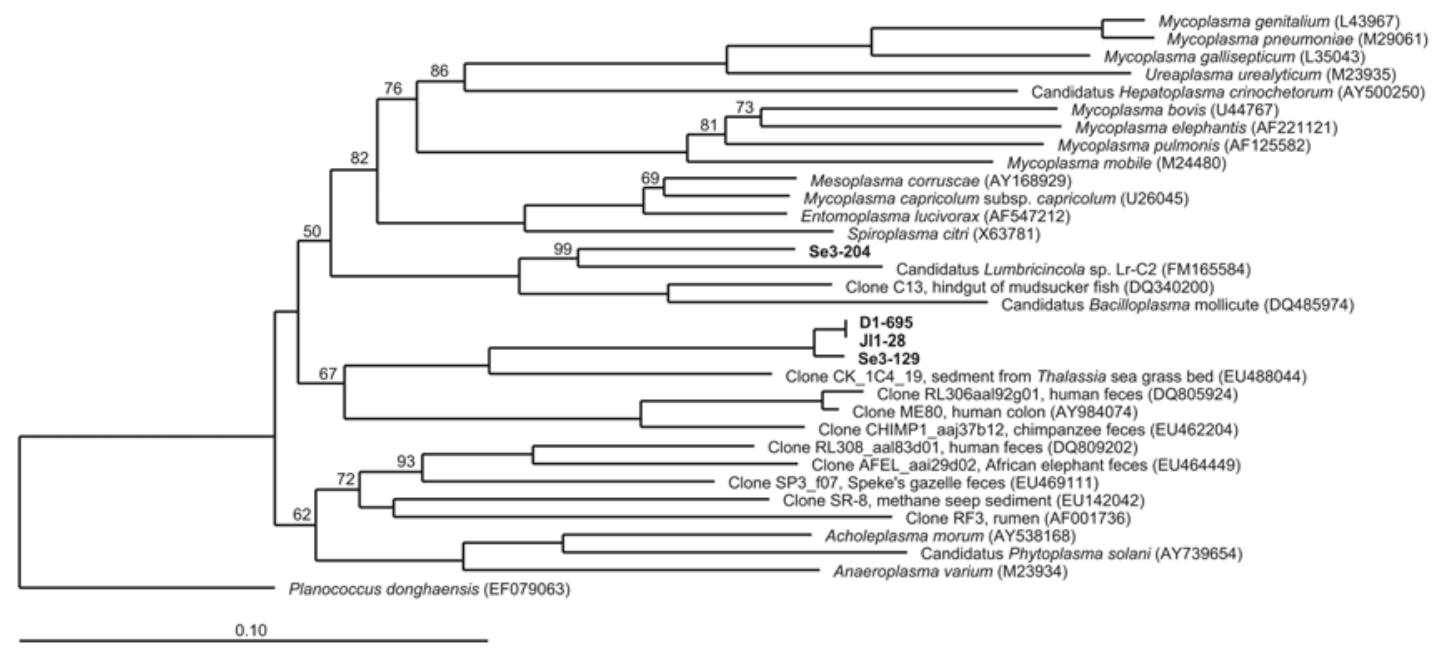

$\Sigma \chi \eta \dot{\mu \alpha}$ 3.7: Neighbor-Joining $\varphi$

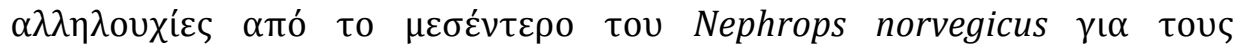

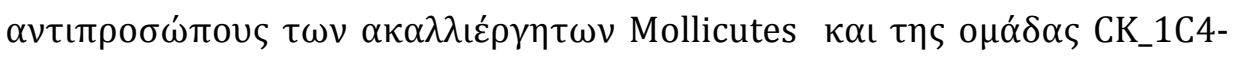

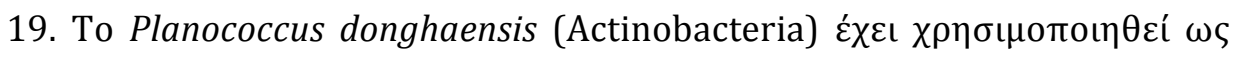

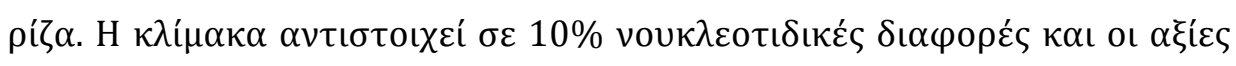

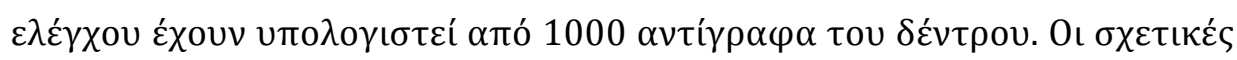

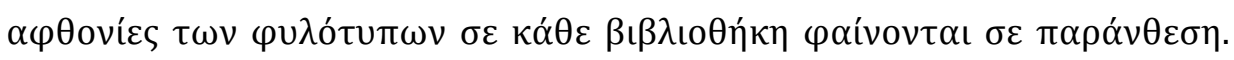

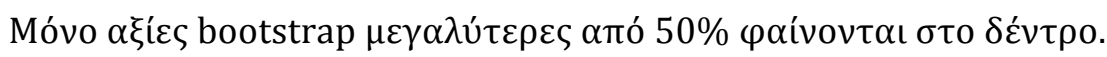




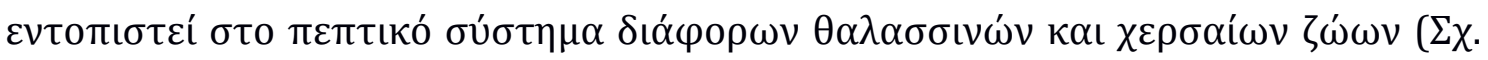

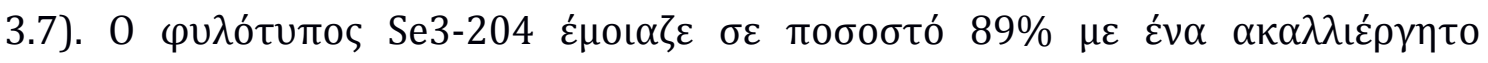

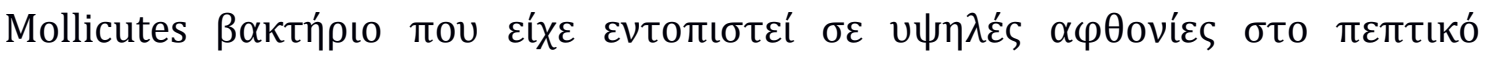

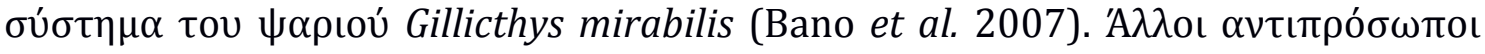

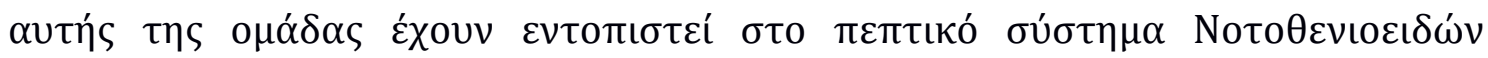

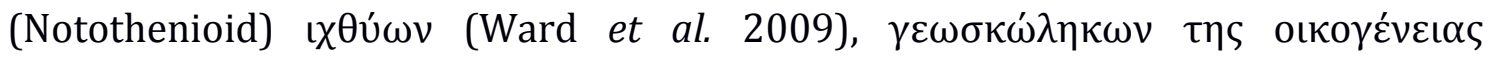

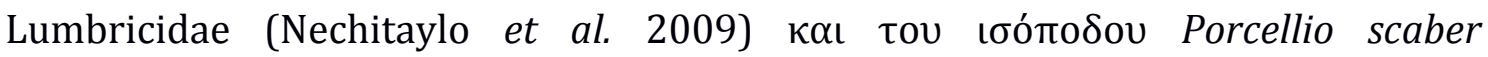
(Kostanjsek et al. 2007).

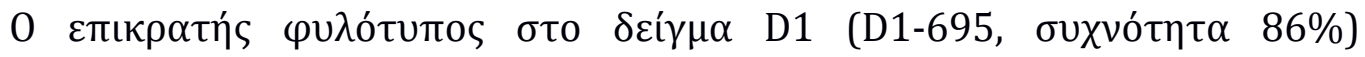

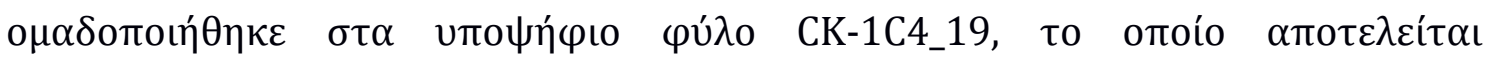

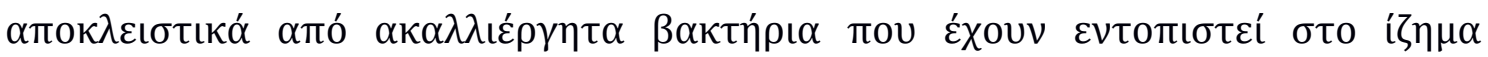

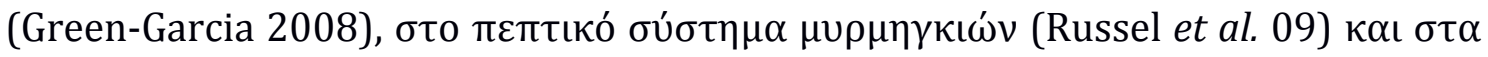

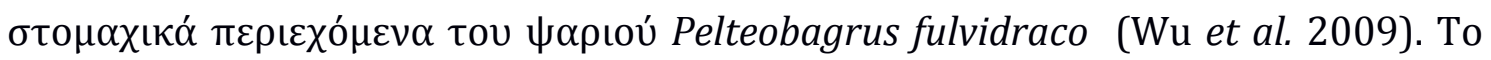

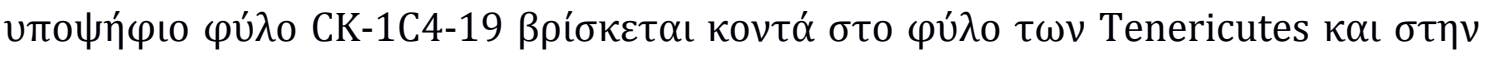

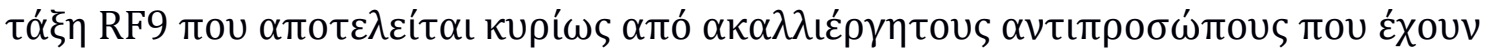

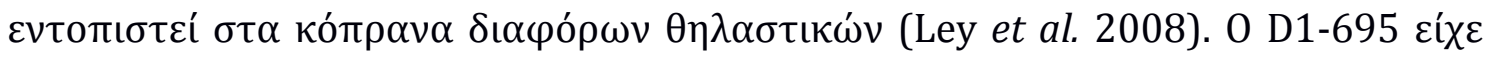

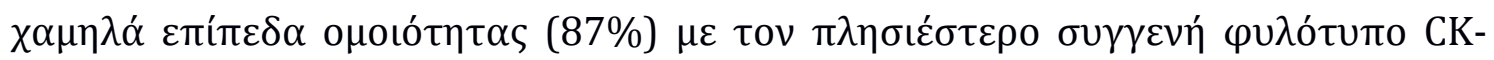

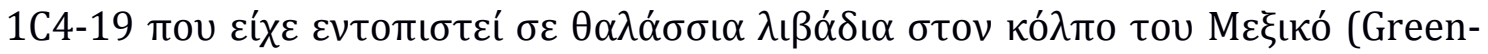

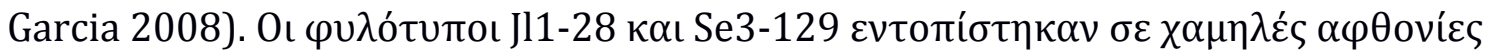

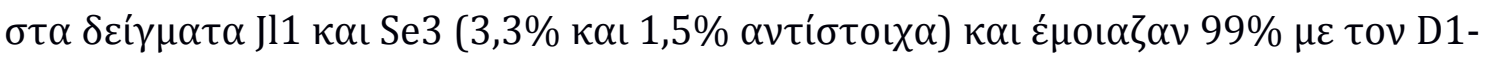

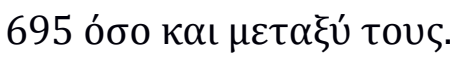

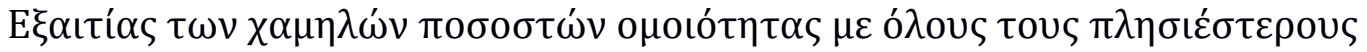

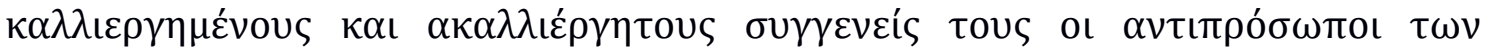




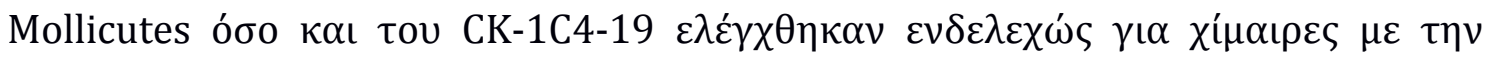

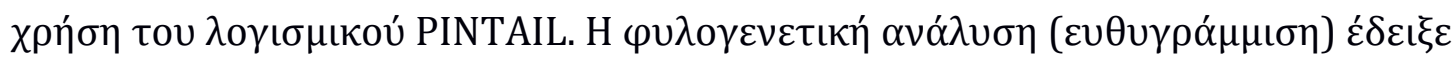

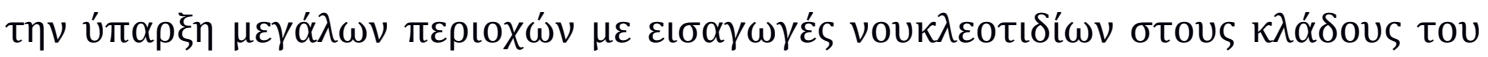

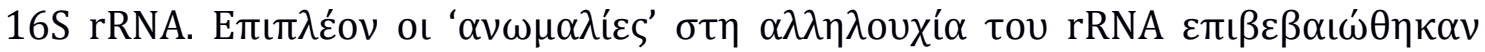

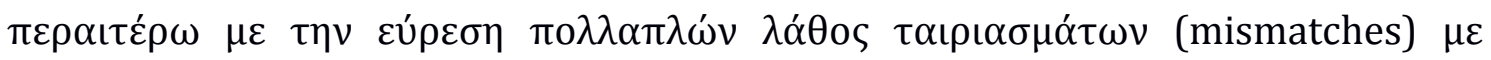

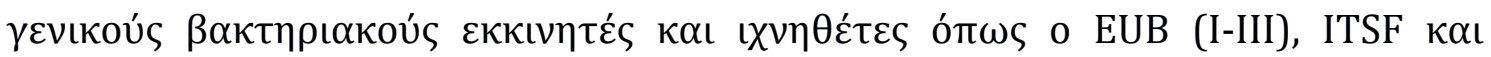
GM5_341f (Пív. 3.4.).

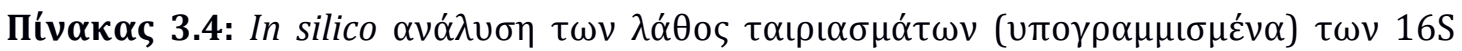

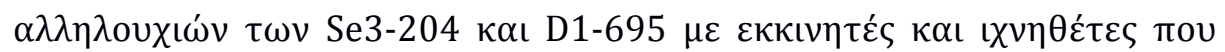

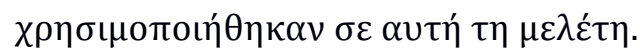

\begin{tabular}{|c|c|c|}
\hline 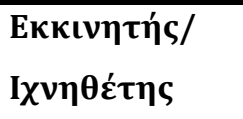 & 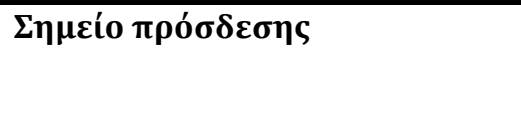 & $\begin{array}{l}\Lambda \alpha \dot{\alpha} \theta 0 \varsigma \\
\tau \alpha \iota \rho ı \alpha ́ \alpha \mu \alpha \tau \alpha\end{array}$ \\
\hline ITSFun & 5'-GTCGTAACAAGGTAGCCGTA-3' & \\
\hline Se3-204 & 5'-GTCGTAACAAGGTATCTCTA-3' & 3 \\
\hline D1-695 & 5'-GTCGTAACAAGGTATC $\underline{\text { ACTA-3' }}$ & 3 \\
\hline GM5_341f & 5'-CCTACGGGAGGCAGCAG-3' & \\
\hline Se3-204 & 5'-TCTACGGAAGGCTGCAG-3' & 3 \\
\hline EUB338 (I-III) & 5'-ACWCCTACGGGWGGCWGC-3' & \\
\hline Se3-204 & 5'-ACTICTACGGAAAGGCTGC-3' & 2 \\
\hline
\end{tabular}

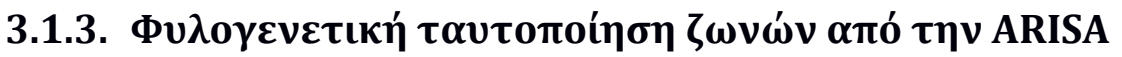

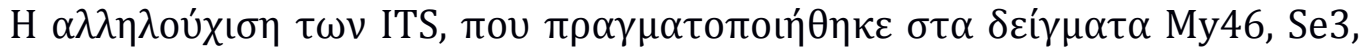

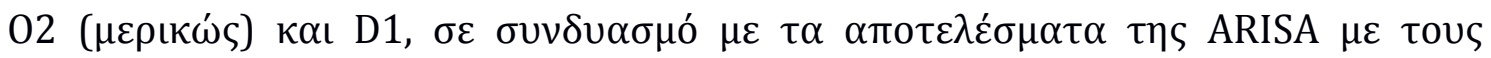

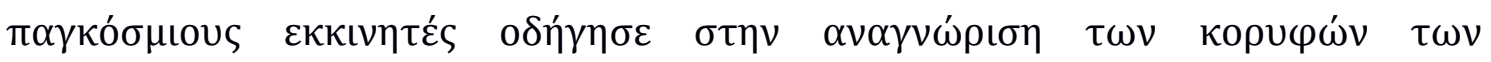




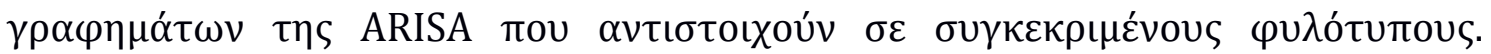

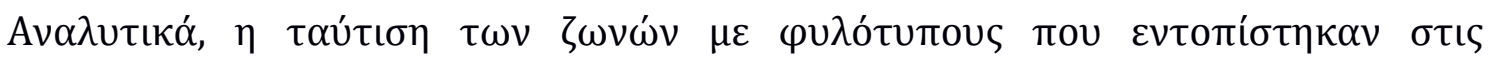

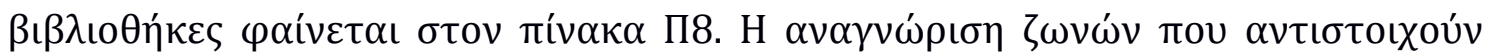

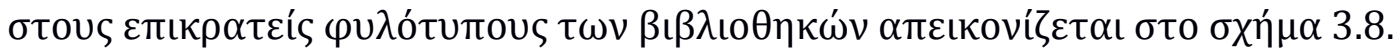

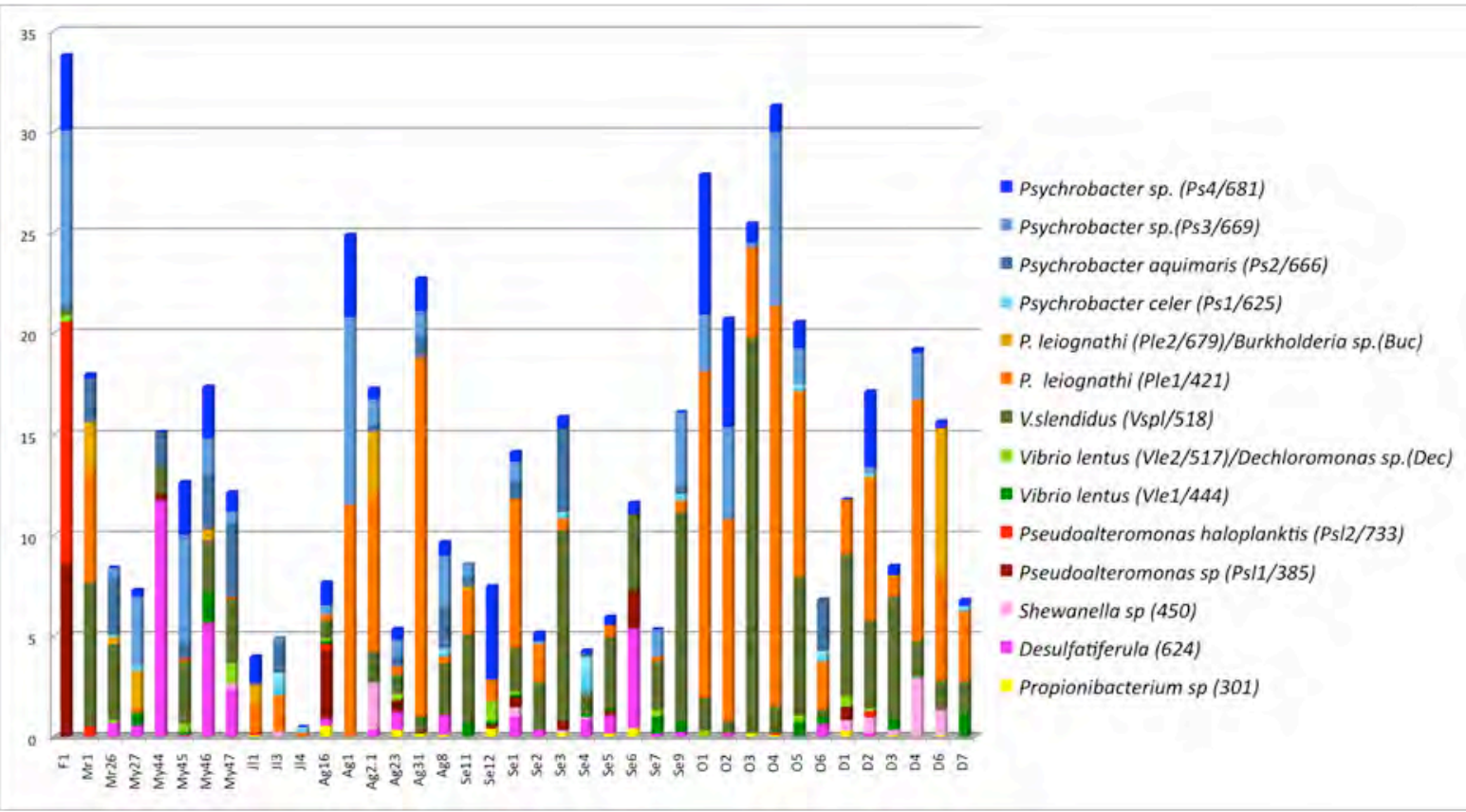

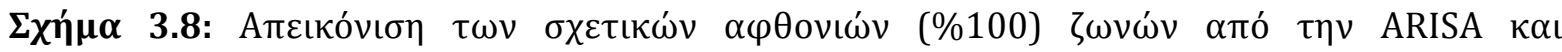

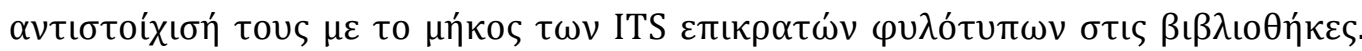

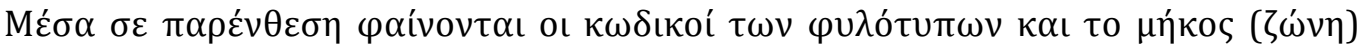
tou ITS.

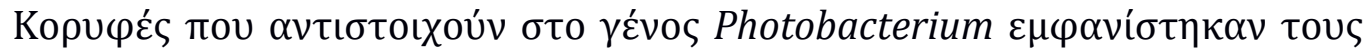

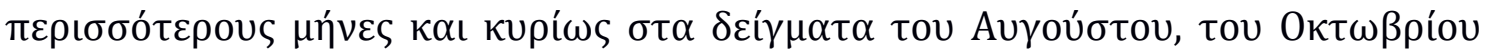

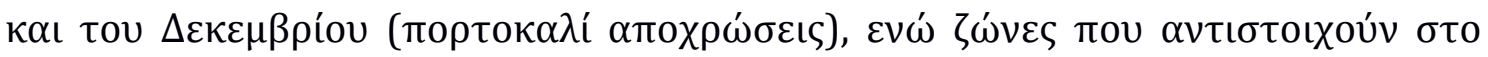

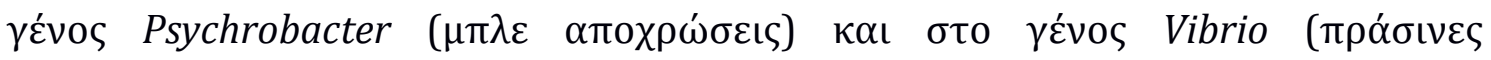

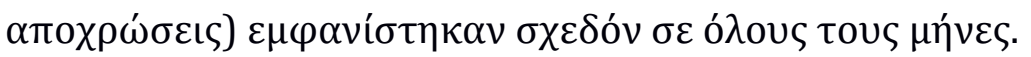




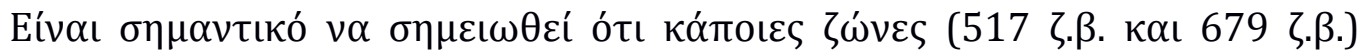

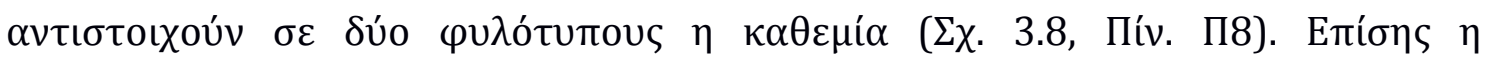

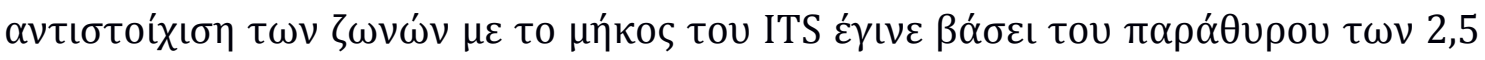

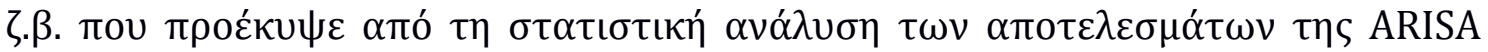

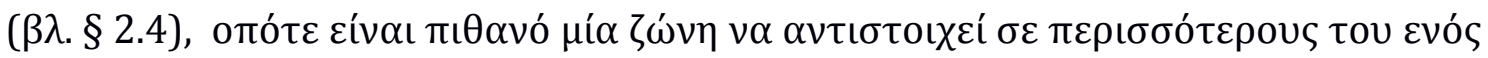

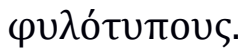

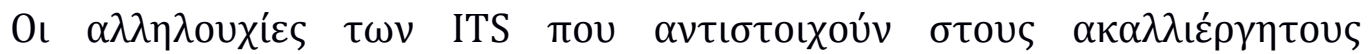

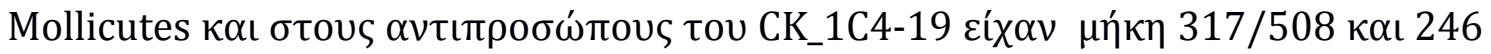

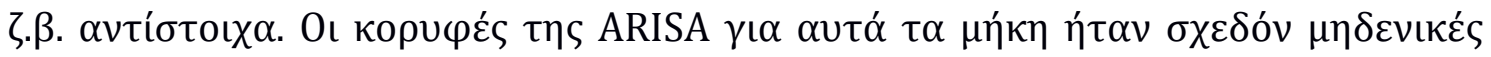

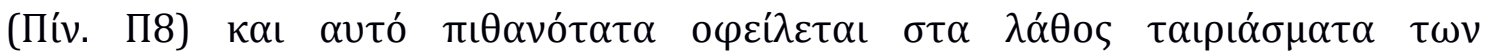

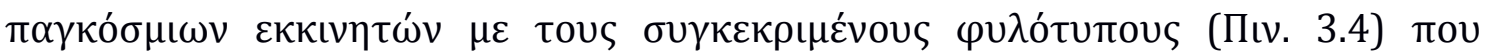

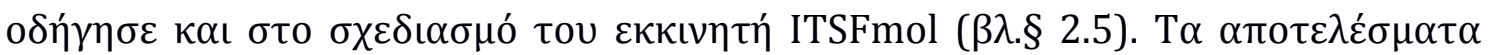

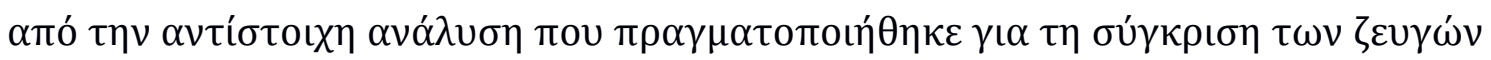

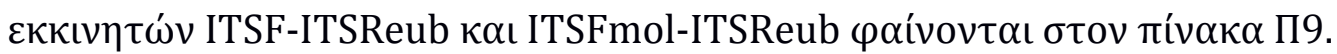

H $\alpha \pi$

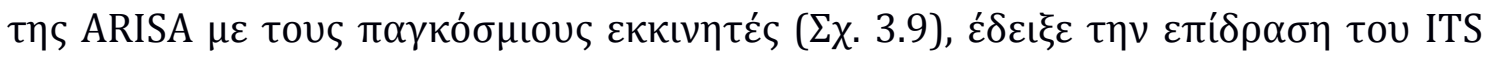

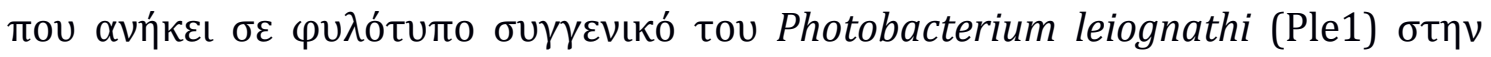

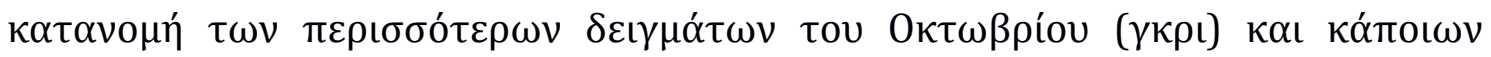

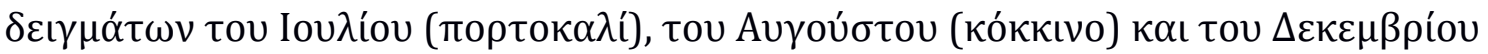

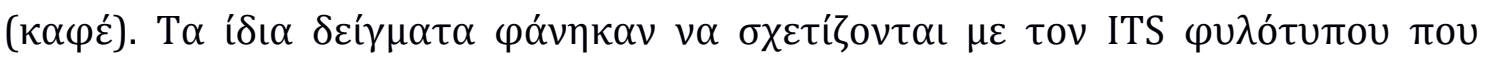

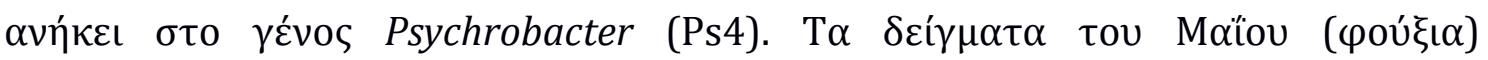

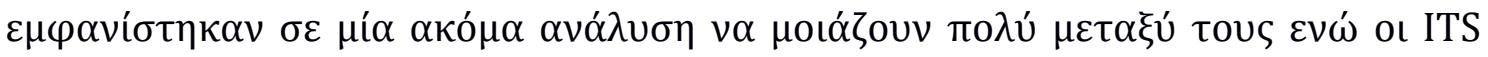

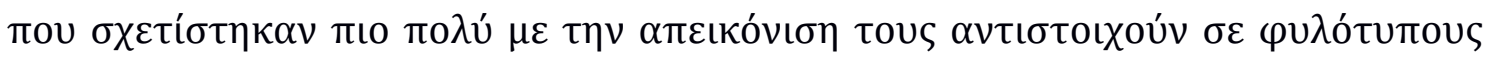

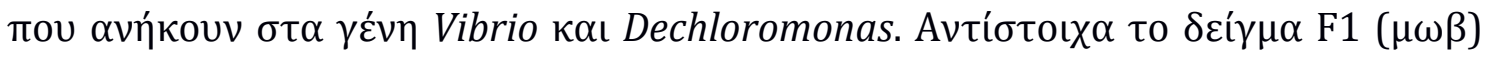




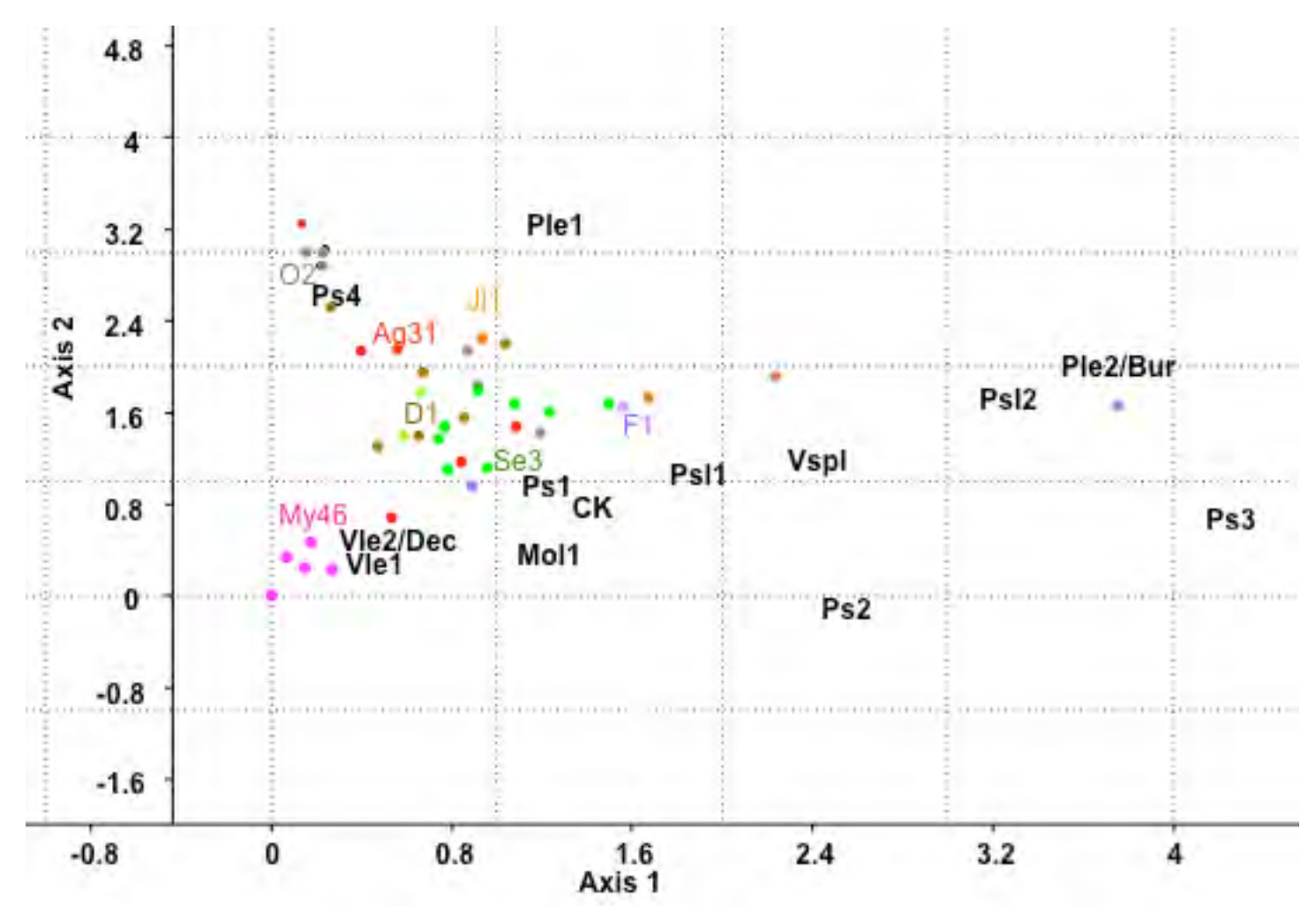

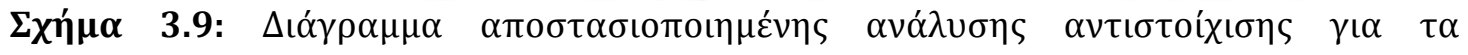

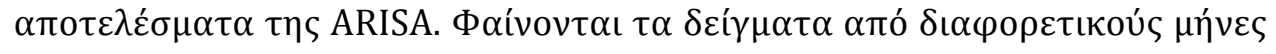

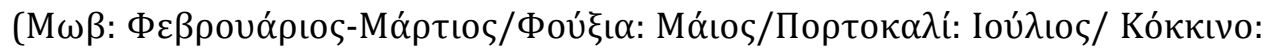

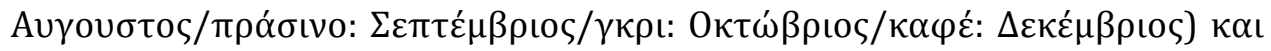

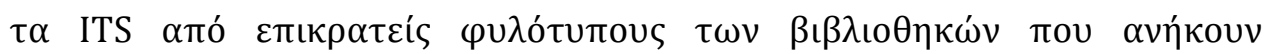
 Psychrobacter sp., Vle: Vibrio lentus, Vspl: Vibrio splendidus, Bur: Burkholderia sp., Mol1: Se3-204, CK: CK_1C4-19, Dec: Dechloromonas sp..

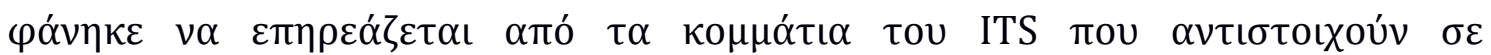

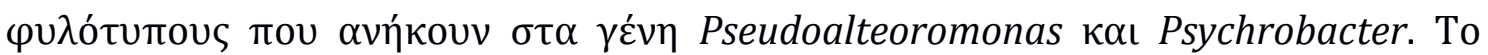

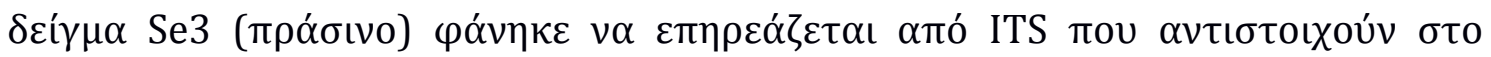

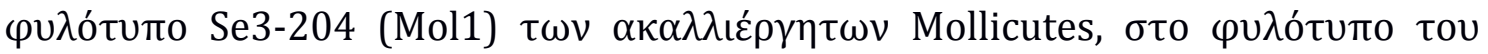

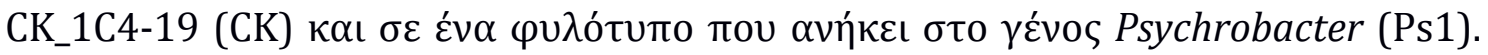

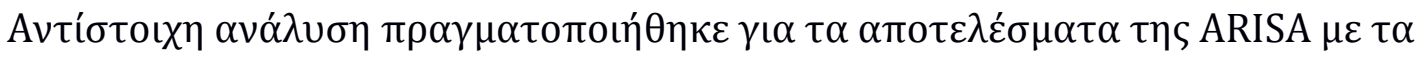




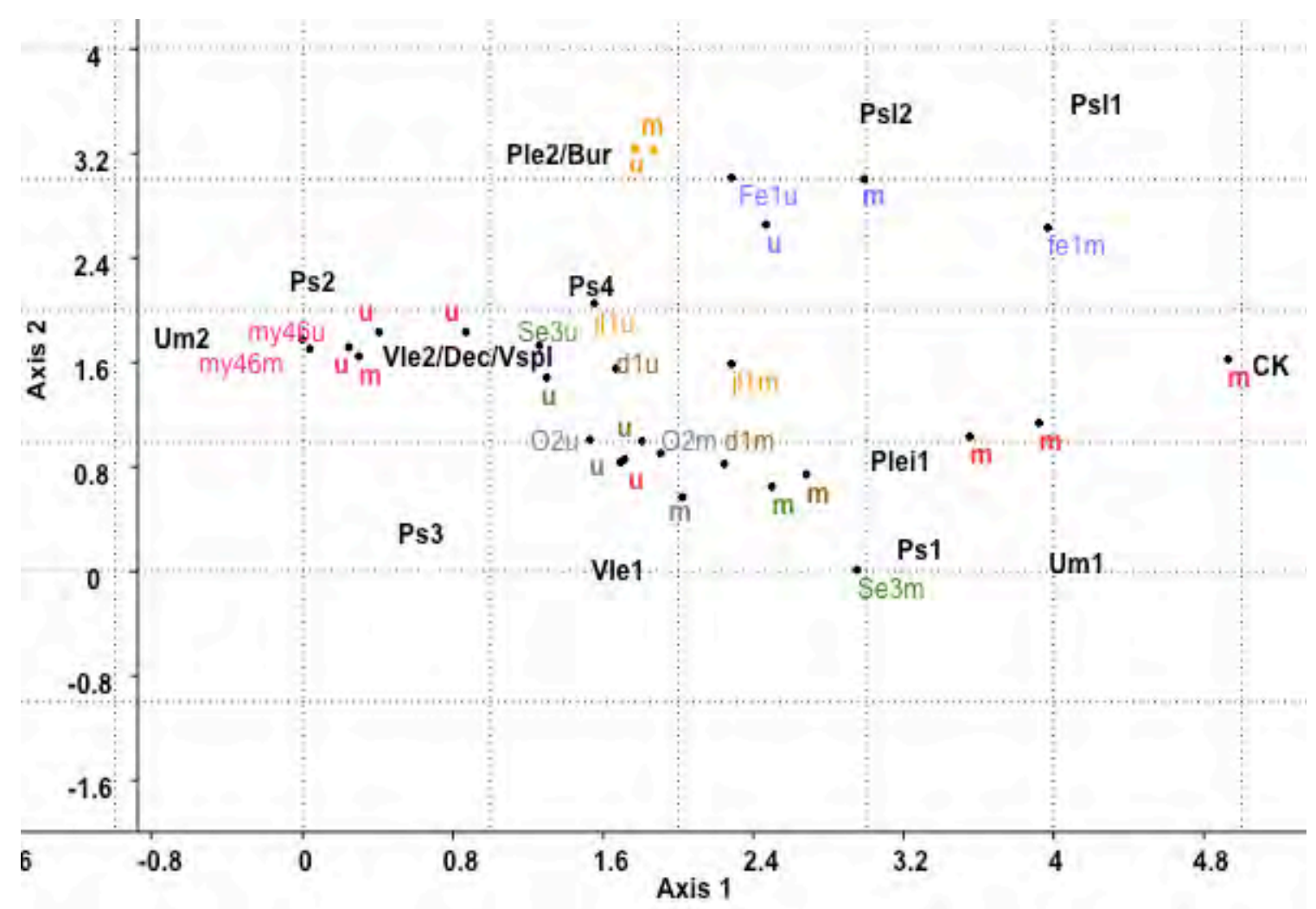

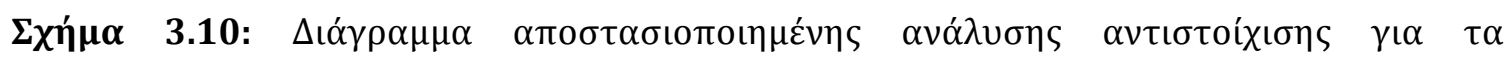

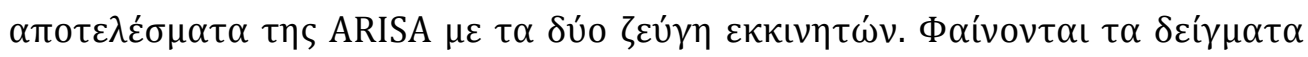

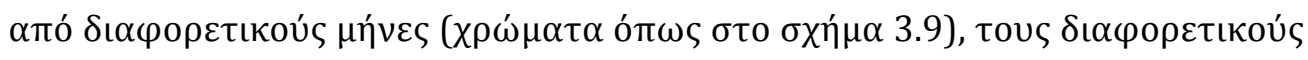

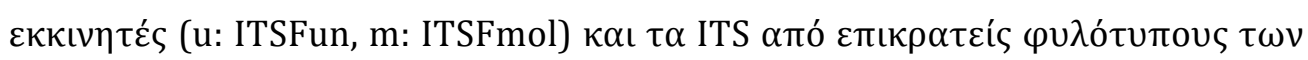

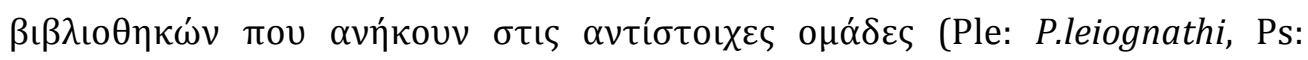
Psychrobacter sp., Vle: Vibrio lentus, Vspl: Vibrio splendidus, Bur: Burkholderia sp., Um: Se3-204, CK: CK_1C4-19, Dec: Dechloromonas sp..

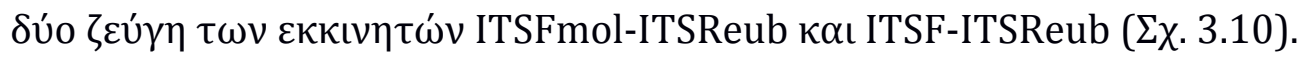

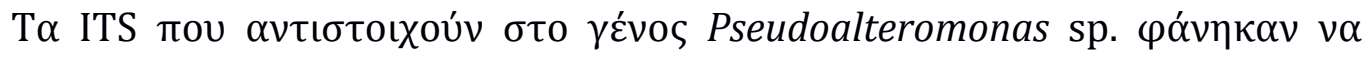

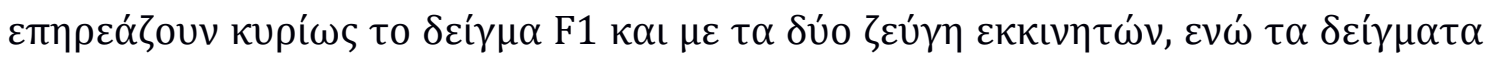

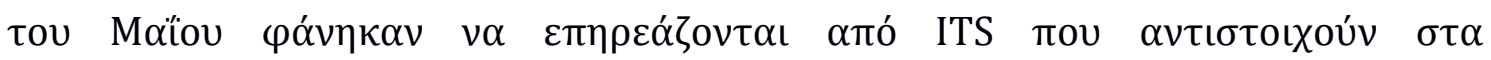

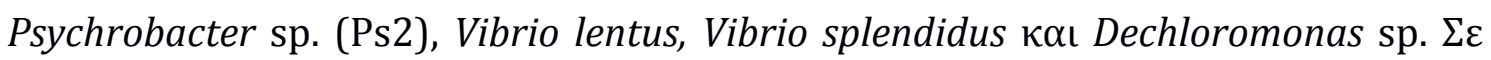

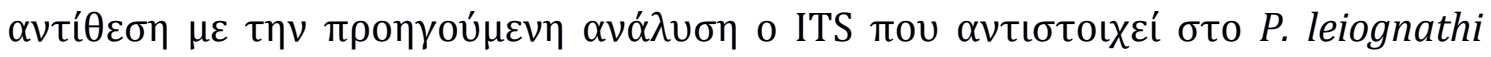

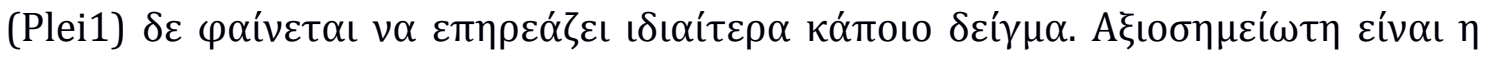




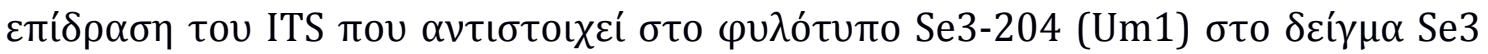

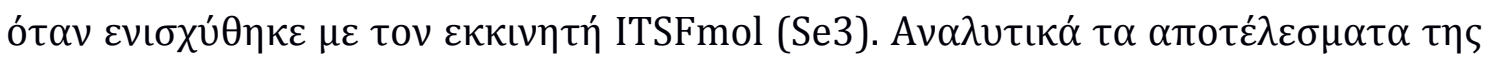

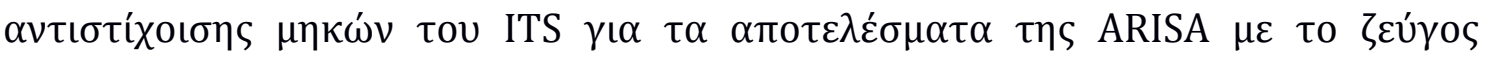

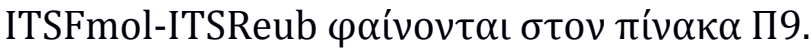

\subsubsection{FISH}

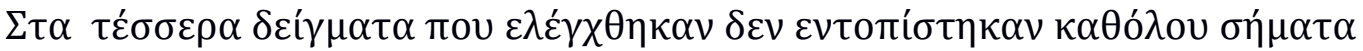

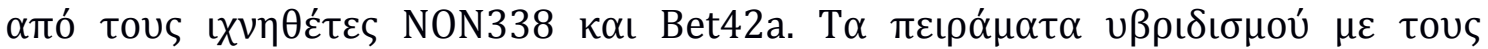

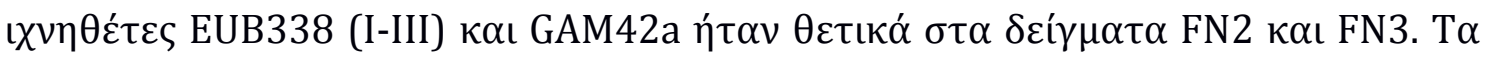

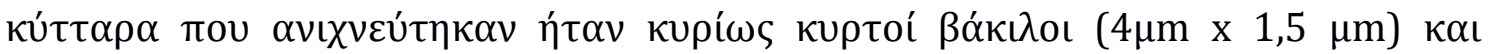

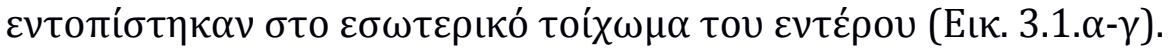

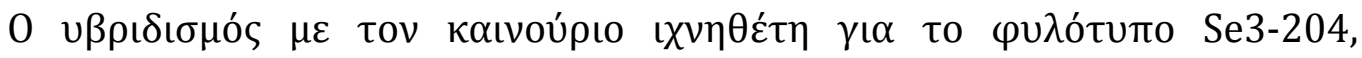

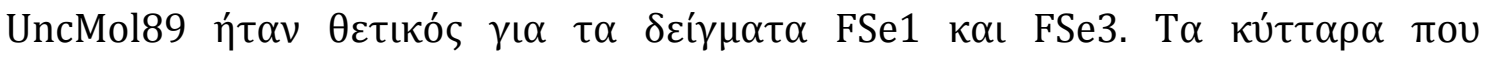

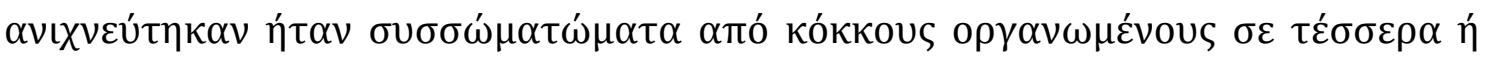

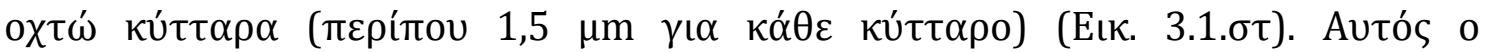


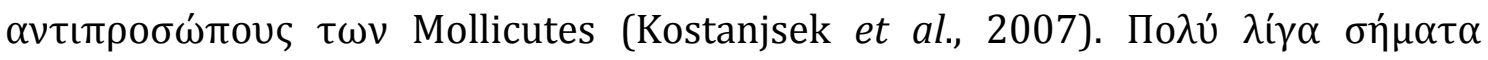

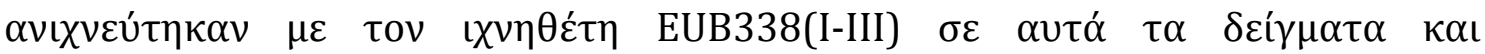

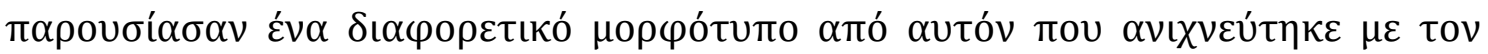

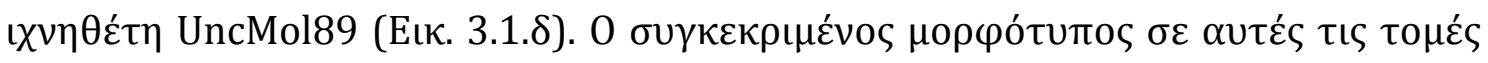

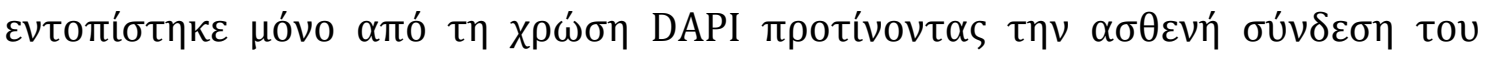

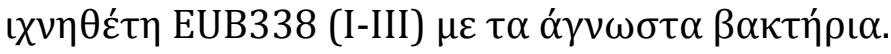




\section{$20 \mu \mathrm{m}$}

$\alpha$
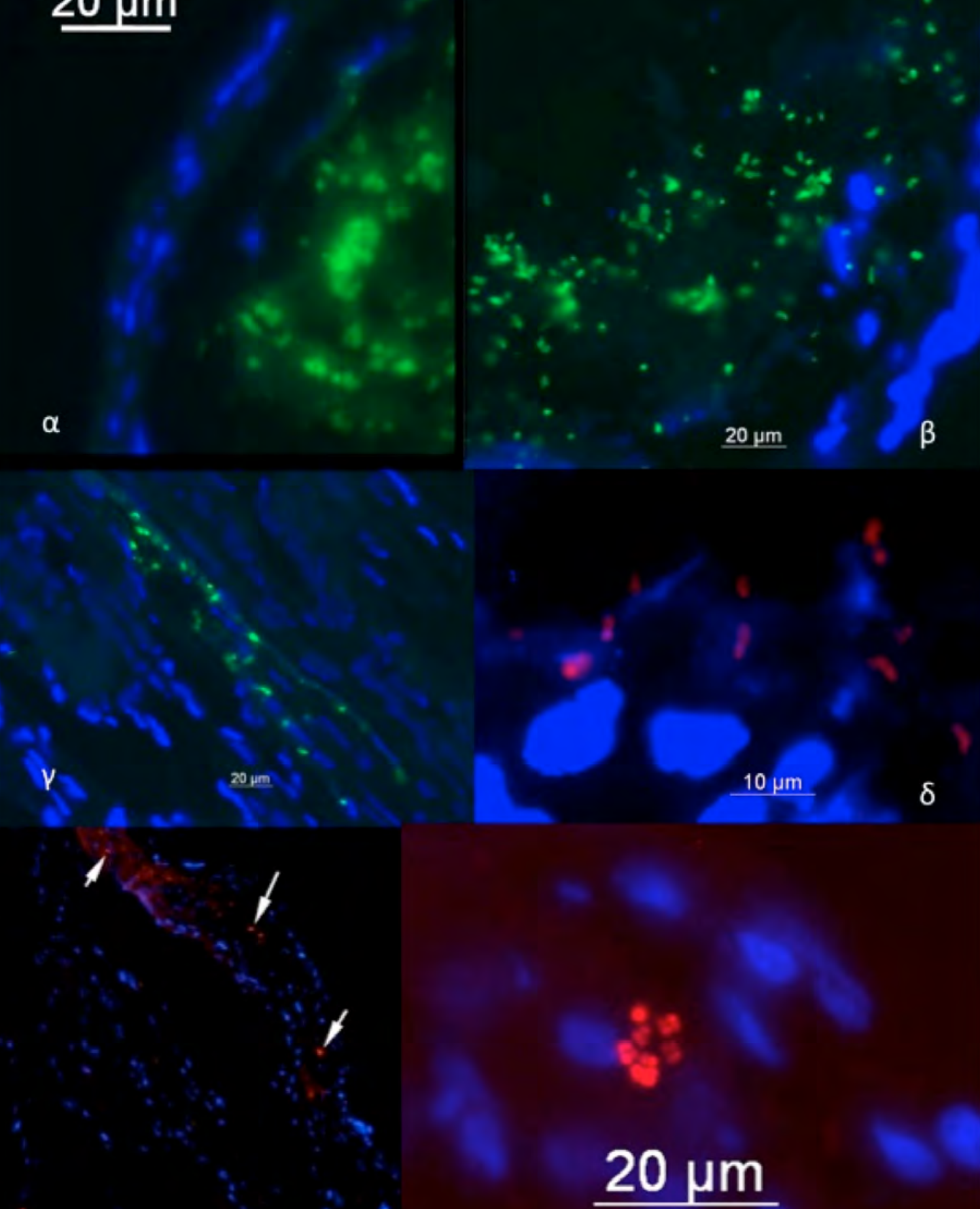

$\varepsilon$
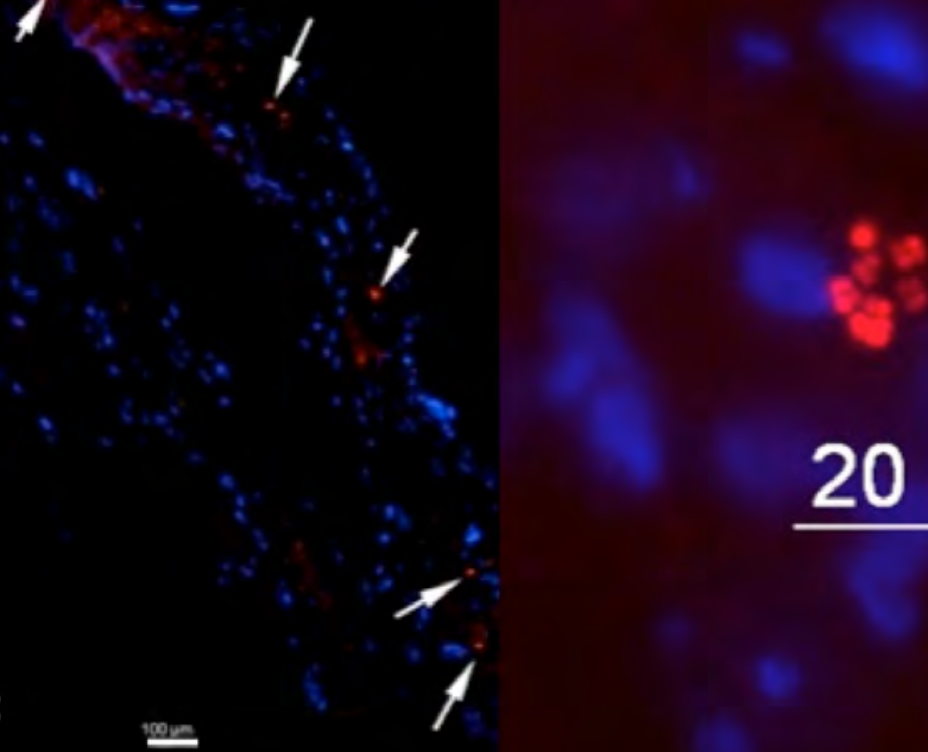

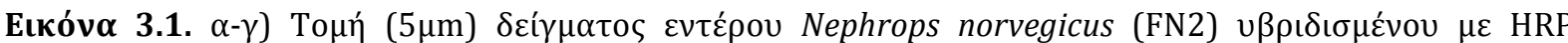

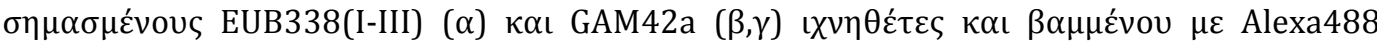

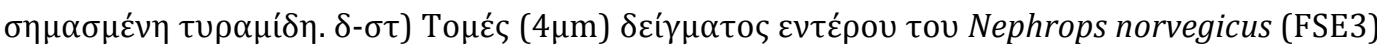

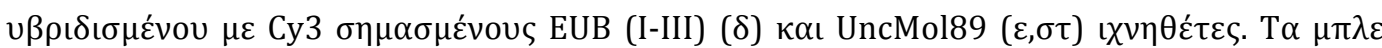

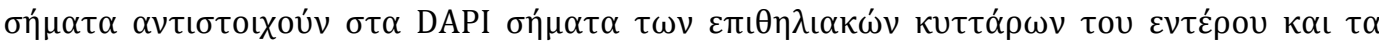

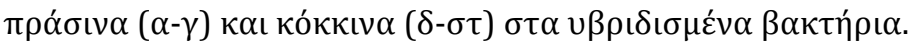




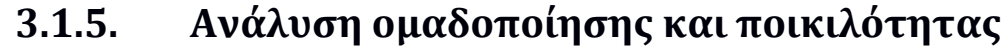

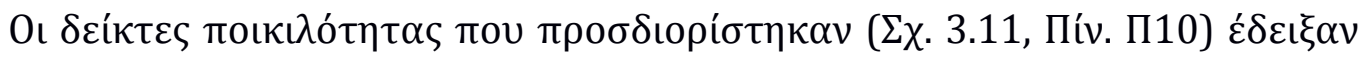

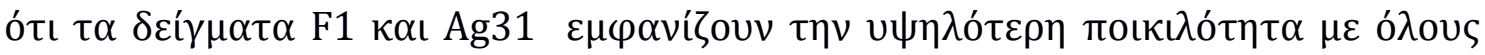

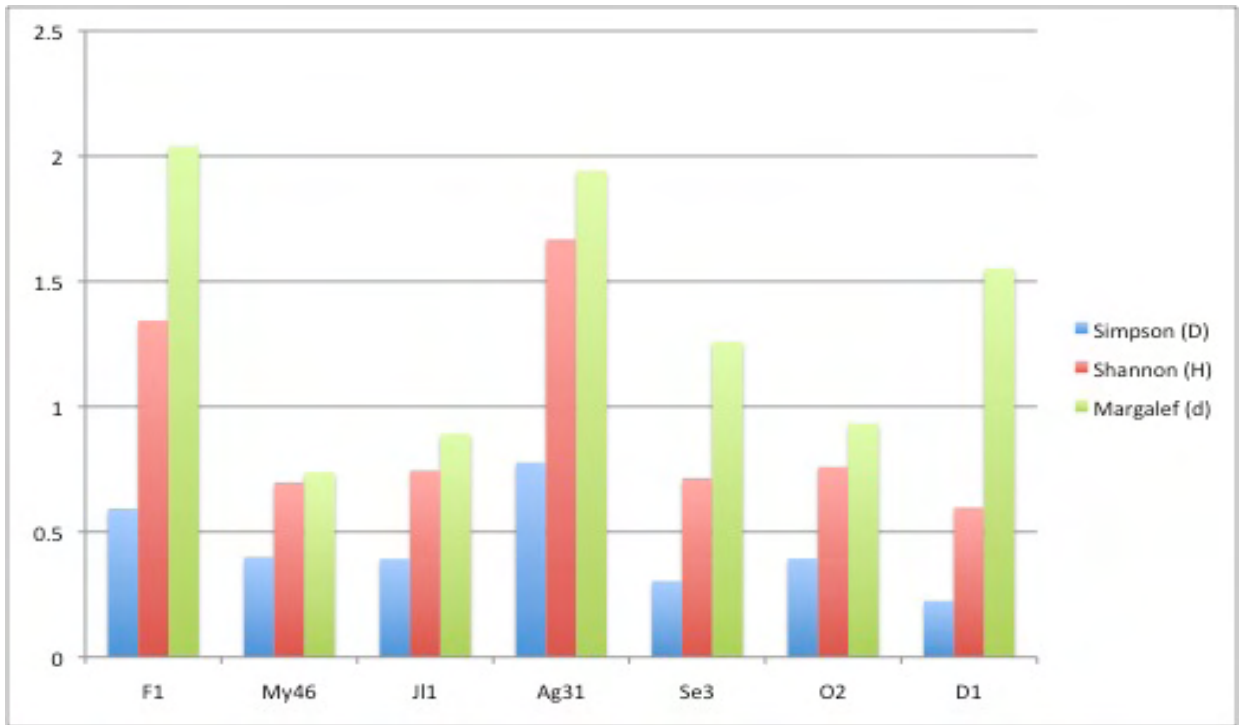

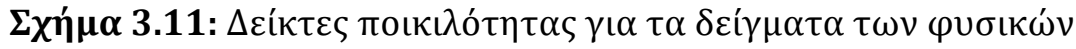

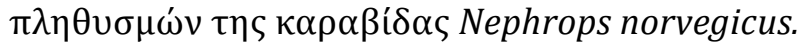

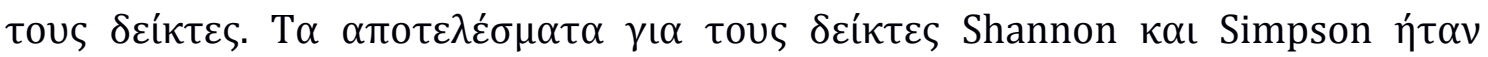

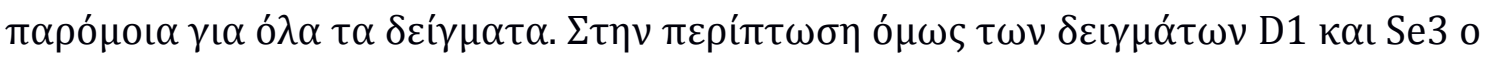

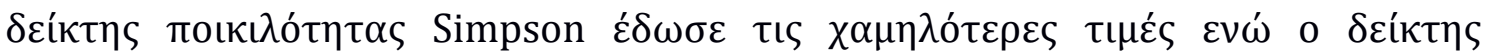

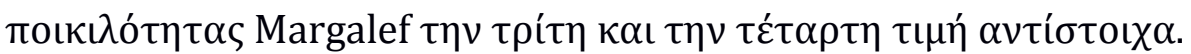

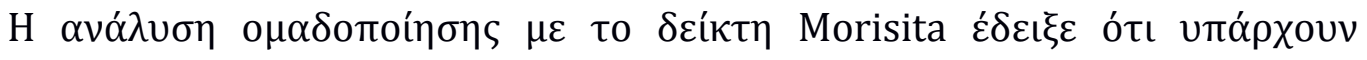

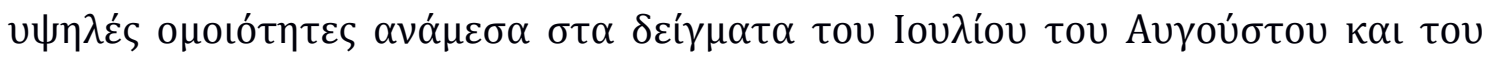

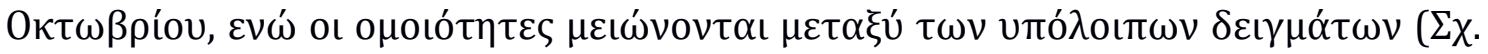

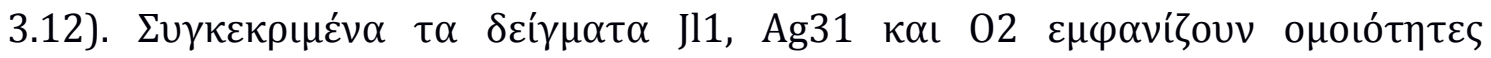




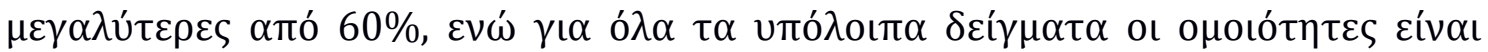

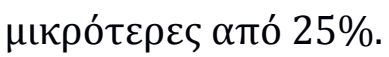

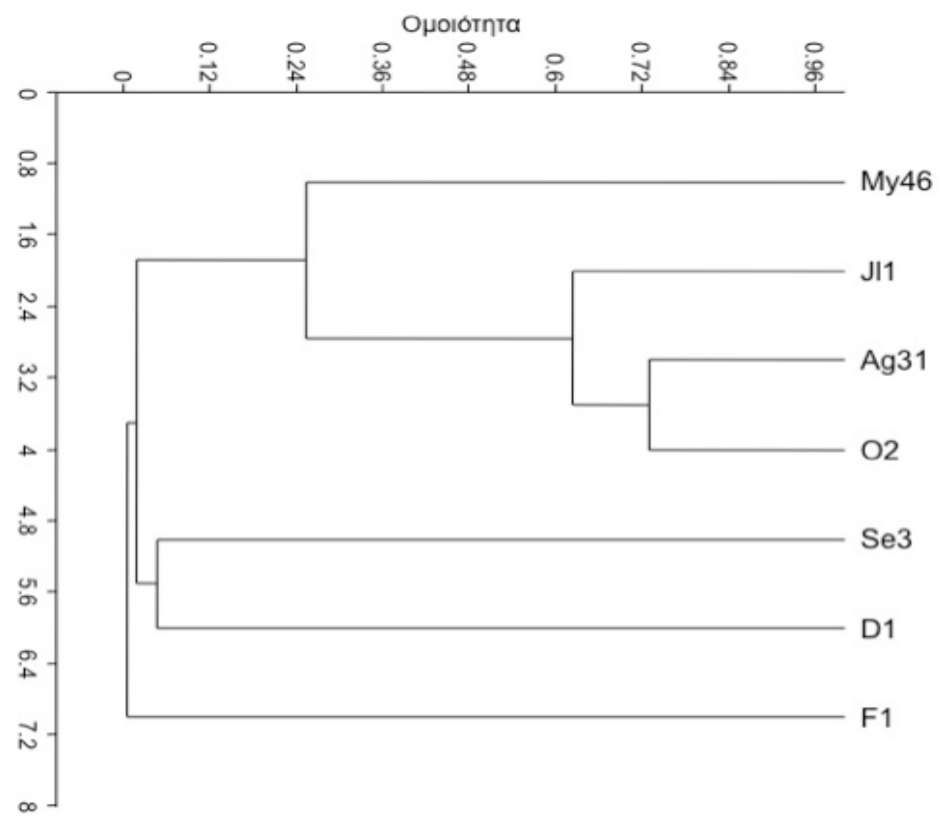

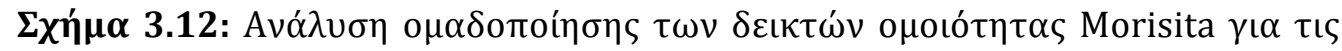

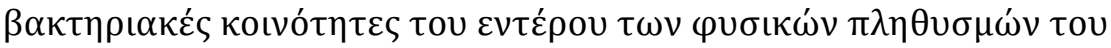
Nephrops norvegicus.

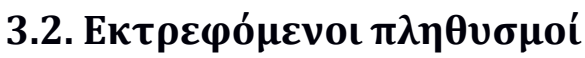

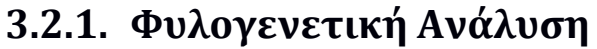

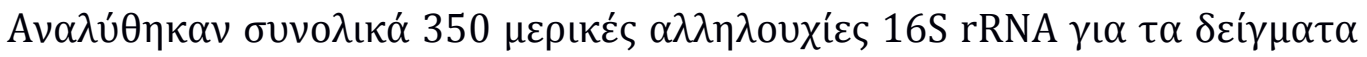
Nat1 (30), Nat2 (25), S6m1 (39), S6m2 (43), M6m1(39), M6m2 (38), P6m1(24),

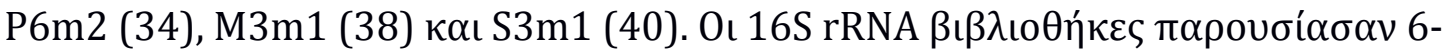




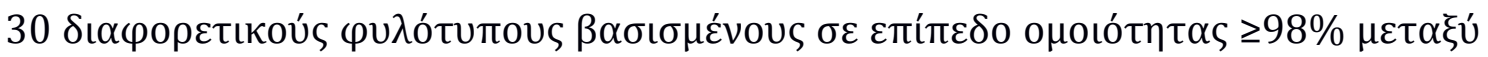

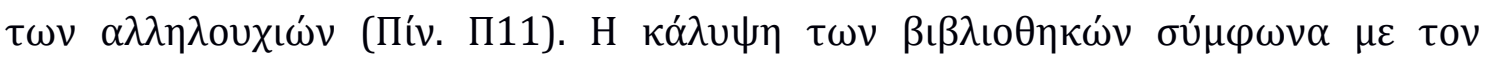

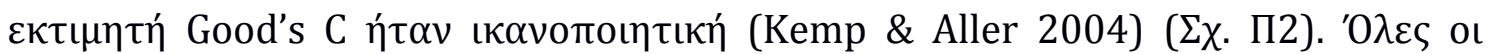

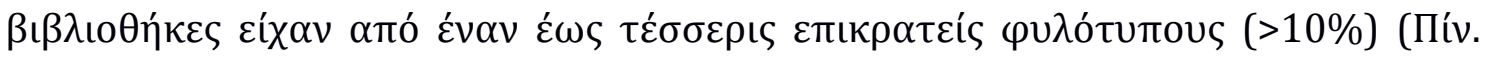

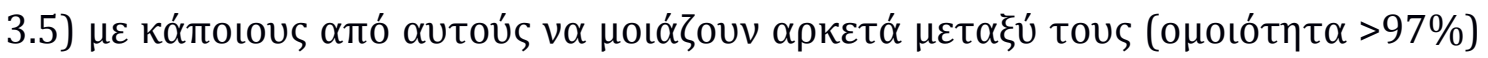
$(\Sigma \chi \cdot 3.13,3.14)$

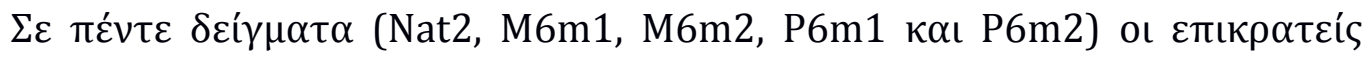

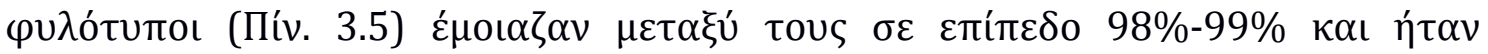

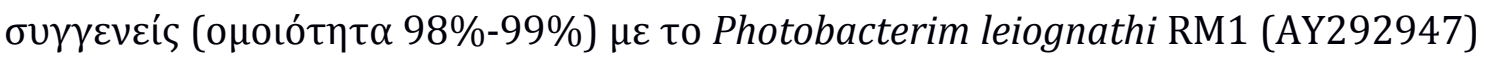

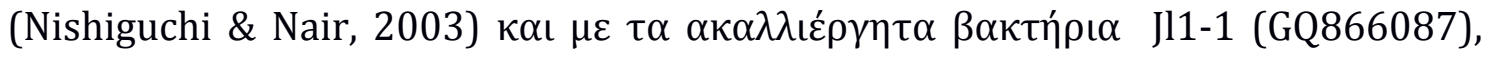

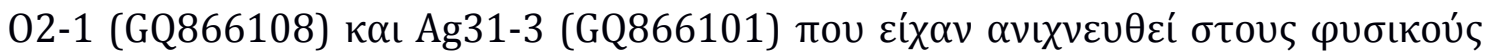
$\pi \lambda \eta \theta v \sigma \mu o u ́ \varsigma(\beta \lambda . \S 3.1 .2)(\Sigma \chi .3 .15)$.

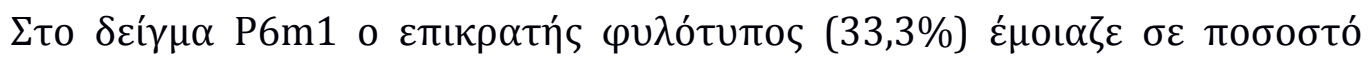

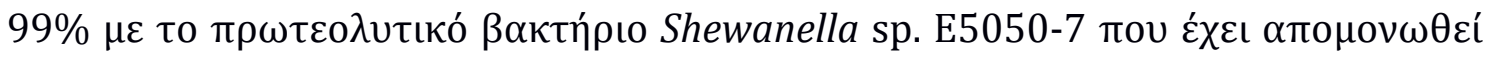

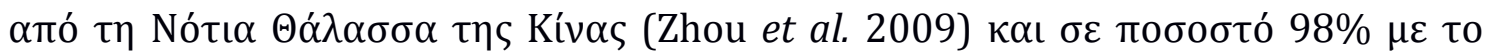

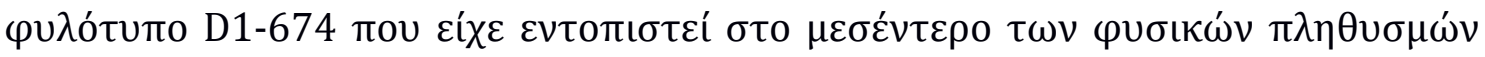

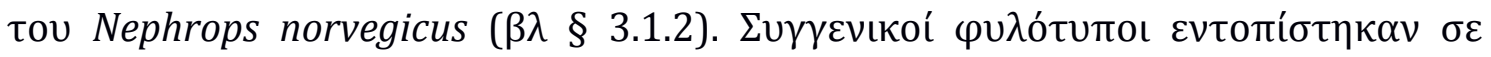

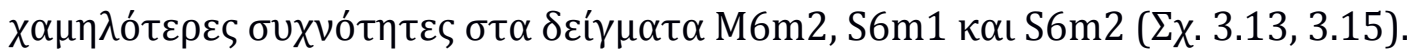

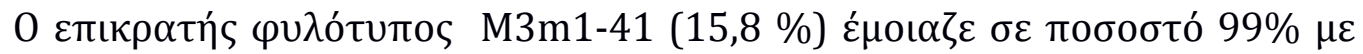

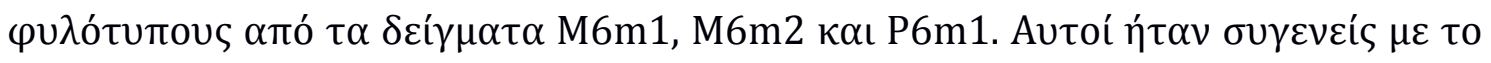

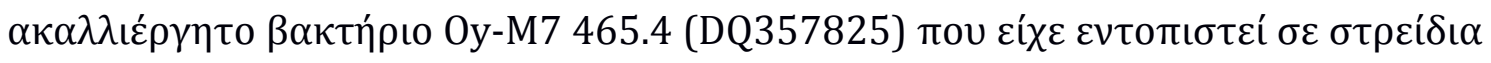

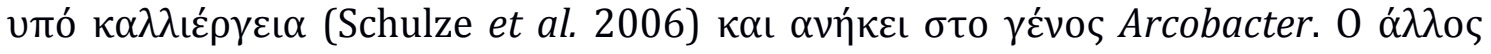

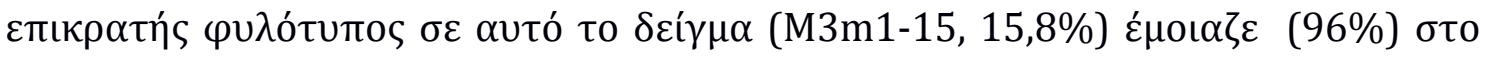

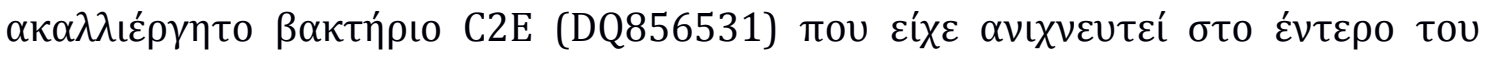




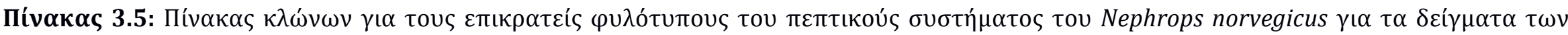

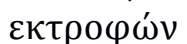

\begin{tabular}{|c|c|c|c|c|}
\hline 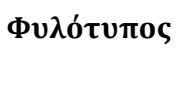 & $\begin{array}{l}\Sigma v \chi v o ́ \tau \eta \tau \alpha \\
(\%)\end{array}$ & 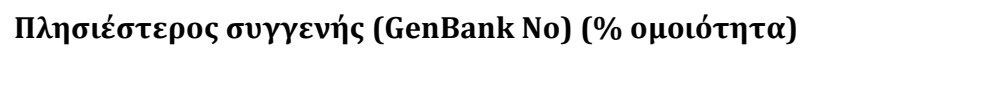 & 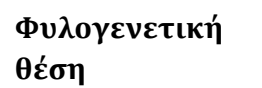 & 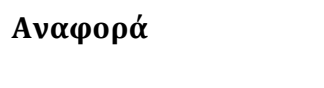 \\
\hline Nat1-12 & 66,6 & 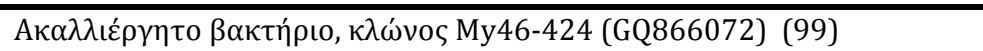 & $\gamma$-Proteobacteria & Meziti et al. 2010 \\
\hline Nat2-8 & 48,0 & 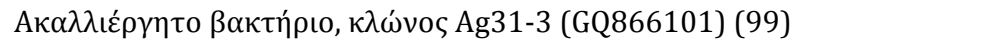 & $\gamma$-Proteobacteria & Meziti et al. 2010 \\
\hline S3m1-3 & 15,0 & 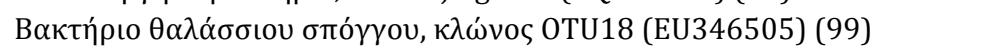 & $\alpha$-Proteobacteria & Sipkema et al. 2011 \\
\hline S3m1-6 & 15,0 & Marinicella litoralis (AB500095) (96) & $\gamma$-Proteobacteria & Romanenko et al. 2010 \\
\hline S3m1-7 & 15,0 & Litoreibacter albidus (AB518881) (99) & $\alpha$-Proteobacteria & Romanenko et al. 2011 \\
\hline S3m1-19 & 12,5 & 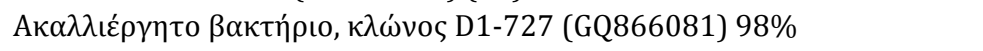 & Actinobacteria & Meziti et al. 2010 \\
\hline S6m1-3 & 12,8 & Marivita cryptomonadis CL-SK44 (EU515919), 99\% & $\alpha$-Proteobacteria & Hwang et al. 2009 \\
\hline S6m1-10 & 12,8 & 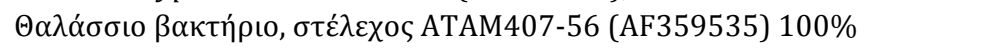 & $\alpha$-Proteobacteria & Hold et al. 2001 \\
\hline S6m1-8 & 10,2 & 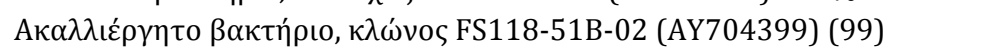 & $\varepsilon$-Proteobacteria & Huber et al. 2006 \\
\hline S6m1-36 & 10,2 & Roseobacter sp. 38.98 (AY870684) (98\%) & $\alpha$-Proteobacteria & Farto et al. 2006 \\
\hline M3m1-15 & 15,8 & 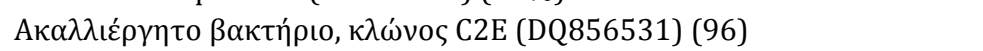 & $\alpha$-Proteobacteria & Li et al. 2007 \\
\hline M3m1-41 & 15,8 & $\varepsilon$ - proteobacterium Oy-M7 clone 465.4 (DQ357825) (96) & $\varepsilon$-Proteobacteria & Schulze et al. 2006 \\
\hline M3m1-25 & 10,5 & 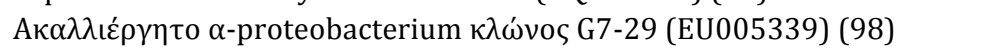 & $\alpha$-Proteobacteria & Unpublished \\
\hline M6m1-4 & 20,5 & 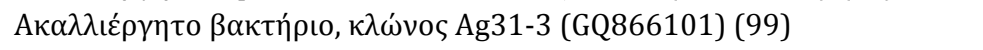 & $\gamma$-Proteobacteria & Meziti et al. 2010 \\
\hline M6m1-56 & 17,9 & 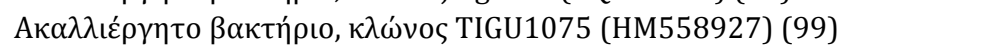 & $\gamma$-Proteobacteria & Suen et al. 2010 \\
\hline M6m1-2 & 12,8 & 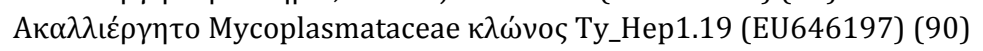 & Tenericutes & Fraune \& Zimmer 2008 \\
\hline M6m2-6 & 28,9 & 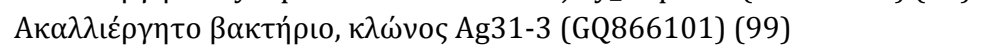 & $\gamma$-Proteobateria & Meziti et al. 2010 \\
\hline M6m2-3 & 15,8 & 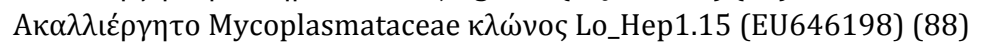 & Tenericutes & Fraune \& Zimmer 2008 \\
\hline M6m2-10 & 13,2 & 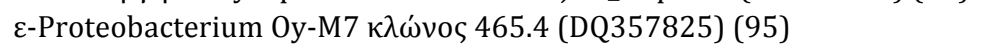 & $\varepsilon$ - Proteobacteria & Schulze et al. 2006 \\
\hline P6m1-12 & 33,3 & 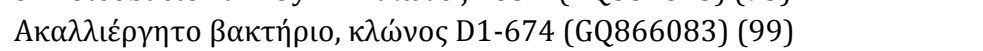 & $\gamma$-Proteobacteria & Meziti et al. 2010 \\
\hline P6m1-11 & 20,8 & 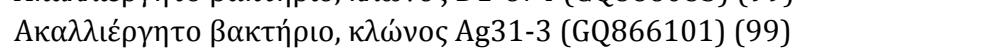 & $\gamma$-Proteobacteria & Meziti et al. 2010 \\
\hline P6m2-2 & 73,5 & 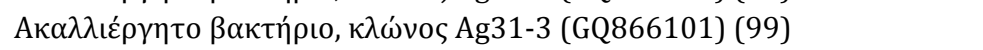 & $\gamma$-Proteobacteria & Meziti et al. 2010 \\
\hline P6m2-18 & 11,8 & Vibrio splendidus PB1-10rrnD (EU091328) (99) & $\gamma$-Proteobacteria & Jensen et al. 2009 \\
\hline
\end{tabular}



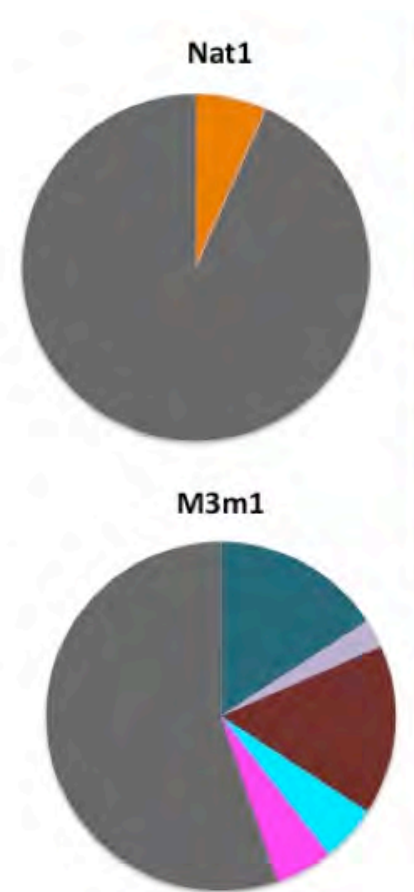

M6m1

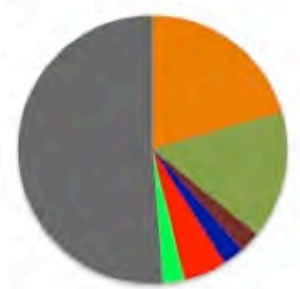

M6m2

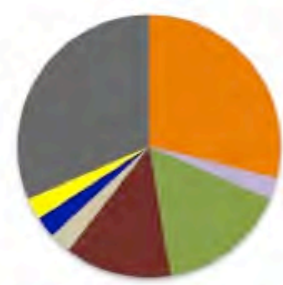

- Photobacterium sp.

- Colwellia sp.

- Arcobacter sp.

- Vibrio sp.

- Phaeobacter sp.

Litoreibacter sp.

- Pseudoalteromonas sp.

- Defluviicoccus sp.

- Candidatus Hepatoplasma

Propionibacterium sp.

Marivita sp.

- Uncultured Bacteroidetes

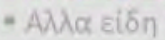

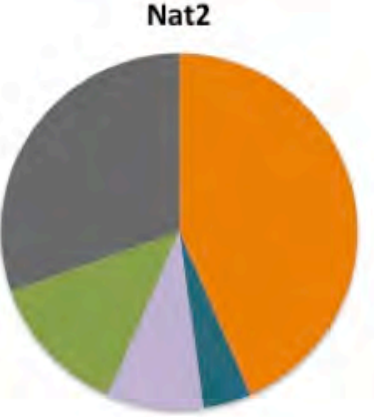

S3m1

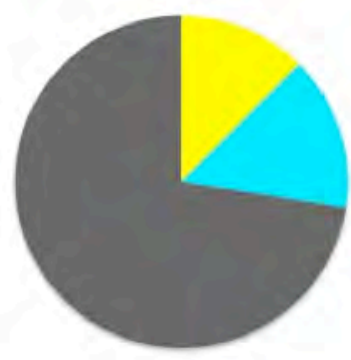

$\mathrm{S} 6 \mathrm{~m} 1$

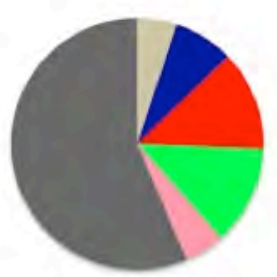

S6m2

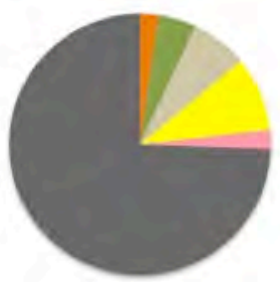

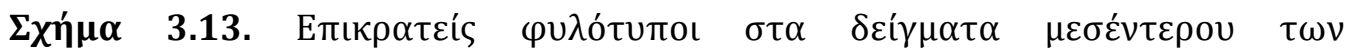

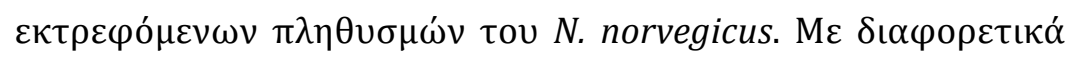

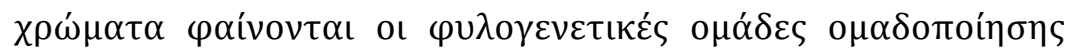

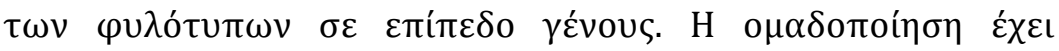

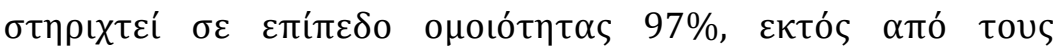

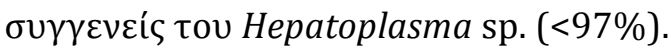


$\Sigma \chi \eta^{\prime} \mu \alpha \quad$ 3.14: Neighbor-Joining

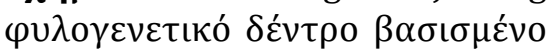

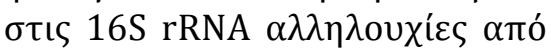

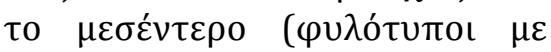

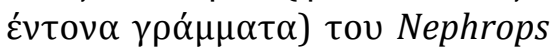
norvegicus. To Aquifex pyrophilus

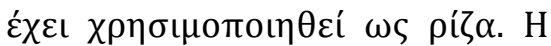

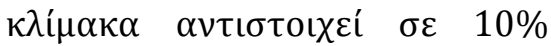

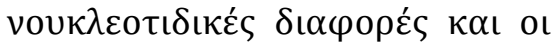

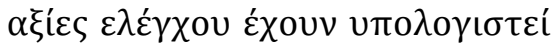

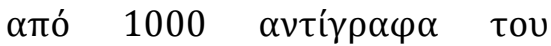

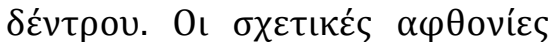

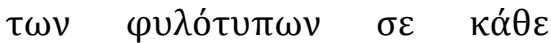

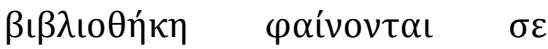
$\pi \alpha \rho \alpha ́ v \theta \varepsilon \sigma \eta$. Móvo $\alpha \xi \dot{\varepsilon} \varepsilon \varsigma$

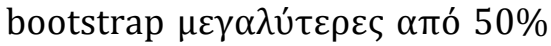

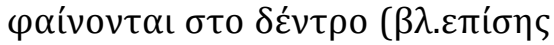

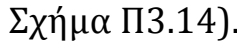

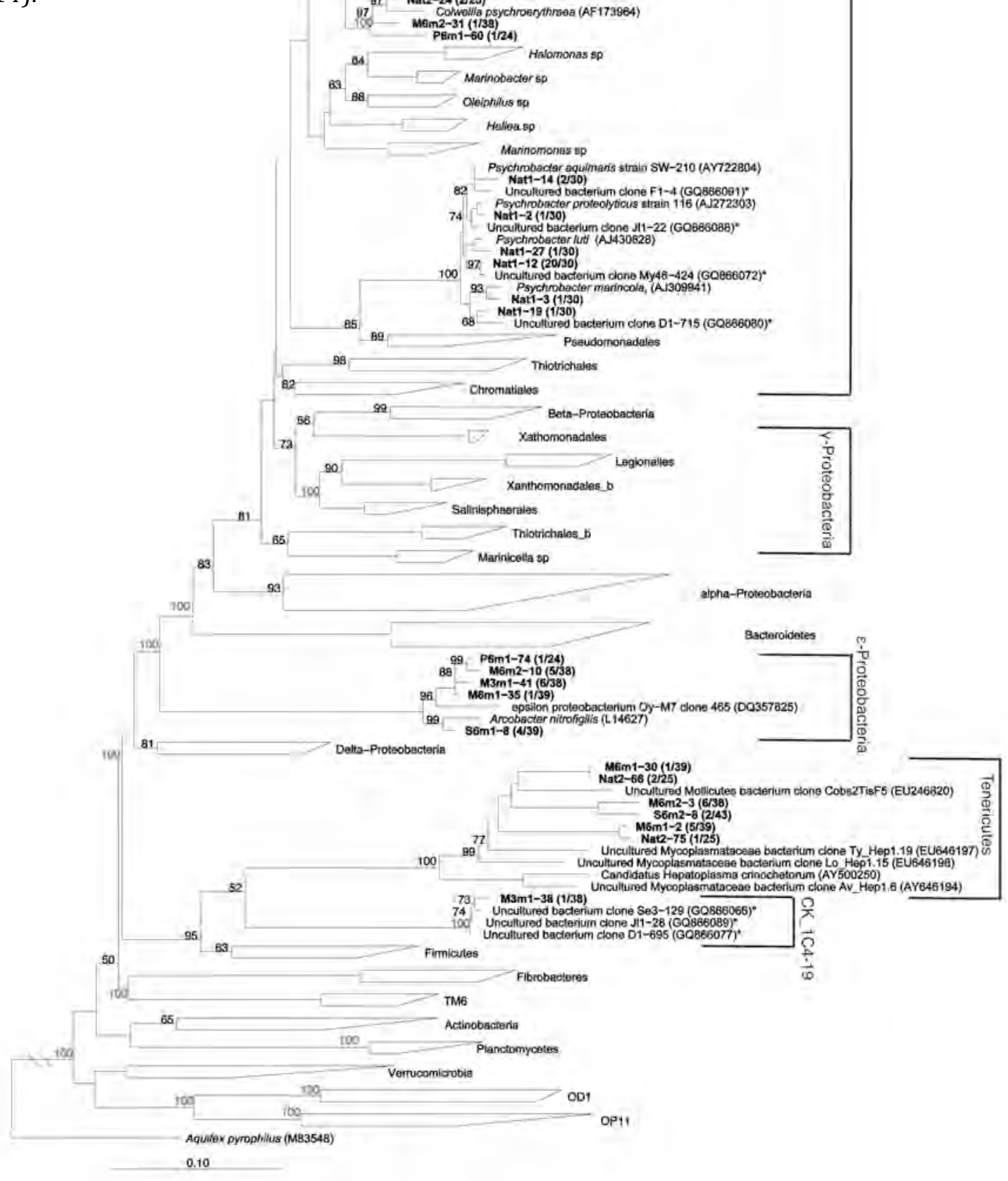




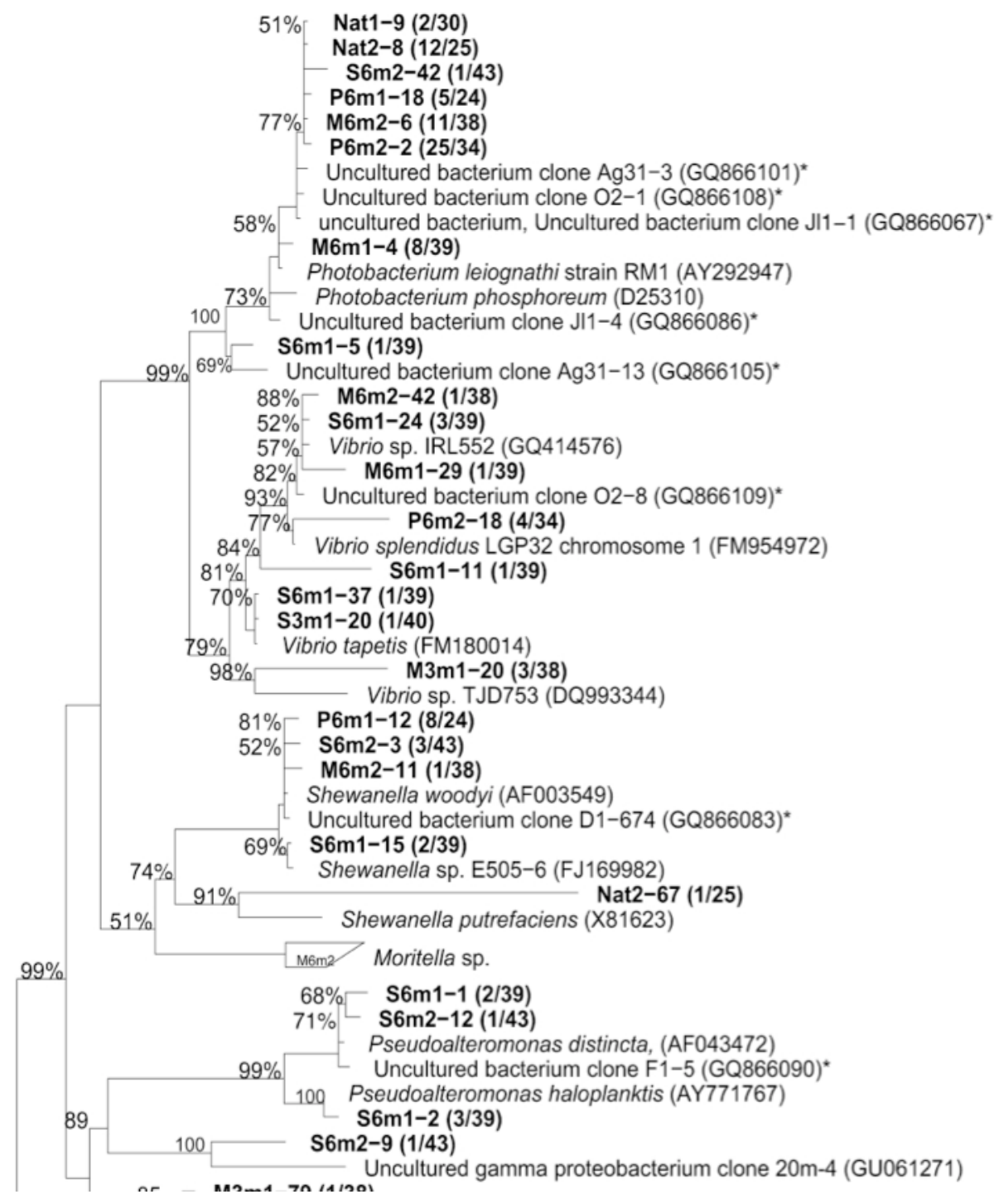

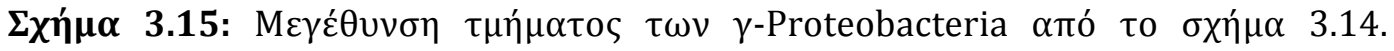

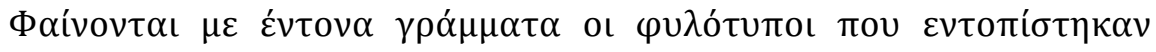

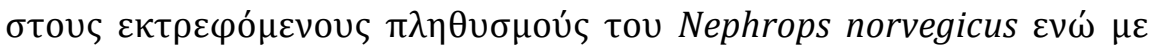

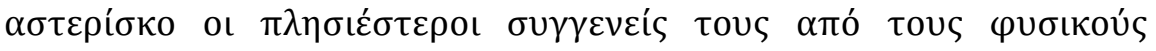
$\pi \lambda \eta \theta v \sigma \mu o u ́ s$

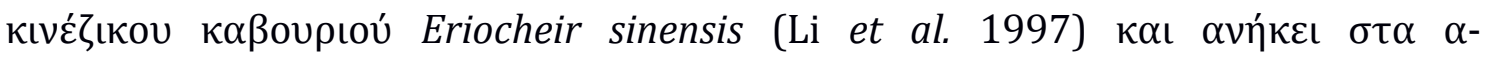

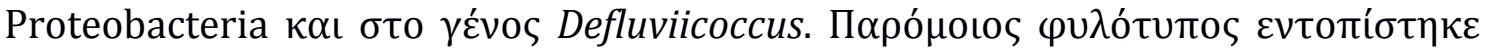

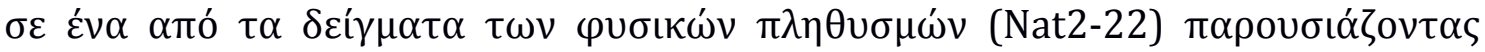
$\chi \alpha \mu \eta \lambda \eta ́$ $\pi \alpha \rho o v \sigma i ́ \alpha(4 \%),(\Sigma \chi .3 .13)$. 


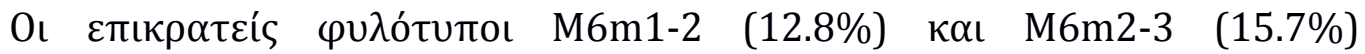

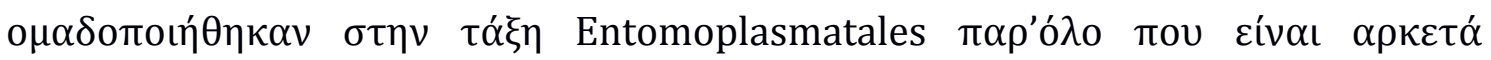

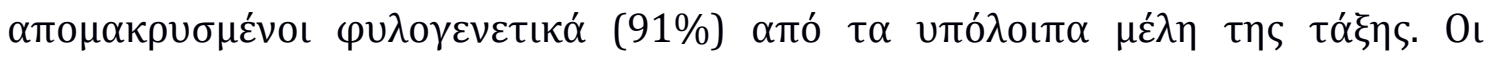

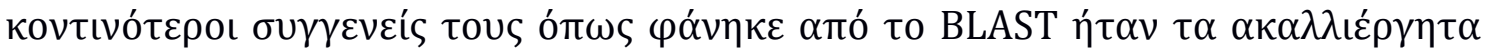

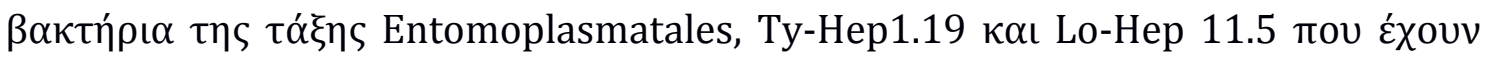

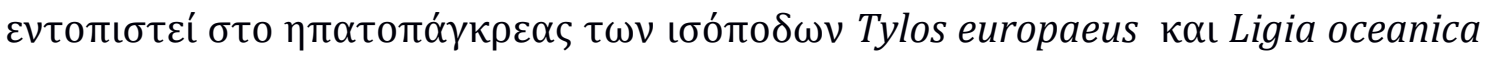

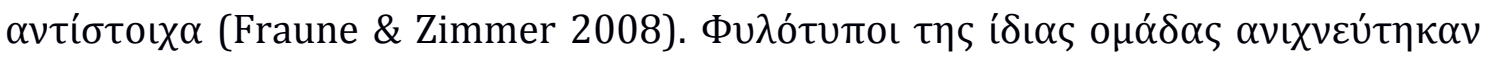

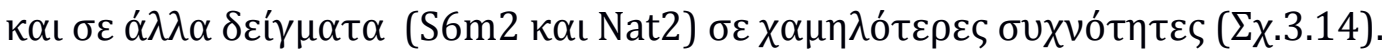

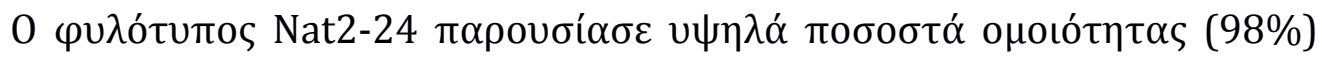

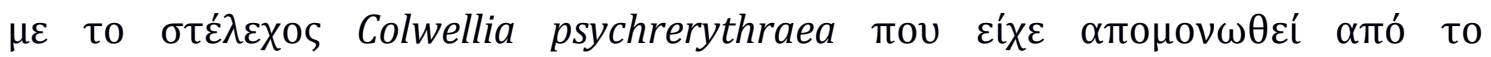

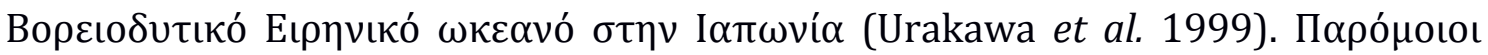


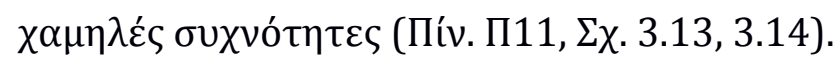

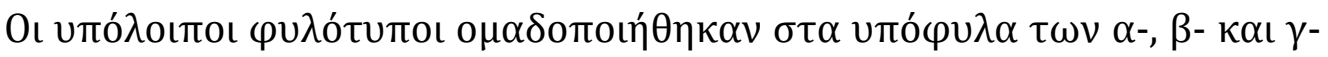
Proteobacteria, $\sigma \tau \alpha \Phi u ́ \lambda \alpha$ Bacteroidetes, Fibrobacteres, Firmicutes, Actinobacteria

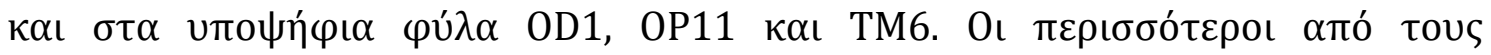

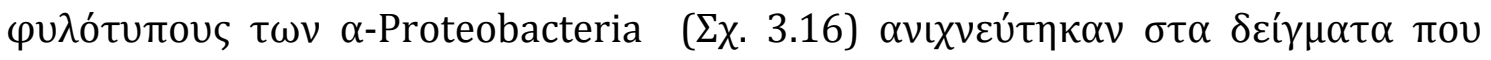

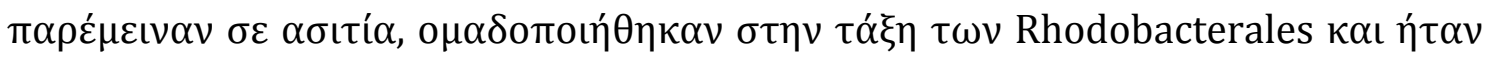
$\pi \alpha \rho$ ó 


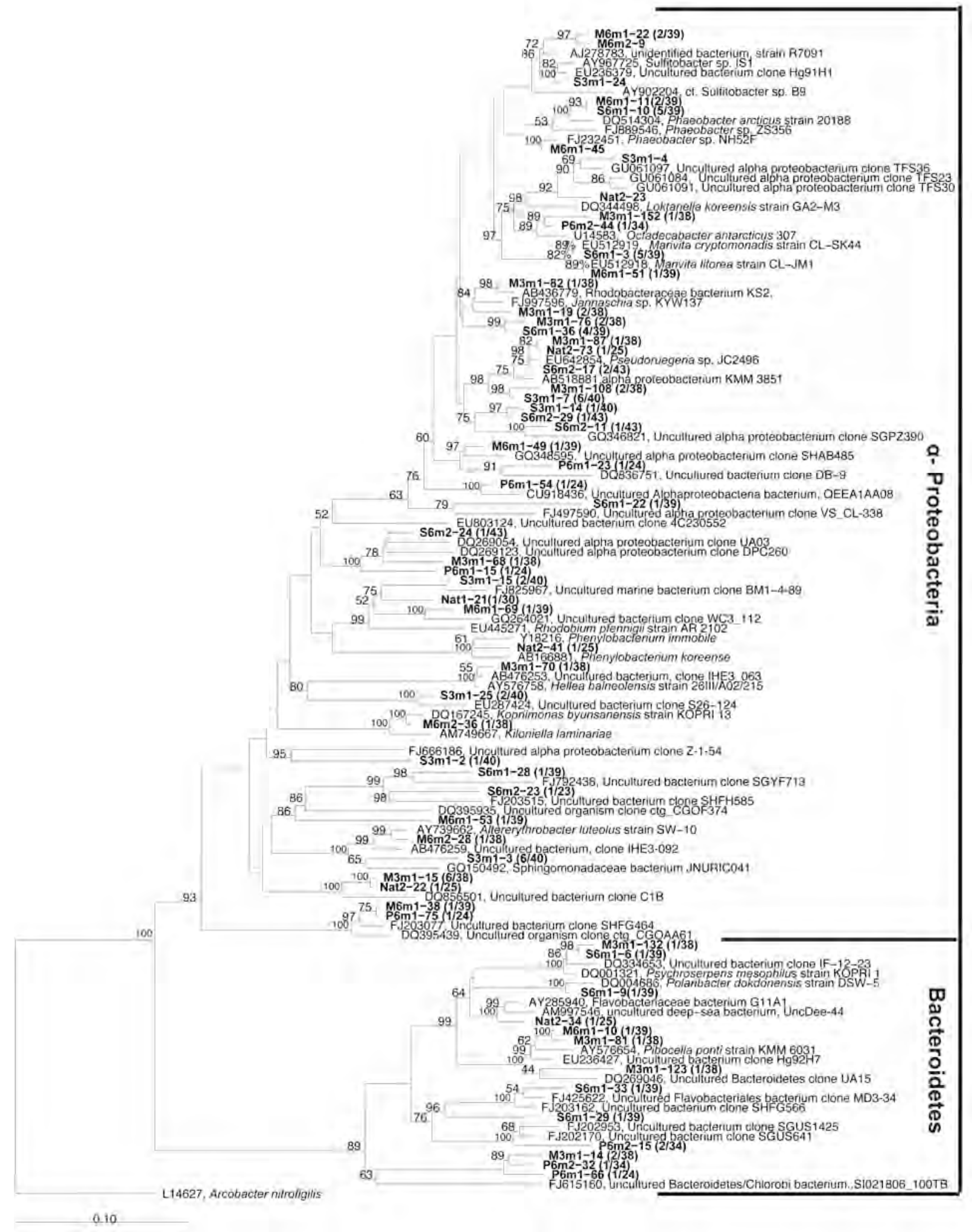

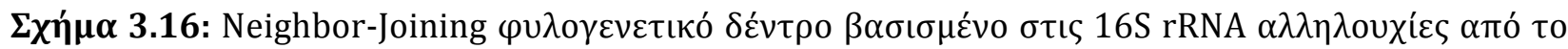

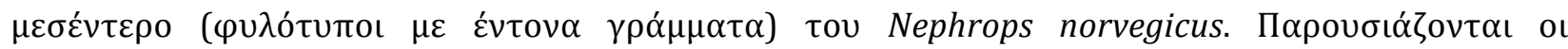

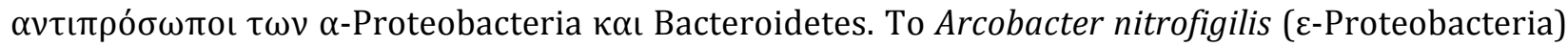

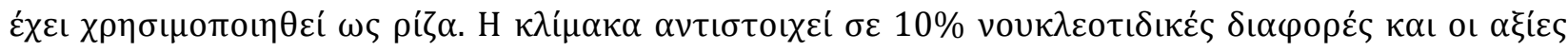

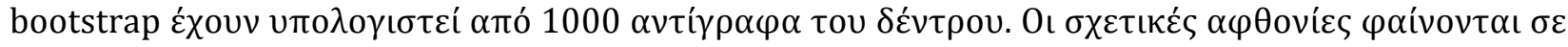

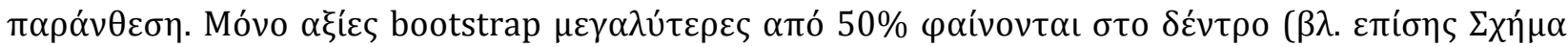
П3.16) 


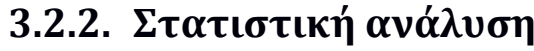

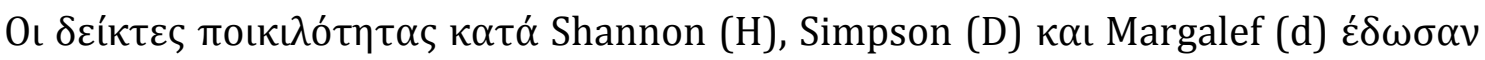

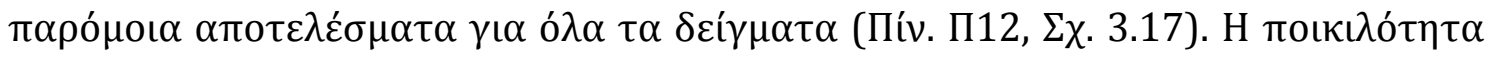

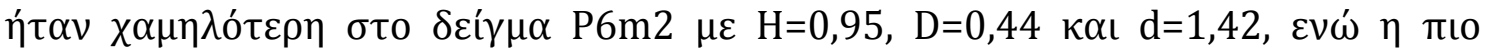

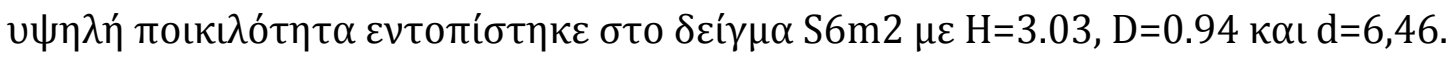

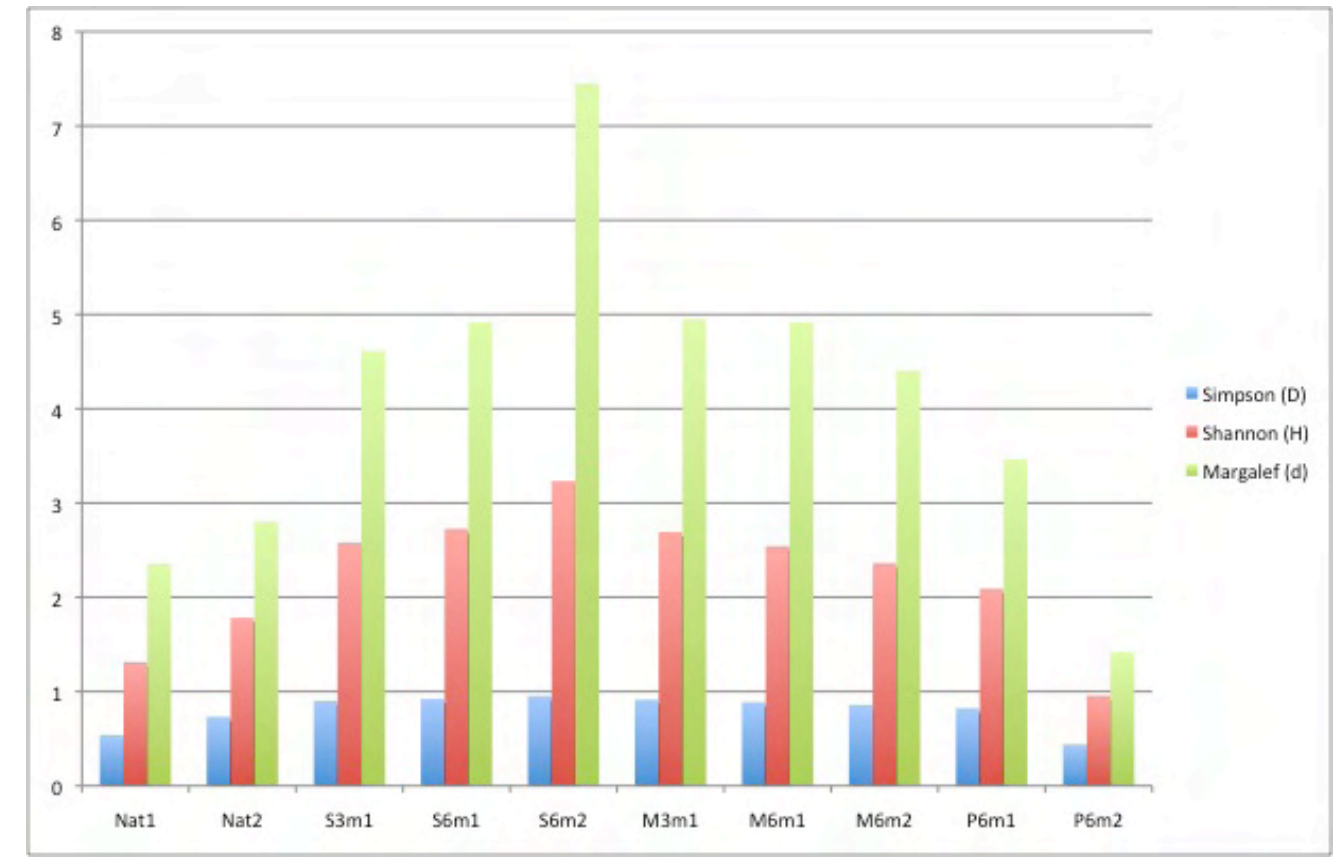

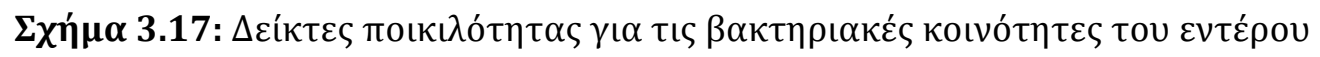

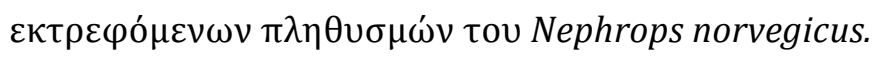

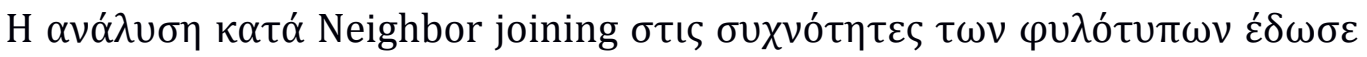

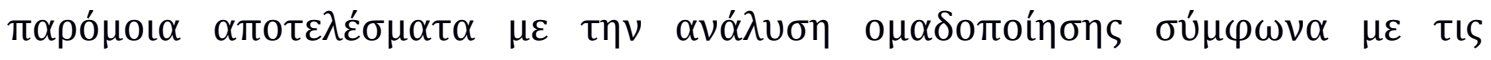

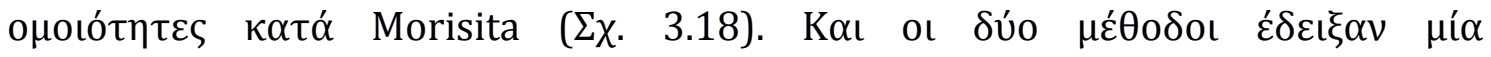

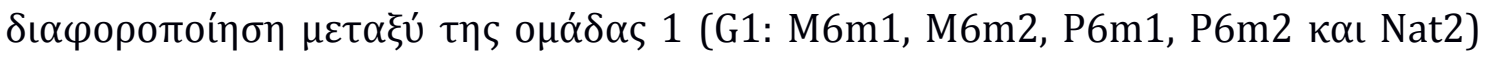

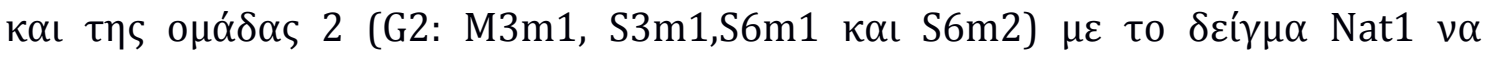




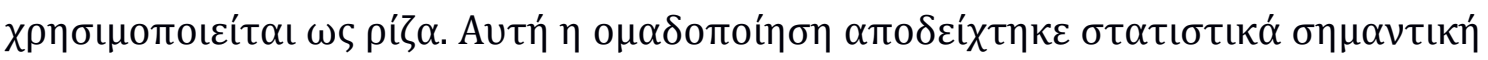

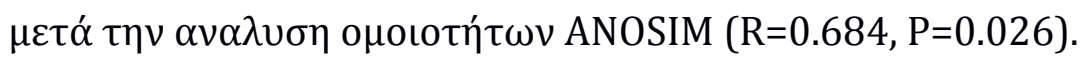

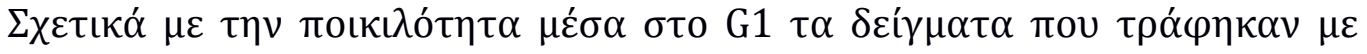

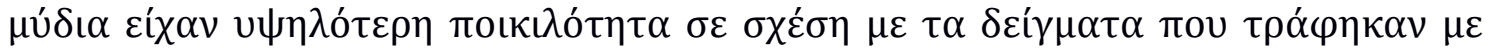
$\sigma u ́ \mu \pi \eta \kappa \tau \alpha$.

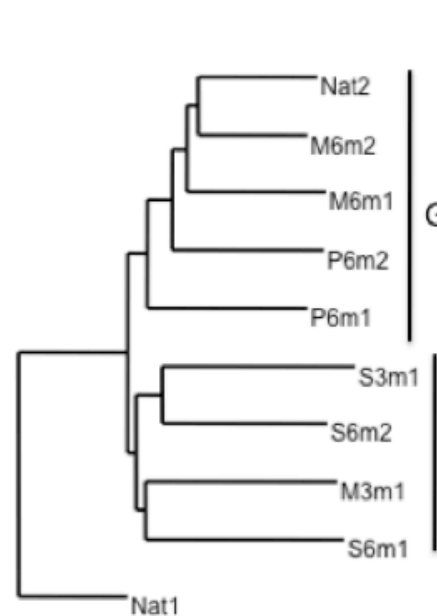

a)

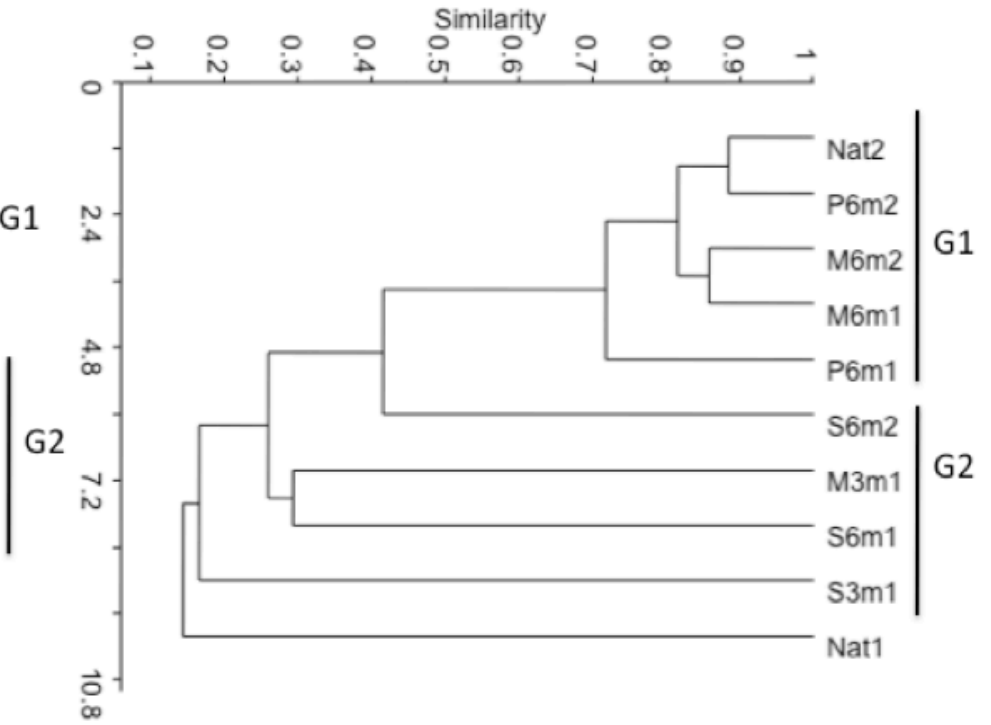

ß)

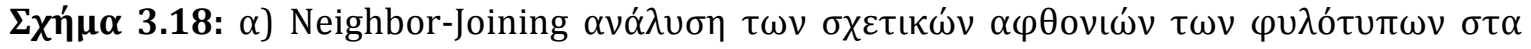

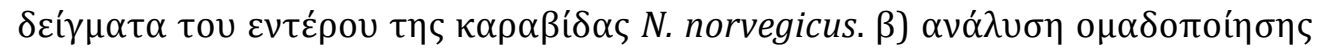

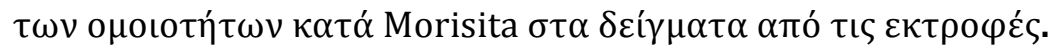

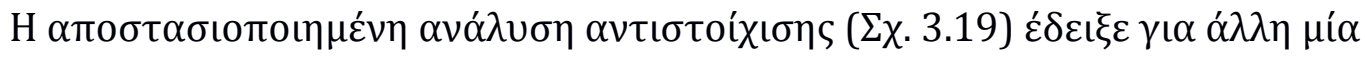

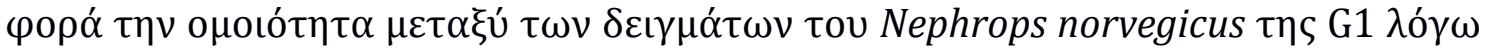

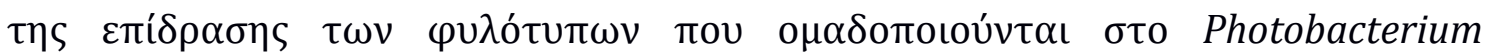

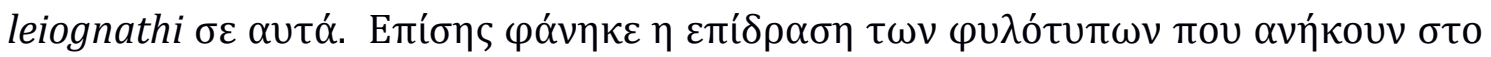

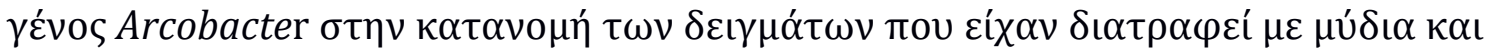

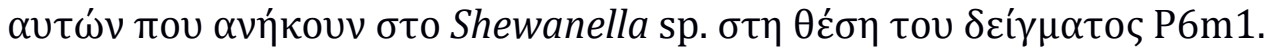




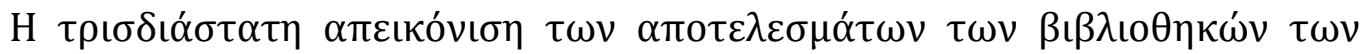

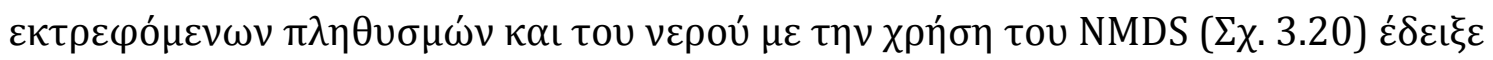

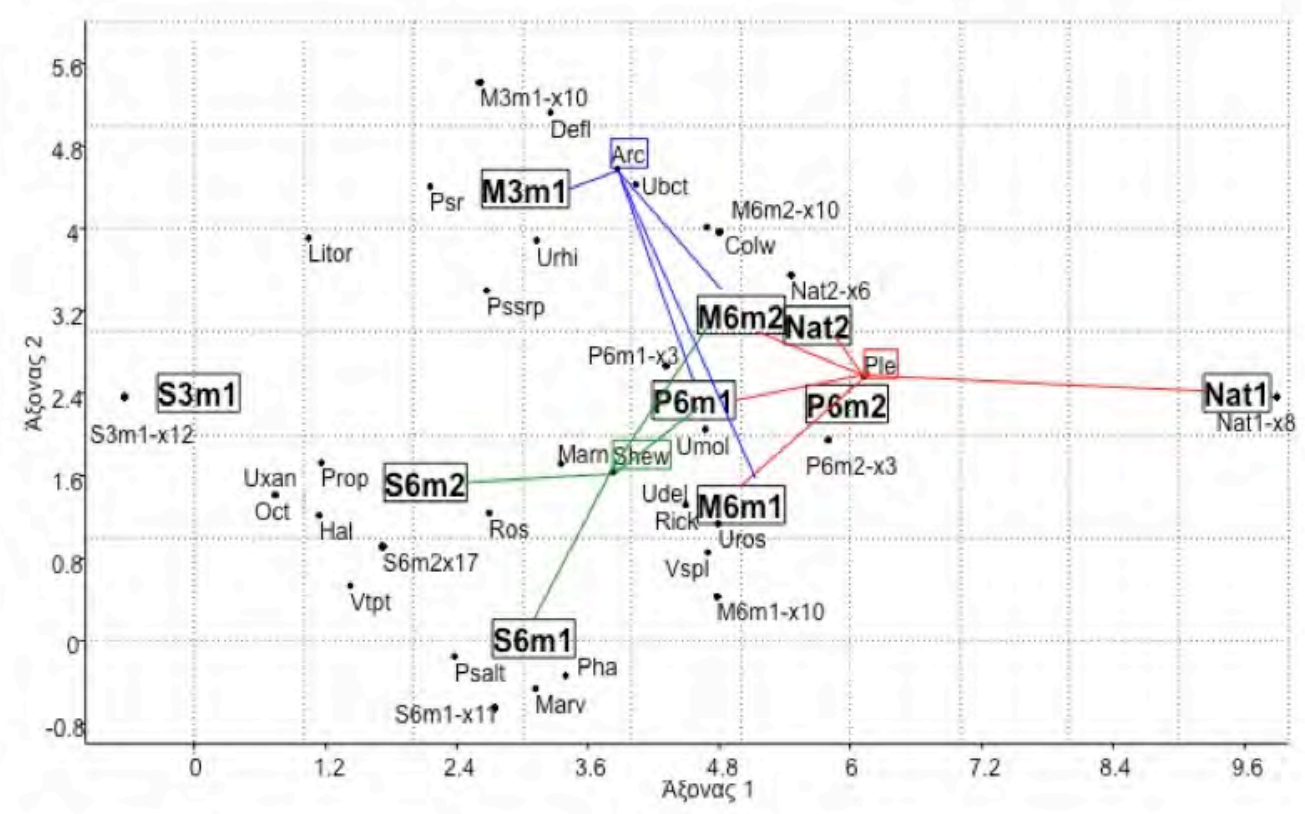

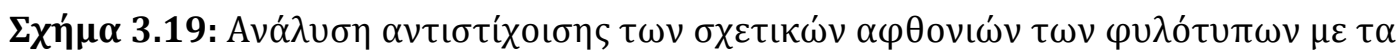

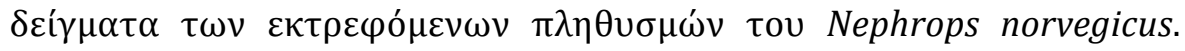

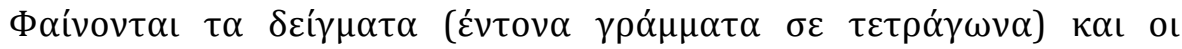

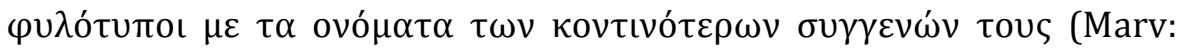
Marivita sp., Psalt: Pseudoalteromonas sp. Pha: Phaeobacter sp., Vtpt: V.tapetis, Hal: Haliea sp., Prop: Propionibacterium sp., Uxan: $\alpha \kappa \alpha \lambda \lambda \iota \varepsilon ́ \rho \gamma \eta \tau o$ xanthomonadales, Oct: Octadecabacter sp., Ros:

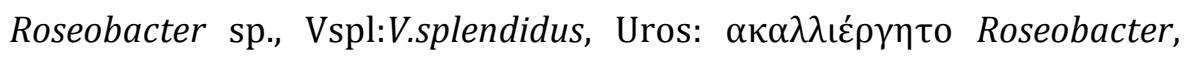

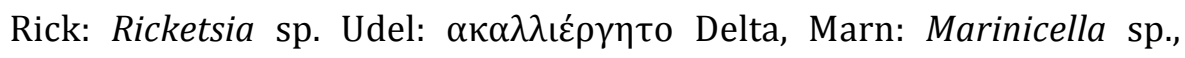

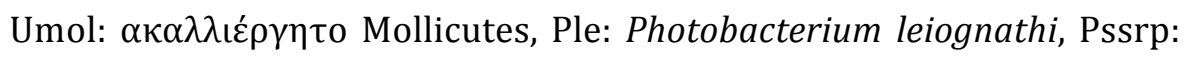
Psychroserpens sp., Urhi: $\alpha \kappa \alpha \lambda \lambda \iota \varepsilon \dot{\varepsilon} \gamma \eta \tau$ Rhizobiales, Litor: Litoreibacter sp., Psr: Pseudoruegeria sp., Colw: Colwellia sp., Arc: Arcobacter sp., Defl:

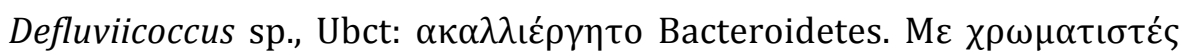

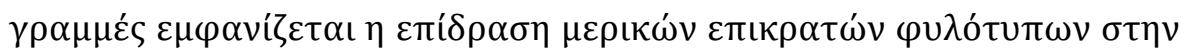
$\kappa \alpha \tau \alpha \nu o \mu \eta \dot{~} \tau \omega \nu \delta \varepsilon \iota \gamma \mu \alpha \dot{\tau} \omega \nu$. 


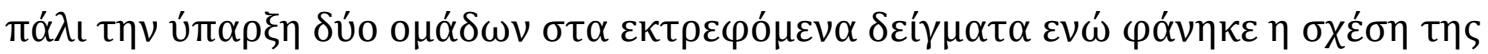

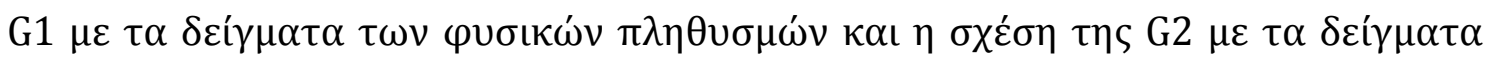

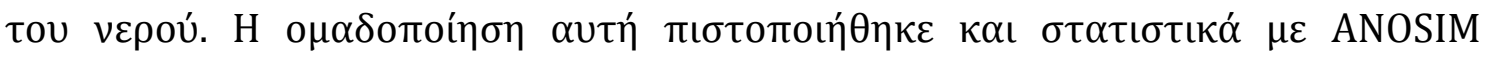
$(\mathrm{R}=0,613, \mathrm{P}=0,004)$.

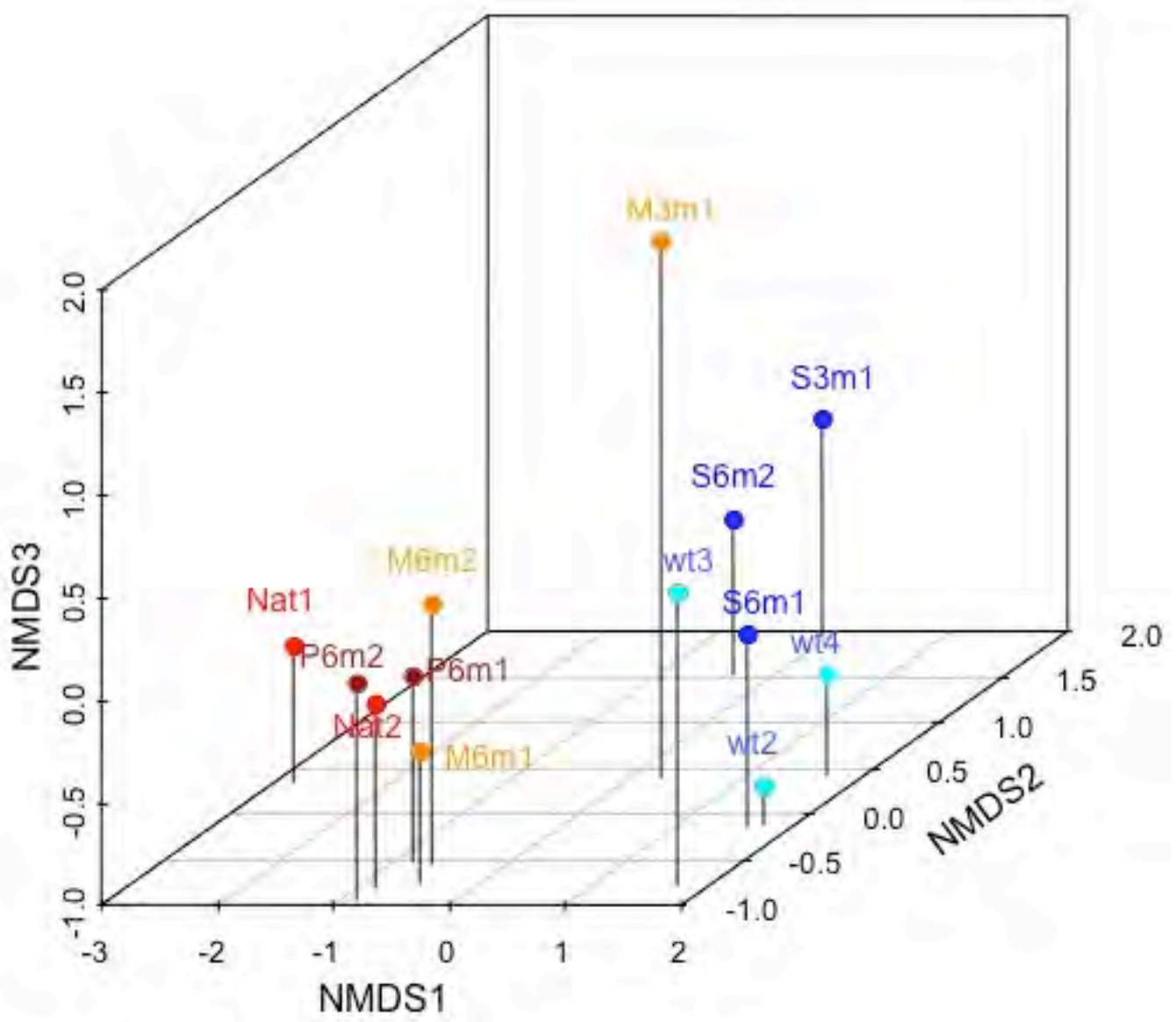

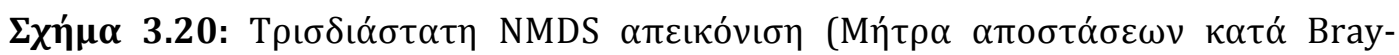

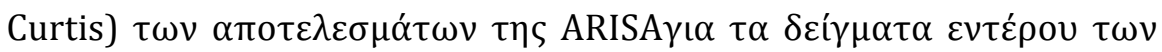

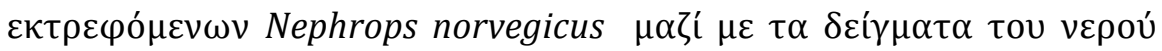

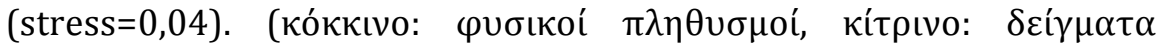

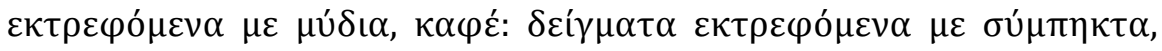

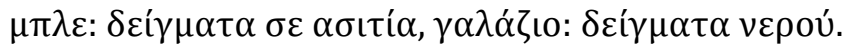




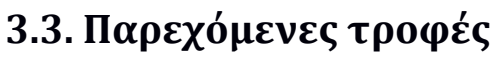

\subsubsection{Mú $\delta \iota \alpha$}

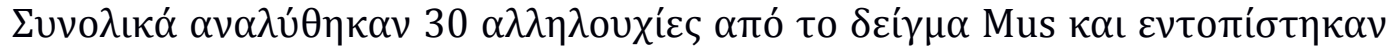

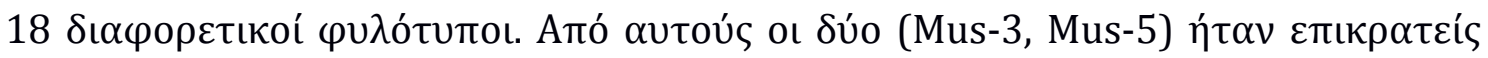

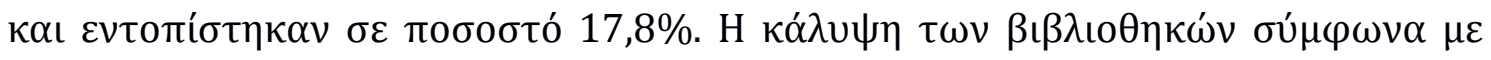

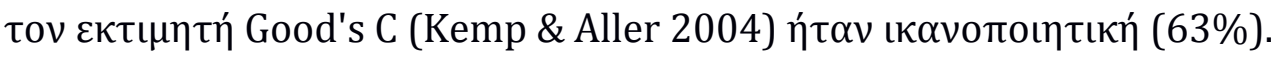

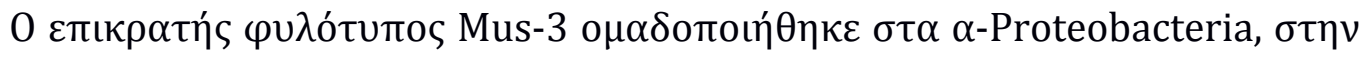

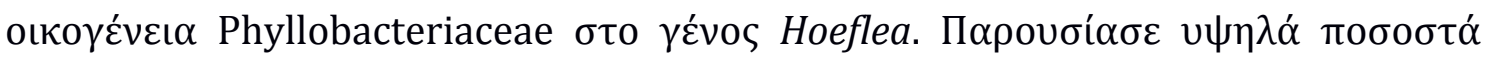

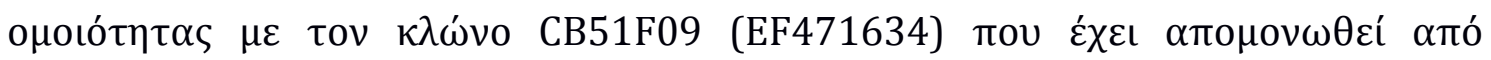

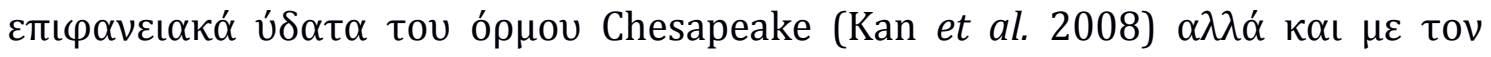

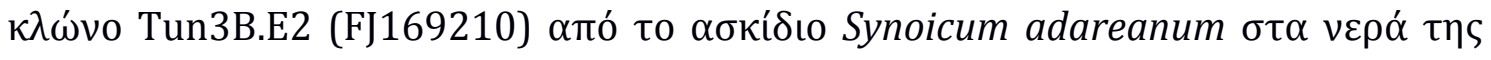

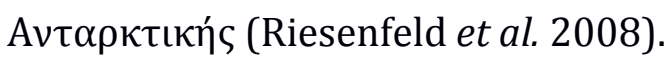

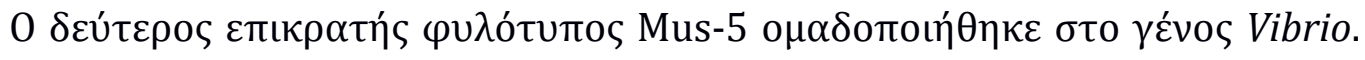

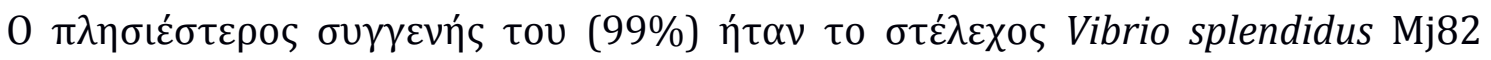

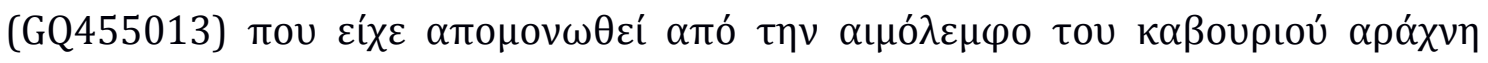

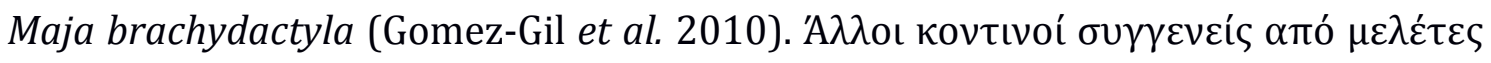

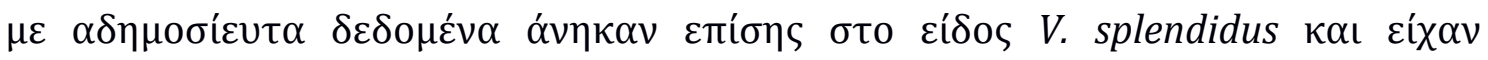

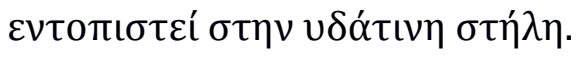

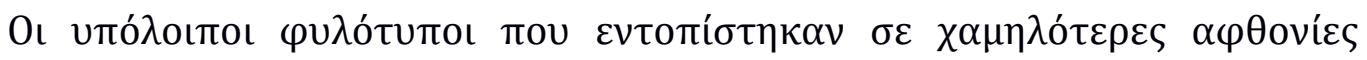

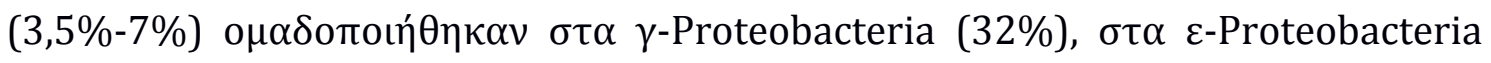
$(10,5 \%) \kappa \alpha \iota ~ \sigma \tau \alpha$ Firmicutes, Bacteroidetes, Flavobacteria, Fusobacteria, OD1 к $\alpha$ ı

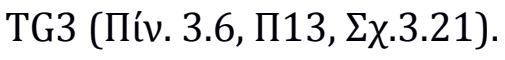




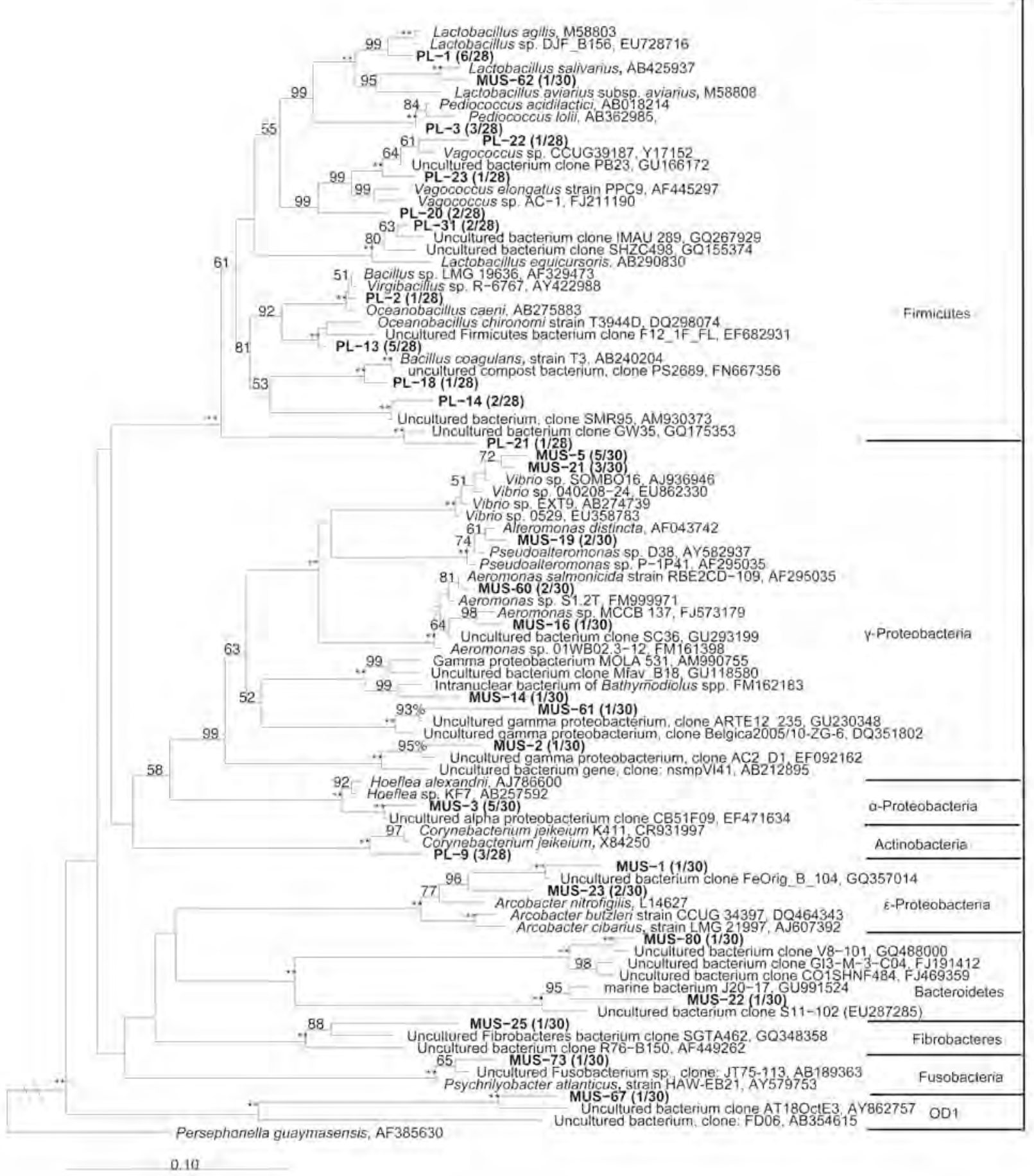

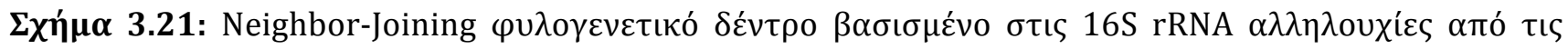

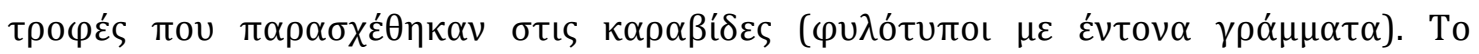

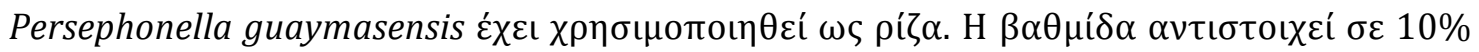

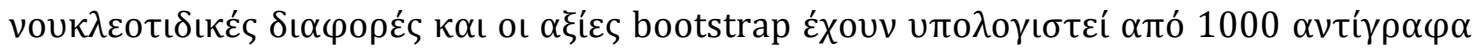

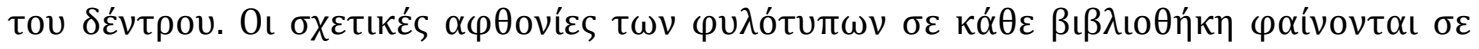

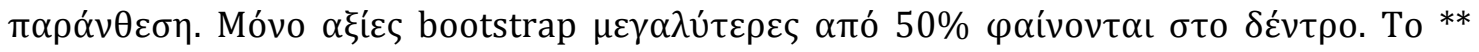

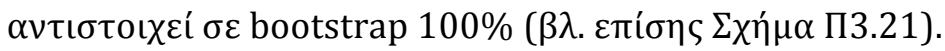




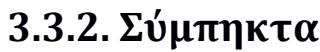

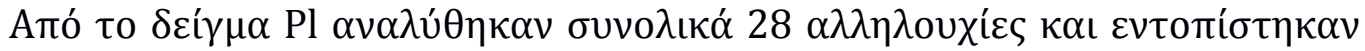

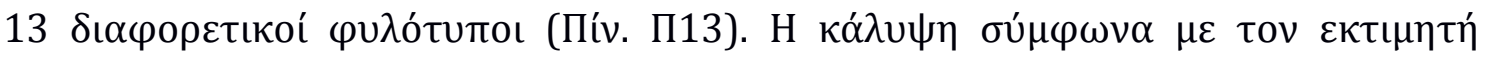

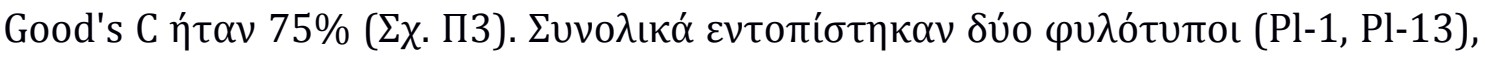

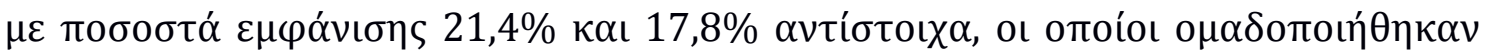

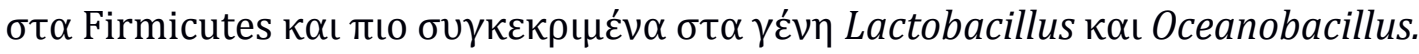

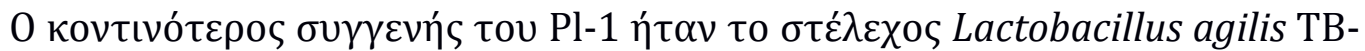

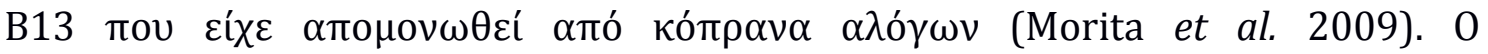

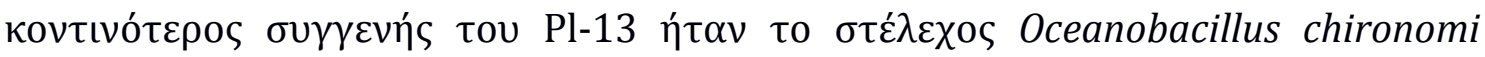

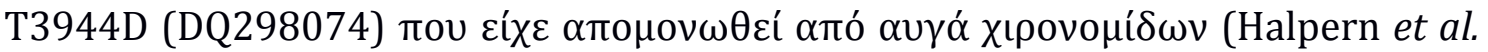
2007).

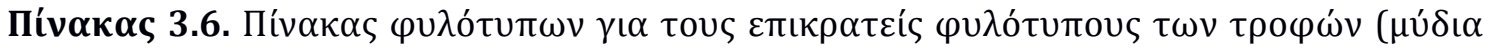

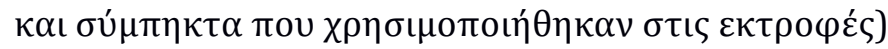

\begin{tabular}{|c|c|c|c|c|}
\hline 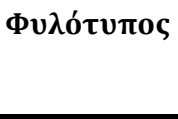 & 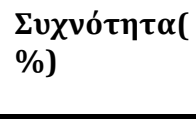 & 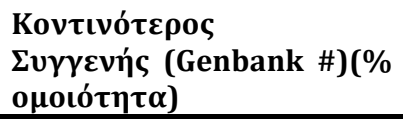 & $\Phi v \lambda{ }^{\prime} \gamma \varepsilon \dot{v \varepsilon \sigma \eta}$ & 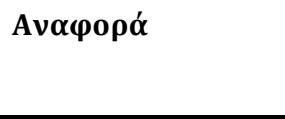 \\
\hline Mus-3 & 16,66 & $\begin{array}{l}\text { Uncultured bacterium clone } \\
\text { Tun3b.E2 (FJ169210) (99) }\end{array}$ & $\alpha$ - Proteobacteria & $\begin{array}{l}\text { Riesenfeld et } a l . \\
2008\end{array}$ \\
\hline Mus-5 & 16,66 & $\begin{array}{l}\text { Vibrio splendidus Mj82 } \\
\text { (GQ455013)(99) }\end{array}$ & $\gamma$-Proteobacteria & $\begin{array}{l}\text { Gomez-Gil et al. } \\
2010\end{array}$ \\
\hline Mus-21 & 10 & $\begin{array}{l}\text { Uncultured Vibrio sp. clone } \\
\text { HG369 (FN646711)(99) }\end{array}$ & $\gamma$-Proteobacteria & 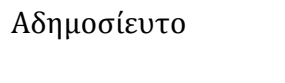 \\
\hline Pl-1 & 21,4 & $\begin{array}{l}\text { Lactobacillus agilis strain: } \\
\text { TB-B13(AB425919)(100) }\end{array}$ & Firmicutes & Morita et al. 2009 \\
\hline Pl-13 & 17,8 & $\begin{array}{l}\text { Uncultured Oceanobacillus } \\
\text { sp. clone O-80-33 } \\
\text { (EU862162)(99) }\end{array}$ & Firmicutes & 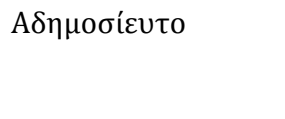 \\
\hline Pl-3 & 10,7 & $\begin{array}{lr}\text { Pediococcus acidilactici } \\
\text { strain DSPV } \\
\text { (FJ751795)(99) }\end{array}$ & Firmicutes & 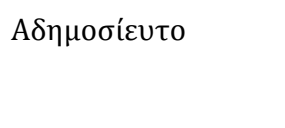 \\
\hline Pl-9 & 10,7 & $\begin{array}{l}\text { Corynebacterium } \\
\text { urealyticum DSM } \\
(\text { AM942444)(99) }\end{array}$ & Actinobacteria & Tauch et al. 2008 \\
\hline
\end{tabular}




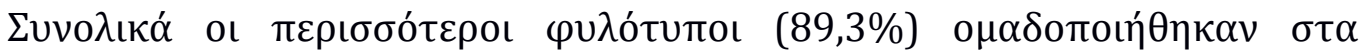

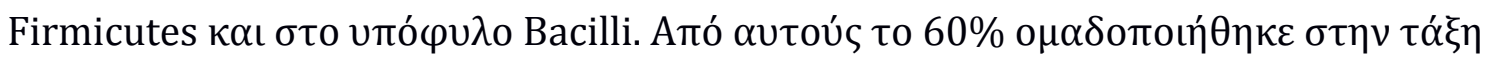

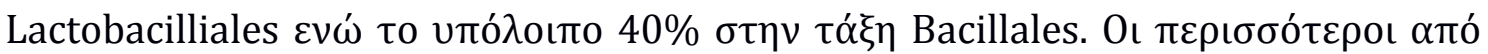

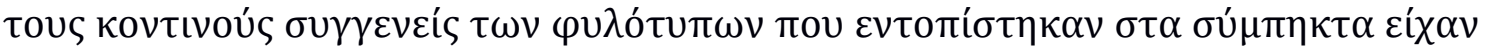

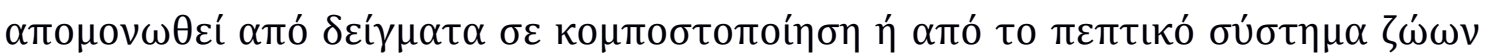

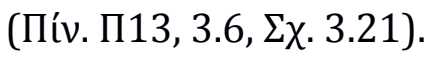

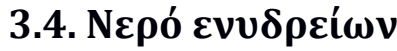

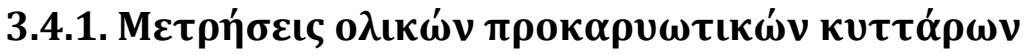

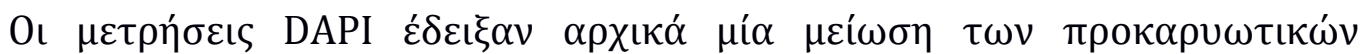

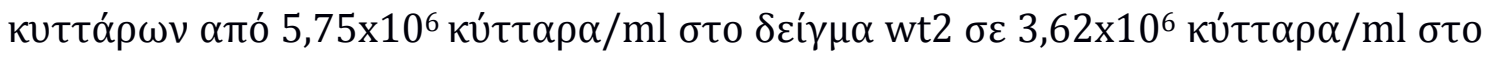

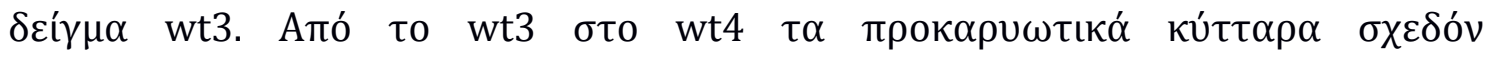

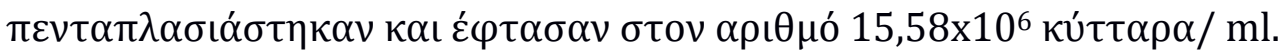

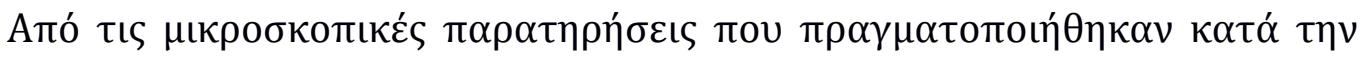

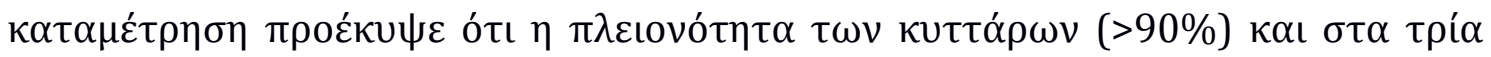

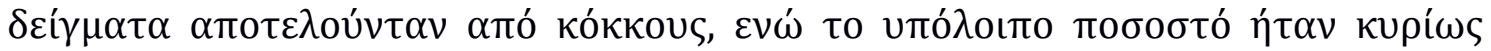

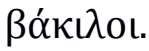

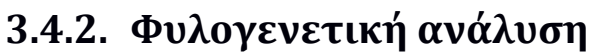

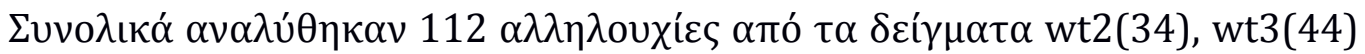

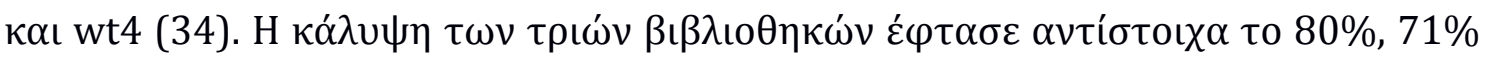




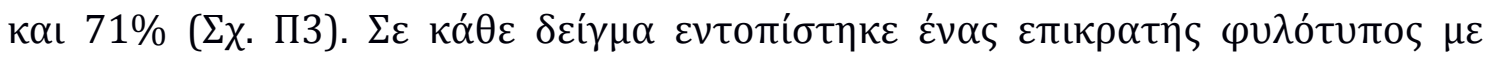

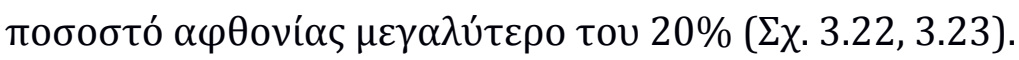

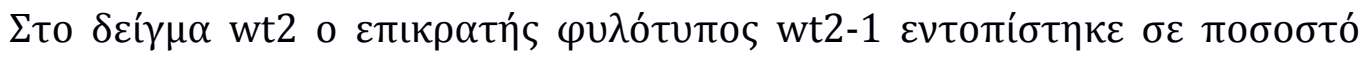

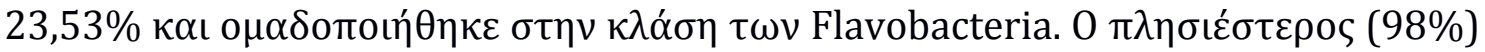

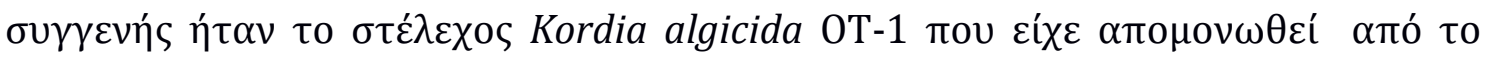

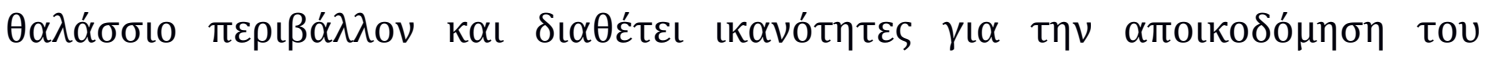

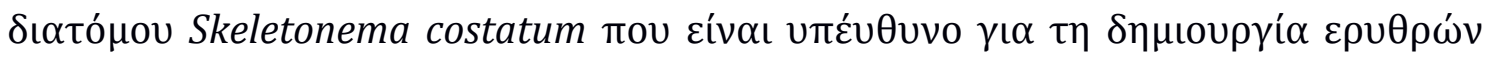

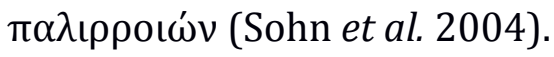
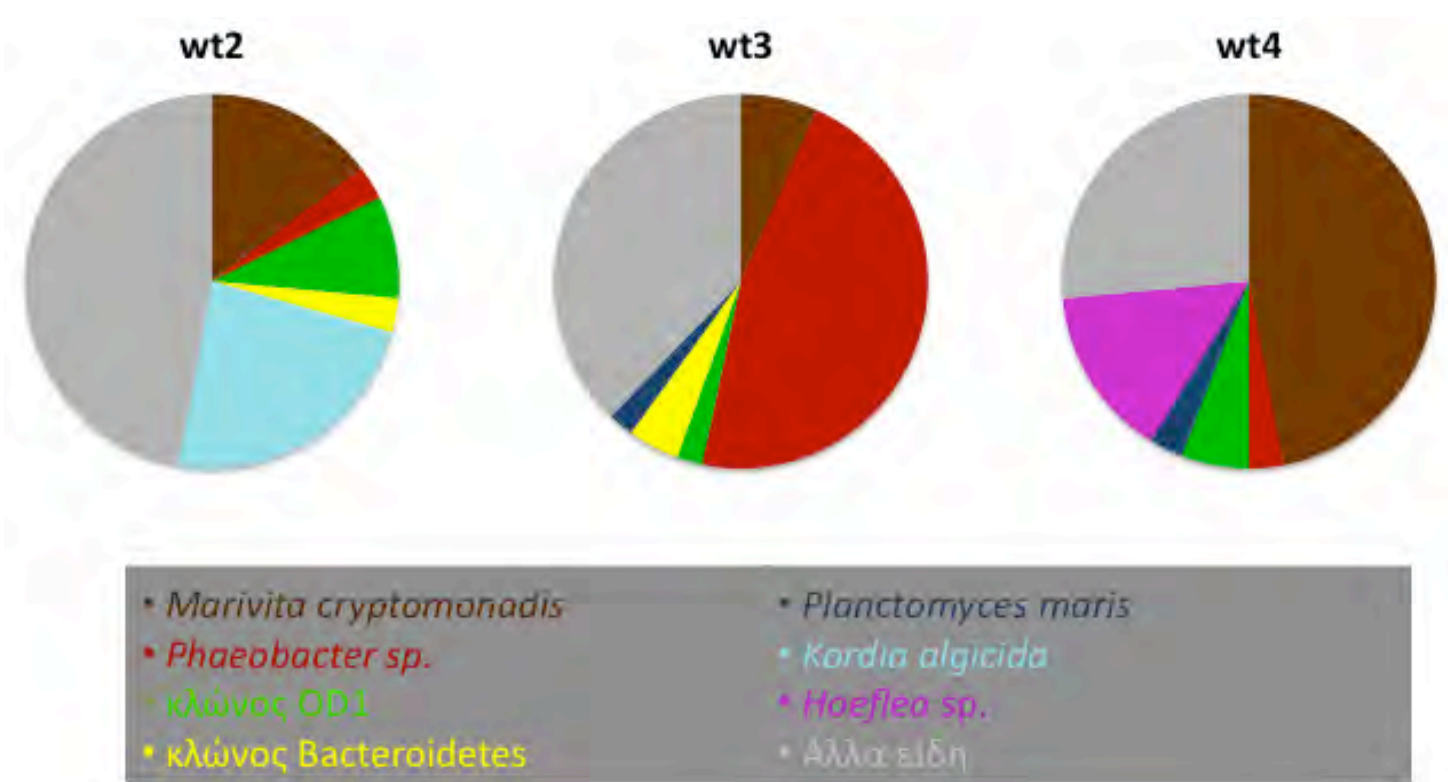

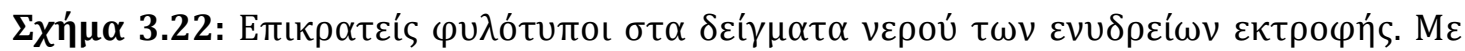

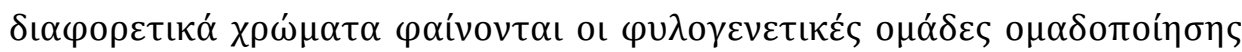

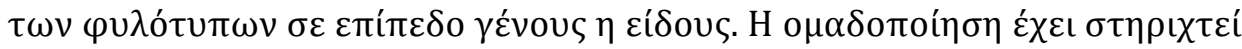

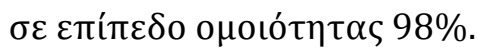




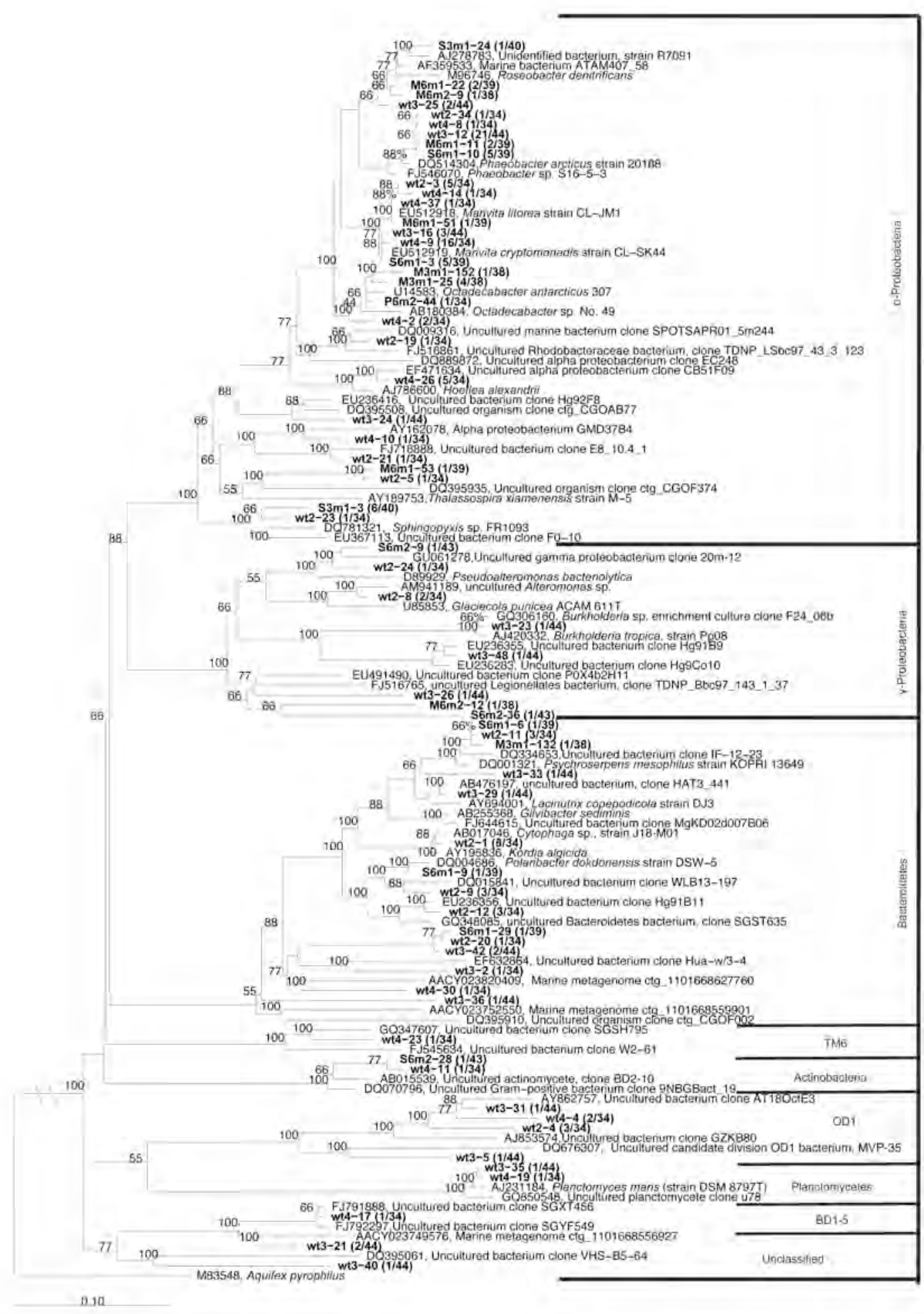

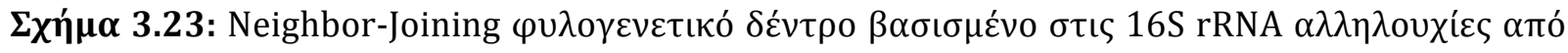

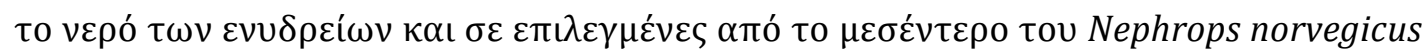
( $\varphi$

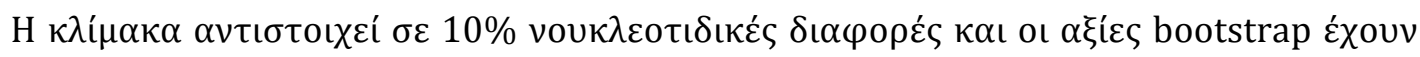

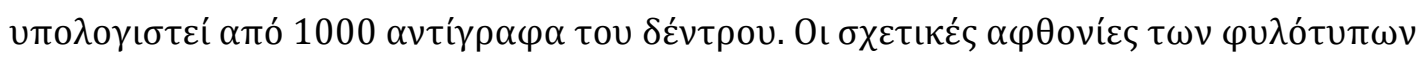

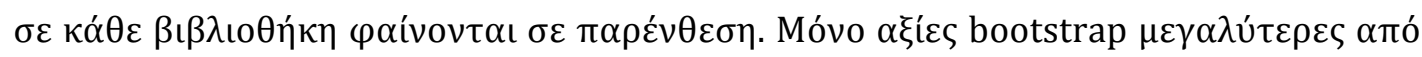

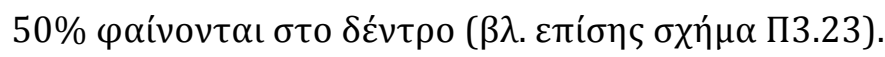




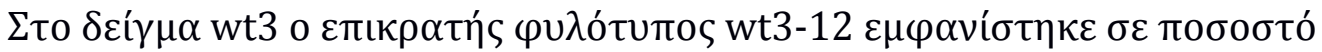

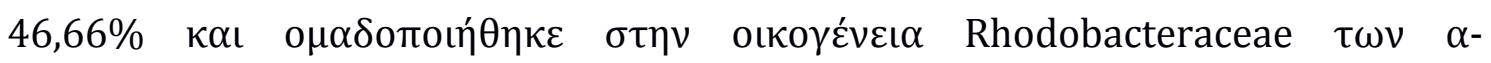

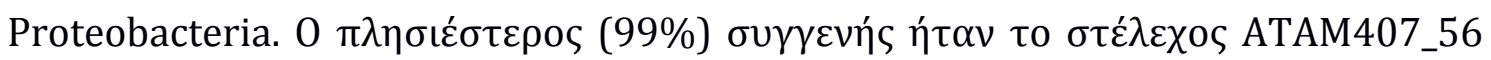

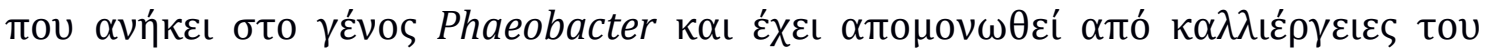

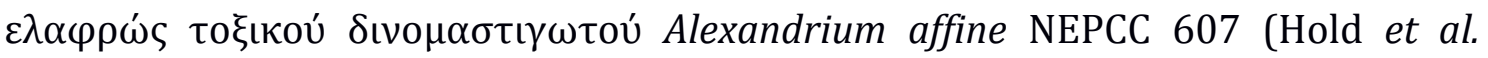
2001).

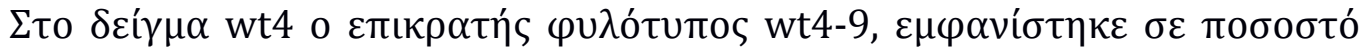

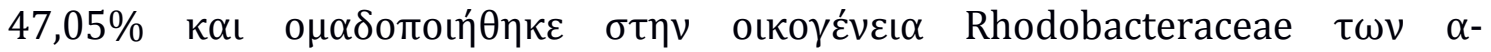

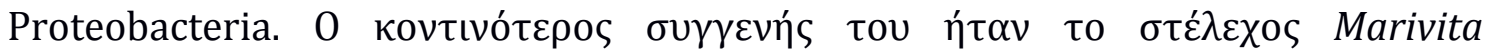

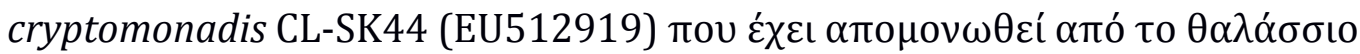

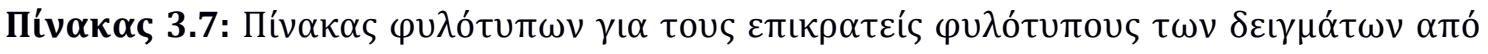

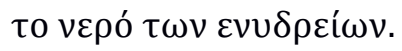

\begin{tabular}{|c|c|c|c|c|}
\hline 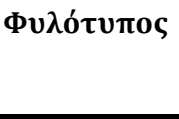 & $\Sigma v \chi v o ́ \tau \eta \tau \alpha(\%)$ & 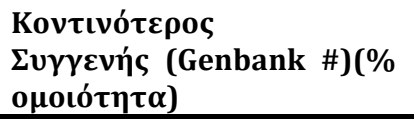 & 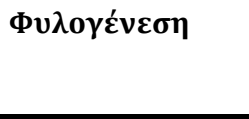 & 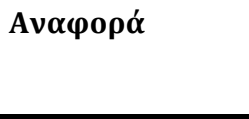 \\
\hline Wt2-1 & 23,52 & $\begin{array}{l}\text { Kordia algicida strain OT-1 } \\
(\mathrm{NR} 027568)(98)\end{array}$ & Bacteroidetes & Sohn et al. 2004 \\
\hline Wt2-3 & 14,70 & $\begin{array}{l}\text { Marivita litorea strain CL- } \\
\text { JM1 (EU512918)(98) }\end{array}$ & $\alpha$-Proteobacteria & Hwang et al. 2009 \\
\hline Wt3-12 & 47,7 & $\begin{array}{l}\text { Marine bacterium } \\
\text { ATAM407-56 } \\
(\text { AF359535)(99) }\end{array}$ & $\alpha$-Proteobacteria & Hold et al. 2001 \\
\hline Wt4-9 & 47,1 & $\begin{array}{l}\text { Marivitaryptomonadis } \\
\text { CL-SK44 ,(EU512919)( 99) }\end{array}$ & $\alpha$-Proteobacteria & Hwang et al. 2009 \\
\hline Wt4-26 & 14,7 & $\begin{array}{l}\text { Uncultured alpha } \\
\text { proteobacterium } \\
\text { CB51F09 (EF471634)(99) }\end{array}$ & $\alpha$-Proteobacteria & Kan et al. 2008 \\
\hline
\end{tabular}

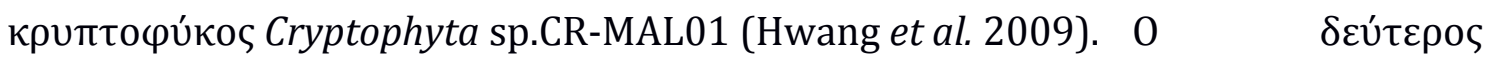

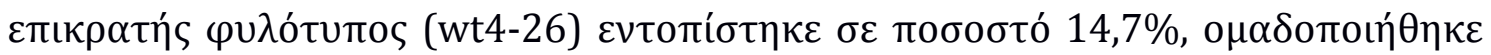

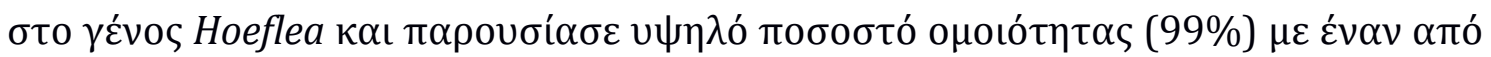

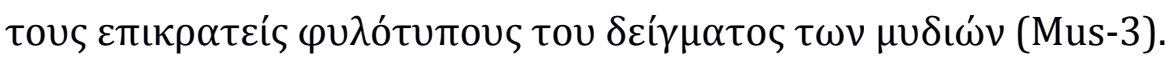




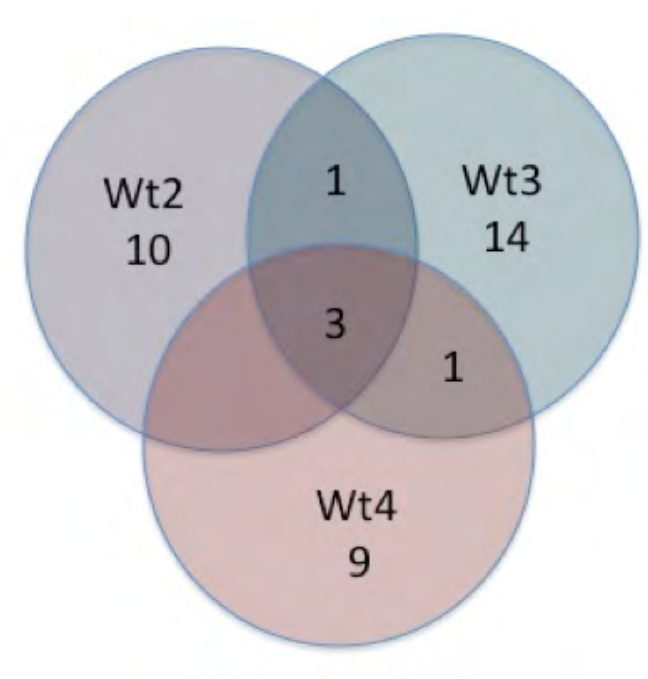

$\Sigma \chi \eta \dot{\mu \alpha}$ 3.24: $\Delta$ เó $\gamma \rho \alpha \mu \mu \alpha$ Venn $\gamma ا \alpha$ tous

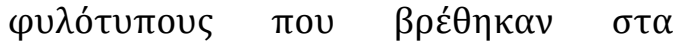

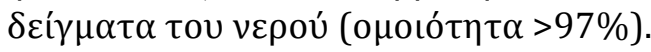

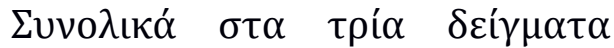

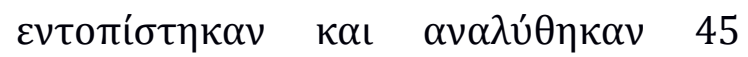
$\delta\llcorner\alpha \varphi о \rho \varepsilon \tau$ เоó $\varphi$

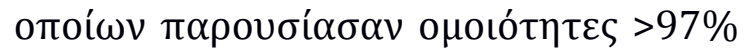
$\mu \varepsilon \tau \alpha \xi u ́$ tous $(\Sigma \chi .3 .22,3.23,3.24)$.

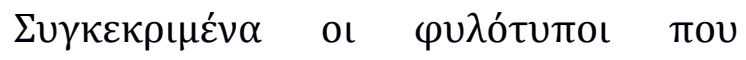

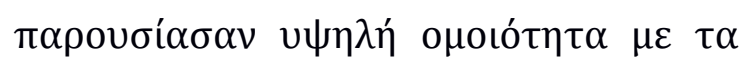

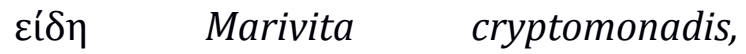
Phaeobacter sp. $\quad$ к $\alpha \iota \quad \mu \varepsilon \quad \varepsilon^{\prime} v \alpha$

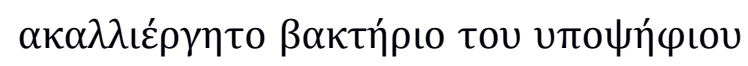

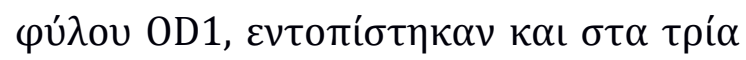
$\delta \varepsilon \dot{\gamma} \gamma \mu \alpha \tau \alpha, \quad \varepsilon v \omega ́$ ol $\varphi$

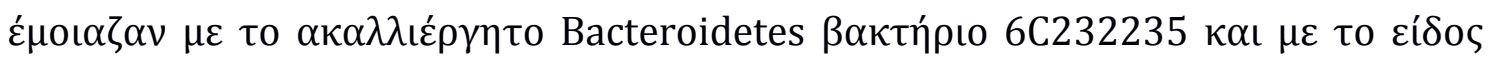

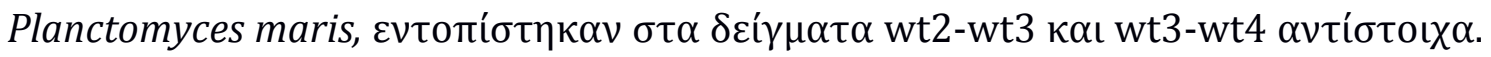

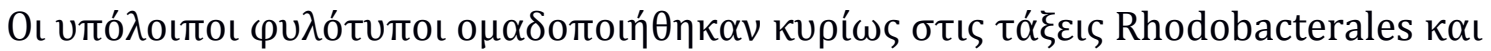

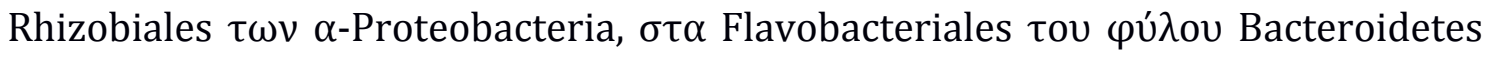

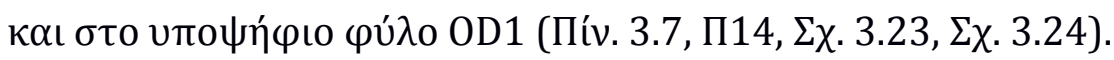

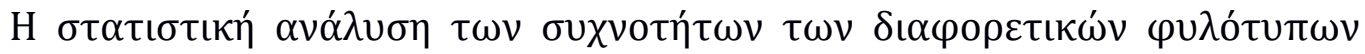
$\varepsilon \dot{\delta \varepsilon \varepsilon \xi \xi \varepsilon \mu \varepsilon \tau \eta \nu ~ \chi \rho \eta ́ \sigma \eta ~ \tau \omega \nu ~ \delta \varepsilon เ \kappa \tau \omega ́ v ~ \kappa \alpha \tau \alpha ́ ~ S h a n n o n ~(H) ~ \kappa \alpha \iota ~ \kappa \alpha \tau \alpha ́ ~ S i m p s o n ~(D) ~ o ́ \tau ı ~ \eta ~}$

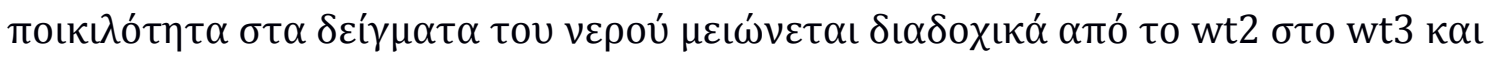

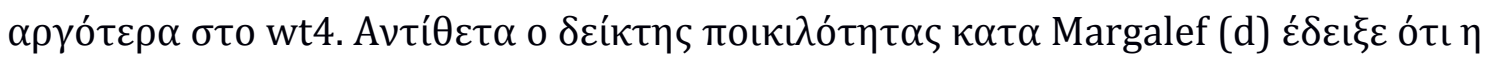

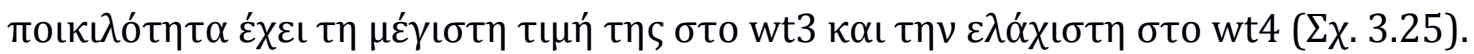




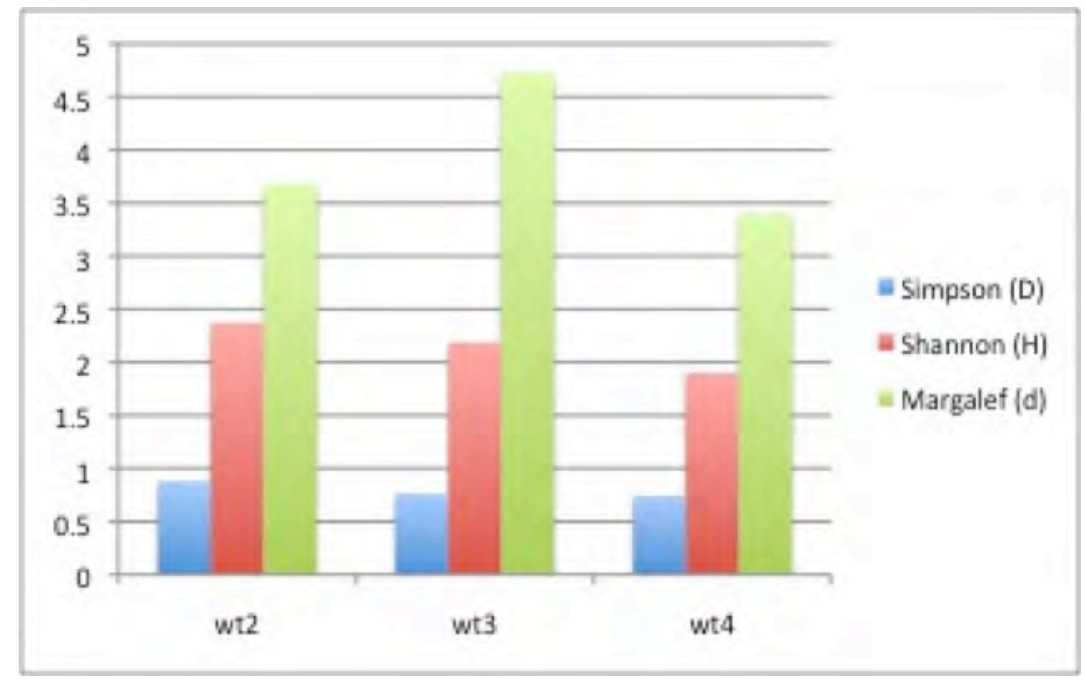

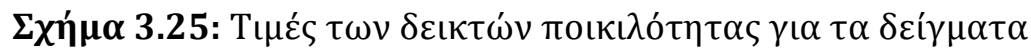

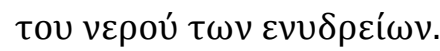




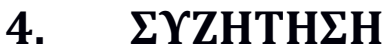

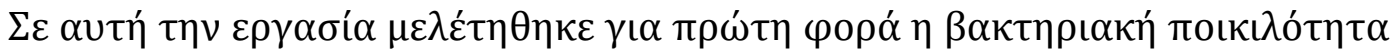

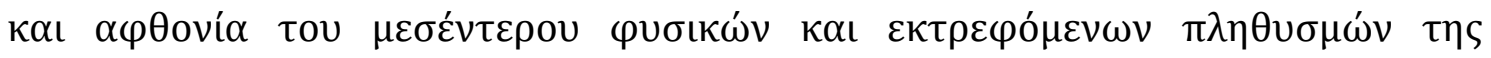

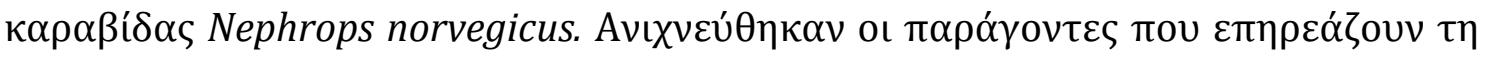

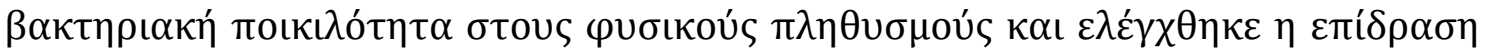

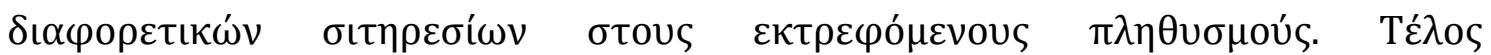

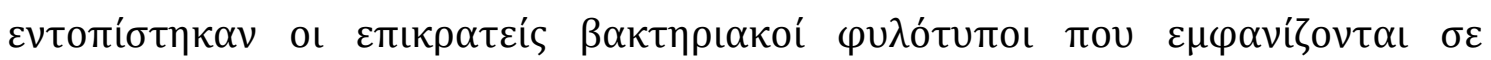

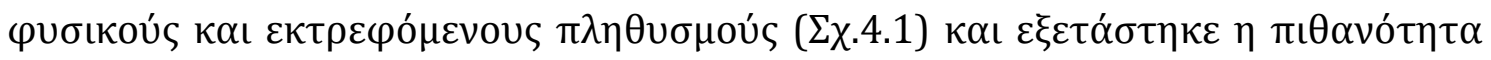

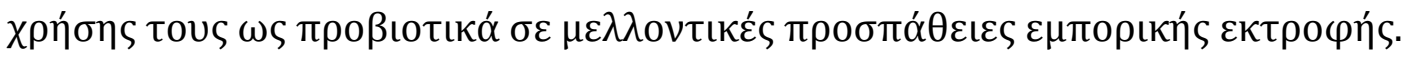

\section{1. Фvбเкоí $\pi \lambda \eta \theta v \sigma \mu o i ́$}

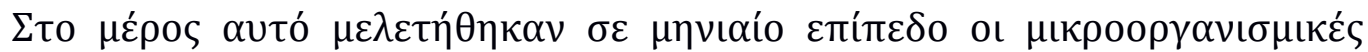

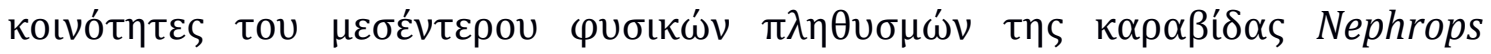

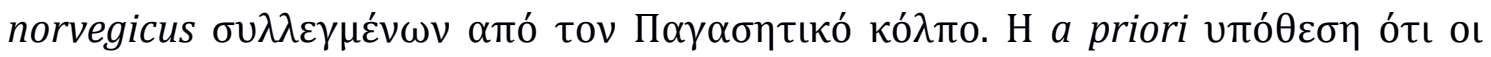

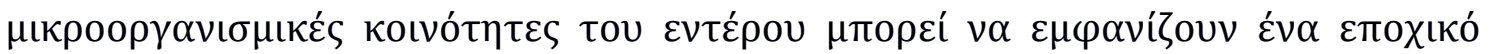

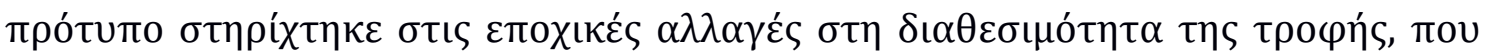

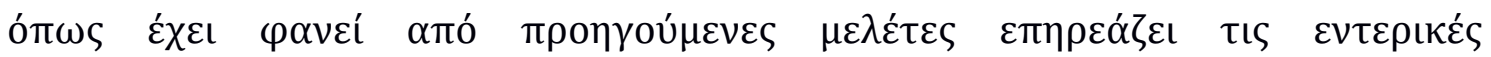

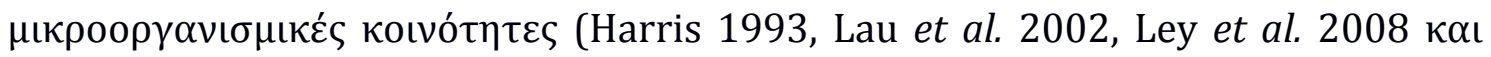
$\left.\alpha v \alpha \varphi \circ \rho \varepsilon^{\prime}\right)$.

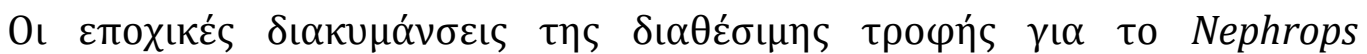

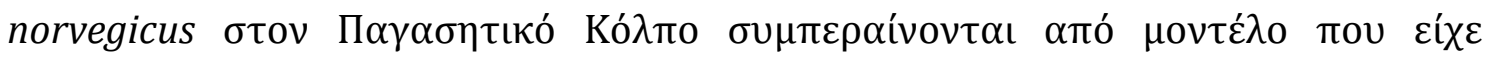

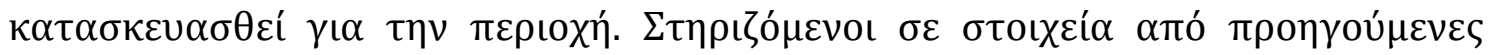

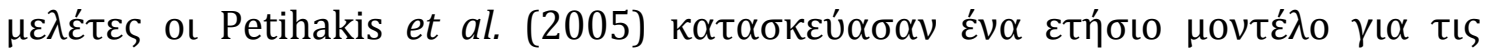




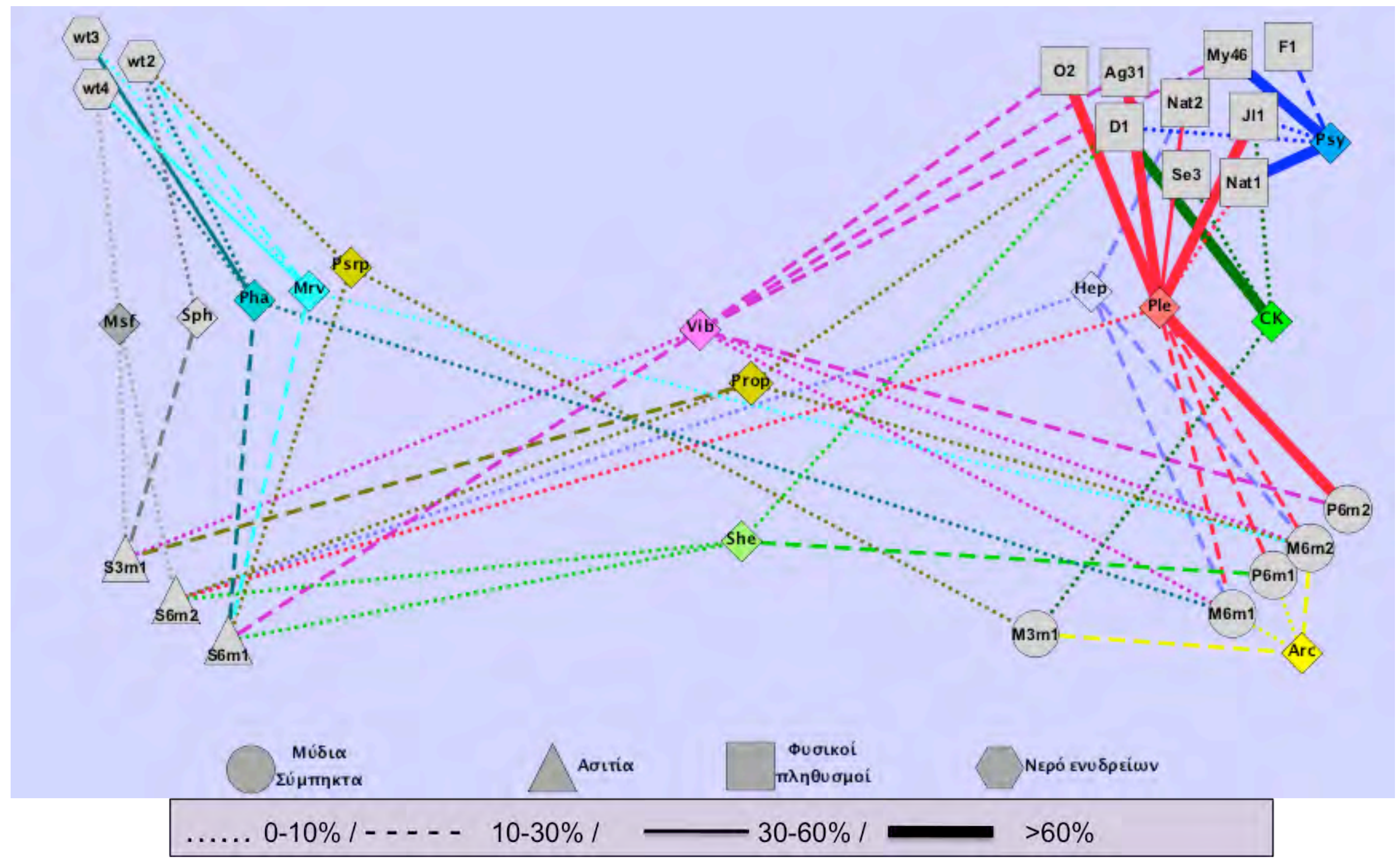

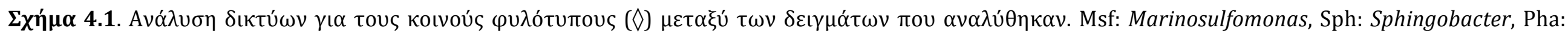
Phaeobacter, Mrv: Marivita, Psrp: Psychroserpens, Prop: Propionibacterium, She: Shewanella, Vib: Vibrio, Ple: Photobacterium leiognathi, CK:

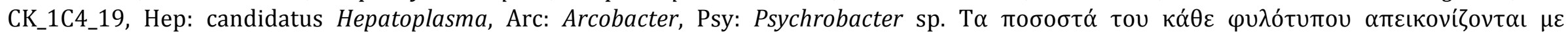
$\delta\llcorner\alpha \varphi о \rho \varepsilon \tau \iota \kappa \varepsilon ́ \varsigma ~ \sigma u v \delta \varepsilon ́ \sigma \varepsilon เ \varsigma$. 


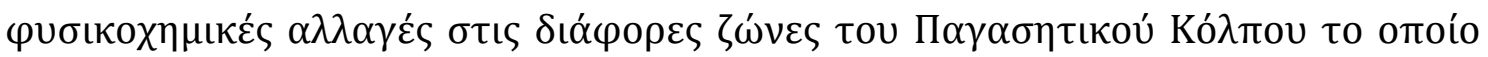

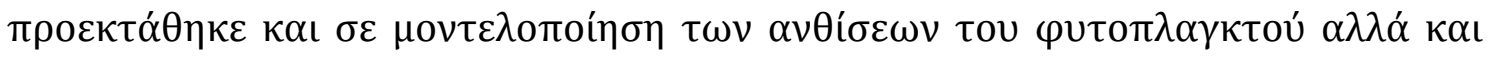

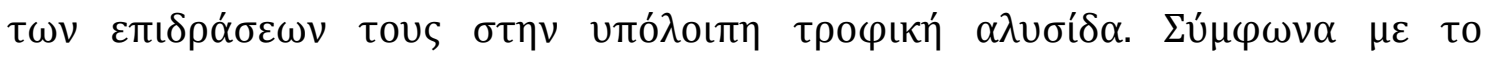

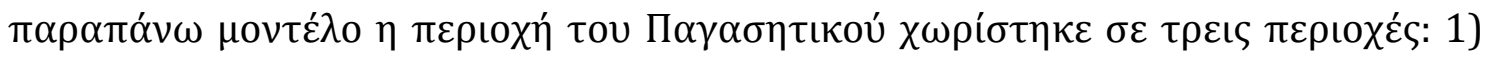

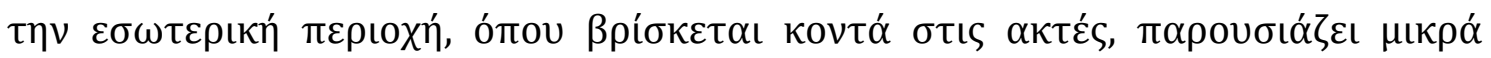

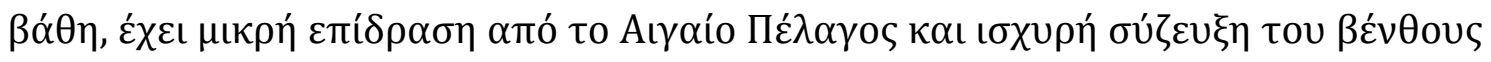

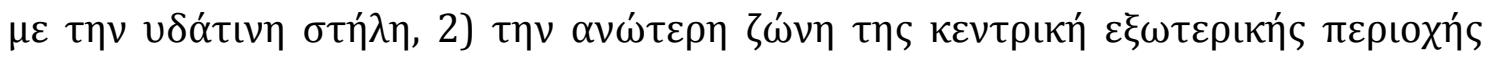

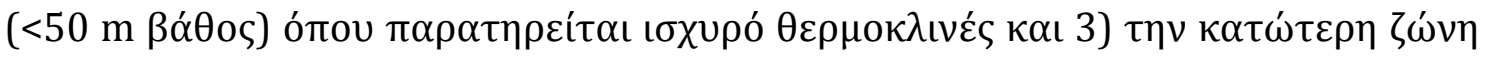

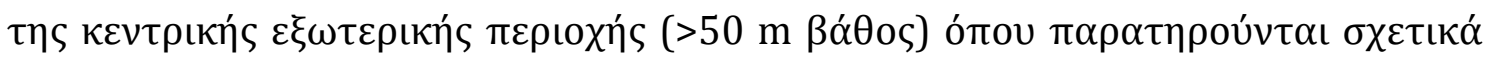

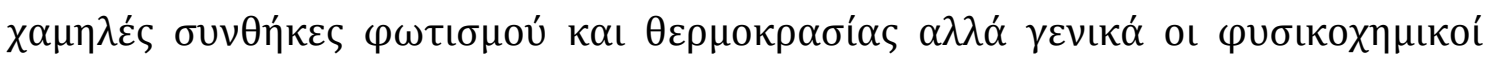

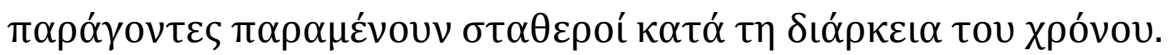

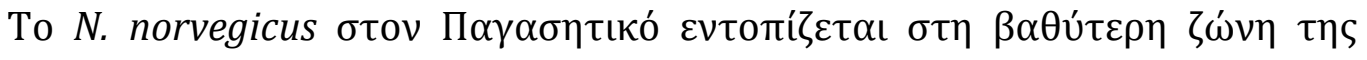

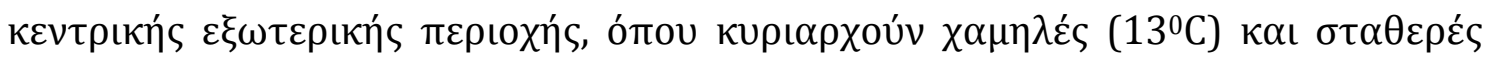

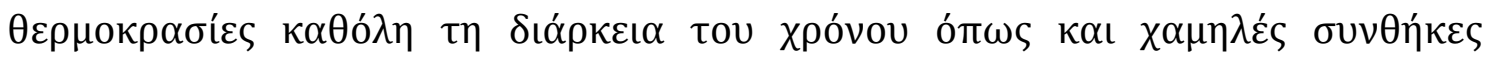

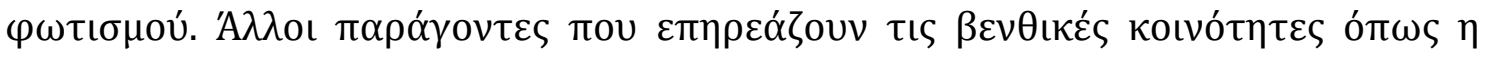

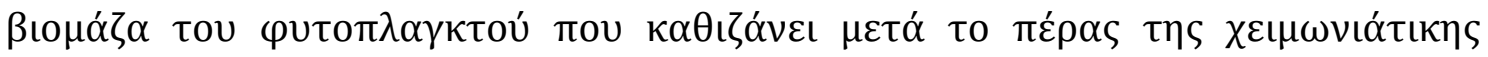

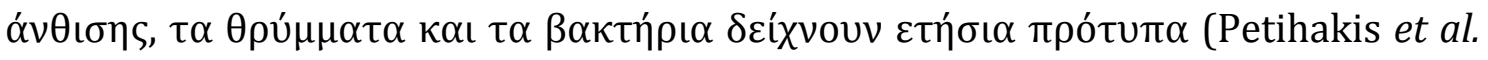

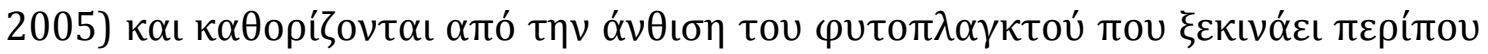

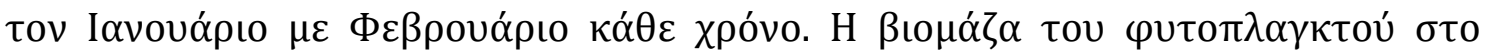

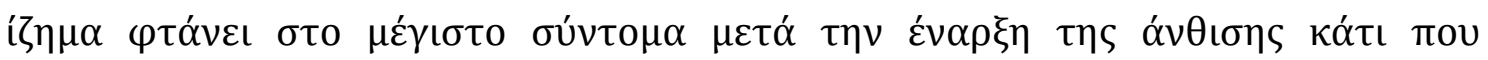

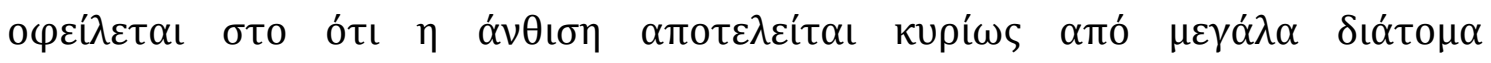

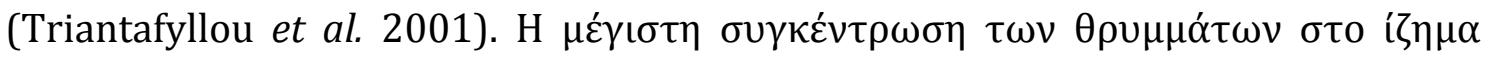

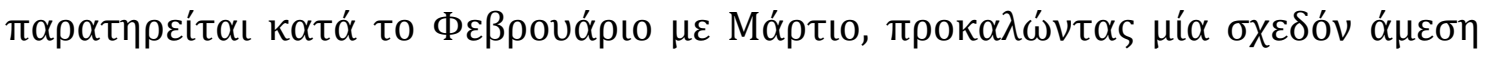

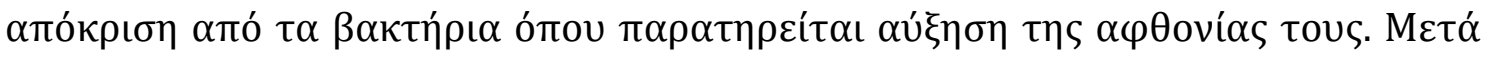




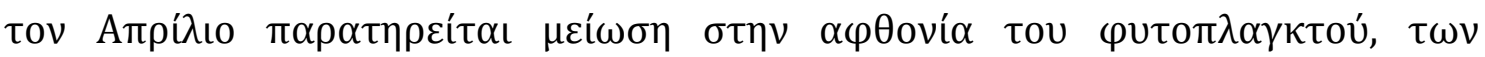

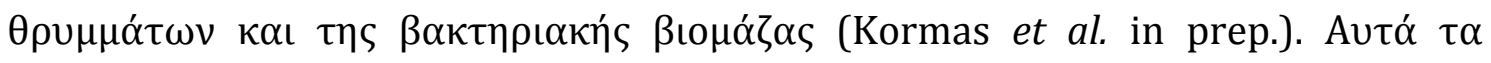

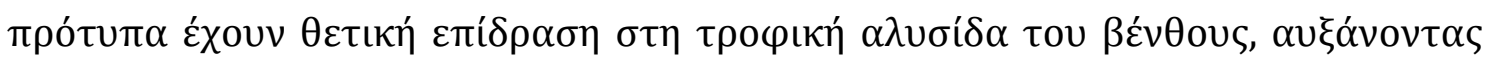

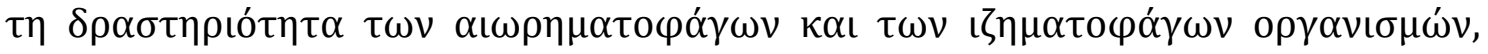

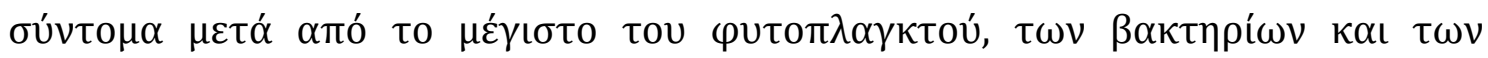

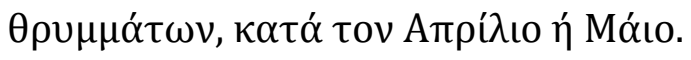

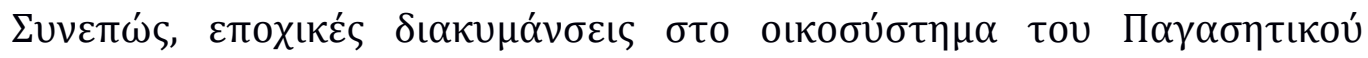

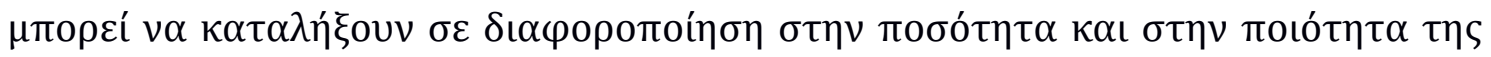

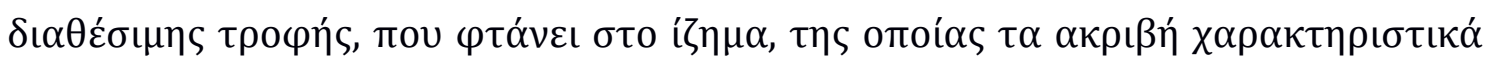

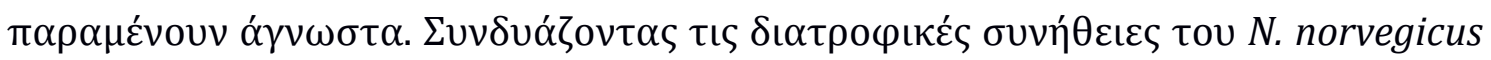

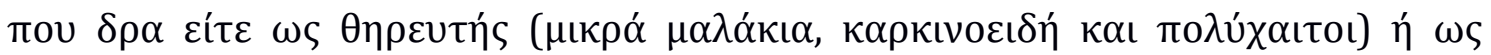

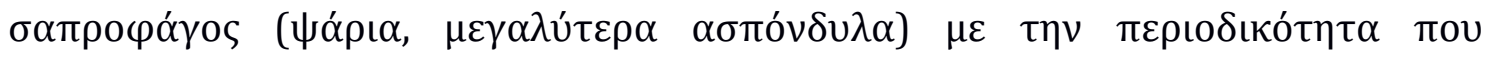

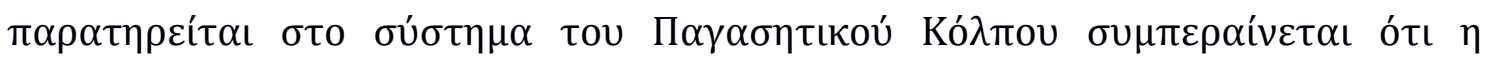

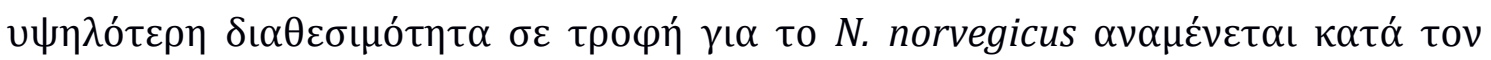

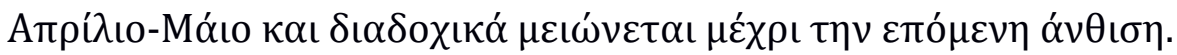

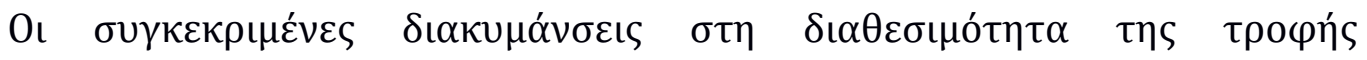

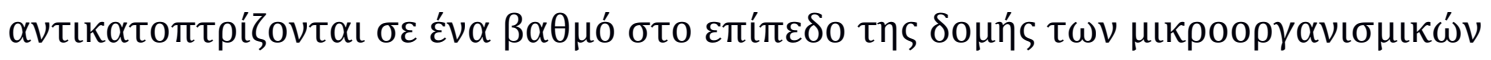

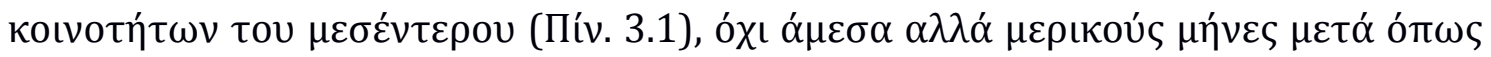

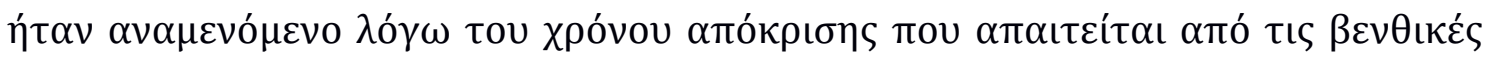

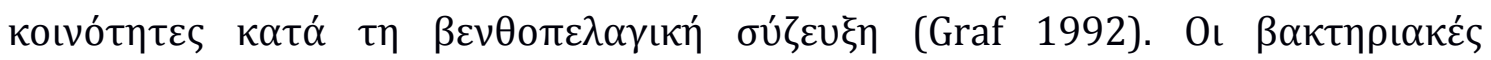

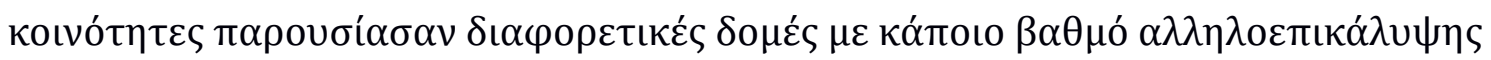

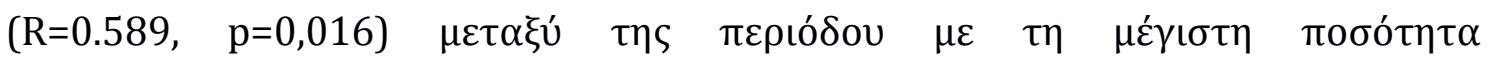

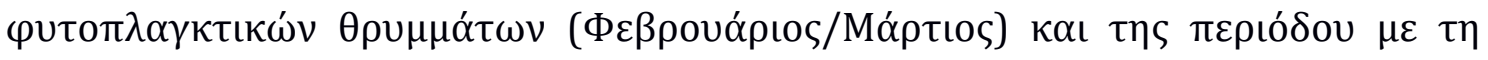

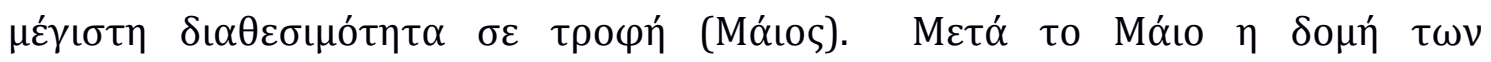




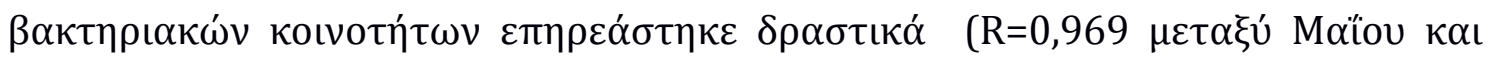

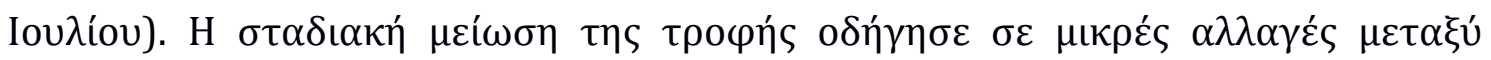

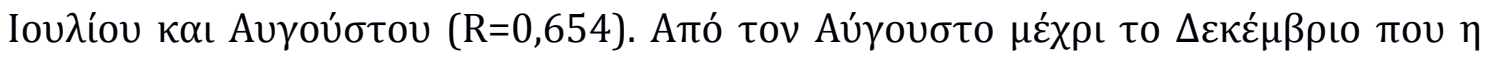

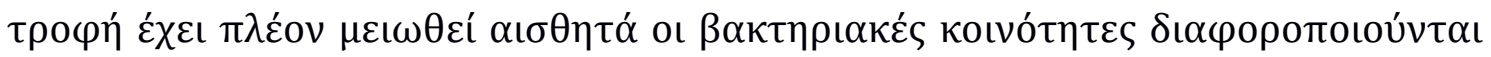

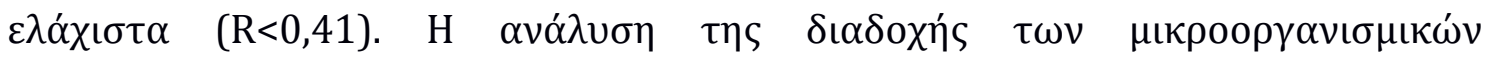

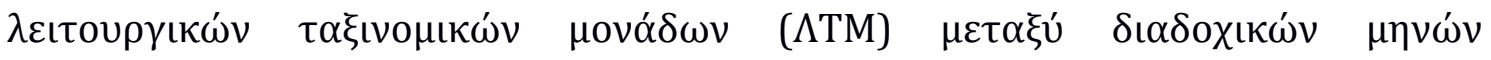

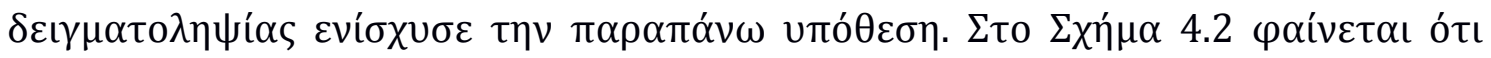

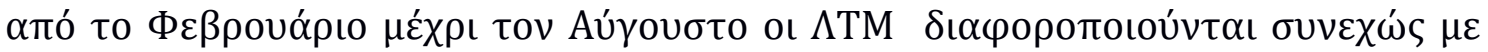

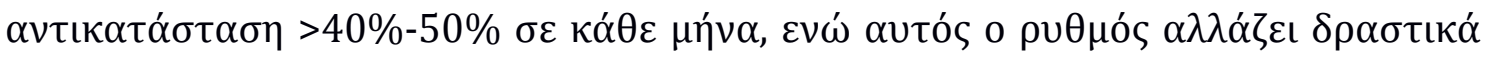

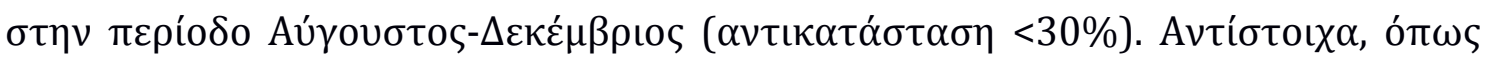

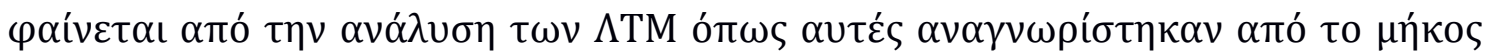

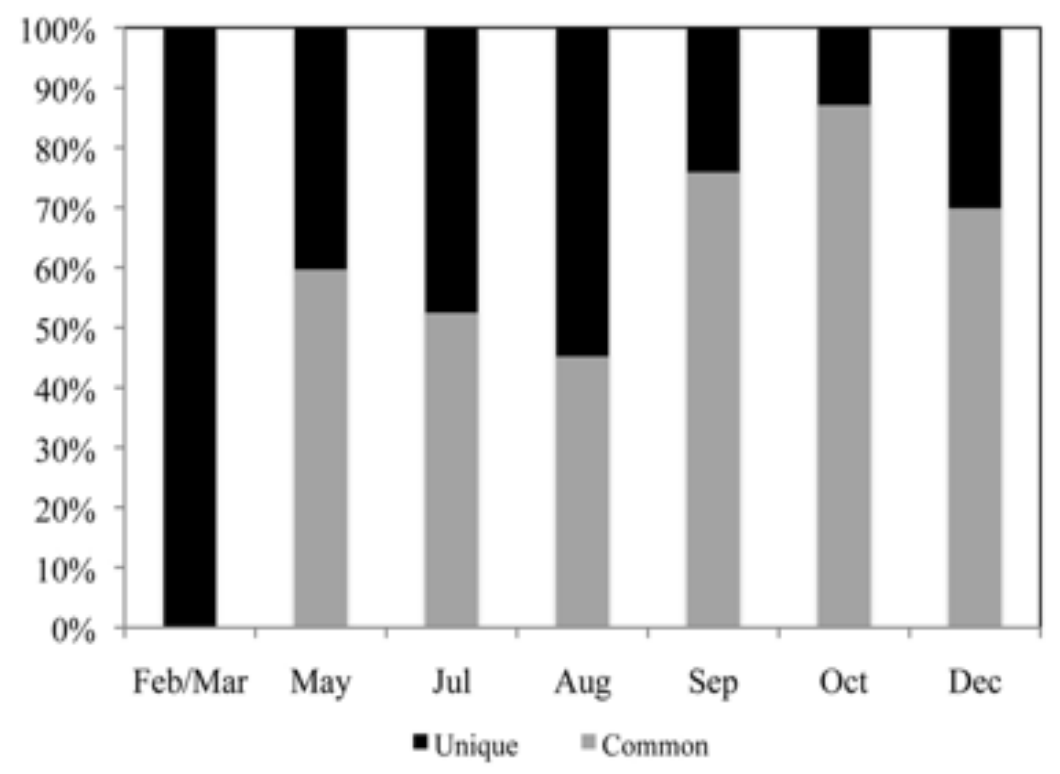

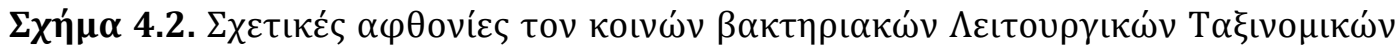

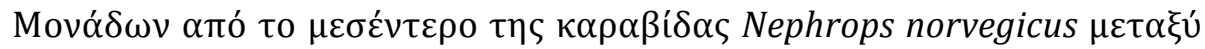

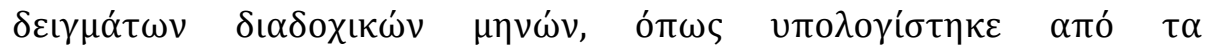

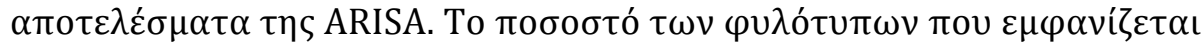

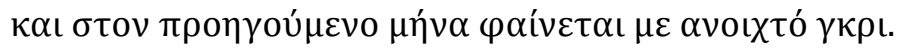




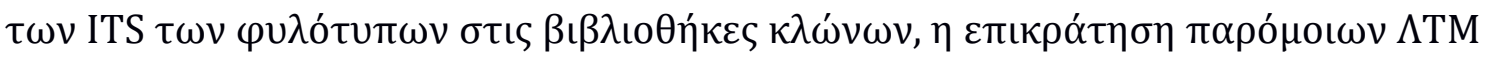

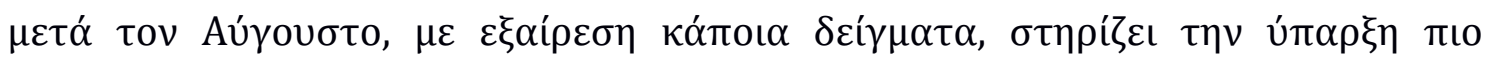

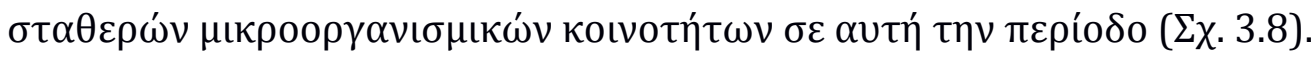

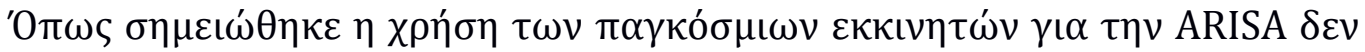

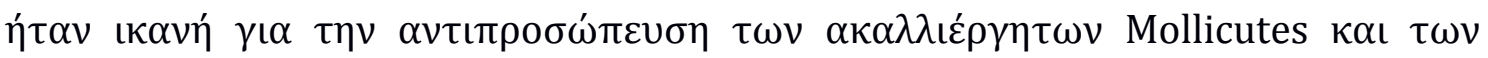

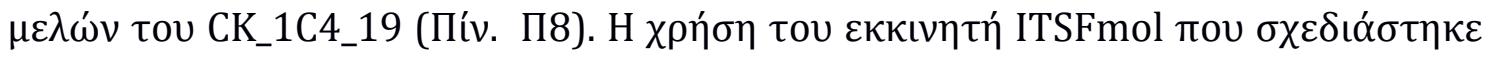

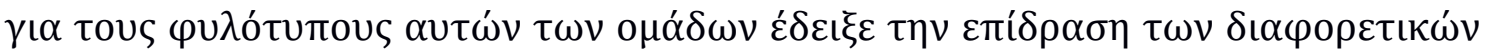

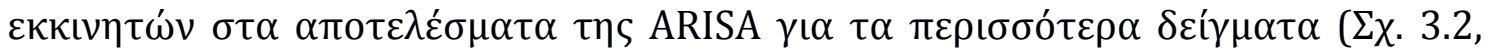

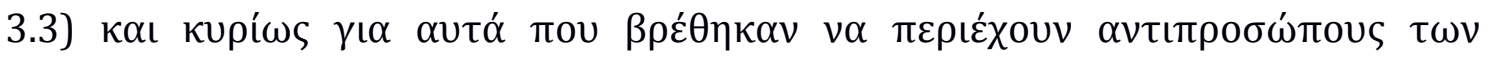

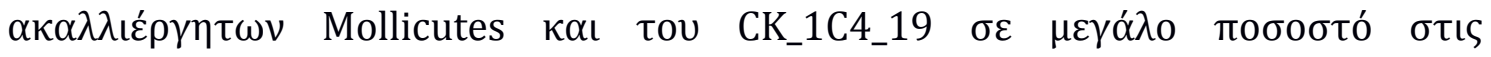

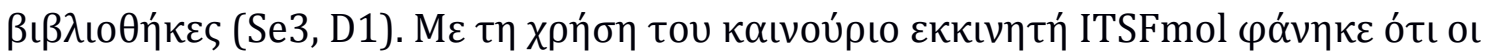

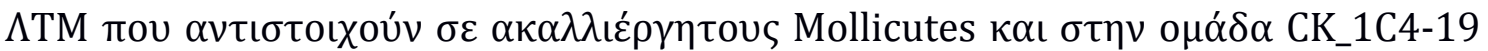

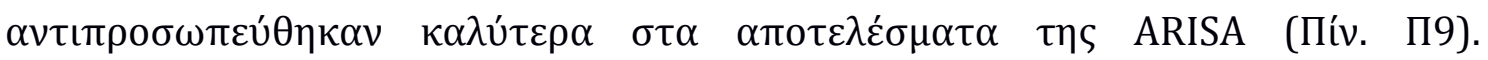

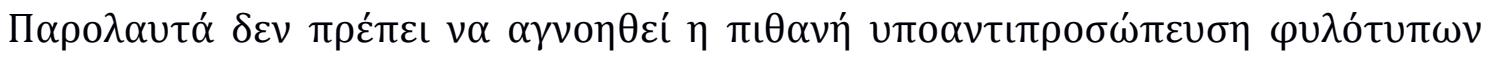

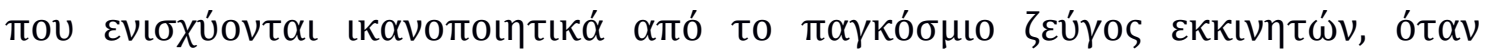

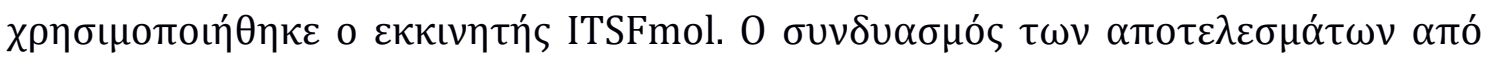

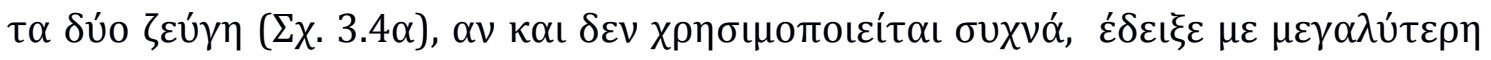

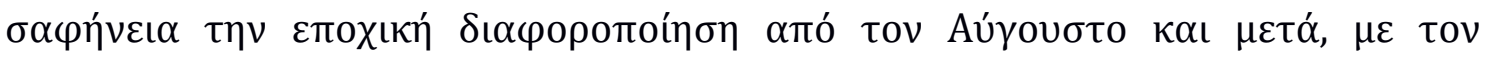

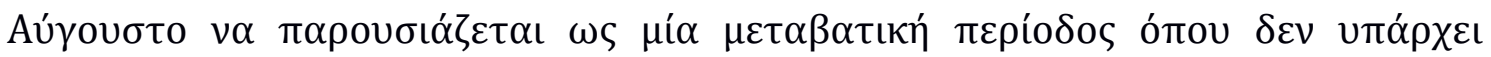

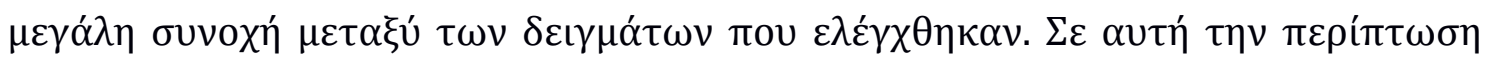

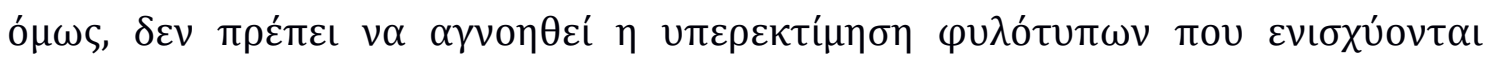

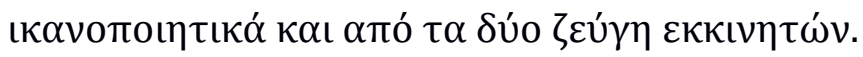

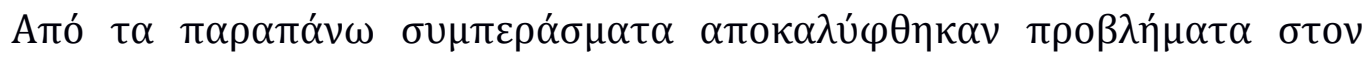

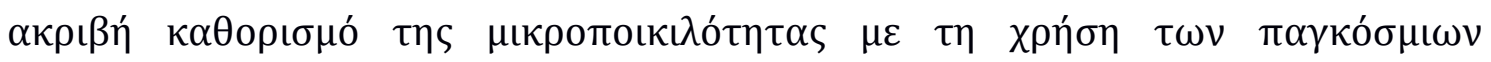




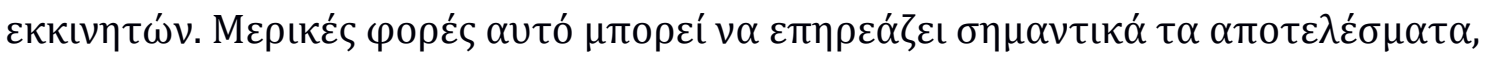

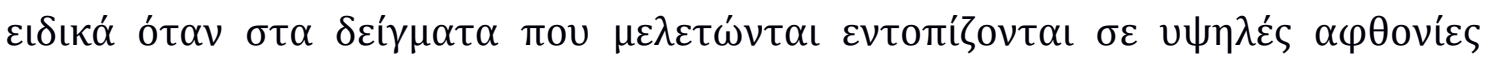

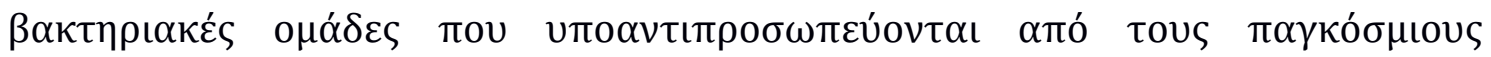

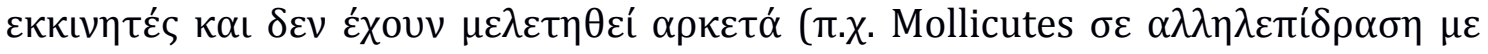

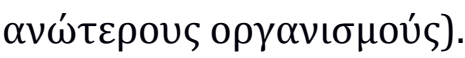

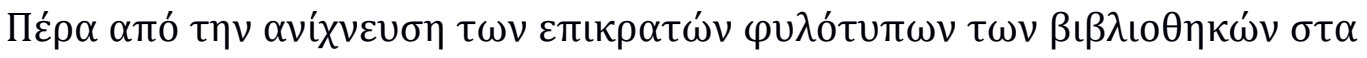

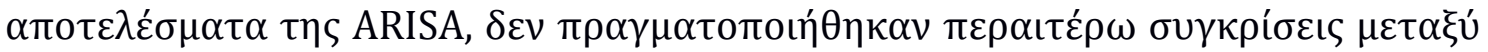

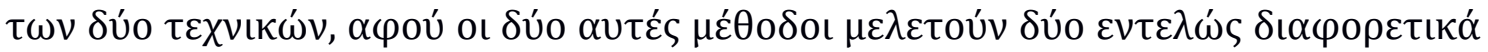

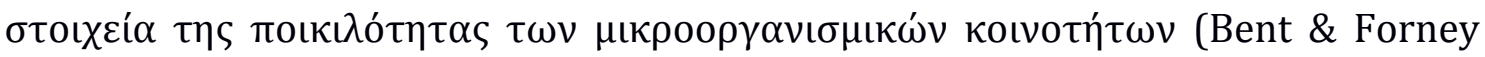

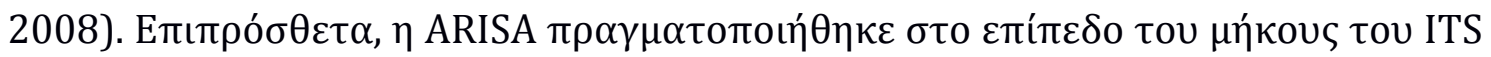
$\varepsilon v \omega ́$ oเ $\beta \iota \beta \lambda เ$ เо

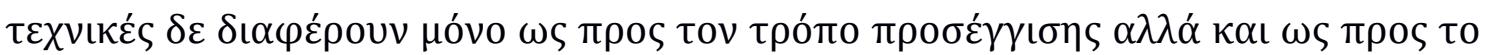

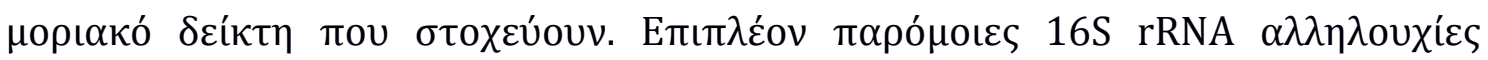

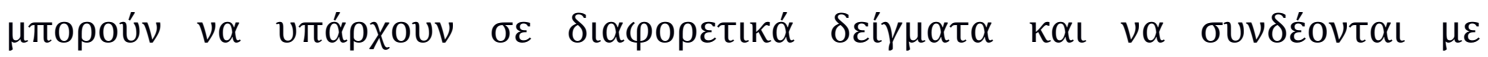

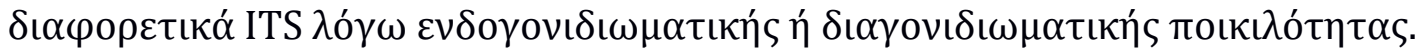

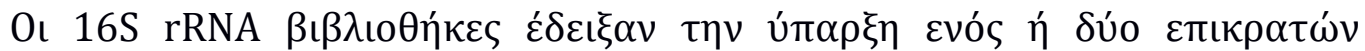

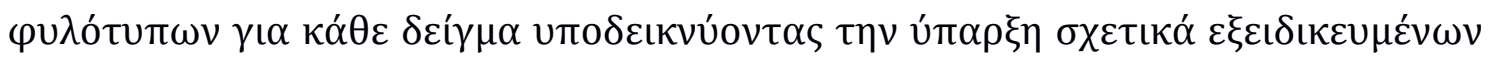

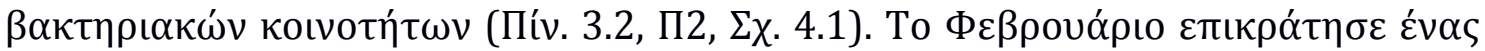

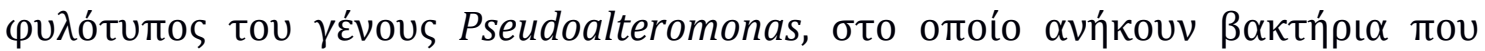

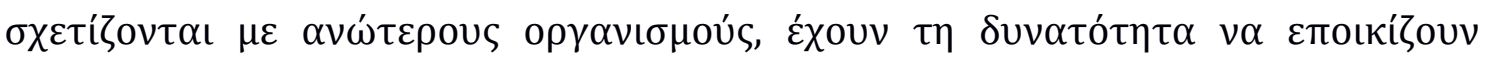

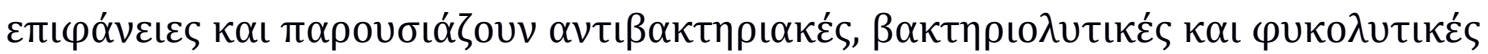

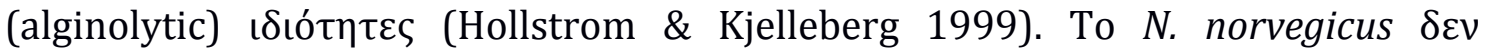

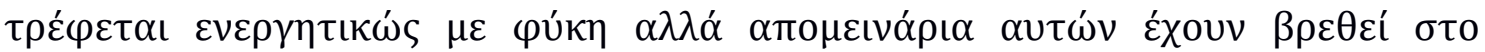

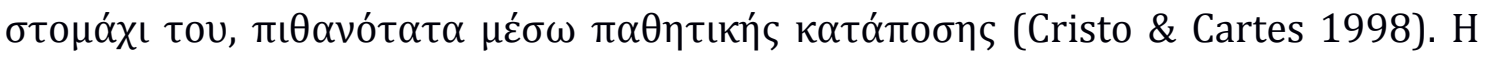




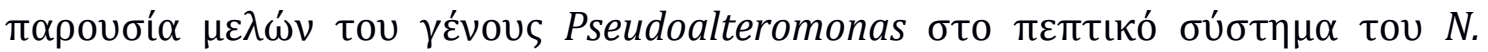

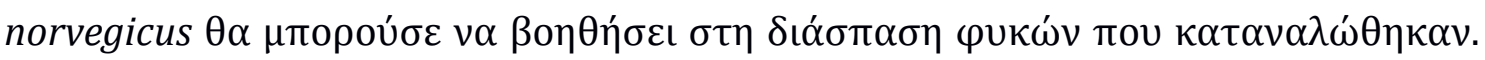

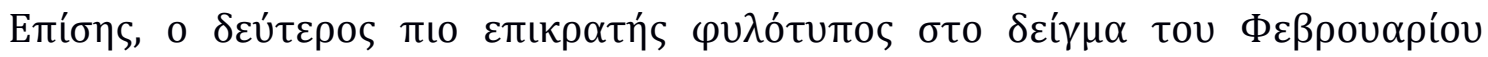

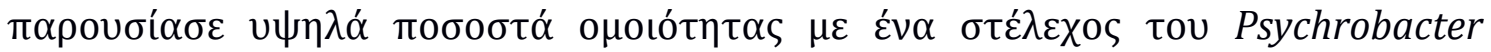

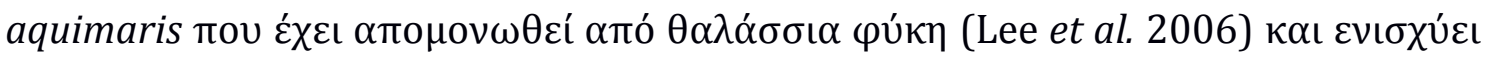

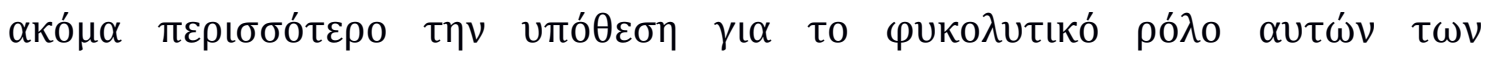

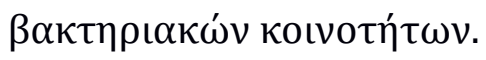

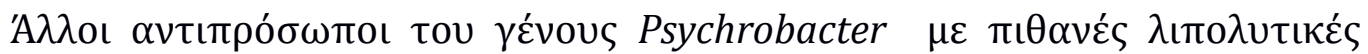

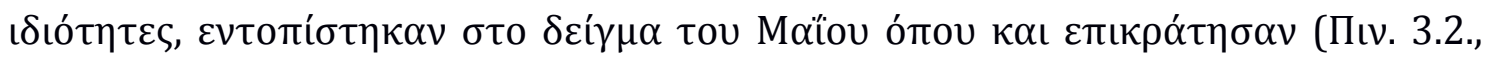

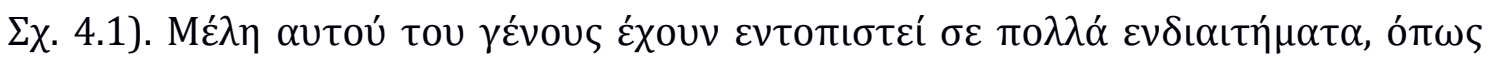

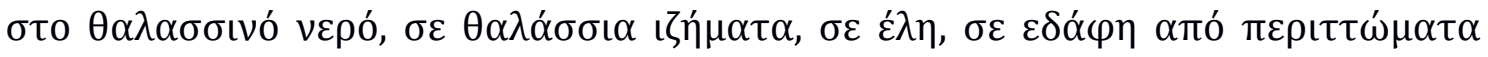

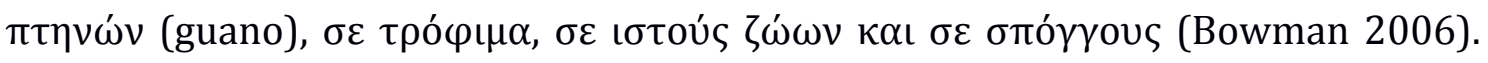

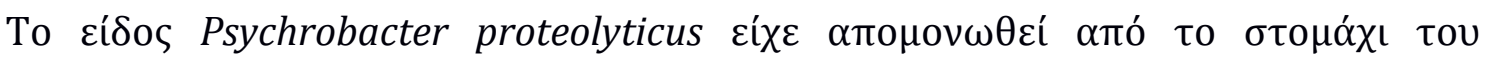

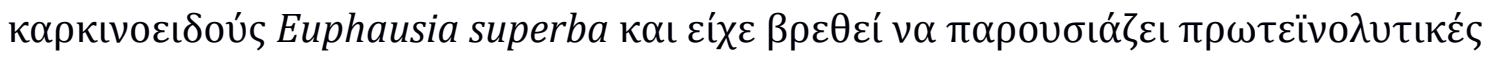

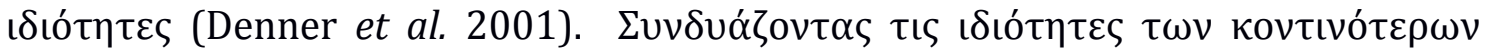

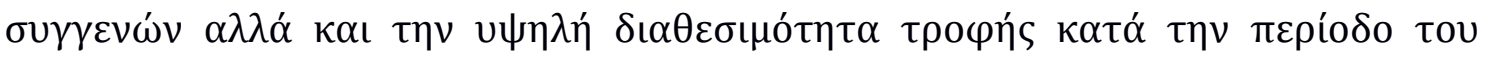

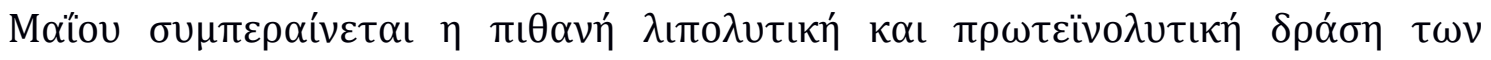

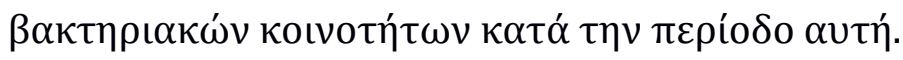

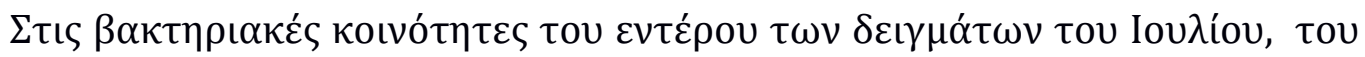

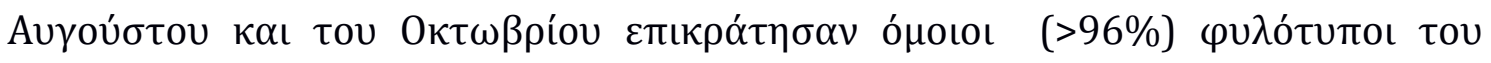

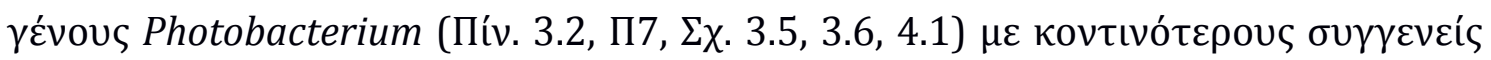

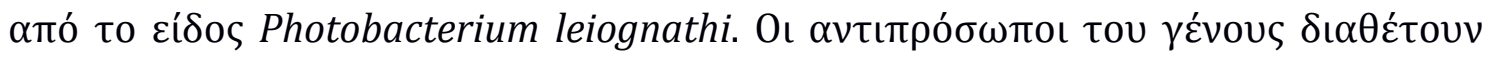

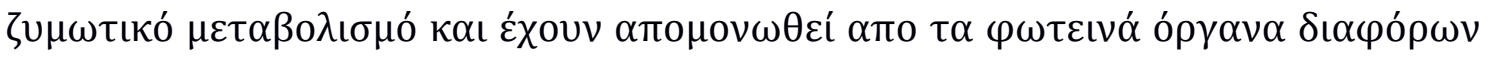

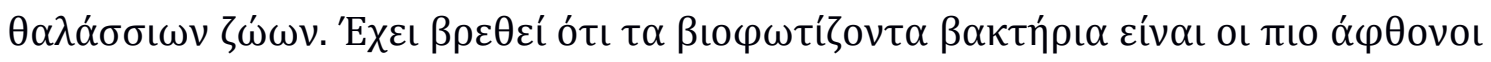




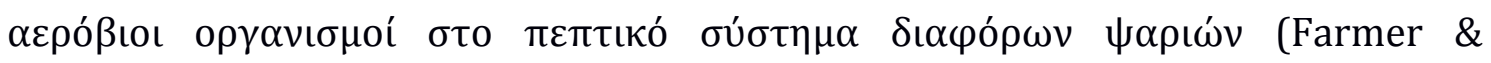

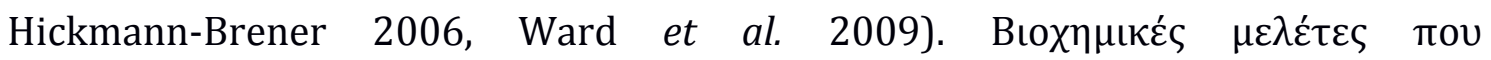

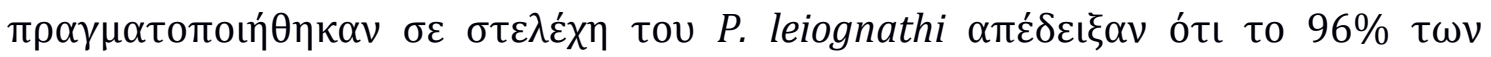

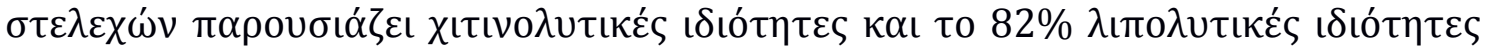

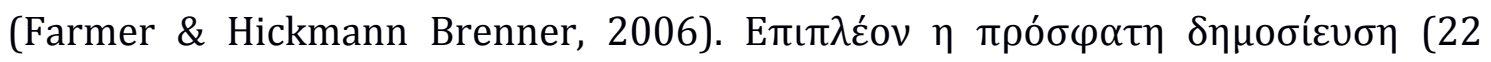

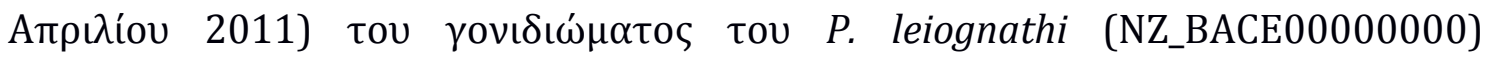

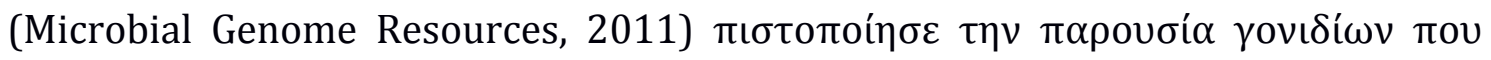

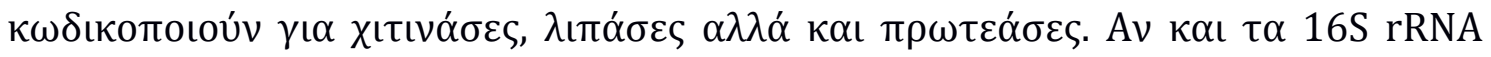

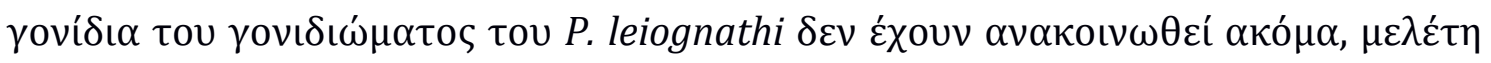

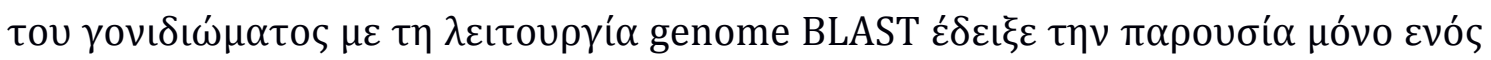

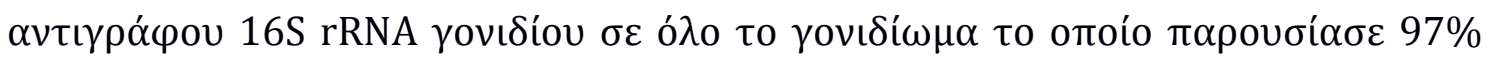

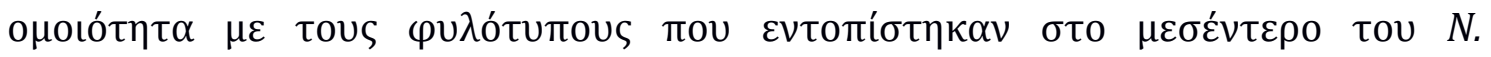

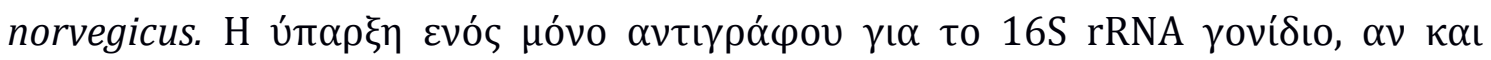

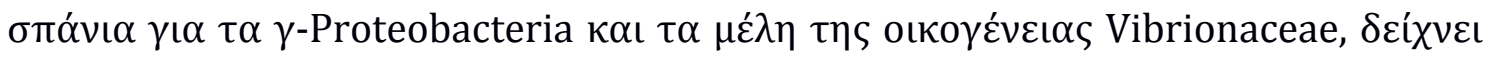

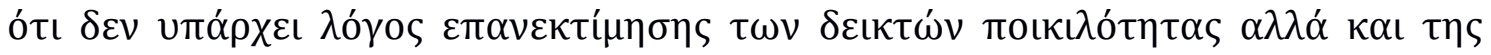
кód $\pi \varepsilon \rho i ́ \pi \tau \omega \sigma \eta \beta$

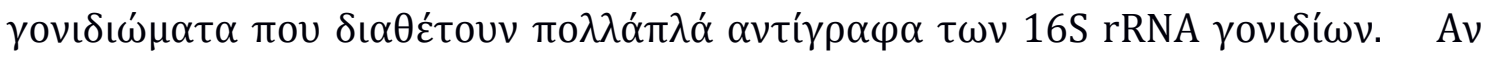

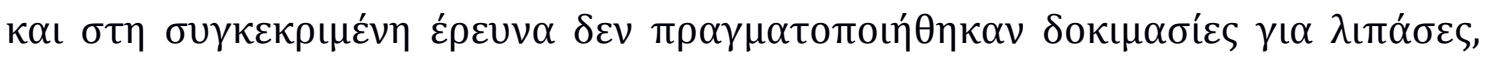

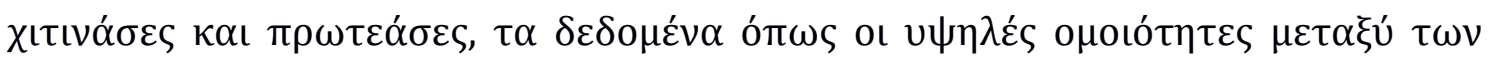

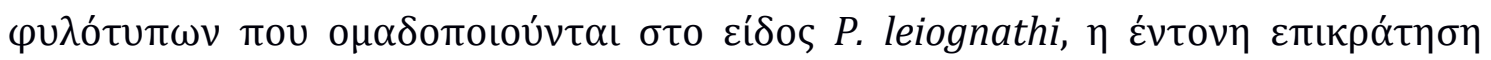

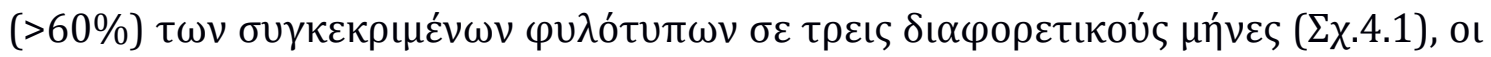

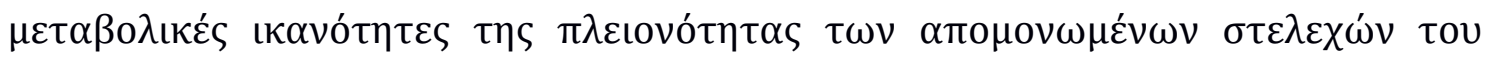

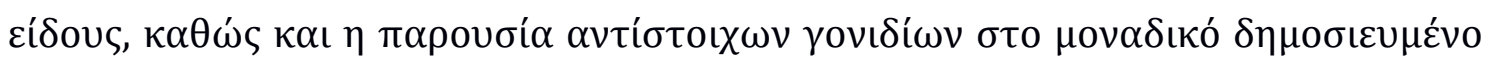




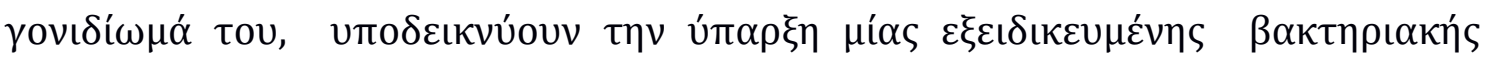

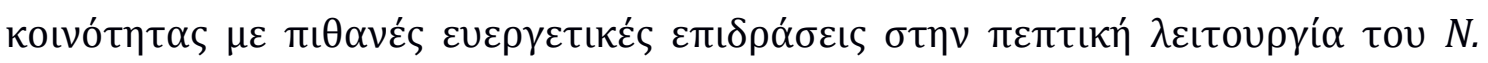

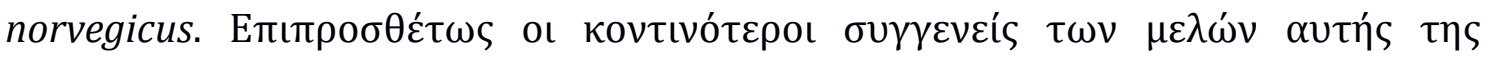

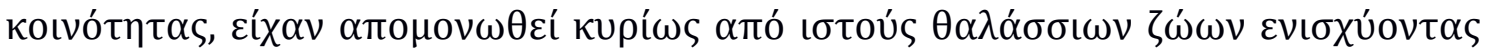

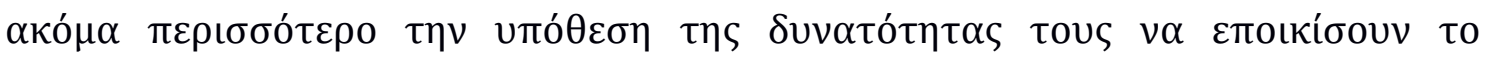
$\mu \varepsilon \sigma \varepsilon ́ v \tau \varepsilon \rho о$ tou N. norvegicus .

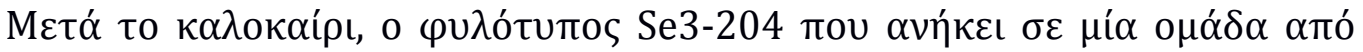

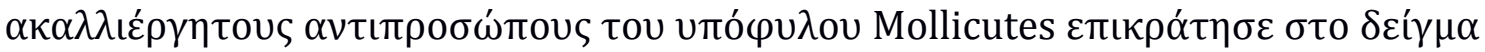

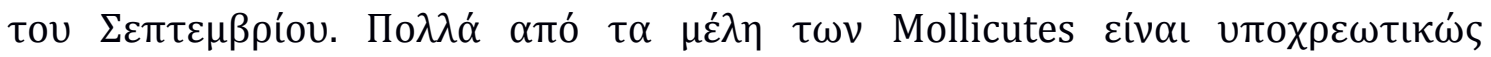

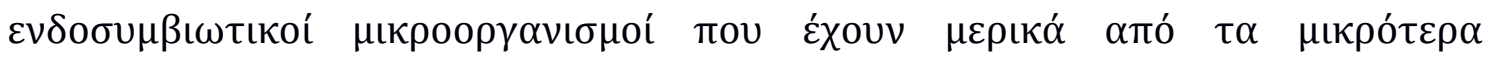

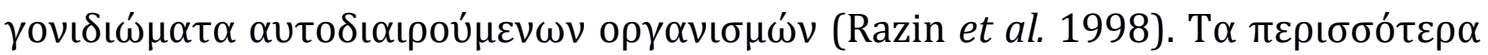

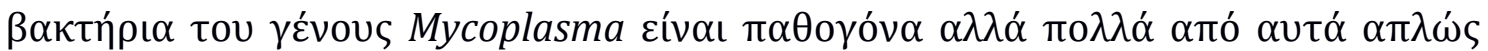

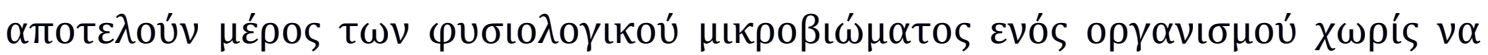

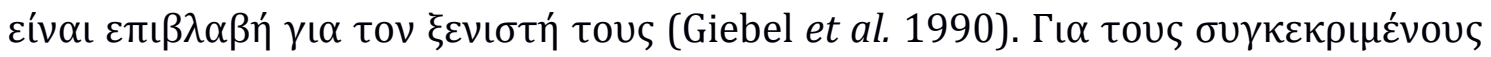

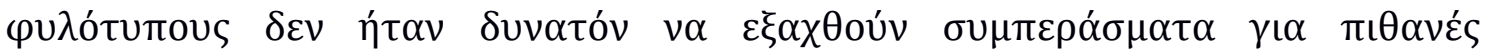

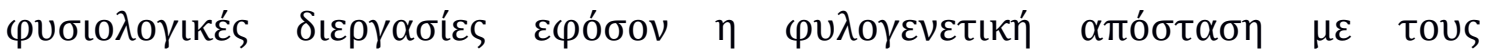

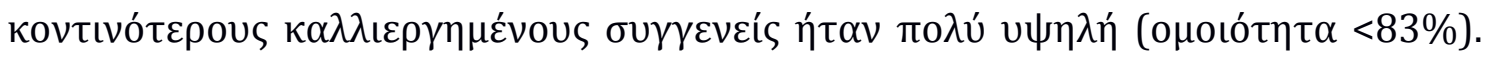

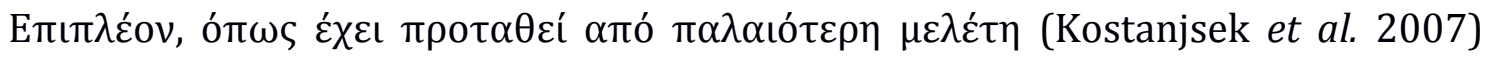

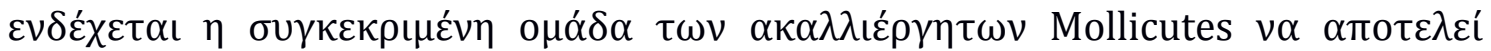

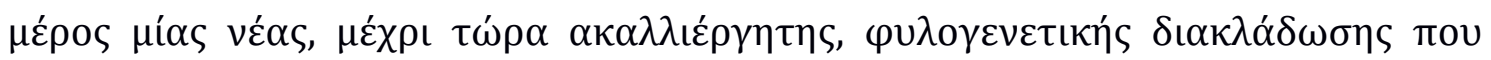

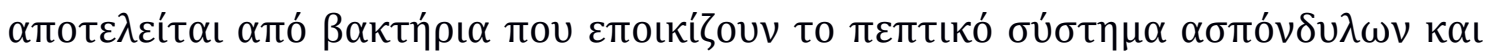

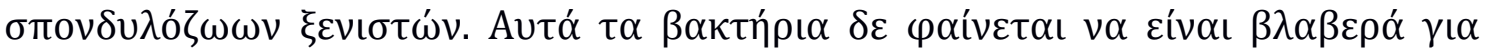

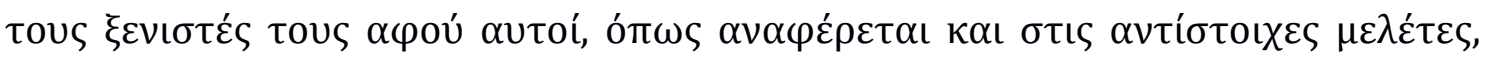




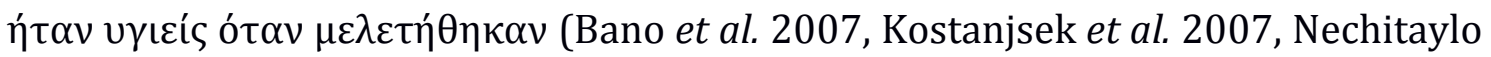
et al. 2009, Ward et al. 2009).

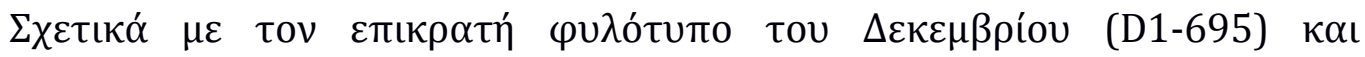

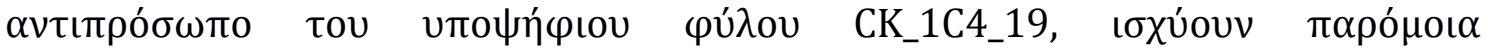

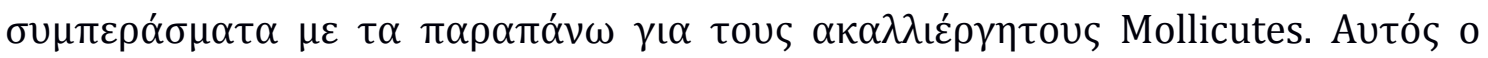

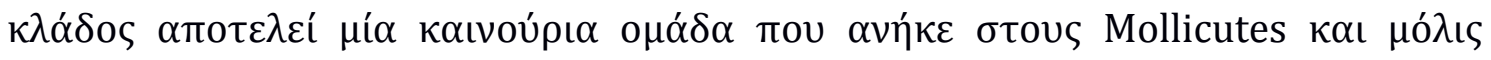

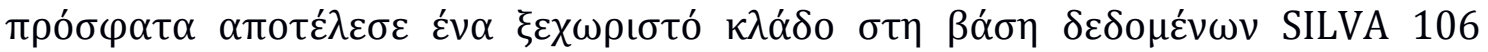
(Pruesse et al. 2007, SILVA 2011).

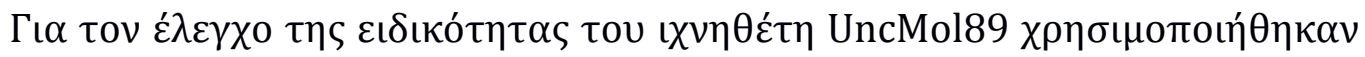

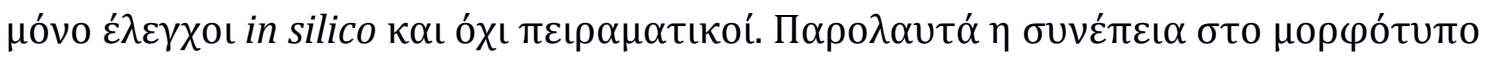

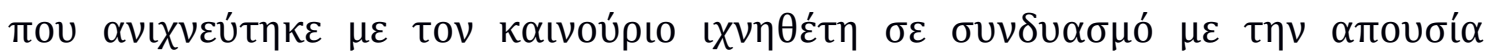

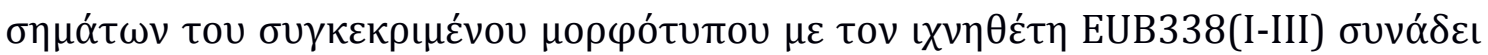

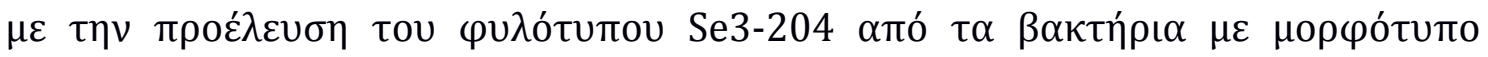

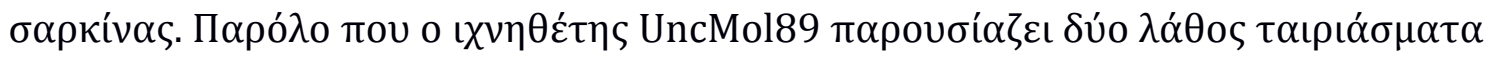

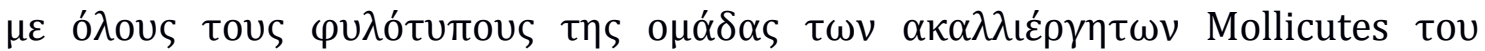

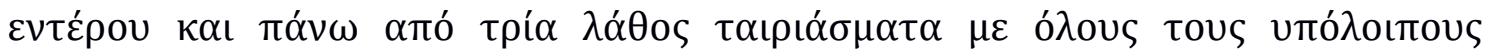

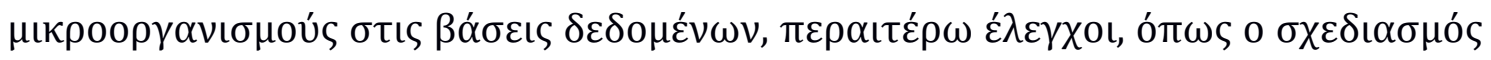

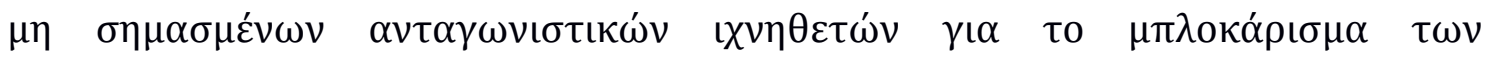

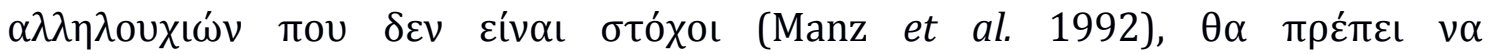

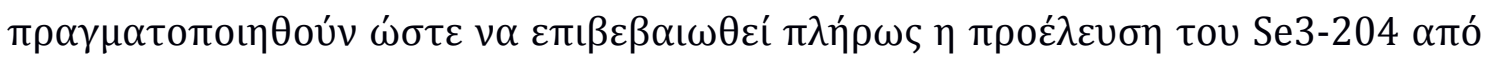

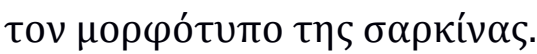

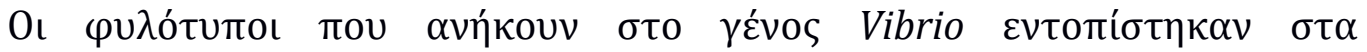

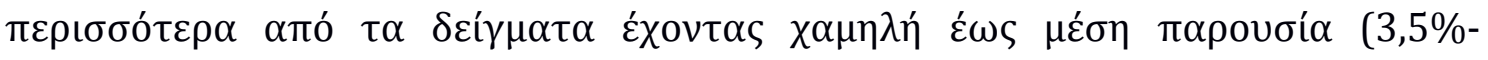

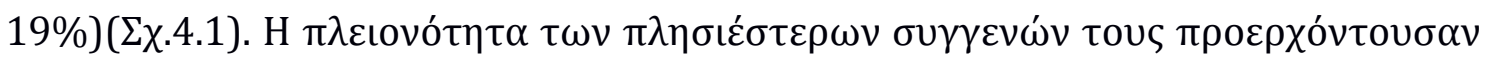




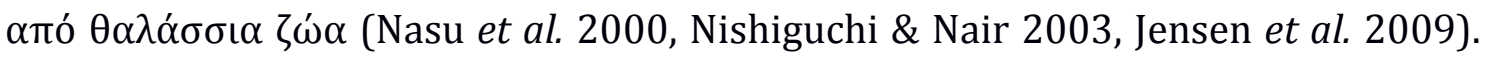

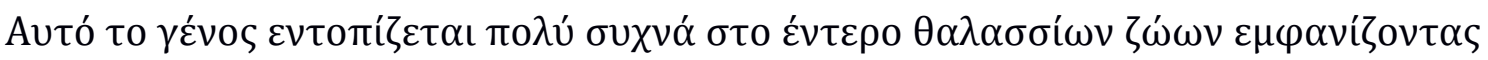

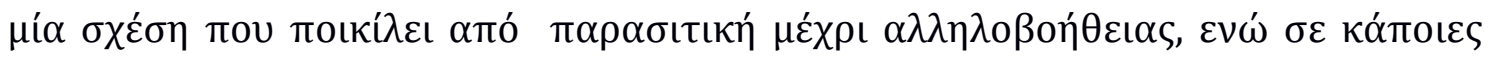

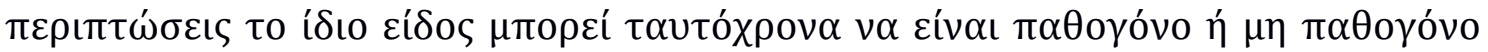

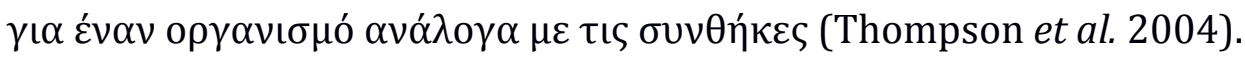

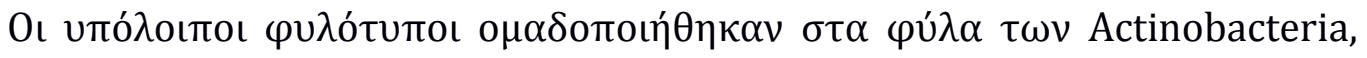
Acidobacteria, Chloroflexi, Firmicutes $\kappa \alpha \iota ~ \sigma \tau \alpha$ vтó $\varphi v \lambda \alpha \tau \omega \nu \alpha-, \beta-\kappa \alpha \iota ~ \delta$ -

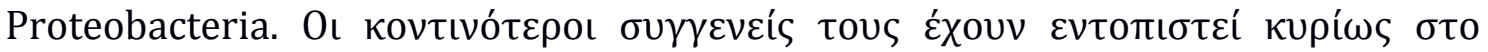

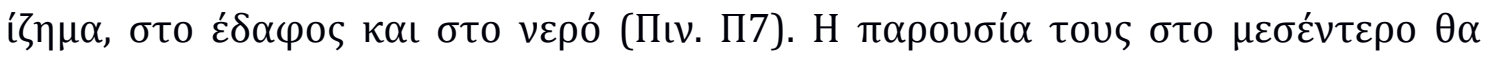

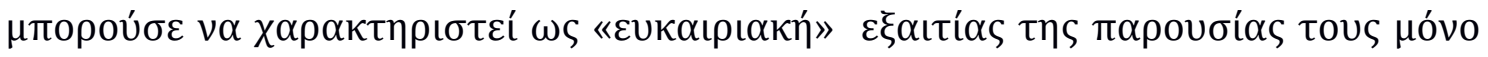

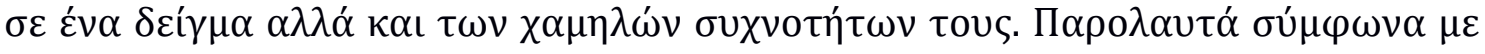

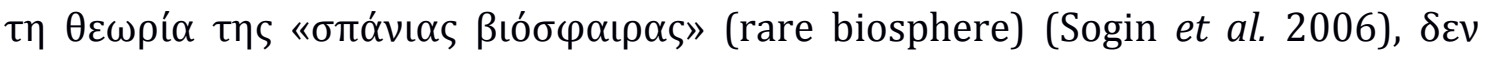

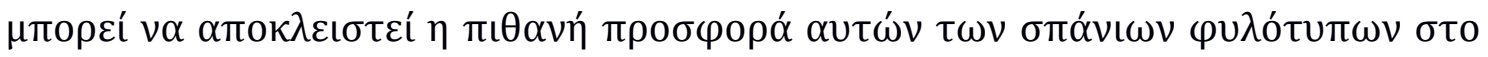

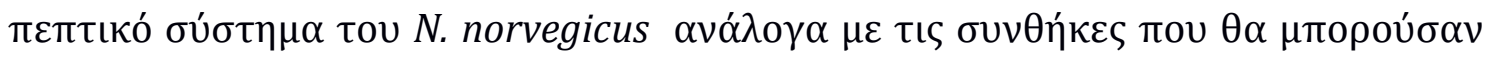

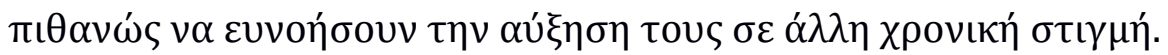

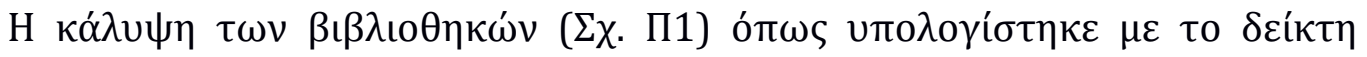

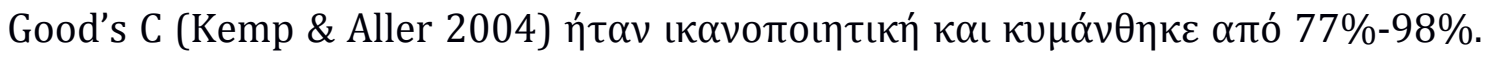

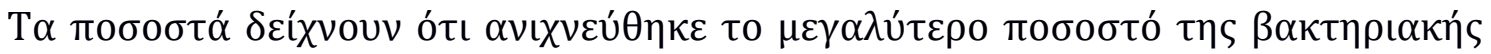

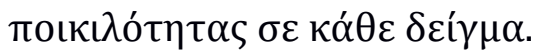

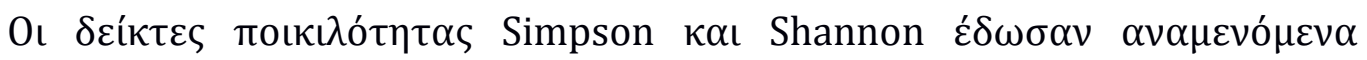

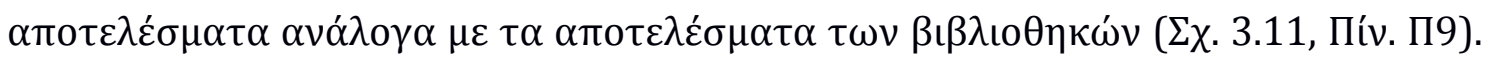

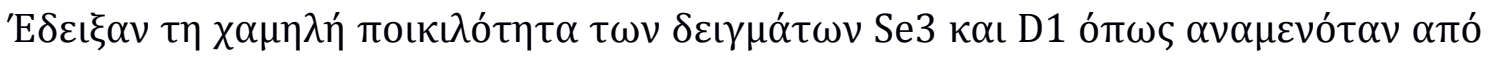

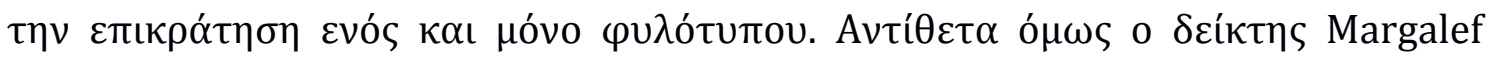

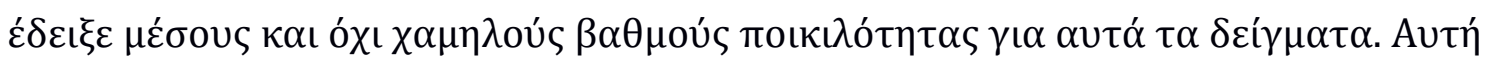




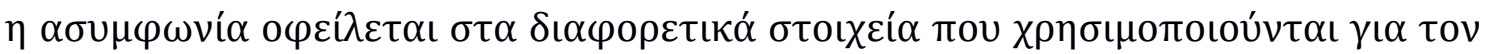

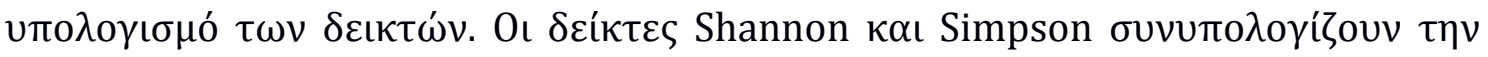

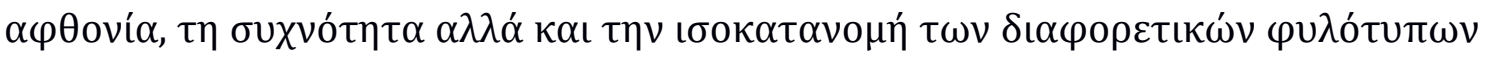

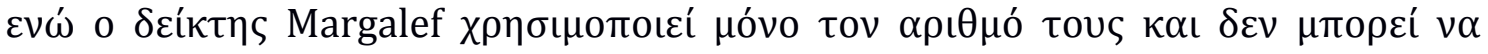

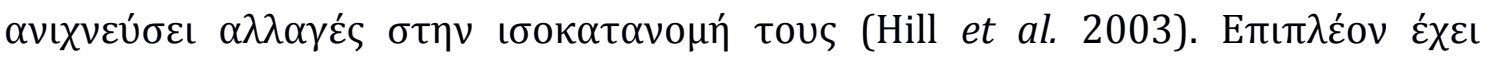

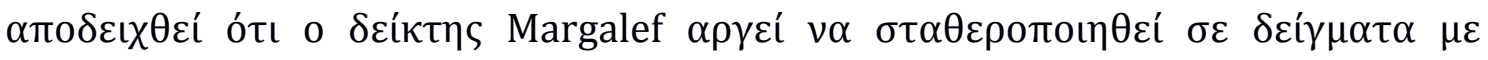

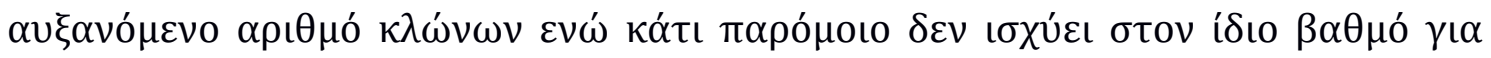

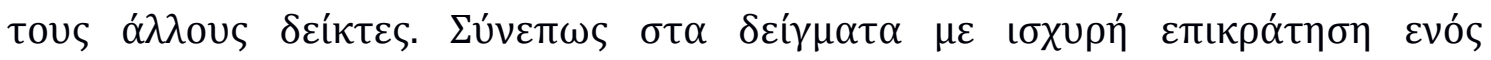

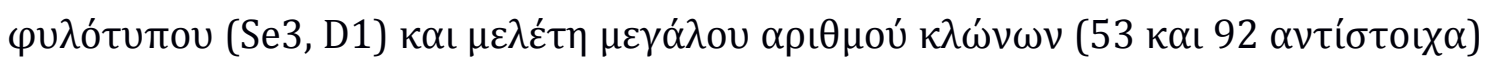

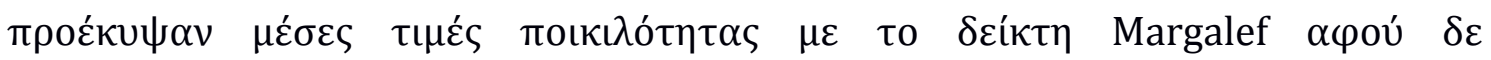

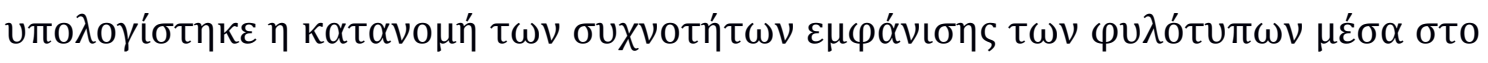
$\delta \varepsilon i ́ \gamma \mu \alpha$.

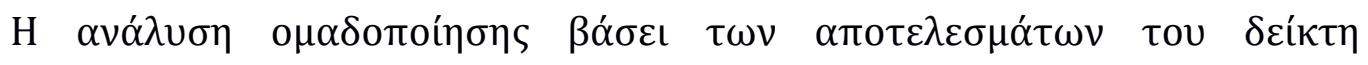

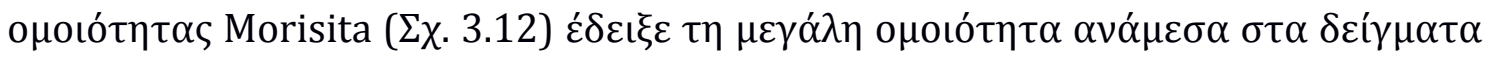

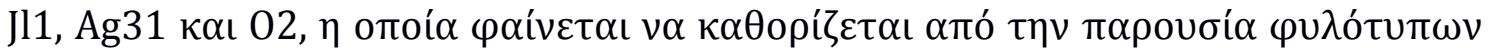

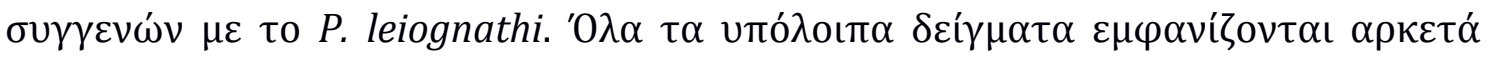

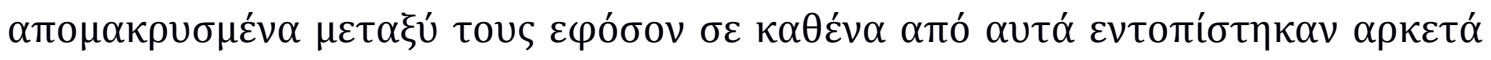

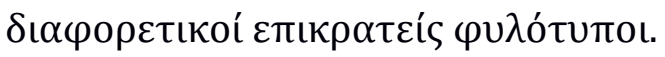

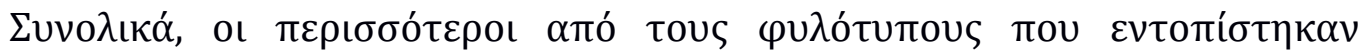

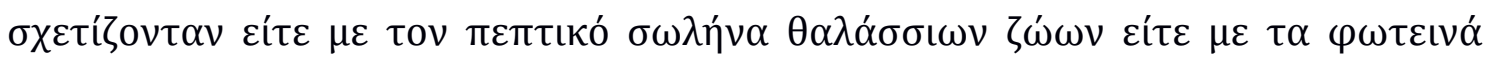

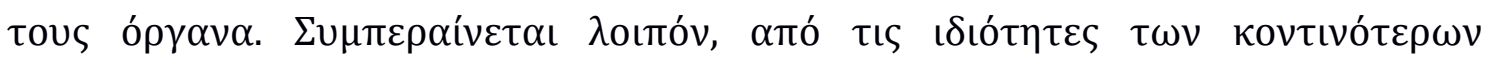

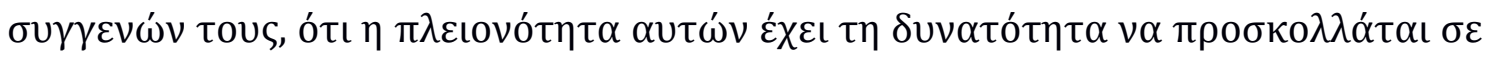

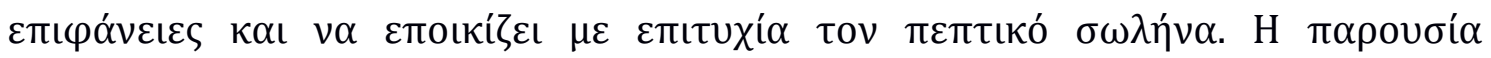

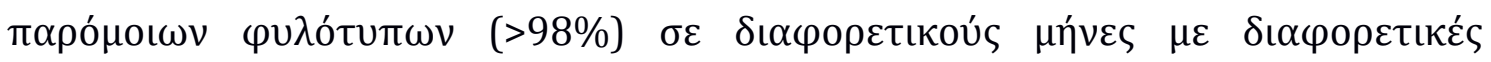




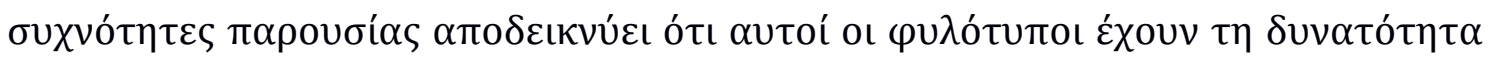

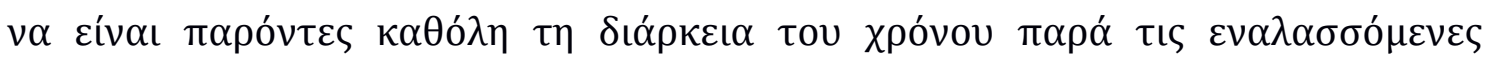

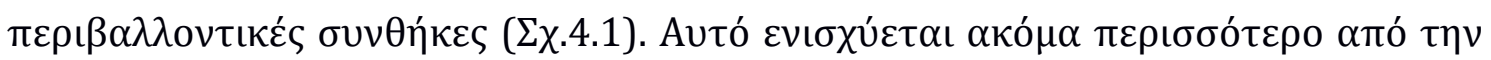

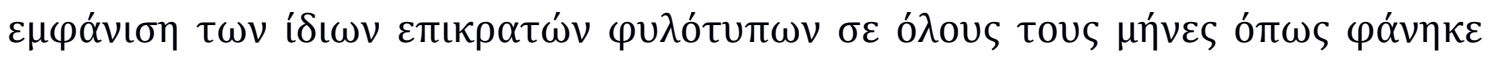

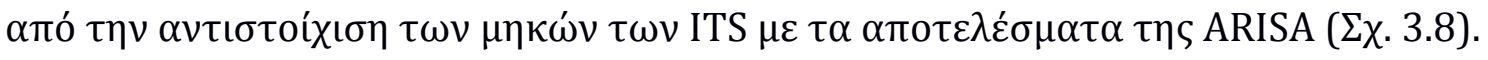

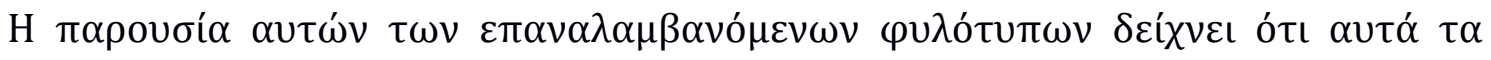

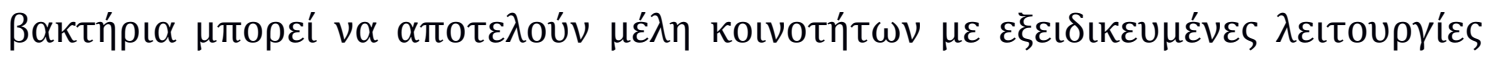

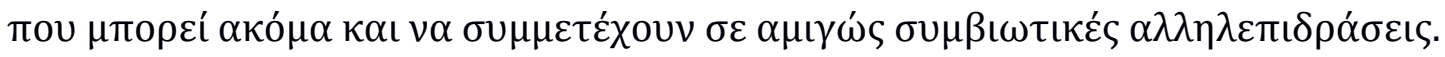

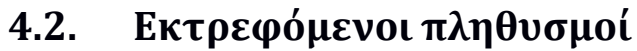

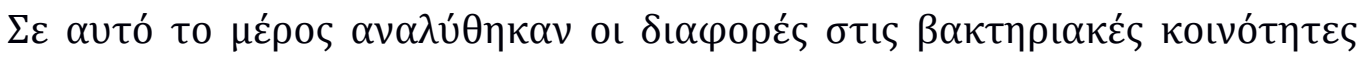

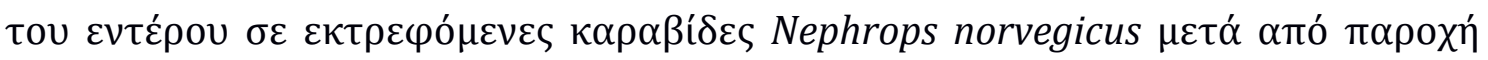

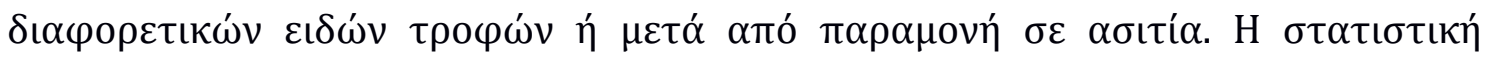

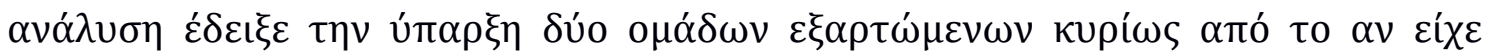

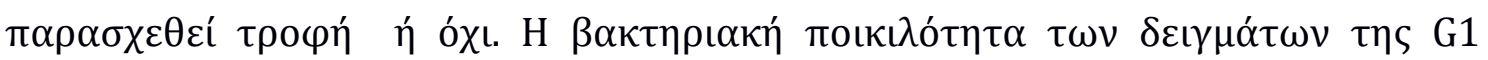

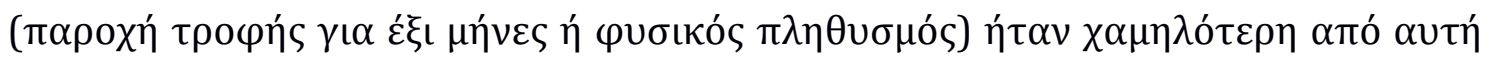

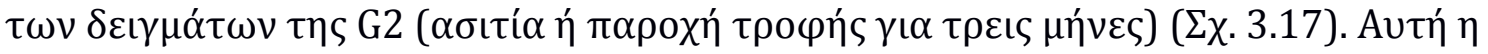

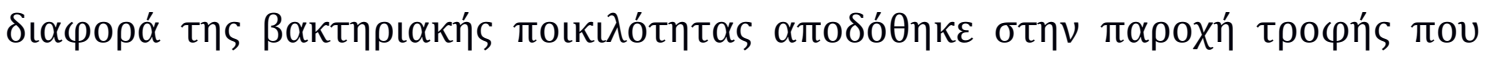

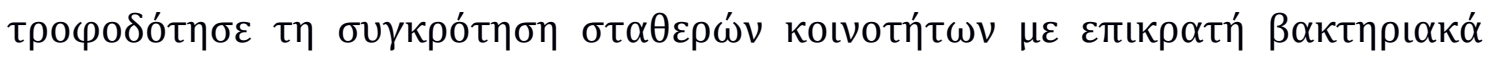

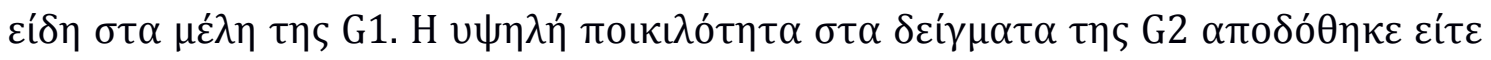

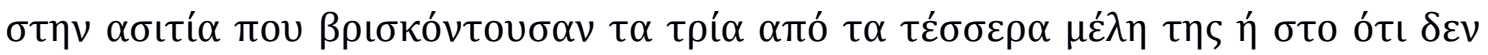

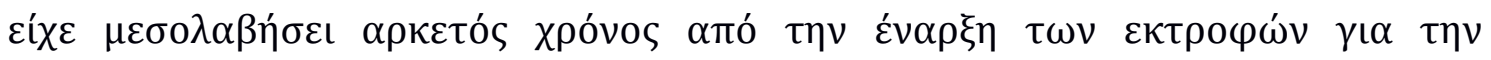




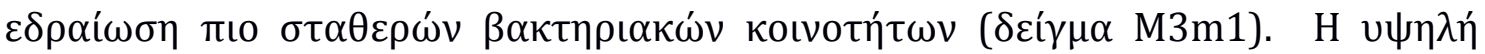

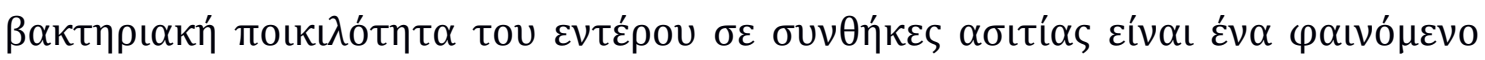

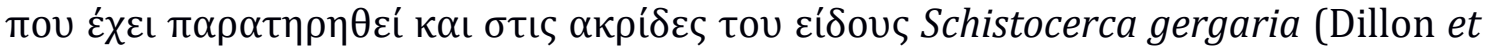

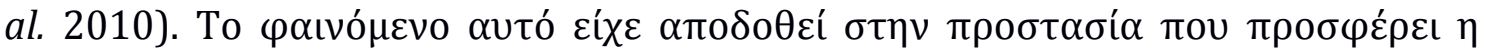

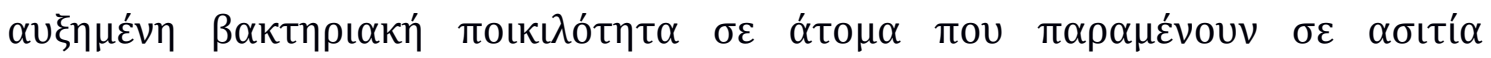

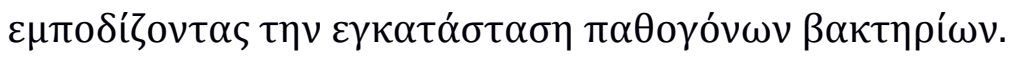

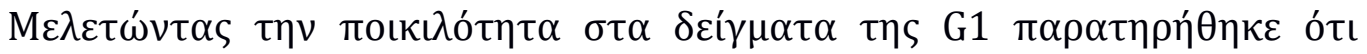

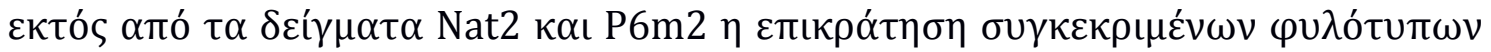

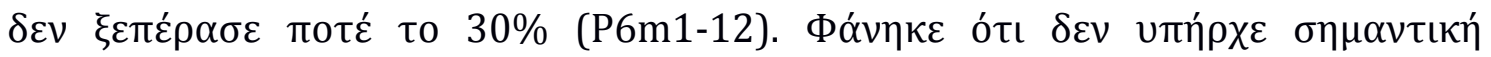

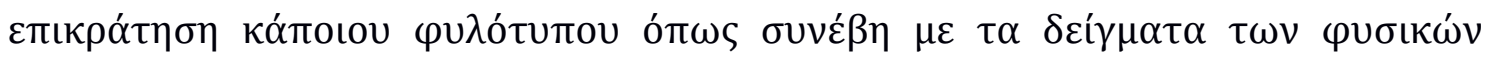

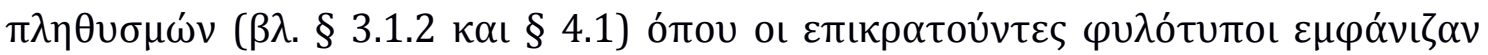

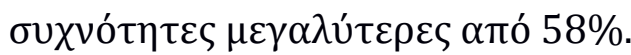

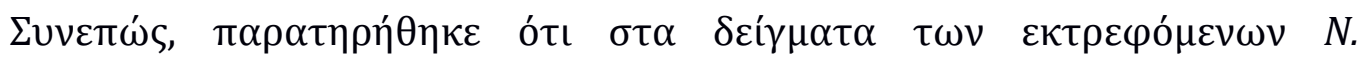

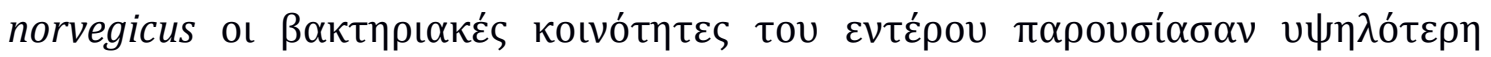

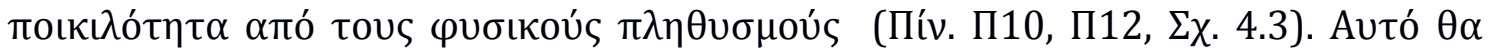

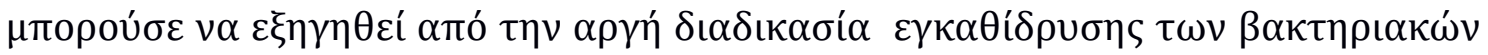

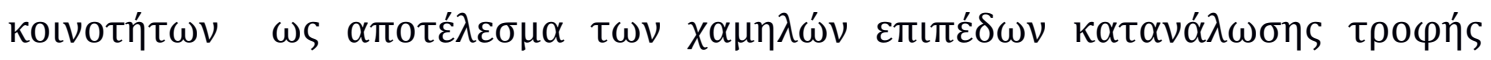

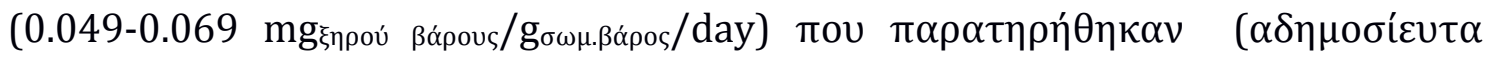

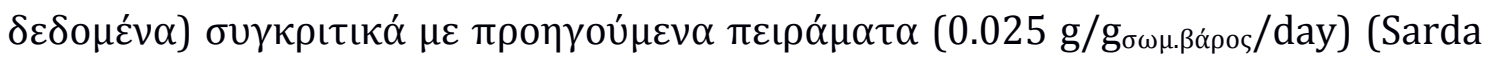
\& Valladares 1990).

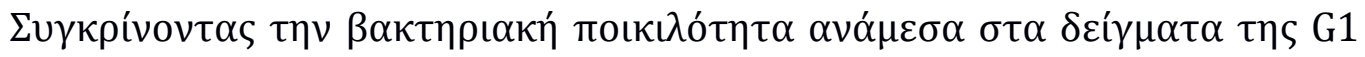

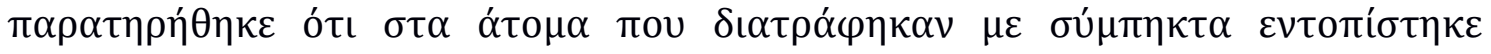

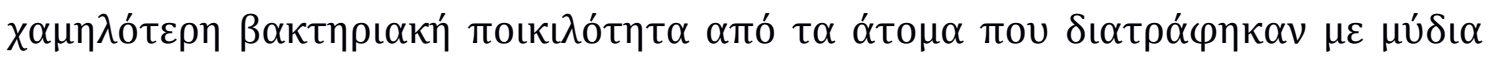
$(\Sigma \chi \cdot 4.3)$ 


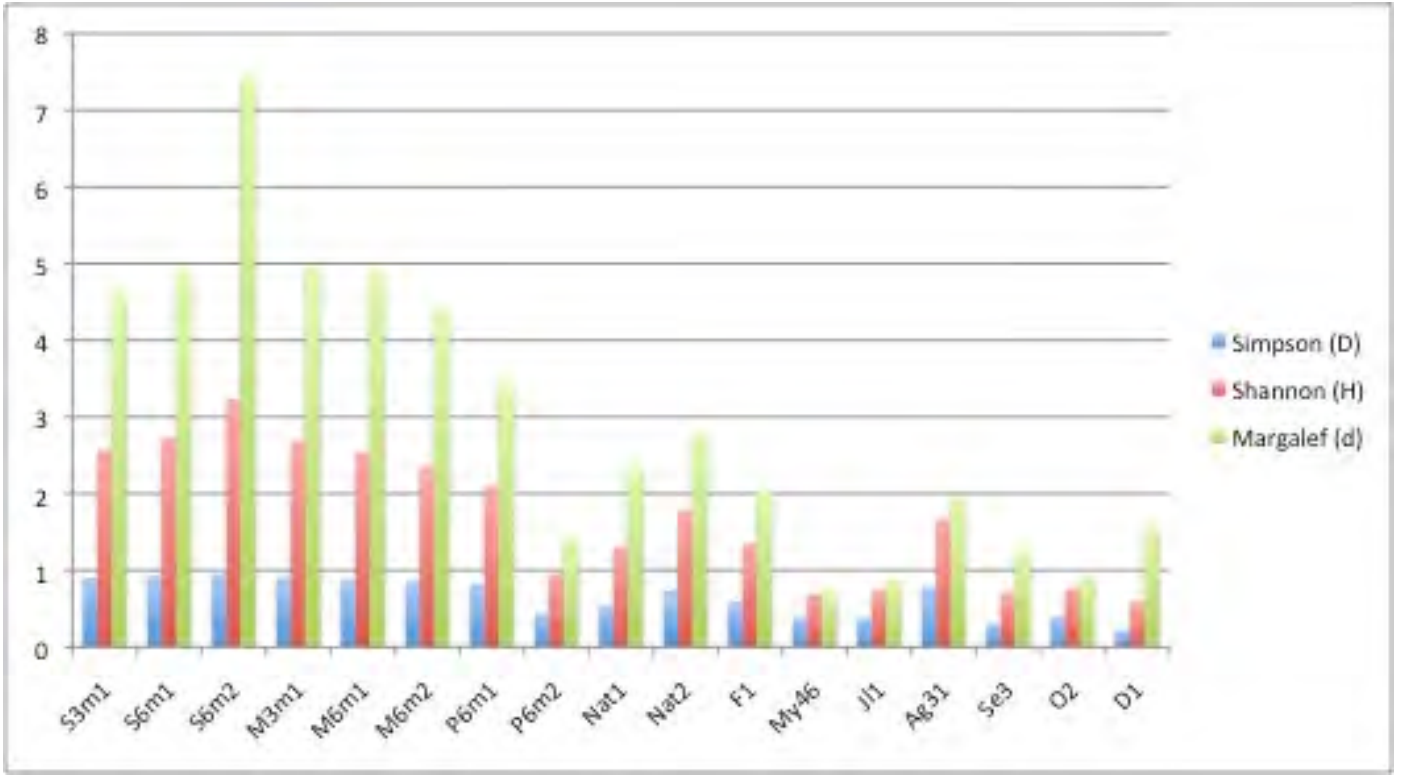

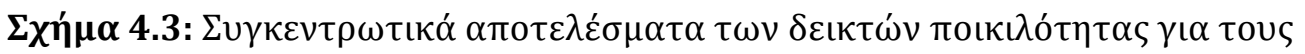

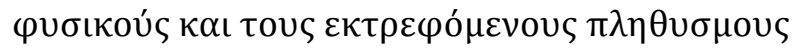

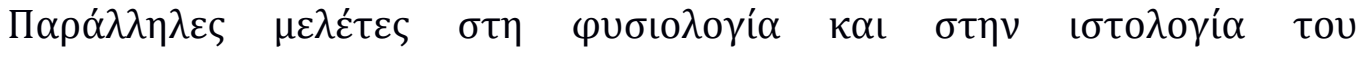

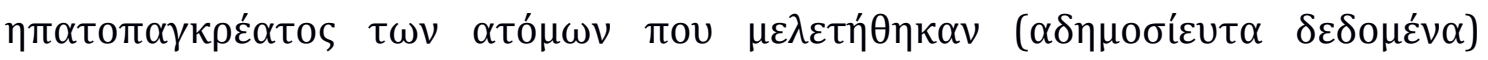

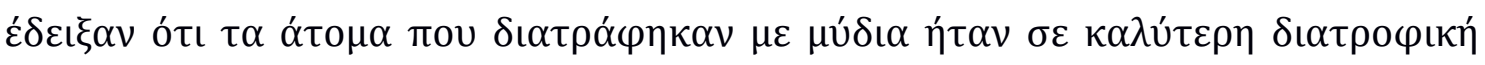

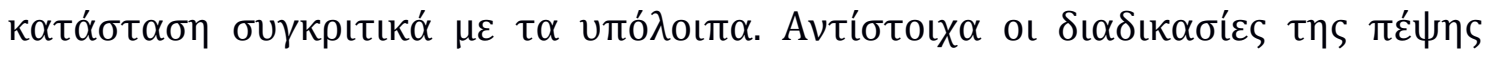

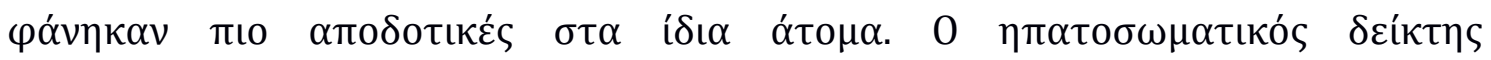

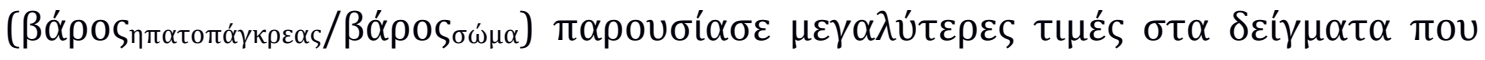

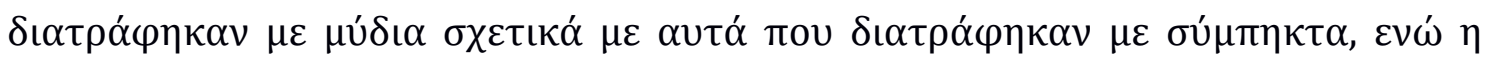

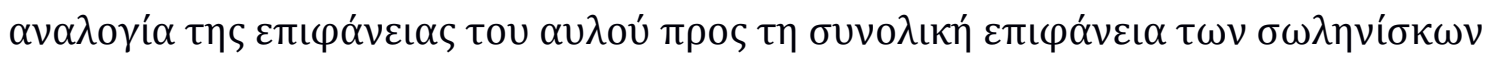

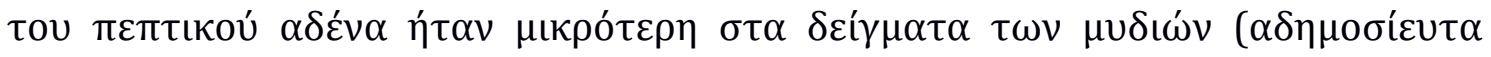

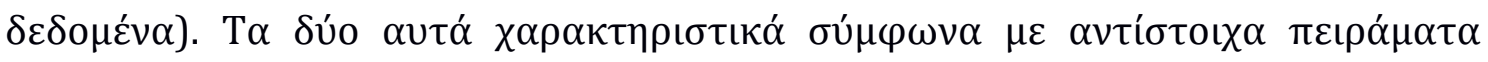

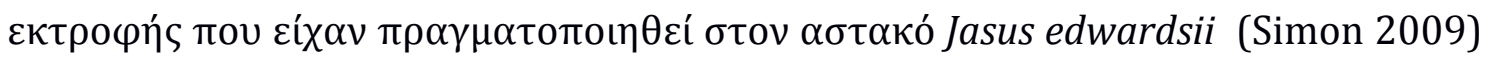

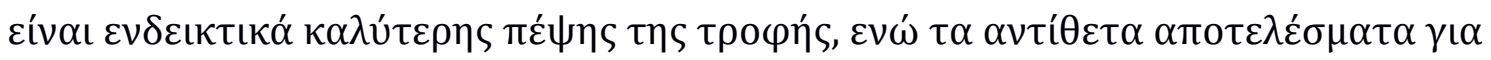




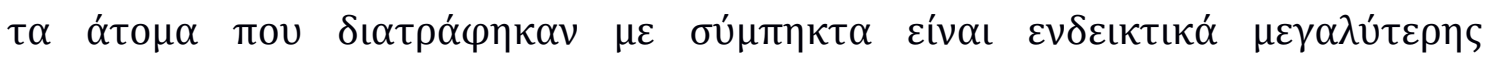

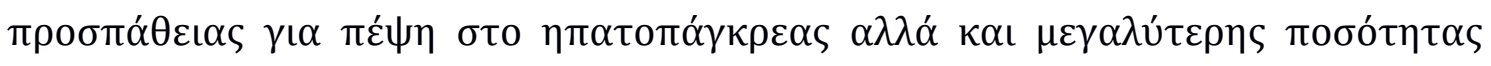

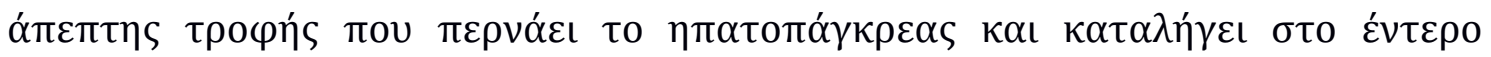

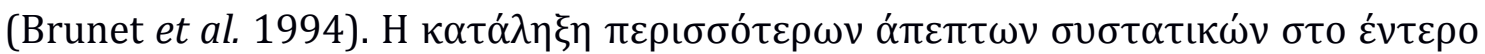

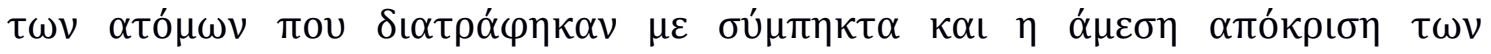

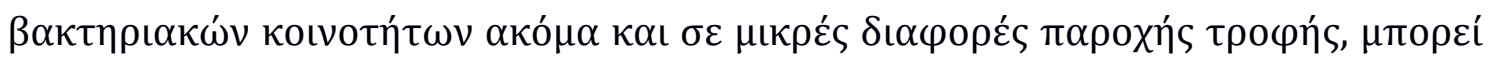

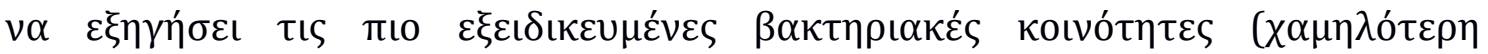

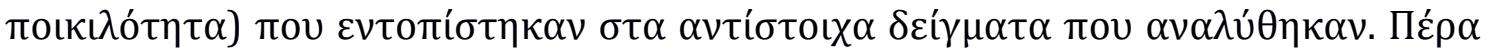

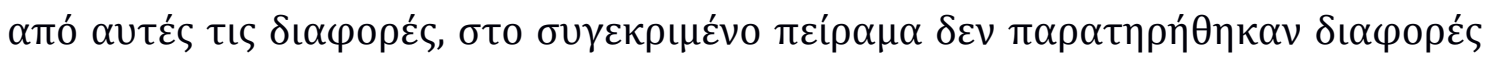

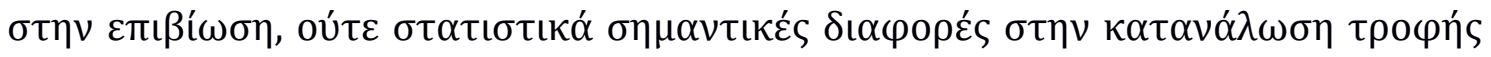

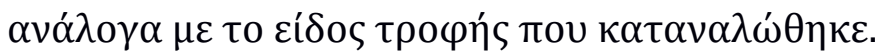

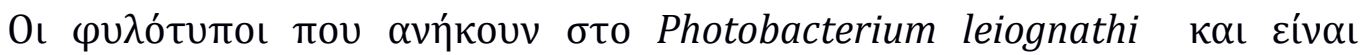

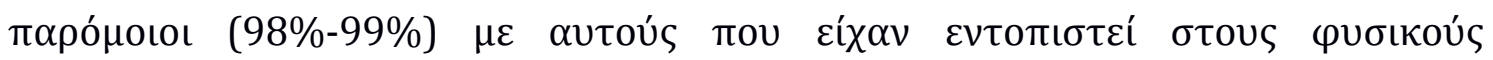

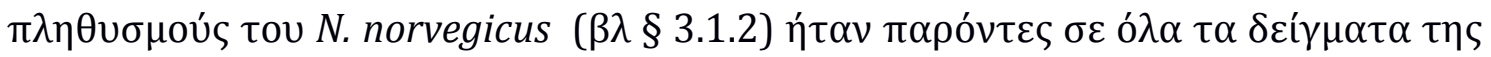

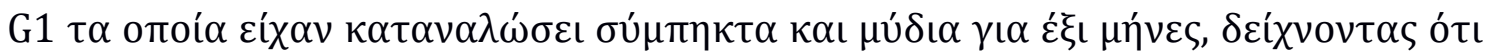

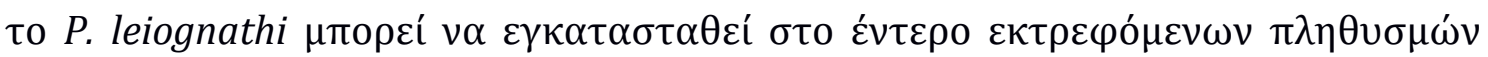

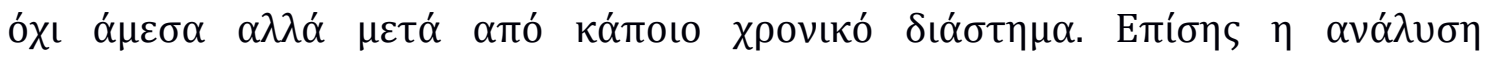

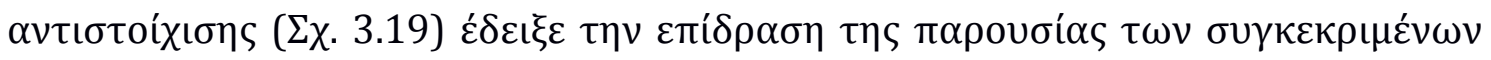

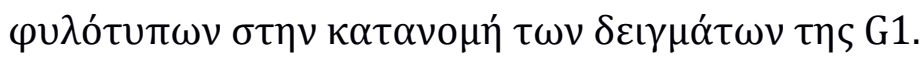

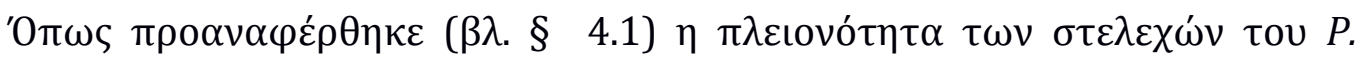

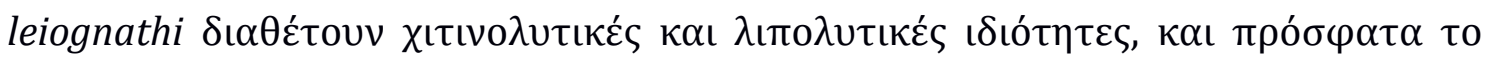

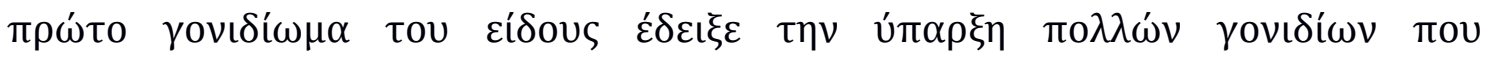

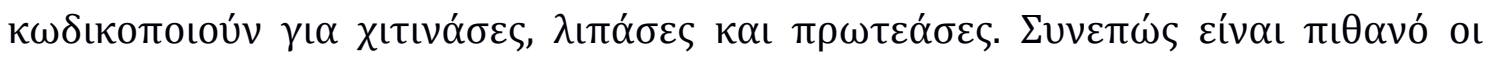

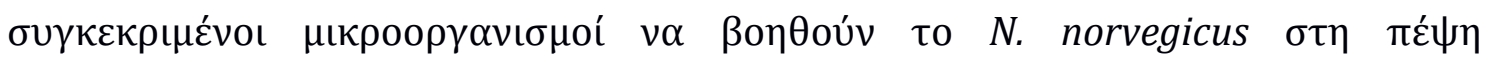




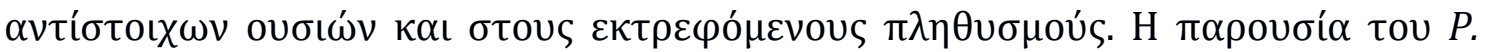

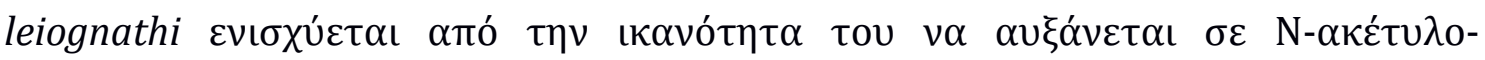

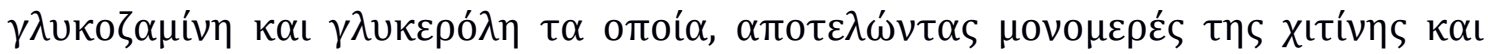

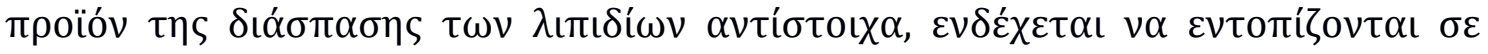

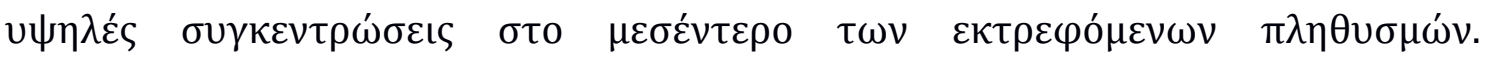

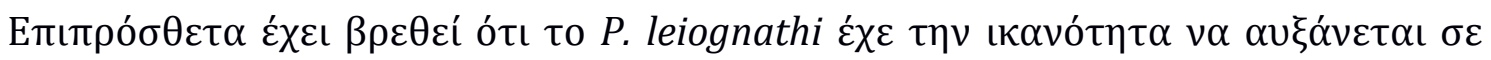

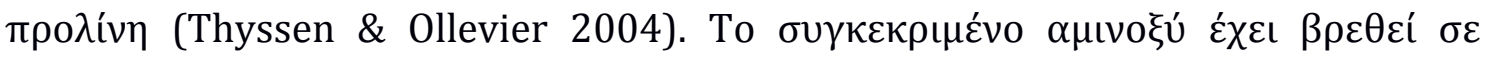

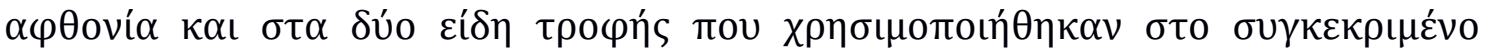

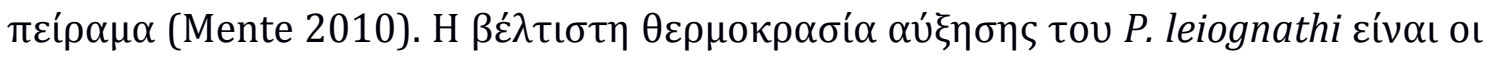

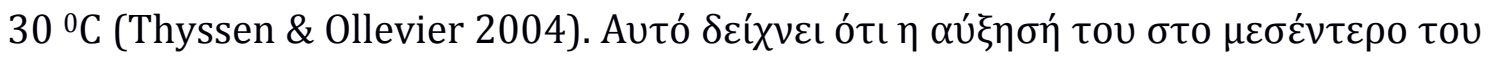

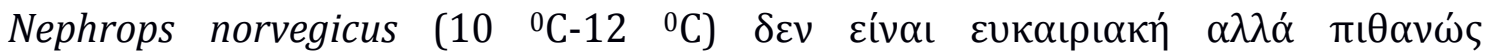

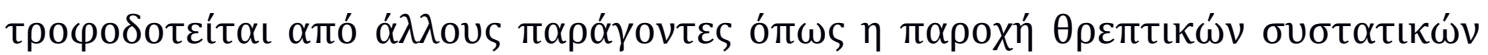

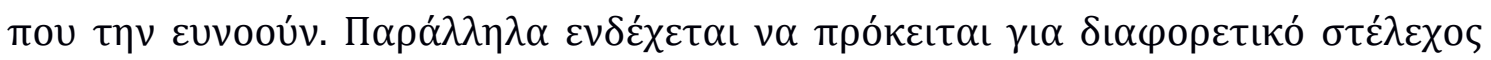

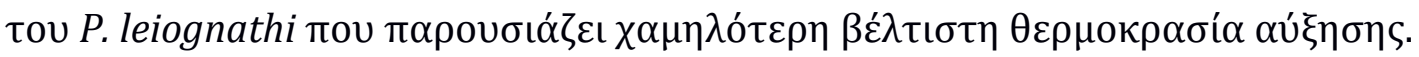

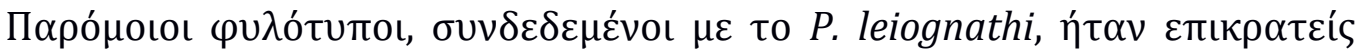

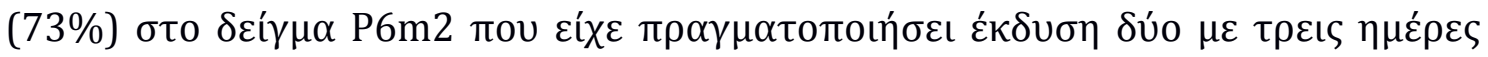

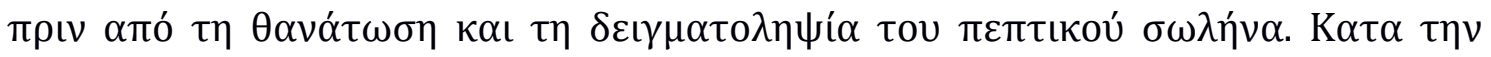

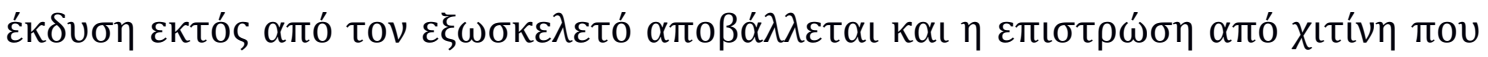

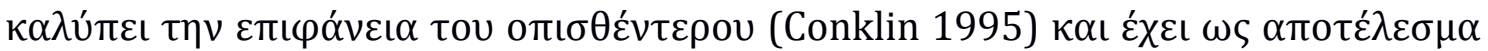

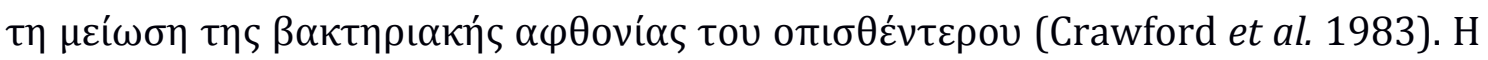

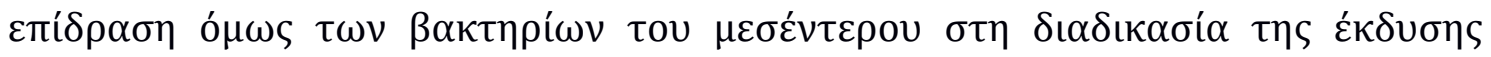

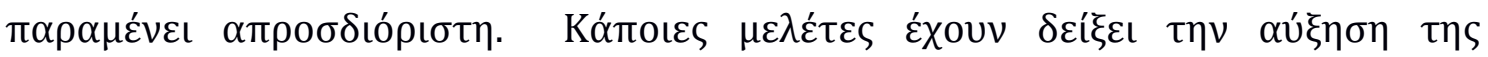

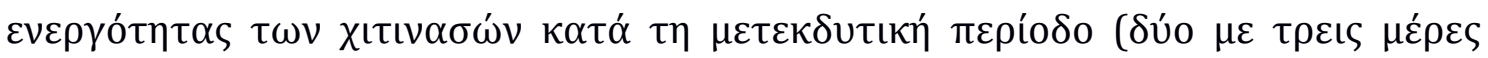

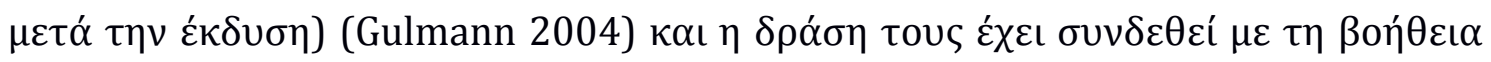




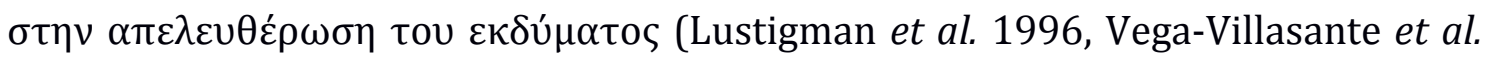

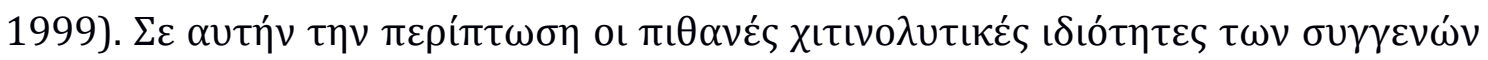

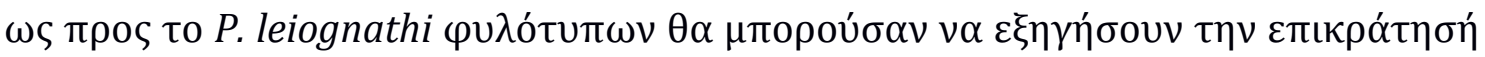

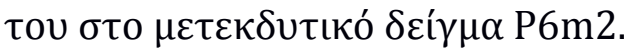

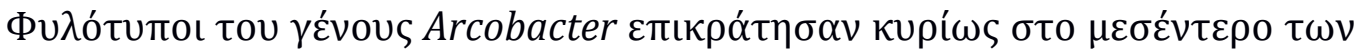

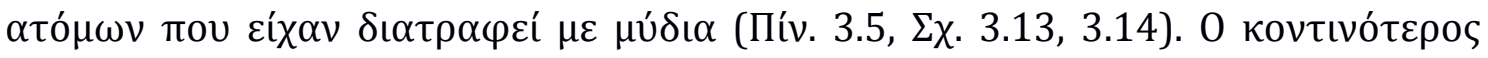

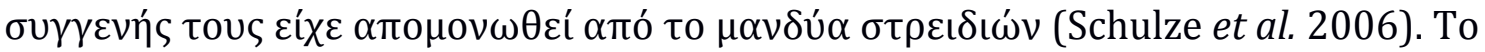

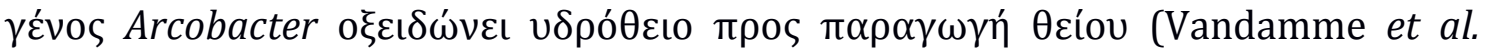

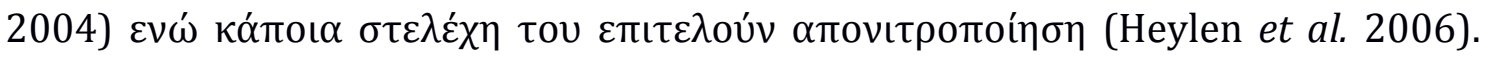

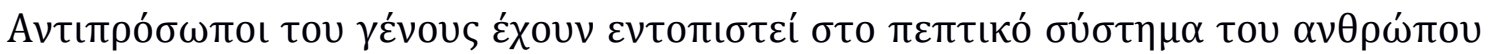

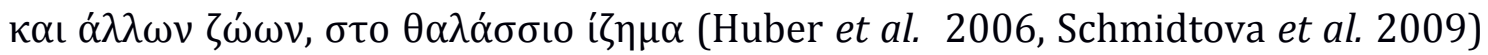

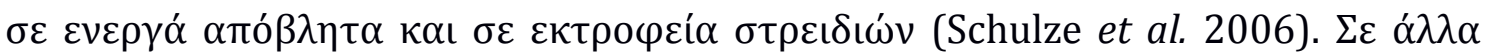

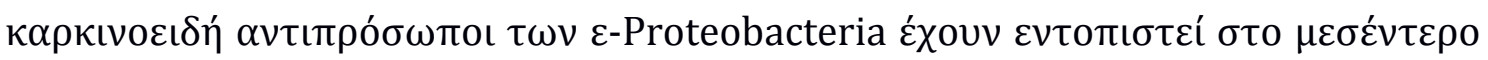

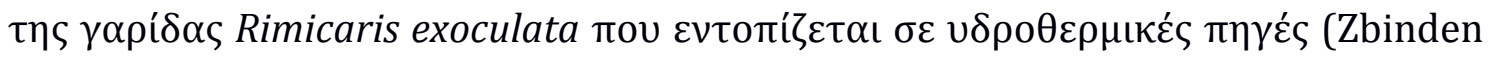

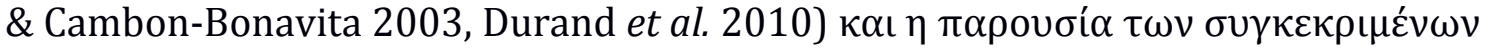

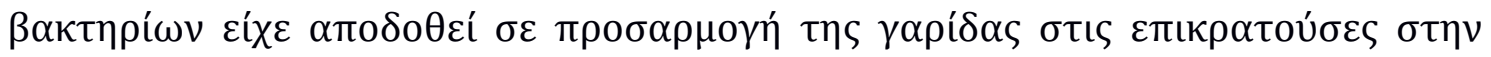

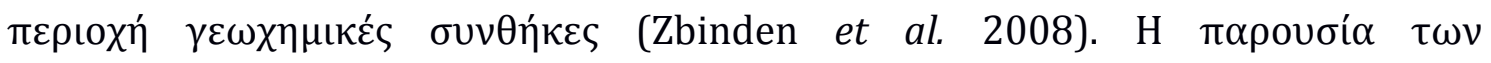

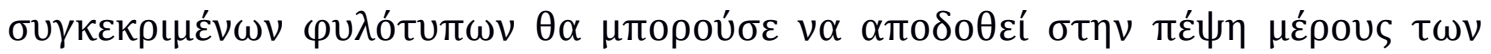

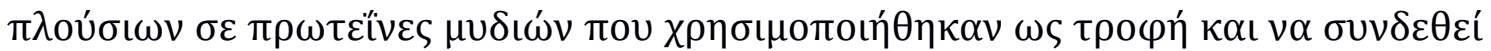

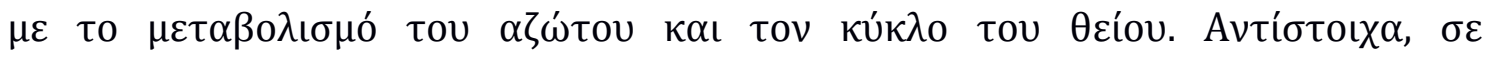

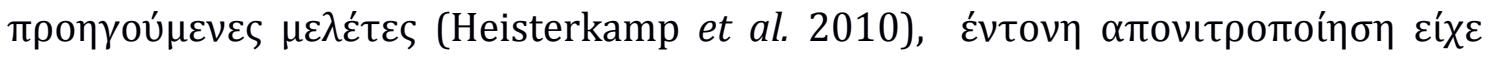

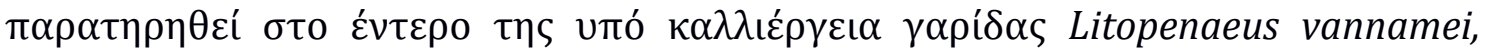

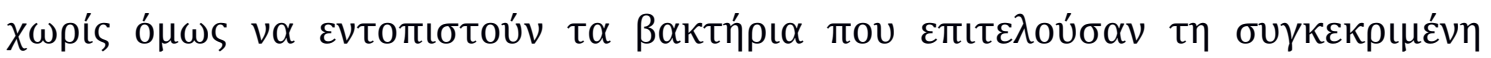
$\delta\llcorner\alpha \delta \iota \kappa \alpha \sigma i ́ \alpha$. 


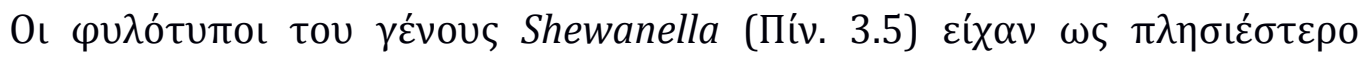

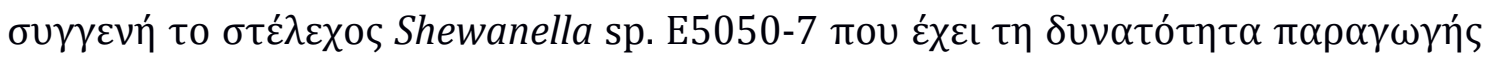

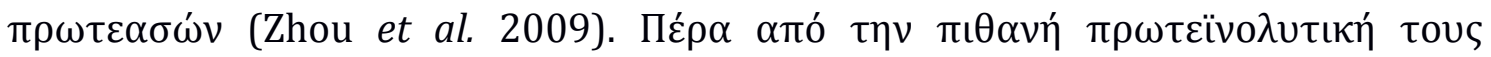

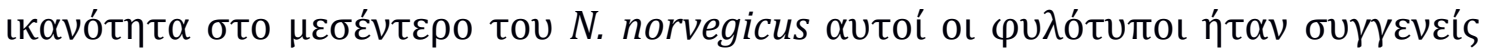

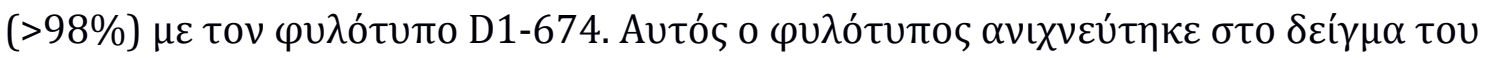

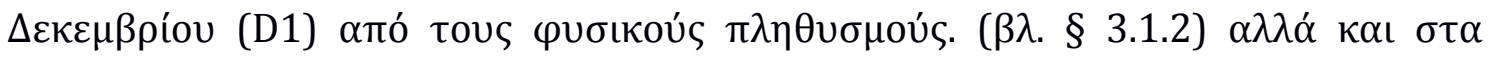

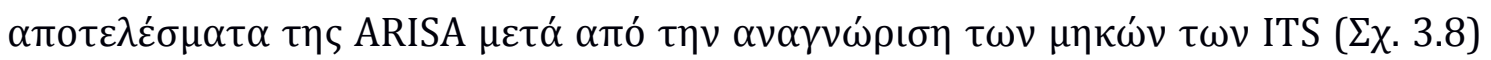

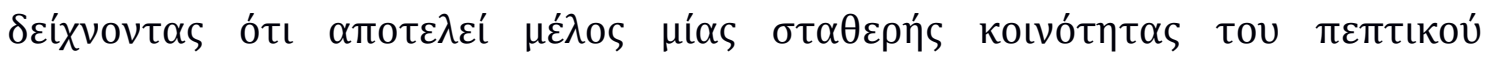

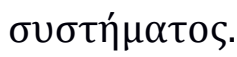

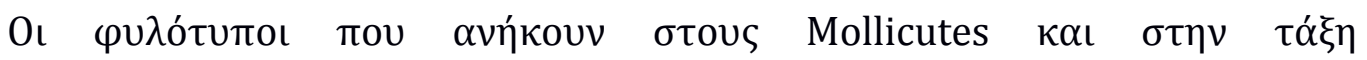

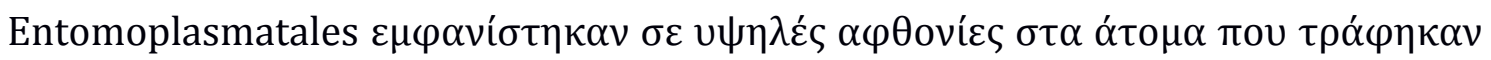

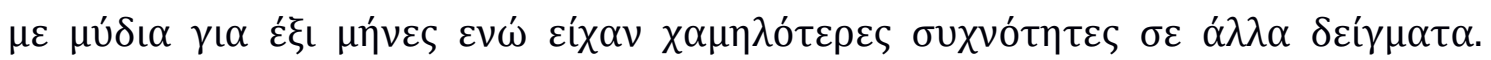

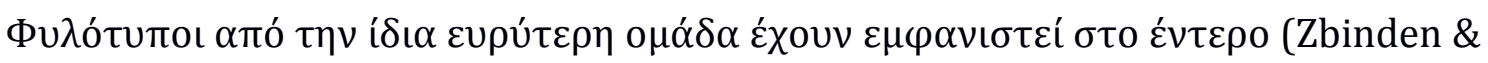
Cambon-Bonavita 2003, Bano et al. 2007, Demiri et al. 2009, Durand et al. 2010)

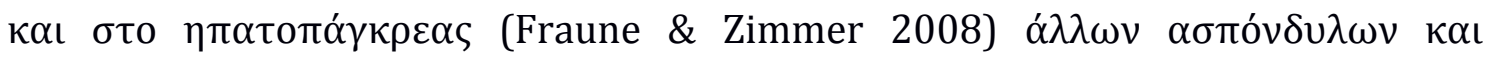

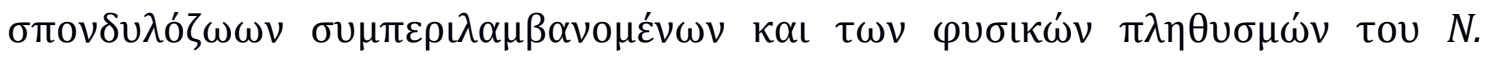

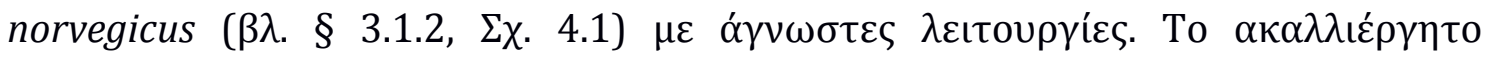

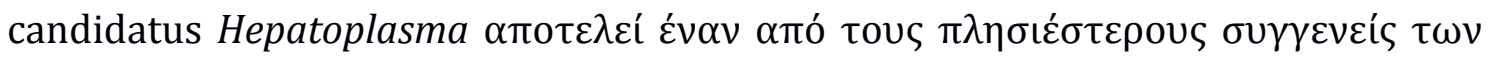

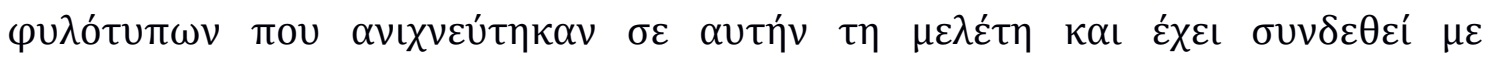

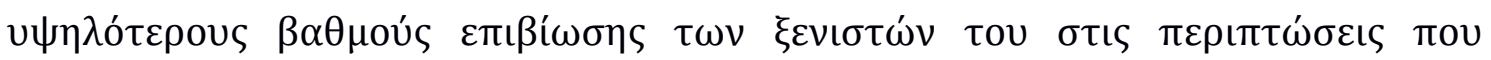

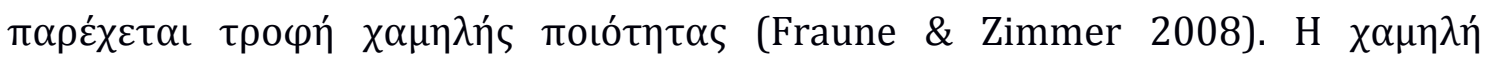

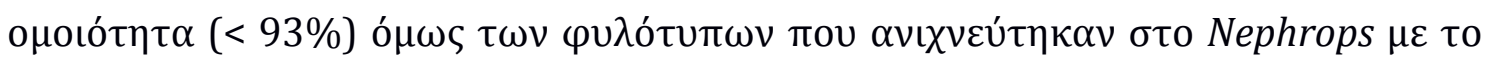

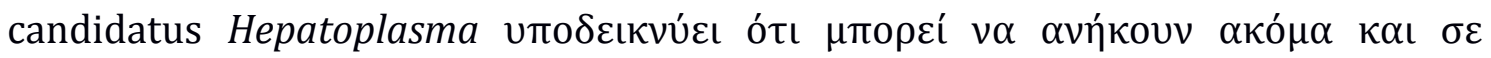

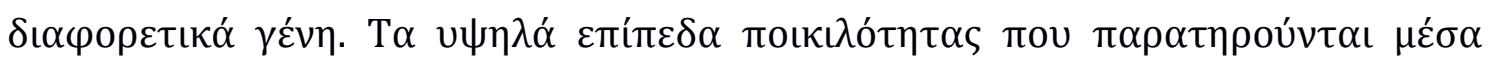




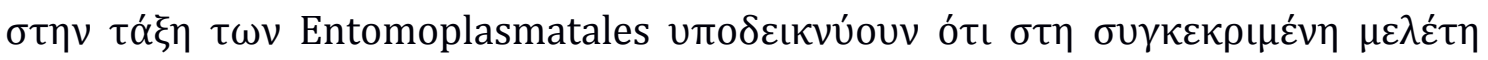

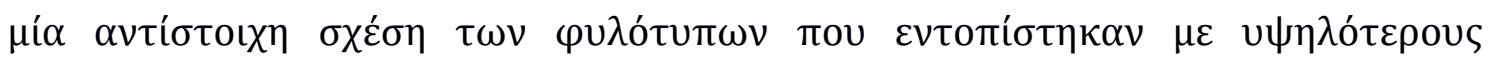

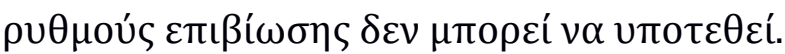

$\Sigma \chi \varepsilon \tau$

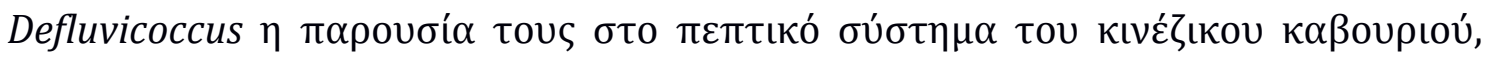

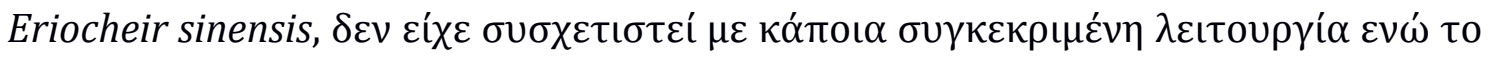

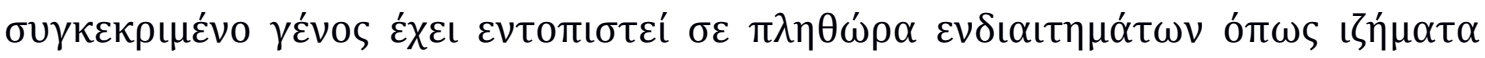

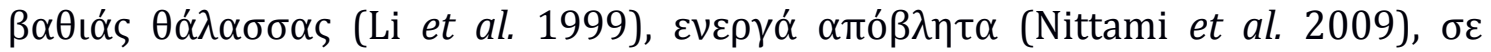

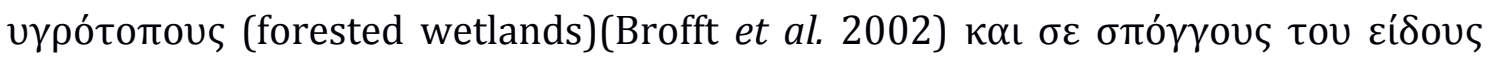

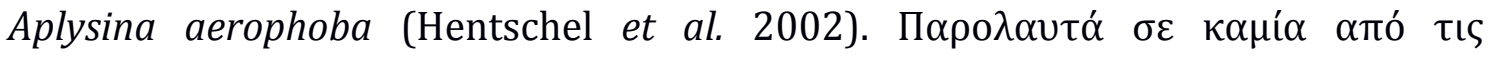

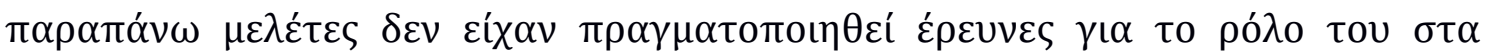

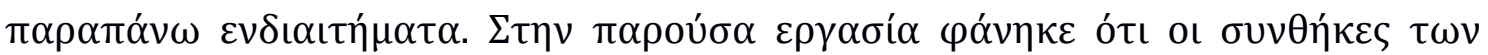

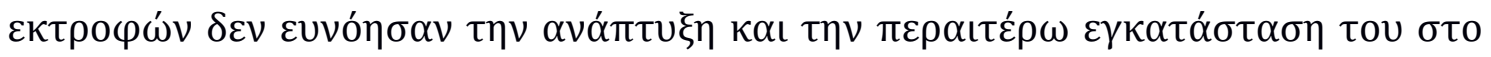

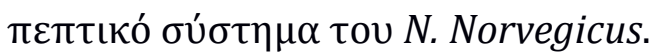

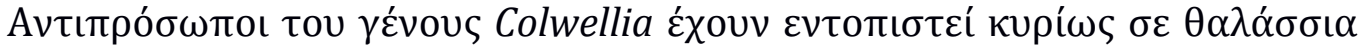

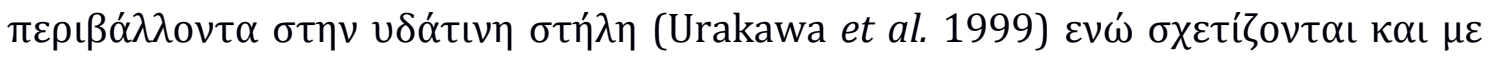

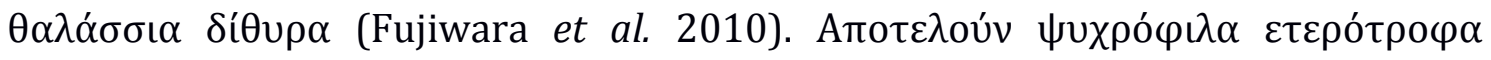

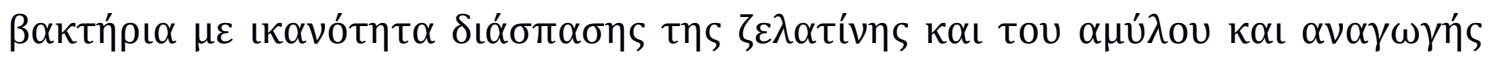

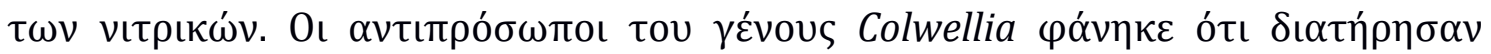

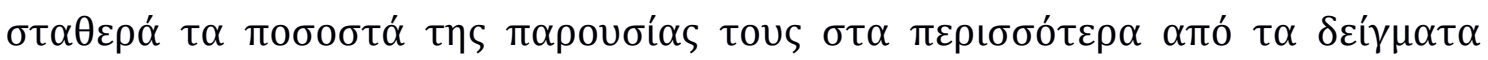

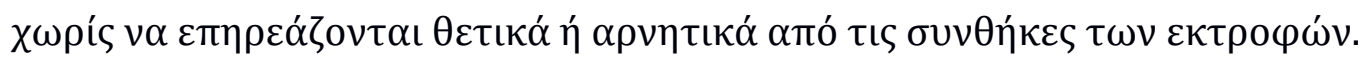

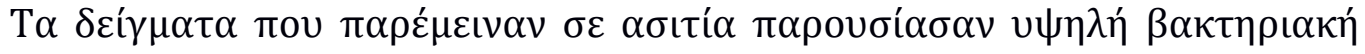

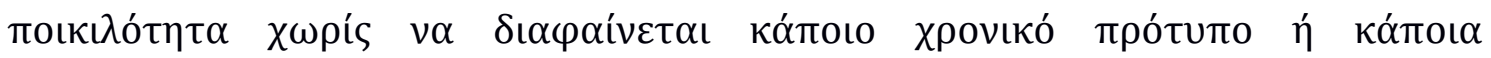

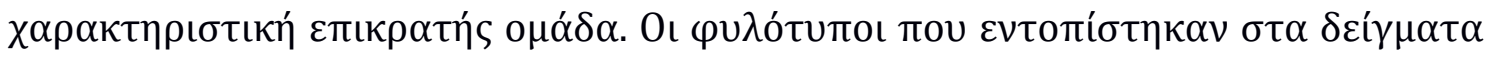




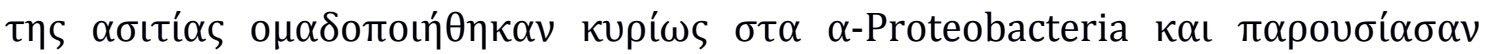

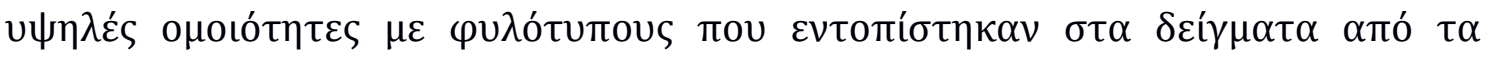

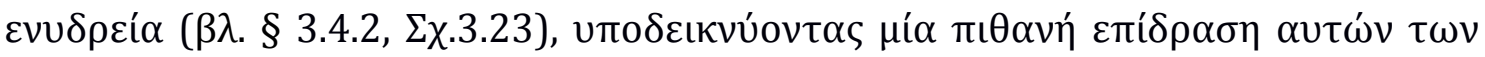

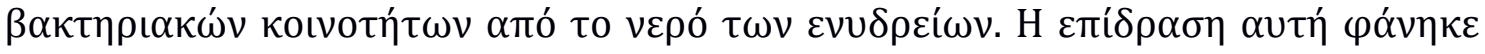

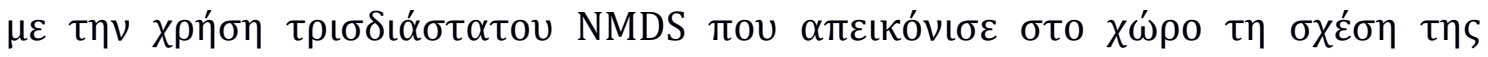

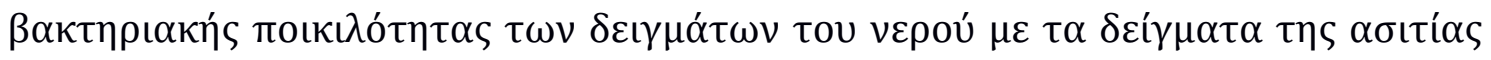

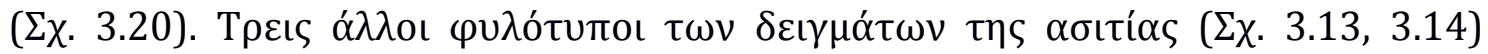

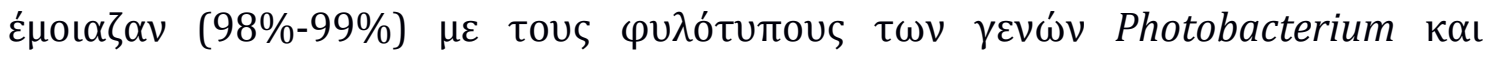

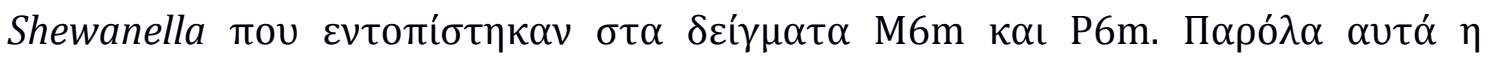

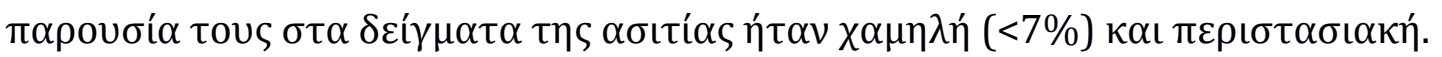

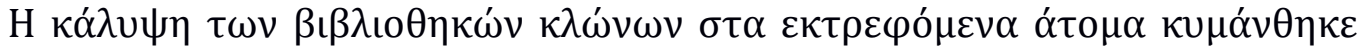

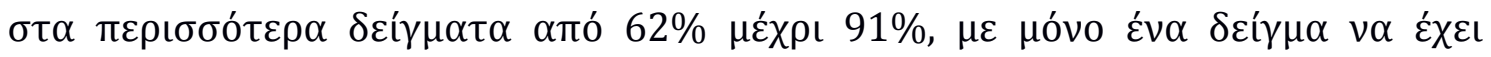

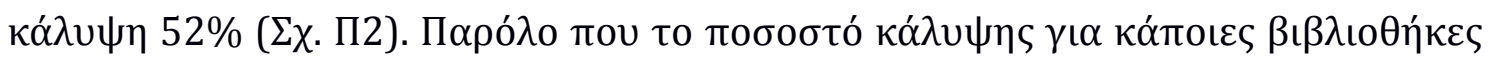

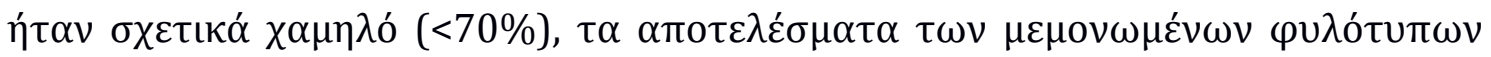

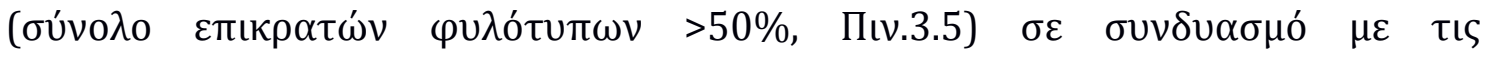
$\alpha \sigma u \mu \pi \tau \omega \tau$

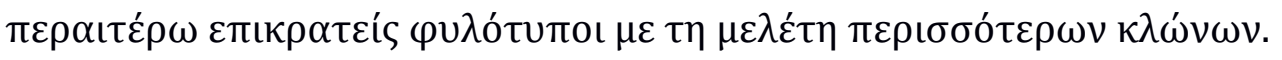

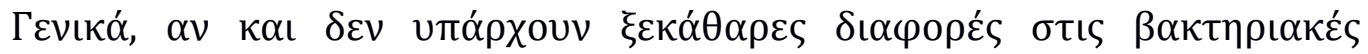

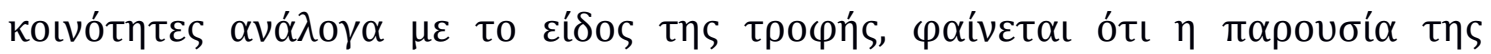

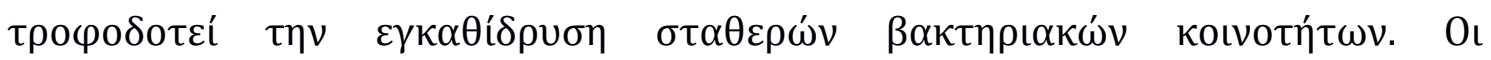

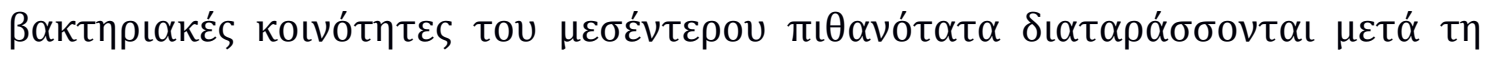

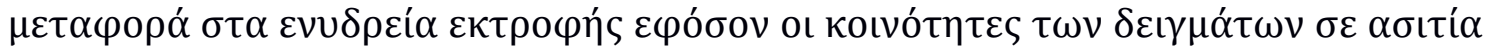

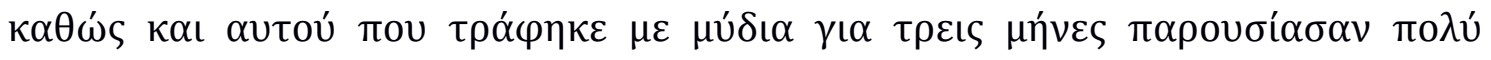




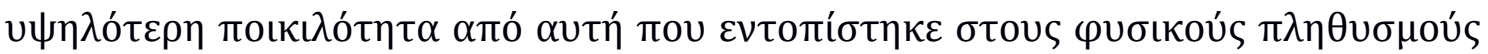
$(\Sigma \chi \cdot 4.3)$

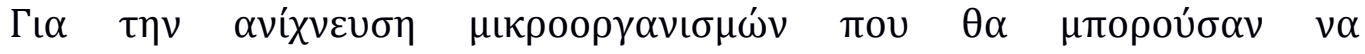

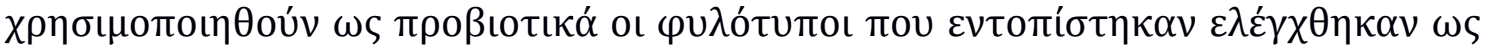

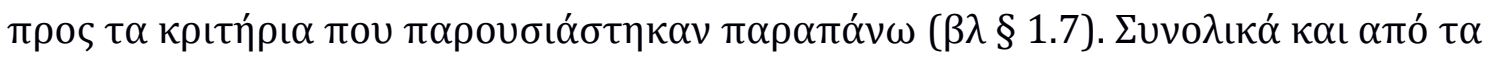

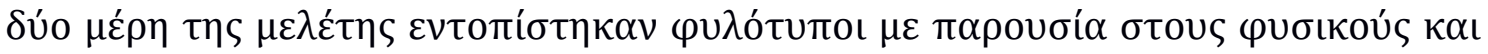

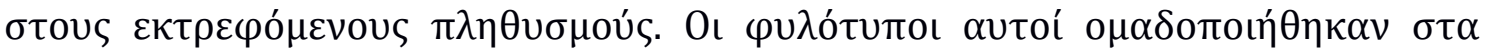

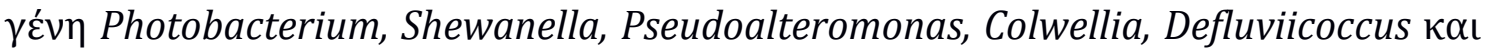

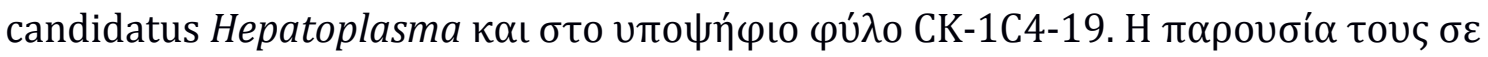

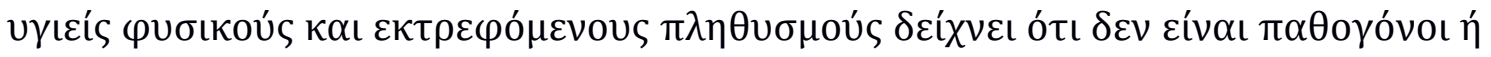

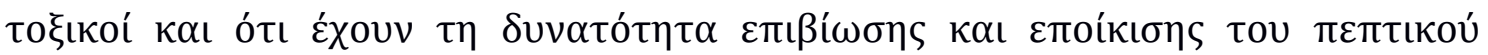

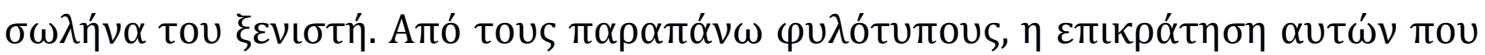

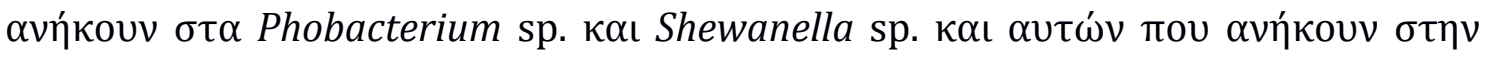

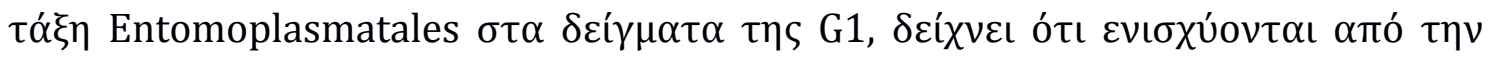

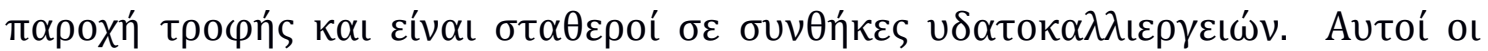

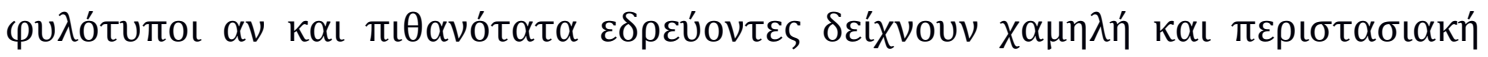

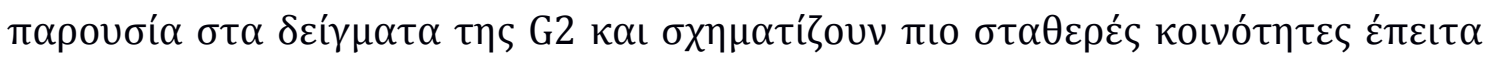

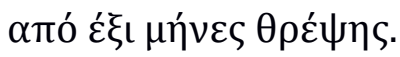

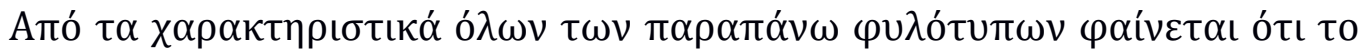

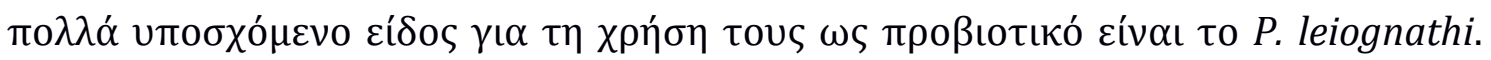

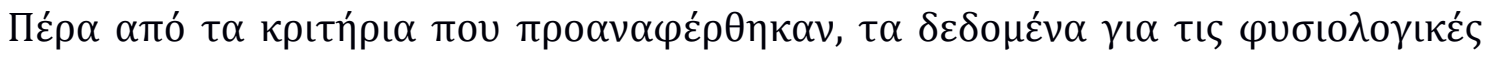

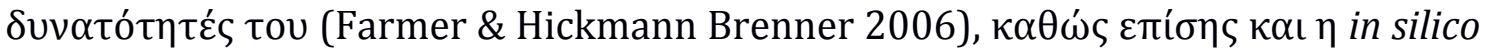

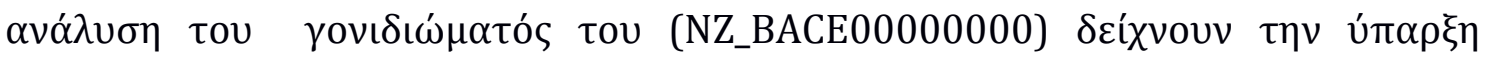

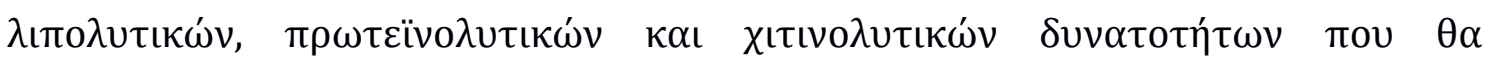




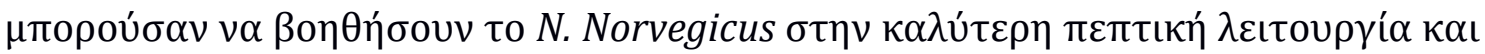
$\varepsilon \pi i \beta i ́ \omega \sigma \eta$.

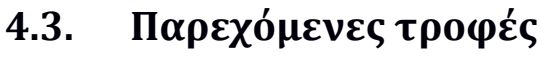

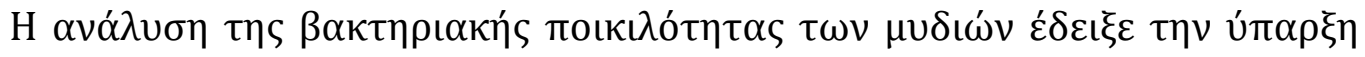

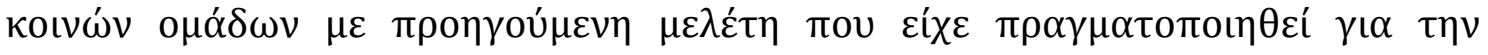

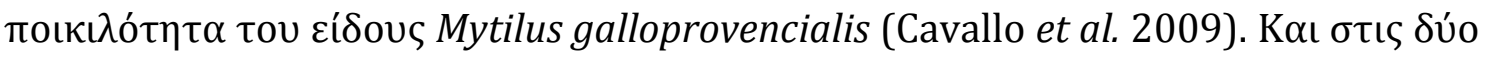

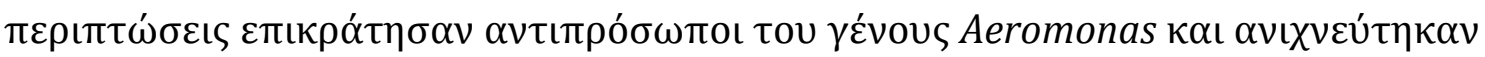

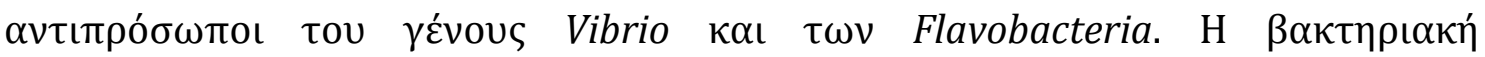

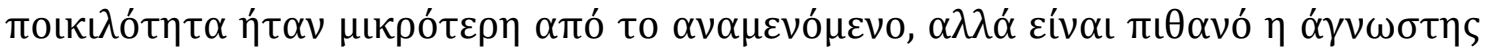

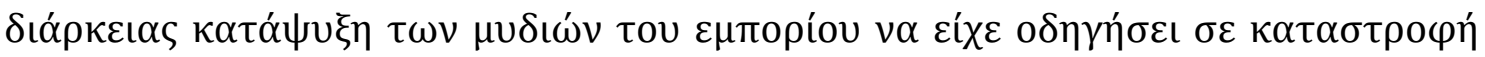

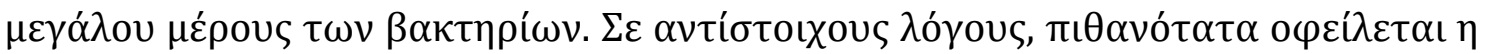

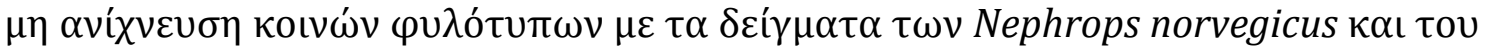

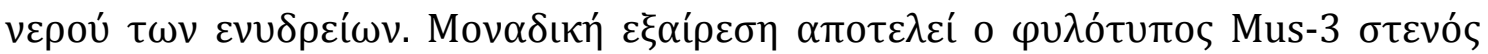

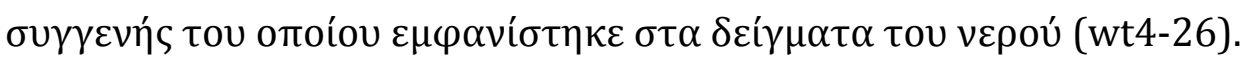

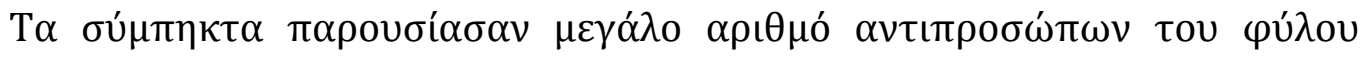

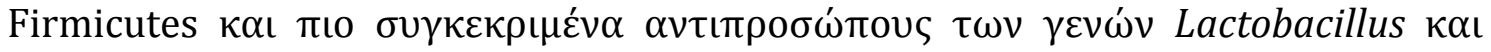

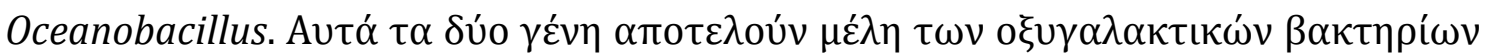

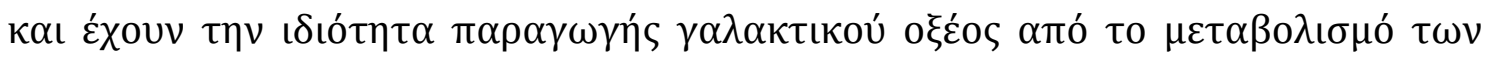

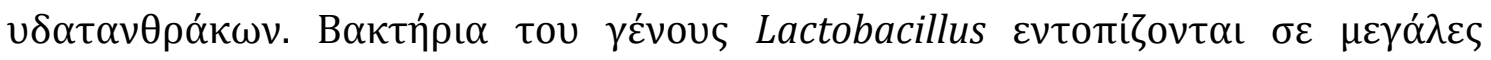

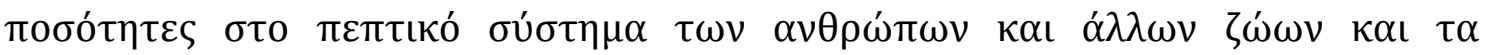




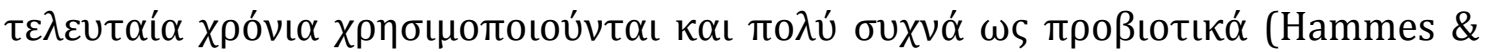
Hertel 2006).

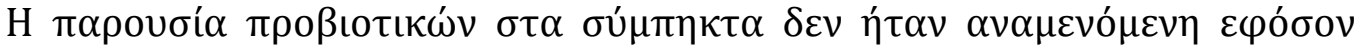

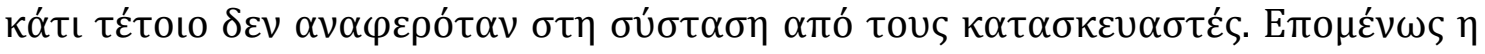

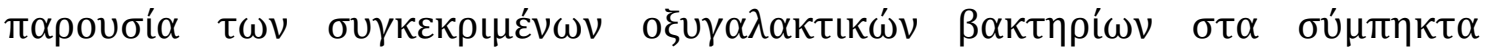

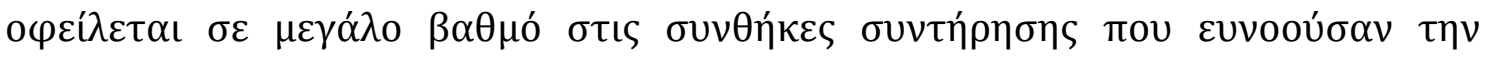

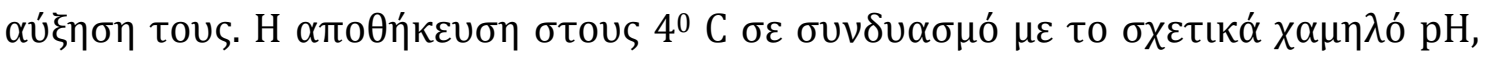

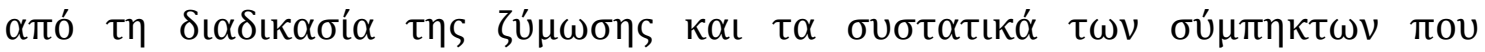

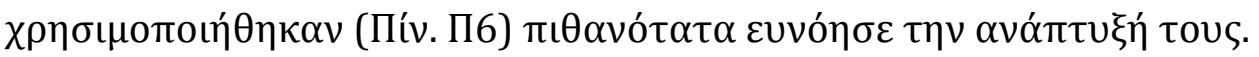

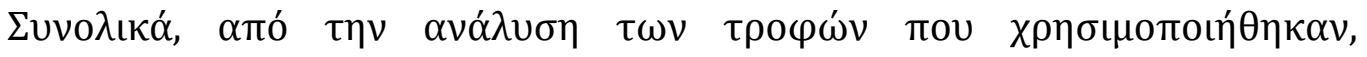

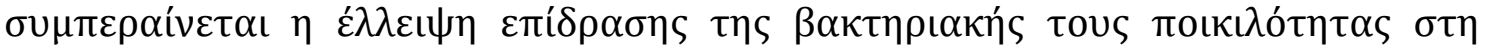

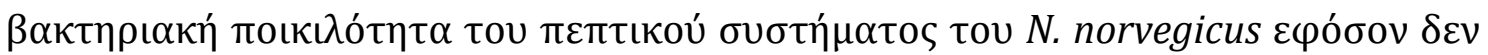
$\alpha v \iota \chi v \varepsilon u ́ \tau \eta \kappa \alpha v$ кoเvoí $\varphi$

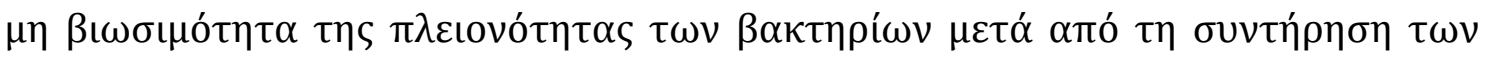

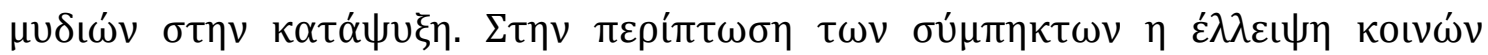

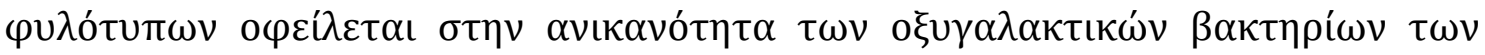

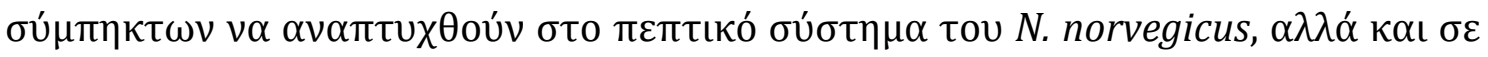

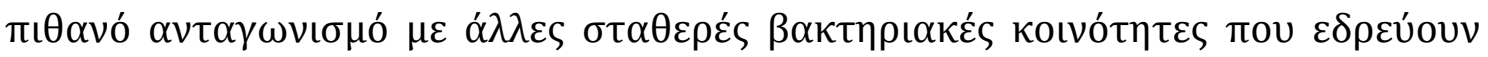

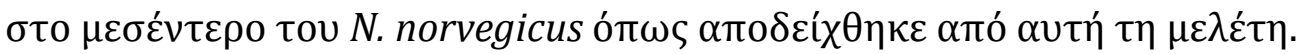

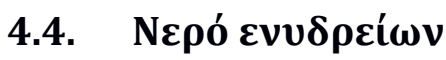

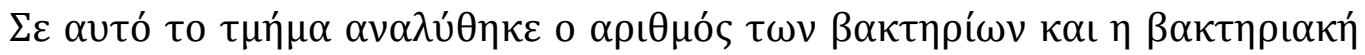

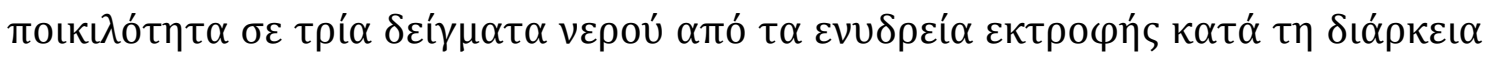




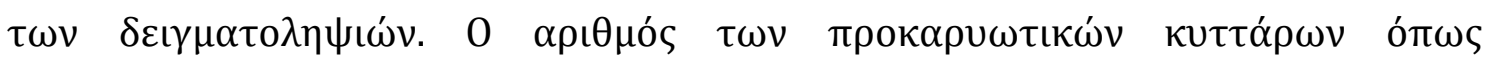

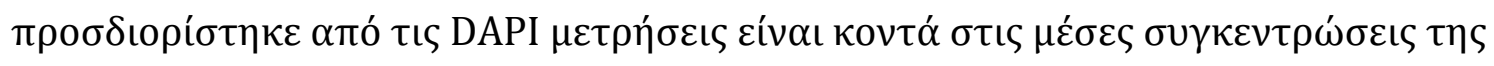

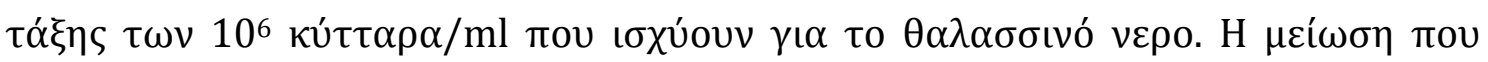

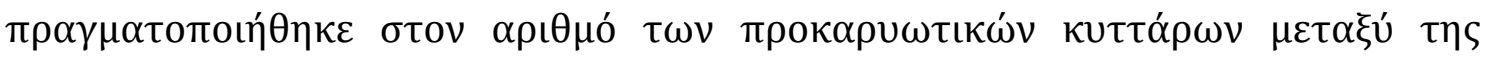

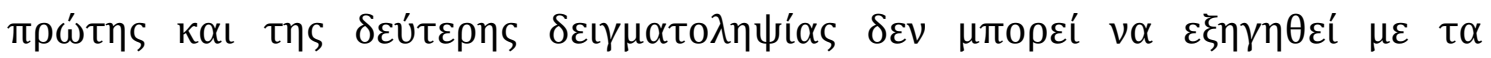

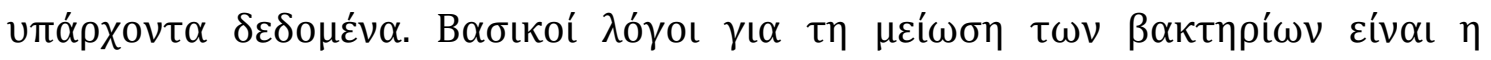

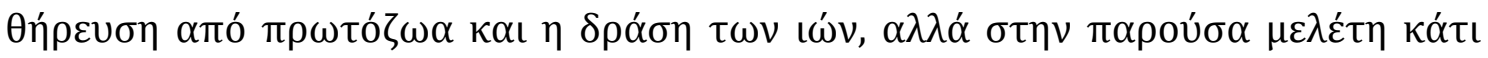

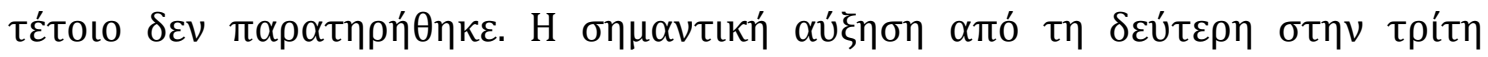

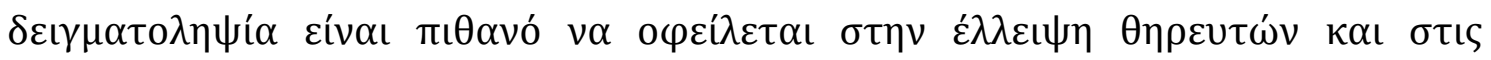

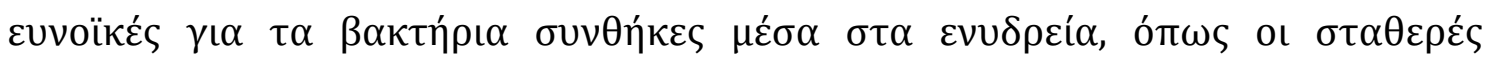

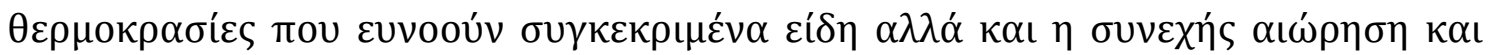

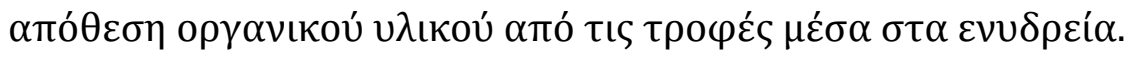

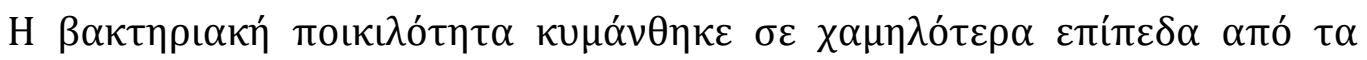

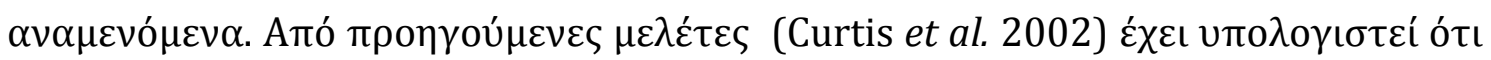

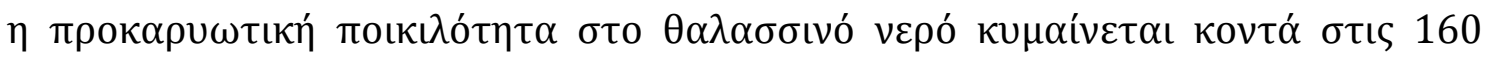

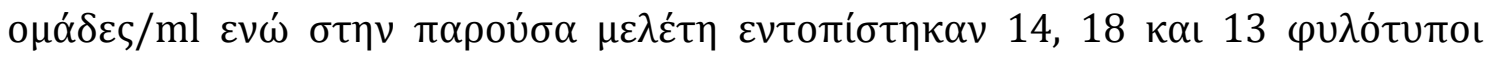

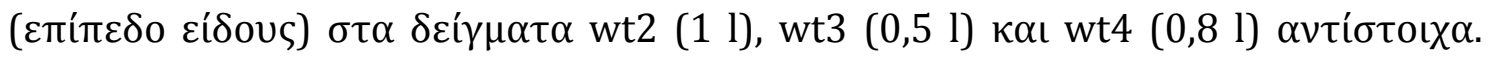

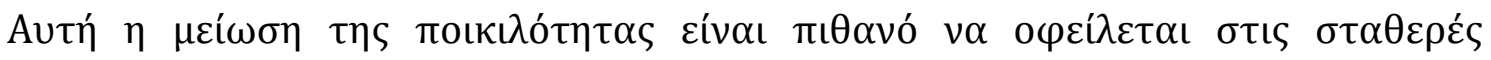

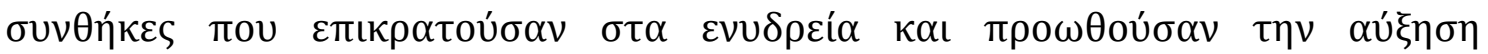

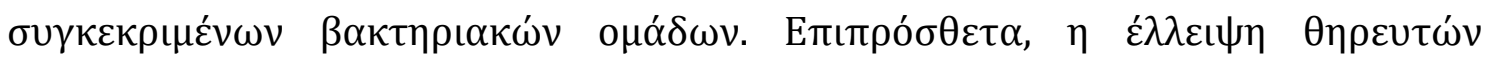

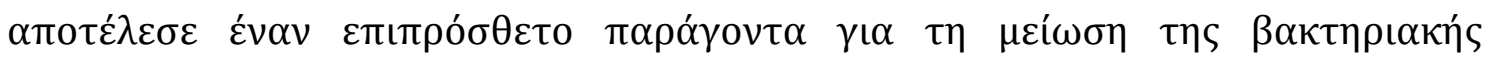

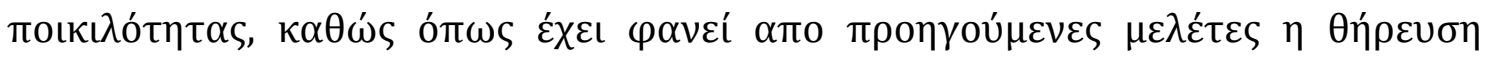

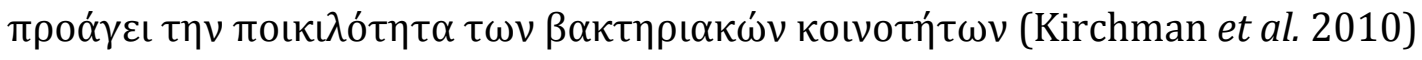




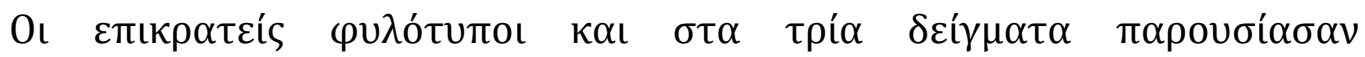

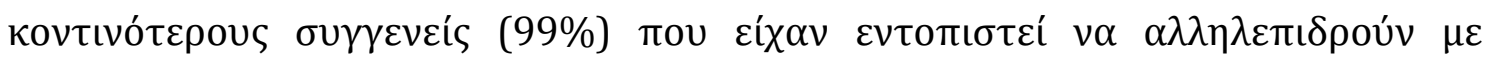

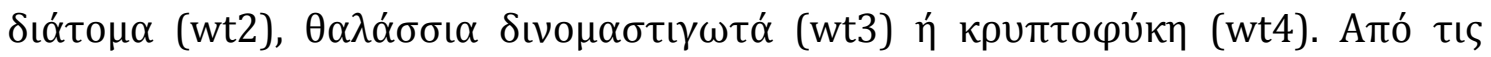

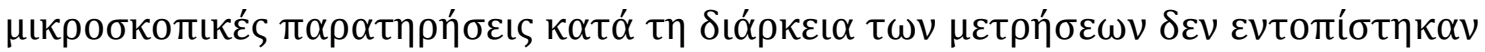

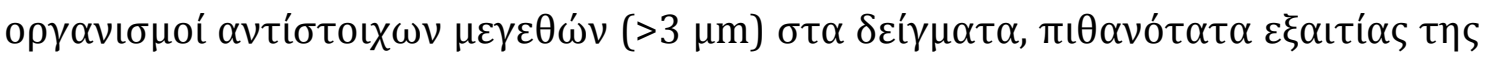

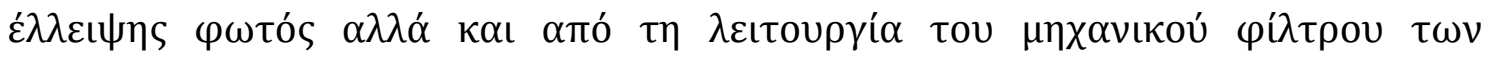

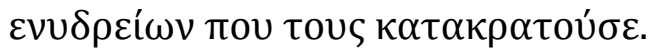

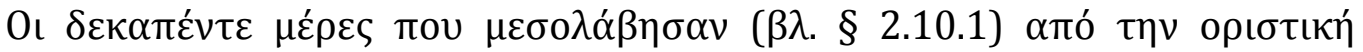

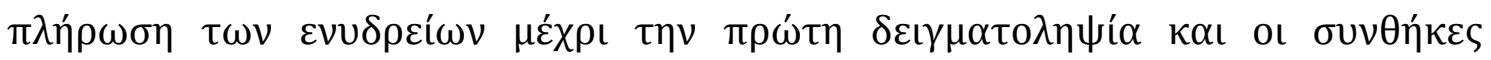

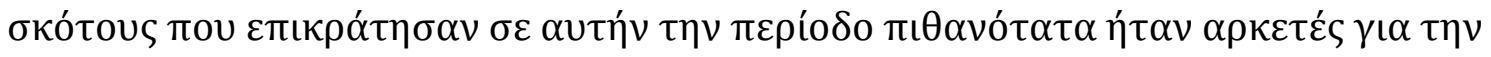

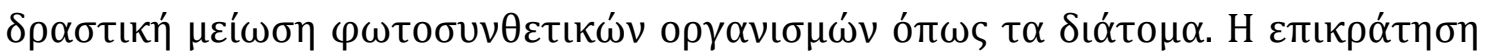

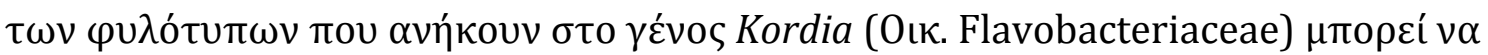

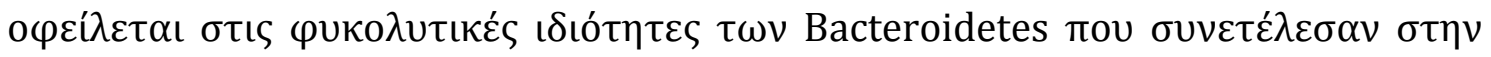

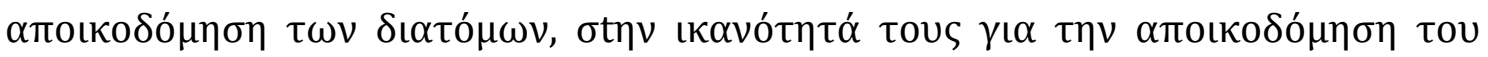

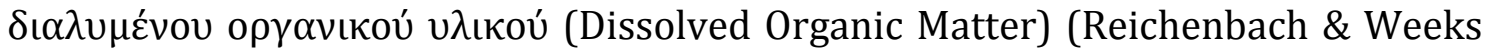

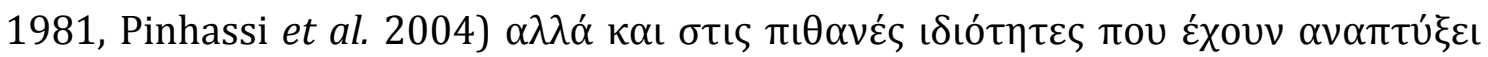

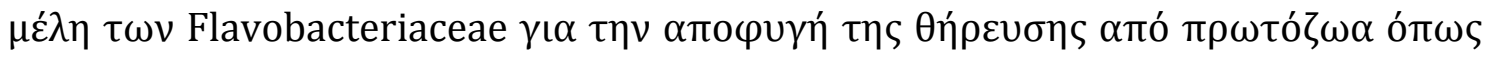

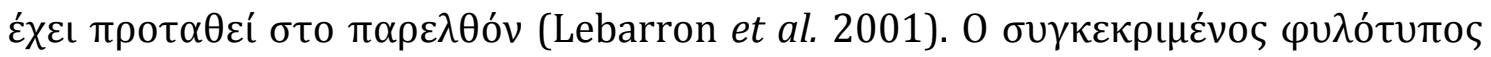

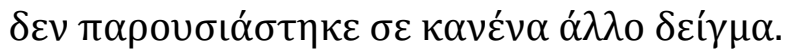

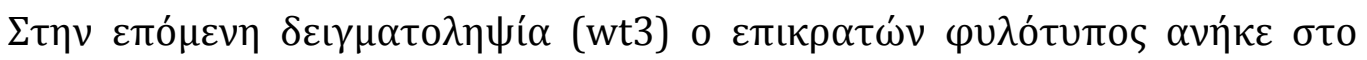

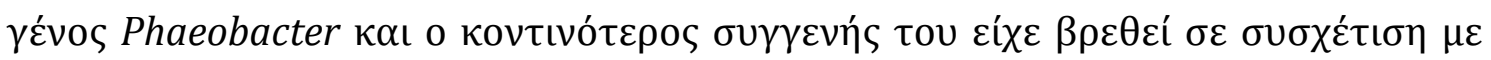

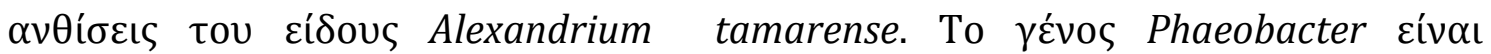

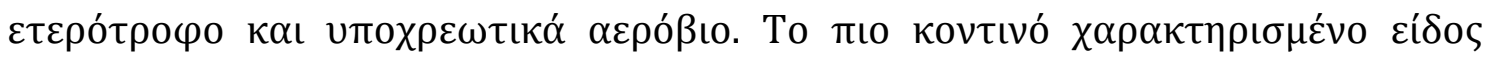

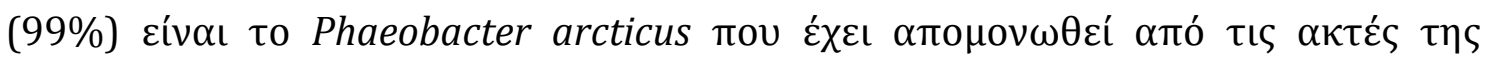




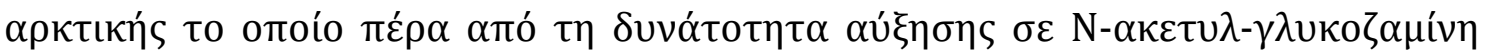

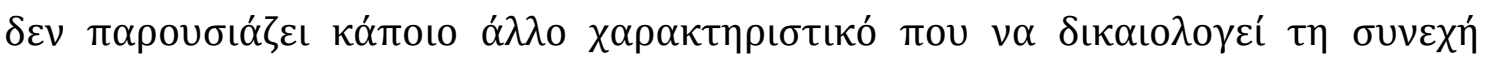

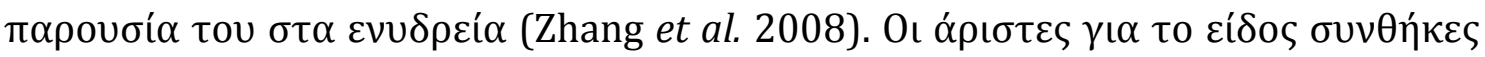

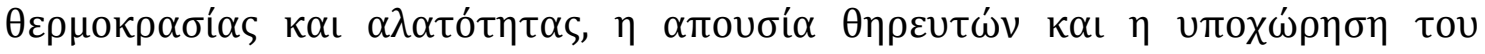

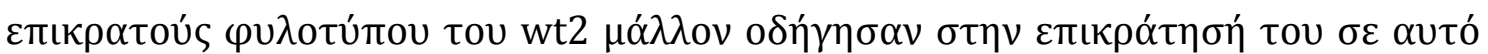

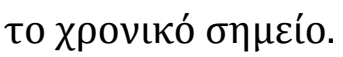

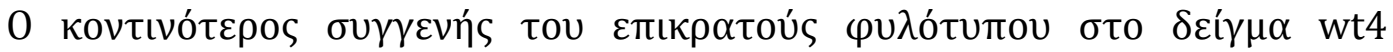

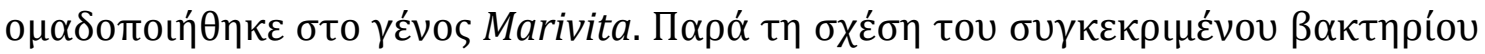

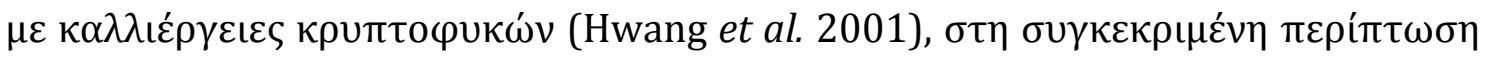

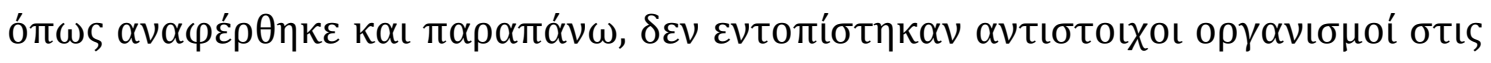

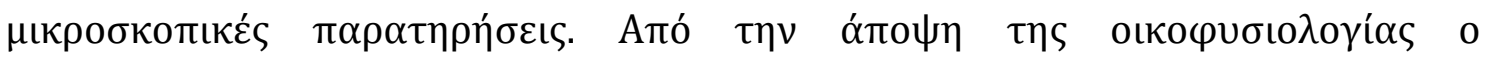

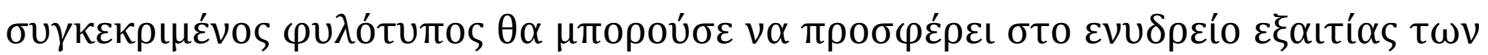

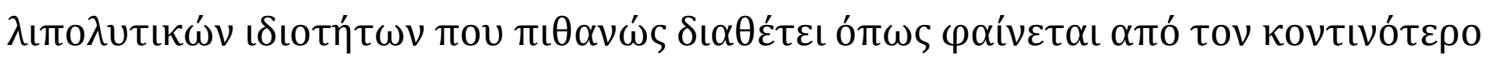

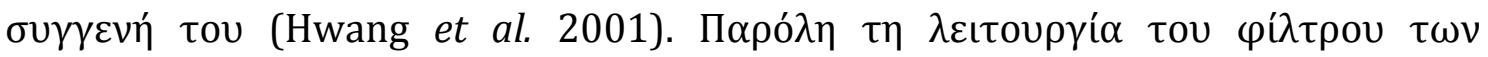

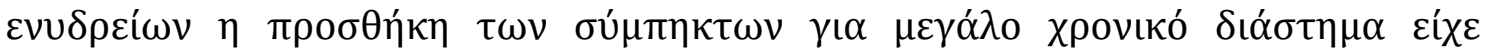

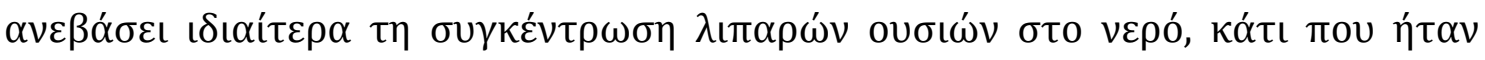

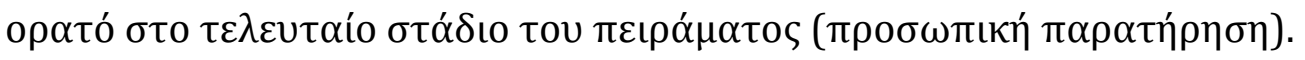

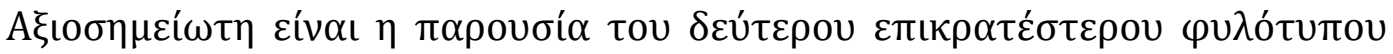

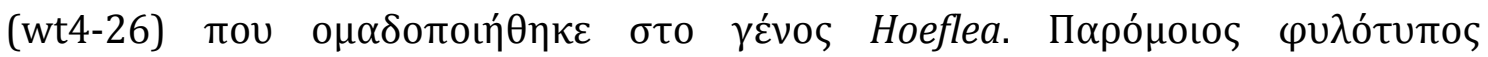

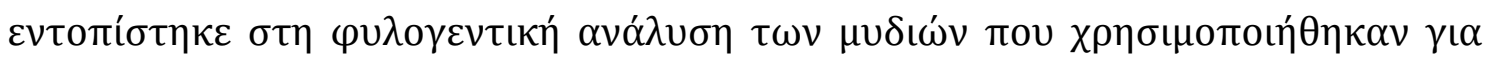

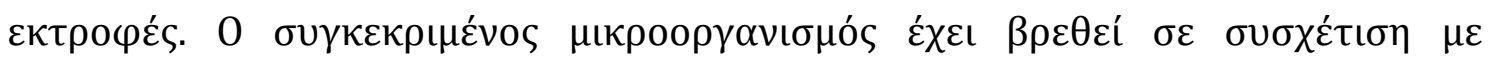

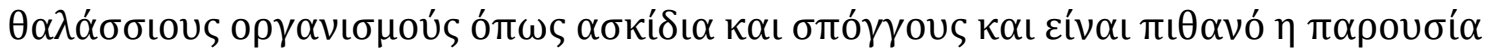

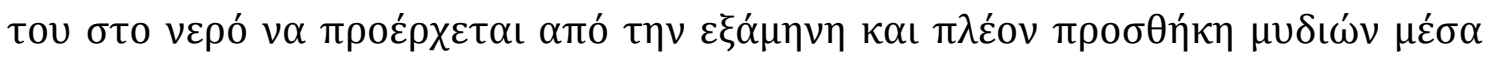
$\sigma \tau \alpha \varepsilon v \cup \delta \rho \varepsilon i ́ \alpha$. 


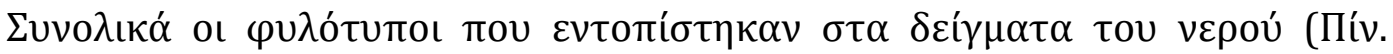

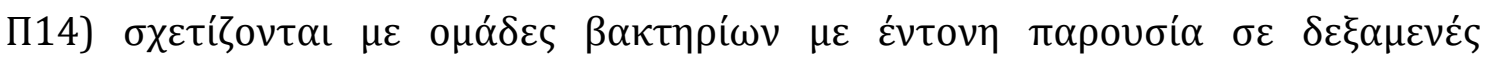

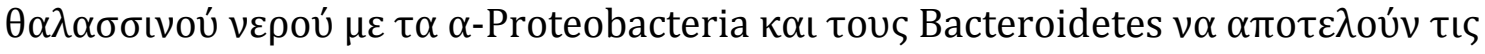

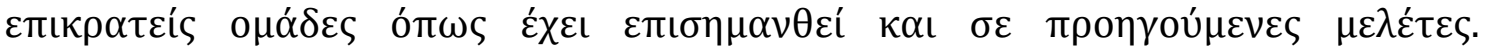

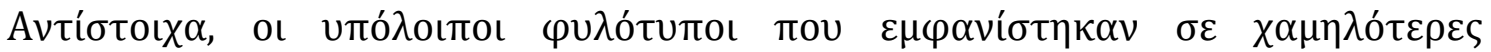

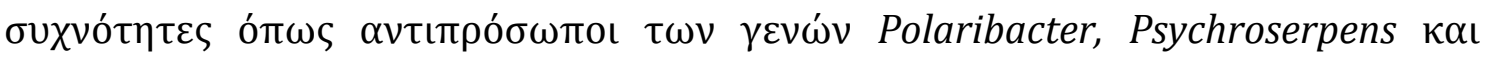

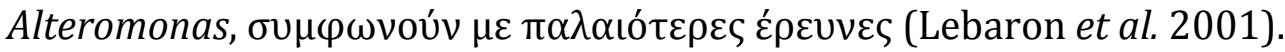

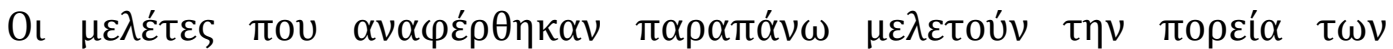

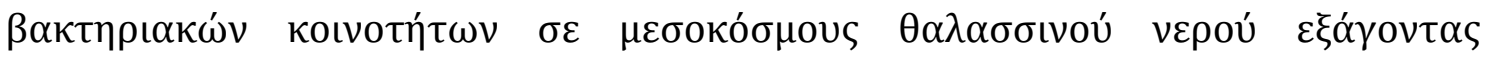

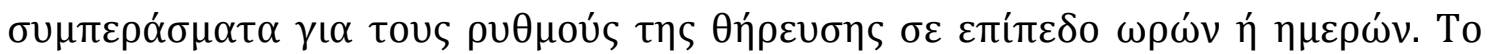

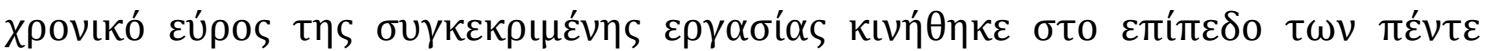

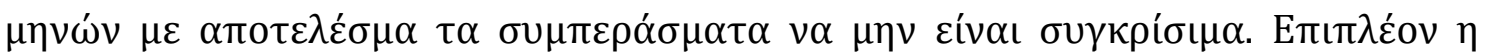

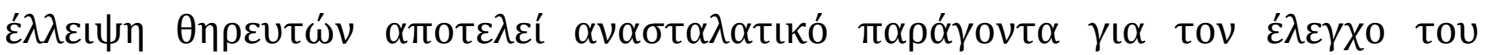

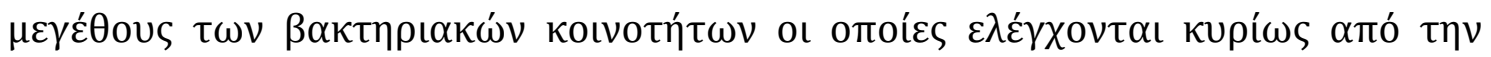

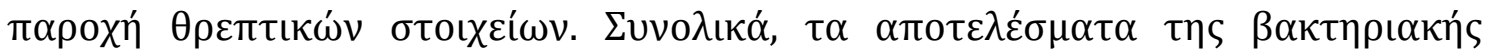

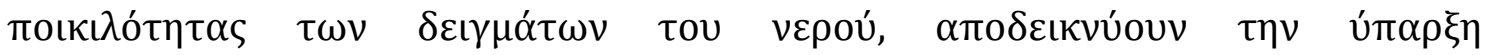

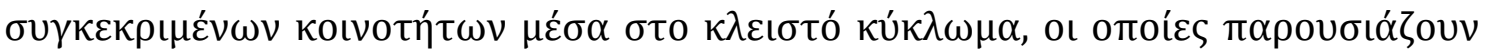

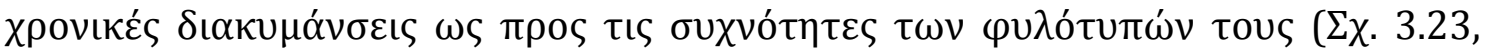
$3.24,3.25)$. 


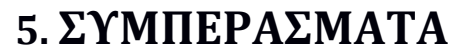

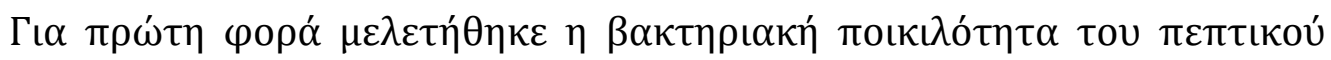

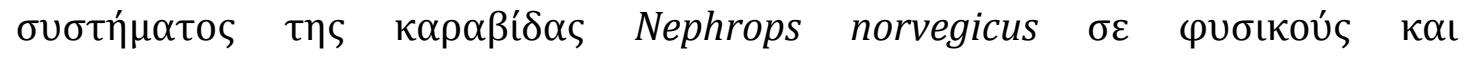

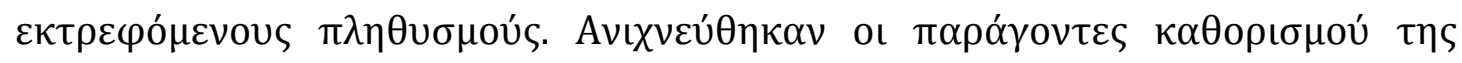

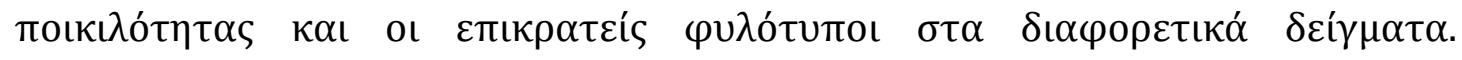

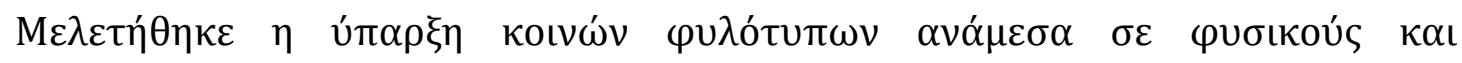

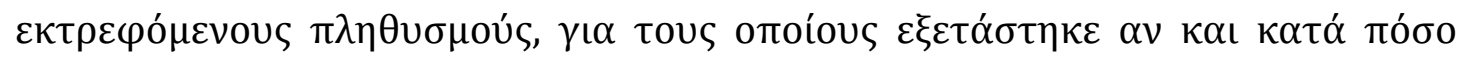

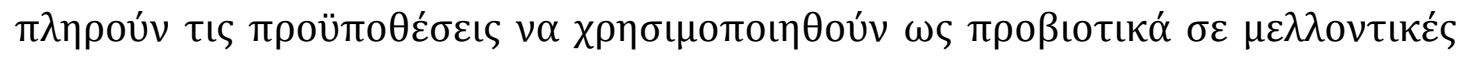

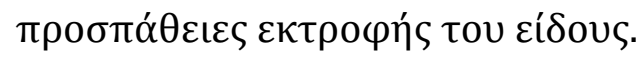

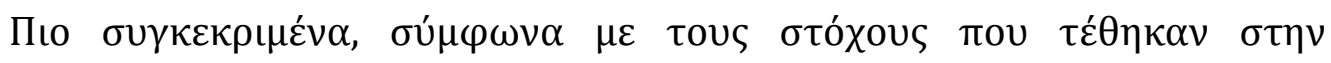

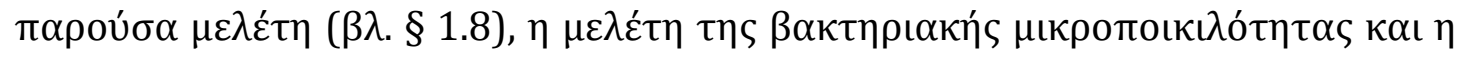

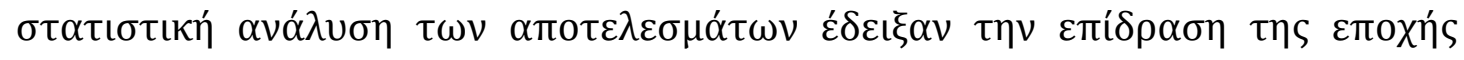

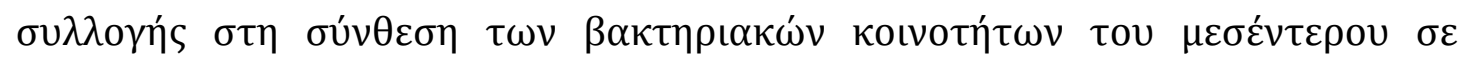

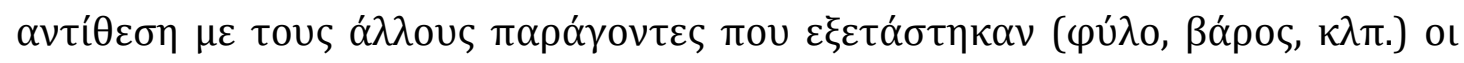

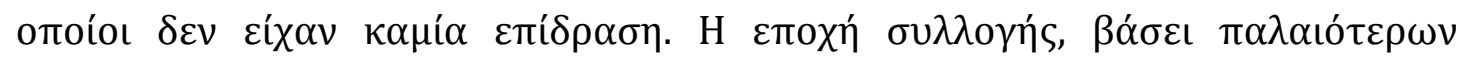

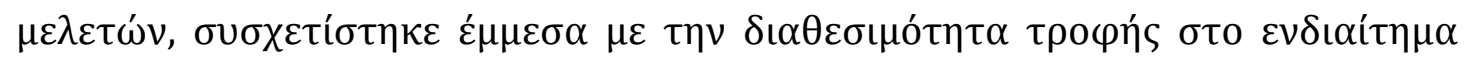

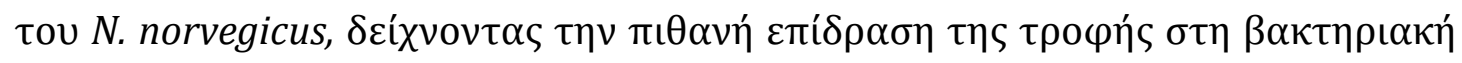

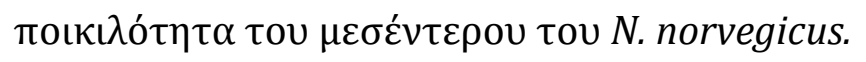

H $\varphi$

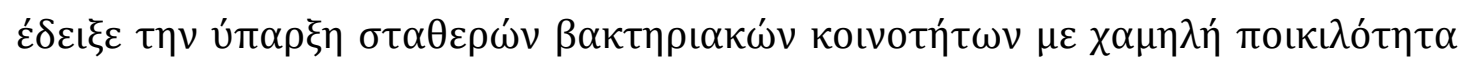

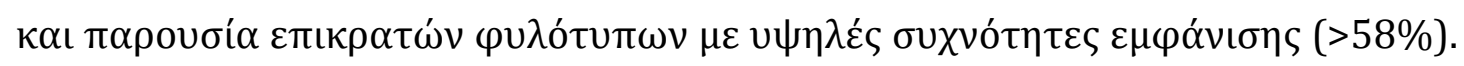

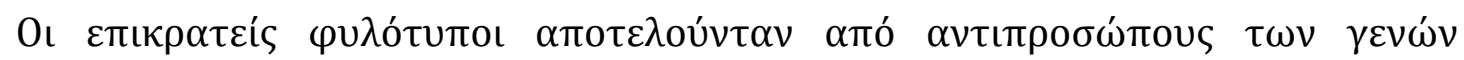




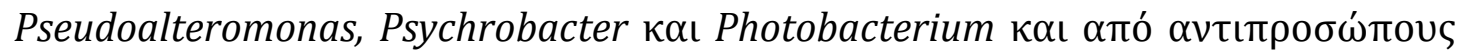

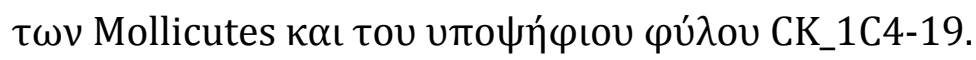

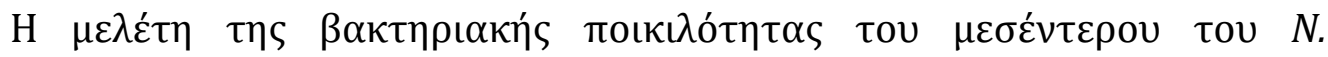

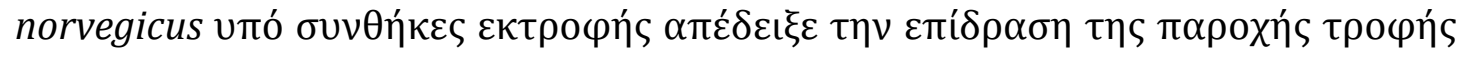

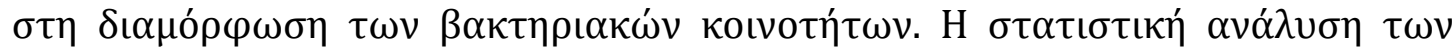

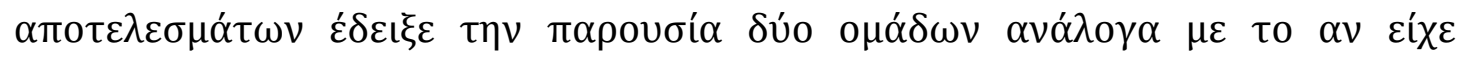

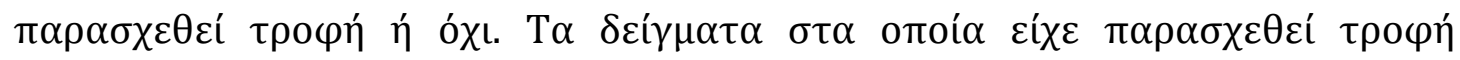

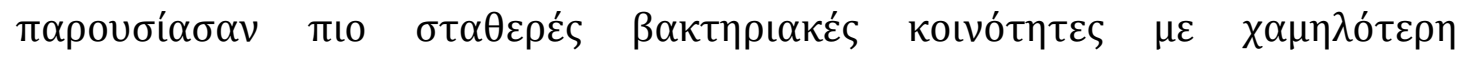

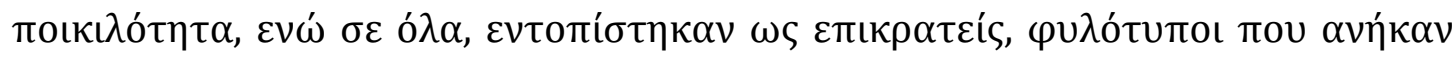

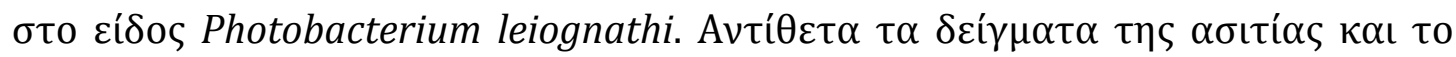

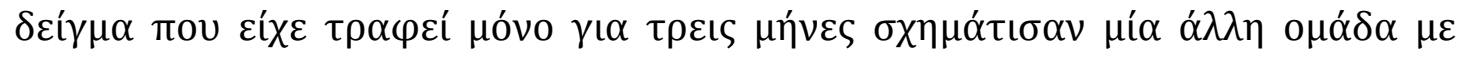

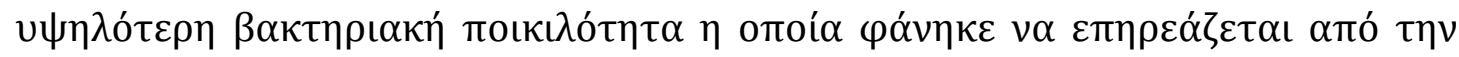

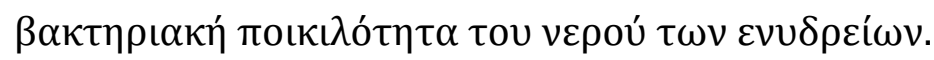

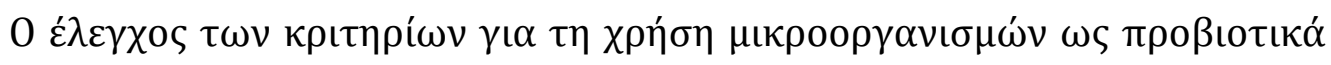

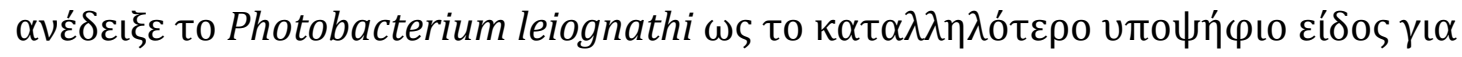

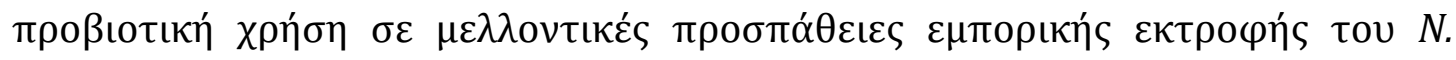

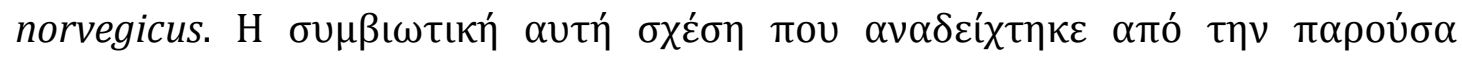

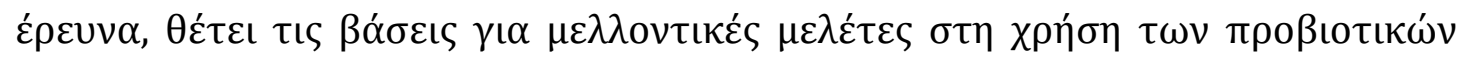

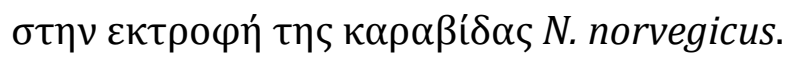




\section{6. ВIВАIОГРАФІА}

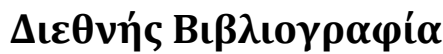

Abello P., Abella A., Adamidou A., Jukic-Peladic S., Majorano P., Spedicato M.T. (2002) Geographical patterns in abundance and population structure of Nephrops norvegicus and Parapenaeus longirostris (Crustacea: Decapoda) along the European Mediterranean coasts. Scientia Marina, 66: 125-141.

Aiken D.E., and Waddy S.L. (1995) Aquaculture. In: . Factor, J.R. (ed). Biology of the Lobster Homarus americanus Academic Press, San Diego: .

Ali A. (2000) Probiotics in fish farming. Evaluation of a bacterial mixture. PhD thesis, Swedish University of Agricultural Sciences.

Alvandi S.V., Vijayan K.K., Santiago T.C., Poornima M., Jithendran K.P., Ali S.A., Rajan J.J.S. (2004) Evaluation of Pseudomonas sp. PM 11 and Vibrio fluvialis PM 17 on immune indices of tiger shrimp, Penaeus monodon. Fish Shellfish Immunology, 17: $115-120$.

Amann R.I., Krumholz L., Stahl D.A. (1990) Fluorescent-oligonucleotide probing of whole cells for determinative, phylogenetic, and environmental studies in microbiology. Journal of Bacteriology, 172: 762-770.

Amann R.I., Ludwig W., Schleifer K.H. (1995) Phylogenetic identification and in situ detection of individual microbial cells without cultivation. Microbiology Reviews, 59: 143-169.

Ashelford K.E., Chuzhanova N.A., Fry J.C., Jones A.J., Weightman A.J. (2005) At least 1 in $2016 \mathrm{~S}$ rRNA sequence records currently held in public repositories is estimated to contain substantial anomalies. Applied and Environmental Microbiology, 71: 7724-7736.

Balcázar J.L., Rojas-Luna T., Cunningham D.P. (2007) Effect of the addition of four potential probiotic strains on the survival of pacific white shrimp (Litopenaeus vannamei) following immersion challenge with Vibrio parahaemolyticus. Journal of Invertebrate Pathology, 96: 147-150.

Bano N., DeRae Smith A., Bennett W., Vasquez L., Hollibaugh J.T. (2007) Dominance of Mycoplasma in the guts of the Long-Jawed Mudsucker, Gillichthys mirabilis, from five California salt marshes. Environmental Microbiology, 9: 2636-2641.

Beckwith T.D., Rose E.J. (1929) Cellulose digestion by organisms from the termite gut. Proceedings of Society of Experimental Biology and Medicine, 27: 4-6.

Behrens S., Fuchs B.M., Mueller F., Amann R. (2003) Is the in situ accessibility of the $16 \mathrm{~S}$ rRNA of Escherichia coli for Cy3-labeled oligonucleotide probes predicted by a three-dimensional structure model of the 30 S ribosomal subunit? Applied and Environmental Microbiology, 69: 4935-4941.

Bell M., Redant F., Tuck I. (2006) Nephrops species. In: Phillips, B. (ed), Lobsters: biology, management, aquaculture and fisheries. Oxford: Blackwell, p. 412-469.

Bent S.J., Forney L.J. (2008) The tragedy of the uncommon: understanding limitations in the analysis of microbial diversity. ISME Journal, 2: 689-695.

Bignell D.E. (1984) The arthropod gut as an environment for microorganisms. In: Anderson, J.M., Raynon, A.D.M., and Walton, D.W.A. (eds), Inverterbratemicrobial interactions. Cambridge: Cambridge University Press, p. 205-227. 
Boer S.I., Hedtkamp S.I.C., van Beusekom J.E.E., Fuhrman J.A., Boetius A., Ramette A. (2009) Time- and sediment depth-related variations in bacterial diversity and community structure in subtidal sands. ISME Journal, 3: 780-791.

Booth J.N. (2006) Jasus species. In: Phillips, B.F. (ed), Lobsters: Biology, management, aquaculture and fisheries. Oxford, UK: Blackwell publishing.

Bowman J.P. (2006) The genus Psychrobacter. In: Dworkin, M., Falkow, S., Rosenberg, E., Schleifer, K-H., Stackenbrandt, E. (eds), The Prokaryotes. New York: Springer, p. 920-930.

Boyle P.J., Mitchell R. (1978) Absence of microorganisms in crustacean digestive tracts. Science, 200: 1157-1159.

Brofft J.E., McArthur J.V., Shimkets L.J. (2002) Recovery of novel bacterial diversity from a forested wetland impacted by reject coal. Environmental Microbiology, 4: 764769.

Brunet M., Arnaud J., Mazza J. (1994) Gut structure and digestive cellular processes in marine Crustacea. Oceanographic Marine Biology Annuals, 32: 335-367.

Cardinale M., Brusetti L., Quatrini P., Borin S., Puglia A.M., Rizzi A., Zanardini E.,Sorlini C., Corselli C., Daffonchio D. (2004) Comparison of different primer sets for use in automated ribosomal intergenic spacer analysis of complex bacterial communities. Applied and Environmental Microbiology, 70: 6147-6156.

Cavallo R.A., Acquaviva M.I., Stabili L. (2009) Culturable heterotrophic bacteria in seawater and Mytilus galloprovincialis from a Mediterranean area (Northern Ionian Sea - Italy). Environmental Monitoring and Assessment, 149: 465-475.

Cavanaugh C.M., Gardiner S.L., Jones M.L., Jannasch H.W., Waterbury J.B. (1981) Prokaryotic cells in the hydrothermal vent tube worm Riftia pachyptila: possible chemoautotrophic symbionts. Science, 213: 340-342.

Ceccaldi H.J. (1989) Anatomy and physiology of digestive tract of crustaceans Decapods reared in aquaculture. Proceedings of Advances in tropical aquaculture conference. Tahiti, pp. 243-259.

Chapman C.J. (1980) Ecology of juvenile and adult Nephrops. In: Cobb, J.S., and Phillips, B.F. (eds), The biology and management of Lobsters. . London: Academic Press, pp. 143-178.

Chiu C.-H., Guu Y.-K., Liu C.-H., Pan T.-M., Cheng W. (2007) Immune responses and gene expression in white shrimp, Litopenaeus vannamei, induced by Lactobacillus plantarum. Fish and Shellfish Immunology, 23: 364-377.

Clarke K.R. (1993) Non-parametric multivariate analyses of changes in community structure. . Australian Journal of Ecology, 18: 117-143.

Clarke K.R., Green R.H. (1988) Statistical Design and Analysis for a Biological Effects Study. Marine Ecology-Progress Series, 46: 213-226.

Colorni A. (1985) A study on the bacterial flora of the giant prawn Macrobrachium rosenbergii, larvae fed with Artemia salina nauplii. Aquaculture, 49: 1-10.

Conklin D.E. (1995) Digestive physiology and Nutrition. In: Factor, I.R. (ed). Biology of the lobster Homarus americanus. USA: Academic Press, pp. 441-458.

Crawford G.S., Minion G.P., Boyers M.D.I.J.I. (1983) Intima morphology, bacterial morphotypes, and effects of annual molt on microflora in the hindgut of the desert millipede, Orthopus ornatus (Girard) (Diplopoda: Spirostreptidae). International Journal of Insect Morphology and Embryology, 12: 301-312.

Cristo M., Cartes J.E. (1998) A comparative study of the feeding ecology of Nephrops norvegicus (L.), (Decapoda : Nephropidae) in the bathyal Mediterranean and the adjacent Atlantic. Scientia Marina, 62: 81-90. 
Curtis T.P., Sloan W.T., Scannell J.W. (2002) Estimating prokaryotic diversity and its limits. Proceedings of the National Academy of Sciences, USA, 99: 10494-10499.

Daims H., Bruhl A., Amann R., Schleifer K.H., Wagner M. (1999) The domain-specific probe EUB338 is insufficient for the detection of all Bacteria: development and evaluation of a more comprehensive probe set. Systematic and Applied Microbiology, 22: 434-444.

Dalmin G., Kathiresan K., Purushothaman A. (2001) Effect of probiotics on bacterial population and health status of shrimp in culture pond ecosystem. Indian Journal of Experimental Biology, 39: 939-942.

Danovaro R., Company J.B., Corinaldesi C., D'Onghia G., Galil B., Gambi C., Gooday A.J., Lampadariou N., Luna G-M., Morigi C., Olu K., Polymenakou P., Ramirez-Llodra E., Sabbatini A., Sarda F., Sibuet M., Tselepides A. (2010) Deep-Sea Biodiversity in the Mediterranean Sea: The known, the unknown and the unknownable. PLoS ONE, 5: e11832.

Deming J.W., Colwell R.R. (1981) Barophilic bacteria associated with deep-sea animals. Bioscience, 31: 507-511.

Demiri A., Meziti A., Papaspyrou S., Thessalou-Legaki M., Kormas K.A. (2009) Abdominal setae and midgut bacteria of the mudshrimp Pestarella tyrrhena. Central European Journal of Biology, 4: 558-566.

Dempsey A.C., Kitting C.L. (1987) Characteristics of bacteria isolated from penaeid shrimp. Crustaceana 52:90-94.

Dempsey A.C., Kitting C.L., Rosson R.A. (1989). Bacterial variability among individual penaeid shrimp digestive tracts. Crustaceana, 56: 266-278.

Denner E.B., Mark B., Busse H.J., Turkiewicz M., Lubitz W. (2001) Psychrobacter proteolyticus sp. nov., a psychrotrophic, halotolerant bacterium isolated from the Antarctic krill Euphausia superba Dana, excreting a cold-adapted metalloprotease. Systematic and Applied Microbiology, 24: 44-53.

Dillon R.J., Webster G., Weightman A.J., Charnley A.K. (2010) Diversity of gut microbiota increases with aging and starvation in the desert locust. Antonie Van Leeuwenhoek International Journal of General and Molecular Microbiology, 97: 69-77.

Donachie S.P., Zdanowski M.K. (1998) Potential digestive function of bacteria in krill Euphausia superba stomach. Aquatic Microbial Ecology, 14: 129-136.

Drach P. (1939). Mue et cycle dintermue chez les crustacees decapodes. . Annuelles Institute Oceanographique, 19: 103-391.

Du H.L., Jiao N.Z., Hu Y.H., Zeng Y.H. (2006) Diversity and distribution of pigmented heterotrophic bacteria in marine environments. FEMS Microbiology Ecology, 57: 92-105.

Dubilier N., Mulders C., Ferdelman T., de Beer D., Pernthaler A., Klein M., Wagner M., Erseus C., Thiermann F., Krieger J., Giere O., Amann R. (2001) Endosymbiotic sulphate-reducing and sulphide-oxidizing bacteria in an oligochaete worm. Nature, 411: 298-302.

Dubilier N., Bergin C. and Lott C. (2008) Symbiotic diversity in marine animals: the art of charnessing chemosynthesis. Nature Reviews Microbiology, 6: 725-740.

Duperron S., Bergin C., Zielinski F., Blazejak A., Pernthaler A., McKiness Z.P., DeChaine E., Cavanaugh C.M., Dubilier N. (2006) A dual symbiosis shared by two mussel species, Bathymodiolus azoricus and Bathymodiolus puteoserpentis (Bivalvia : Mytilidae), from hydrothermal vents along the northern Mid-Atlantic Ridge. Environmental Microbiology, 8: 1441-1447. 
Durand L., Zbinden M., Cueff-Gauchard V., Duperron S., Roussel E.G., Shillito B., CambonBonavita M.A. (2010) Microbial diversity associated with the hydrothermal shrimp Rimicaris exoculata gut and occurrence of a resident microbial community. FEMS Microbiology Ecology, 71: 291-303.

Farmer A.S. (1973) Age and growth in Nephrops norvegicus (Decapoda, Nephropidae). Marine Biology, 23: 315-325.

Farmer A.S. (1974) A new technique applied to the chromosomes of Nephrops norvegicus (L.) (DECA-PODA, NEPHROPIDAE). Crustaceana, 27: 17-21.

Farmer A.S.D. (1975) Synopsis of data on the Norway lobster Nephrops norvegicus (Linnaeus, 1758). FAO fisheries synopsis, 112: 1-97.

Farmer J., J. III, and Hickman-Brenner (2006) The genera Vibrio and Photobacterium. In: Dworkin, M., Falkow, S., Rosenberg, E., Schleifer, K.-H., and Stackenbrandt, E. (eds), The Prokaryotes. . New York: Springer, p 508-563.

Farto R., Guisande J.A., Armada S.P., Prado S., Nieto T.P. (2006) An improved and rapid biochemical identification of indigenous aerobic culturable bacteria associated with galician oyster production. Journal of Shellfish Research, 25: 1059-1065.

Farzanfar A. (2006). The use of probiotics in shrimp aquaculture. FEMS Immunology and Medical Microbiology, 48: 149-158.

Figueiredo M.J., Vilela M.H. (1972) On the artificial culture of Nephrops norvegicus reared from the egg. Aquaculture, 1: 173-180.

Fisher M.M., Triplett E.W. (1999) Automated approach for ribosomal intergenic spacer analysis of microbial diversity and its application to freshwater bacterial communities. Applied and Environmental Microbiology, 65: 4630-4636.

Flint H.J., Bayer E.A., Rincon M.T., Lamed R., White B.A. (2008) Polysaccharide utilization by gut bacteria: potential for new insights from genomic analysis. Nature Reviews Microbiology, 6: 121-131.

Fraune S., Zimmer M. (2008) Host-specificity of environmentally transmitted Mycoplasma-like isopod symbionts. Environmental Microbiology, 10: 24972504.

Fuchs B.M., Woebken D., Zubkov M.V., Burkill P., Amann R. (2005) Molecular identification of picoplankton populations in contrasting waters of the Arabian Sea. Aquatic Microbial Ecology, 39: 145-157.

Fujiwara Y., Kawato M., Noda C., Kinoshita G., Yamanaka T.E.A. (2010) Extracellular and Mixotrophic Symbiosis in the Whale-Fall Mussel Adipicola pacifica: A Trend in Evolution from Extra- to Intracellular Symbiosis. PLoS ONE, 5: e11808. doi:11810.11371/journal.pone.0011808.

Fuller R. (1991) Probiotics in human medicine. Gut, 32: 439-442.

Fuller R. (1992) Probiotics: History and Development of Probiotics. New York: Chapman \& Hall.

Giebel J., Binder A., Kirchhoff H. (1990) Isolation of Mycoplasma moatsii from the Intestine of Wild Norway Rats (Rattus norvegicus). Veterinary Microbiology, 22: 23-29.

Giovannoni S.J., Britschgi T.B., Moyer C.L., Field K.G. (1990) Genetic diversity in Sargasso Sea bacterioplankton. Nature, 345: 60-63.

Glöckner F.O., Fuchs B.M., Amann R. (1999) Bacterioplankton compositions of lakes and oceans: a first comparison based on fluorescence in situ hybridization. Applied and Environmental Microbiology, 65: 3721-3726. 
Gomez-Gil B., Roque A., Lacuesta B., Rotllant G. (2010) Diversity of vibrios in the haemolymph of the spider crab Maja brachydactyla. Journal of Applied Microbiology, 109: 918-926.

Graf G. (1992) Benthic-pelagic coupling: A benthic view. Oceanography and marine biology: an annual review, 30: 149-190.

Graham N., Ferro R.S.T. (2004) The Nephrops Fisheries of the Northeast Atlantic and Mediterranean - A Review and Assessment of Fishing Gear Design. ICES Cooperative Research Report, 270.

Green-Garcia A.M. (2008) Characterization of the Lucinid bivalve-bacteria symbiotic system: the significance of the geochemical habitat on bacterial symbiont diversity and phylogeny. In M. Sc. Thesis. Louisiana state University.

Greenacre M.J. (1984) Theory and applications of correspondence analysis. London: Academic Press.

Guarner F., Schaafsma G.J. (1998) Probiotics. International Journal of Food Microbiology, 39: 237-238.

Gulmann L.K. (2004) Gut associated microbial sympbionts of the marsh fiddler crab, Uca pugnax. PhD thesis, Woods Hole Oceanographic Institution. Massachussetes.

Halpern M., Landsberg O., Raats D., Rosenberg E. (2007) Culturable and VBNC Vibrio cholerae: Interactions with chironomid egg masses and their bacterial population. Microbial Ecology, 53: 285-293.

Hammer Ø., Harper D.A.T., Ryan P.D. (2001) Past: paleontological statistics software package for education and data analysis. Palaeontologia Electronica, 4: 1-9.

Hammes W.P., Hertel C. (2006) The Genera Lactobacillus and Carnobacterium. In: Dworkin, M. (ed). The Prokaryotes. New York: Springer, p. 320-403.

Harris J.M. (1993) The Presence, Nature, and Role of Gut Microflora in Aquatic invertebrates: A Synthesis. Microbial Ecology, 25: 195-231.

Harris J.M., Seiderer L.J., Lucas M.I. (1991) Gut microflora of two saltmarsh detritivore Thalassinid prawns, Upogebia africana and Callianassa kraussi. Microbial Ecology, 21: 63-82.

Hayward P.J., Ryland J.S. (1990) The Marine Fauna of the British Isles and North-West Europe, I, Introduction and Protozoans to Arthropods. Oxford: Clarendon Press, p.489-552.

Hehemann J.-H., Correc G., Barbeyron T., Helbert W., Czjzek M., Michel G. (2010) Transfer of carbohydrate-active enzymes from marine bacteria to Japanese gut microbiota. Nature, 464: 908-912.

Heisterkamp I.M., Schramm A., de Beer D., Stief P. (2010) Nitrous oxide production associated with coastal marine inverterbrates. Marine Ecology Progress Series, 415: 1-9.

Hentschel U., Hopke J., Horn M., Friedrich A.B., Wagner M., Hacker J., Moore B.S. (2002) Molecular evidence for a uniform microbial community in sponges from different oceans. Applied and Environmental Microbiology, 68: 4431-4440.

Heylen K., Vanprays B., Wittebolle L., Verstraete W., Boon N., De Vos P. (2006) Cultivation of denitrifying bacteria: Optimization of isolation conditions and diversity study. Applied and Environmental Microbiology, 72: 2637-2643.

Hill T.C.J., Walsh K.A., Harris J.A., Moffett B.F. (2003) Using ecological diversity measures with bacterial communities. FEMS Microbiology Ecology, 43: 1-11.

Hold G.L., Smith E.A., Rappe M.S., Maas E.W., Moore E.R.B., Stroempl C., Stephen J.R., Prosser J.I., Birkbeck T.H., Gallacher S. (2001) Characterization of bacterial 
communities associated with toxic and non-toxic dinoflagellates: Alexandrium spp. and Scrippsiella trochoidea. FEMS Microbiology Ecology, 37: 161-173.

Holmstrom C., Kjelleberg S. (1999) Marine Pseudoalteromonas species are associated with higher organisms and produce biologically active extracellular agents. FEMS Microbiology Ecology, 30: 285-293.

Hood M.A., Meyers S.P., Colmer A.R. (1971) Bacteria of the digestive tract of the white shrimp Penaeus setiferus. Bacteriology, 71: 48.

Huber A.J., Johnson H.P., Butterfield D.A., Baross J.A. (2006) Microbial life in ridge flank crystal fluids. Environmental Microbiology, 8: 88-99.

Hwang C.Y., Bae G.D., Yih W., Cho B.C. (2009) Marivita cryptomonadis gen.nov., sp. nov. and Marivita litorea sp. nov., of the family Rhodobacteraceae, isolated from marine habitats. International Journal of Systematic and Evolutionary Microbiology, 59: 1568-1575.

Irianto A., Austin B. (2002) Probiotics in aquaculture. Journal of Fish Diseases, 25: 633642.

Irianto A., Austin B. (2003) Use of dead probiotic cells to control furunculosis in rainbow trout, Onchorhynchus mykiss. Journal of Fish Diseases, 26: 59-62.

Jensen S., Frost P., Torsvik V.L. (2009) The nonrandom microheterogeneity of 16S rRNA genes in Vibrio splendidus may reflect adaptation to versatile lifestyles. FEMS Microbiology Letters, 294: 207-215.

Jung S.Y., Lee M.H., Oh T.K., Park Y.H., Yoon J.H.(2005) Psychrobacter cibarius sp nov., isolated from jeotgal, a traditional Korean fermented seafood. International Journal of Systematic and Evolutionary Microbiology, 55: 577-582.

Kan J., Evans S.E., Chen F., Suzuki M.T. (2008) Novel estuarine bacterioplankton in rRNA operon libraries from the Chesapeake Bay. Aquatic Microbial Ecology, 51: 55-56.

Kane M.D., Poulsen L.K., Stahl D.A. (1993) Monitoring the Enrichment and Isolation of Sulfate-Reducing Bacteria by Using Oligonucleotide Hybridization Probes Designed from Environmentally Derived 16s Ribosomal-Rna Sequences. Applied and Environmental Microbiology, 59: 682-686.

Kemp P.F., Aller J.Y. (2004) Estimating prokaryotic diversity: When are 16S rDNA libraries large enough? Limnology and Oceanography-Methods, 2: 114-125.

Kim C.-J., Yoon S.-K., Kim H.-I., Park Y.-H., Oh H.-M. (2006) Effect of Spirulina platensis and probiotics as feed additives on growth of shrimp Fenneropenaeus chinensis. Journal of Microbiolgy and Biotechnology, 16: 1248-1254.

Kirchman D.L., Cottrell M.T., Lovejoy C. (2010) The structure of bacterial communities in the western Arctic Ocean as revealed by pyrosequencing of 16S rRNA genes. Environmental Microbiology, 12: 1132-1143.

Kormas K.A. (2011) Interpreting diversity of Proteobacteria based on 16S rRNA gene copy number. In Proteobacteria: Phylogeny, metabolic diversity and ecological effects. Sezenna, M.L. (ed). New York: Nova Publishers, pp. 73-89.

Kostanjsek R., Strus J., Avgustin G. (2007) "Candidatus Bacilloplasma" a novel lineage of Mollicutes associated with the hindgut wall of the terrestrial isopod Porcellio scaber (Crustacea : Isopoda). Applied and Environmental Microbiology, 73: 5566-5573.

Kruskal J.B. (1964) Multidimensional scaling by optimizing a goodness of fit to a nonmetric hypothesis. . Psychometrics 29: 1-28.

Lane D.J. (1991 ) 16S/23S rRNA sequencing. In Nucleic Acid Techniques in Bacterial Systematics. Stackenbrandt, E., and Goodfellow, M. (eds). Chichester: John Wiley \& Sons, pp. 115-175. 
Larkin M.A., Blackshields G., Brown N.P., Chenna R., McGettigan P.A., McWilliam H., Valentin F., Wallace I.M., Wilm A., Lopez R., Thompson J.D., Gibson T.J., Higgins D.G. (2007) Clustal W and Clustal X version 2.0. Bioinformatics, 23: 2947-2948.

Lau W.W.Y., Jumars P.A., Armbrust E.V. (2002) Genetic diversity of attached bacteria in the hindgut of the deposit-feeding shrimp Neotrypaea (formerly Callianassa) californiensis (Decapoda : Thalassinidae). Microbial Ecology, 43: 455-466.

Lebaron P., Servais P., Troussellier M., Courties C., Muyzer G., Bernard L., Schaefer H., Pukall R., Stackebrandt E., Guindulain T., Vives-Rego J. (2001) Microbial community dynamics in Mediterranean nutrient-enriched seawater mesocosms: changes in abundances, activity and composition. FEMS Microbiology Ecology, 34: 255-266.

Lee Y.K., Jung H.J., Lee H.K. (2006) Marine bacteria associated with the Korean brown alga, Undaria pinnatifida. Journal of Microbiol, 44: 694-698.

Legendre P., Legendre L. (1998) Numerical Ecology. Amsterdam: Elsevier, p. 671-672.

Ley R.E., Turnbaugh P.J., Klein S., Gordon J.I. (2006) Microbial ecology: human gut microbes associated with obesity. Nature, 444: 1022-1023.

Ley R.E., Hamady M., Lozupone C., Turnbaugh P.J., Ramey R.R., Bircher J.S., Schlegel M.L., Tucker T.A., Schrenzel M.D., Knight R., Gordon J.I. (2008) Evolution of mammals and their gut microbes. Science, 320: 1647-1651.

Li J., Tan B., Mai K., Ai Q., Zhang W., Xu W., Liufu Z., Hongming M. (2006) Comparative study between probiotic bacterium Arthrobacter XE-7 and chloramphenicol on protection of Penaeus chinensis post-larvae from pathogenic vibrios. Aquaculture, 253: 140-147.

Li K., Guan W., Wei G., Liu B., Xu J., Zhao L., Zhang Y. (2007) Phylogenetic analysis of intestinal bacteria in the Chinese mitten crab (Eriocheir sinensis). Journal of Applied Microbiology, 103: 675-682.

Li L., Kato C., Horikoshi K. (1999) Bacterial diversity in deep-sea sediments from different depths. Biodiversity and Conservation, 8: 659-677.

Lin H.Z., Guo Z., Yang Y., Zheng W., Li Z.J. (2004) Effect of dietary probiotics on apparent digestibility coefficients of nutrients of white shrimp Litopenaeus vannamei Boone. Aquaculture Research, 35: 1441-1447.

Ludwig W., Strunk O., Westram R., Richter L., Meier H., Yadhukumar, Buchner A., Lai T., Steppi S., Jobb G., Foerster W., Brettske I., Gerber S., Ginhart A.W., Gross O., Grumann S., Hermann S., Jost R., Koenig A., Liss T., Luessmann R., May M., Nonhoff B., Reichel B., Strehlow R., Stakatakis A., Stuckmann N., Vilbig A., Lenke M., Ludwig T., Bode A., Schleifer K.-H. (2004) ARB: a software environment for sequence data. Nucleic Acids Research, 32: 1363-1371.

Lustigman S., McKerrow J.H., Shah K., Lui J., Huima T., Hough M., Brotman B. (1996) Cloning of a Cysteine Protease Required for the Molting of Onchocerca volvulus Third Stage Larvae. Journal of Biological Chemistry, 271: 30181-30189.

Maltagliati F., Camilli L., Biagi F., Abbiati M. (1998) Genetic structure of Norway lobster, Nephrops norvegicus (L.) (Crustacea: Nephropidae), from the Mediterranean Sea. Scientia Marina, 62: 91-99.

Manz W., Amann R., Ludwig W., Wagner M., Schleifer K.-H. (1992) Phylogenetic oligodeoxynucleotide probes for the major subclasses of Proteobacteria: problems and solutions. . Systematic and Applied Microbiology, 15: 593-600.

Margalef R. (1958) Temporal succession and spatial heterogeneity in phytoplankton. . In Perspectives in marine biology. Berkeley and Los Angeles: Univ. Calif. Press., p. 323-350. 
Medigue C., Krin E., Pascal G., Barbe V., Bernsel A., Bertin P.N., Cheung F., Cruveiller S., D’Amico S., Duilio A., Fang G., Feller G., Ho C., Mangenot S., Marino G., Nilsson J., PArrilli E., Rocha E.P.C., Rouy Z., Sekowska A., Tutino M.L., Vallenet D., von Heijne G., Danchin A. (2005) Coping with cold: the genome of the versatile marine Antarctica bacterium Pseudoalteromonas haloplanktis TAC125. Genome Res, 15: 1325-1335.

Mente E., Karapanagiotidis I.T., Logothetis P., Vafidis D., Malandrakis E., Neofitou N., Exadactylos A., Stratakos A. (2009) The reproductive cycle of Norway lobster. Journal of Zoology, 278: 324-332.

Mente E. (2010) Survival, food consumption and growth of Norway lobster (Nephrops norvegicus) kept in laboratory conditions. Integrative Zoology, 5: 256-263.

Meziti A., Ramette A., Mente E., Kormas K.A. (2010) Temporal shifts of the Norway lobster (Nephrops norvegicus) gut bacterial communities. FEMS Microbiology Ecology, 74: 472-484.

Morita H., Nakano A., Shimazu M., Toh H., Nakajima F., Nagayama M., Hisamatsu S., Kato Y., Takagi M., Takami H., Akita H., Matsumoto M., Masaoka T., Murakami M. (2009) Lactobacillus hayakitensis, L. equigenerosi and L. equi, predominant lactobacilli in the intestinal flora of healthy thoroughbreds. Animal Science Journal, 80: 339-346.

Muyzer G., Teske A., Wirsen C.O., Jannasch H.W. (1995) Phylogenetic-Relationships of Thiomicrospira Species and Their Identification in Deep-Sea Hydrothermal Vent Samples by Denaturing Gradient Gel-Electrophoresis of 16s rDNA Fragments. Archives of Microbiology, 164: 165-172.

Nasu H., Iida T., Sugahara T., Yamaichi Y., Park K.S., Yokoyama K., Makino K., Shinagawa H., Honda T. (2000) A filamentous phage associated with recent pandemic Vibrio parahaemolyticus 03:K6 strains. Journal of Clinical Microbiology, 38: 2156-2161.

Nayak S.K. (2010) Role of gastrointestinal microbiota in fish. Aquaculture Research, 41: 1553-1573.

Nechitaylo T.Y., Timmis K.N., Golyshin P.N. (2009) 'Candidatus Lumbricincola', a novel lineage of uncultured Mollicutes from earthworms of family Lumbricidae. Environmental Microbiology, 11: 1016-1026.

Nicosia F., Lavalli K. (1999) Homarid lobster hatcheries: their history and role in research, management, and aquaculture. Marine Fisheries Review, 61: 1-57.

Nishiguchi M.K., Nair V.S. (2003) Evolution of symbiosis in the Vibroonaceae: a combined approach using molecules and physiology. International Journal of Systematic and Evolutionary Microbiology, 53: 2019-2026.

Nittami T., McIlroy S., Seviour E.M., Schroeder S., Seviour R.J. (2009) Candidatus Monilibacter spp., common bulking filaments in activated sludge, are members of Cluster III Defluviicoccus. Systematic and Applied Microbiology, 32: 480-489.

Olsen G.J., Lane D.J., Giovannoni S.J., Pace N.R., Stahl. D.A. (1986) Microbial ecology and evolution: a ribosomal RNA approach. Annual Review of Microbiology, 40: 337365.

Orozco-Medina C., Maeda-Martínez A.M., López-Cortés A. (2002) Effect of aerobic Grampositive heterotrophic bacteria associated with Artemia franciscana cysts on the survival and development of its larvae. Aquaculture, 213: 15-29.

Passamonti M., Mantovani B., Scali V., Froglia C. (1997) Allozymic characterization of Scottish and Aegean populations of Nephrops norvegicus. Journal of the Marine Biological Association of the United Kingdom, 77: 727-735. 
Petihakis G., Triantafyllou G., Pollani A., Koliou A., Theodorou A. (2005) Field data analysis and application of a complex water column biogeochemical model in different areas of a semi-enclosed basin: towards the development of an ecosystem management tool. Marine Environmental Research, 59: 493-518.

Phillips B.F., Melville-Smith R. (2006) Panulirus species. In: Phillips, B.F. (ed), Lobsters: Biology, management, aquaculture and fisheries. Oxford, UK: Blackwell publishing.

Pinhassi J., Sala M.M., Havskum H., Peters F., Guadayol O., Malits A., Marrasse C.L. (2004) Changes in bacterioplankton composition under different phytoplankton regimens. Applied and Environmental Microbiology, 70: 6753-6766.

Pinn E.H., Nickell L.A., Rogerson A., Atkinson R.J.A. (1999) Comparison of gut morphology and gut microflora of seven species of mud shrimp (Crustacea: Decapoda: Thalassinidea). Marine Biology, 133: 103-114.

Price H.J., Boyd K.R., Boyd C.M. (1988) Omnivorous feeding behaviour of the Antarctic krill Euphausia superba. Marine Biology, 97: 67-77.

Pruesse E., Quast C., Knittel K., Fuchs B.M., Ludwig W., Peplies J., Glockner F.O. (2007) SILVA: a comprehensive online resource for quality checked and aligned ribosomal RNA sequence data compatible with ARB. Nucleic Acids Res, 35: 7188-7196.

Pryde S.E., Duncan S.H., Hold G.L., Stewart C.S., Flint H.J. (2002) The microbiology of butyrate formation in the human colon. FEMS Microbiology Letters, 217: 133139.

Ramette A. (2007) Multivariate analyses in microbial ecology. FEMS Microbiology Ecology, 62: 142-160.

Ramette A. (2009) Quantitative Community Fingerprinting Methods for Estimating the Abundance of Operational Taxonomic Units in Natural Microbial Communities. Applied and Environmental Microbiology, 75: 2495-2505.

Rao C.R. (1964) The use and interpretation of principal component analysis in applied research. Sankhya A, 26: 329-358.

Razin S., Yogev D., Naot Y. (1998) Molecular biology and pathogenicity of mycoplasmas. Microbiology and Molecular Biology Reviews, 62: 1094-+.

Reichenbach H., Weeks O. (1981) The Flavobacteium-Cytophaga group. Weinheim, Germany: Verlag Chemie.

Rengpipat S., Tunyamum A., Fast A.W., Piyatiratitivoraku S., Menasveta P. (2003) Enhanced growth and resistance to vibrio challenge in pond-reared black tiger shrimp Penaeus monodon fed a Bacillus probiotic. Diseases of Aquatic Organisms, 55: 169-173.

Rice A.L., Chapman C.J. (1971) Observations on the burrows and burrowing behaviour of two mud-dwelling decapod crustaceans, Nephrops norvegicus and Goneplax rhomboides. Marine Biology, 10: 330-342.

Riesenfeld C.S., Murray A.E., Baker B.J. (2008) Characterization of the Microbial Community and Polyketide Biosynthetic Potential in the Palmerolide-Producing Tunicate Synoicum adareanum. Journal of Natural Products, 71: 1812-1818.

Romanenko L.A., Tanaka N., Frolova G.M., Mikhailov V.V. (2010) Marinicella litoralis gen. nov., sp. nov., a gammaproteobacterium isolated from coastal seawater. International Journal of Systematic and Evolutionary Microbiology, 60: 16131619. 
Romanenko L.A., Tanaka N., Uchino M., Kalinovskaya N.I., Mikhailov V.V. (2008) Diversity and Antagonistic Activity of Sea lee Bacteria Isolated from the Sea of Japan. Microbes and Environments, 23: 209-214.

Romanenko L.A., Tanaka N., Frolova G.M., Svetashev V.I., Mikhailov V.V. (2011) Litoreibacter albidus gen. nov., sp. nov. and Litoreibacter janthinus sp. nov., members of the class Alphaproteobacteria isolated from the seashore. International Journal of Systematic and Evolutionary Microbiology, 61.

Rosa R., Morais S., Calado R., Narciso L., Nunes M.L. (2003) Biochemical changes during the embryonic development of Norway lobster, Nephrops norvegicus. Aquaculture, 221: 507-522.

Rotllant G., Charmantier-Daures M., Charmantier G., Anger K., Sarda F. (2001) Effects of diet on Nephrops norvegicus (L.) larval and postlarval development, growth, and elemental composition. Journal of Shellfish Research, 20: 347-352.

Russell J.A., Moreau C.S., Goldman-Huertas B., Fujiwara M., Lohman D.J., and Pierce N.E. (2009) Bacterial gut symbionts are tightly linked with the evolution of herbivory in ants. Proceedings of the National Academy of Sciences of the United States of America, 106: 21236-21241.

Sarda F. (1983) Determinación de los estados de intermuda en Nephrops norvegicus (L.), mediante la observación de los pléopodos. Investigacion Pesquera, 47: 95-112.

Sarda F. (1991) Reproduction and moult synchronism in Nephrops norvegicus (L.) (Decapoda, Nephropidae) in the western Mediterranean: is spawning annual or biennial? . Crustaceana, 60: 186-199.

Sarda F. (1995) A review (1967-1990) of some aspects of the life history of Nephrops norvegicus. ICES marine science symposia, 199: 78-88.

Sarda F., Valladares F.J. (1990) Gastric Evacuation of Different Foods by Nephrops Norvegicus (Crustacea, Decapoda) and Estimation of Soft-Tissue Ingested, Maximum Food-Intake and Cannibalism in Captivity. Marine Biology, 104: 2530 .

Sawabe T., Narita M., Tanaka R., Onji M., Tajima K., Ezura Y. (2000) Isolation of Pseudoalteromonas elyakovii strains from spot-wounded fronds of Laminaria japonica. Nippon Suisan Gakkaishi, 66: 249-254.

Schmidtova J., Hallam S.J., Baldwin S.A. (2009) Phylogenetic diversity of transition and anoxic zone bacterial communities within a near-shore anoxic basin: Nitinat lake. Environmental Microbiology, 11: 3233-3251.

Schulze A.D., Alabi A.O., Tattersall-Sheldrake A.R., Miller K.M. (2006) Bacterial diversity in a marine hatchery: Balance between pathogenic and potentially probiotic bacterial strains. Aquaculture, 256: 50-73.

Shannon C.E., Weaver W. (1949) The Mathematical Theory of Communication. Urbana: University of Illinois Press.

Simon C.J. (2009) Digestive enzyme response to natural and formulated diets in cultured juvenile spiny lobster, Jasus edwardsii. Aquaculture, 294: 271-281.

Simpson E.H. (1949) Measurement of diversity. Nature, 163: 688.

Sipkema D., Schippers K., Maalcke W.J., Yang Y., Salim S., Blanch H.W. (2011) Multiple Approaches To Enhance the Cultivability of Bacteria Associated with the Marine Sponge Haliclona gellius sp. Applied and Environmental Microbiology, 77: 21302140.

Smith C.J., Papadopoulou K.-N. (2003) Burrow density and stock size fluctuations of Nephrops norvegicus in a semi-enclosed bay. ICES Journal of Marine Science, 60:798-805. 
Sogin M.L., Morrison H.G., Huber J.A., Welch D.M., Huse S.M., Neal P.R., Arrieta J.M., Herndl G.H. (2006) Microbial diversity in the deep sea and the underexplored 'rare biosphere'. Proceedings of the National Academy of Sciences, USA, 103: 12115-12120.

Sohn J.H., Lee J.-H., Yi H., Chun J., Bae K.S., Ahn T.-Y., Kim S.-J. (2004) Kordia algicida gen. nov., sp. nov., an algicidal bacterium isolated from red tide. International Journal of Systematic and Evolutionary Microbiology, 54: 675-680

Stamatis C., Triantafyllidis A., Moutou K.A., Mamuris Z. (2004) Mitochondrial DNA variation in northeast atlantic and mediterranean populations of norway lobster, Nephrops norvegicus. Molecular Ecology, 13: 1377-1390.

Suen G., Scott J.J., Aylward F.O., Adams S.M., Tringe S.G., Pinto-Tomas A.A., Foster C.E., Pauly M., Weimer P.J., Barry K.W., Goodwin L.A. Bouffard P., Li L., Osterberger J., Harkins T.T., Slater S.C., Donohue T.J., Currie C.R. (2010) An insect herbivore microbiome with high plant biomass-degrading capacity. PLoS Genetics, 6: e1001129.

Tauch A., Trost E., Tilker A., Ludewig U., Schneiker S., Goesmann A., Arnold W., Bekel T., Brinkoff K., Brune I., Goetker S., Kalinowski J., Kamp P.-B., Pereira Lobo F., Viehoever P., Weisshaar B., Soriano F., Droege M., Puehler A. (2008) The lifestyle of Corynebacterium urealyticum derived from its complete genome sequence established by pyrosequencing. Journal of Biotechnology, 136: 11-21.

Thompson F.L., Iida T., Swings J. (2004) Biodiversity of vibrios. Microbiology and Molecular Biology Reviews, 68: 403-431

Thyssen A., Ollevier F. (2004) The Family Vibrionaceae, The genus Photobacterium. In: Garrity, G.M. (ed), Bergey's Manual of Systematic Bacteriology. Michigan, USA: Springer, p. 546-552.

Triantafyllou G., Petihakis G., Dounas C., Theodorou A. (2001) Assessing marine ecosystem response to nutrient inputs. Marine Pollution Bulletin, 43: 175-186.

Tsukamoto H., Takakura Y., Mine T., Yamamoto T. (2008) Photobacterium sp. JT-ISH-224 produces two sialyltransferases, alpha-/beta-galactoside alpha2,3sialyltransferase and beta-galactoside alpha2,6-sialyltransferase. Journal of Biochemistry, 143: 187-197.

Tuck I.D., Atkinson R.J., Chapman C.J. (2000) Population biology of the Norway lobster, Nephrops norvegicus (L.) in the Firth of Clyde, Scotland. II. Fecundity and size at onset of sexual maturity. ICES Journal of Marine Science, 57: 1227-1239.

Turnbaugh P.J., Ley R.E., Mahowald M.A., Magrini V., Mardis E.R., Gordon J.I. (2006) An obesity-associated gut microbiome with increased capacity for energy harvest. Nature, 444: 1027-1031.

Urakawa H., Kita-Tsukamoto K., Ohwada K. (1999) Restriction fragment length polymorphism analysis of psychrophilic and psychrotrophic Vibrio and Photobacterium from the north-western Pacific Ocean and Otsuchi Bay, Japan. Canadian Journal of Microbiology, 45: 67-76.

Vandamme P., Dewhirst F.E., Paster B.J., On S.L.W. (2004) Genus II. Arcobacter. In: Garrity, G.M. (ed). Bergey's Manual of Bacteriology. Michigan, USA: Springer, p. 1161-1165.

Vaseeharan B., Ramasamy P. (2003) Control of pathogenic Vibrio spp. by Bacillus subtilis BT23, a possible probiotic treatment for black tiger shrimp Penaeus monodon. Letters in Applied Microbiology, 36: 83-87.

Vega-Villasante F., Fernandez I., Preciado R., Oliva M., Torvar D. (1999) The activity of digestive enzymes during the molting stages of the arched swimming Callinectes 
arcuatus, Ordway, 1963 (Crustacea: Decapoda: Portunidae). Bulletin of Marine Science, 65: 1-9.

Verschuere L., Rombout G., Sorgeloos P., Verstraete W. (2000) Probiotic bacteria as biological control agents in aquaculture. Microbiology and Molecular Biology Reviews, 64: 655-671.

Ward N.L., Steven B., Penn K., Methe B.A., Detrich W.H. (2009) Characterization of the intestinal microbiota of two Antarctic notothenioid fish species. Extremophiles, 13: 679-685.

Woese C.R., Fox G.E. (1977) Phylogenetic structure of the Prokaryotic domain: The primary Kingdoms. Proceedings of the National Academy of Sciences, USA, 74: 5088-5090.

Wolda H. (1981) Similarity indices, sample size and diversity. Oecologia, 50: 296-302.

Wu S., Gao T., Zheng Y., Wang W., Cheng Y., Wang G. (2010) Microbial diversity of intestinal contents and mucus in yellow catfish (Pelteobagrus fulvidraco). Aquaculture, 303: 1-7.

Yonge C.M. (1924) Studies on the Comparative Physiology of Digestion: II. The Mechanism of Feeding, Digestion, and Assimilation in Nephrops Norvegicus. Journal of Experimental Biology, 1: 343-389.

Zbinden M., Cambon-Bonavita M.A. (2003) Occurrence of Deferribacterales and Entomoplasmatales in the deep-sea Alvinocarid shrimp Rimicaris exoculata gut. FEMS Microbiology Ecology, 46: 23-30.

Zbinden M., Shilito B., Le Bris N., de Villardi de Montlaur C., Roussel E., Guyot F., Gaill F., Cambon-Bonavita M.-A. (2008) New insights on the metabolic diversity among the epibiotic microbial community of the hydrothermal shrimp Rimicaris exoculata. Journal of Experimental Marine Biology and Ecology, 359: 131-140.

Zhang D.-C., Li H.-R., Xin Y.-H., Liu H.-C., Chi Z.-M., Zhou P.-J., Yu Y. (2008) Phaeobacter arcticus sp. nov., a psychrophilic bacterium isolated from the Arctic. International Journal of Systematic and Evolutionary Microbiology, 58: 13841387.

Zhou M.-Y., Chen X.-L., Zhao H.-L., Dang H.-Y., Luan X.-W., Zhang X.-Y., He H.-L., Zhou B.C., Zhang Y.-Z. (2009) Diversity of both cultivable protease-producing bacteria and their extracellular proteases in the sediments of the south china Sea. Microbial Ecology, 58: 582-590.

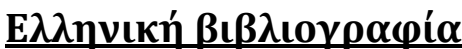

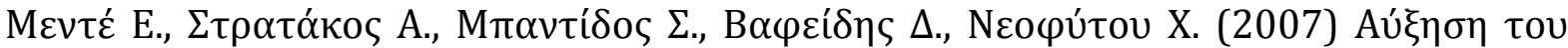

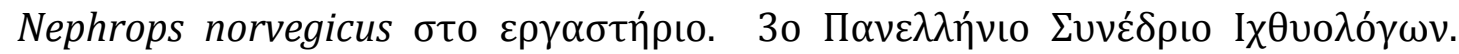

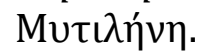

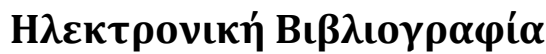

BLAST (2011):

http://blast.ncbi.nlm.nih.gov/Blast.cgi?PAGE=Nucleotides\&PROGRAM=blastn\& MEGABLAST $=$ on\&BLAST PROGRAMS=megaBlast\&PAGE TYPE=BlastSearch\&SH OW DEFAULTS=on\&LINK LOC=blasthome $(01 / 05-01 / 09 / 2011)$

FAO (2011): FAO Fisheries and Aquaculture Department

http://www.fao.org/docrep/009/A0699e/A0699E00.HTM (04/10/2011) 
Microbial Genome Resources (2011):

http://www.ncbi.nlm.nih.gov/sites/entrez?db=genome\&cmd=Retrieve\&dopt=0 verview\&list uids $=7287(05 / 10 / 2011)$

SILVA (2011): http://www.arb-silva.de/browser/ (15/06/2011)

SPADE (2010): http://chao.stat.nthu.edu.tw/softwareCE.html (01/06/2011)

Zimmer C. (2011): the wired atlas of the human ecosystem:

http://www.wired.com/magazine/2011/09/mf microbiome/ (15/10/2011) 


\section{ABSTRACT}

The Norway lobster Nephrops norvegicus, also known as Dublin bay prawn is a Decapod Crustacean of the Family Nephropidae that is widely distributed in silty areas, of the continental shelf and the upper continental slope, of the Northeast Atlantic and the Mediterranean Sea. The Norway lobster is a highly commercial species in the areas where it is found. Efforts for the commercial rearing of the species have not succeeded yet due to lack of knowledge on the nutritional habits of the species on rearing conditions and on its exact nutritional demands.

This thesis studied for the first time the gut bacterial diversity of Nephrops norvegicus. Studies were performed in natural and reared populations, where different food sources were provided. The project targeted in finding the factors that shape gut bacterial diversity and in detecting symbiotic, non toxic and non pathogenic bacterial species that can influence the digestive functions of $N$. norvegicus and could potentially be used as probiotics in future rearing efforts.

For the study of the natural populations $N$. norvegicus individuals were collected from Pagasitikos gulf during different months in 2007. The digestive tract was extracted and evacuated, followed by DNA extraction. DNA was analyzed with the use of molecular tools studying $16 \mathrm{~S}$ rRNA and the Intergenic Transcribed Spacer (ITS). The 16S rRNA diversity was studied with the use of clone libraries and Fluorescence in Situ Hybridization (FISH) while the ITS diversity was studied with the use of clone libraries and Automated Ribosomal Intergenic Spacer Analysis (ARISA). The study of the ITS diversity with the use of ARISA showed that 
gut bacterial microdiversity was influenced by sampling season while other factors that were checked such as sex or weight had no influence. Seasonal differences in Pagasitikos gulf, and mostly in the areas where $N$. norvegicus is found, are based in changes in food availability and quality and not in physicochemical factors. In this area temperature and salinity are almost stable all over the year, while changes are observed in the quantity and the quality of the organic matter from upper layers that finally influences the trophic webs in the bottom.

Further analysis of selected samples from all collection months with 16SITS clone libraries showed the dominance of specific phylotypes $(>58 \%)$ in each one of them. The dominant phylotypes in the samples of February/March, May, July, August and October clustered in the genera of Pseudoalteromonas, Psychrobacter and Photobacterium while the ones in the samples of September and December clustered in an uncultured Mollicutes group and in the candidate division CK-1C4-19 respectively. The presence of $\gamma$-Proteobacteria in August samples and of uncultured Mollicutes in September samples was further verified with the FISH results. For the detection of cells belonging to $\gamma$-Proteobacteria, probe GAM42a was used while for the cells belonging to the dominant Mollicute phylotype a specific probe was designed.

The combination of ARISA results with the ITS lengths of the dominant phylotypes from the clone libraries further confirmed the presence of Photobacterium sp. and Psychrobacter sp. representatives in most of the samples studied. However the presence of the Mollicutes and CK-1C4-19 phylotypes was underestimated in the ARISA as a result of mismatches of their sequences with the primers used. 
Rearing experiments were performed in order to confirm the effect of feeding in gut bacterial diversity. $N$. norvegicus individuals were divided in three groups and were kept for six months in rearing tanks where three different kinds of food were checked (S: starvation, M: mussels, P: pellets). Mussels and pellets were provided in the respective groups three times per week. In order to study the gut bacterial diversity, $N$. norvegicus samplings were performed at the beginning of the experiment (t0) and after three (t1) and six months (t2). At the same time water samples from the rearing tanks and samples from the food provided were analyzed.

All the samples were analyzed using 16S rRNA clone libraries. Samples from the initial time point ( $\mathrm{t} 0$ ) had similar results with the natural populations showing low bacterial diversities and dominant phylotypes with closest relatives belonging to the genera Psychrobacter and Photobacterium. All samples after six month rearing with pellets and mussels showed higher bacterial diversity than wild samples. However all of them had dominant phylotypes, with frequencies ranging from $22 \%$ to $73.5 \%$, clustering in the species Photobacterium leiognathi and showing high percentages of similarity (>98\%) with the respective Photobacterium like phylotypes of the wild populations. Previous microbiological and genomic studies have shown the presence of lipolytic, proteinolytic and chitinolytic enzymes in P. leiognathi enhancing the hypothesis of its assistance in N. norvegicus digestive activity.

Starvation samples as well as the three months mussel reared sample showed higher bacterial diversity than all other samples. As it was proved after statistical analysis their bacterial diversity was mostly influenced by the tanks' 
water bacterial diversity. Statistical analysis for all samples showed the existence of two groups depending mostly on whether food was provided or not regardless of its kind. The basic discriminative characteristic between these two groups was the presence of phylotypes clustering in P. leiognathi.

For the first time, it was proved that food provision is an important factor for the shaping of gut bacterial communities of $N$. norvegicus. The factors that shape gut bacterial diversity, as well as abundant and potentially symbiotic microorganisms were detected. Bacterial phylotypes clustering in P. leiognathi with potential chitinolytic, proteinolytic and lipolytic activities were found abundant in the gut of wild and reared populations of $N$. norvegicus suggesting their use as probiotics in future rearing efforts.

Keywords: Nephrops norvegicus, midgut, Bacteria, 16S rRNA, rearing, Photobacterium, probiotics 


\section{ПАРАРТНМА}




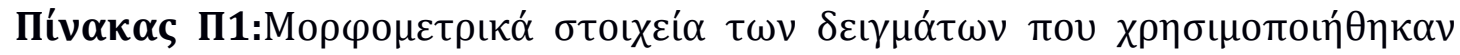

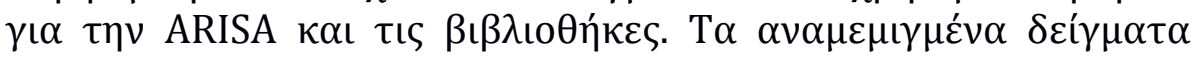

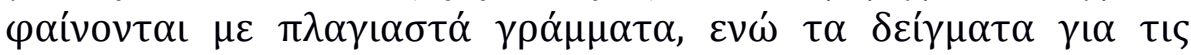

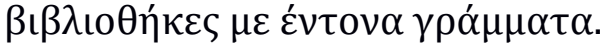

\begin{tabular}{|c|c|c|c|c|c|}
\hline$\Delta \varepsilon^{\prime} \gamma \mu \alpha$ & Mńvos & 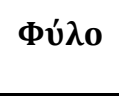 & $\begin{array}{l}\text { Bópos } \\
\sigma \omega ́ \mu \alpha \tau o \zeta\end{array}$ & 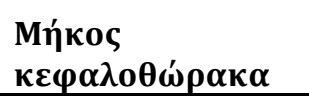 & $\begin{array}{l}\Pi \lambda \alpha^{\prime} \tau 0 S \\
\kappa \varepsilon \varphi \alpha \lambda 0 \theta \omega ́ \rho \alpha \kappa \alpha\end{array}$ \\
\hline F40 & 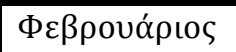 & A & 89,91 & 50,21 & 27,47 \\
\hline F44 & 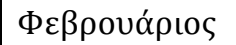 & A & 44,71 & 26,77 & 24,5 \\
\hline $\begin{array}{l}F 1 \\
(F 40 / F 44)\end{array}$ & 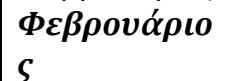 & $A$ & 67,31 & 38,49 & 25,985 \\
\hline Mr4 & 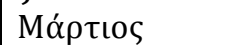 & $\Theta$ & 17,98 & 36,04 & 18,25 \\
\hline Mr10 & 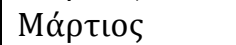 & $\Theta$ & 26,31 & 36,4 & 18,6 \\
\hline $\begin{array}{l}\text { Mr1(Mr4/ } \\
\text { Mr10) }\end{array}$ & 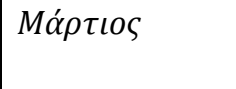 & $\Theta$ & 22,145 & 36,22 & 18,425 \\
\hline Mr26 & 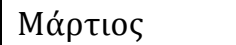 & A & 30,35 & 40,6 & 22,47 \\
\hline My27 & Mó́tos & $\Theta$ & 12,47 & 27,32 & 13,95 \\
\hline My44 & Mó́tos & A & 49,79 & 42,75 & 21,84 \\
\hline My45 & Mó́tos & A & 27,69 & 36,15 & 18,89 \\
\hline My46 & Mó́tos & A & 29,2 & 37,22 & 17,64 \\
\hline My47 & Mó́tos & A & 39,21 & 40,36 & 21,3 \\
\hline Jl1 & Iov́גıos & $\Theta$ & 38,51 & 38,54 & 20,51 \\
\hline $\mathrm{Jl3}$ & 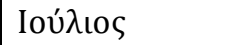 & $\Theta$ & 65,28 & 48,13 & 25,22 \\
\hline Jl14 & 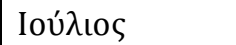 & A & 59,9 & 44,79 & 23,18 \\
\hline Jl17 & 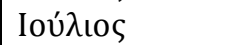 & A & 50,38 & 41,92 & 20,78 \\
\hline Jl19 & 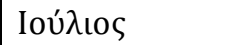 & A & 39,73 & 40,16 & 19,65 \\
\hline $\begin{array}{l}J l 4(J l 14 / J I \\
17 / J 119)\end{array}$ & Ioúגıoৎ & $A$ & 50 & 42,29 & 21,2 \\
\hline Ag4 & 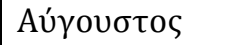 & $\Theta$ & 22,53 & 35,18 & 17,94 \\
\hline Ag6 & 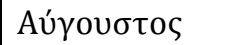 & $\Theta$ & 28,35 & 35,94 & 18,67 \\
\hline Ag9 & 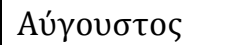 & $\Theta$ & 43,54 & 42,53 & 21,39 \\
\hline $\begin{array}{l}\operatorname{Ag1}(\operatorname{Ag} 4 / A \\
g 6 / A g 9)\end{array}$ & 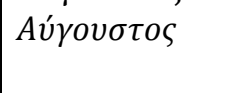 & $\Theta$ & 31,47 & 37,88 & 19,33 \\
\hline Ag2,1 & 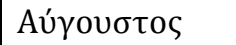 & $\Theta$ & 30,66 & 36,4 & 18,3 \\
\hline Ag8 & 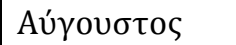 & $\Theta$ & 23,72 & 32,93 & 16,53 \\
\hline Ag16 & 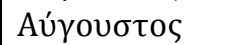 & $\Theta$ & 22,05 & 35,66 & 18 \\
\hline Ag23 & 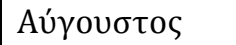 & $\Theta$ & 31,48 & 37,41 & 18,63 \\
\hline Ag31 & 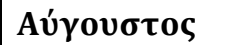 & $\mathbf{A}$ & 52,58 & 42,46 & 21,34 \\
\hline Se1 & $\Sigma \varepsilon \pi \tau \varepsilon \dot{\varepsilon} \mu \beta \rho$ & A & 43,3 & 40,32 & 22,74 \\
\hline Se2 & $\Sigma \varepsilon \pi \tau \varepsilon \dot{\mu} \mu \beta \rho$ เо & A & 68,9 & 44,96 & 27,17 \\
\hline Se3 & $\Sigma \varepsilon \pi \tau \varepsilon \dot{\varepsilon} \mu \beta \rho เ о \varsigma$ & $\mathbf{A}$ & 22,297 & 31,47 & 17,59 \\
\hline Se4 & 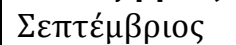 & $\Theta$ & 45,85 & 40,92 & 20,53 \\
\hline Se5 & $\Sigma \varepsilon \pi \tau \varepsilon \dot{\varepsilon} \mu \beta \rho \rho^{\prime}$ & $\Theta$ & 27,5 & 35,04 & 17,83 \\
\hline Se6 & $\Sigma \varepsilon \pi \tau \varepsilon \dot{\varepsilon} \mu \beta \rho \circ$ & $\Theta$ & 21,61 & 31,67 & 19,32 \\
\hline Se7 & $\Sigma \varepsilon \pi \tau \varepsilon \dot{\varepsilon} \mu \beta \rho$ & $\Theta$ & 36,04 & 38,9 & 19,98 \\
\hline Se9 & 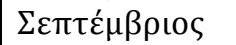 & $\Theta$ & 31,51 & 37,58 & 19,35 \\
\hline Se11 & $\sum \varepsilon \pi \tau \varepsilon \dot{\varepsilon} \mu \beta \rho$ & A & 28,88 & 35,33 & 17,83 \\
\hline Se12 & $\Sigma \varepsilon \pi \tau \varepsilon \dot{\varepsilon} \mu \beta \rho \iota{ }^{\prime}$ & A & 32,41 & 37,94 & 18,45 \\
\hline 01 & Окти́ßріоц & $\Theta$ & 36,71 & 38,36 & 19,21 \\
\hline 02 & 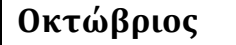 & $\Theta$ & 24,8 & 35,61 & 16,44 \\
\hline 03 & 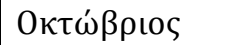 & $\Theta$ & 17,37 & 29,97 & 14,91 \\
\hline 04 & 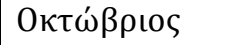 & $\Theta$ & 11,85 & 28,59 & 13,34 \\
\hline 05 & 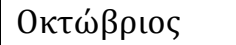 & A & 29,12 & 36,84 & 17,72 \\
\hline 06 & 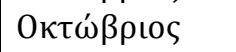 & A & 27,76 & 35,71 & 17,35 \\
\hline D1 & $\Delta \varepsilon \kappa \varepsilon ́ \mu \beta \rho t o \zeta$ & $\Theta$ & 22,06 & 35,25 & 17,3 \\
\hline D2 & $\Delta \varepsilon \kappa \varepsilon \dot{\varepsilon} \mu \beta \rho\llcorner о \varsigma$ & $\Theta$ & 21,99 & 32,12 & 16,23 \\
\hline
\end{tabular}




\begin{tabular}{|c|c|c|c|c|c|}
\hline$\Delta \varepsilon^{\prime} \gamma \mu \alpha$ & Mńvas & $\Phi u ́ \lambda$ o & $\begin{array}{l}\text { Bópos } \\
\sigma \omega ́ \mu \alpha \tau o s\end{array}$ & $\begin{array}{l}\text { Мท́ко૬ } \\
\kappa \varepsilon \varphi \alpha \lambda о \theta \omega ́ \rho \alpha \kappa \alpha\end{array}$ & 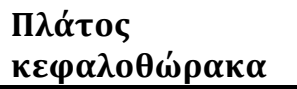 \\
\hline D3 & 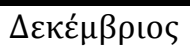 & $\Theta$ & 20,77 & 32,18 & 15,51 \\
\hline D4 & $\Delta \varepsilon \kappa \varepsilon \dot{\varepsilon} \mu \beta \rho ı$ & A & 32,78 & 40,05 & 19,48 \\
\hline D6 & $\Delta \varepsilon \kappa \varepsilon ́ \mu \beta \rho ı \varsigma$ & A & 59,47 & 45,88 & 24,09 \\
\hline D7 & $\Delta \varepsilon \kappa \varepsilon ́ \mu \beta \rho$ & $A$ & 18,85 & 35 & 17,32 \\
\hline
\end{tabular}

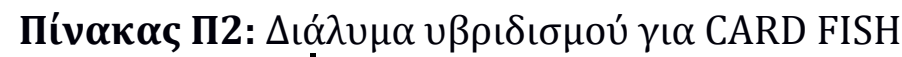

\begin{tabular}{|c|c|c|c|c|c|}
\hline FA\% & 20 & 30 & 40 & 50 & 60 \\
\hline $\mathrm{NaCl} 5 \mathrm{M}$ & & & $3.6 \mathrm{ml}$ & & \\
\hline $\begin{array}{c}\text { Tris-HCl } 1 \text { M } \\
\text { pH=8 }\end{array}$ & & & $0.4 \mathrm{ml}$ & & \\
\hline SDS $20 \%$ & & & $20 \mu \mathrm{l}$ & & \\
\hline Фор$\mu \alpha \mu i ́ \delta$ เo (ml) & 4 & 6 & 8 & 10 & 12 \\
\hline 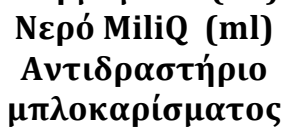 & 10 & 8 & $\begin{array}{c}6 \\
2 \mathrm{ml}\end{array}$ & 4 & 2 \\
\hline
\end{tabular}

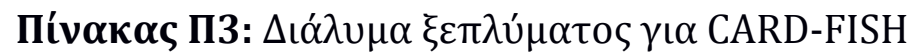

\begin{tabular}{|c|c|c|c|c|c|}
\hline FA\% & 20 & 30 & 40 & 50 & 60 \\
\hline EDTA 0.5M & & & $0.5 \mathrm{ml}$ & & \\
\hline Tris-HCl 1M & & & $1.0 \mathrm{ml}$ & & \\
\hline $\mathrm{NaCl} 5 \mathrm{M}(\mu \mathrm{l})$ & 2150 & 1020 & 460 & 180 & 40 \\
\hline $\begin{array}{c}\text { Nepó MiliQ } \\
\text { SDS 20\% }\end{array}$ & & П & $\begin{array}{c}\varepsilon \tau \varepsilon \lambda{ }_{\mathrm{tK}} \\
25 \mu \mathrm{l}\end{array}$ & & \\
\hline
\end{tabular}

\begin{tabular}{|c|c|c|c|c|c|c|c|c|}
\hline FA\% & 0 & 10 & 20 & 30 & 40 & 50 & 60 & 70 \\
\hline $\mathrm{NaCl}$ 5M & \multicolumn{8}{|c|}{$360 \mu \mathrm{l}$} \\
\hline Tris-HCl 1M & \multicolumn{8}{|c|}{$40 \mu \mathrm{l}$} \\
\hline 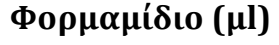 & 0 & 0.2 & 0.4 & 0.6 & 0.8 & 1 & 1.2 & 1.4 \\
\hline $10 \%$ SDS & \multicolumn{8}{|c|}{$2 \mu \mathrm{l}$} \\
\hline 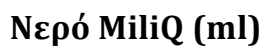 & 1.6 & 1.4 & 1.2 & 1 & 0.8 & 0.6 & 0.4 & 0.2 \\
\hline
\end{tabular}

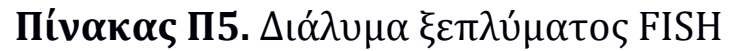

\begin{tabular}{lllllllll} 
FA\% & $\mathbf{0}$ & $\mathbf{1 0}$ & $\mathbf{2 0}$ & $\mathbf{3 0}$ & $\mathbf{4 0}$ & $\mathbf{5 0}$ & $\mathbf{6 0}$ & $\mathbf{7 0}$ \\
\hline Tris-HCl 1M, pH=8 & & & & & $1 \mathrm{ml}$ & & & - \\
\hline NaCl 5 M (ml) & 9 & 4.5 & 2.15 & 1.02 & 0.46 & 0.18 & 0.04 & 0.35 \\
EDTA 0.5 M (ml) & - & - & 0.5 & 0.5 & 0.5 & 0.5 & 0.5 &
\end{tabular}


SDS $10 \%$

MiliQ water
$50 \mu \mathrm{l}$

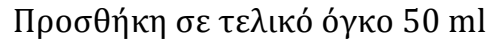

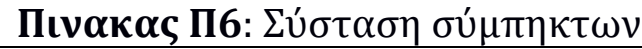

\begin{tabular}{|c|c|}
\hline 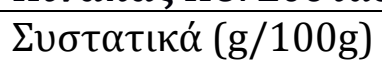 & $\Sigma u^{\prime} \mu \pi \eta \kappa \tau \alpha$ \\
\hline $\mathrm{I} \chi \theta v \alpha^{\prime} \lambda \varepsilon v \rho \alpha$ & 63.0 \\
\hline 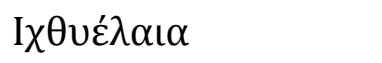 & 9.0 \\
\hline 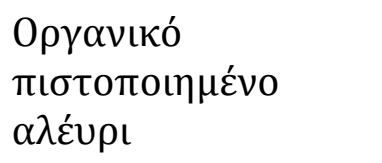 & 26.5 \\
\hline $\begin{array}{l}A v \alpha ́ \lambda v \sigma \eta \kappa \alpha \tau \alpha ́ \\
\pi \rho o \sigma \varepsilon ́ \gamma \gamma \iota \sigma \eta\end{array}$ & $\Sigma u^{\prime} \mu \pi \eta \kappa \tau \alpha$ \\
\hline$\Upsilon \gamma \rho \alpha \sigma i ́ \alpha(\%)$ & 10 \\
\hline 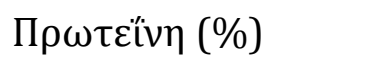 & 45 \\
\hline$\Lambda \iota \pi i \delta ı \alpha(\%)$ & 14 \\
\hline 'Ives (\%) & 0.6 \\
\hline 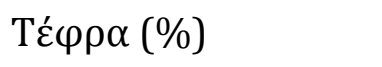 & 11 \\
\hline 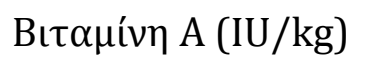 & 16000 \\
\hline 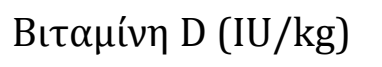 & 1600 \\
\hline 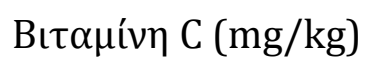 & 715 \\
\hline 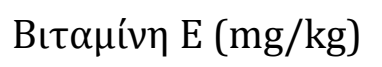 & 830 \\
\hline 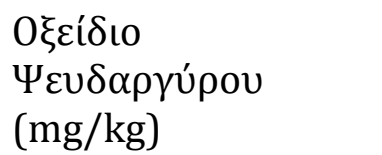 & 107 \\
\hline 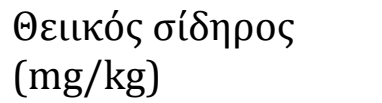 & 267 \\
\hline 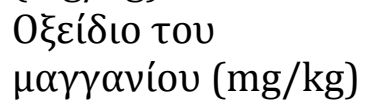 & 110 \\
\hline 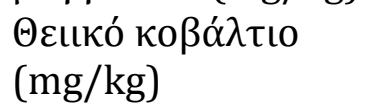 & 8 \\
\hline 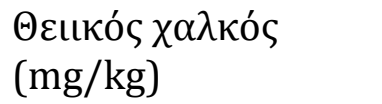 & 9 \\
\hline Ivoбıเó $\lambda \eta$ & 300 \\
\hline$P(\%)$ & 1.5 \\
\hline
\end{tabular}




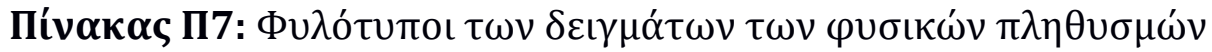

\begin{tabular}{|c|c|c|c|c|c|c|}
\hline 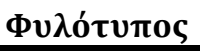 & $\Sigma v \chi v o ́ \tau \eta \tau \alpha$ & 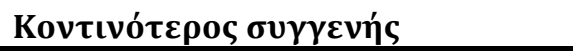 & GenBank \# & 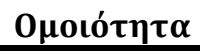 & $\Delta \varepsilon \varepsilon^{\prime} \gamma \mu \alpha \varepsilon v \tau o \pi t \sigma \mu o v ́$ & 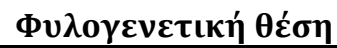 \\
\hline F1-5 & $58,1 \%$ & Pseudoalteromonas sp, NPO-Jl-58 & AY745828 & $99 \%$ & 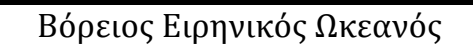 & $\gamma$-Proteobacteria \\
\hline F1-4 & $12,9 \%$ & $\begin{array}{l}\text { Psychrobacter aquimaris, KOPRI } \\
24929\end{array}$ & EF101547 & $99 \%$ & $\begin{array}{l}\text { Фаıючи́коৎ Undaria } \\
\text { pinnatifida }\end{array}$ & $\gamma$-Proteobacteria \\
\hline F1-7 & $6,4 \%$ & 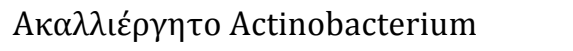 & EU374093 & $97 \%$ & $\Theta \alpha \lambda \alpha \alpha_{\sigma} \sigma \sigma \iota \iota^{\prime} \zeta \eta \mu \alpha$ & Actinobacteria \\
\hline F1-2 & $3,2 \%$ & 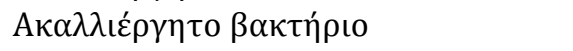 & FJ203056 & $96 \%$ & 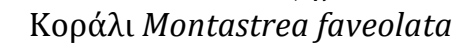 & $\gamma$-Proteobacteria \\
\hline F1-6 & $3,2 \%$ & 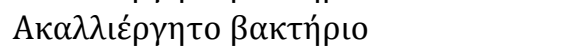 & FJ545576 & $96 \%$ & 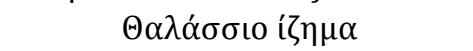 & Acidobacteria \\
\hline F1-18 & $3,2 \%$ & 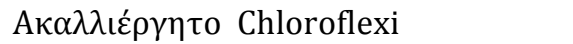 & DQ811871 & $98 \%$ & 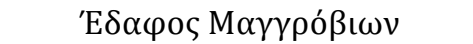 & Chloroflexi \\
\hline F1-19 & $3,2 \%$ & Ак $\alpha \lambda \lambda ı \dot{\varepsilon} \rho \gamma \eta \tau о$ Acidobacterium & DQ395041 & $96 \%$ & 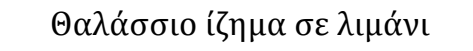 & Acidobacteria \\
\hline F1-20 & $3,2 \%$ & $\alpha \kappa \alpha \lambda \lambda \iota \varepsilon ́ \rho \gamma \eta \tau o$ Actinobacterium & EF208654 & $96 \%$ & $\mathrm{~A} \mu \mu \omega \dot{\delta} \delta \varepsilon \varsigma i ́ \zeta \eta \mu \alpha$ & Actinobacteria \\
\hline F1-26 & $3,2 \%$ & 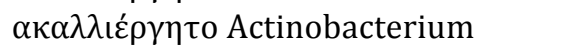 & EU374093 & $99 \%$ & $\Theta \alpha \lambda \alpha ́ \sigma \sigma เ \iota^{i} \zeta \eta \mu \alpha$ & Actinobacteria \\
\hline F1-28 & $3,2 \%$ & 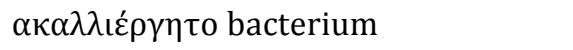 & FJ695595 & $98 \%$ & Ko $\alpha^{\prime} \lambda ı$ Acropora digitifera & $\gamma$-Proteobacteria \\
\hline My46-424 & $74,1 \%$ & Psychrobacter sp, Pi 2-33 & AB365059 & $99 \%$ & 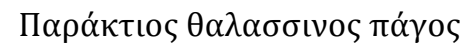 & $\gamma$-Proteobacteria \\
\hline My46-492 & $19,0 \%$ & Vibrio lentus $\sigma \tau \varepsilon \dot{\lambda} \lambda \chi \chi \circ \varsigma$ Sat201 & AY292936 & $99 \%$ & Sepiola atlantica & $\gamma$-Proteobacteria \\
\hline My46-460 & $3,4 \%$ & 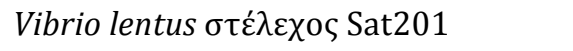 & AY292936 & $99 \%$ & Sepiola atlantica & $\gamma$-Proteobacteria \\
\hline My46-442 & $1,7 \%$ & Planococcus rifitiensis strain M8 & AJ493659 & $99 \%$ & 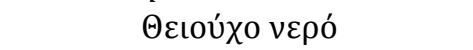 & Firmicutes \\
\hline My46-484 & $1,7 \%$ & 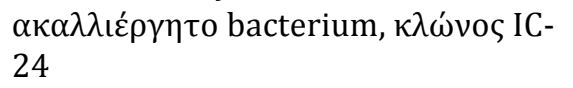 & AB255057 & $98 \%$ & $\Delta \mathrm{t} \alpha \beta \rho \omega \mu \varepsilon^{\prime} v o \tau \sigma \iota \varepsilon^{\prime} \nu \tau o$ & Firmicutes \\
\hline Jl1-36 & $73,3 \%$ & Photobacterium leiognathi & AY292917 & $99 \%$ & $\Sigma \tau \varepsilon^{\prime} \lambda \varepsilon \chi 0 \varsigma$ & $\gamma$-Proteobacteria \\
\hline Jl1-4 & $13,3 \%$ & Photobacterium sp, JT-ISH-224 & AB293986 & $99 \%$ & 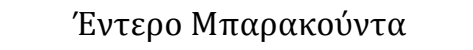 & $\gamma$-Proteobacteria \\
\hline Jl1-1 & $6,6 \%$ & $\begin{array}{l}\text { Photobacterium leiognathi strain } \\
\text { RM1 }\end{array}$ & AY292947 & $99 \%$ & 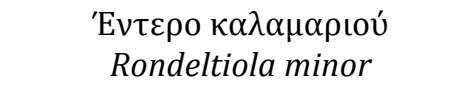 & $\gamma$-Proteobacteria \\
\hline Jl1-22 & $3,3 \%$ & Psychrobacter aquimaris & EF101547 & $99 \%$ & $\begin{array}{l}\text { Фаıочúкоธ Undaria } \\
\text { pinnatifida }\end{array}$ & $\gamma$-Proteobacteria \\
\hline Jl1-28 & $3,3 \%$ & 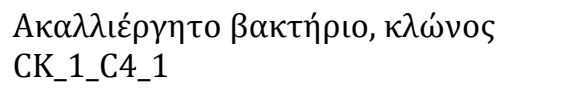 & EU488044 & $87 \%$ & 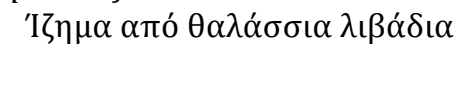 & CK-1C4-19 \\
\hline Ag31-3 & $31,9 \%$ & 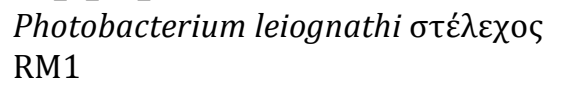 & AY292947 & $99 \%$ & 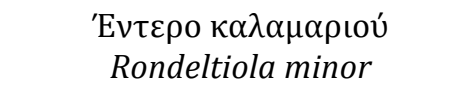 & $\gamma$-Proteobacteria \\
\hline Ag31-6 & $27,3 \%$ & Photobacterium leiognathi & AY292917 & $98 \%$ & $\Sigma \tau \varepsilon \dot{\lambda} \varepsilon \chi \chi \varsigma$ & $\gamma$-Proteobacteria \\
\hline Ag31-15 & $13,6 \%$ & Vibrio lentus & AY292936 & $98 \%$ & Sepiola atlantica & $\gamma$-Proteobacteria \\
\hline
\end{tabular}




\begin{tabular}{|c|c|c|c|c|c|c|}
\hline 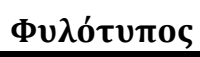 & $\Sigma v \chi v o ́ \tau \eta \tau \alpha$ & 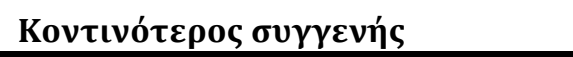 & GenBank \# & 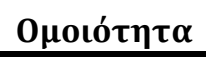 & $\Delta \varepsilon \dot{\gamma} \gamma \mu \alpha \varepsilon v \tau о \pi เ \sigma \mu o v ́$ & 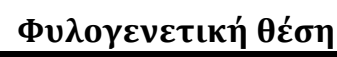 \\
\hline Ag31-2 & $9,1 \%$ & 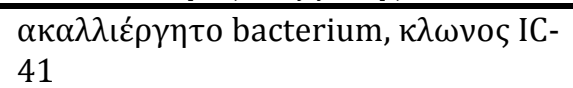 & AB255065 & $99 \%$ & $\Delta \iota \alpha \beta \rho \omega \mu \varepsilon^{\prime} \nu 0 \tau \sigma \iota \varepsilon^{\prime} v \tau o$ & $\gamma$-Proteobacteria \\
\hline Ag31-1 & $4,5 \%$ & $\begin{array}{l}\text { Vibrio parahaemolyticus RIMD } \\
2210633\end{array}$ & BA000031 & $99 \%$ & $\Sigma \tau \varepsilon ́ \lambda \varepsilon \chi \circ \varsigma$ & $\gamma$-Proteobacteria \\
\hline Ag31-21 & $4,5 \%$ & $\begin{array}{l}\text { Vibrio splendidus LGP32 } \\
\text { chromosome } 1\end{array}$ & FM954972 & $98 \%$ & $\Sigma \tau \varepsilon ́ \lambda \varepsilon \chi \circ \varsigma$ & $\gamma$-Proteobacteria \\
\hline Ag31-22 & $4,5 \%$ & $\begin{array}{l}\text { Vibrio salmonicida } \sigma \tau \varepsilon \dot{\lambda} \varepsilon \chi 0 \varsigma \text { PB1- } \\
\text { 8rrnB }\end{array}$ & EU091324 & $99 \%$ & 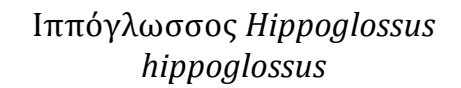 & $\gamma$-Proteobacteria \\
\hline Ag31-13 & $4,5 \%$ & Photobacterium sp, HAR23 & AB038031 & $99 \%$ & 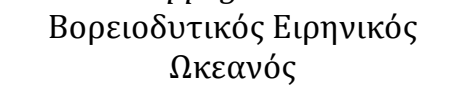 & $\gamma$-Proteobacteria \\
\hline Se3-204 & $83,0 \%$ & $\alpha \kappa \alpha \lambda \lambda \iota \varepsilon ́ \rho \gamma \eta \tau o$ Mollicutes & DQ340200 & $90 \%$ & 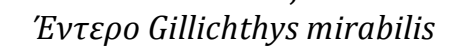 & Mollicutes \\
\hline Se3-206 & $5,6 \%$ & $\begin{array}{l}\text { Desulfatibacillus olefinivorans } \\
\sigma \tau \varepsilon ́ \lambda \varepsilon \chi 0 \varsigma \text { LM2801 }\end{array}$ & DQ826724 & $87 \%$ & $\Sigma \tau \varepsilon \dot{\lambda} \varepsilon \bar{\chi} \bigcirc$ & $\delta$-Proteobacteria \\
\hline Se3-199 & $3,8 \%$ & Ralstonia sp, FRA01 & AF098288 & $99 \%$ & 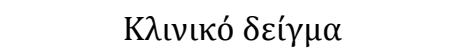 & $\beta$-Proteobacteria \\
\hline Se3-178 & $3,8 \%$ & Dechloromonas $\mathrm{sp}, \mathrm{HZ}$ & AF479766 & $98 \%$ & 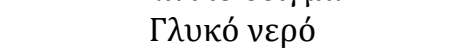 & $\beta$-Proteobacteria \\
\hline Se3-129 & $1,9 \%$ & 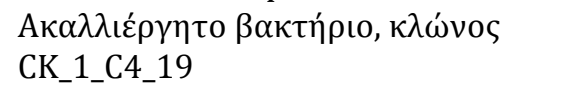 & EU488044 & $87 \%$ & 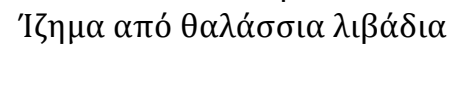 & CK-1C4-19 \\
\hline Se3-157 & $1,9 \%$ & 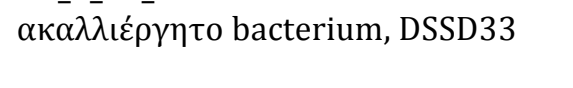 & AY328732 & $99 \%$ & 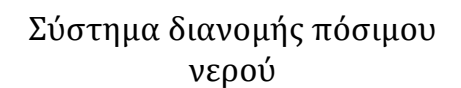 & $\alpha$-Proteobacteria \\
\hline $02-1$ & $76,0 \%$ & $\begin{array}{l}\text { Photobacterium leiognathi } \sigma \tau \varepsilon \dot{\lambda} \varepsilon \chi \chi \circ \varsigma \\
\text { RM1 }\end{array}$ & AY292947 & $99 \%$ & 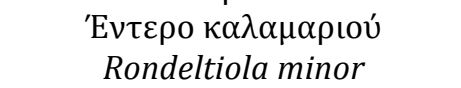 & $\gamma$-Proteobacteria \\
\hline $02-8$ & $16,0 \%$ & $\begin{array}{l}\text { Vibrio splendidus } \sigma \tau \varepsilon \dot{\lambda} \lambda \varepsilon \chi 0 \varsigma \text { PB1- } \\
10 \mathrm{rrnH}\end{array}$ & EU091332 & $99 \%$ & $\Sigma \tau \dot{\lambda} \lambda \varepsilon \chi \circ \varsigma$ & $\gamma$-Proteobacteria \\
\hline $02-23$ & $4,0 \%$ & 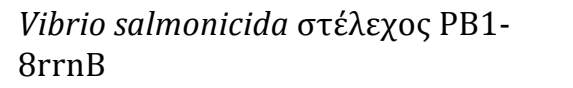 & EU091324 & $99 \%$ & $\Sigma \tau \varepsilon ́ \lambda \varepsilon \chi 0 \varsigma$ & $\gamma$-Proteobacteria \\
\hline $02-37$ & $4,0 \%$ & 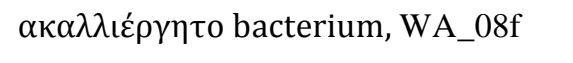 & EF123487 & $98 \%$ & Ko $\rho \alpha ́ \lambda ı$ Siderastrea siderata & $\gamma$-Proteobacteria \\
\hline D1-695 & $86,0 \%$ & 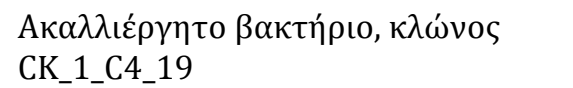 & EU488044 & $87 \%$ & 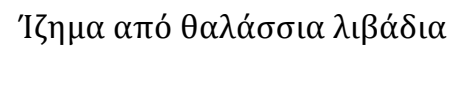 & CK-1C4-19 \\
\hline D1-668 & $2,2 \%$ & Dechloromonas sp, $\mathrm{HZ}$ & AF479766 & $99 \%$ & 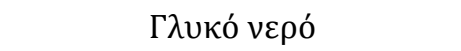 & $\beta$-Proteobacteria \\
\hline D1-669 & $2,2 \%$ & 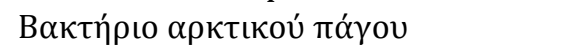 & AF468382 & $99 \%$ & 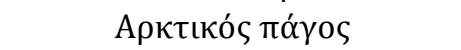 & $\gamma$-Proteobacteria \\
\hline D1-715 & $2,2 \%$ & Psychrobacter celer & EF101550 & $98 \%$ & $\begin{array}{c}\text { Фаเори́коร Undaria } \\
\text { pinnatifida }\end{array}$ & $\gamma$-Proteobacteria \\
\hline D1-727 & $2,2 \%$ & 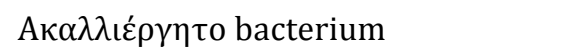 & EU137440 & $99 \%$ & Oropsyla hirsuta ( $\left.\psi v \dot{\lambda} \lambda \lambda_{\circ}\right)$ & Actinobacteria \\
\hline
\end{tabular}




\begin{tabular}{|c|c|c|c|c|c|c|}
\hline 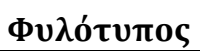 & 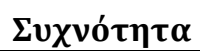 & 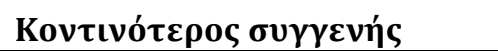 & GenBank \# & 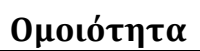 & $\Delta \varepsilon i ́ \gamma \mu \alpha \varepsilon v \tau o \pi t \sigma \mu o v ́$ & 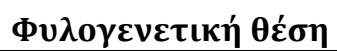 \\
\hline & & Oh3137A10B & & & 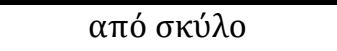 & \\
\hline D1-684 & $2,2 \%$ & Psychromonas japonica & AB304804 & $98 \%$ & 'I $\eta \mu \alpha \sigma \varepsilon$ whale falls & $\gamma$-Proteobacteria \\
\hline D1-674 & $1,1 \%$ & Shewanella woodyi ATCC 51908 & СР000961 & $99 \%$ & 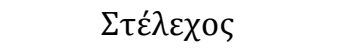 & $\gamma$-Proteobacteria \\
\hline D1-700 & $1,1 \%$ & Burkholderia phytofirmans PsJN & СР001053 & $98 \%$ & 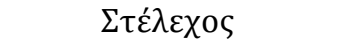 & $\beta$-Proteobacteria \\
\hline
\end{tabular}

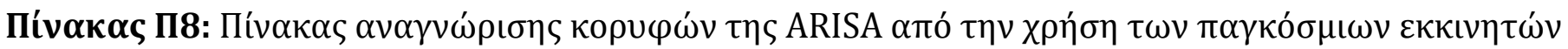

\begin{tabular}{|c|c|c|c|c|c|c|c|c|c|c|c|c|c|c|c|c|c|c|}
\hline 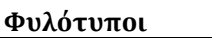 & 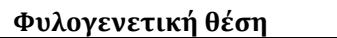 & F1 & Mr1 & Mr26 & My27 & My44 & My45 & My46 & My47 & Jl1 & $\mathrm{Jl3}$ & $\mathrm{Jl4}$ & Ag16 & Ag1 & Ag2,1 & Ag23 & Ag31 & Ag8 \\
\hline $\begin{array}{l}\text { Se3-129/D1- } \\
\text { 695/ J11-28 }\end{array}$ & CK_1C4-19 (246) & - & & - & - & - & - & - & - & - & - & - & 0,10 & - & - & - & - & \\
\hline My46-442 & Planococcus sp,(273) & - & - & - & - & - & - & 0,33 & - & - & - & - & 0,32 & - & - & - & - & - \\
\hline Мy46-484 & $\begin{array}{l}\text { Planomicrobium sp (295) } \\
\text { Propionibacterium sp }\end{array}$ & - & - & - & - & - & - & - & - & - & - & - & - & - & - & - & - & - \\
\hline D1-727 & $(301)$ & - & - & - & - & - & - & - & - & 0,10 & - & - & 0,56 & - & - & 0,36 & 0,17 & 0,12 \\
\hline Se3-204 & Mollicutes 1 (317) & - & 0,09 & - & - & - & - & - & - & 0,50 & 1,54 & 18,65 & 1,59 & 0,18 & - & 0,51 & 0,36 & 0,46 \\
\hline F1-6 & Acidobacterium (320) & - & - & - & - & - & - & - & - & - & 1,32 & 9,41 & 9,62 & - & - & - & - & - \\
\hline F1-20 & $\begin{array}{l}\text { Actinobacterium (367) } \\
\text { Pseudoalteromonas sp }\end{array}$ & - & 2,23 & - & - & - & - & - & - & - & - & - & 0,24 & - & - & - & - & - \\
\hline F1-5 & $(385)$ & 8,63 & - & - & - & 0,29 & - & - & - & - & - & - & 3,42 & - & - & 0,62 & 0,11 & - \\
\hline F1-26 & $\begin{array}{l}\text { Actinobacterium (390) } \\
\text { Photobacterium leiognathi }\end{array}$ & - & - & 1,17 & - & - & - & - & - & 0,28 & - & 0,14 & 0,25 & - & - & - & - & - \\
\hline $\mathrm{NO2-2}$ & $(421)$ & - & 5,57 & - & 0,28 & - & 0,14 & - & 0,11 & 1,43 & 1,80 & 0,22 & 0,30 & 11,53 & 7,76 & 0,40 & 17,77 & 0,34 \\
\hline My46-492 & Vibrio lentus (444) & - & - & - & 0,65 & - & 0,15 & 1,53 & - & - & - & - & 0,19 & - & - & - & - & - \\
\hline D1-674 & Shewanella sp (450) & - & - & - & - & - & - & - & 0,29 & - & 0,27 & - & - & - & 2,34 & - & - & - \\
\hline F1-7 & actinobacterium (455) & - & 0,10 & 2,89 & 2,75 & - & 0,29 & - & 0,22 & 0,12 & - & 0,59 & - & 2,51 & 0,51 & - & - & - \\
\hline F1-18 & chloroflexi (489) & 0,28 & - & - & - & - & - & - & 0,94 & 0,80 & - & - & 7,66 & 0,12 & 0,21 & 3,35 & 2,15 & 0,78 \\
\hline $\begin{array}{l}\text { Se3-204 } \\
\text { My46-460/Se3- } \\
\text { 178/D1-668 }\end{array}$ & $\begin{array}{l}\text { Mollicutes (508) } \\
\text { Vibrio lentus } \\
\text { (517)/Dechloromonas sp, }\end{array}$ & 0,33 & - & $\begin{array}{l}0,95 \\
0,17\end{array}$ & - & - & 4,80 & 1,93 & 5,57 & 0,18 & - & - & 0,62 & - & 0,73 & 1,00 & 0,73 & 1,95 \\
\hline NO2-10 & V,slendidus (518) & 0,27 & 7,13 & 3,73 & - & 1,42 & 3,09 & 2,60 & 3,19 & - & - & - & 0,88 & - & 1,56 & 0,94 & 0,75 & 2,57 \\
\hline Se3-206 & Desulfatiferula (624) & - & - & 0,69 & 0,56 & 11,75 & 0,10 & 5,65 & 2,36 & - & - & - & 0,34 & - & 0,35 & 0,86 & - & 0,97 \\
\hline D1-715 & Psychrobacter celer (625) & - & - & 0,10 & 0,26 & - & - & - & - & - & 1,10 & 0,27 & - & - & - & - & - & 0,37 \\
\hline
\end{tabular}




\begin{tabular}{|c|c|c|c|c|c|c|c|c|c|c|c|c|c|c|c|c|c|c|}
\hline 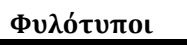 & 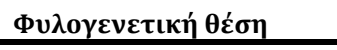 & F1 & Mr1 & Mr26 & My27 & My44 & My45 & My46 & My47 & $\mathrm{Jl1}$ & $\mathrm{Jl} 3$ & $\mathrm{Jl4}$ & Ag16 & Ag1 & Ag2,1 & Ag23 & Ag31 & Ag8 \\
\hline F1-2 & $\begin{array}{l}\text { Unc gamma (639) } \\
\text { Psychrobacter aquimaris }\end{array}$ & - & - & - & 0,60 & & - & 0,40 & - & & 0,17 & & - & 5,50 & 1,93 & & 0,18 & - \\
\hline F1-4 & $(666)$ & 0,17 & 2,11 & 2,79 & - & 1,68 & 0,75 & 2,74 & 3,67 & 0,10 & 1,52 & - & - & - & 0,31 & 0,51 & 1,08 & 2,09 \\
\hline $\begin{array}{l}\text { My46-424b } \\
\text { NO2- } \\
46(679 b p)\end{array}$ & $\begin{array}{l}\text { Psychrobacter sp, }(669) \\
\text { Photobacterium leiognathi } \\
(679)\end{array}$ & 8,74 & - & 0,54 & 3,40 & - & 5,39 & 1,79 & 0,54 & - & 0,21 & - & 0,48 & 9,27 & 1,23 & 0,84 & 1,25 & 2,54 \\
\hline D1-700 (679 & Burkholderia sp (679) & - & 2,43 & 0,38 & 1,80 & - & - & 0,53 & - & 0,93 & - & - & - & - & 3,15 & - & - & - \\
\hline My46-424a & Psychrobacter sp, (681 & - & - & 0,37 & 3,55 & 1,68 & 1,76 & - & 0,52 & - & 3,87 & 1,02 & - & - & 0,17 & 2,74 & - & 0,54 \\
\hline Se3-157 & $\begin{array}{l}\text { Uncultured alpha (707) } \\
\text { Pseudoalteromonas }\end{array}$ & - & - & 4,15 & - & 2,00 & - & - & - & 0,34 & 0,12 & - & - & - & 0,89 & 0,34 & 0,17 & 2,10 \\
\hline F1-29 & haloplanktis (733) & 11,93 & 0,51 & - & - & - & - & - & & 0,13 & - & & 0,28 & - & - & - & - & - \\
\hline
\end{tabular}

\begin{tabular}{|c|c|c|c|c|c|c|c|c|c|c|c|c|c|c|c|c|c|c|c|c|c|c|c|}
\hline 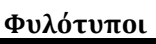 & 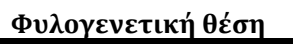 & Se11 & Se12 & Se1 & Se2 & Se3 & Se4 & Se5 & Se6 & Se7 & Se9 & 01 & 02 & 03 & 04 & 05 & 06 & D1 & D2 & D3 & D4 & D6 & D7 \\
\hline Se3-129 & CK_1C4-19 (246) & - & - & - & - & - & - & - & - & - & - & - & - & - & - & - & - & - & - & - & - & - & - \\
\hline My46-442 & $\begin{array}{l}\text { Planococcus sp.(273) } \\
\text { Planomicrobium sp }\end{array}$ & - & 0.1 & - & - & - & 0.1 & 0.1 & 0.1 & - & 0.1 & - & - & - & - & - & - & - & 0.1 & 0.1 & - & - & - \\
\hline My46-484 & $\begin{array}{l}\text { (295) } \\
\text { Propionibacterium sp }\end{array}$ & - & - & - & - & - & - & - & - & - & - & - & - & 0.1 & - & - & - & - & - & - & - & 0.2 & - \\
\hline D1-727 & $(301)$ & - & 0.4 & - & - & 0.2 & - & 0.2 & 0.4 & - & - & - & - & 0.2 & 0.1 & - & - & 0.3 & - & 0.1 & - & 0.1 & - \\
\hline Se3-204 & $\begin{array}{l}\text { Mollicutes } 1 \text { (317) } \\
\text { Acidobacterium sp. }\end{array}$ & 0.6 & 3.8 & 0.2 & 0.2 & 1.0 & 0.3 & 0.6 & 0.9 & 5.3 & 2.4 & 0.2 & 0.2 & 1.3 & 0.7 & 0.8 & - & 0.7 & 5.5 & 0.5 & - & 0.2 & 0.3 \\
\hline F1-6 & $(320)$ & - & - & - & - & - & - & - & - & 3.5 & 0.8 & - & - & - & - & - & - & 2.1 & - & - & - & - & - \\
\hline F1-20 & $\begin{array}{l}\text { Actinobacterium (367) } \\
\text { Pseudoalteromonas sp }\end{array}$ & 0.3 & 0.3 & - & - & 0.1 & - & 0.2 & 0.3 & - & 0.2 & - & - & - & - & - & - & - & 0.2 & 0.1 & - & - & - \\
\hline F1-5 & $(385)$ & - & 0.2 & 0.5 & - & 0.4 & - & 0.2 & 1.9 & - & - & - & - & - & - & - & - & 0.7 & - & - & - & - & - \\
\hline F1-26 & $\begin{array}{l}\text { Actinobacterium (390) } \\
\text { Photobacterium }\end{array}$ & - & - & - & - & 0.2 & - & - & - & - & - & - & - & - & - & - & - & 0.2 & - & - & - & 0.3 & - \\
\hline NO2-2 & leiognathi (421) & 2.3 & 1.1 & 7.4 & 1.9 & 0.6 & - & 0.6 & - & 0.3 & 0.5 & 16.2 & 10.1 & 4.5 & 19.9 & 9.2 & 2.4 & 2.7 & 7.0 & 1.0 & 11.9 & 5.3 & 3.5 \\
\hline My46-492 & Vibrio lentus (444) & 0.7 & 0.2 & 0.1 & - & - & 0.2 & 0.1 & - & 0.9 & 0.5 & - & - & - & - & 0.8 & 0.4 & - & - & 0.5 & 0.1 & - & 1.2 \\
\hline D1-674 & Shewanella sp (450) & - & - & 0.5 & - & 0.2 & 0.1 & - & - & - & - & - & - & - & - & - & - & 0.5 & 0.8 & 0.3 & 2.9 & 1.2 & - \\
\hline F1-7 & actinobacterium (455) & 0.3 & 1.2 & 0.3 & - & - & 0.3 & 0.1 & 0.6 & 0.6 & 0.2 & 1.3 & 1.7 & 0.6 & 4.7 & 0.7 & 0.1 & 0.7 & 0.3 & 0.5 & - & 0.9 & 0.4 \\
\hline F1-18 & chloroflexi (489) & 2.7 & 5.0 & 1.5 & 0.8 & 2.7 & 0.9 & 2.5 & 4.6 & 0.4 & 3.9 & 0.9 & 0.3 & 2.2 & 0.7 & 1.4 & 0.5 & 2.6 & 1.3 & 0.9 & 0.4 & 0.3 & 1.5 \\
\hline Se3-204 & Mollicutes (508) & 0.6 & 0.5 & 0.2 & 0.4 & 0.5 & 0.2 & 0.6 & 1.0 & - & 0.7 & 0.3 & - & 0.3 & 0.4 & 0.5 & 0.2 & 0.2 & 0.4 & 0.4 & 0.2 & - & 0.2 \\
\hline My46- & Vibrio lentus & - & 1.0 & 0.2 & - & - & - & - & - & 0.3 & - & 0.3 & - & - & - & 0.3 & - & 0.5 & 0.1 & - & - & - & - \\
\hline
\end{tabular}




\begin{tabular}{|c|c|c|c|c|c|c|c|c|c|c|c|c|c|c|c|c|c|c|c|c|c|c|c|}
\hline 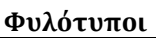 & 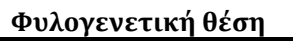 & Se11 & Se12 & Se1 & $\mathrm{Se} 2$ & Se3 & Se4 & Se5 & Se6 & Se7 & Se9 & 01 & 02 & 03 & 04 & 05 & 06 & D1 & D2 & D3 & D4 & D6 & D7 \\
\hline $\begin{array}{l}460 / \mathrm{Se} 3- \\
178\end{array}$ & $\begin{array}{l}\text { (517)/Dechloromonas } \\
\text { sp. }\end{array}$ & & & & & & & & & & & & & & & & & & & & & & \\
\hline NO2-10 & V.slendidus (518) & 4.4 & - & 2.1 & 2.4 & 9.5 & 1.0 & 3.6 & 3.8 & 2.4 & 10.4 & 1.6 & 0.6 & 19.6 & 1.3 & 6.9 & 0.3 & 7.0 & 4.4 & 6.1 & 1.8 & 1.5 & 1.5 \\
\hline Se3-206 & $\begin{array}{l}\text { Desulfatiferula (624) } \\
\text { Psychrobacter celer }\end{array}$ & - & - & 1.0 & 0.3 & - & 0.9 & 0.9 & 4.9 & 0.2 & 0.2 & - & 0.2 & - & - & - & 0.6 & - & 0.2 & - & - & - & - \\
\hline D1-715 & (625) & - & - & - & - & 0.3 & 1.8 & - & - & - & 0.3 & - & - & - & - & 0.3 & 0.5 & - & 0.2 & - & - & - & 0.2 \\
\hline F1-2 & $\begin{array}{l}\text { Unc gamma (639) } \\
\text { Psychrobacter }\end{array}$ & - & - & - & 0.3 & - & - & - & - & - & - & 1.3 & 1.4 & - & 3.8 & 1.3 & - & - & 0.4 & - & 3.6 & 0.9 & 0.2 \\
\hline $\begin{array}{l}\text { F1-4 } \\
\text { My46- }\end{array}$ & aquimaris (666) & 0.4 & - & 0.9 & - & 4.2 & 0.2 & - & - & - & 0.4 & - & 0.1 & - & - & - & 2.6 & - & - & - & - & - & - \\
\hline $\begin{array}{l}424 b \\
\text { NO2-46 }\end{array}$ & $\begin{array}{l}\text { Psychrobacter sp.(669) } \\
\text { Photobacterium } \\
\text { leiognathi }(679\end{array}$ & 0.7 & - & 1.0 & 0.2 & - & - & - & - & 1.3 & 3.6 & 2.8 & 4.4 & 0.2 & 8.6 & 1.8 & - & - & 0.3 & - & 2.3 & - & - \\
\hline $\begin{array}{l}\text { D1-700 } \\
\text { My46- }\end{array}$ & Burkholderia sp (679) & 0.2 & - & - & - & - & - & - & - & - & - & - & - & - & - & - & - & - & 0.1 & 0.1 & - & 7.3 & - \\
\hline $424 a$ & $\begin{array}{l}\text { Psychrobacter sp. (681 } \\
\text { Uncultured alpha }\end{array}$ & 0.1 & - & - & - & - & - & - & - & - & 0.3 & - & - & - & - & - & - & - & 0.3 & 0.1 & - & - & - \\
\hline Se3-157 & $\begin{array}{l}\text { (707) } \\
\text { Pseudoalteromonas }\end{array}$ & - & 1.1 & 0.5 & 5.1 & 1.5 & 0.3 & 0.5 & 0.9 & 0.2 & 0.4 & - & 0.3 & 2.0 & - & 0.8 & - & 0.3 & 0.2 & 0.3 & - & 0.2 & 0.1 \\
\hline F1-29 & haloplanktis (733) & - & - & - & - & - & - & - & - & - & - & - & - & - & 0.1 & - & - & - & 0.3 & - & - & - & - \\
\hline
\end{tabular}

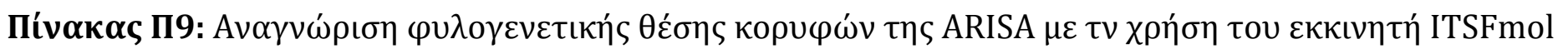

\begin{tabular}{|c|c|c|c|c|c|c|c|c|c|c|c|c|c|c|c|c|c|}
\hline 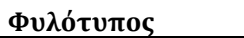 & 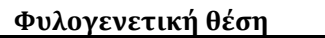 & fe1u & fe1m & mr26u & mr26m & my46u & my46m & my47u & my $47 m$ & jl1u & jl1m & $j 13 u$ & jl3m & ag1u & ag1m & ag23u & ag23m \\
\hline $\begin{array}{l}\text { Se3-129/D1-695/ } \\
\text { Jl1-28 }\end{array}$ & CK_1C4-19 (246) & 0.11 & - & - & - & - & - & - & - & - & - & - & - & - & - & - & 1.34 \\
\hline My46-442 & Planococcus sp.(273) & 0.12 & - & 0.13 & - & 0.54 & - & - & - & - & - & - & - & - & 0.32 & - & - \\
\hline My46-484 & $\begin{array}{l}\text { Planomicrobium sp (295) } \\
\text { Propionibacterium sp }\end{array}$ & 1.86 & 0.34 & - & - & - & - & - & - & 0.49 & - & - & - & 0.80 & - & 9.55 & - \\
\hline D1-727 & $(301)$ & - & - & - & - & - & - & - & - & - & - & - & - & - & - & - & 0.38 \\
\hline Se3-204a & Mollicutes 1 (317) & - & 1.20 & - & 0.29 & - & - & - & - & 0.18 & 1.82 & - & - & - & 5.78 & 0.36 & 2.26 \\
\hline F1-20 & $\begin{array}{l}\text { Actinobacterium (367) } \\
\text { Pseudoalteromonas sp }\end{array}$ & - & - & 0.64 & 1.20 & - & - & - & - & - & - & - & - & - & - & - & - \\
\hline F1-5 & $(385)$ & 7.32 & 16.17 & 0.17 & - & - & - & - & - & - & 0.10 & - & - & - & - & - & - \\
\hline F1-26 & Actinobacterium (390) & - & 0.18 & 0.25 & 1.23 & - & - & - & - & - & - & 1.20 & 1.37 & - & - & - & 0.45 \\
\hline
\end{tabular}




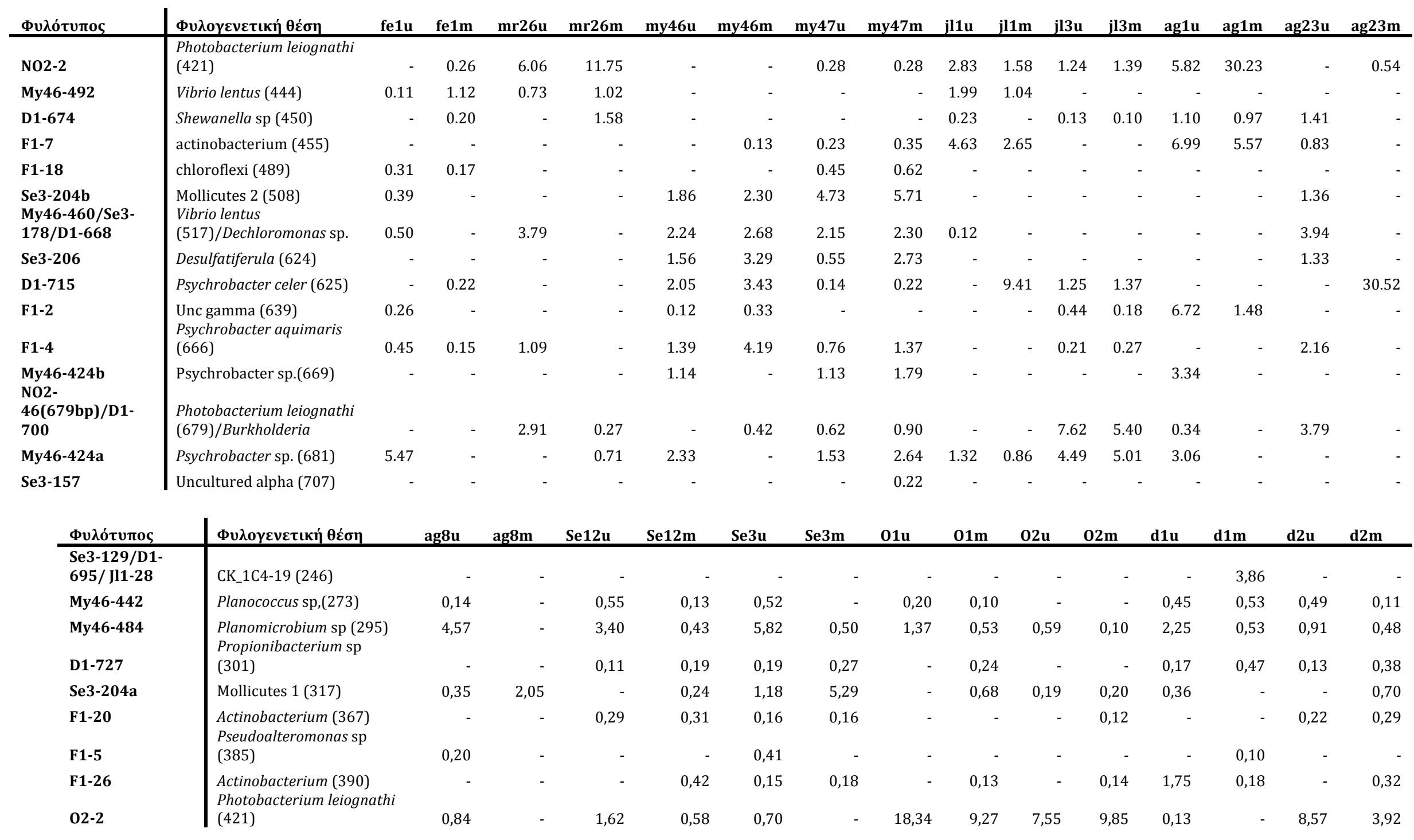




\begin{tabular}{|c|c|c|c|c|c|c|c|c|c|c|c|c|c|c|c|}
\hline 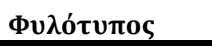 & 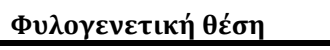 & ag8u & ag8m & Se12u & Se12m & Se3u & Se3m & 01u & $01 \mathrm{~m}$ & $02 u$ & $02 \mathrm{~m}$ & d1u & d1m & d2u & d2m \\
\hline My46-492 & Vibrio lentus (444) & 0,19 & - & - & - & 0,34 & - & 0,17 & - & 1,13 & 0,29 & 0,50 & - & - & - \\
\hline D1-674 & Shewanella sp (450) & - & - & 0,22 & - & 1,34 & 0,22 & - & - & - & - & 0,44 & 12,79 & 0,49 & - \\
\hline F1-7 & actinobacterium (455) & 0,19 & . & 0,91 & - & 0,26 & 0,15 & 1,57 & 0,55 & 2,04 & 1,67 & 7,10 & - & - & 0,78 \\
\hline F1-18 & chloroflexi (489) & 0,22 & - & - & - & - & - & - & - & - & - & - & 4,18 & - & - \\
\hline $\begin{array}{l}\text { Se3-204b } \\
\text { My46-460/Se3- } \\
\text { 178/D1-668 }\end{array}$ & $\begin{array}{l}\text { Mollicutes } 2 \text { (508) } \\
\text { Vibrio lentus } \\
\text { (517)/Dechloromonas sp, }\end{array}$ & $\begin{array}{l}2,63 \\
5,09\end{array}$ & - & 18,11 & - & 12,30 & - & 0,28 & 0,14 & 0,32 & 0,17 & 8,11 & - & 4,09 & - \\
\hline Se3-206 & Desulfatiferula (624) & 0,46 & - & 1,17 & 2,50 & - & - & - & 0,70 & 0,16 & - & - & 0,19 & - & 0,11 \\
\hline D1-715 & Psychrobacter celer (625) & - & . & - & 20,08 & 1,08 & 6,16 & - & 5,55 & 0,12 & 4,17 & 0,33 & 5,55 & 0,11 & 14,16 \\
\hline F1-2 & $\begin{array}{l}\text { Unc gamma (639) } \\
\text { Psychrobacter aquimaris }\end{array}$ & 57 & - & 000 & 0,23 & - & - & 1,21 & 0,44 & 1,18 & 1,24 & 120 & 020 & 0,23 & 0,31 \\
\hline F1-4 & $(666)$ & 5,74 & - & 0,88 & - & 2,84 & - & 0,25 & - & - & 0,13 & 1,39 & 0,30 & 0,20 & - \\
\hline $\begin{array}{l}\text { My46-424b } \\
\text { NO2- } \\
46(679 b p) / D 1- \\
700\end{array}$ & $\begin{array}{l}\text { Psychrobacter sp,(669) } \\
\text { Photobacterium leiognathi } \\
(679\end{array}$ & 0,47 & - & 0,41 & - & - & - & - & - & 2,81 & 2,83 & 0,14 & 2,07 & 0,12 & - \\
\hline My46-424a & Psychrobacter sp, (681 & - & . & - & - & - & - & 9,52 & 2,56 & 6,26 & 7,09 & - & - & 3,09 & 1,25 \\
\hline Se3-157 & Uncultured alpha (707) & - & . & - & 0,32 & - & 0,30 & - & 0,20 & & - & - & 0,31 & 0,50 & 0,52 \\
\hline
\end{tabular}

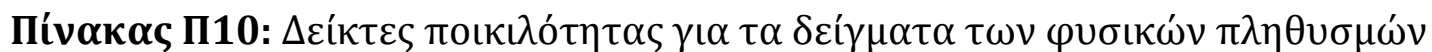

\begin{tabular}{l|rrrrrrr} 
& \multicolumn{1}{|c}{ | 1} & \multicolumn{1}{c}{ My46 } & J11 & \multicolumn{1}{c}{ Ag31 } & \multicolumn{1}{c}{ Se3 } & \multicolumn{1}{c}{ 02 } & \multicolumn{1}{c}{ D1 } \\
\hline Simpson (D) & 0,5931 & 0,3995 & 0,3924 & 0,7769 & 0,304 & 0,3936 & 0,2249 \\
Shannon (H) & 1,344 & 0,6971 & 0,7449 & 1,668 & 0,7142 & 0,7593 & 0,5976 \\
Margalef (d) & 2,038 & 0,7388 & 0,8909 & 1,941 & 1,259 & 0,932 & 1,552
\end{tabular}




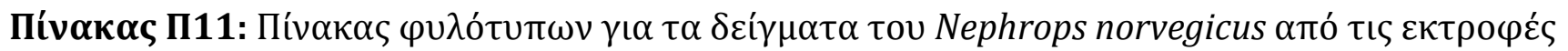

\begin{tabular}{|c|c|c|c|c|c|c|}
\hline 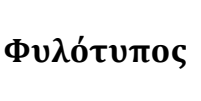 & 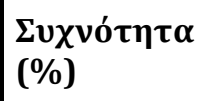 & 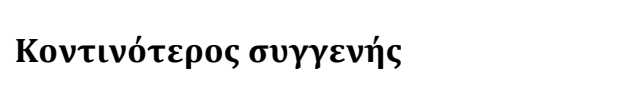 & Genbank \# & 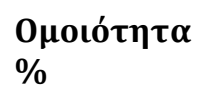 & $\Delta \varepsilon \dot{\gamma} \gamma \mu \alpha \varepsilon v \tau о \pi t \sigma \mu o v ́$ & 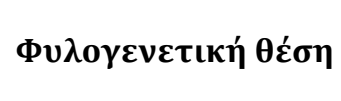 \\
\hline Nat1-12 & 66,6 & 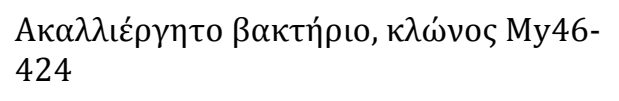 & GQ866072 & 99 & 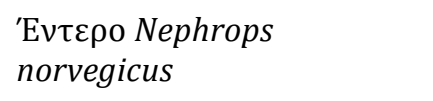 & $\gamma$-Proteobacteria \\
\hline Nat1-14 & 6,6 & $\begin{array}{l}\text { Psychrobacter aquimaris strain } \\
\text { KOPRI } 24929\end{array}$ & EF101547 & 98 & $\begin{array}{l}\text { Фаıочúкоs Undaria } \\
\text { pinnatifida }\end{array}$ & $\gamma$-Proteobacteria \\
\hline Nat1-9 & 6,6 & 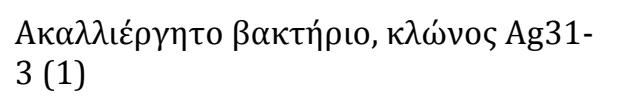 & GQ866101 & 99 & 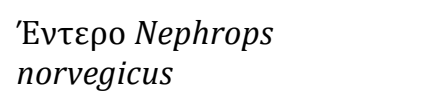 & $\gamma$-Proteobacteria \\
\hline Nat1-3 & 3,3 & Psychrobacter sp. mp2 & AJ551116 & 99 & 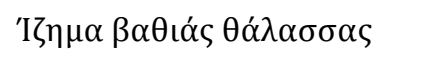 & $\gamma$-Proteobacteria \\
\hline Nat1-21 & 3,3 & 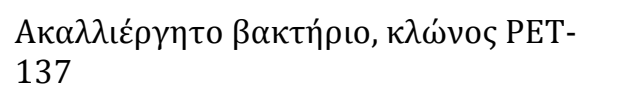 & JF344261 & 95 & 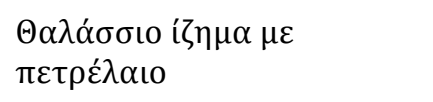 & $\alpha$-Proteobacteria \\
\hline Nat1-19 & 3,3 & 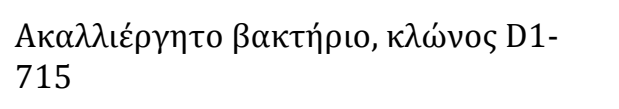 & GQ866080 & 97 & 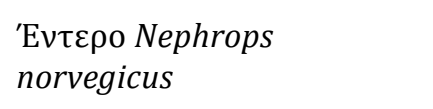 & $\gamma$-Proteobacteria \\
\hline Nat2-8 & 48 & 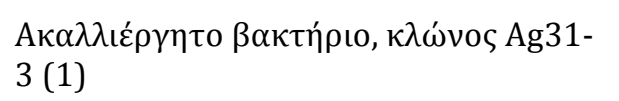 & GQ866101 & 99 & 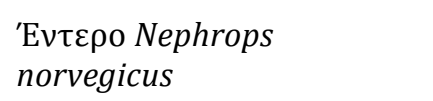 & $\gamma$-Proteobacteria \\
\hline Nat2-24 & 8 & Colwellia psychroerythraea (2) & AB011364 & 95 & 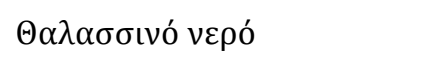 & $\gamma$-Proteobacteria \\
\hline Nat2-66 & 8 & 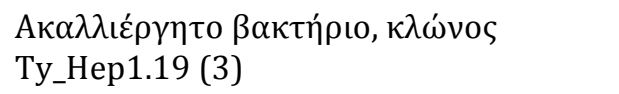 & EU646197 & 91 & 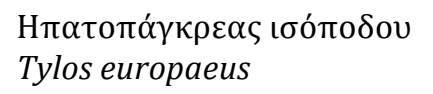 & Tenericutes \\
\hline
\end{tabular}




\begin{tabular}{|c|c|c|c|c|c|c|}
\hline 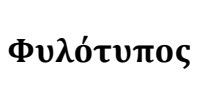 & $\begin{array}{l}\Sigma v \chi v o ́ \tau \eta \tau \alpha \\
(\%)\end{array}$ & 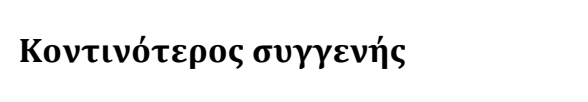 & Genbank \# & 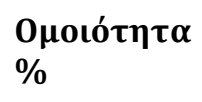 & 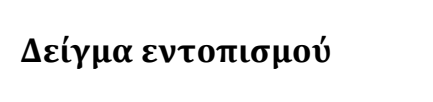 & 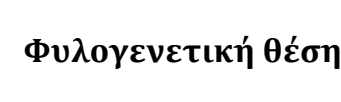 \\
\hline Nat2-35 & 8 & Acinetobacter sp. 8A12N2 & HQ246231 & 99 & 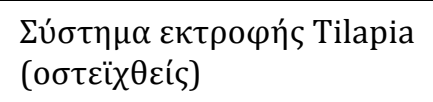 & $\gamma$-Proteobacteria \\
\hline Nat2-22 & 4 & 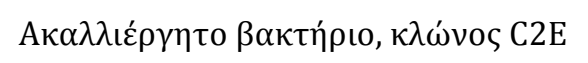 & DQ856531 & 97 & 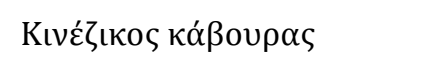 & $\alpha$-Proteobacteria \\
\hline Nat2-23 & 4 & 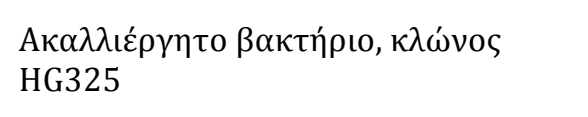 & FN646739 & 96 & 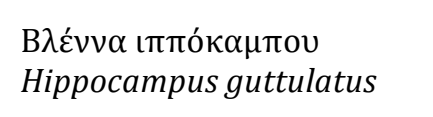 & $\alpha$-Proteobacteria \\
\hline Nat2-34 & 4 & 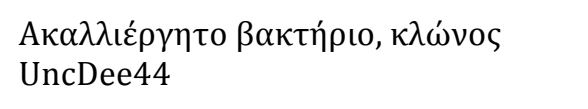 & AM997546 & 96 & 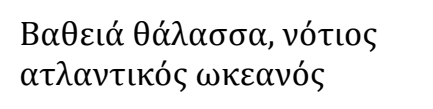 & Bacteroidetes \\
\hline Nat2-41 & 4 & 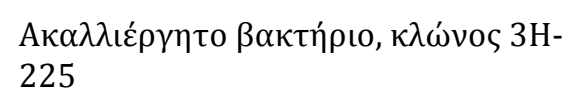 & EU786124 & 99 & 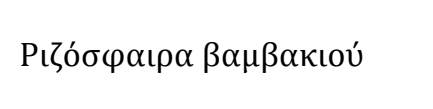 & $\alpha$-Proteobacteria \\
\hline Nat2-67 & 4 & $\begin{array}{l}\text { Shewanella xiamenensis strain CAIM } \\
1494\end{array}$ & HM584096 & 96 & $\begin{array}{l}\text { Bót } \rho \alpha \chi \circ \varsigma \text { Rana } \\
\text { catesbeiana }\end{array}$ & $\gamma$-Proteobacteria \\
\hline Nat2-73 & 4 & Pseudoruegeria sp. JC2225 & EU642855 & 99 & $\Sigma \tau \varepsilon \dot{\lambda} \lambda \varepsilon \chi \circ \varsigma$ & $\alpha$-Proteobacteria \\
\hline Nat2-75 & 4,3 & 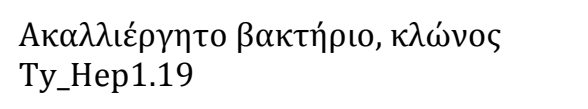 & EU646197 & 90 & 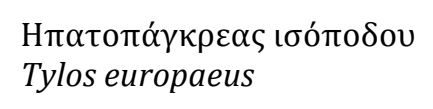 & Tenericutes \\
\hline S3m1-6 & 15 & Marinicella litoralis & AB500095 & 96 & 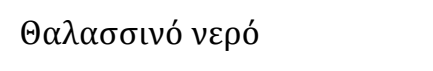 & $\gamma$-Proteobacteria \\
\hline S3m1-7 & 15 & Litoreibacter albidus KMM3851 & AB518881 & 99 & 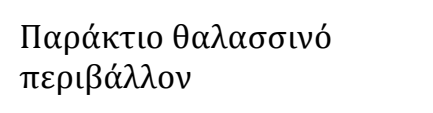 & $\alpha$-Proteobacteria \\
\hline S3m1-3 & 15 & 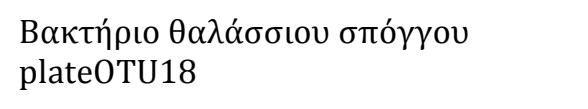 & EU346505 & 99 & 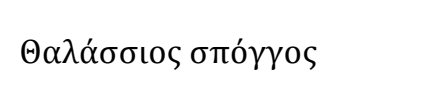 & $\alpha$-Proteobacteria \\
\hline S3m1-19 & 12,5 & 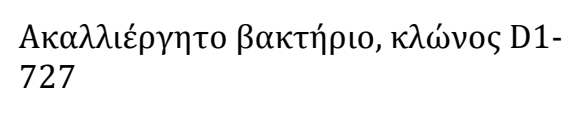 & GQ866081 & 98 & 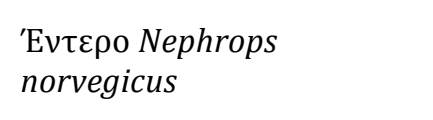 & Actinobacteria \\
\hline S3m1-25 & 5 & 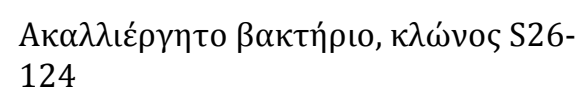 & EU287424 & 98 & 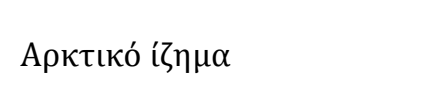 & $\alpha$-Proteobacteria \\
\hline
\end{tabular}




\begin{tabular}{|c|c|c|c|c|c|c|}
\hline 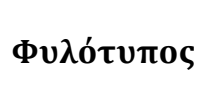 & $\begin{array}{l}\Sigma v \chi v o ́ \tau \eta \tau \alpha \\
(\%)\end{array}$ & 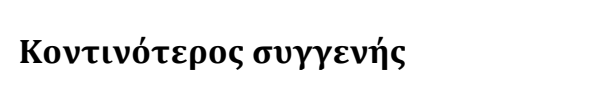 & Genbank \# & 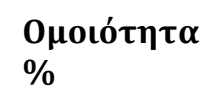 & $\Delta \varepsilon \dot{\gamma} \gamma \mu \alpha \varepsilon v \tau 0 \pi \iota \sigma \mu o v ́$ & 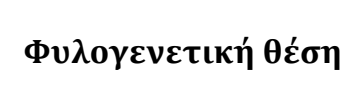 \\
\hline S3m1-5 & 5 & Halomonas subterranea strain ZG16 & EF144148 & 99 & 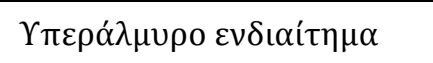 & $\gamma$-Proteobacteria \\
\hline S3m1-15 & 5 & 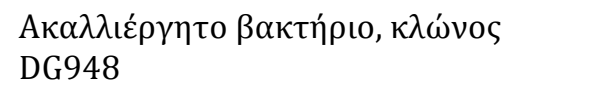 & AY258091 & 100 & Gymnodinium catenatum & Alpha \\
\hline S3m1-2 & 2,5 & 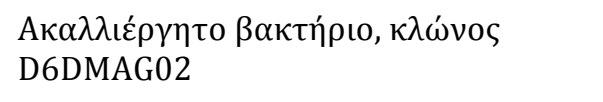 & HQ216328 & 99 & 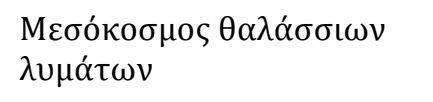 & $\alpha$-Proteobacteria \\
\hline S3m1-4 & 2,5 & 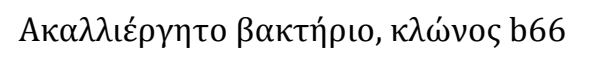 & EU919786 & 97 & 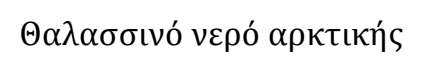 & $\alpha$-Proteobacteria \\
\hline S3m1-8 & 2,5 & 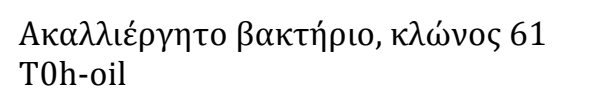 & FM242237 & 99 & $' \mathrm{I} \zeta \eta \mu \alpha$ & $\gamma$-Proteobacteria \\
\hline S3m1-10 & 2,5 & 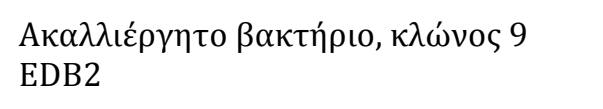 & AM882553 & 97 & 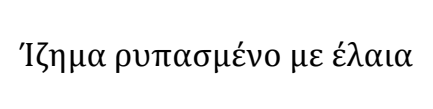 & $\gamma$-Proteobacteria \\
\hline S3m1-11 & 2,5 & 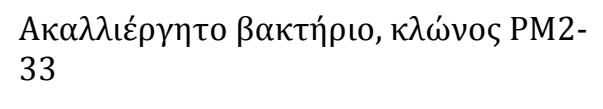 & EF215821 & 97 & 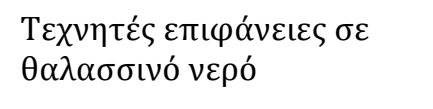 & $\gamma$-Proteobacteria \\
\hline S3m1-14 & 2,5 & Octadecabacter sp. NF3-22 & FJ196058 & 97 & 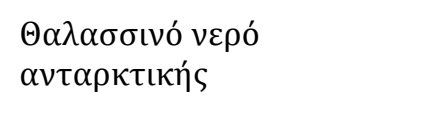 & $\alpha$-Proteobacteria \\
\hline S3m1-18 & 2,5 & 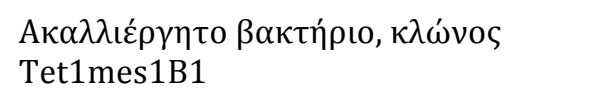 & EU290251 & 97 & 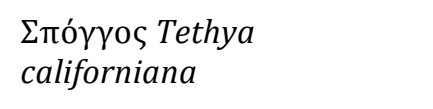 & Verrucomicrobia \\
\hline S3m1-20 & 2,5 & Vibrio tapetis strain LP2 & AY129278 & 99 & 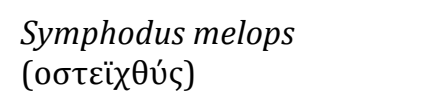 & $\gamma$-Proteobacteria \\
\hline S3m1-24 & 2,5 & 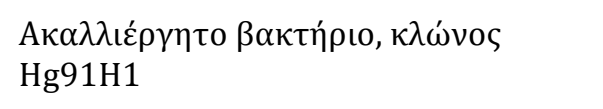 & EU236379 & 99 & $\begin{array}{l}\text { Haliclona cf. Gellius sp. } \\
\text { sponge }\end{array}$ & $\alpha$-Proteobacteria \\
\hline S3m1-29 & 2,5 & Marinomonas sp. SC22 & FN433057 & 99 & 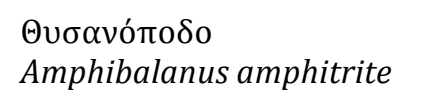 & $\gamma$-Proteobacteria \\
\hline S3m1-42 & 2,5 & 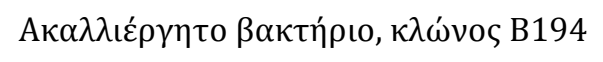 & EU328045 & 99 & 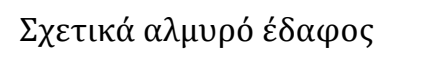 & $\gamma$-Proteobacteria \\
\hline
\end{tabular}




\begin{tabular}{|c|c|c|c|c|c|c|}
\hline 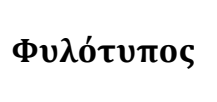 & $\begin{array}{l}\Sigma v \chi v o ́ \tau \eta \tau \alpha \\
(\%)\end{array}$ & 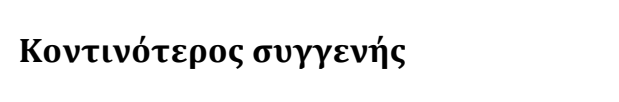 & Genbank \# & 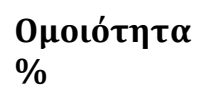 & 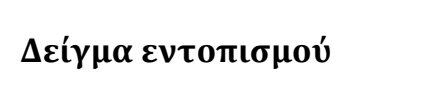 & 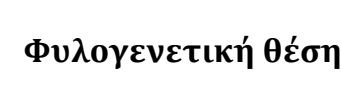 \\
\hline S6m1-2 & 7,6 & $\begin{array}{l}\text { Pseudoalteromonas translucida strain } \\
\text { KMM } 520\end{array}$ & NR025655 & 99 & 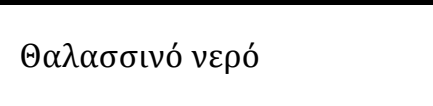 & $\gamma$-Proteobacteria \\
\hline S6m1-3 & 12,8 & Marivita cryptomonadis CL-SK44 & EU512919 & 99 & Cryptophyta sp. CR-MAL01 & $\alpha$-Proteobacteria \\
\hline S6m1-10 & 12,8 & 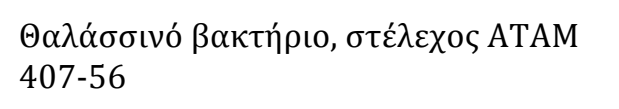 & AF359535 & 100 & 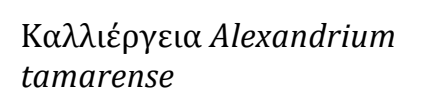 & $\alpha$-Proteobacteria \\
\hline S6m1-8 & 10,2 & 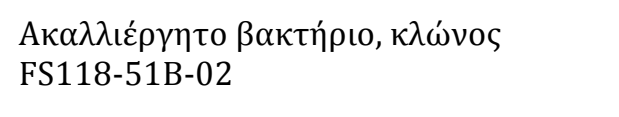 & AY704399 & 99 & 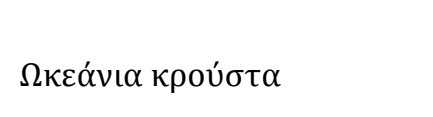 & $\varepsilon$-Proteobacteria \\
\hline S6m1-36 & 10,2 & Roseobacter sp. 38.98 & AY870684 & 98 & $\Sigma \tau \rho \varepsilon i \delta \delta \_$ & $\alpha$-Proteobacteria \\
\hline S6m1-15 & 5,1 & 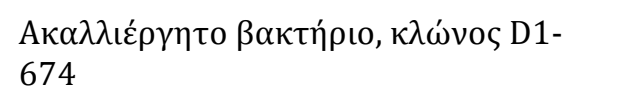 & GQ866083 & 99 & 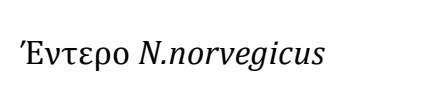 & $\gamma$-Proteobacteria \\
\hline S6m1-1 & 5,1 & Pseudoalteromonas sp. P58 & EU935093 & 99 & 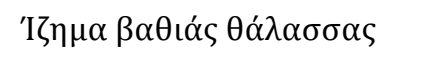 & $\gamma$-Proteobacteria \\
\hline S6m1-24 & 7,7 & Vibrio splendidus isolate PB1-10rrnH & EU091332 & 99 & 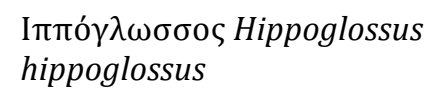 & $\gamma$-Proteobacteria \\
\hline S6m1-5 & 2,6 & Photobacterium leiognathi strain LN101 & AY292944 & 99 & 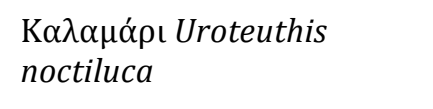 & $\gamma$-Proteobacteria \\
\hline S6m1-6 & 2,6 & $\begin{array}{l}\text { Psychroserpens mesophilus strain } \\
\text { KOPRI } 13649\end{array}$ & DQ001321 & 98 & $\Theta \alpha \lambda \alpha ́ \sigma \sigma ı$ biofilm & Bacteroidetes \\
\hline S6m1-9 & 2,6 & $\begin{array}{l}\text { Polaribacter dokdonensis strain } \\
\text { MED152 }\end{array}$ & DQ481463 & 99 & 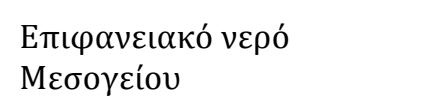 & Bacteroidetes \\
\hline S6m1-11 & 2,6 & Vibrio gallaecicus strain CECT 7244 & EU541605 & 99 & 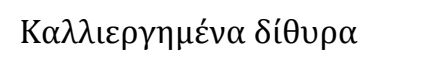 & $\gamma$-Proteobacteria \\
\hline S6m1-22 & 2,6 & 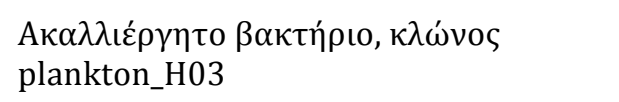 & FJ664810 & 92 & & $\alpha$-Proteobacteria \\
\hline
\end{tabular}




\begin{tabular}{|c|c|c|c|c|c|c|}
\hline 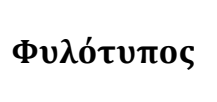 & $\begin{array}{l}\Sigma v \chi v o ́ \tau \eta \tau \alpha \\
(\%)\end{array}$ & 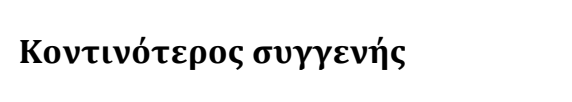 & Genbank \# & 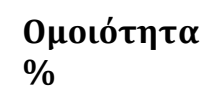 & 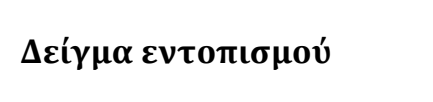 & 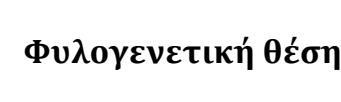 \\
\hline S6m1-28 & 2,6 & 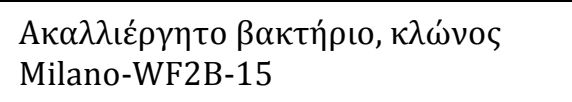 & AY592903 & 99 & 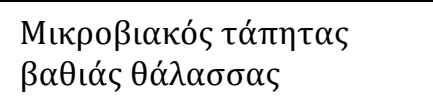 & $\alpha$-Proteobacteria \\
\hline S6m1-29 & 2,6 & 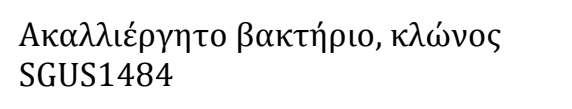 & FJ202554 & 90 & 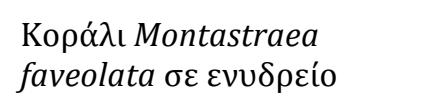 & Bacteroidetes \\
\hline S6m1-33 & 2,6 & 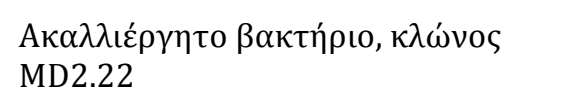 & FJ403071 & 97 & Montastrea faveolata & Bacteroidetes \\
\hline S6m1-35 & 2,6 & Marinomonas sp. 139Z-17 & GU584157 & 98 & 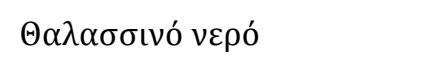 & $\gamma$-Proteobacteria \\
\hline S6m1-37 & 2,6 & Vibrio tapetis strain LP2 & AY129278 & 99 & 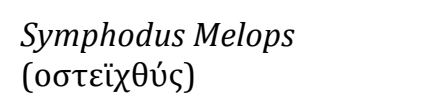 & $\gamma$-Proteobacteria \\
\hline S6m1-38 & 2,6 & 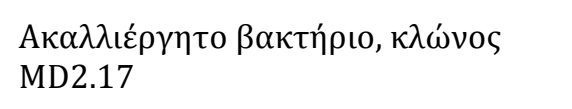 & FJ403066 & 94 & Montastrea faveolata & $\delta$-Proteobacteria \\
\hline S6m2-2 & 9,3 & 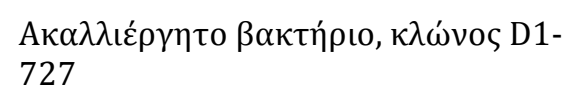 & GQ866081 & 98 & 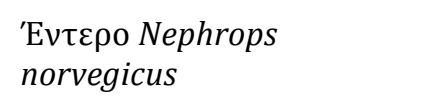 & Actinobacteria \\
\hline S6m2-13 & 4,6 & 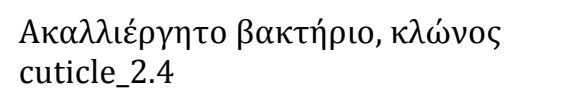 & HQ111166 & 99 & 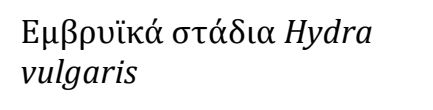 & $\beta$-Proteobacteria \\
\hline S6m2-16 & 9,3 & $\begin{array}{l}\text { Stenotrophomonas maltophilia strain } \\
\text { ATCC } 53510\end{array}$ & FJ976090 & 99 & 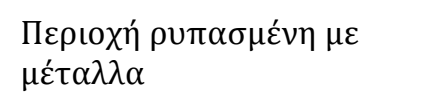 & $\gamma$-Proteobacteria \\
\hline S6m2-15 & 4,6 & 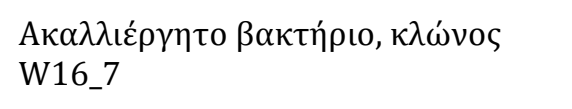 & FR729092 & 100 & 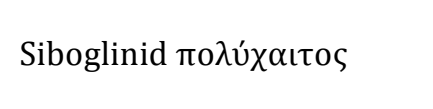 & $\beta$-Proteobacteria \\
\hline S6m2-3 & 7 & 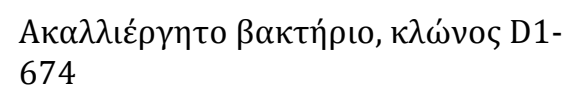 & GQ866083 & 99 & 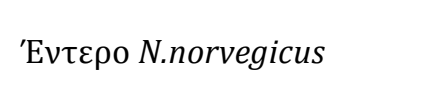 & $\gamma$-Proteobacteria \\
\hline S6m2-8 & 4,6 & 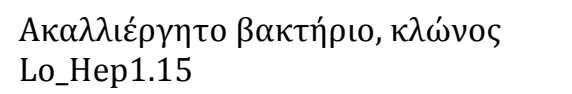 & EU646198 & 89 & 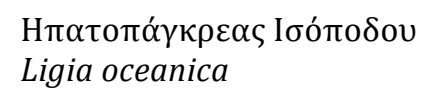 & Tenericutes \\
\hline
\end{tabular}




\begin{tabular}{|c|c|c|c|c|c|c|}
\hline 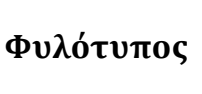 & 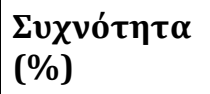 & 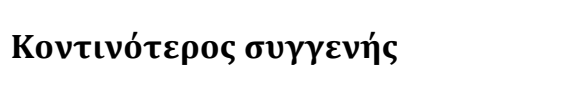 & Genbank \# & 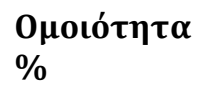 & 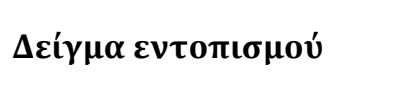 & 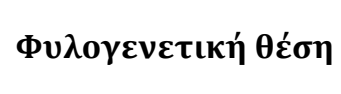 \\
\hline S6m2-59 & 4,6 & 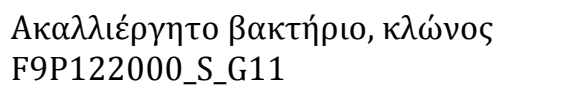 & HQ673860 & 90 & 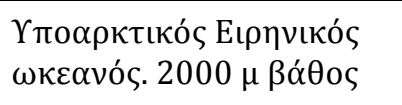 & Actinobacteria \\
\hline S6m2-1 & 4,6 & 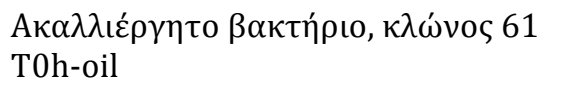 & FM242237 & 99 & 'I $\zeta \eta \mu \alpha$ & $\gamma$-Proteobacteria \\
\hline S6m2-17 & 4,6 & Litoreibacter albidus KMM3851 & AB518881 & 98 & 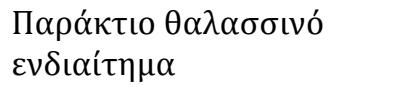 & $\alpha$-Proteobacteria \\
\hline S6m2-9 & 2,3 & 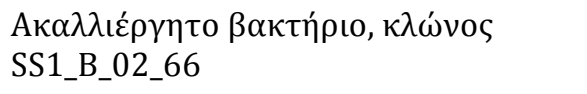 & EU050781 & 96 & 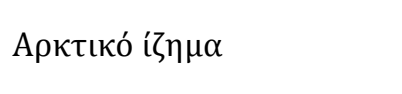 & $\gamma$-Proteobacteria \\
\hline S6m2-11 & 2,3 & 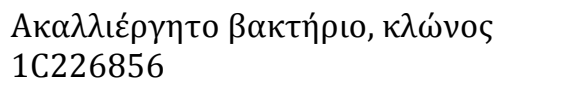 & EU799275 & 98 & 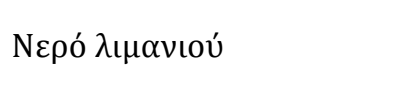 & $\alpha$-Proteobacteria \\
\hline S6m2-12 & 2,3 & 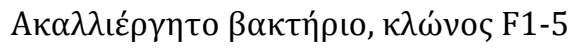 & GQ866090 & 99 & 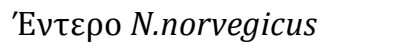 & $\gamma$-Proteobacteria \\
\hline S6m2-14 & 2,3 & 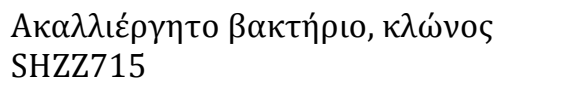 & HQ163591 & 97 & $\begin{array}{l}\Upsilon \delta \alpha \dot{\tau} \tau \iota v \eta \sigma \tau \eta^{\prime} \lambda \eta, 120 \mu \\
\beta \alpha \alpha \theta \varsigma\end{array}$ & $\gamma$-Proteobacteria \\
\hline S6m2-21 & 2,3 & $\begin{array}{l}\text { Marinomonas blandensis strain MED } \\
121\end{array}$ & DQ403809 & 96 & 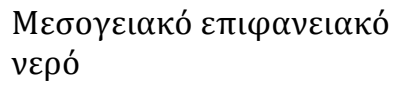 & $\gamma$-Proteobacteria \\
\hline S6m2-23 & 2,3 & 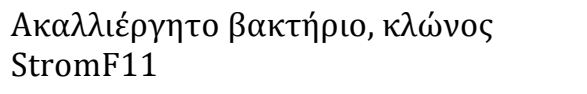 & EU918014 & 92 & $\Sigma \tau \rho \omega \mu \alpha \tau о \lambda i ́ \tau \eta s$ & $\alpha$-Proteobacteria \\
\hline S6m2-24 & 2,3 & 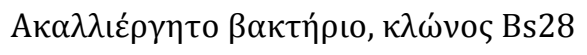 & EF137383 & 96 & 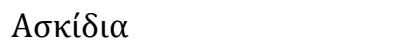 & $\alpha$-Proteobacteria \\
\hline S6m2-28 & 2,3 & 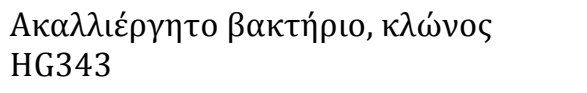 & FN646757 & 97 & 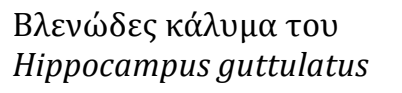 & Actinobacteria \\
\hline S6m2-29 & 2,3 & Octadecabacter sp. NF3-22 & FJ196058 & 97 & 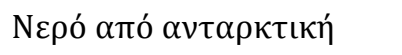 & $\alpha$-Proteobacteria \\
\hline S6m2-34 & 2,3 & 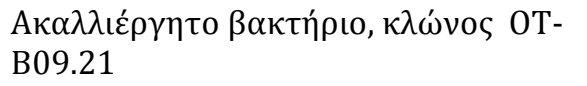 & AB252434 & 98 & $\Theta \alpha \lambda \alpha ́ \sigma \sigma ı \iota^{\prime} \zeta \mu \alpha$ & $\delta$-Proteobacteria \\
\hline
\end{tabular}




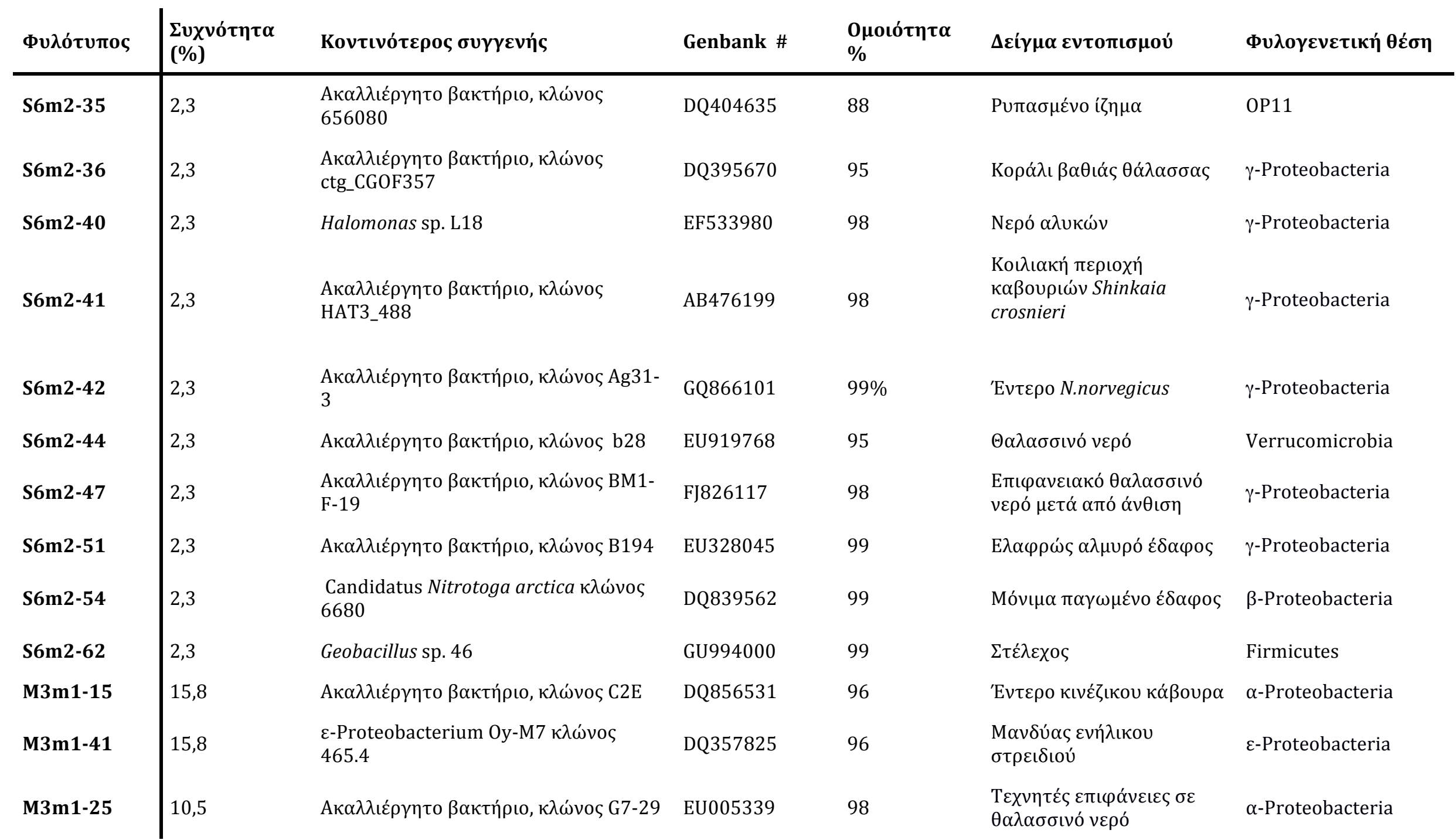




\begin{tabular}{|c|c|c|c|c|c|c|}
\hline 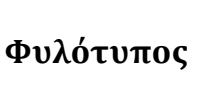 & $\begin{array}{l}\Sigma v \chi v o ́ \tau \eta \tau \alpha \\
(\%)\end{array}$ & 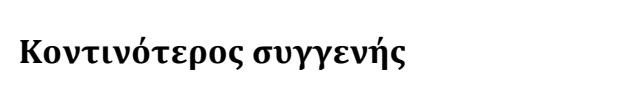 & Genbank \# & 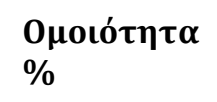 & $\Delta \varepsilon i ́ \gamma \mu \alpha \varepsilon \varepsilon \tau 0 \pi \iota \sigma \mu о v ́$ & 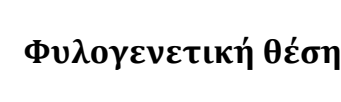 \\
\hline M3m1-20 & 7,9 & Vibrio sp. r17 & AB470928 & 95 & Kopódı Montipora sp. & $\gamma$-Proteobacteria \\
\hline M3m1-14 & 5,3 & Persicivirga sp. PHSCD-1 & HM854017 & 87 & Laminaria sp. & Bacteroidetes \\
\hline M3m1-19 & 5,3 & Rhodobacteraceae $\beta \alpha \kappa \tau$ т́ $\rho$ เо 183 & AJ810844 & 97 & 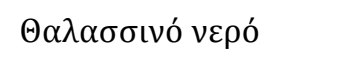 & $\alpha$-Proteobacteria \\
\hline M3m1-108 & 5,3 & Litoreibacter albidus KMM3851 & AB518881 & 96 & 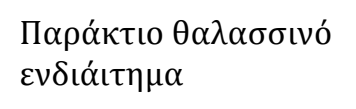 & $\alpha$-Proteobacteria \\
\hline M3m1-76 & 5,3 & Roseobacter sp. 38.98 (12) & AY870684 & 97 & $\Sigma \tau \rho \varepsilon^{\prime} \delta ı \alpha$ & $\alpha$-Proteobacteria \\
\hline M3m1-38 & 2,6 & 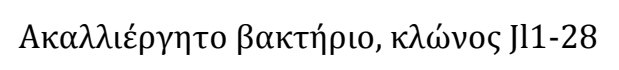 & GQ866089 & 99 & 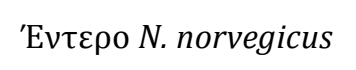 & CK-1C4-19 \\
\hline M3m1-68 & 2,6 & 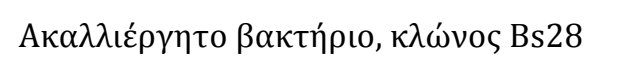 & EF137383 & 96 & 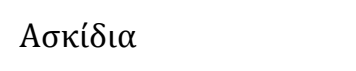 & $\alpha$-Proteobacteria \\
\hline M3m1-70 & 2,6 & $\begin{array}{l}\text { Hellea balneolensis strain } \\
\text { 26III/A02/215 }\end{array}$ & AY576758 & 99 & $\Sigma \tau \varepsilon ́ \lambda \varepsilon \chi \circ \varsigma$ & $\alpha$-Proteobacteria \\
\hline M3m1-79 & 2,6 & Colwellia psychroerythraea & AB011364 & 98 & $\Theta \alpha \lambda \alpha \sigma \sigma i v o ́$ vepó & $\gamma$-Proteobacteria \\
\hline M3m1-81 & 2,6 & Pibocella ponti strain KMM 6031 & NR_025821 & 95 & $\begin{array}{l}\text { Фúкоц Acrosiphonia } \\
\text { sonderi }\end{array}$ & Bacteroidetes \\
\hline M3m1-82 & 2,6 & Roseovarius sp. E4-1 & FN377714 & 99 & 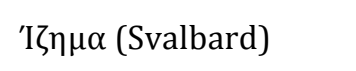 & $\alpha$-Proteobacteria \\
\hline M3m1-87 & 2,6 & Litoreibacter albidus KMM3851 & AB518881 & 98 & 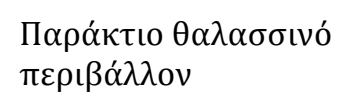 & $\alpha$-Proteobacteria \\
\hline M3m1-121 & 2,6 & 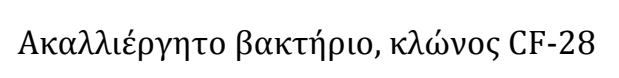 & FJ535311 & 98 & 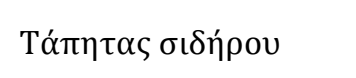 & $\gamma$-Proteobacteria \\
\hline M3m1-123 & 2,6 & 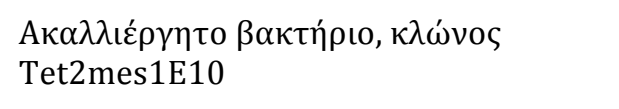 & EU290363 & 94 & 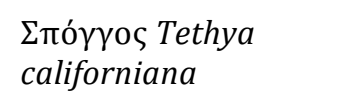 & Bacteroidetes \\
\hline M3m1-132 & 2,6 & $\begin{array}{l}\text { Psychroserpens mesophilus strain } \\
\text { KOPRI } 13649\end{array}$ & DQ001321 & 97 & $\Theta \alpha \lambda \alpha ́ \sigma \sigma ı$ biofilm & Bacteroidetes \\
\hline
\end{tabular}




\begin{tabular}{|c|c|c|c|c|c|c|}
\hline 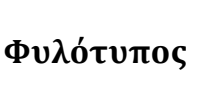 & 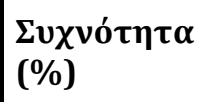 & 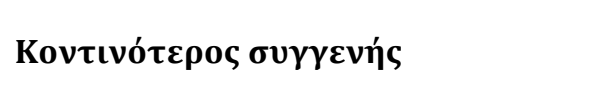 & Genbank \# & 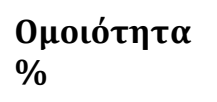 & 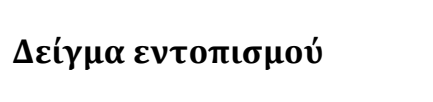 & 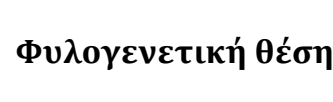 \\
\hline M3m1-152 & 2,6 & 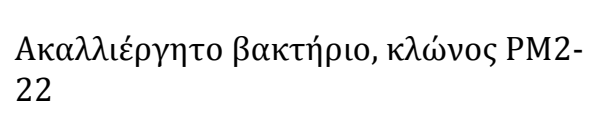 & EF215810 & 99 & 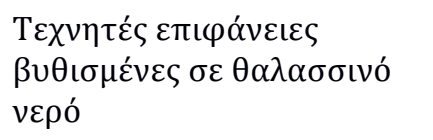 & $\alpha$-Proteobacteria \\
\hline M6m1-4 & 20,5 & 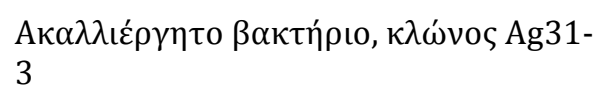 & GQ866101 & $99 \%$ & 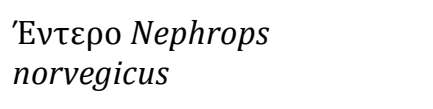 & $\gamma$-Proteobacteria \\
\hline M6m1-56 & 17,9 & 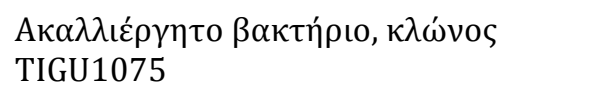 & HM558927 & 99 & 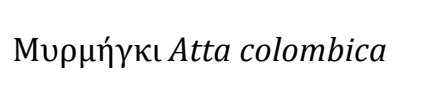 & $\gamma$-Proteobacteria \\
\hline M6m1-2 & 12,8 & 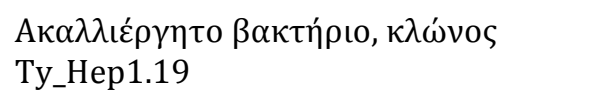 & EU646197 & 90 & $\begin{array}{l}\eta \pi \alpha \tau о \pi \alpha ́ \gamma \kappa \rho \varepsilon \alpha \varsigma ~ T y l o s \\
\text { europeus }\end{array}$ & Tenericutes \\
\hline M6m1-22 & 5,1 & 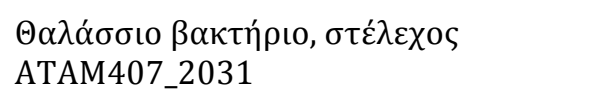 & AF359536 & 98 & $\begin{array}{l}\text { K } \alpha \lambda \lambda \lambda_{1 \varepsilon ́} \rho \gamma \varepsilon ı \alpha \text { Alexandrium } \\
\text { tamarense }\end{array}$ & $\alpha$-Proteobacteria \\
\hline M6m1-11 & 5,1 & 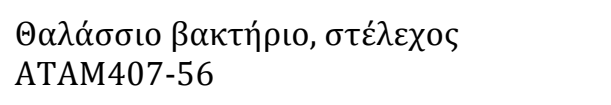 & AF359535 & 99 & $\begin{array}{l}\text { K } \alpha \lambda \lambda \iota \varepsilon ́ \rho \gamma \varepsilon ı \alpha \text { Alexandrium } \\
\text { tamarense }\end{array}$ & $\alpha$-Proteobacteria \\
\hline M6m1-29 & 2,6 & Vibrio splendidus isolate PB1-10rrnD & EU091328 & 99 & $\begin{array}{l}\text { Iтாó } \lambda \lambda \omega \sigma \sigma o \varsigma \text { Hippoglossus } \\
\text { hippoglossus }\end{array}$ & $\gamma$-Proteobacteria \\
\hline M6m1-30 & 2,6 & 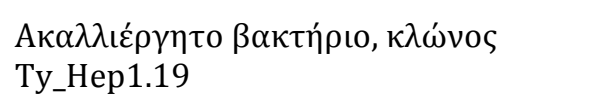 & EU646197 & 91 & 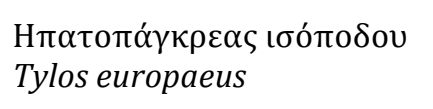 & Tenericutes \\
\hline M6m1-35 & 2,6 & 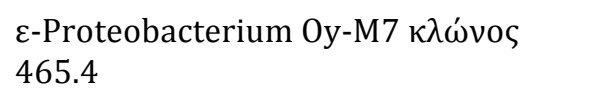 & DQ357825 & 96 & 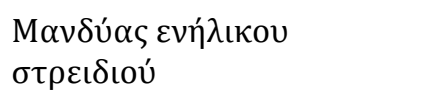 & $\varepsilon$-Proteobacteria \\
\hline M6m1-38 & 2,6 & 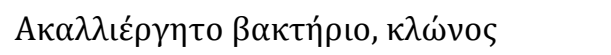 & FJ203077 & 98 & 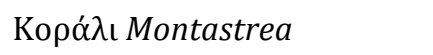 & $\alpha$-Proteobacteria \\
\hline
\end{tabular}




\begin{tabular}{|c|c|c|c|c|c|c|}
\hline 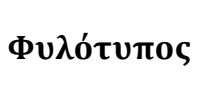 & 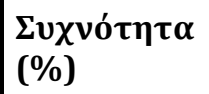 & 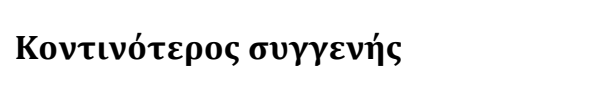 & Genbank \# & 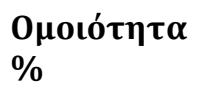 & $\Delta \varepsilon \dot{\gamma} \gamma \mu \alpha \varepsilon v \tau 0 \pi \iota \sigma \mu o v ́$ & 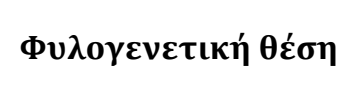 \\
\hline & & SHFG464 & & & falveolata & \\
\hline M6m1-45 & 2,6 & $\begin{array}{l}\text { Leisingera nanhaiensis DSM } 24252 \\
\text { strain NH52F }\end{array}$ & FJ232451 & 99 & 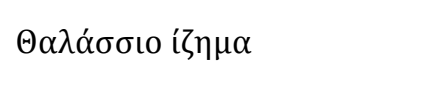 & $\alpha$-Proteobacteria \\
\hline M6m1-49 & 2,6 & 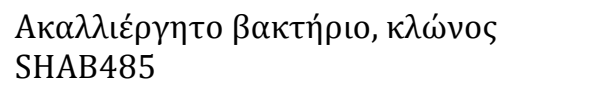 & GQ348595 & 97 & 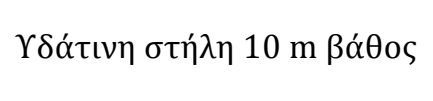 & $\alpha$-Proteobacteria \\
\hline M6m1-51 & 2,6 & Marivita litorea strain CL-JM1 & EU512918 & 100 & 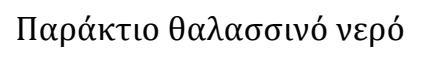 & $\alpha$-Proteobacteria \\
\hline M6m1-53 & 2,6 & 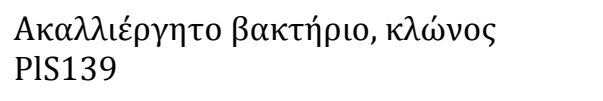 & AY779705 & 89 & 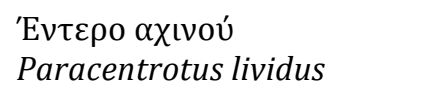 & $\alpha$-Proteobacteria \\
\hline M6m1-69 & 2,6 & 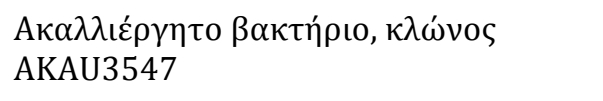 & DQ125562 & 98 & 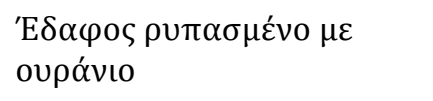 & $\alpha$-Proteobacteria \\
\hline M6m2-6 & 28,9 & 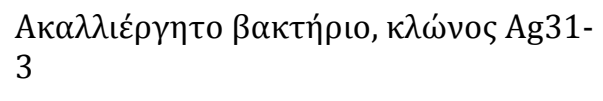 & GQ866101 & $99 \%$ & 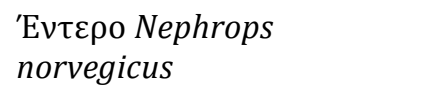 & $\gamma$-Proteobacteria \\
\hline M6m2-3 & 15,8 & 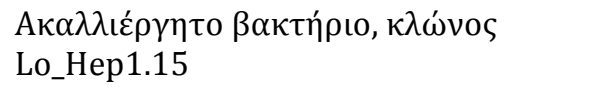 & EU646198 & 88 & 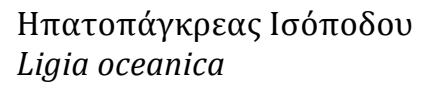 & Tenericutes \\
\hline M6m2-10 & 13,2 & 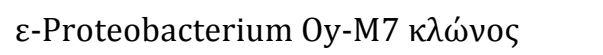 & DQ357825 & 95 & 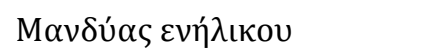 & $\varepsilon$-Proteobacteria \\
\hline
\end{tabular}




\begin{tabular}{|c|c|c|c|c|c|c|}
\hline 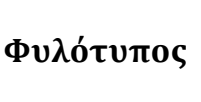 & 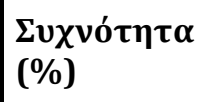 & 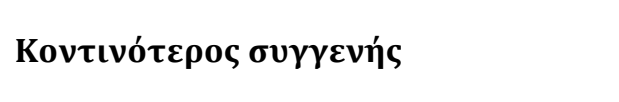 & Genbank \# & 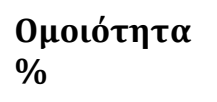 & 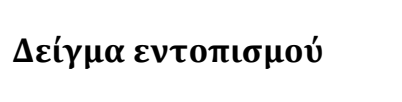 & 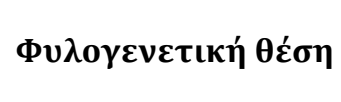 \\
\hline & & 465.4 & & & $\sigma \tau \rho \varepsilon ı$ เoú & \\
\hline M6m2-4 & 7,9 & 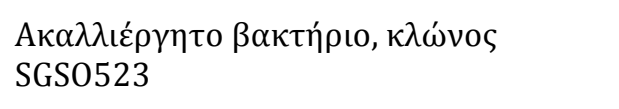 & GQ347793 & 99 & 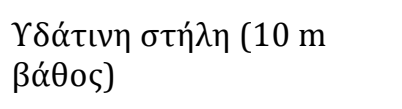 & $\gamma$-Proteobacteria \\
\hline M6m2-1 & 2,6 & 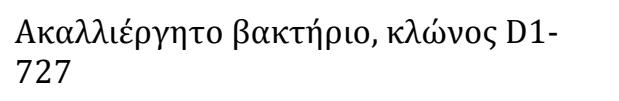 & GQ866081 & 99 & 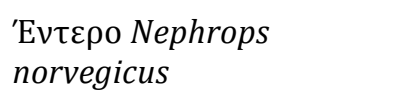 & Actinobacteria \\
\hline M6m2-8 & 2,6 & $\begin{array}{l}\text { Staphylococcus epidermidis strain } \\
\text { HNR19 }\end{array}$ & EU373384 & 99 & 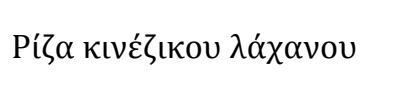 & Firmicutes \\
\hline M6m2-9 & 2,6 & 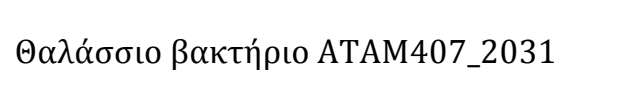 & AF359536 & 97 & $\begin{array}{l}\text { K } \alpha \lambda \lambda \_\varepsilon ́ \rho \gamma \varepsilon ı \alpha \text { Alexandrium } \\
\text { tamarense }\end{array}$ & $\alpha$-Proteobacteria \\
\hline M6m2-11 & 2,6 & 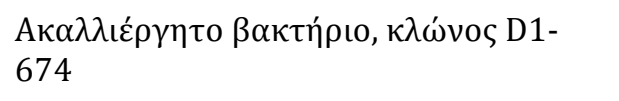 & GQ866083 & 98 & 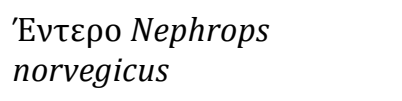 & $\gamma$-Proteobacteria \\
\hline M6m2-25 & 2,6 & Moritella sp. ODA02 & AB011353 & 98 & 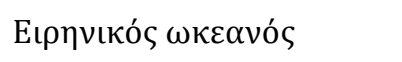 & $\gamma$-Proteobacteria \\
\hline M6m2-28 & 2,6 & $\begin{array}{l}\text { Altererythrobacter luteolus strain SW- } \\
109\end{array}$ & AY739662 & 98 & 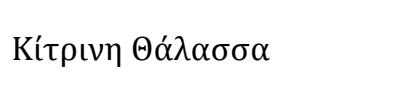 & $\alpha$-Proteobacteria \\
\hline M6m2-31 & 2,6 & Colwellia psychroerythraea & AB011364 & 97 & 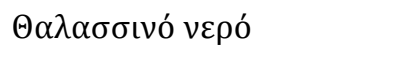 & $\gamma$-Proteobacteria \\
\hline M6m2-36 & 2,6 & $\begin{array}{l}\text { Kopriimonas byunsanensis strain KOPRI } \\
13522\end{array}$ & DQ167245 & 99 & $\Theta \alpha \lambda \alpha ́ \sigma \sigma \iota$ biofilm & $\alpha$-Proteobacteria \\
\hline
\end{tabular}




\begin{tabular}{|c|c|c|c|c|c|c|}
\hline 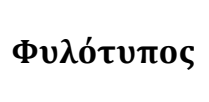 & $\begin{array}{l}\Sigma v \chi v o ́ \tau \eta \tau \alpha \\
(\%)\end{array}$ & 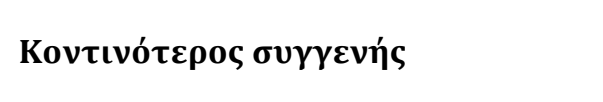 & Genbank \# & 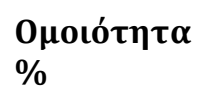 & $\Delta \varepsilon \dot{\gamma} \gamma \mu \alpha \varepsilon v \tau 0 \pi \iota \sigma \mu o v ́$ & 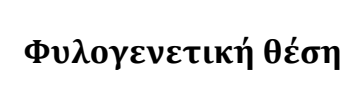 \\
\hline M6m2-38 & 2,6 & 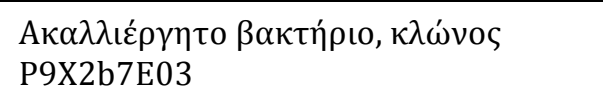 & EU491195 & 92 & 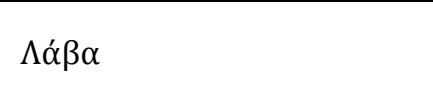 & TM6 \\
\hline M6m2-42 & 2,6 & Vibrio splendidus isolate PB1-10rrnB & EU091326 & 99 & 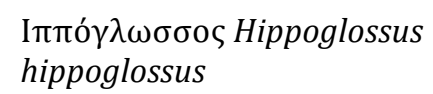 & $\gamma$-Proteobacteria \\
\hline P6m1-12 & 33,3 & 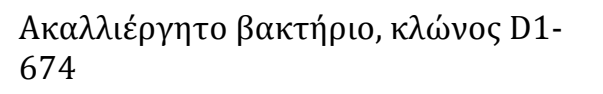 & GQ866083 & 99 & 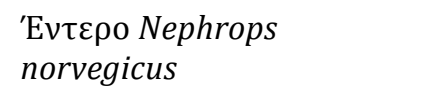 & $\gamma$-Proteobacteria \\
\hline P6m1-18 & 20,8 & 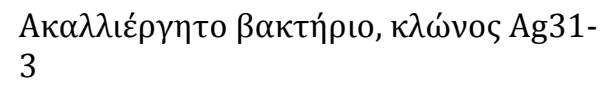 & GQ866101 & $99 \%$ & 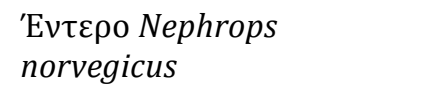 & $\gamma$-Proteobacteria \\
\hline P6m1-72 & 8,3 & 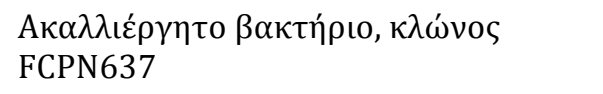 & EF515914 & 94 & 'E $\delta \alpha \varphi \circ \lambda_{\imath \iota} \beta \alpha \delta\llcorner$ เо & Fibrobacteres \\
\hline P6m1-15 & 4,2 & 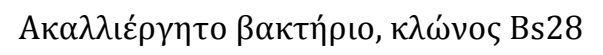 & EF137383 & 95 & 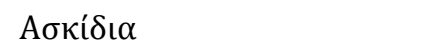 & $\alpha$-Proteobacteria \\
\hline P6m1-23 & 4,2 & 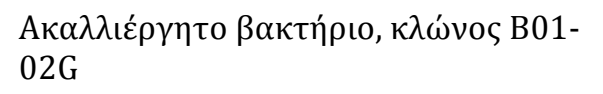 & FJ542926 & 96 & 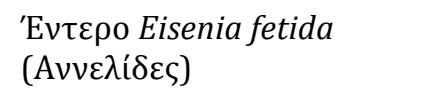 & $\alpha$-Proteobacteria \\
\hline P6m1-54 & 4,2 & 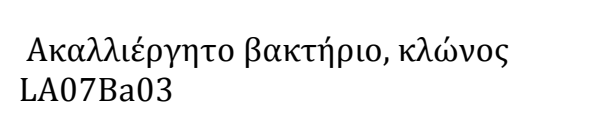 & GU133211 & 91 & 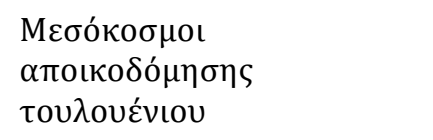 & $\alpha$-Proteobacteria \\
\hline P6m1-58 & 4,2 & $\begin{array}{l}\text { Marinomonas blandensis strain MED } \\
121\end{array}$ & DQ403809 & 98 & 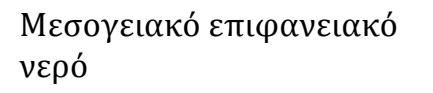 & $\gamma$-Proteobacteria \\
\hline P6m1-60 & 4,2 & Colwellia psychroerythraea & AB011364 & 95 & 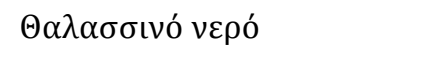 & $\gamma$-Proteobacteria \\
\hline P6m1-61 & 4,2 & 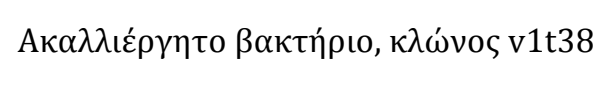 & FM165265 & 99 & 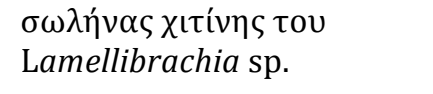 & $\delta$-Proteobacteria \\
\hline P6m1-66 & 4,2 & Persicivirga sp. PHSCD-1 & HM854017 & 87 & Laminaria sp. & Bactroidetes \\
\hline P6m1-74 & 4,2 & 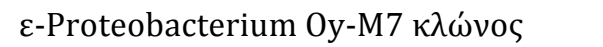 & DQ357825 & 96 & 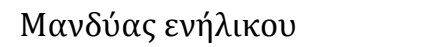 & $\varepsilon$-Proteobacteria \\
\hline
\end{tabular}




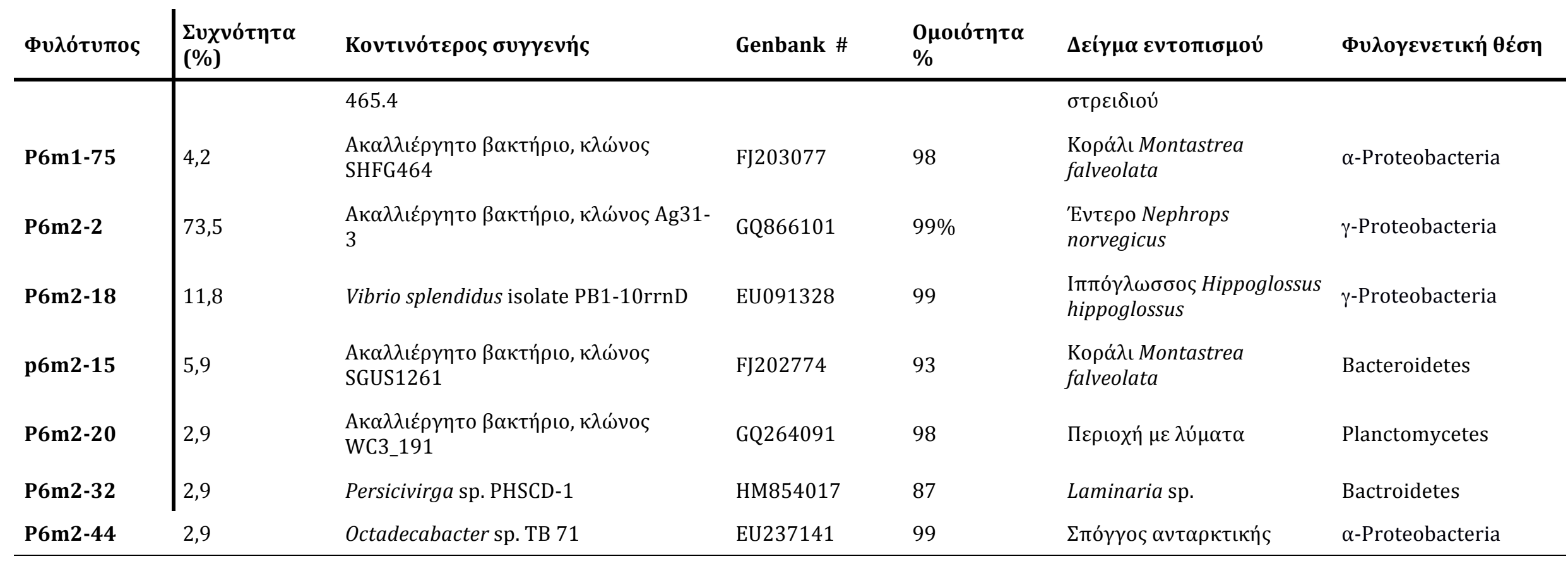

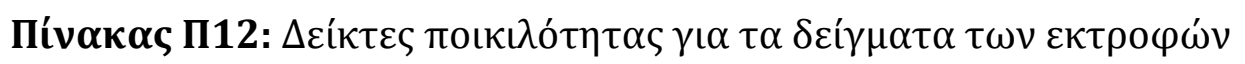

\begin{tabular}{l|rrrrrrrrrr} 
& Nat1 & Nat2 & \multicolumn{1}{c|}{ S3m1 } & S6m1 & S6m2 & M3m1 & M6m1 & M6m2 & P6m1 & P6m2 \\
\hline Simpson_1_D & 0,54 & 0,7328 & 0,9025 & 0,9218 & 0,954 & 0,9141 & 0,8876 & 0,8587 & 0,8229 & 0,4394 \\
Shannon_H & 1,312 & 1,783 & 2,577 & 2,726 & 3,233 & 2,693 & 2,541 & 2,362 & 2,092 & 0,9557 \\
Margalef & 2,352 & 2,796 & 4,608 & 4,913 & 7,444 & 4,948 & 4,913 & 4,399 & 3,461 & 1,418
\end{tabular}




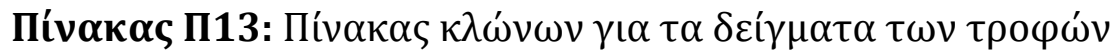

\begin{tabular}{|c|c|c|c|c|c|c|}
\hline Фиגótuтоs & 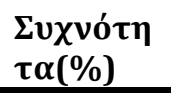 & 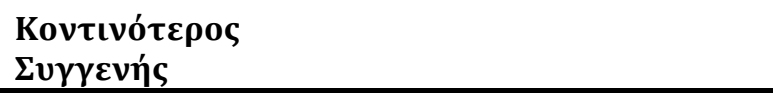 & Genbank \# & 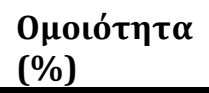 & 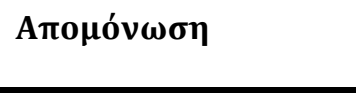 & 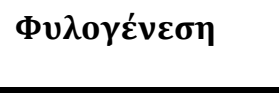 \\
\hline Mus1-3 & 16,66 & 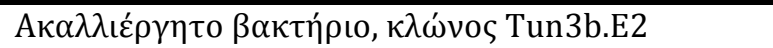 & FJ169210 & 99 & 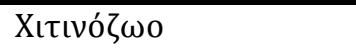 & $\alpha$-Proteobacteria \\
\hline Mus1-5 & 16,66 & Vibrio sp. $к \lambda \omega \nu$ os $\mathrm{H} 02 \mathrm{C} 48$ & HQ161443 & 99 & 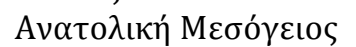 & $\gamma$-Proteobacteria \\
\hline Mus1-21 & 10 & 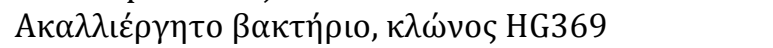 & FN646711 & 99 & Ітпо́к $\alpha \mu \pi о \varsigma$ & $\gamma$-Proteobacteria \\
\hline Mus1-19 & 6,66 & Pseudoalteromonas sp. SDMRI-B9 & JF268254 & 99 & Кора́dıı & $\gamma$-Proteobacteria \\
\hline Mus1-23 & 6,66 & 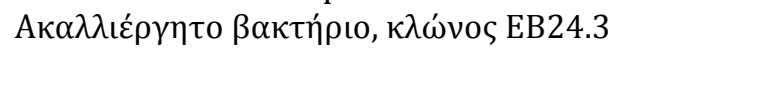 & EU573099 & 97 & 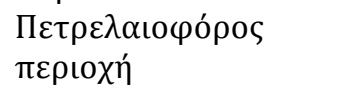 & $\varepsilon$-Proteobacteria \\
\hline Mus1-60 & 6,66 & Aeromonas hydrophila strain IPC6 & HQ108173 & 98 & 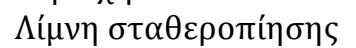 & $\gamma$-Proteobacteria \\
\hline Mus1-1 & 3,33 & 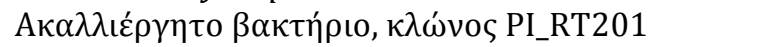 & AY580421 & 98 & 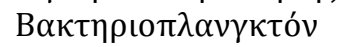 & $\varepsilon$-Proteobacteria \\
\hline Mus1-2 & 3,33 & 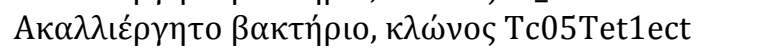 & EU290395 & 98 & 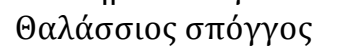 & $\gamma$-Proteobacteria \\
\hline Mus1-14 & 3,33 & Intranuclear bacterium of Bathymodiolus spp & FM162194 & 97 & Bathymodiolus spp. & $\gamma$-Proteobacteria \\
\hline Mus1-16 & 3,33 & Aeromonas veronii strain CYJ102 & FJ940846 & 99 & 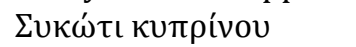 & $\gamma$-Proteobacteria \\
\hline Mus1-22 & 3,33 & 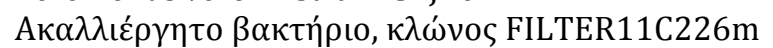 & EU346429 & 94 & 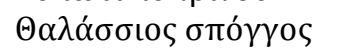 & Bacteroidetes \\
\hline Mus1-25 & 3,33 & 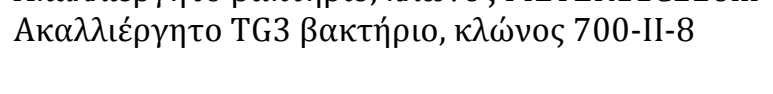 & AB256016 & 97 & 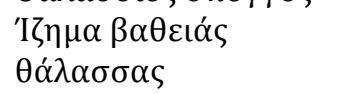 & TG3 \\
\hline Mus1-61 & 3,33 & 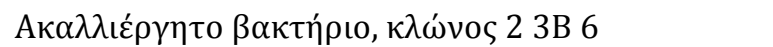 & FJ800218 & 95 & 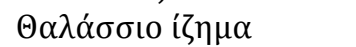 & $\gamma$-Proteobacteria \\
\hline Mus1-62 & 3,33 & Lactobacillus sp. C56 & FJ611792 & 99 & 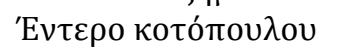 & Fimicutes \\
\hline Mus1-67 & 3,33 & 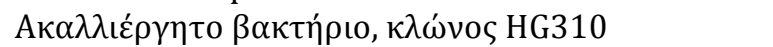 & FN646724 & 93 & 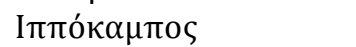 & oD1 \\
\hline Mus1-73 & 3,33 & 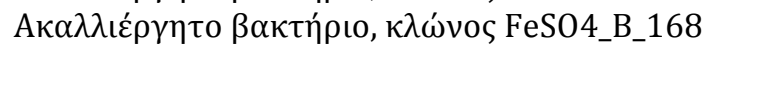 & GQ356983 & 99 & 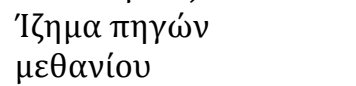 & Fusobacteria \\
\hline Mus1-80 & 3,33 & 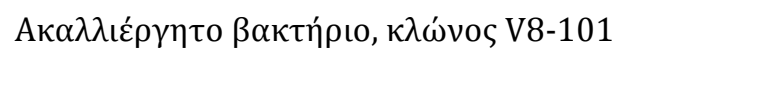 & GQ488000 & 99 & 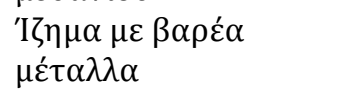 & Bacteroidetes \\
\hline PI-1 & 21,4 & Lactobacillus agilis strain: TB-B13. & AB425919 & 100 & 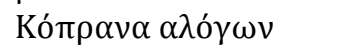 & Firmicutes \\
\hline Pl-13 & 17,8 & 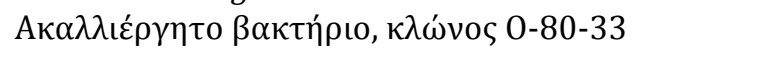 & EU862162 & 99 & 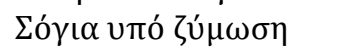 & Firmicutes \\
\hline Pl-3 & 10,7 & Pediococcus acidilactici strain DSPV 358T & FJ751795 & 99 & 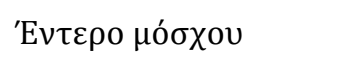 & Firmicutes \\
\hline Pl-9 & 10,7 & Corynebacterium urealyticum DSM 7109 & AM942444 & 99 & $\Sigma \tau \varepsilon \dot{\lambda} \varepsilon \chi \propto \varsigma$ & Actinobacteria \\
\hline PI-20 & 7,1 & 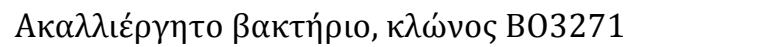 & FN667450 & 99 & 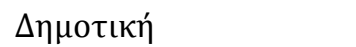 & Firmicutes \\
\hline
\end{tabular}




\begin{tabular}{|c|c|c|c|c|c|c|}
\hline Pl-31 & 7,1 & $\begin{array}{l}\text { Lactobacillus delbrueckii strain: YIT } 0080 \text { (= ATCC } \\
\text { 9649) }\end{array}$ & AB008207 & 99 & $\Sigma \tau \varepsilon \lambda \varepsilon \chi \circ \varsigma$ & Firmicutes \\
\hline Pl-2 & 3,6 & Oceanobacillus sp. AvH 7 & HQ316193 & 99 & 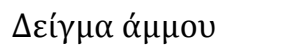 & Firmicutes \\
\hline Pl-14 & 7,1 & 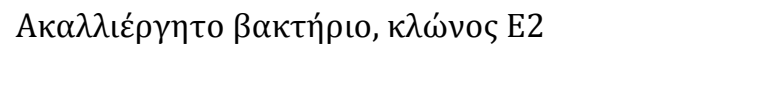 & AM500764 & 99 & 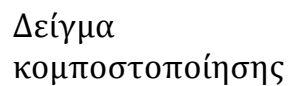 & Firmicutes \\
\hline Pl-18 & 3,6 & 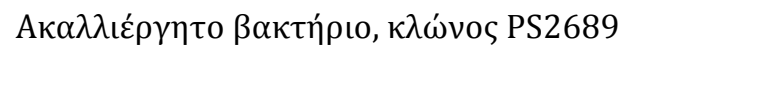 & FN667356 & 99 & 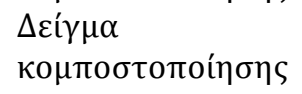 & Firmicutes \\
\hline Pl-21 & 3,6 & 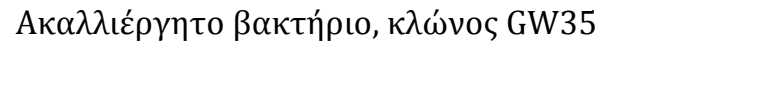 & GQ175353 & 99 & 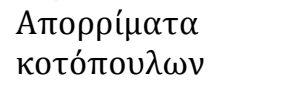 & Firmicutes \\
\hline Pl-22 & 3,5 & Vagococcus lutrae isolate F01595 & DQ395281 & 99 & 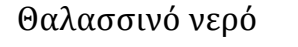 & Firmicutes \\
\hline Pl-23 & 3,5 & Vagococcus lutrae strain CAIM 1759 & HM584114 & 99 & - & Firmicutes \\
\hline
\end{tabular}

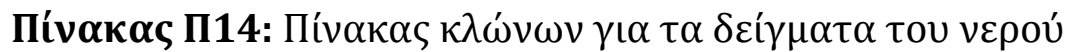

\begin{tabular}{|c|c|c|c|c|c|c|}
\hline 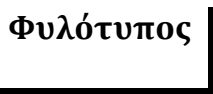 & $\Sigma v \chi v o ́ \tau \eta \tau \alpha(\%)$ & 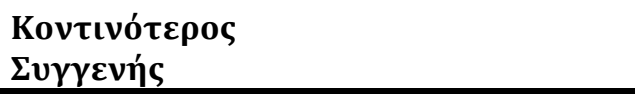 & Genbank \# & 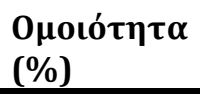 & 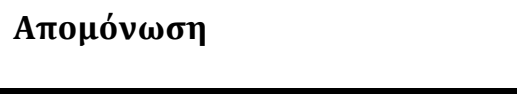 & $\Phi v \lambda{ }^{\prime} \gamma \varepsilon \dot{v \varepsilon \sigma \eta}$ \\
\hline Wt2-1 & 23,52 & Kordia algicida strain 0T-1 & NR_027568 & 98 & $\Theta \alpha \lambda \alpha^{\prime} \sigma \sigma ı \pi \varepsilon \rho \iota \beta \alpha^{\prime} \lambda \lambda$ ov & Bacteroidetes \\
\hline Wt2-3 & 14,70 & Marivita litorea strain CL-JM1 & EU5̄12918 & 98 & 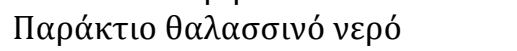 & Alpha \\
\hline Wt2-4 & 8,8 & 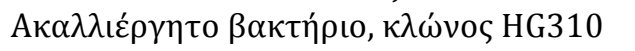 & FN646724 & 90 & 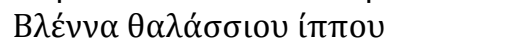 & OD1 \\
\hline Wt2-8 & 5,8 & 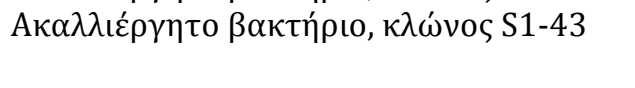 & EF491304 & 99 & 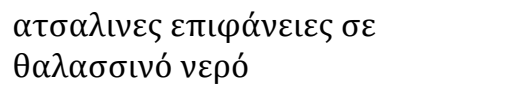 & Gamma \\
\hline Wt2-9 & 8,8 & Polaribacter sp. K20-5 & HM010403 & 96 & 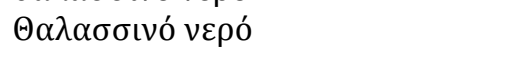 & Bacteroidetes \\
\hline Wt2-11 & 8,8 & $\begin{array}{l}\text { Psychroserpens mesophilus strain KOPRI } \\
13649\end{array}$ & DQ001321 & 98 & 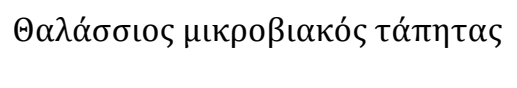 & Bacteroidetes \\
\hline Wt2-12 & 8,8 & 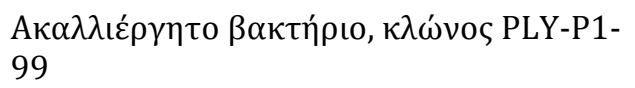 & AY354728 & 96 & 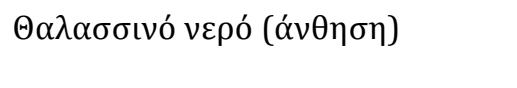 & Bacteroidetes \\
\hline Wt2-5 & 2,9 & 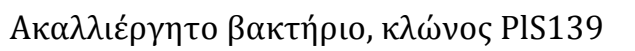 & AY770705 & 89 & 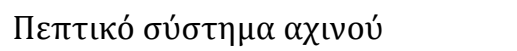 & Alpha \\
\hline Wt2-19 & 2,9 & 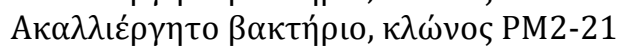 & EF215890 & 98 & 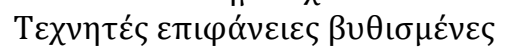 & Alpha \\
\hline
\end{tabular}




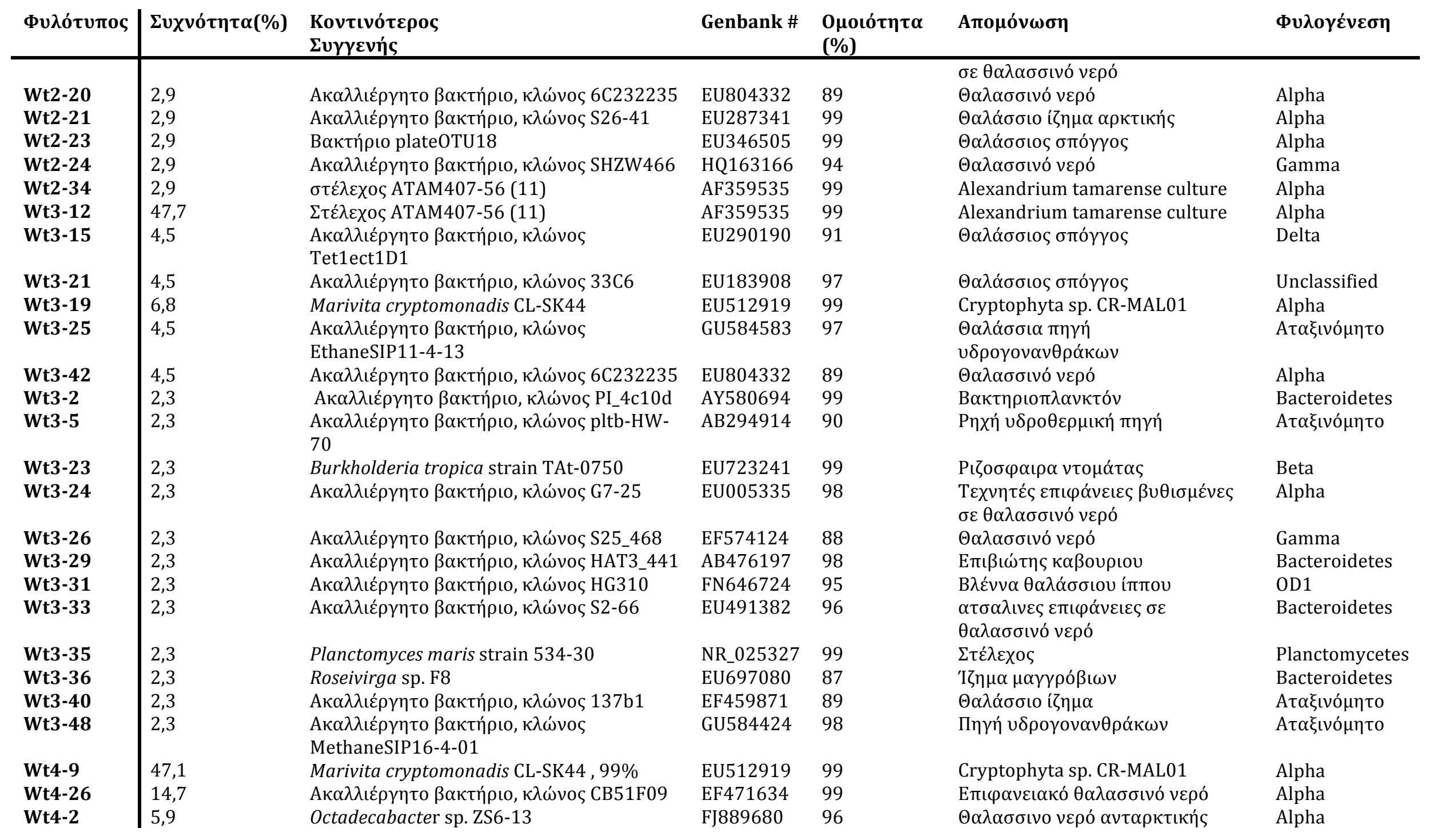




\begin{tabular}{|c|c|c|c|c|c|c|}
\hline 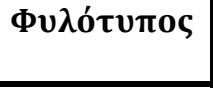 & $\Sigma v \chi v o ́ \tau \eta \tau \alpha(\%)$ & 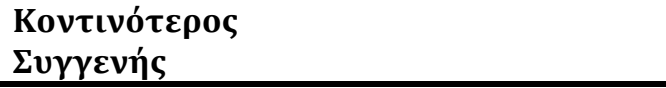 & Genbank \# & 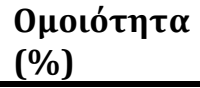 & 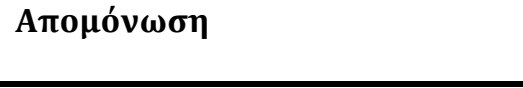 & 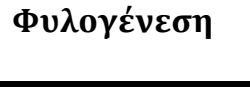 \\
\hline Wt4-4 & 5,9 & 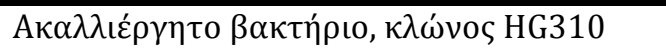 & FN646724 & 93 & 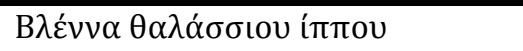 & OD1 \\
\hline Wt4-8 & 2,9 & 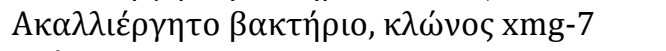 & HM116850 & 99 & 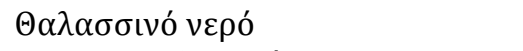 & Alpha \\
\hline Wt4-10 & 2,9 & $\Sigma \tau \varepsilon ́ \lambda \varepsilon \chi 0 \varsigma$ GMD37B4 & AY162078 & 95 & В $\alpha \kappa \tau \eta \rho ı т \lambda \alpha \nu \kappa \tau o ́ \nu$ & Alpha \\
\hline Wt4-11 & 2,9 & 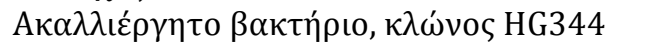 & FN646758 & 97 & 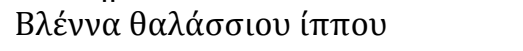 & 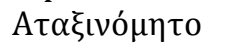 \\
\hline Wt4-14 & 2,9 & Marivita litorea strain CL-JM1 & EU512918 & 97 & Coastal seawater & Alpha \\
\hline Wt4-17 & 2,9 & 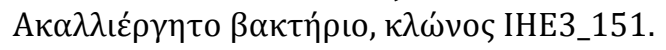 & $\mathrm{AB} 476267$ & 97 & 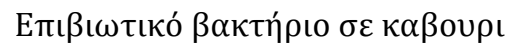 & BD1-5 \\
\hline Wt4-19 & 2,9 & Planctomyces maris strain 534-30 & NR_025327 & 99 & 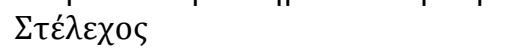 & Planctomycetes \\
\hline Wt4-23 & 2,9 & 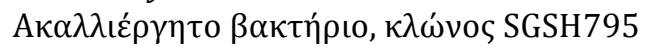 & GQ347607 & 95 & 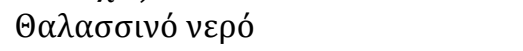 & TM6 \\
\hline Wt4-30 & 2,9 & 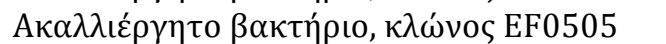 & EF657862 & 91 & 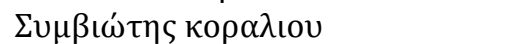 & Bacteroidetes \\
\hline Wt4-37 & 2,9 & 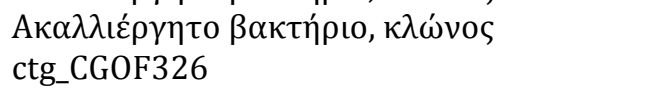 & DQ395877 & 95 & 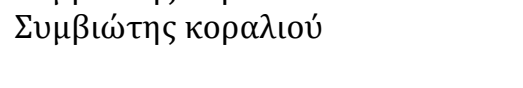 & Alpha \\
\hline
\end{tabular}



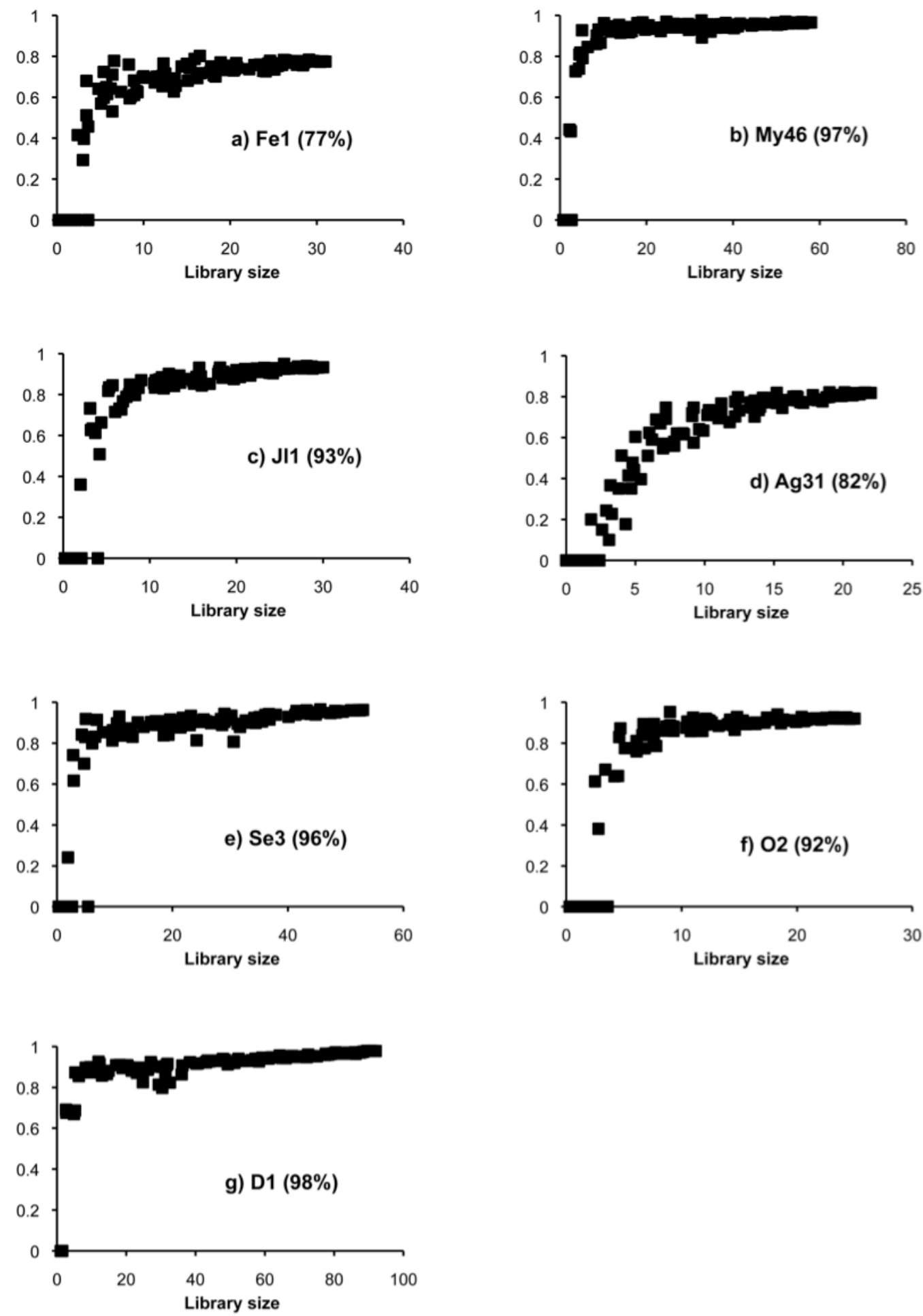

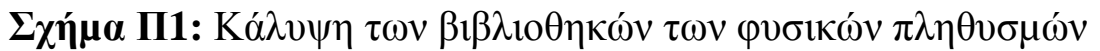



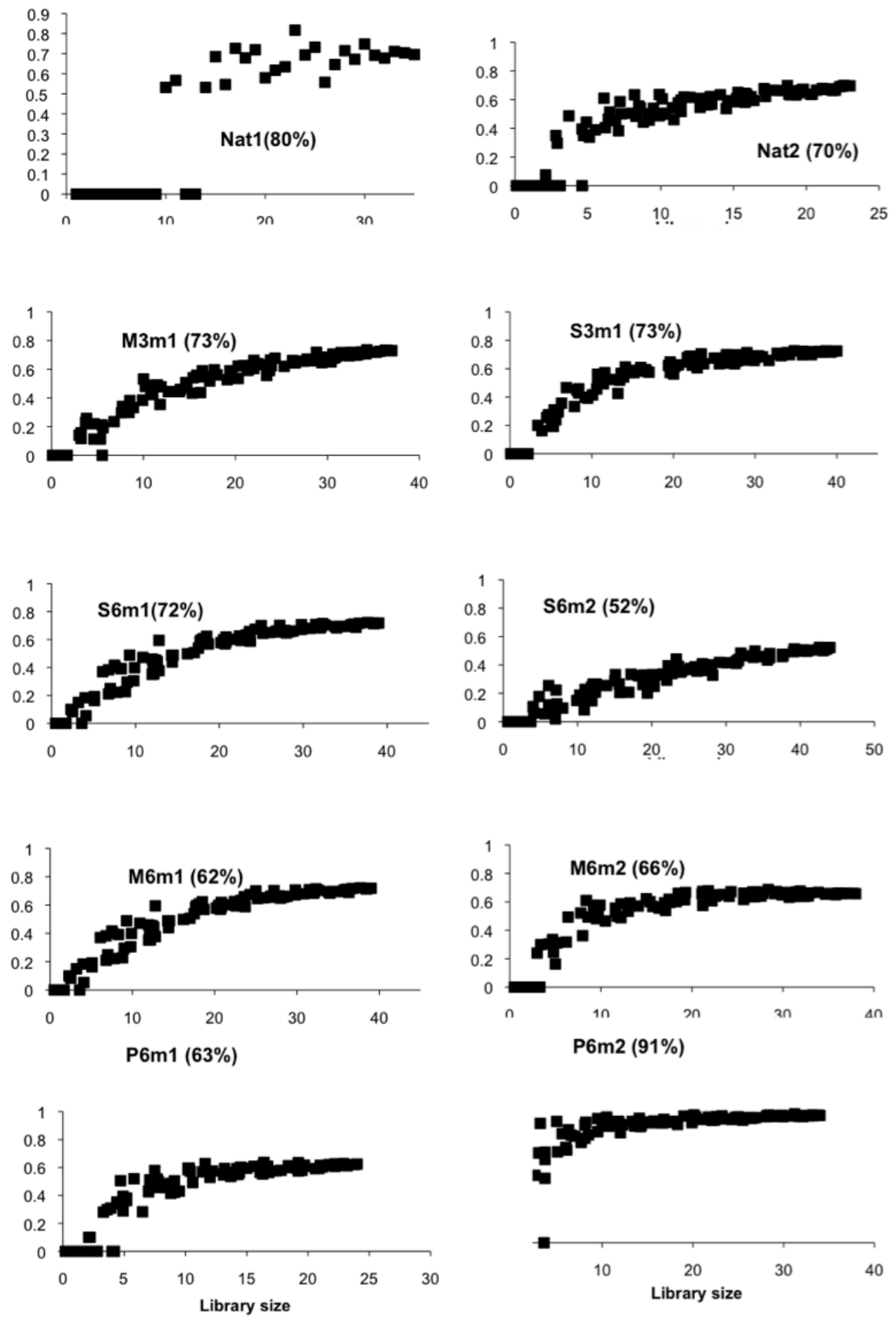

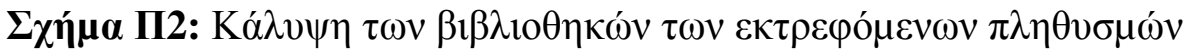



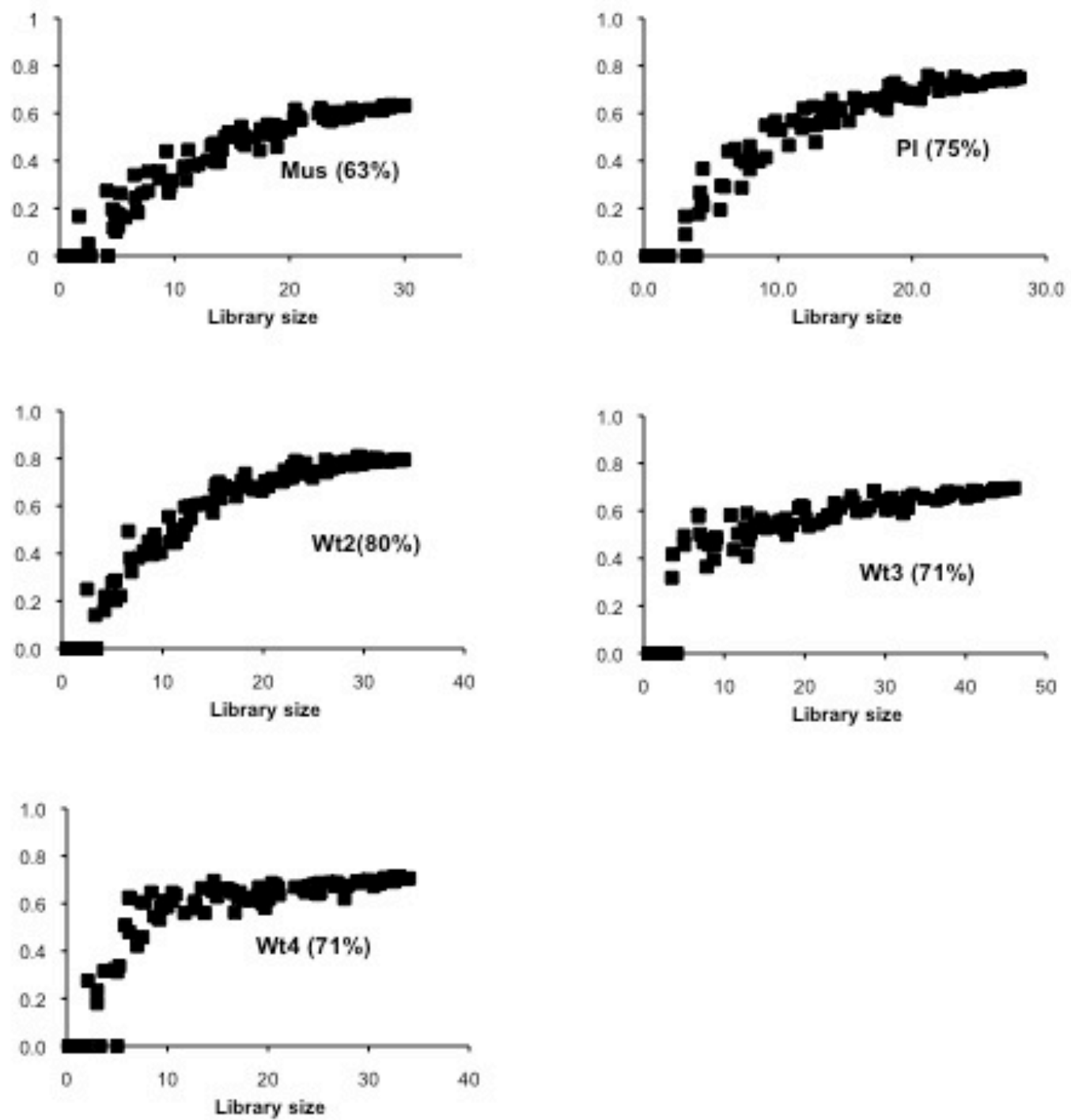

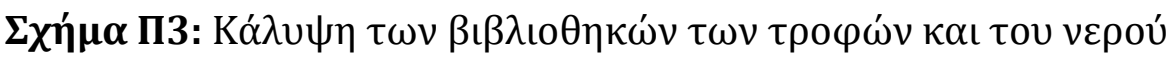




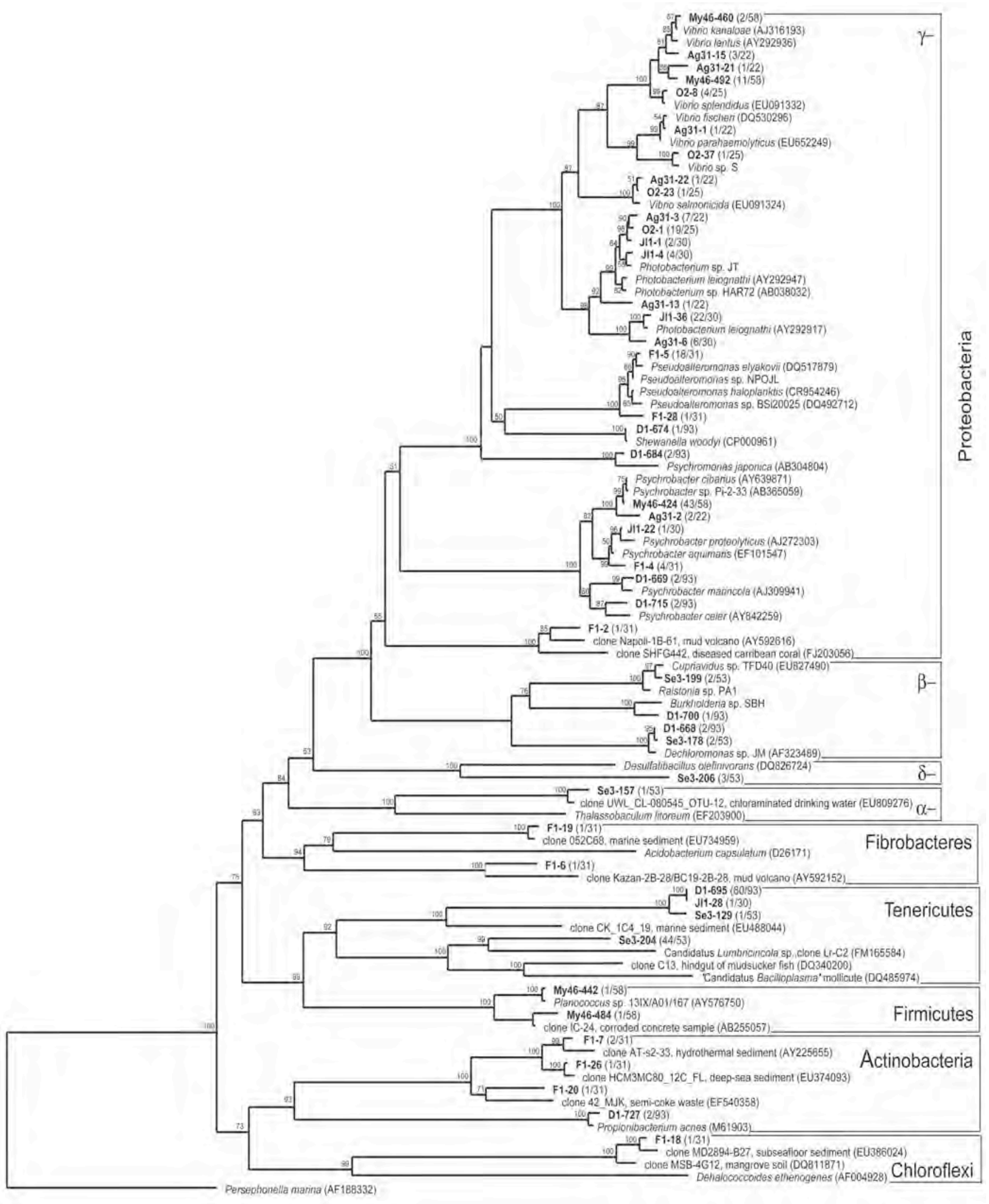

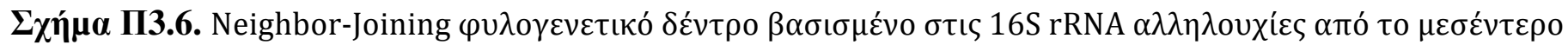

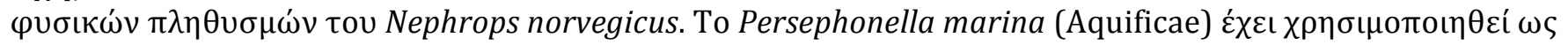

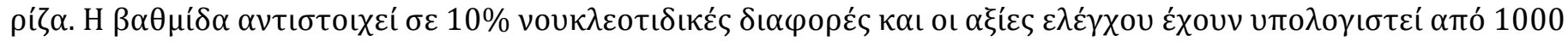

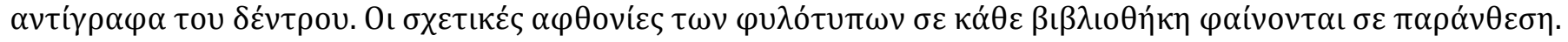

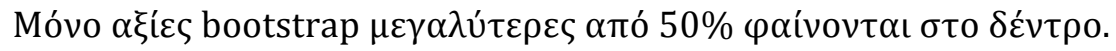


$\Sigma \chi \eta ் \mu \alpha$ П3.14: Neighbor-Joining

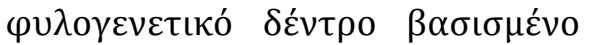

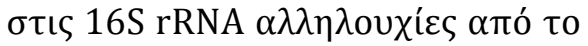
$\mu \varepsilon \sigma \varepsilon ́ v \tau \varepsilon \rho о$ ( $\varphi$ $\gamma \rho \alpha \dot{\mu} \mu \alpha \tau \alpha)$ tou Nephrops norvegicus. To Aquifex pyrophilus

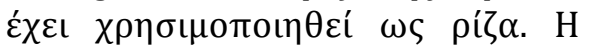

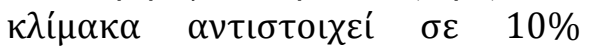

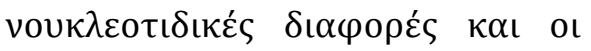

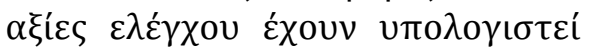

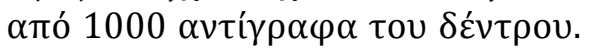

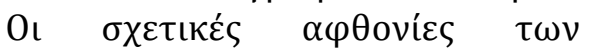

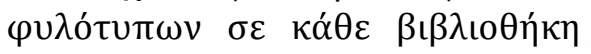

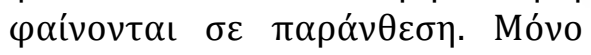
$\alpha \xi \hat{\xi} \varepsilon \varsigma$ bootstrap $\mu \varepsilon \gamma \alpha \lambda u ́ \tau \varepsilon \rho \varepsilon \varsigma \alpha \pi$ ó

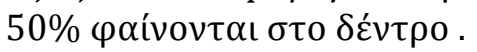

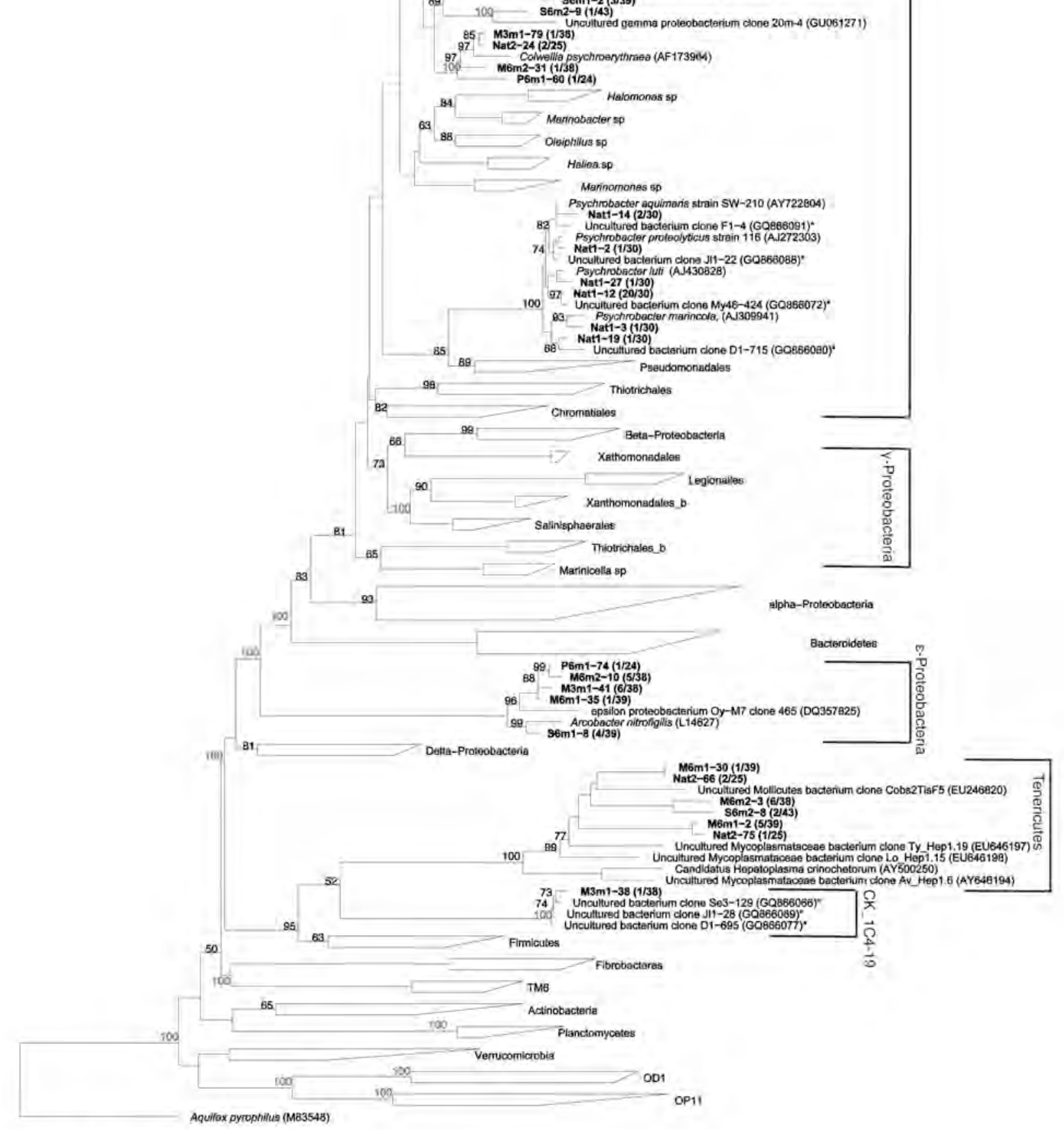




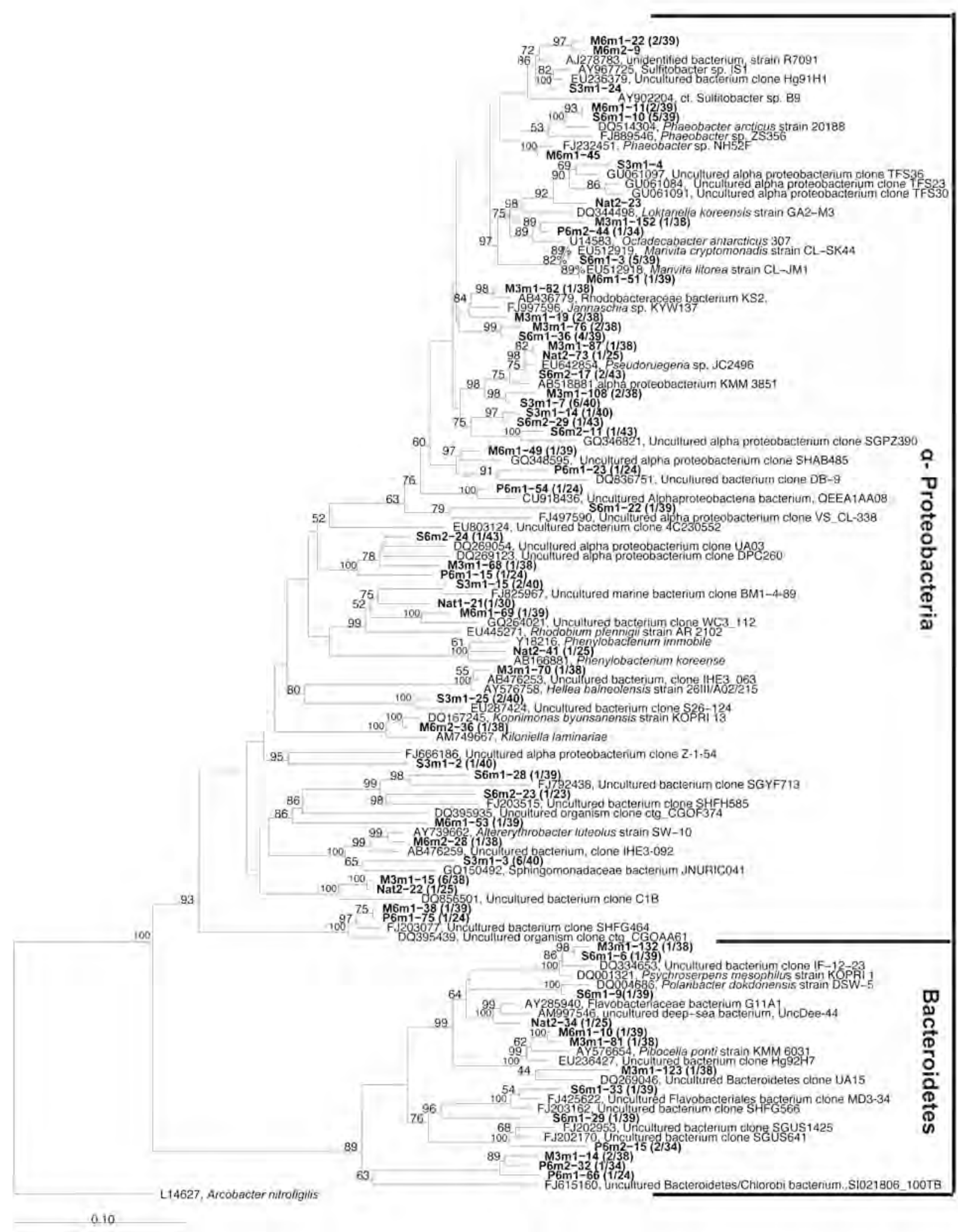

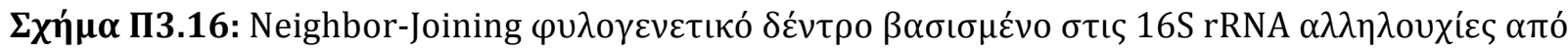

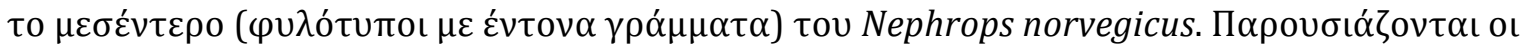

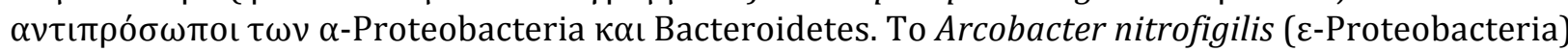

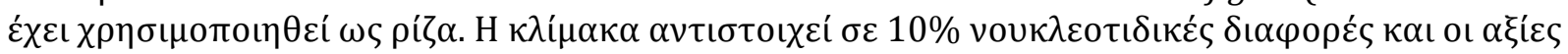

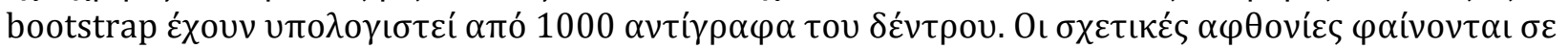

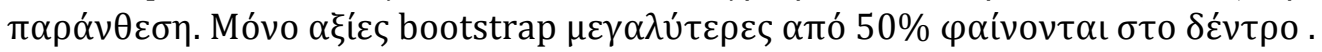




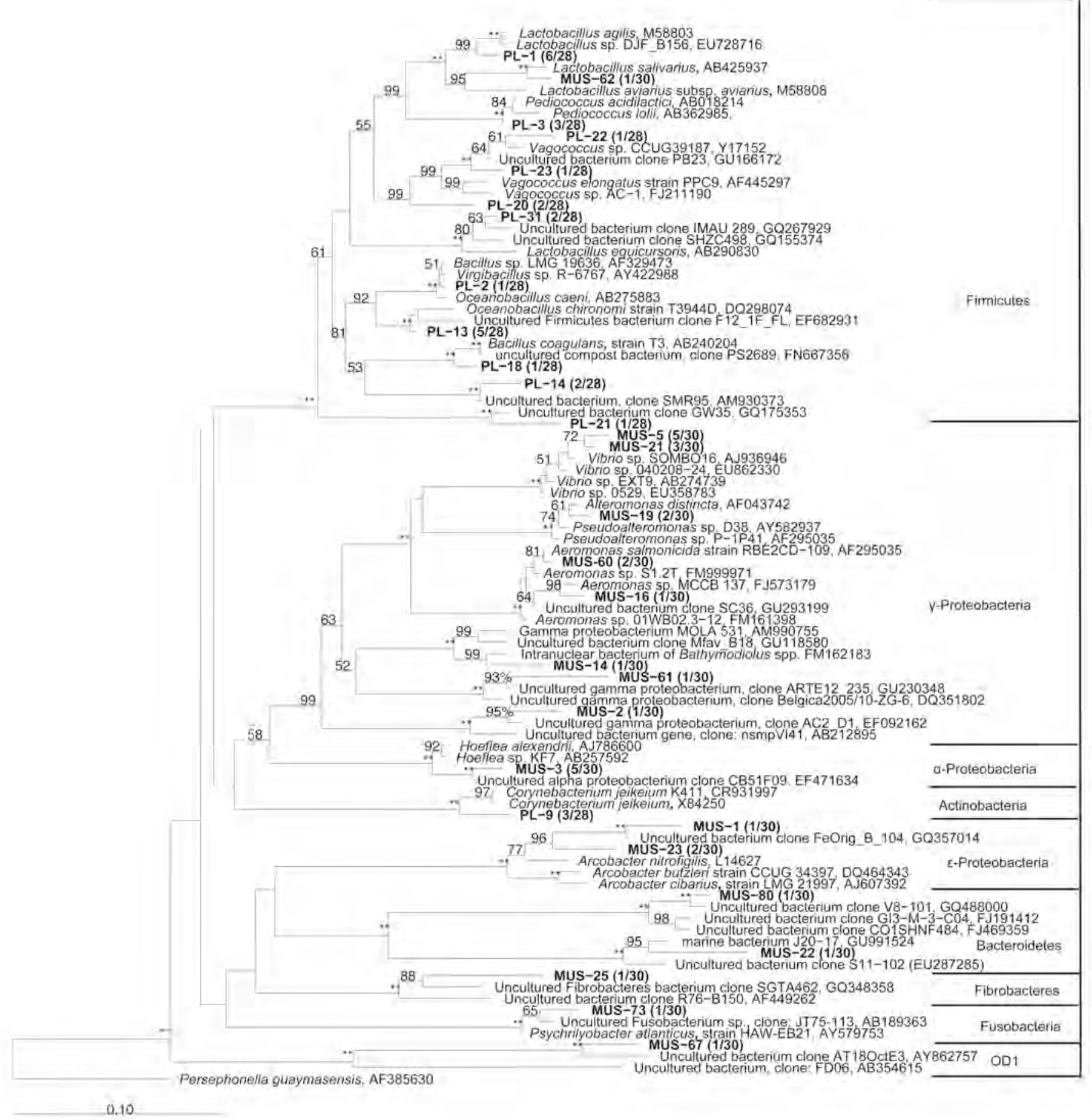

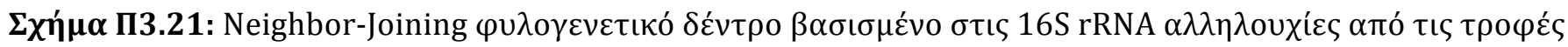

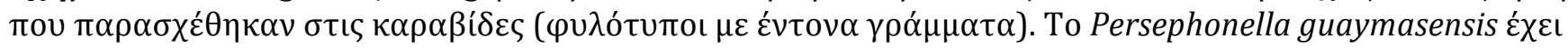

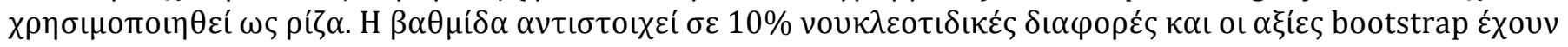

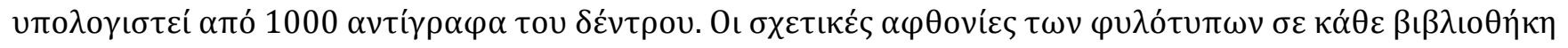

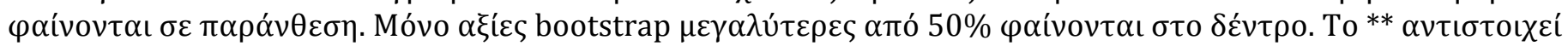
$\sigma \varepsilon$ bootstrap $100 \%$. 


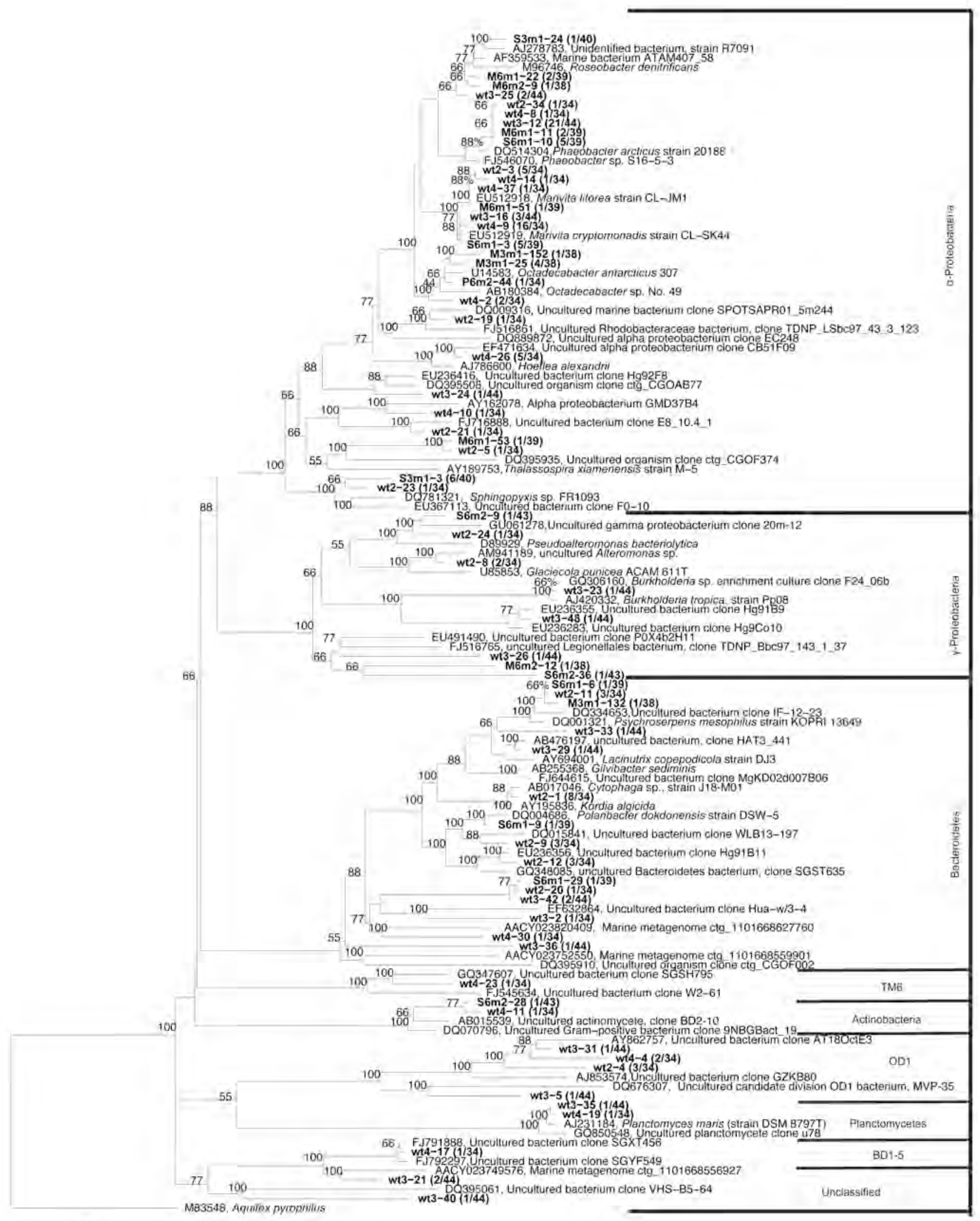

0.10

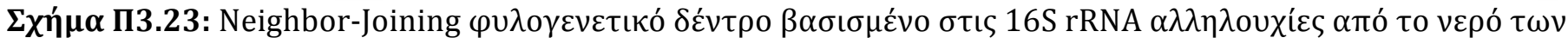

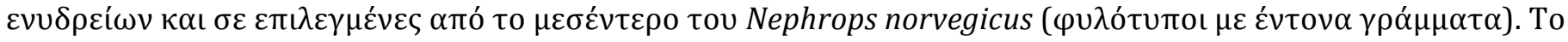

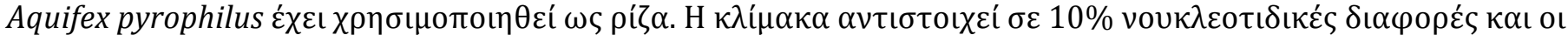

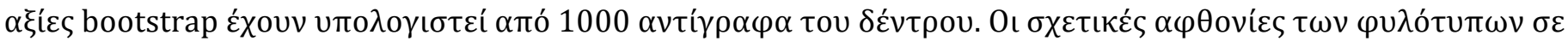

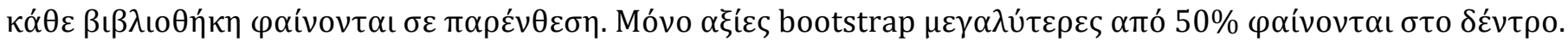




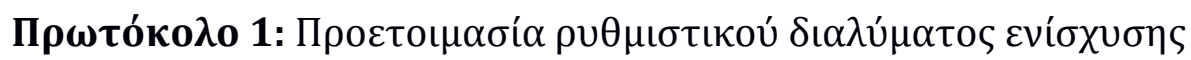

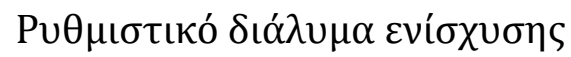

1) $4 \mathrm{ml} 10 \mathrm{XPBS}$

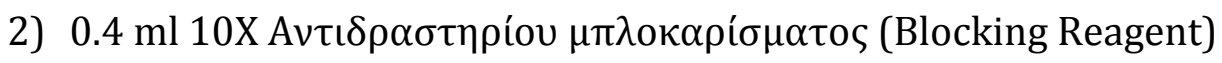

3) $16 \mathrm{ml} 5 \mathrm{M} \mathrm{NaCl}$

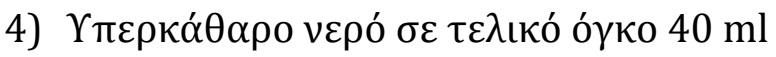

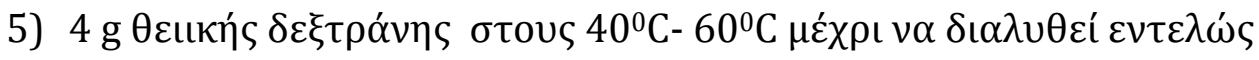

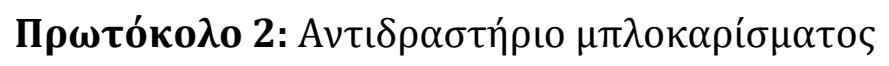

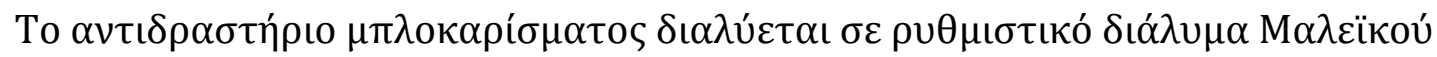
oł́ścs.

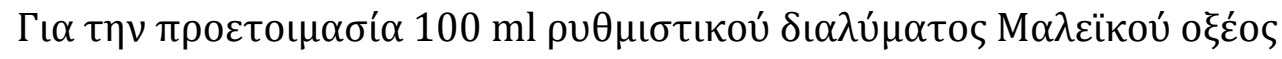

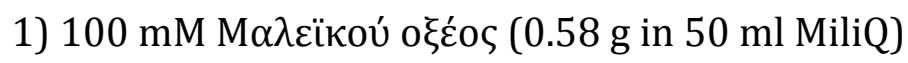

2) $150 \mathrm{mM} \mathrm{NaCl}$ (óүкоৎ $50 \mathrm{ml}$ )

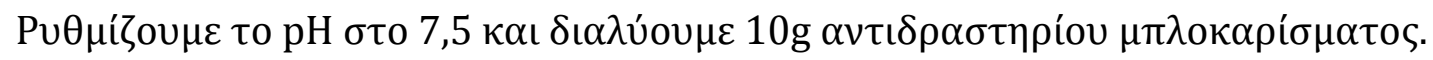

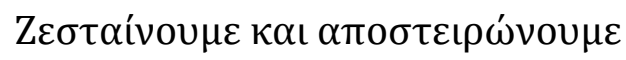




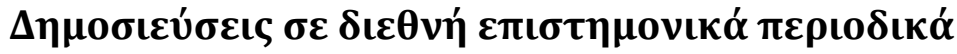

Meziti A., Ramette A., Mente E., Kormas K.A. (2010). Temporal shifts of the Norway lobster (Nephrops norvegicus) gut bacterial communities. FEMS Microbiology Ecology, 74: 472484.

Meziti A., Mente E., Kormas K.A. (2012). Gut bacteria associated with different diets in reared Nephrops norvegicus. In preparation. 


\title{
Temporal shifts of the Norway lobster (Nephrops norvegicus) gut bacterial communities
}

\author{
Alexandra Meziti ${ }^{1,2}$, Alban Ramette ${ }^{2}$, Eleni Mente ${ }^{1,3}$ \& Konstantinos Ar. Kormas ${ }^{1}$ \\ ${ }^{1}$ Department of Ichthyology and Aquatic Environment, University of Thessaly, Magnisia, Greece; ${ }^{2}$ Max Planck Institute for Marine Microbiology, Bremen, \\ Germany; and ${ }^{3}$ School of Biological Sciences, University of Aberdeen, Aberdeen, Scotland, UK
}

\author{
Correspondence: Konstantinos Ar. Kormas, \\ Department of Ichthyology and Aquatic \\ Environment, University of Thessaly, 38446 \\ Nea Ionia, Magnisia, Greece. \\ Tel.: +30242109 3082; fax: +30242109 \\ 3157; e-mail: kkormas@uth.gr
}

Received 7 March 2010; revised 20 July 2010; accepted 30 July 2010

Final version published online 10 September 2010.

DOI:10.1111/j.1574-6941.2010.00964.x

Editor: Julian Marchesi

\section{Keywords}

Bacteria; FISH; ARISA; 16 S rRNA gene; gut; Nephrops norvegicus.

\begin{abstract}
The aim of this study was to investigate the gut bacterial communities of Nephrops norvegicus individuals, using a suite of molecular tools consisting of automated ribosomal intergenic spacer analysis, $16 \mathrm{~S}$ rRNA gene-internal transcribed spacer clone libraries and FISH. The animals were collected from Pagasitikos Gulf, Greece, during different months of the year. The diversity of the gut bacterial communities was found to mostly vary with sampling time, which could be related to temporal variations in food supply. The $16 \mathrm{~S}$ rRNA gene diversity analysis showed dominance of specific phylotypes for each month studied. February, May, July, August and October samples were rich in sequences related to the gammaproteobacterial genera Pseudoalteromonas, Psychrobacter and Photobacterium. September and December samples were dominated by phylotypes affiliated with uncultured representatives of Mollicutes, which are generally associated with the intestinal tracts of various animals. The presence of Gammaproteobacteria and uncultured Mollicutes in August and September samples, respectively, was further confirmed by FISH. None of the morphometric parameters considered was related to the temporal pattern of dominant bacterial communities.
\end{abstract}

\section{Introduction}

The Norway lobster Nephrops norvegicus (also called Dublin bay prawn) is a burrowing decapod crustacean living at $20-800 \mathrm{~m}$ depth. It is widely distributed on muddy substrata throughout the northeast Atlantic Ocean from Iceland in the north to Morocco in the south, at the British coasts and in the Mediterranean Sea and has a high commercial importance in these areas (Bell, 2009). However, concerns about possible depletion and recruitment failure for Nephrops fisheries have been expressed due to overexploitation and insufficient or inappropriate management strategies. Nephrops norvegicus feeds mainly on fish, molluscs, crustaceans, polychaetes, echinoderms and foraminifera (Cristo \& Cartes, 1998). Differences in diet are attributed mainly to differences in prey abundance rather than to differences in prey preference. Nephrops norvegicus is considered to be an opportunistic predator and a scavenger. It has been suggested that it can act as a suspension feeder. This occurs when food resources are limited (Loo et al., 1993). In captivity, cannibalism has also been observed when food supply is insufficient
(Sarda \& Valladares, 1990) and when other individuals are vulnerable, for example due to molting (A. Meziti, I. Karapanagiotidis, K. Kormas \& E. Mente, pers. commun.).

The digestive system of $N$. norvegicus consists of three parts: (1) the foregut, consisting of the esophagus, the cardiac and the pyloric stomach, (2) the midgut, which includes the hepatopancreas and the intestine till the sixth abdominal segment and (3) the hindgut, which is the last very short chitin-lined part after the midgut leading to the anus. The main part of the food assimilation occurs in the upper part of the midgut either by enzymes excreted from the hepatopancreas or by mechanical disruption from the gastric mill. The midgut is the main absorbing organ, and no absorption occurs in the short hindgut (Yonge, 1924).

Gut microorganisms are considered very important for the nutrition of many animal species including crustaceans (Harris, 1993). The microorganisms grow in a stable environment inside the animal gut, while the host usually benefits either from microbially mediated digestion of ingested food or from important nutrients. However, the microbial 
colonization of $N$. norvegicus has not been studied in detail as yet.

Most of the available studies have focused on the hindgut microbiota of other Decapoda, mainly Thalassinidae, and have found thriving microbial communities because no assimilation of nutrients occurs there and consequently no competition with the hosts (Lau et al., 2002 and references therein). However, over the last years, there has been an increasing interest on the shifts of gut microbial communities driven by the nutritional habits of the host (Ley et al., 2008), making the parts of the intestinal tract where nutrient absorption takes place, such as the Decapoda midgut, appropriate systems for the investigation of microorganism-host interactions.

Pagasitikos Gulf, Greece, is a major N. norvegicus fishing ground. According to models constructed by Petihakis et al. (2005), the sediment surface of the central-external area $<50 \mathrm{~m}$ depth is characterized by rather constantly low temperatures $\left(13^{\circ} \mathrm{C}\right.$ average) and dim light conditions. Other factors influencing the benthic communities, such as settling phytoplankton biomass, detritus and bacteria, show annual patterns (Petihakis et al., 2005) and are regulated by the phytoplankton bloom starting between January and February each year. Phytoplankton biomass reaches its maximum near the sediment soon after the bloom initiation. Maximum detritus concentrations reach the sediment surface during February and March, causing an almost immediate bacterial response (i.e. increased abundance). After April, a decrease is observed for phytoplankton, detritus and bacterial biomass (Petihakis et al., 2005). These patterns have positive effects on the benthic food web by increasing the activity of suspension and deposit feeders soon after the peaks of phytoplankton, bacteria and detritus around April or May.

The aim of this study was to investigate whether temporal changes, which reflect different food supply, as discussed above, influence the gut microbial communities along with other morphological factors. For this purpose, midgut samples from $N$. norvegicus individuals were collected during different months from the same habitat and were investigated by $16 \mathrm{~S}$ rRNA gene diversity and internal transcribed spacer (ITS) analysis. To the knowledge of the authors, there is no similar study for the molecular analysis of the gut microbial communities in N. norvegicus. Midgut was selected for analysis as the gut part where additional nutrient absorption from the host could occur and a relationship of mutualism with the bacteria could evolve. The examination of the gut bacterial communities provides useful insights into the feeding and nutritional behavior of the animal, especially in commercial rearing efforts that are suggested as alternative to the overexploitation and insufficient or inappropriate management strategies of $N$. norvegicus.

\section{Materials and methods}

\section{Nephrops norvegicus collection and morphometric analysis}

Samples were collected from Pagasitikos Gulf (Greece) from an area determined by four points: North $\left(39^{\circ} 17.34^{\prime} \mathrm{N}\right.$, $\left.23^{\circ} 02.27^{\prime} \mathrm{E}\right)$, West $\left(39^{\circ} 15.55^{\prime} \mathrm{N}, 22^{\circ} 56.45^{\prime} \mathrm{E}\right)$, South $\left(39^{\circ}\right.$ $\left.10.33^{\prime} \mathrm{N}, 23^{\circ} 00.59^{\prime} \mathrm{E}\right)$ and East $\left(39^{\circ} 09.14^{\prime} \mathrm{N}, 23^{\circ} 08.52^{\prime} \mathrm{E}\right)$. Samples were collected monthly in 2007, apart from January, April, June and November, when sampling was not possible due to bad weather conditions or fishing restrictions. Individual, large traps $(60 \mathrm{~cm} \times 45 \mathrm{~cm} \times 30 \mathrm{~cm})$ were used to avoid stress on the animals during capture. The traps were left at the bottom of the sea for a maximum of $7 \mathrm{~h}$ before retrieving them. Only live animals upon retrieval of the traps were kept for the current work until return to the laboratory $(\leq 2 \mathrm{~h})$. The sampling depth varied from 60 to $88 \mathrm{~m}$. After collection, the $N$. norvegicus individuals were immediately transferred on ice to the laboratory, and animal sex, weight, carapace length and width and abdominal width were measured (Supporting Information, Table S1). In total, 53 gut samples were collected for further analysis. Encoding of the samples according to collection month is shown in Table S2.

\section{Midgut isolation}

The animals were dissected using sterile lancets and the midgut was extracted using sterile forceps. In order to exclude as many as possible transient bacterial cells ingested with food particles, the extracted midgut was emptied by applying mechanical force and by rinsing in autoclaved particle-free sea water (filtered by $0.2-\mu \mathrm{m}$ pore size). All dissecting tools were alcohol flame sterilized between each individual sample.

\section{DNA extraction and tissue fixation}

DNA extraction was performed on 49 gut tissues from all sampling months using the QIAamp DNA Mini Kit (Qiagen Inc.) following the manufacturer's standard protocol. Some of the gut tissues deriving from the same collection month and from animals of the same sex were pooled for the DNA extraction due to low tissue mass (Table S1). At the final step, DNA was diluted in $100 \mu \mathrm{L}$ of elution buffer, provided with the kit, and was stored at $-20^{\circ} \mathrm{C}$. Because of low numbers of animals available, only samples from August and September were fixed in $4 \%$ formaldehyde in sterile $1 \times$ phosphate-buffered saline (PBS) $(137 \mathrm{mM} \mathrm{NaCl}$, $2.7 \mathrm{mM} \mathrm{KCl}, 10 \mathrm{mM} \mathrm{Na} \mathrm{HPO}_{4}, 2 \mathrm{mM} \mathrm{KH}_{2} \mathrm{PO}_{4}$ ) at $4{ }^{\circ} \mathrm{C}$ for $3 \mathrm{~h}$. Samples were rinsed three times in $1 \times \mathrm{PBS}$ for $10 \mathrm{~min}$ and were washed in sterile double-distilled water. Samples were stored in $1.5 \mathrm{~mL} 1 \times$ PBS : ethanol (1:1). In total, 39 
DNA samples were further analyzed as a result of the pooled tissues and four gut tissues were used for FISH.

\section{Automated ribosomal intergenic spacer analysis (ARISA), nonmetric multidimensional scaling (NMDS) analysis and environmental interpretation}

The gut bacterial community structure was estimated by ARISA (Fisher \& Triplett, 1999). PCR amplification was conducted in triplicates using primers ITSF ( $5^{\prime}$-GTCGTAA CAAGGTAGCCGTA- $\left.3^{\prime}\right)$ and ITSReub (5'-GCCAAGG CATCCACC-3') (Cardinale et al., 2004). Primer ITSF was labeled with FAM fluorochrome at the $5^{\prime}$ end. The PCR conditions consisted of an initial denaturation step at $94{ }^{\circ} \mathrm{C}$ for $3 \mathrm{~min}$, followed by 30 cycles of $94^{\circ} \mathrm{C}$ for $45 \mathrm{~s}, 55^{\circ} \mathrm{C}$ for $45 \mathrm{~s}, 72^{\circ} \mathrm{C}$ for $90 \mathrm{~s}$ and a final extension at $72{ }^{\circ} \mathrm{C}$ for $5 \mathrm{~min}$. PCR-amplified fragments were purified with Sephadex G-50 Superfine (Sigma-Aldrich, Germany) and the preparation for capillary electrophoresis separation was performed as described previously (Boer et al., 2009). ARISA profiles were analyzed using the GENEMAPPER software v 3.7 (Applied Biosystems Inc., Carlsbad, CA). The total peak area per sample was normalized to one, and only fragments above a threshold of 50 fluorescence units and between 100 and $1000 \mathrm{bp}$ length were considered. GENEMAPPER output files were further analyzed by custom R scripts (Ramette, 2009). To account for size calling imprecision, samples were binned with automatic and interactive binner as described previously (Ramette, 2009). A 'window' of 2.5 bp was selected as the frame offering the highest pairwise similarities among samples and was further subjected to multivariate analyses.

Triplicate profiles were merged to only keep bands that appeared at least twice among the replicates of a given sample (Boer et al., 2009; Ramette, 2009). Unconstrained ordinations were performed to graphically illustrate the relationships between different samples using three-dimensional NMDS (Kruskal, 1964), implemented in $\mathrm{R}$ (version 2.7.0). NMDS ordination attempts to place all samples in a three-dimensional space such that their ordering relationships (here based on a Bray-Curtis similarity matrix) are preserved. Hence, the closer the samples are in the resulting ordination, the more similar their overall gut bacterial communities are. Kruskal's stress value reflects the difficulty involved in fitting the samples' relationships into a threedimensional ordination space. To determine whether season and morphometric data could significantly explain the variation in bacterial community structure, a redundancy analysis (RDA) [reviewed in Ramette (2007)] was implemented in $\mathrm{R}$ and its significance was assessed by 999 permutation tests. The a priori hypothesis that gut microbial communities differ between different seasons was tested using RDA and the nonparametric ANOSIM (Clarke \& Green,
1988). ANOSIM generates a test statistic, $R$, that ranges from -1 to 1 . The magnitude of $R$ is indicative of the degree of separation between groups, with a score of 1 indicating complete separation and 0 indicating no separation (Clarke, 1993). $R$ is unlikely to be substantially smaller than 0 because this would indicate that similarities within groups are systematically lower than those among groups. The critical significance level used for ANOSIM results was $P=0.05$. Since multiple pairwise comparisons (21) were performed, the Bonferroni correction was applied, decreasing the critical significance level to $0.0024(0.05 / 21)$. It has to be mentioned that the Bonferroni correction has often been judged to be overly conservative (Legendre \& Legendre, 1998), and so care should be taken when applied to ecological data. Only results from the ANOSIM method are presented because both methods gave the same conclusions.

\section{Cloning and sequencing of 16S rRNA genes and ITS}

Bacterial 16S rRNA gene and the ITS were amplified from samples F1, My46, J11, Ag31, Se3, O2 and D1 using the 27f BAC (5'-AGAGTTTGATCMTGGTCAG-3') (Lane, 1991) and ITSReub primers. Expected amplicon sizes varied from 1800 to $2400 \mathrm{bp}$ due to variation in ITS size. PCR conditions were $2 \mathrm{~min}$ at $94^{\circ} \mathrm{C}$, followed by 10 cycles with $30 \mathrm{~s}$ at $94^{\circ} \mathrm{C}$, $1 \mathrm{~min}$ at $55^{\circ} \mathrm{C}$ and $3 \mathrm{~min}$ at $72^{\circ} \mathrm{C}$, followed by $13-20$ cycles of $30 \mathrm{~s}$ at $94{ }^{\circ} \mathrm{C}, 30 \mathrm{~s}$ at $55^{\circ} \mathrm{C}$ and $3 \mathrm{~min}(+5 \mathrm{~s}$ in each cycle) at $72{ }^{\circ} \mathrm{C}$ and finally $7 \mathrm{~min}$ at $72^{\circ} \mathrm{C}$. PCR cycles were adjusted when needed to decrease nonspecific products. In samples, J11, Ag31 and O2, where PCR products were not enough to allow for further analysis, a nested PCR was performed with primers GM3 (5'-AGAGTTTGATCMTGGC-3') (Muyzer et al., 1995) and GM4 (5'-TACCTTGTTACGACTT-3') (Kane et al., 1993). The expected amplicon size was 1550-1600 bp. PCR conditions involved an initial denaturation at $94^{\circ} \mathrm{C}$ for $3 \mathrm{~min}$, followed by $10-13$ cycles of $94^{\circ} \mathrm{C}$ for $1 \mathrm{~min}, 44^{\circ} \mathrm{C}$ for $1 \mathrm{~min}, 72^{\circ} \mathrm{C}$ for $3 \mathrm{~min}$ and a final extension at $72{ }^{\circ} \mathrm{C}$ for $5 \mathrm{~min}$. The total cycles for all samples for both PCRs varied from 25 cycles in Se3 to 37 cycles in Ag31 and O2. PCR products were purified using QIAquick PCR Purification Kit (Qiagen Inc., Germany) and were cloned directly using TOPO TA Kit for sequencing (Invitrogen Inc., Germany) with chemically competent cells. The insert size was checked via PCR with M13f-M13R vector-binding primers. Positive clones were grown overnight in $1.5 \mathrm{~mL}$ of Luria-Bertani medium containing ampicillin $\left(100 \mu \mathrm{g} \mathrm{mL}^{-1}\right)$, and plasmids were prepared from the pelleted cells with a QIAprep Miniprep Kit (Qiagen Inc.). Plasmids were partially sequenced with primers GM4 and ITSReub (the latter used only for the 16S-ITS libraries). After alignment with SEQUenCHer 4.6 (Gene Codes Corporation) and CLustalw (Larkin et al., 2007), manual correction, elimination of 
chimeras using PINTAIL (Ashelford et al., 2005) and visual examination of the alignments, clones were grouped based on a $16 \mathrm{~S}$ rRNA gene similarity cutoff of $99 \%$ and representatives from each group were fully sequenced using primers GM5_341f, GM1R and 907R (Muyzer et al., 1995). Sequencing reactions were performed using ABI BigDye and an ABI PRISM 3100 Genetic Analyzer (Applied Biosystems Inc.). Sequences were checked for closest relatives using the BLAST application and all sequences were checked for chimeras using PINTAIL. 16S rRNA gene sequences were aligned using the ARB software (Ludwig et al., 2004) and the SILVA aligner application (Pruesse et al., 2007). 16S rRNA gene distance matrices were calculated with the Jukes-Cantor formula and were clustered using the neighbor-joining method. The phylogenetic tree was created in ARB software (Ludwig et al., 2004). Bootstrap values were obtained from 1000 replicates using similar parameters. The length of the ITS sequences was used for the identification of ARISA peaks and for the study of the microdiversity of dominant $16 \mathrm{~S}$ rRNA genederived phylotypes (data not presented here). All 16S rRNA gene sequences from this study have been deposited under GenBank numbers GQ866066-GQ866111.

\section{FISH}

Fixed tissues were washed in optimal cutting temperature (OCT) cryomicrotome medium (HISTO Service, Germany) for $5 \mathrm{~h}$ at $4{ }^{\circ} \mathrm{C}$ and were embedded in specific plastic wells filled with OCT overnight at $-20^{\circ} \mathrm{C}$. The frozen embedded samples were sliced horizontally to the gut walls with a cryomicrotome (Microm GmbH, Germany) into 4-5- $\mu \mathrm{m}$ thick slices. Slices were collected on Menzel-Gläser Polysine Microscope Slides (Menzel-Gläser, Germany). Catalyzed reporter deposition-FISH was performed only in sample FN2 following a protocol described previously (Fuchs et al., 2005) with the following modifications. For the deactivation of endogenous peroxidases, slides were incubated for $30 \mathrm{~min}$ with $0.5 \%$ sodium dodecyl sulfate in methanol, followed by two washing steps of $10 \mathrm{~min}$ in $1 \times \mathrm{PBS}$ and of $3 \mathrm{~min}$ in sterile double-distilled water. Hybridization buffer was prepared for the respective formamide concentrations as described previously (Fuchs et al., 2005). Sections were prehybridized for $15 \mathrm{~min}$ at $46^{\circ} \mathrm{C}$ by adding hybridization buffer only. Hybridization was followed by the addition of horseradish peroxidase-labeled probe $\left(50 \mathrm{ng} \mu \mathrm{L}^{-1}\right)$ in a $1: 50$ dilution with the respective hybridization buffer. Samples FN3, FSe1 and FSe3 were analyzed following the basic FISH protocol (Duperron et al., 2006) with the appropriate concentrations of formamide (Table S3). Basic FISH was preferentially used in most of our samples because it yielded better results in terms of clearer signals, less background and the better calculation of cell dimensions. General Cy3labeled probes were used for the in situ identification of
Bacteria, Alpha-, Beta- and Gammaproteobacteria; NON338 was used for the detection of false-positive signals (Table S3). Cy3-labeled GAM42a probe was combined with nonlabeled BET42a, and vice versa, as described before (Manz et al., 1992). The current specificity of these probes has recently been reviewed (Amann \& Fuchs, 2008). A specific probe targeting the $16 \mathrm{~S}$ rRNA gene sequence of the dominant in September samples phylotype (Se3-204) clustering with Mollicutes was designed using the ARB 'probe design' function (Ludwig et al., 2004). This probe (UncMol89 $5^{\prime}$-CGTTCGCCACTAACACCAAAATC- $3^{\prime}$ ) was designed in order to be specific for this phylotype and to have two or more mismatches with the sequences found in the SILVA database (Pruesse et al., 2007). The probe design considered the accessibility of $16 \mathrm{~S}$ rRNA gene probe target sites reported elsewhere (Behrens et al., 2003) and the position of the specific for the Candidatus Bacilloplasma probe (Kostanjsek et al., 2007). Optimal stringency conditions were determined for the new probe by checking the signal intensities at increasing formamide concentrations $(0-40 \%)$. The highest formamide concentration where signals were detected was $40 \%$. However, these signals were weak, and finally $35 \%$ was chosen for the rest of the analysis. Four gut samples were analyzed, two from August 2007 (FN2, FN3) and two from September 2007 (FSe1, FSe3). Samples were visualized using an Axioplan II Imaging epifluorescence microscope (Carl Zeiss, Jena, Germany), and pictures were realized using software AXIOvisIon Rel. 4.7.2 (Carl Zeiss).

\section{Results}

\section{Analysis of shifts in community structure}

The ARISA profiles generated from the bacterial communities associated with the midgut tissue of $N$. norvegicus showed high variability between samples. None of the ARISA fragments were present in all samples and the number of operational taxonomic units (OTUs) varied between 43 (sample My44) and 147 (D2) (average 91). When the complexity of the community profile was reduced by NMDS (Fig. 1), sampling time emerged as an important factor (ANOSIM $R=0.5531$, $P<0.001)$. Morphometric factors did not significantly explain changes in microbial community structures $(P>0.05)$. Further pairwise comparisons showed that gut bacterial communities varied significantly $(P<0.05)$ between almost all months (Table 1 ), with either strong community differentiation $(R>0.75)$ or with differentiated, yet overlapping structure $(0.50<R<0.75)$. The $R$ values for the ANOsIm tests between August, September, October and December indicated largely overlapping structure $(R<0.5)$, with only the exception of the December-September comparison. No group separation was observed between the sample pairs February/March-July $(P=0.198)$ and August-October 

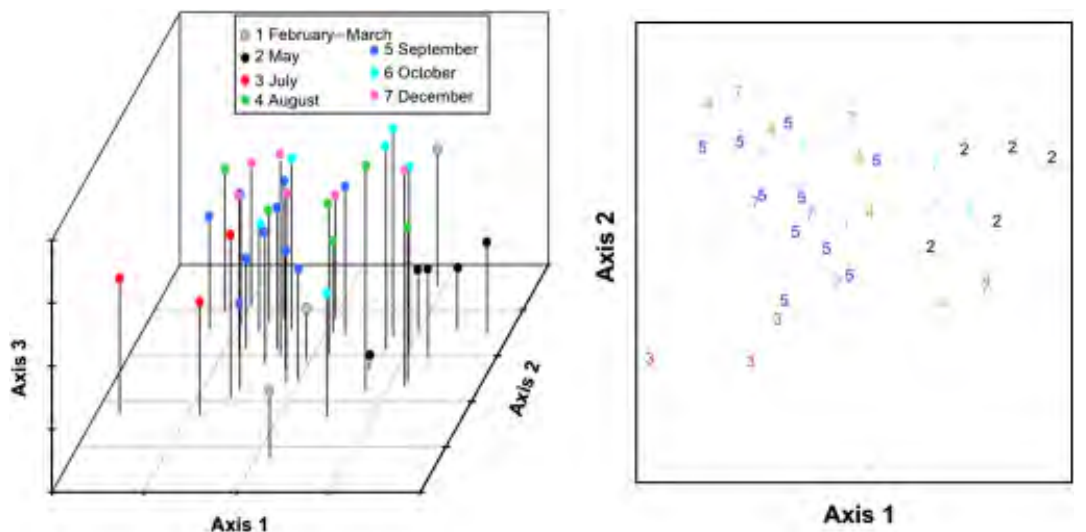

Axis 1

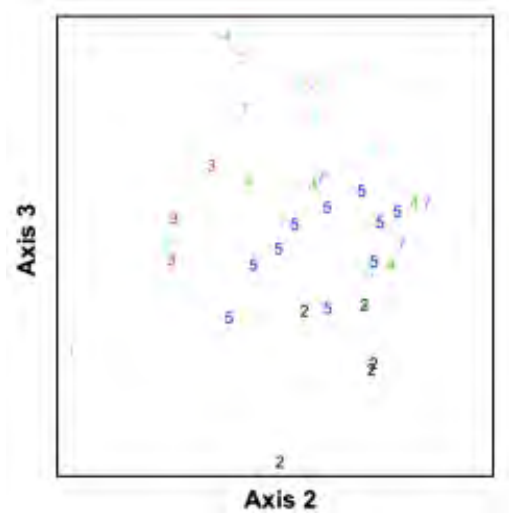

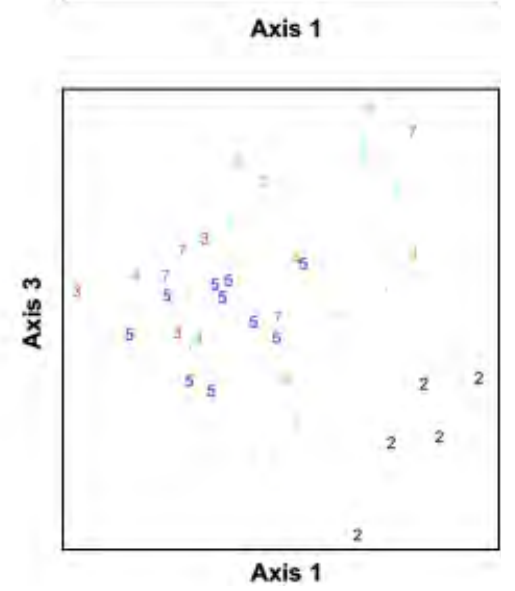

Fig. 1. NMDS ordination plots (Bray-Curtis distance matrix) of merged ARISA profiles for the Nephrops norvegicus gut-derived samples (ordination stress $=0.17$ ). Each month is indicated by a different color with filled circle or number: (1) gray, February-March; (2) black, May; (3) red, July; (4) green, August; (5) blue, September; (6) light blue, October; and (7) pink, December. (a) provides three-dimensional representation of the results, (b)-(d) represent separate two-dimensional plots for all combinations of the three NMDS axes. NMDS axes are arbitrary, so that plots may arbitrarily be rotated, centered or inverted.

Table 1. ANOSIM results for the a priori determined temporal groups

\begin{tabular}{|c|c|c|c|c|c|c|c|}
\hline & February/March & May & July & August & September & October & December \\
\hline February/March & & 0.0166 & 0.1983 & 0.0484 & 0.0083 & 0.0231 & 0.0105 \\
\hline May & 0.5897 & & 0.0183 & 0.0044 & 0.0002 & 0.0026 & 0.0023 \\
\hline August & $0.3765^{*}$ & 0.6053 & 0.6543 & & 0.0138 & 0.0916 & 0.0154 \\
\hline September & 0.7208 & 0.9389 & 0.8403 & $0.2933^{*}$ & & 0.0043 & $\underline{0.0003}$ \\
\hline October & 0.5000 & 0.8720 & 0.6975 & $0.1611^{*}$ & $0.4028^{*}$ & & $\overline{0.0302}$ \\
\hline December & 0.6111 & 0.9227 & 0.7654 & $0.3870^{*}$ & 0.5206 & $0.2796^{*}$ & \\
\hline
\end{tabular}

*Largely overlapping groups.

Significant noncorrected $P$-values $(P<0.05)$ are indicated with italics, significant Bonferroni corrected $P$-values $(P<0.0024)$ are underlined $($ upper right triangle) and $R$ values are in bold (lower left triangle).

$(P=0.092)$. When applying a Bonferroni correction for the 21 comparisons being made, a clear separation only occurred between all pairwise comparisons involving May, September and December samples. As mentioned above, the Bonferroni correction has often been judged to be overly conservative (Legendre \& Legendre, 1998), and so care should be taken when applied to ecological data.

\section{Phylogenetic analysis}

Seven samples from different sampling seasons (Table S2) were further analyzed with the construction of clone libraries. A total of 311 full-length $16 \mathrm{~S}$ rRNA gene sequences were determined for samples F1 (31), MY46 (58), J11 (30), Ag31 (22), Se3 (53), O2 (25) and D1 (92). In each clone library, 5-10 different phylotypes were detected based on a cutoff similarity of 99\% (Table 2, Fig. 2). All clone libraries had a satisfactory coverage (Fig. S1) according to Good's C estimator (Kemp \& Aller, 2004). Almost all clone libraries had coverage higher than $90 \%$, apart from February (77\%) and August (82\%).

Except for clone library Ag31 that had two dominant phylotypes with a frequency of $31 \%$ and $27 \%$, all clone libraries were dominated by a single phylotype being present 
Table 2. $16 \mathrm{~S}$ rRNA gene phylotypes detected in midgut samples of Nephrops norvegicus individuals

\begin{tabular}{|c|c|c|c|c|c|}
\hline Phylotype & Frequency (\%) & Name of closest relative & GenBank accession no. & Similarity (\%) & Phylogenetic affiliation \\
\hline F1-5 & 58.1 & Pseudoalteromonas sp. NPO-JI-58 & AY745828 & 99 & Gammaproteobacteria \\
\hline $\mathrm{F} 1-4$ & 12.9 & Psychrobacter aquimaris, KOPRI 24929 & EF101547 & 99 & Gammaproteobacteria \\
\hline F1-7 & 6.4 & Uncultured actinobacterium & EU374093 & 97 & Actinobacteria \\
\hline F1-2 & 3.2 & Uncultured bacterium & FJ203056 & 96 & Gammaproteobacteria \\
\hline F1-6 & 3.2 & Uncultured bacterium & FJ545576.1 & 96 & Acidobacteria \\
\hline F1-18 & 3.2 & Uncultured Chloroflexi & DQ811871 & 98 & Chloroflexi \\
\hline F1-19 & 3.2 & Uncultured acidobacterium & DQ395041 & 96 & Acidobacteria \\
\hline $\mathrm{F} 1-20$ & 3.2 & Uncultured actinobacterium & EF208654 & 96 & Actinobacteria \\
\hline F1-26 & 3.2 & Uncultured actinobacterium & EU374093 & 99 & Actinobacteria \\
\hline F1-28 & 3.2 & Uncultured bacterium & FJ695595 & 98 & Gammaproteobacteria \\
\hline My46-424 & 74.1 & Psychrobacter sp. Pi 2-33 & AB365059 & 99 & Gammaproteobacteria \\
\hline My46-492 & 19.0 & Vibrio lentus & AY292936 & 99 & Gammaproteobacteria \\
\hline My46-460 & 3.4 & Vibrio lentus & AY292936 & 99 & Gammaproteobacteria \\
\hline My46-442 & 1.7 & Planococcus rifitiensis strain M8 & AJ493659 & 99 & Firmicutes \\
\hline My46-484 & 1.7 & Uncultured bacterium & AB255057 & 98 & Firmicutes \\
\hline Jl1-36 & 73.3 & Photobacterium leiognathi & AY292917 & 99 & Gammaproteobacteria \\
\hline$J 1-4$ & 13.3 & Photobacterium sp. JT-ISH-224 & AB293986 & 99 & Gammaproteobacteria \\
\hline J11-1 & 6.6 & Photobacterium leiognathi strain RM1 & AY292947 & 99 & Gammaproteobacteria \\
\hline Jl1-22 & 3.3 & Psychrobacter aquimaris & EF101547 & 99 & Gammaproteobacteria \\
\hline J11-28 & 3.3 & Uncultured bacterium clone CK_1_C4_1 & EU488044 & 87 & Firmicutes \\
\hline Ag31-3 & 31.9 & Photobacterium leiognathi strain RM1 & AY292947 & 99 & Gammaproteobacteria \\
\hline Ag31-6 & 27.3 & Photobacterium leiognathi & AY292917 & 98 & Gammaproteobacteria \\
\hline Ag31-15 & 13.6 & Vibrio lentus & AY292936 & 98 & Gammaproteobacteria \\
\hline Ag31-2 & 9.1 & Uncultured bacterium & AB255065 & 99 & Gammaproteobacteria \\
\hline Ag31-1 & 4.5 & Vibrio parahaemolyticus RIMD 2210633 & BA000031 & 99 & Gammaproteobacteria \\
\hline Ag31-21 & 4.5 & Vibrio splendidus LGP32 chromosome 1 & FM954972 & 98 & Gammaproteobacteria \\
\hline Ag31-22 & 4.5 & Vibrio salmonicida isolate PB1-8rrnB & EU091324 & 99 & Gammaproteobacteria \\
\hline Ag31-13 & 4.5 & Photobacterium sp. HAR23 & AB038031 & 99 & Gammaproteobacteria \\
\hline Se3-204 & 83.0 & Uncultured Mollicutes & DQ340200 & 90 & Mollicutes \\
\hline Se3-206 & 5.6 & Desulfatibacillus olefinivorans strain LM2801 & DQ826724 & 87 & Deltaproteobacteria \\
\hline Se3-199 & 3.8 & Ralstonia sp. FRA01 & AF098288 & 99 & Betaproteobacteria \\
\hline Se3-178 & 3.8 & Dechloromonas sp. $\mathrm{HZ}$ & AF479766 & 98 & Betaproteobacteria \\
\hline Se3-129 & 1.9 & Uncultured bacterium clone CK_1_C4_19 & EU488044 & 87 & Mollicutes \\
\hline Se3-157 & 1.9 & Uncultured bacterium & AY328732 & 99 & Alphaproteobacteria \\
\hline O2-1 & 76.0 & Photobacterium leiognathi strain RM1 & AY292947 & 99 & Gammaproteobacteria \\
\hline $02-8$ & 16.0 & Vibrio splendidus isolate PB1-10rrnH & EU091332 & 99 & Gammaproteobacteria \\
\hline $02-23$ & 4.0 & Vibrio salmonicida isolate PB1-8rrnB & EU091324 & 99 & Gammaproteobacteria \\
\hline O2-37 & 4.0 & Uncultured bacterium & EF123487 & 98 & Gammaproteobacteria \\
\hline D1-695 & 86.0 & Uncultured bacterium clone CK_1_C4_1 & EU488044 & 87 & Firmicutes \\
\hline D1-668 & 2.2 & Dechloromonas sp. HZ & AF479766 & 99 & Betaproteobacteria \\
\hline D1-669 & 2.2 & Arctic sea ice bacterium & AF468382 & 99 & Gammaproteobacteria \\
\hline D1-715 & 2.2 & Psychrobacter celer & EF101550 & 98 & Gammaproteobacteria \\
\hline D1-727 & 2.2 & Uncultured bacterium & EU137440 & 99 & Actinobacteria \\
\hline D1-684 & 1.1 & Psychromonas japonica & AB304804 & 98 & Gammaproteobacteria \\
\hline D1-674 & 1.1 & Shewanella woodyi ATCC 51908 & СР000961 & 99 & Gammaproteobacteria \\
\hline D1-700 & 1.1 & Burkholderia phytofirmans PsJN & CP001053 & 98 & Betaproteobacteria \\
\hline
\end{tabular}

For sample code, see text. Closest relative determined via BLAST top score and phylogenetic affiliation via tree reconstruction (see Fig. 2).

with a frequency of $56-87 \%$. All clone libraries were either dominated by sequences affiliated with Gammaproteobacteria (F1, My46, J11, Ag31, O2) or Mollicutes (Se3, D1). Other phylotypes detected in lower abundances were clustered with Firmicutes, Actinobacteria, Acidobacteria, Chloroflexi, Alpha-,
Beta- and Deltaproteobacteria. Sample F1 had one dominant phylotype (F1-5, frequency 58.06\%) with 99\% similarity with Pseudoalteromonas sp. NPO-Jl-58, a heterotroph isolated from the North Pacific Ocean (Du et al., 2006). The two closest known species were 


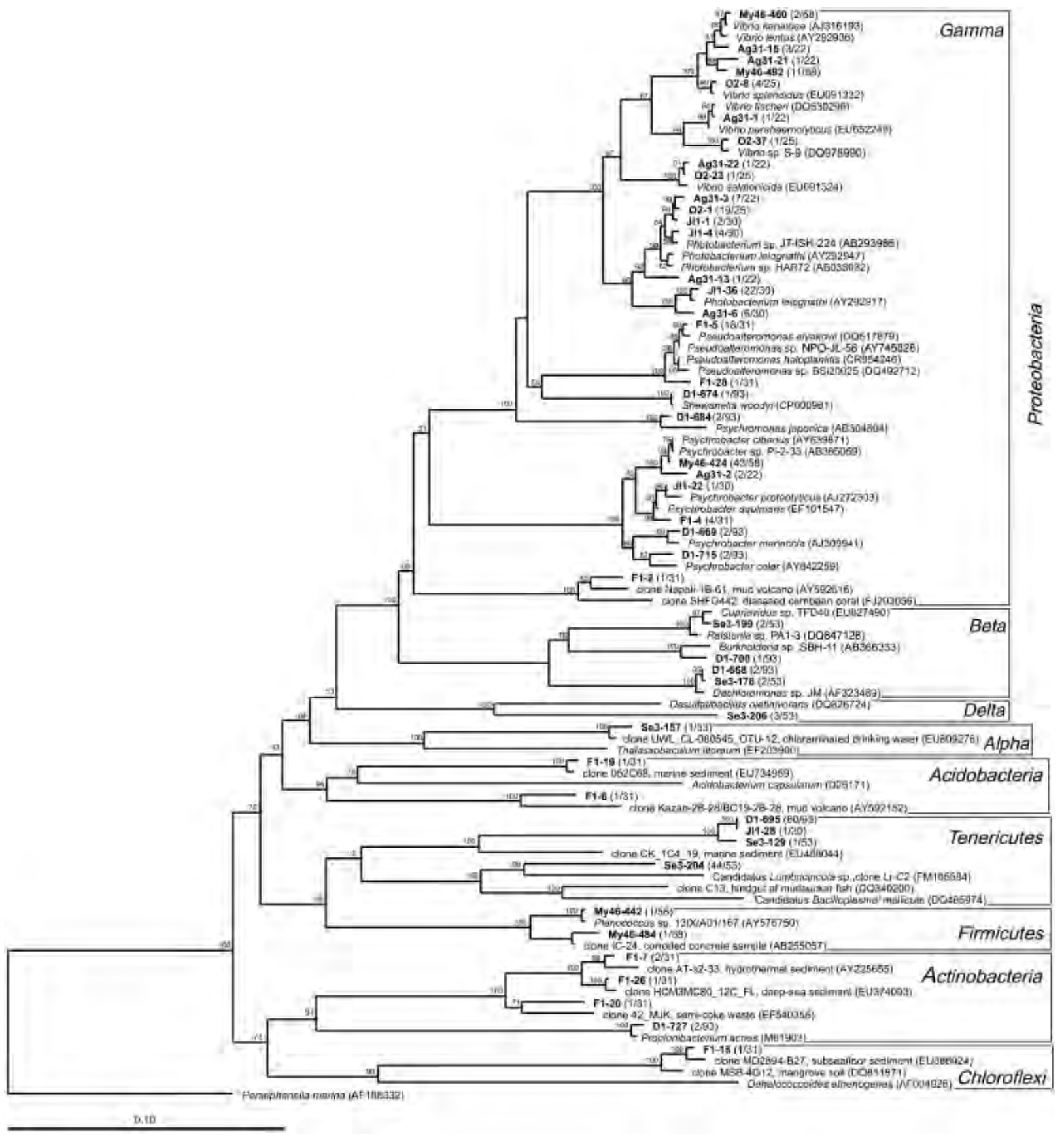

Fig. 2. Neighbor-joining tree based on $16 \mathrm{~S}$ rRNA gene sequences from the midgut of Nephrops norvegicus. Persephonella marina (Aquificae) was used as an outgroup. The bar corresponds to $10 \%$ nucleotide difference and bootstrap values were calculated from 1000 replicate trees. Relative abundances of retrieved phylotypes in each clone library are shown in parentheses. Only bootstrap values over $50 \%$ are shown in the tree.

Pseudoalteromonas haloplanktis TAC125 (Medigue et al., 2005) and Pseudoalteromonas elyakovii (Sawabe et al., 2000), exhibiting alginolytic activity.

Sample My46 had one dominant phylotype (My46-424, frequency $72 \%$ ) with $99 \%$ similarity with Psychrobacter sp. Pi 2-33 (Romanenko et al., 2008). This strain was isolated from coastal sea ice, and although not fully characterized, it was positively tested for lipolytic activity and the reduction of nitrate to nitrite. The closest fully characterized relative was Psychrobacter cibarius strain JG-220 isolated from fermented seafood (Jung et al., 2005), exhibiting lipolytic activity as well. A closely related phylotype 
(Ag31-2, 98\% similarity) was detected in Ag31 in a lower frequency $(9 \%)$.

Samples J11, Ag31 and O2 were dominated by phylotypes clustering with the genus Photobacterium and showing $>96 \%$ similarity between them (Table S4). The closest cultured relatives were a free-living strain, Photobacterium leiognathi RM1, isolated from the light organs of the squid Rondeletiola minor (Nishiguchi \& Nair, 2003), Photobacterium sp. JT-ISH-224, isolated from the gut of the Japanese barracuda (Tsukamoto et al., 2008) and Photobacterium sp. HAR23, isolated from the north-west Pacific Ocean water column (Urakawa et al., 1999).

Sample Se3 was clearly dominated by phylotype Se3-204 ( $83 \%$ frequency), which clustered in a group of uncultured Mollicutes affiliated as a sister group of the Mycoplasma clade and consisting of bacteria found in the intestinal tracts of various terrestrial and marine animals (Fig. S2). Se3-204 was only $89 \%$ similar with an uncultured Mollicutes bacterium detected in high abundances in the gut of the long-jawed mudsucker Gillichtys mirabilis (Bano et al., 2007). Other representatives of this group have been found in the intestine of notothenioid fishes (Ward et al., 2009), the gut of Lumbricidae earthworms (Nechitaylo et al., 2009) and the isopod Porcellio scaber (Kostanjsek et al., 2007).

The dominant phylotype of sample D1 (D1-695, frequency $86 \%$ ) fell within a highly divergent group of uncultured Mollicutes (Fig. S2). The representatives of the group, showing high heterogeneity between them, originate from various habitats including mammals' feces (Ley et al., 2008), humans' intestinal tract where they were related to obesity (Ley et al., 2006) and sediment samples (GreenGarcia, 2008). D1-695 showed low similarity (87\%) with its closest relative, phylotype CK_1C4_19, found in sea-grass bed sediment from the Gulf of Mexico (Green-Garcia, 2008). Phylotypes J11-28 and Se3-129, found in low abundances in samples Jl1 and Se3 (3.3\% and 1.5\%, respectively), were $99 \%$ similar with D1-695 and with each other. Because of the low percentages of similarity with their closest relatives, both phylotypes were thoroughly checked for chimeras with the PINTAIL tool. The phylogenetic analysis (alignment) showed the presence of stem areas with large insertions. Moreover, the 'abnormalities' in the 16S rRNA gene sequences were further confirmed with the mismatches found with universal bacterial primers and probes such as EUB (I-III), ITSF and GM5_341f (Table S5).

\section{FISH}

No signals were detected with the negative control NON338 and with probe BET42a. Hybridization experiments with probes EUB338 (I-III) and GAM42a were positive in samples FN2 and FN3. The cells detected were mostly curved rods $(4 \mu \mathrm{m} \times 1.5 \mu \mathrm{m})$ and were located in the internal gut wall (Fig. 3a). Hybridization experiments with the newly designed UncMol89 probe, targeting phylotype Se3-204, were positive in FSe1 and FSe3. The cells detected were packages of cocci organized in groups of four cells or eight cells (each cell around $1.5 \mu \mathrm{m}$ ) (Figs $3 \mathrm{~b}$ and S3), a sarcina morphotype, which is not consistent with that of known Mollicutes. They were located in the internal wall surface of the gut and showed a patchy distribution. Such distribution has been described previously for another uncultured gut Mollicutes (Kostanjsek et al., 2007). Very few signals were detected with probe EUB338 (I-III) in samples FSe1 and FSe3 and showed a different morphotype to the sarcina detected with probe UncMol89. The sarcina morphotype was detected on the same sections only through DAPI signals, suggesting the weak binding of EUB338 (I-III) to the unknown bacteria.

\section{Discussion}

This study analyzed the temporal variability throughout a year of gut microbial communities of $N$. norvegicus individuals along with their morphometric characteristics in order
Fig. 3. (a) Gut section (thickness $5 \mu \mathrm{m}$ ) of Nephrops norvegicus sample FN2 (August) hybridized with horseradish peroxidase-labeled GAM42a probe specific for Gammaproteobacteria, and stained with Alexa488-labeled tyramide. Blue signal corresponds to DAPI signal from the gut-wall epithelial cells and green signal corresponds to the hybridized bacteria. (b) Gut section (thickness $4 \mu \mathrm{m}$ ) of $N$. norvegicus sample FSE3 (September) hybridized with Cy3-labeled probe UncMol89 specific for uncultured Mollicutes phylotype Se3-204 detected in this study. Blue signal corresponds to DAPI signal from the gut-wall epithelial cells and red signal corresponds to the hybridized bacteria.
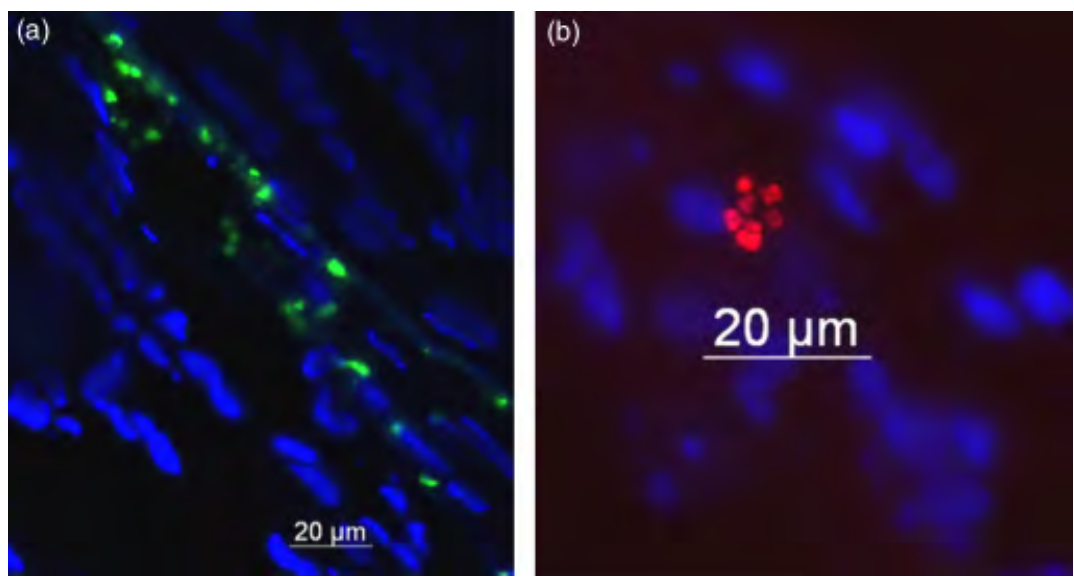
to reveal the factors that might influence their gut microbial diversity. None of the morphological factors could explain significantly the bacterial community shifts, while a temporal pattern of variation was observed (Fig. 1; Table 1). The $a$ priori hypothesis that gut microbial communities may show a temporal pattern was based on the temporal changes in food availability, which has been shown to potentially influence the gut microbiota (Harris, 1993; Lau et al., 2002; Ley et al., 2008 and references therein). Hence, ecosystem seasonal fluctuations (e.g. phytoplankton bloom) result in differential food availability and quality, whose exact components are still to be determined. Combining the feeding behavior of $N$. norvegicus acting either as a predator (small mollusks, crustaceans and polychaetes) or as a scavenger (fish, bigger invertebrates), with the periodicity observed in the system of Pagasitikos Gulf, it is assumed that the highest quality of available food for $N$. norvegicus would occur around April-May and would slowly decrease till the next bloom.

Interestingly, the above-mentioned fluctuations in food quality and quantity were reflected at the level of microbial community structure (Table 1), not at the onset of the food changes, but a few months later. Indeed, bacterial communities presented a different structure but some degree of overlap ( $R=0.5897 ; P=0.0166)$ between February/March (maximum phytodetritus season) and May (highest food quality and availability). However, it was only after May that the structures of the gut bacterial communities were drastically affected $(R=0.969$ between May and July). July-August could further represent a transition zone where food still decreases in abundance or quality and thus slight changes in community structures are still observed $(R=0.6543)$. From August to December, when food becomes scarce, there is no drastic change in diet and the gut bacterial communities would not differentiate considerably $(R<0.41)$. These hypotheses were also supported by analyzing the microbial OTU turnover between any two consecutive months (Fig. 4). Between February and August, bacterial communities became gradually more and more dissimilar ( $>40-55 \%$ replacement of the OTUs each time), while after August, no further variation was evidenced ( $<30 \%$ turnover).

Noticeably, the results of the clone libraries and FISH experiments, for representatives of these groups, showed that December and September samples were dominated by uncultured Mollicutes while August and October samples contained more Gammaproteobacteria. The in silico analysis for both Mollicutes dominant phylotypes showed that the ITSF primer used in this study for ARISA had three mismatches with the binding site (Table S5). The ITS length found for the two dominant phylotypes, Se3-204 and D1695, was 317 and $246 \mathrm{bp}$, respectively. These two ITS sizes were found in the ARISA tables showing very low relative area values $(<0.5 \%)$ (data not shown in this study).

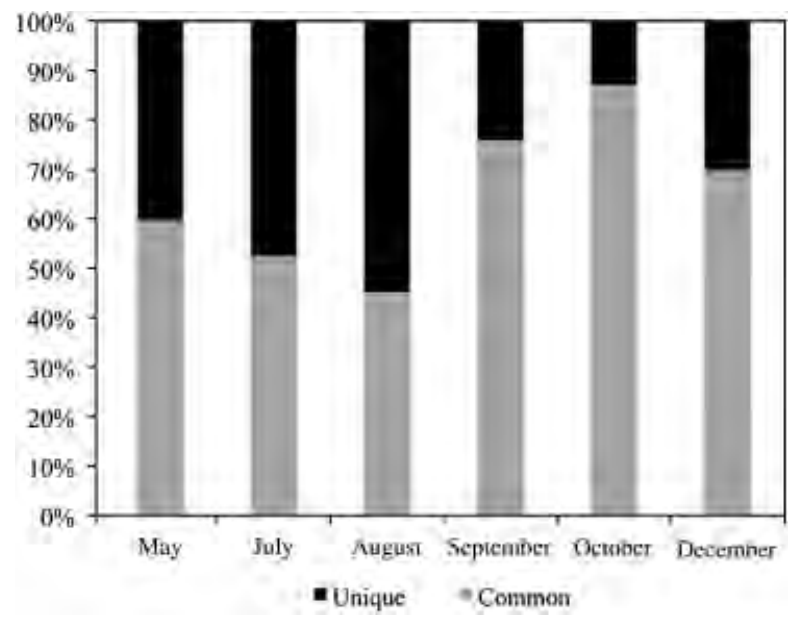

Fig. 4. Relative abundance of common bacterial OTUs from the midgut of Nephrops norvegicus between two consecutive months' samples as calculated from the ARISA experiments. The percentage of phylotypes that is shared with the previous month's samples is designated with light gray. February/March is not shown because there was no previous month with which it could be compared.

Consequently, the Mollicutes' microdiversity is not totally represented in the NMDS plot due to PCR and primer biases. In order to design a novel forward ARISA primer targeting at the same time Mollicutes and the rest of the Bacteria, a primer with three degenerate positions at the $3^{\prime}$ primer end with one of them being degenerate for more than two nucleotides (Table S5) would be needed. Because this could lead to additional PCR biases, the testing of such a primer will be addressed in our future research. Apart from this technical consideration, further comparisons between the two techniques (ARISA and clone libraries) are not presented, because the two approaches describe fundamentally different components of the diversity of microbial communities (Bent \& Forney, 2008). In addition, ARISA was performed on the ITS level, while sequencing was performed on the $16 \mathrm{~S}$ and ITS levels; therefore, not only the techniques differed but also the targeted molecular markers. It is then not surprising that a discrepancy between the clone libraries and the ARISA results may be evidenced for OTU numbers. Indeed, it is conceivable that a given $16 \mathrm{~S}$ rRNA gene sequence might occur in many samples, but could be associated with different ITS sequences (representing intra- or intergenomic variability), the latter being associated only with specific samples.

The clone libraries analysis showed one or two dominant phylotypes for each sample (Table 2), suggesting rather specialized bacterial communities. February was dominated by a Pseudoalteromonas-like phylotype. Members of this genus are associated with higher organisms, have the ability of easily colonizing surfaces and display antibacterial, bacteriolytic, agarolytic and algicidal activities (Holmstrom \& 
Kjelleberg, 1999). Although N. norvegicus does not feed on algae, remains of plants can be found in its stomach (Cristo \& Cartes, 1998) probably due to passive ingestion. The presence of Pseudoalteromonas-like organisms could be related to the presence of algal material in the intestine of $N$. norvegicus assisting in its decomposition. In addition, the second most dominant phylotype in the same sample was closely related to a Psychrobacter aquimaris strain, originating from a marine alga, enhancing the inferred alginolytic character of the bacterial community.

Another Psychrobacter-like phylotype, with potential lipolytic activity, dominated in May. Members of this genus have been found in a wide range of environments, such as sea ice, sea water, marine sediments, salt marshes, ornithogenic soils, food microbiota, animal tissues and sponges (Bowman, 2006). For example, Psychrobacter proteolyticus has been isolated from the gut of the krill Euphausia superba (Denner et al., 2001). The lipolytic activity inferred from its closest relatives, combined with the expected high food availability, indicates that lipid digestion is feasible.

The July, August and October communities were dominated by highly similar phylotypes of the genus Photobacterium (Tables 2 and S4). Representatives of this genus show fermentative metabolism and are isolated from the light organs of several marine animals. However, bioluminescent bacteria are ubiquitous in fish intestinal tracts, being the most abundant aerobes in such habitats (Farmer \& Hickman-Brenner, 2006; Ward et al., 2009). Biochemical studies proved chitinase activity and lipase activity in $96 \%$ and $82 \%$ of $P$. leiognathi strains, respectively (Farmer \& HickmanBrenner, 2006). Strains of Photobacterium phosphoreum have characteristics similar to that of $P$. leiognathi including chitinase activity. Although we did not investigate lipase or chitinase activity in our samples, the dominance $(>60 \%)$ of almost identical phylotypes in three different months reveals a specialized community. The phylotypes detected were closely related, as described above, to Photobacterium sp. isolated from tissues of marine animals enhancing the hypothesis of their ability to colonize the N. norvegicus gut.

After the summer, phylotypes Se3-204 and D1-695 clustered within two groups of uncultured Mollicutes (Fig. S2). Many members of Mollicutes are obligate intracellular symbionts that possess the smallest genomes of self-replicating organisms (Razin et al., 1998). Some mycoplasmas are known pathogens, but many species appear to be simply part of the natural microbiota of their hosts and seem to have no harmful effects on the host (Giebel et al., 1990). No physiological properties can be inferred for the found Mollicutes-related phylotypes, because they share $<83 \%$ similarity with cultured representatives. Moreover, it has been suggested that members from the two groups of uncultured Mollicutes to which the phylotypes of this study belonged (Fig. S2) may represent only a small part of an as-yet-uncultured bacterial lineage colonizing the intestines of various arthropods and vertebrate hosts (Kostanjsek et al., 2007). Importantly, these bacteria do not seem to have a parasitic relationship with their hosts, because in most cases, the animals that harbored were healthy (Bano et al., 2007; Kostanjsek et al., 2007; Nechitaylo et al., 2009; Ward et al., 2009).

The FISH specificity of UncMol89, targeting Se3-204 was only tested in silico and not empirically. However, the consistent sarcina morphotype detected with UncMol89 and the expected absence of EUB338 (I-III) signals of this specific morphotype (Table S5) argues in favor of sequence Se3-204 originating from the sarcina morphotypes. Although probe UncMol89 had minimum two mismatches with the 'closely' related gut uncultured Mollicutes and more than three mismatches to all other known sequences, additional experiments such as the design of nonlabeled competitor probes for the blocking of non-target-binding sites (Manz et al., 1992) should be used in future experiments to ensure the origin of the Se3-204 phylotype from the sarcina cells.

Other ways to better test the specificity of UncMol89 would be the design of a modified UncMol89 with two to three mismatches to the Se3-204 phylotype where diminished probe signaling from the sarcina cells would be expected. Alternatively, UncMol89 could be tested against cultured organisms with two or more mismatches to test whether probe signaling disappears. The absence of signals from the sarcina cells when using EUB338 (I-III) could be explained by the two central mismatches of the probe with the 16S rRNA gene sequence of the phylotype Se3-204 (Table S5). The design of modified EUB probes correcting the mismatches with Se3-204 and resulting in better probe signalling for the sarcina cells would further confirm their origin from Se3-204.

Phylotypes clustering within the genus Vibrio were present in most of the samples, showing low (3.5\%) to moderate $(19.0 \%)$ relative abundance. The majority of their closest relatives originated from marine animals (Nasu et al., 2000; Nishiguchi \& Nair, 2003; Jensen et al., 2009). This genus is often found in associations with the gut of marine animals varying from parasitism to mutualism and showing diverse metabolic capabilities (Thompson et al., 2004).

The rest of the phylotypes clustered within the groups of Actinobacteria, Acidobacteria, Chloroflexi, Firmicutes, Alpha-, Beta- and Deltaproteobacteria. These phyla dominate frequently in sediment samples. Their low presence could be attributed to remains of sediment particles of ingested food that were not removed by the mechanical evacuation and the rinsing of the gut.

Overall, most of the phylotypes found were associated either with the intestinal tract of marine animals or with their light organs. Consequently, it is most likely that these 
phylotypes have the ability to attach to tissue surfaces and successfully colonize the midgut. Some of the phylotypes could also be pathogens, although all animals collected were healthy. The presence of highly similar $(\geq 99 \%)$ phylotypes in different months at varying frequencies (Tables 2 and S4, Fig. 2) indicates that at least the dominant phylotypes may be present throughout the year, despite fluctuating environmental conditions. The existence of these recurring phylotypes indicates that these bacteria might be representative of specific communities or even participate in symbiotic interactions, i.e. resident bacteria, with $N$. norvegicus. More specifically, the Gammaproteobacteria detected are universally dispersed in different animals and varying habitats and appear in $N$. norvegicus' gut at different abundances throughout the year. The hypothesis of the temporal effect of the trophic state of the overlying water column on the gut microbial communities, which this study supports, needs to be further investigated with feeding experiments and estimation of prey availability or phytodetritus flux.

\section{Acknowledgements}

We acknowledge support from the International Max Planck Research School of Marine Microbiology (MarMic), the Deutscher Academischer Austausch Dienst (DAAD). Part of this research was cofunded by the European Social Fund \& National Resources EPEAK II-PYTHAGORAS II. Rudolf Amann is acknowledged for advice on FISH and for his comments on the manuscript. We also thank two anonymous reviewers for their comments.

\section{References}

Amann R \& Fuchs BM (2008) Single-cell identification in microbial communities by improved fluorescence in situ hybridization techniques. Nat Rev Microbiol 6: 339-348.

Ashelford KE, Chuzhanova NA, Fry JC, Jones AJ \& Weightman AJ (2005) At least 1 in 20 16S rRNA sequence records currently held in public repositories is estimated to contain substantial anomalies. Appl Environ Microb 71: 7724-7736.

Bano N, DeRae Smith A, Bennett W, Vasquez L \& Hollibaugh JT (2007) Dominance of Mycoplasma in the guts of the LongJawed Mudsucker, Gillichthys mirabilis, from five California salt marshes. Environ Microbiol 9: 2636-2641.

Behrens S, Ruhland C, Inacio J, Huber H, Fonseca A, SpencerMartins I, Fuchs BM \& Amann R (2003) In situ accessibility of small-subunit rRNA of members of the domains Bacteria, Archaea, and Eucarya to Cy3-labeled oligonucleotide probes. Appl Environ Microb 69: 1748-1758.

Bell MC, Redant F \& Tuck I (2006) Nephrops species. Lobsters: biology, management, aquaculture and fisheries (Phillips BF, ed), pp. 412-469. Blackwell, Oxford.
Bent SJ \& Forney LJ (2008) The tragedy of the uncommon: understanding limitations in the analysis of microbial diversity. ISME J 2: 689-695.

Boer SI, Hedtkamp SIC, van Beusekom JEE, Fuhrman JA, Boetius A \& Ramette A (2009) Time- and sediment depth-related variations in bacterial diversity and community structure in subtidal sands. ISME J 3: 780-791.

Bowman JP (2006) The genus Psychrobacter. The Prokaryotes (Dworkin M, Falkow S, Rosenberg E, Schleifer K-H \& Stackenbrandt E, eds), pp. 920-930. Springer, New York.

Cardinale M, Brusetti L, Quatrini P, Borin S, Puglia AM, Rizzi A, Zanardini E, Sorlini C, Corselli C \& Daffonchio D (2004) Comparison of different primer sets for use in automated ribosomal intergenic spacer analysis of complex bacterial communities. Appl Environ Microb 70: 6147-6156.

Clarke KR (1993) Non-parametric multivariate analyses of changes in community structure. Aust J Ecol 18: 117-143.

Clarke KR \& Green RH (1988) Statistical design and analysis for a biological effects study. Mar Ecol-Prog Ser 46: 213-226.

Cristo M \& Cartes JE (1998) A comparative study of the feeding ecology of Nephrops norvegicus (L.), (Decapoda: Nephropidae) in the bathyal Mediterranean and the adjacent Atlantic. Sci Mar 62: 81-90.

Denner EB, Mark B, Busse HJ, Turkiewicz M \& Lubitz W (2001) Psychrobacter proteolyticus sp. nov., a psychrotrophic, halotolerant bacterium isolated from the Antarctic krill Euphausia superba Dana, excreting a cold-adapted metalloprotease. Syst Appl Microbiol 24: 44-53.

Du HL, Jiao NZ, Hu YH \& Zeng YH (2006) Diversity and distribution of pigmented heterotrophic bacteria in marine environments. FEMS Microbiol Ecol 57: 92-105.

Duperron S, Bergin C, Zielinski F, Blazejak A, Pernthaler A, McKiness ZP, DeChaine E, Cavanaugh CM \& Dubilier N (2006) A dual symbiosis shared by two mussel species, Bathymodiolus azoricus and Bathymodiolus puteoserpentis (Bivalvia: Mytilidae), from hydrothermal vents along the northern Mid-Atlantic Ridge. Environ Microbiol 8: 1441-1447.

Farmer JJ III \& Hickman-Brenner FW (2006) The genera Vibrio and Photobacterium. The Prokaryotes (Dworkin M, Falkow S, Rosenberg E, Schleifer K-H \& Stackenbrandt E, eds), pp. 508-563. Springer, New York.

Fisher MM \& Triplett EW (1999) Automated approach for ribosomal intergenic spacer analysis of microbial diversity and its application to freshwater bacterial communities. Appl Environ Microb 65: 4630-4636.

Fuchs BM, Woebken D, Zubkov MV, Burkill P \& Amann R (2005) Molecular identification of picoplankton populations in contrasting waters of the Arabian Sea. Aquat Microb Ecol 39: 145-157.

Giebel J, Binder A \& Kirchhoff H (1990) Isolation of Mycoplasma moatsii from the intestine of wild Norway rats (Rattus norvegicus). Vet Microbiol 22: 23-29.

Green-Garcia AM (2008) Characterization of the Lucinid bivalvebacteria symbiotic system: the significance of the geochemical 
habitat on bacterial symbiont diversity and phylogeny. MSc Thesis, Louisiana State University, Baton Rouge, LA.

Harris JM (1993) The presence, nature, and role of gut microflora in aquatic invertebrates: a synthesis. Microb Ecol 25: 195-231.

Holmstrom C \& Kjelleberg S (1999) Marine Pseudoalteromonas species are associated with higher organisms and produce biologically active extracellular agents. FEMS Microbiol Ecol 30: 285-293.

Jensen S, Frost P \& Torsvik VL (2009) The nonrandom microheterogeneity of 16S rRNA genes in Vibrio splendidus may reflect adaptation to versatile lifestyles. FEMS Microbiol Lett 294: 207-215.

Jung SY, Lee MH, Oh TK, Park YH \& Yoon JH (2005) Psychrobacter cibarius sp. nov., isolated from jeotgal, a traditional Korean fermented seafood. Int J Syst Evol Micr 55: 577-582.

Kane MD, Poulsen LK \& Stahl DA (1993) Monitoring the enrichment and isolation of sulfate-reducing bacteria by using oligonucleotide hybridization probes designed from environmentally derived $16 \mathrm{Ss}$ ribosomal-RNA sequences. Appl Environ Microb 59: 682-686.

Kemp PF \& Aller JY (2004) Estimating prokaryotic diversity: when are $16 \mathrm{~S}$ rDNA libraries large enough? Limnol OceanogrMeth 2: 114-125.

Kostanjsek R, Strus J \& Avgustin G (2007) 'Candidatus Bacilloplasma,' a novel lineage of Mollicutes associated with the hindgut wall of the terrestrial isopod Porcellio scaber (Crustacea: Isopoda). Appl Environ Microb 73: 5566-5573.

Kruskal JB (1964) Multidimensional scaling by optimizing a goodness of fit to a nonmetric hypothesis. Psychometrika 29: $1-28$.

Lane DJ (1991) 16S/23S rRNA sequencing. Nucleic acid techniques in bacterial systematics (Stackenbrandt E \& Goodfellow M, eds), pp. 115-175. John Wiley \& Sons, Chichester.

Larkin MA, Blackshields G, Brown NP et al. (2007) Clustal W and clustal X version 2.0. Bioinformatics 23: 2947-2948.

Lau WWY, Jumars PA \& Armbrust EV (2002) Genetic diversity of attached bacteria in the hindgut of the deposit-feeding shrimp Neotrypaea (formerly Callianassa) californiensis (Decapoda: Thalassinidae). Microb Ecol 43: 455-466.

Legendre P \& Legendre L (1998) Numerical Ecology, 2nd edn. Elsevier, Amsterdam.

Ley RE, Turnbaugh PJ, Klein S \& Gordon JI (2006) Microbial ecology: human gut microbes associated with obesity. Nature 444: 1022-1023.

Ley RE, Hamady M, Lozupone C et al. (2008) Evolution of mammals and their gut microbes. Science 320: 1647-1651.

Loo LO, Baden SP \& Ulmestrand M (1993) Suspension-feeding in adult Nephrops norvegicus (L.) and Homarus gammarus (L.) (decapoda). Neth J Sea Res 31: 291-297.

Ludwig W, Strunk O, Westram R et al. (2004) ARB: a software environment for sequence data. Nucleic Acids Res 32: 1363-1371.
Manz W, Amann R, Ludwig W, Wagner M \& Schleifer K-H (1992) Phylogenetic oligodeoxynucleotide probes for the major subclasses of Proteobacteria: problems and solutions. Syst Appl Microbiol 15: 593-600.

Medigue C, Krin E, Pascal G et al. (2005) Coping with cold: the genome of the versatile marine Antarctica bacterium Pseudoalteromonas haloplanktis TAC125. Genome Res 15: 1325-1335.

Muyzer G, Teske A, Wirsen CO \& Jannasch HW (1995) Phylogenetic-relationships of Thiomicrospira species and their identification in deep-sea hydrothermal vent samples by denaturing gradient gel-electrophoresis of $16 \mathrm{~S}$ rDNA fragments. Arch Microbiol 164: 165-172.

Nasu H, Iida T, Sugahara T, Yamaichi Y, Park KS, Yokoyama K, Makino K, Shinagawa H \& Honda T (2000) A filamentous phage associated with recent pandemic Vibrio parahaemolyticus O3:K6 strains. J Clin Microbiol 38: 2156-2161.

Nechitaylo TY, Timmis KN \& Golyshin PN (2009) 'Candidatus Lumbricincola', a novel lineage of uncultured Mollicutes from earthworms of family Lumbricidae. Environ Microbiol 11: 1016-1026.

Nishiguchi MK \& Nair VS (2003) Evolution of symbiosis in the Vibroonaceae: a combined approach using molecules and physiology. Int J Syst Evol Micr 53: 2019-2026.

Petihakis G, Triantafyllou G, Pollani A, Koliou A \& Theodorou A (2005) Field data analysis and application of a complex water column biogeochemical model in different areas of a semi-enclosed basin: towards the development of an ecosystem management tool. Mar Environ Res 59: 493-518.

Pruesse E, Quast C, Knittel K, Fuchs BM, Ludwig W, Peplies J \& Glockner FO (2007) SILVA: a comprehensive online resource for quality checked and aligned ribosomal RNA sequence data compatible with ARB. Nucleic Acids Res 35: 7188-7196.

Ramette A (2007) Multivariate analyses in microbial ecology. FEMS Microbiol Ecol 62: 142-160.

Ramette A (2009) Quantitative community fingerprinting methods for estimating the abundance of operational taxonomic units in natural microbial communities. Appl Environ Microb 75: 2495-2505.

Razin S, Yogev D \& Naot Y (1998) Molecular biology and pathogenicity of mycoplasmas. Microbiol Mol Biol R 62 1094-1156.

Romanenko LA, Tanaka N, Uchino M, Kalinovskaya NI \& Mikhailov VV (2008) Diversity and antagonistic activity of sea ice bacteria isolated from the sea of Japan. Microbes Environ 23: 209-214.

Sarda F \& Valladares FJ (1990) Gastric evacuation of different foods by Nephrops norvegicus (Crustacea: Decapoda) and estimation of soft-tissue ingested, maximum food-intake and cannibalism in captivity. Mar Biol 104: 25-30.

Sawabe T, Tanaka R, Iqbal MM, Tajima K, Ezura Y, Ivanova EP \& Christen R (2000) Assignment of Alteromonas elyakovii KMM $162(\mathrm{~T})$ and five strains isolated from spot-wounded fronds of 
Laminaria japonica to Pseudoalteromonas elyakovii comb. nov. and the extended description of the species. Int J Syst Evol Micr 50: 265-271.

Thompson FL, Iida T \& Swings J (2004) Biodiversity of vibrios. Microbiol Mol Biol R 68: 403-431.

Tsukamoto H, Takakura Y, Mine T \& Yamamoto T (2008)

Photobacterium sp. JT-ISH-224 produces two

sialyltransferases, alpha-/beta-galactoside alpha2,3sialyltransferase and beta-galactoside alpha2,6sialyltransferase. J Biochem 143: 187-197.

Urakawa H, Kita-Tsukamoto K \& Ohwada K (1999) Restriction fragment length polymorphism analysis of psychrophilic and psychrotrophic Vibrio and Photobacterium from the northwestern Pacific Ocean and Otsuchi Bay, Japan. Can J Microbiol 45: 67-76.

Ward NL, Steven B, Penn K, Methe BA \& Detrich WH (2009) Characterization of the intestinal microbiota of two Antarctic notothenioid fish species. Extremophiles 13: 679-685.

Yonge CM (1924) Studies on the comparative physiology of digestion: II. The mechanism of feeding, digestion, and assimilation in Nephrops norvegicus. J Exp Biol 1: 343-389.

\section{Supporting Information}

Additional Supporting Information may be found in the online version of this article:

Figure S1. Clone library coverage based on Good's C estimator.
Figure S2. Neighbour-joining tree based on the Mollicutes 16S rRNA gene sequences from the midgut of Nephrops norvegicus.

Figure S3. Gut section (thickness $4 \mu \mathrm{m}$ ) of Nephrops norvegicus sample FSE3 (September) hybridized with Cy3-labeled probe UncMol89 specific for uncultured Mollicutes phylotype Se3-204 detected in this study.

Table S1. Morphometric data for the samples used for ARISA and clone libraries analysis.

Table S2. Samples used for DNA extraction and FISH (all DNA samples were used for ARISA analysis).

Table S3. Oligonucleotide probes used in the study.

Table S4. Pairwise comparisons of the 16S rRNA gene sequence similarities (\%) of Photobacterium sp.-like sequences and their closest relatives: (1) Photobacterium leiognathi strain RM1 (AY292947), (2) P. leiognathi (AY292917), (3) Photobacterium sp. HAR23 (AB038031), (4) Photobacterium sp. Jt-ISH-224 (AB293986).

Table S5. In silico detected mismatches (underlined) of phylotypes Se3-204 and D1-695 with primers and probe EUB338 (I-III) used in this study with.

Please note: Wiley-Blackwell is not responsible for the content or functionality of any supporting materials supplied by the authors. Any queries (other than missing material) should be directed to the corresponding author for the article. 
Table S1. Morphometric data for the samples used for ARISA and clone libraries analysis. Pooled samples are designated with italics, and samples analyzed with clone libraries are in boldface.

\begin{tabular}{|c|c|c|c|c|c|c|}
\hline Sample & Month & Sex & $\begin{array}{l}\text { Body weight } \\
\text { (g) }\end{array}$ & $\begin{array}{l}\text { Carapace length } \\
\text { (mm) }\end{array}$ & $\begin{array}{l}\text { Carapace width } \\
\text { (mm) }\end{array}$ & $\begin{array}{l}\text { Abdominal width } \\
\text { (mm) }\end{array}$ \\
\hline F40 & February & $\mathrm{M}$ & 89.91 & 50.21 & 27.47 & 24.57 \\
\hline F44 & February & M & 44.71 & 26.77 & 24.5 & 23.48 \\
\hline F1 (F40/F44) & February & $M$ & 67.31 & 38.49 & 25.985 & 24.025 \\
\hline $\mathrm{Mr} 4$ & March & $\mathrm{F}$ & 17.98 & 36.04 & 18.25 & 17.59 \\
\hline Mr10 & March & $\mathrm{F}$ & 26.31 & 36.4 & 18.6 & 19.8 \\
\hline Mr1(Mr4/Mr10) & March & $F$ & 22.145 & 36.22 & 18.425 & 18.695 \\
\hline Mr26 & March & M & 30.35 & 40.6 & 22.47 & 21.18 \\
\hline My27 & May & $\mathrm{F}$ & 12.47 & 27.32 & 13.95 & 15.33 \\
\hline My44 & May & M & 49.79 & 42.75 & 21.84 & 21.83 \\
\hline My45 & May & M & 27.69 & 36.15 & 18.89 & 18.65 \\
\hline My46 & May & $\mathbf{M}$ & 29.2 & 37.22 & 17.64 & 19.22 \\
\hline My47 & May & M & 39.21 & 40.36 & 21.3 & 20.63 \\
\hline Jl1 & July & $\mathbf{F}$ & 38.51 & 38.54 & 20.51 & 22.57 \\
\hline $\mathrm{Jl3}$ & July & $\mathrm{F}$ & 65.28 & 48.13 & 25.22 & 26.82 \\
\hline Jl14 & July & M & 59.9 & 44.79 & 23.18 & 23.12 \\
\hline Jl17 & July & M & 50.38 & 41.92 & 20.78 & 21.41 \\
\hline Jl19 & July & M & 39.73 & 40.16 & 19.65 & 20.37 \\
\hline Jl4(Jl14/Jl17/Jl19) & July & $M$ & 50 & 42.29 & 21.2 & 21.63 \\
\hline Ag4 & August & $\mathrm{F}$ & 22.53 & 35.18 & 17.94 & 19.8 \\
\hline Ag6 & August & $\mathrm{F}$ & 28.35 & 35.94 & 18.67 & 19 \\
\hline Ag9 & August & $\mathrm{F}$ & 43.54 & 42.53 & 21.39 & 23.33 \\
\hline Ag1(Ag4/Ag6/Ag9) & August & $F$ & 31.47 & 37.88 & 19.33 & 20.71 \\
\hline Ag2.1 & August & $\mathrm{F}$ & 30.66 & 36.4 & 18.3 & 20.5 \\
\hline Ag8 & August & $\mathrm{F}$ & 23.72 & 32.93 & 16.53 & 18.4 \\
\hline Ag16 & August & $\mathrm{F}$ & 22.05 & 35.66 & 18 & 20.46 \\
\hline Ag23 & August & $\mathrm{F}$ & 31.48 & 37.41 & 18.63 & 21.4 \\
\hline Ag31 & August & $\mathbf{M}$ & 52.58 & 42.46 & 21.34 & 21.88 \\
\hline Se1 & September & M & 43.3 & 40.32 & 22.74 & 21.83 \\
\hline Se2 & September & M & 68.9 & 44.96 & 27.17 & 27.09 \\
\hline Se3 & September & $\mathbf{M}$ & 22.297 & 31.47 & 17.59 & 17 \\
\hline Se4 & September & $\mathrm{F}$ & 45.85 & 40.92 & 20.53 & 20.45 \\
\hline Se5 & September & $\mathrm{F}$ & 27.5 & 35.04 & 17.83 & 18.32 \\
\hline Se6 & September & $\mathrm{F}$ & 21.61 & 31.67 & 19.32 & 18.67 \\
\hline Se7 & September & $\mathrm{F}$ & 36.04 & 38.9 & 19.98 & 22.5 \\
\hline Se9 & September & $\mathrm{F}$ & 31.51 & 37.58 & 19.35 & 21.22 \\
\hline Se11 & September & M & 28.88 & 35.33 & 17.83 & 18.51 \\
\hline Se12 & September & M & 32.41 & 37.94 & 18.45 & 17.51 \\
\hline $\mathrm{O} 1$ & October & $\mathrm{F}$ & 36.71 & 38.36 & 19.21 & 22.11 \\
\hline 02 & October & $\mathbf{F}$ & 24.8 & 35.61 & 16.44 & 19.75 \\
\hline O3 & October & $\mathrm{F}$ & 17.37 & 29.97 & 14.91 & 16.85 \\
\hline O4 & October & F & 11.85 & 28.59 & 13.34 & 13.9 \\
\hline O5 & October & M & 29.12 & 36.84 & 17.72 & 18.53 \\
\hline O6 & October & M & 27.76 & 35.71 & 17.35 & 18.12 \\
\hline D1 & December & $\mathbf{F}$ & 22.06 & 35.25 & 17.3 & 19.86 \\
\hline D2 & December & $\mathrm{F}$ & 21.99 & 32.12 & 16.23 & 18.18 \\
\hline D3 & December & $\mathrm{F}$ & 20.77 & 32.18 & 15.51 & 17.17 \\
\hline D4 & December & M & 32.78 & 40.05 & 19.48 & 20.27 \\
\hline D6 & December & M & 59.47 & 45.88 & 24.09 & 23.1 \\
\hline D7 & December & M & 18.85 & 35 & 17.32 & 17.87 \\
\hline
\end{tabular}


Table S2. Samples used for DNA extraction and FISH (all DNA samples were used for ARISA analysis). Samples used for the construction of the clone libraries are indicated by bold letters. F: February, Mr: March, My: May, Jl: July, Ag: August, Se: September, O: October, D: December, FN: fixed August samples, FSe: fixed September samples

\begin{tabular}{llll}
\hline Month & Sample code & Pooled samples & Fixed samples \\
\hline February & - & F1 (F40/F44) & \\
March & Mr26 & Mr1 (Mr4/Mr10) & \\
May & My27/My44/My45/My46/My47 & & \\
July & Jl1/Jl3 & Jl4 (J114/Jl17/J119) & \\
August & Ag2.1/Ag8/Ag16/Ag23/Ag31 & Ag1 (Ag4/Ag6/Ag9) & FN2/FN3 \\
September & Se1/Se2/Se3/Se4/Se5/Se6/Se7/Se9/Se11/Se12 & & FSe3/FSe1 \\
October & O1/O2/O3/O4/O5/O6 & & \\
December & D1/D2/D3/D4/D6/D7 & & \\
\hline
\end{tabular}


Table S3. Oligonucleotide probes used in the study. FA: formamide concentration.

\begin{tabular}{|c|c|c|c|c|c|}
\hline Probe & Group specificity & Sequence (5’ $\rightarrow$ 3’) & Escherichia coli $16 \mathrm{~S}$ or $23 \mathrm{~S}$ rRNA position & FA\% & Reference \\
\hline EUB338 (I-III) & Almost all Bacteria & GCWGCCWCCCGTAGGWGT & $338-355$ & 35 & Daims, et al., 1999 \\
\hline NON338 & Negative control & ACTCCTACGGGAGGCAGC & - & 35 & Amann, et al., 1990 \\
\hline GAM42a & $\gamma$-Proteobacteria & GCCTTCCCACATCGTTT & helix 42a, 23S rRNA & 35 & Manz et al., 1992 \\
\hline BET42a & $\beta$-Proteobacteria & GCCTTCCCACTTCGTTT & helix 42a, $23 \mathrm{~S}$ rRNA & 35 & Manz et al., 1992 \\
\hline $\operatorname{ALF968}^{(*)}$ & $\alpha$-Proteobacteria & GGTAAGGTTCTGCGCGTT & 968 & 35 & Gloeckner, et al., 1999 \\
\hline UncMol89 & Uncult. Mollicutes & CGTTCGCСACTAACACСАAАATC & 89 & 35 & This study \\
\hline
\end{tabular}

*: ALF968 has low specificity as determined from database search. However in this study no signals were detected with this probe so no further checking was performed

Amann RI, Krumholz L \& Stahl DA (1990) Fluorescent-oligonucleotide probing of whole cells for determinative, phylogenetic, and environmental studies in microbiology. $J$ Bacteriol 172: 762-770.

Daims H, Bruhl A, Amann R, Schleifer KH \& Wagner M (1999) The domain-specific probe EUB338 is insufficient for the detection of all Bacteria: Development and evaluation of a more comprehensive probe set. Syst Appl Microbiol 22: 434-444.

Glockner FO, Fuchs BM \& Amann R (1999) Bacterioplankton compositions of lakes and oceans: a first comparison based on fluorescence in situ hybridization. Appl Environ Microbiol 65: 3721-3726.

Manz W, Amann R, Ludwig W, Wagner M \& Schleifer K-H (1992) Phylogenetic oligodeoxynucleotide probes for the major subclasses of Proteobacteria: problems and solutions. . Syst Appl Microbiol 15: 593-600. 
Table S4. Pairwise comparisons of the 16S rRNA gene sequence similarities (\%) of Photobacterium sp.-like sequences and their closest relatives, 1: Photobacterium leiognathi strain RM1 (AY292947), 2: P. leiognathi (AY292917), 3: Photobacterium sp. HAR23 (AB038031), 4: Photobacterium sp. Jt-ISH-224 (AB293986). Highest similarity, 99\%, is indicated in boldface. For sample codes see text.

\begin{tabular}{|c|c|c|c|c|c|c|c|c|c|c|c|}
\hline 1 & 2 & 3 & 4 & J11-36 & Jl1-4 & Jl1-1 & Ag31-3 & Ag31-6 & Ag31-13 & O2-1 & phylotypes \\
\hline \multirow[t]{11}{*}{ - } & 96 & 99 & 98 & 96 & 99 & 99 & 99 & 96 & 97 & 99 & 1 \\
\hline & - & 96 & 96 & 99 & 96 & 96 & 96 & 98 & 96 & 96 & 2 \\
\hline & & - & 98 & 96 & 99 & 99 & 98 & 97 & 98 & 99 & 3 \\
\hline & & & - & 96 & 99 & 99 & 98 & 96 & 97 & 99 & 4 \\
\hline & & & & - & 96 & 96 & 96 & 98 & 96 & 96 & Jl1-36 \\
\hline & & & & & - & 98 & 98 & 96 & 97 & 98 & Jl1-4 \\
\hline & & & & & & - & 99 & 96 & 97 & 99 & Jl1-1 \\
\hline & & & & & & & - & 96 & 97 & 99 & Ag31-3 \\
\hline & & & & & & & & - & 96 & 96 & Ag31-6 \\
\hline & & & & & & & & & - & 97 & Ag31-13 \\
\hline & & & & & & & & & & - & O2-1 \\
\hline
\end{tabular}


Table S5. In silico detected mismatches (underlined) of phylotypes Se3-204 and D1-695 with primers and probe EUB338 (I-III) used in this study with.

\begin{tabular}{lll}
\hline Primer/Probe & Primer sequence/Probe binding site & \\
\hline ITSF & 5'-GTCGTAACAAGGTAGCCGTA-3' & \\
Se3-204 & 5'-GTCGTAACAAGGTATCTCTA-3’ & 3 mismatches \\
D1-695 & 5'-GTCGTAACAAGGTATCACTA-3' & 3 mismatches \\
& & \\
GM5_341f & 5'-CCTACGGGAGGCAGCAG-3' & \multirow{2}{*}{ 3 mismatches } \\
Se3-204 & 5'-TCTACGGAAGGCTGCAG-3' & \\
EUB338 (I-III) & 5'-ACWCCTACGGGWGGCWGC-3' & \\
Se3-204 & 5'-ACTTCTACGGAAGGCTGC-3' & 2 mismatches \\
\hline
\end{tabular}



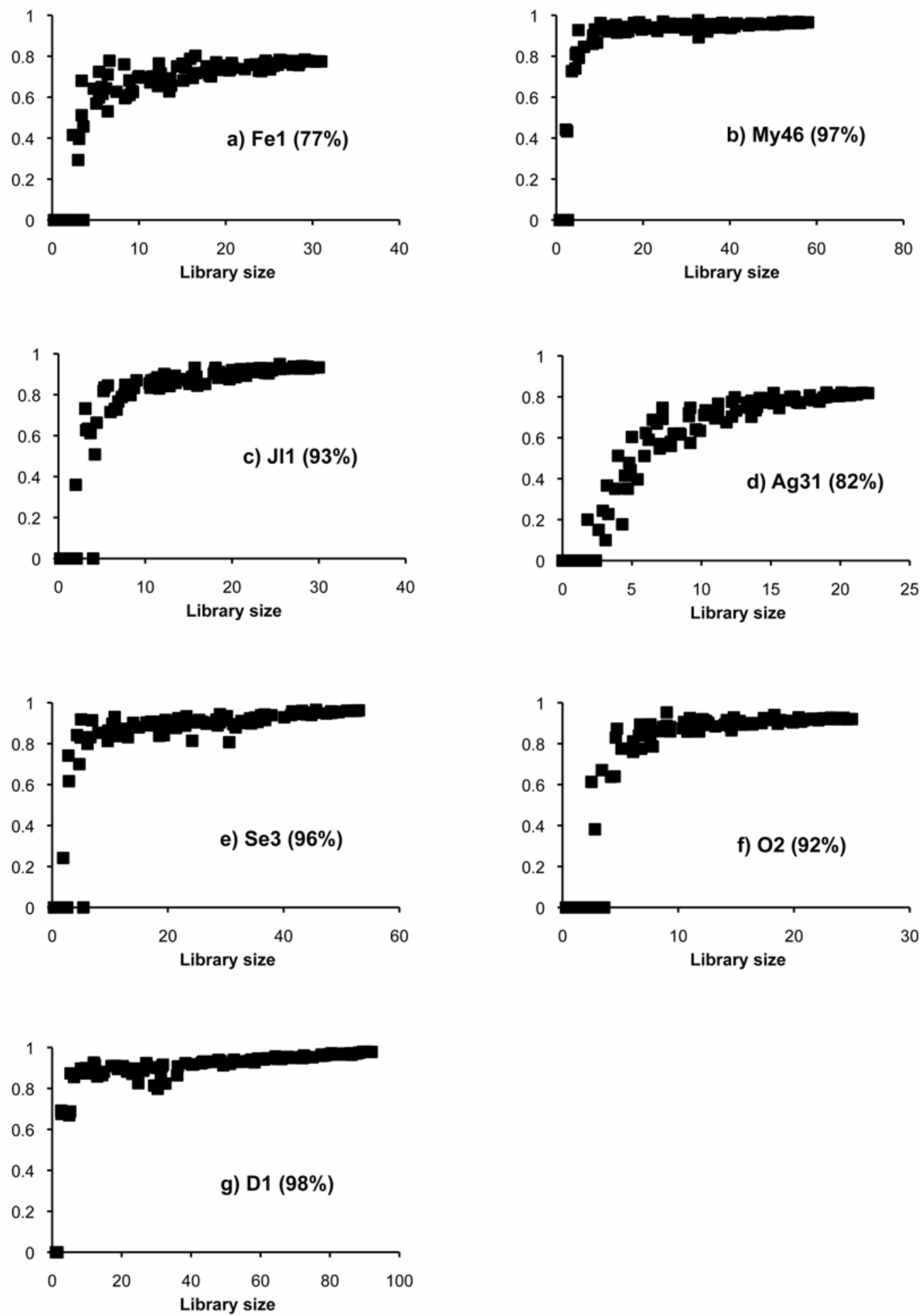

Figure S1. Clone library coverage based on Good's C estimator. The coverage value is indicated for each sample in parenthesis. 


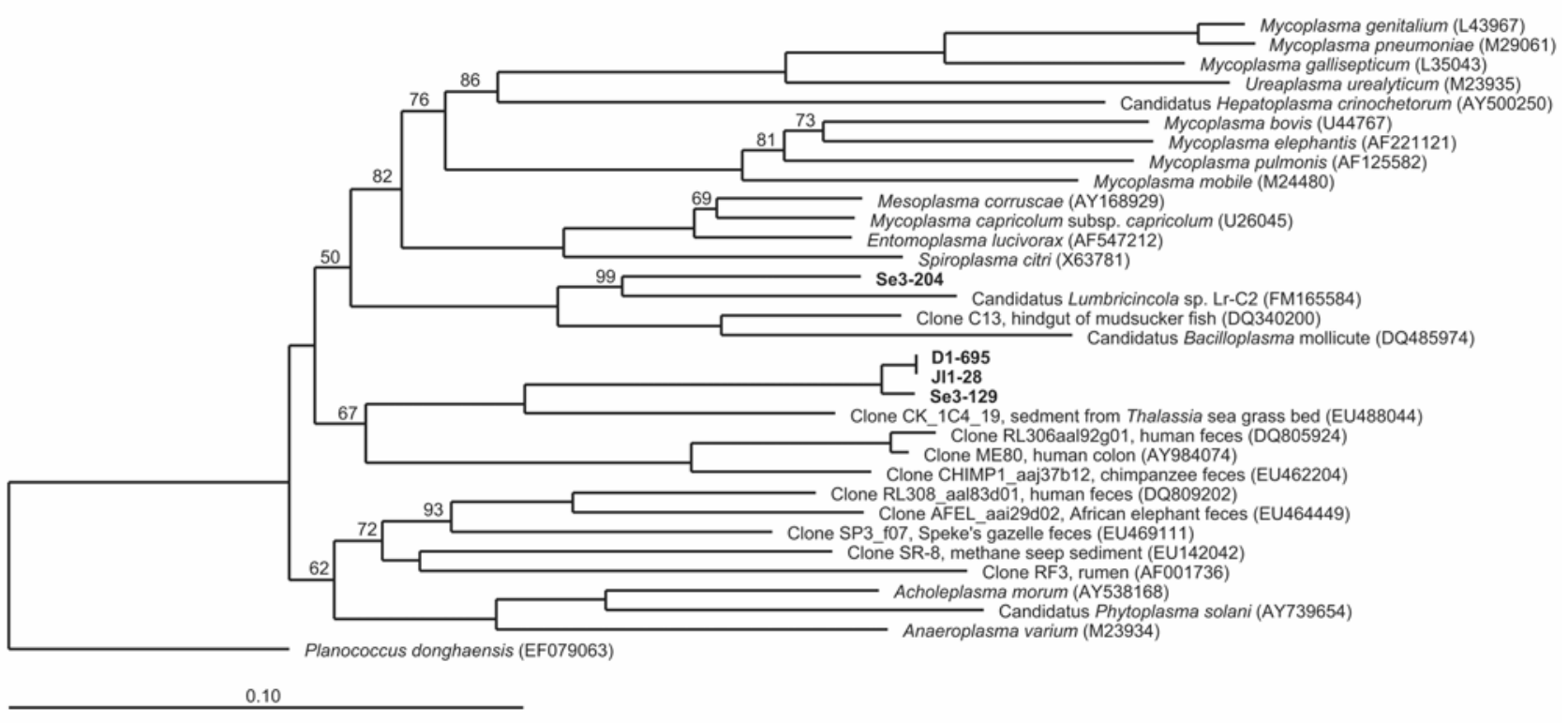

Figure S2. Neighbour-joining tree based on the Mollicutes 16S rRNA sequences from the midgut of Nephrops norvegicus. Planococcus donghaensis (Firmicutes) was used as an outgroup. The bar corresponds to $10 \%$ nucleotide difference and bootstrap values were calculated from 1000 replicate trees. Only bootstrap values over $50 \%$ are shown in the tree. 


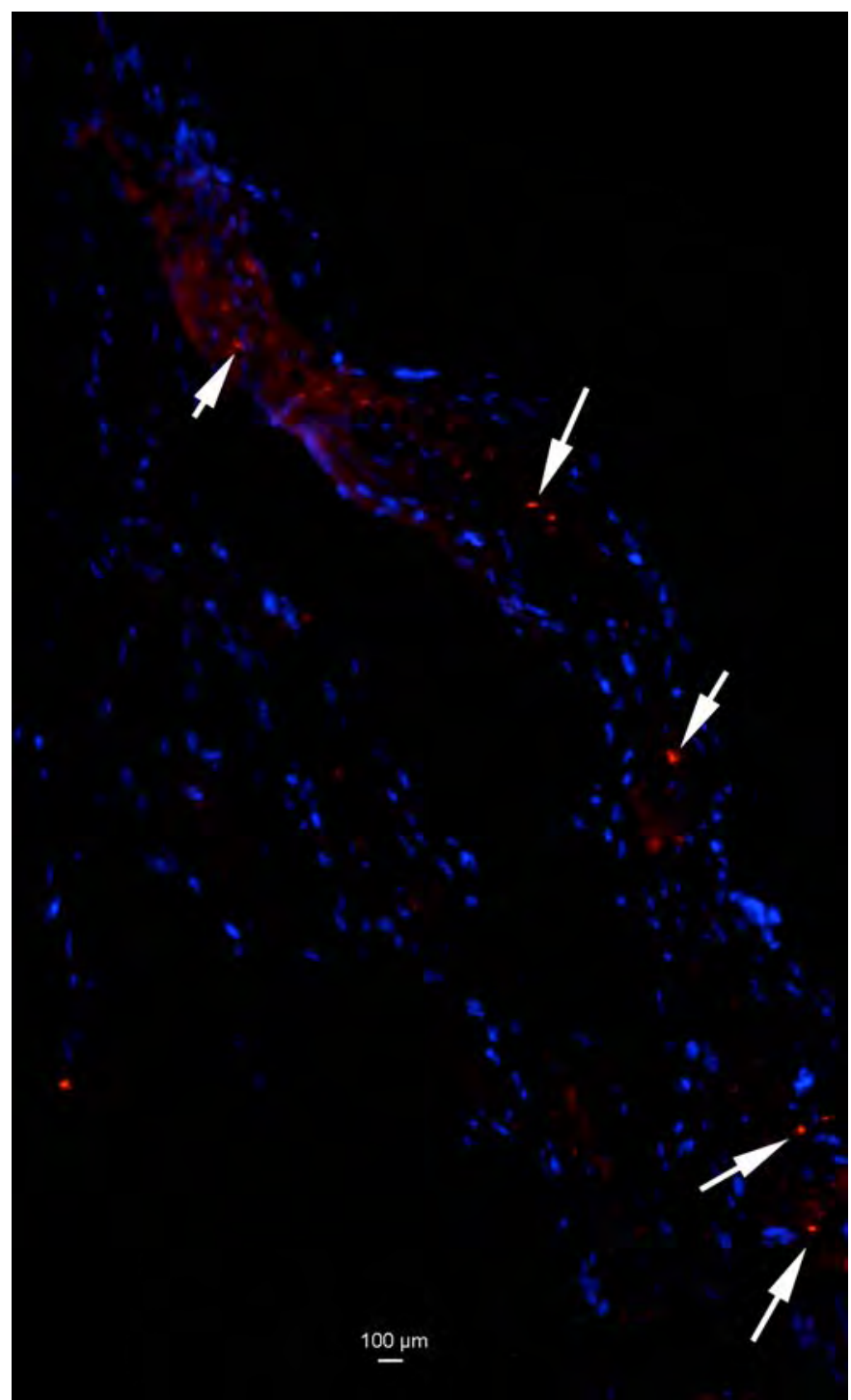

Figure S3. Gut section (thickness $4 \mu \mathrm{m}$ ) of Nephrops norvegicus sample FSE3 (September) hybridized with Cy3 labeled probe UncMol89 specific for uncultured Mollicutes phylotype Se3-204 detected in this study. Arrows indicate sarcina-like aggregates of Mollicutes. Blue signal corresponds to DAPI signal from the gut wall epithelial cells and red signal to the hybridized bacteria 


\section{Gut bacteria associated with different diets in reared Nephrops norvegicus}

Alexandra Meziti, Eleni Mente, Konstantinos Ar. Kormas

\section{ABSTRACT}

The impact of different diets on the gut microbiota of reared Nephrops norvegicus was investigated, based on the bacterial 16S rRNA gene diversity. $N$. norvegicus specimens were collected from Pagasitikos Gulf (Greece) and were kept in rearing tanks in the laboratory, reflecting in situ conditions, for six months. Animals were divided into three groups based on the supplied diet: frozen natural food (mussels, Group M), dry formulated pellet (Group P) and starvation (Group S). Gut samples were collected at the initiation of the experiment, and after three and six months. Water samples from the tanks were analyzed at all $N$. norvegicus sampling points as well as samples from the food provided. All samples were analyzed by studying the 16S rRNA bacterial diversity. Statistical analysis in gut samples showed the presence of two groups (G1 and G2) depending on whether food was provided or not ( $\mathrm{M}$ and P samples vs. S samples). No significant differences were detected between $\mathrm{M}$ and $\mathrm{P}$ samples. NMDS analysis in gut and water samples' bacterial communities revealed that starvation samples as well as three months mussel fed sample were more similar to the tanks' water samples than with the rest of the gut samples. Multiple diversity indices revealed higher diversity in the starvation samples. M samples were dominated by groups of $\gamma^{-}, \varepsilon$ - Proteobacteria and Tenericutes, while P samples were mostly dominated by $\gamma$-Proteobacteria. Phylotypes clustering in Photobacterium leiognathi, Shewanella sp. and the 
order Entomoplasmatales were abundant in the M and P samples but had low abundance in S samples. Our study showed that starvation of the animals induces a rather opportunistic bacterial community in the $N$. norvegicus gut, while food-induced changes resulted in the selection of specific species, which occur in the natural population, and might be related to the animal's nutrition. 